\title{
Global Structure of an Elliptic Fibration
}

\author{
By \\ Noboru NAKAYAMA*
}

\begin{abstract}
$\partial$-étale topology is introduced for analytic spaces with boundary as an analog of étale topology for schemes. A locally projective elliptic fibration is bimeromorphically considered as a torsor in the $\partial$-étale topology of the associated basic elliptic fibration. The related $\partial$-étale cohomology groups have much information on the structure of elliptic fibrations. In particular, an answer to Ueno's extension problem, a relation to Tate-Shafarevich groups and their finiteness properties, characterizations of projective and Kähler elliptic fibrations, and a generalization of logarithmic transformation to arbitrary dimension are obtained. This article is a revised version of [N5].
\end{abstract}

\section{Contents}

$\S 0$. Introduction

$\S 0.1$. Background

§0.2. Global structure: The results

$\S 0.3$. Notation

$\S 1 . \quad \partial$-space

$\S 1.1$. Category of $\partial$-spaces

$\S 1.2$. Profinite fundamental group

$\S 2 . \quad \partial$-étale Cohomology

\section{$\S 2.1$. Sheaf}

§2.2. Čech cohomology and right derived functor cohomology

$\S 2.3$. Coherent sheaf

Received June 1, 2001.

2000 Mathematics Subject Classification(s): 14J15, 14D06, 14E30, 32J27, 14D07, 32G20, 14J27.

Key words: elliptic fibration, minimal model, variation of Hodge structure.

* Research Institute for Mathematical Sciences, Kyoto University, Kyoto 606-8502, Japan. e-mail: nakayama@kurims.kyoto-u.ac.jp 
$\S 3 . \quad$ Cohomology Groups of Toroidal Embeddings

$\S 3.1$. Torus embedding

§3.2. Normal varieties with boundary

$\S 3.3$. Toroidal embedding

§3.4. $\partial$-spaces associated with toroidal embeddings

$\S 3.5$. Reflexive sheaf and parabolic structure

$\S 4$. Local Nature of Variation of Hodge Structure

$\S 4.1$. Monodromy and periods

$\S 4.2$. Canonical extension

$\S 4.3$. Group cohomology

$\S 4.4$. $\partial$-étale cohomology

$\S 5$. Elliptic Fibration

$\S 5.1$. Weierstrass model

$\S 5.2$. Classification problem

§5.3. Minimal basic elliptic fibration over $S^{\circ}$

$\S 5.4$. Fundamental diagram

$\S 5.5$. Elliptic fibration having local meromorphic sections

$\S 6$. Description of Global Structure

$\S 6.1$. Fundamental diagram in $\partial$-étale topology

$\S 6.2$. Calculation of cohomology groups

$\S 6.3$. Description of $\mathcal{E}(S, D, H)$

$\S 7$. Applications

$\S 7.1$. Extension of elliptic fibrations

§7.2. Tate-Shafarevich group

$\S 7.3$. Minimal models with trivial canonical divisor

§7.4. Kähler morphism

$\S 7.5$. New description of logarithmic transformations

§7.6. Logarithmic transformation on elliptic surfaces

References

\section{$\S 0 . \quad$ Introduction}

An elliptic fibration is a proper surjective morphism $f: X \rightarrow S$ of normal complex analytic varieties with general fibers being elliptic curves. In this paper, we fix the base space $S$ and consider the classification of elliptic fibrations up to the bimeromorphic equivalence relation over $S$. We assume that there is a Zariski-open subset $S^{\star} \subset S$ such that the open immersion is a toroidal 
embedding [K6]. Let $f^{\star}$ be the restriction $X^{\star}:=f^{-1} S^{\star} \rightarrow S^{\star}$ of $f$. Assume that $X$ is non-singular and that $f^{\star}$ is bimeromorphically equivalent to a smooth elliptic fibration over $S^{\star}$. Then the sheaf $H(f):=R^{1} f_{*}^{\star} \mathbb{Z}_{X^{\star}}$ forms a variation of Hodge structure of rank two and of weight one. $H(f)$ depends only on the bimeromorphic equivalence class of $f: X \rightarrow S$. This is naturally $\mathbb{Z}$-polarized by the trace map $R^{2} f_{*}^{\star} \mathbb{Z}_{X^{\star}} \rightarrow \mathbb{Z}_{S^{\star}}$ and induces a period mapping from the universal covering space of $S^{\star}$ into the upper-half plane $\mathbb{H}:=\{z \in \mathbb{C} \mid \operatorname{Im} z>0\}$ and a compatible monodromy representation $\pi_{1}\left(S^{\star}\right) \rightarrow \mathrm{SL}(2, \mathbb{Z})$. As a method of classification, we fix also a variation of Hodge structure $H$ of rank two and of weight one on $S^{\star}$ and consider marked elliptic fibrations $(f: X \rightarrow S, \phi)$ associated with $(S, D, H)$, where $D:=S \backslash S^{\star}$, defined as follows: $f: X \rightarrow S$ is an elliptic fibration from a non-singular variety such that its restriction $f^{\star}=\left.f\right|_{S^{\star}}$ is bimeromorphically equivalent to a smooth elliptic fibration; $\phi$ is an isomorphism $H(f) \leadsto H$ as variations of Hodge structure. Two marked elliptic fibrations are called mutually bimeromorphically equivalent if there is a bimeromorphic mapping between them over $S$ that commutes with each markings $\phi$. We denote by $\mathcal{E}(S, D, H)$ the set of bimeromorphic equivalence classes of marked elliptic fibrations $(f: X \rightarrow S, \phi)$ associated with $(S, D, H)$ such that, locally over $S, f$ is bimeromorphically equivalent to a projective morphism. We also consider its subset $\mathcal{E}^{\operatorname{proj}}(S, D, H)$ consisting of all the marked elliptic fibrations $(f: X \rightarrow S, \phi)$ such that $f$ is bimeromorphically equivalent over $S$ to a projective morphism.

There is an elliptic fibration $\boldsymbol{p}: \boldsymbol{B}(H) \rightarrow S$ uniquely up to the bimeromorphic equivalence relation over $S$ satisfying the following three conditions:

- $\boldsymbol{p}$ is smooth over $S^{\star}$;

- There is a meromorphic section $S \cdots \rightarrow \boldsymbol{B}(H)$ of $\boldsymbol{p}$;

- There is an isomorphism $\phi: H(\boldsymbol{p}) \leadsto H$.

The $\boldsymbol{p}$ is called a basic elliptic fibration associated with $H$. The element $(\boldsymbol{p}: \boldsymbol{B}(H) \rightarrow S, \phi) \in \mathcal{E}(S, D, H)$ is independent of the choice of $\phi$. The restriction

$$
\boldsymbol{p}^{\star}:=\left.\boldsymbol{p}\right|_{S^{\star}}: \boldsymbol{B}(H)^{\star}:=\boldsymbol{p}^{-1}\left(S^{\star}\right) \rightarrow S^{\star}
$$

is uniquely determined up to isomorphisms over $S^{\star}$ is called a smooth basic elliptic fibration. A construction of $\boldsymbol{B}(H)^{\star}$ from the data of period mapping and monodromy representation is explained in [K7]. For a given section $\boldsymbol{\sigma}^{\star}: S^{\star} \rightarrow \boldsymbol{B}(H)^{\star}, \boldsymbol{p}^{\star}$ has a group structure with $\boldsymbol{\sigma}^{\star}$ being zero: $\boldsymbol{p}^{\star}$ is a group object in the category $\mathcal{A} n / S^{\star}$ of complex analytic spaces over $S^{\star}$. 
For a given meromorphic section $\boldsymbol{\sigma}: S \cdots \rightarrow \boldsymbol{B}(H)$, the sheaf $\mathfrak{S}_{H / S}$ of germs of meromorphic sections has an abelian group structure with $\boldsymbol{\sigma}$ being zero. There is an identification $\mathcal{E}\left(S^{\star}, \emptyset, H\right) \longleftrightarrow H^{1}\left(S^{\star}, \mathfrak{S}_{H / S}\right)$. This means that any smooth elliptic fibration is a torsor of a smooth basic elliptic fibration. Let $\mathcal{E}_{0}(S, D, H) \subset \mathcal{E}(S, D, H)$ be the subset consisting of all elliptic fibrations $f: X \rightarrow S$ such that $f$ admits meromorphic sections locally on $S$. We infer that if $\operatorname{dim} S=1$, then $\mathcal{E}_{0}(S, D, H)$ is identified with $H^{1}\left(S, \mathfrak{S}_{H / S}\right)$ by Kodaira's theory of elliptic surfaces. It is not enough to use $\mathfrak{S}_{H / S}$ for describing $\mathcal{E}(S, D, H)$. We introduce a new category $\partial \mathrm{sP}$ of $\partial$-spaces containing the category $\mathcal{A} n$ of analytic spaces and a Grothendieck topology called $\partial$-étale topology on it. The $\partial \mathrm{sP}$ is the localization of the category $\mathcal{A B}$ of analytic spaces with boundary by $\partial$-isomorphisms. The pair $(S, D)$ defines a $\partial$-space $\underline{S}$ and $H$ defines a similar sheaf $\mathfrak{S}_{H / \underline{S}}$ of $\underline{S}$ in the $\partial$-étale topology. We shall show that $\mathcal{E}(S, D, H)$ is regarded as a subgroup of $H^{1}\left(\underline{S}, \mathfrak{S}_{H / \underline{S}}\right)$ and that $\mathcal{E}^{\operatorname{proj}}(S, D, H)$ is identified with $H^{1}\left(\underline{S}, \mathfrak{S}_{H / \underline{S}}\right)_{\text {tor }}$ (cf. 5.2.5, 6.3.2, 6.3.4 and 6.3.8). The following results are also obtained by the $\partial$-étale cohomology theory:

(1) An elliptic fibration $f: X \rightarrow S$ is bimeromorphically equivalent to a projective morphism if and only if there is a prime divisor of $X$ dominating $S$ (cf. 6.3.8).

(2) The description of local structure of projective elliptic fibrations (cf. 6.2.12 and 6.3.9).

(3) The answer to the following question posed by Ueno (cf. [F4, II, 1.15]) is negative: Does a smooth elliptic fibration $Y \rightarrow \boldsymbol{\Delta}^{2} \backslash\{(0,0)\}$ admitting no holomorphic sections extend to an elliptic fibration over $\Delta^{2}$ ? Here $\boldsymbol{\Delta}^{2}$ stands for the two-dimensional unit polydisc (cf. 0.3). A proof in a general form is given in Section 7.1.

(4) Tate-Shafarevich groups in algebraic situation are described by some $\partial$ étale cohomology groups (cf. 6.2.11, 7.2.1 and 7.2.2). Some results of Gross [G5] related to the boundedness of Calabi-Yau manifolds are generalized in Section 7.2 .

(5) A generalization of a result of Miyaoka [M5], [M6] is given in the following form in 7.4.4: For a compact complex manifold $X$ having an elliptic fibration over a d-dimensional compact Kähler manifold $S, X$ is bimeromorphically equivalent to a compact Kähler manifold if and only if $H^{2 d}(S, \mathbb{C}) \rightarrow H^{2 d}(X, \mathbb{C})$ is not zero. 
(6) The induced homomorphism $H^{0}\left(\underline{S}, \mathfrak{Q}_{H / \underline{S}}\right) \rightarrow H^{1}\left(\underline{S}, \mathfrak{S}_{H / \underline{S}}\right)$ from the distinguished triangle (6.8) describes logarithmic transformations (cf. Section $7.5)$.

(7) A logarithmic transform of a modular elliptic surface [S8] along its singular fiber is still a projective surface. On the other hand, a logarithmic transform of the modular elliptic surface along some smooth fiber is not projective. These are shown in Section 7.6.

\section{§0.1. Background}

We shall recall the background of the study of elliptic fibrations.

\section{Kodaira's theory}

First of all, we recall Kodaira's theory of elliptic surfaces ([K7], [K8]). This treats the case $S$ is a non-singular curve, i.e., elliptic fibrations over a non-singular curve. We recall this along the following four parts:

(I) Minimal Model. An elliptic surface is called minimal, if there exist no exceptional curves of the first kind in any fibers. This is equivalent to the condition that some multiple of the canonical bundle is the pullback of a line bundle on the base curve $S$. The minimal model of an elliptic surface is obtained by a successive contraction of exceptional curves of the first kind contained in fibers. If two elliptic surfaces are mutually bimeromorphically equivalent over the base curve, then their minimal models are isomorphic to each other. Thus the study of elliptic surfaces is reduced to that of minimal elliptic surfaces. A fiber $f^{-1}(s)=X \times_{S}\{s\}$ of an elliptic surface is called a singular fiber, if $f$ is not a smooth morphism along the fiber. Kodaira classified singular fibers of minimal elliptic surfaces by numerical calculation [K7]. The list of types is as follows: $\mathrm{I}_{a}, \mathrm{I}_{a}^{*},{ }_{m} \mathrm{I}_{a}, \mathrm{II}, \mathrm{II}^{*}$, III, III* ${ }^{*}, \mathrm{IV}, \mathrm{IV}^{*}$, where $a \geq 0$ and $m \geq 2$.

(II) Basic fibration. Let $H$ be a $\mathbb{Z}$-polarized variation of Hodge structure of rank two and of weight one on $S^{\star}$ and let $\boldsymbol{p}^{\star}: \boldsymbol{B}(H)^{\star} \rightarrow S^{\star}$ be the associated smooth basic elliptic surface with a section $\boldsymbol{\sigma}^{\star}: S^{\star} \rightarrow \boldsymbol{B}(H)^{\star}$. Kodaira constructed an extension $\boldsymbol{p}: \boldsymbol{B}(H) \rightarrow S$ of $\boldsymbol{p}^{\star}$ as a minimal elliptic surface and an extension $\boldsymbol{\sigma}: S \rightarrow \boldsymbol{B}(H)$ of $\boldsymbol{\sigma}^{\star}$. The fibration $\boldsymbol{p}$ is uniquely determined up to isomorphisms over $S$, which is called the basic fibration, basic family, or basic elliptic surface, etc. We recall briefly the construction. Assume that the base curve $S$ is a unit disc $\boldsymbol{\Delta}$ and $S^{\star}$ is the punctured disc $\boldsymbol{\Delta} \backslash\{0\}$. First, suppose that the order of monodromy of $H$ is finite. For the order $m$, let $\boldsymbol{\Delta}^{\prime} \ni t \mapsto t^{m} \in \boldsymbol{\Delta}$ be the finite cyclic covering. Then the monodromy of the 
pullback of $H$ is trivial. Thus the associated smooth basic elliptic surface defined over the punctured disc $\boldsymbol{\Delta}^{\prime} \backslash\{0\}$ canonically extends to a smooth basic elliptic surface $\boldsymbol{B}^{\prime} \rightarrow \boldsymbol{\Delta}^{\prime}$. The Galois group $\mathbb{Z} / m \mathbb{Z}$ acts holomorphically on $\boldsymbol{B}^{\prime}$ and the quotient space is a partial compactification of $\boldsymbol{B}(H)^{\star}$. By taking a resolution of the quotient singularities and by contracting exceptional curves of the first kind, we have the minimal elliptic surface $\boldsymbol{B}(H)$. Next, suppose that the order of monodromy of $H$ is infinite. Then we may assume that the monodromy matrix is unipotent by the same argument as above to a suitable cyclic covering. In this case, Kodaira's description of $\boldsymbol{B}(H)$ is interpreted by means of toroidal embedding. $\boldsymbol{B}(H)^{\star}$ is regarded as the quotient of $\mathbb{C}^{\star} \times S^{\star}$ by a suitable action of $\mathbb{Z}$ induced from $H$. There is a toroidal embedding $\mathbb{C}^{\star} \times S^{\star} \subset \mathcal{X}$ over $S^{\star} \subset S$ such that the fiber over $\{0\}$ is an infinite chain of smooth rational curves and that the action of $\mathbb{Z}$ holomorphically extends to a properly discontinuous and fixed point free action on $\mathcal{X}$. The quotient $\mathbb{Z} \backslash \mathcal{X}$ is the minimal elliptic surface $\boldsymbol{B}(H)$. (cf. [A], [N1], [N4]).

(III) Torsor. Let $\boldsymbol{B}(H)^{\sharp}$ be the maximal open subset of $\boldsymbol{B}(H)$ at which the fibration $\boldsymbol{p}: \boldsymbol{B}(H) \rightarrow S$ is smooth. Then the restriction $\boldsymbol{p}^{\sharp}: \boldsymbol{B}(H)^{\sharp} \rightarrow S$ has a group structure over $S$ which is an extension of $\boldsymbol{p}^{\star}$. There is a relative action of $\boldsymbol{p}^{\sharp}$ on $\boldsymbol{p}$ compatible with the open immersion $\boldsymbol{B}(H)^{\sharp} \subset \boldsymbol{B}(H)$. The $\boldsymbol{p}^{\sharp}$ is the so-called Néron model [N7]. Let $\mathfrak{S}_{H / S}$ be the sheaf of germs of sections of $\boldsymbol{p}^{\sharp}$. The cohomology group $H^{1}\left(S, \mathfrak{S}_{H / S}\right)$ is identical to the set of isomorphism classes of torsors of $\boldsymbol{p}^{\sharp}$. By the action of $\boldsymbol{p}^{\sharp}$ on $\boldsymbol{p}$, an element $\eta \in H^{1}\left(S, \mathfrak{S}_{H / S}\right)$ defines a minimal elliptic surface $\boldsymbol{p}^{\eta}: \boldsymbol{B}(H)^{\eta} \rightarrow S$. If a minimal elliptic surface has a section over a neighborhood of arbitrary point of $S$, then it is isomorphic to $\boldsymbol{B}^{\eta}(H) \rightarrow S$ for some $\eta \in H^{1}\left(S, \mathfrak{S}_{H / S}\right)$.

(IV) Multiple fiber. A multiple fiber is a fiber which has no reduced components. It is of type ${ }_{m} \mathrm{I}_{a}$ with $m \geq 2$. A non-multiple fiber appears as a fiber of a minimal basic fibration. A multiple fiber $f^{-1}(s)$ turns to be non-multiple by a suitable ramified covering over a neighborhood of $s$. In other words, a multiple fiber is locally obtained as a fiber of the quotient of basic elliptic surfaces. Kodaira fixed a finite Galois covering $S^{\prime} \rightarrow S$ and considered the set of isomorphism classes of minimal elliptic surfaces $f: X \rightarrow S$ over $S$ with an isomorphism $H(f) \leadsto H$ such that the pullback by $S^{\prime} \rightarrow S$ turns to be an elliptic surface without multiple fibers. This set is described as a suitable cohomology group determined by $S^{\prime}, H$, and the Galois group of $S^{\prime} \rightarrow S$. In [K8], Kodaira gave another description of multiple fibers by means of logarithmic transformation. The logarithmic transformation does not change the complement of the given fiber but a neighborhood of the fiber. A multiple fiber is obtained by 
a logarithmic transformation from a non-multiple fiber. The induced isomorphism between the complements of fibers is written by a logarithmic function and is not algebraic: it does not extend to a bimeromorphic mapping.

As a consequence, an elliptic surface is constructed by a variation of Hodge structure $H$, the twist by an element $\eta \in H^{1}\left(S, \mathfrak{S}_{H / S}\right)$, and by logarithmic transformations. This theory is one of the origins of studies of degenerations of curves, surfaces, and abelian varieties. In the study of purely algebraic elliptic fibrations, there are other important objects such as Néron models, Tate-Shafarevich groups, and Mordell-Weil groups.

\section{Generalization to higher dimension}

A degeneration should be a fibration over a curve or, more generally, a flat fibration. Non-flat fibrations are not yet studied well. Secondly, we recall known results on elliptic fibrations over a higher dimensional base space $S$ along the same four parts.

(I) Minimal Model. The minimal model theory for higher dimensional projective varieties was developed in 1980's, but the main difficult conjectures, flip and abundance conjectures, are proved only in dimension up to three (cf. [M7], [K4], [M8], [K3]). Under the assumption of the flip conjecture, the construction of minimal model is generalized to the case of relatively projective morphisms of complex analytic varieties [N2]. A projective elliptic fibration $f: X \rightarrow S$ is called minimal if $X$ has only terminal singularities and the canonical $\mathbb{Q}$-line bundle $K_{X}$ is relatively nef: $K_{X} \cdot \gamma \geq 0$ for any curves $\gamma$ contained in fibers. However, the minimal model for a given variety is not necessarily uniquely determined if the dimension is greater than two. These minimal models are connected by a successive operations called flops. Projective elliptic fibrations over a surface are studied by the method of minimal model theory in [N4, Appendix]. As a consequence, the study is reduced to that of standard elliptic fibrations, which are equi-dimensional and locally $\mathbb{Q}$-factorial over the base surfaces.

There are interesting examples of non-projective elliptic fibrations. For example, an elliptic fibration over a two-dimensional polydisc is smooth outside the origin but the central fiber is isomorphic to a Hopf surface or its multiple (cf. [N4, Section 3]).

(II) Basic fibration. Suppose that $S$ is non-singular and that $S \backslash S^{\star}$ is a normal crossing divisor. Let $H$ be a $\mathbb{Z}$-polarized variation of Hodge structure of rank two and of weight one defined over $S^{\star}$. In the case $S$ is a surface, Kawai [K1] constructed an extension $\boldsymbol{p}: \boldsymbol{B}(H) \rightarrow S$ of the smooth basic elliptic 
fibration $\boldsymbol{p}^{\star}: \boldsymbol{B}(H)^{\star} \rightarrow S^{\star}$ as a projective elliptic fibration with holomorphic section. Ueno [U1] generalized the construction to arbitrary dimension and obtained a desingularization of $\boldsymbol{B}(H)$. But the induced fibration is not always minimal in the sense of minimal model theory. A Weierstrass model $\mathbf{p}: \mathbf{W} \rightarrow S$ is defined as a relative effective divisor of a $\mathbb{P}^{2}$-bundle over $S$ whose local defining equation is expressed as a Weierstrass form: $\mathrm{Y}^{2} \mathrm{Z}=\mathrm{X}^{3}+\boldsymbol{\alpha} \mathrm{XZ}^{2}+\boldsymbol{\beta} \mathrm{Z}^{3}$ for a homogeneous coordinate (X : Y : Z) of $\mathbb{P}^{2}$ and for suitable functions $\boldsymbol{\alpha}, \boldsymbol{\beta}$ locally defined over $S$. For the precise definition, see [N3] or Section 5.1. The following theorem is easily shown in algebraic situation and is proved also in analytic case $[\mathrm{N} 3,2.1]$ :

Theorem. Let $X \rightarrow S$ be an elliptic fibration admitting a meromorphic section $S \cdots \rightarrow X$. Then there is a bimeromorphic mapping $X \cdots \rightarrow \mathbf{W}$ into a Weierstrass model which sends the meromorphic section to the canonical section $\mathrm{X}=\mathrm{Z}=0$.

The author showed in [N3, Section 2] that a Weierstrass model $\mathbf{W}(H)$ is canonically constructed from a given variation of Hodge structure $H$ as an extension of $\boldsymbol{B}(H)^{\star}$ and that $\mathbf{W}(H)$ has only rational Gorenstein singularities with relatively trivial canonical sheaf. Hence $\mathbf{W}(H)$ is a minimal model with only canonical singularities. If $S$ is a curve, then $\mathbf{W}(H)$ is obtained from the minimal elliptic surface $\boldsymbol{B}(H)$ by contractions of irreducible components of singular fibers away from the zero section. We define a basic elliptic fibration $\boldsymbol{p}: \boldsymbol{B}(H) \rightarrow S$ associated with $H$ to be an elliptic fibration admitting a meromorphic section and an isomorphism $H(\boldsymbol{p}) \simeq H$.

In purely algebraic situation, for a given algebraic elliptic fibration $\mathrm{f}: \mathrm{X} \rightarrow$ $\mathrm{S}$, the generic fiber $\mathrm{X}_{\eta}$ is a smooth projective curve of genus one defined over the function field $\mathbb{C}(\eta)=\mathbb{C}(\mathrm{S})$. It is called an elliptic curve only when it contains a $\mathbb{C}(\eta)$-valued point, equivalently, $\mathbf{f}$ has a rational section. The Jacobian $\mathrm{J}\left(\mathrm{X}_{\eta}\right)$ of $\mathrm{X}_{\eta}$ is well-defined over $\mathbb{C}(\eta)$ and it extends to a basic elliptic fibration $\mathrm{J} \rightarrow \mathrm{S}$. The Jacobian $\mathrm{J}\left(\mathrm{X}_{\eta}\right)$ is considered as an invariant instead of the variation of Hodge structure $H$. A basic fibration is therefore called a Jacobian fibration in some articles.

Miranda [M4] studied minimal models for elliptic fibrations over surfaces having global sections by using Weierstrass equations. He obtained a resolution of singularities of the Weierstrass model by blowing up the base surface and by looking at the change of equations. The obtained threefold gives a minimal and flat elliptic fibration over the blown up surface.

(III) Torsor. Ueno [U1] described the set of elliptic fibrations over a surface $S$ which have meromorphic sections locally over $S$ as a suitable cohomology 
group similar to $H^{1}\left(S, \mathfrak{S}_{H / S}\right)$. In the definition of cohomology group, there is a delicate thing over double points of the discriminant locus. In purely algebraic situation, Tate-Shafarevich group $\amalg_{S}\left(B_{\eta}\right)$ corresponds to the cohomology group. Here, $\mathrm{p}: \mathrm{B} \rightarrow \mathrm{S}$ is an algebraic basic elliptic fibration associated with $H, \eta$ is the generic point of $\mathrm{S}$, and $\mathrm{B}_{\eta}$ is the elliptic curve over $\operatorname{Spec} \mathbb{C}(\eta)$. The Tate-Shafarevich group classifies birational equivalence classes of pairs

$$
\left(\mathrm{f}: \mathrm{X} \rightarrow \mathrm{S}, \quad \Phi: \mathrm{X}_{\eta} \times_{\mathbb{C}(\eta)} \mathrm{X}_{\eta} \rightarrow \mathrm{B}_{\eta} \times_{\mathbb{C}(\eta)} \mathrm{X}_{\eta}\right)
$$

consisting of a projective elliptic fibration $f$ and an isomorphism $\Phi$ between elliptic fibrations over $X_{\eta}$ via the second projections such that $f$ admits a section étale locally over $S$ and that $\Phi$ sends the diagonal to the zero section. The group is expressed as the étale cohomology group $H^{1}\left(\mathrm{~S}_{\text {ét }}, \iota_{*} \mathrm{~B}_{\eta}\right)$ for the étale sheaf $\mathrm{B}_{\eta}$ over Spec $\mathbb{C}(\eta)$ and for $\iota: \operatorname{Spec} \mathbb{C}(\eta) \rightarrow \mathrm{S}($ cf. [D5]).

(IV) Multiple fiber. Ueno [U2] constructed some examples of multiple fibers. He performed a logarithmic transformation along a smooth divisor in the case local monodromies are trivial. The logarithmic transformation is also studied by Fujimoto [F4]. Let $\mathrm{p}: \mathrm{B} \rightarrow \mathrm{S}$ be an algebraic basic elliptic fibration that is smooth over $S^{\star} \subset S$. Then Tate-Shafarevich group $\amalg_{S^{\star}}\left(B_{\eta}\right)$ over $S^{\star}$ describes projective elliptic fibrations over $S$ that are smooth over $S^{\star}$. Thus a fibration having multiple fibers belongs to the complement $\amalg_{S^{\star}}\left(B_{\eta}\right) \backslash \amalg_{S}\left(B_{\eta}\right)$. Dolgachev and Gross [D5], [G4] considered Tate-Shafarevich groups in order to analyze multiple fibers.

\section{Local structure}

In local situation on the base space $S$, projective elliptic fibrations $f: X \rightarrow$ $S$ that are smooth outside a fixed normal crossing divisor $D$ are bimeromorphically classified in [N4]. Thirdly, we recall results obtained in [N4]. Assume that $S$ is a unit polydisc $\boldsymbol{\Delta}^{d}$ and that $D$ is a union of coordinate hyperplanes. In the case the variation of Hodge structure $H$ on $S^{\star}=S \backslash D$ has only unipotent monodromies, a toric model (or a smooth model) is constructed as a non-singular minimal model of $\boldsymbol{B}(H) \rightarrow S$. The construction is a generalization of that by Nakamura [N1] for degeneration of principal abelian varieties. The flops between toric models are described by suitable graphs. Further the following result is obtained:

Theorem ([N4, Section 4]). Let $f: X \rightarrow S=\Delta^{d}$ be a projective elliptic fibration smooth over $S \backslash D$. Then there is a finite ramified covering $S^{\prime}=\boldsymbol{\Delta}^{d} \rightarrow S$ such that

(1) $S^{\prime} \rightarrow S$ is ramified only along $D$, 
(2) the pullback $X \times_{S} S^{\prime} \rightarrow S^{\prime}$ is bimeromorphically equivalent to a toric (or a smooth) model over $S^{\prime}$.

An essential idea of the proof is considering torsors over $S \backslash \operatorname{Sing} D$. The corresponding results to the four parts above are as follows:

(I) A minimal model is constructed as a toric or a smooth model in the case $H$ has only unipotent monodromies.

(II) The variations of Hodge structure $H$ on $S^{\star}$ are classified. The list of types of monodromy of $H$ is as follows (cf. 4.1.1): $\mathrm{I}_{0}, \mathrm{I}_{0}^{(*)}, \mathrm{II}^{(*)}, \mathrm{III}^{(*)}, \mathrm{IV}_{+}^{(*)}$, $\mathrm{IV}_{-}^{(*)}, \mathrm{I}_{(+)}, \mathrm{I}_{(+)}^{(*)}$. The period functions are written explicitly in each types. A basic elliptic fibration $\boldsymbol{B}(H) \rightarrow S$ can be chosen to be a relatively minimal model over $S \backslash \operatorname{Sing} D$.

(III) and (IV) Even if there are no multiple fibers over $D \backslash \operatorname{Sing} D$, the fibration may not admit a section. But, there is a finite Galois covering $S^{\prime} \rightarrow S$ ramified only along $D$ such that the pullback admits a meromorphic section. The covering depends on the monodromy of $H$ and the multiplicities of fibers over $D \backslash \operatorname{Sing} D$. Let $G$ be the Galois group of $S^{\prime} \rightarrow S$ and let $\mathfrak{S}^{\prime}$ be the sheaf of germs of meromorphic sections of the associated basic elliptic fibration $\boldsymbol{B}^{\prime} \rightarrow S^{\prime}$. Then an elliptic fibration corresponds to a cohomology class of $H^{1}\left(G, H^{0}\left(S^{\prime}, \mathfrak{S}^{\prime}\right)\right)$ for such a covering $S^{\prime} \rightarrow S$. The possible Galois actions on $\boldsymbol{B}^{\prime}$, which are sometimes only meromorphic actions, are classified. Thus we can list all the collision of singular fibers including multiple fibers. For an ideal classification of germs of projective elliptic fibrations, we need to obtain a suitable quotient by such meromorphic action and to describe its minimal model.

\section{$\S 0.2$. Global structure: The results}

The purpose of this paper is a globalization of the local classification in [N4]. We shall explain our results more precisely than before. We fix a normal complex analytic variety $S$ and a reduced effective divisor $D$ such that $S^{\star}:=$ $S \backslash D \subset S$ is a toroidal embedding. We also fix a $\mathbb{Z}$-polarized variation of Hodge structure $H$ of rank two and weight one defined on $S^{\star}$. Our aim is to describe the set $\mathcal{E}(S, D, H)$ of all the bimeromorphic equivalence classes of pairs $(f: X \rightarrow S, \phi)$ consisting of an elliptic fibration $f: X \rightarrow S$ and a marking $\phi$ satisfying the following conditions:

- The restriction $\left.f\right|_{f^{-1} S^{\star}}: f^{-1} S^{\star} \rightarrow S^{\star}$ is bimeromorphically equivalent to a smooth elliptic fibration $f^{\prime \star}: X^{\prime \star} \rightarrow S^{\star}$ over $S^{\star}$;

- Locally over $S, f$ is bimeromorphically equivalent to a projective morphism; 
- $\phi$ is an isomorphism $H(f):=H\left(f^{\prime \star}\right):=R^{1}{f^{\prime \star}}_{*} \mathbb{Z}_{X^{\prime \star}} \leadsto H$ of variations of Hodge structure.

Note that, in algebraic situation, the definition of $\mathcal{E}(S, D, H)$ looks like that of Tate-Shafarevich group $\amalg_{S^{\star}}\left(B_{\eta}\right)$, where $B \rightarrow S$ is a basic elliptic fibration inducing $H$ as a variation of Hodge structure. However, information only from the complex analytic space $S^{\star}=S \backslash D$ and from $H$ is not enough to determine $\mathcal{E}(S, D, H)$ in the complex analytic situation (cf. 5.2).

In order to treat multiple fibers, we introduce the category $\partial \mathrm{sP}$ of $\partial$-spaces and a Grothendieck topology named $\partial$-étale topology on it; Let $[X, B]$ be a pair of complex analytic space $X$ and its nowhere-dense analytic subset $B$. An object of $\partial \mathrm{sP}$ is an equivalence class $(X, B)$ of such pairs $[X, B]$. A morphism $f:[X, B] \rightarrow[Y, D]$ is called a $\partial$-étale morphism, if $f: X \rightarrow Y$ has only discrete fibers, $B=f^{-1}(D)$, and if $X \backslash B \rightarrow Y \backslash D$ is a local isomorphism. The $\partial-$ étale morphisms define the $\partial$-étale topology on $\partial \mathrm{sP}$. We next establish $\partial$-étale cohomology theory. As a result, these cohomology groups can be calculated by Čech cocycles and there exist Leray's spectral sequences for morphisms in $\partial$ sp. We define the space $\operatorname{sp}(\underline{X})$ of a $\partial$-space $\underline{X}:=(X, B)$ as a reduced analytic space and define the stalk $F_{x}$ of a sheaf $F$ at a point $x \in \operatorname{sp}(\underline{X})$. The stalk has a structure of discrete $\hat{\pi}_{1}^{\text {loc }}(\underline{X} ; x)$-module, where $\hat{\pi}_{1}^{\text {loc }}(\underline{X} ; x)$ is the local profinite fundamental group at $x$. Let $\varepsilon: \underline{X}=(X, B) \rightarrow X=(X, \emptyset)$ be the canonical morphism and assume that $X$ is homeomorphic to $\operatorname{sp}(\underline{X})$. Then the stalk of $R^{p} \varepsilon_{*} F$ at a point $x \in X$ is isomorphic to the continuous group cohomology $H_{\text {cont. }}^{p}\left(\hat{\pi}_{1}^{\text {loc }}(\underline{X} ; x), F_{x}\right)$. In particular, $H^{p}(\underline{X}, F) \simeq H^{p}\left(X, \varepsilon_{*} F\right)$ for any sheaves $F$ of $\mathbb{Q}$-vector spaces. In the case $X \backslash B \subset X$ is a toroidal embedding, $H^{p}(\underline{X}, \mathbb{Z})$ are calculated in 3.4.2.

Let $(f: X \rightarrow S, \phi)$ be a marked elliptic fibration as before. Then there is a $\partial$-étale covering family

$$
\left\{\underline{U}_{\lambda}:=\left(U_{\lambda}, \Delta_{\lambda}\right) \rightarrow \underline{S}:=(S, D)\right\}_{\lambda \in \Lambda},
$$

such that each $X \times{ }_{S} U_{\lambda} \rightarrow U_{\lambda}$ has a meromorphic section. This is proved in 6.3.4 by a different method from [N4, Section 4]. Thus we have bimeromorphic mappings $X \times{ }_{S} U_{\lambda} \cdots \rightarrow \boldsymbol{B}(H) \times{ }_{S} U_{\lambda}$, where $\boldsymbol{p}: \boldsymbol{B}(H) \rightarrow S$ is a fixed basic elliptic fibration associated with $H$. The induced meromorphic transition mapping

$$
\boldsymbol{B}(H) \times_{S}\left(U_{\lambda} \times_{S} U_{\mu}\right) \cdots \rightarrow \boldsymbol{B}(H) \times_{S}\left(U_{\lambda} \times{ }_{S} U_{\mu}\right)
$$

over $U_{\lambda} \times{ }_{S} U_{\mu}$ is expressed as the translation mapping of a meromorphic section of $\boldsymbol{B}(H) \times_{S}\left(U_{\lambda} \times_{S} U_{\mu}\right)$, since it preserves the marking phi. Let $\mathfrak{S}_{H / \underline{S}}$ be the sheaf in $\partial$-étale topology on $\underline{S}$ of germs of meromorphic sections of 
$\boldsymbol{p}: \boldsymbol{B}(H) \rightarrow S$. Then the marked elliptic fibration defines an element in $H^{1}\left(\underline{S}, \mathfrak{S}_{H / \underline{S}}\right)$. Roughly speaking, such elliptic fibrations look like torsors of the basic elliptic fibration with respect to $\partial$-étale topology. We have:

Theorem $(6.3 .2,6.3 .4,6.3 .8)$. There is a natural injection $\mathcal{E}(S, D, H)$ $\hookrightarrow H^{1}\left(\underline{S}, \mathfrak{S}_{H / \underline{S}}\right)$ under which the subset $\mathcal{E}^{\operatorname{proj}}(S, D, H)$ is identified with the torsion part $H^{1}\left(\underline{S}, \mathfrak{S}_{H / \underline{S}}\right)_{\text {tor }}$.

Therefore, a projective elliptic fibration is really constructed from a torsion element of $H^{1}\left(\underline{S}, \mathfrak{S}_{H / \underline{S}}\right)$. For the calculation of $H^{1}\left(\underline{S}, \mathfrak{S}_{H / \underline{S}}\right)$, the fundamental diagram Figure 3 (cf. 6.1 .5 ) is important. In particular, the exact sequence

$$
0 \rightarrow \underline{j}_{*} H \rightarrow \mathcal{L}_{H / \underline{S}} \rightarrow \mathfrak{S}_{H / \underline{S}} \rightarrow \mathfrak{T}_{H / \underline{S}} \rightarrow 0
$$

and the distinguished triangle (6.8)

$$
\cdots \stackrel{+1}{\rightarrow} \tau_{\leq 1} R \underline{j}_{*} H \rightarrow \mathcal{L}_{H / \underline{S}} \oplus \mathfrak{Q}_{H / \underline{S}}[-1] \rightarrow \mathfrak{S}_{H / \underline{S}} \stackrel{+1}{\longrightarrow} \cdots
$$

are very useful. Here, $\underline{j}: S^{\star} \hookrightarrow \underline{S}$ is the $\partial$-open immersion and $\mathcal{L}_{H / \underline{S}}$ is the invertible sheaf determined as the $\mathrm{Gr}_{\mathcal{F}}^{0}$ of the canonical extension of $H$ with respect to the filtration $\mathcal{F}$ induced from the Hodge filtration (cf. 4.2.1). The sheaf $\mathfrak{T}_{H / S}$ is a subsheaf of $R^{1} \underline{j}_{*} H$ defined in the argument preceding 6.1.5 and $\mathfrak{Q}_{H / \underline{S}}=R^{1} \underline{j}_{*} H / \mathfrak{T}_{H / \underline{S}}$. If $\bar{S}^{*}$ is a curve, $\mathfrak{T}_{H / \underline{S}}$ is the sheaf of the groups of irreducible components of singular fibers of Néron model. It is not clear $H^{1}\left(\underline{S}, \mathfrak{S}_{H / \underline{S}}\right)=\mathcal{E}(S, D, H)$. The example 6.3 .14 is a candidate of an element of the complement of $\mathcal{E}(S, D, H)$.

The construction of this paper is as follows: The category of $\partial$-spaces is introduced in Section 1, and the $\partial$-étale cohomology theory is developed in Section 2. In Section 3, some $\partial$-étale cohomology groups are calculated in the case of toroidal embeddings. Further, a relation between reflexive sheaves in the $\partial$-étale topology and parabolic sheaves is mentioned in Section 3.5. Section 4 is devoted to the study of local nature of variation of Hodge structures of rank two and of weight one which are defined on the open part of a toroidal embedding. The set $\mathcal{E}(S, D, H)$ is introduced and a fundamental diagram Figure 1 in the usual topology is obtained in Section 5. The essential use of $\partial$-étale cohomology for determining $\mathcal{E}(S, D, H)$ appears in Section 6 , where most essential results are derived. Section 7 is devoted to the applications. Section 7.1 treats extension problems, especially the problem of Ueno. In Sections 7.2 and 7.3, the Tate-Shafarevich group in purely algebraic situation is interpreted by means of the $\partial$-étale cohomology theory, and some results of Dolgachev and Gross in [D5], [G4], [G5] are generalized. Kähler morphisms are studied in 
Section 7.4. Besides a generalization of Miyaoka's result, a characterization of cohomologically Kähler elliptic fibration is given. The notion of logarithmic transformation is interpreted as a homomorphism of some $\partial$-étale cohomology groups in Section 7.5. Some partial results on the problem when the logarithmic transform of a basic elliptic surface is projective are obtained in Section 7.6.

\section{Acknowledgement}

The author expresses his gratitude to Professors Akira Fujiki, Yoshio Fujimoto, Yoichi Miyaoka, Shigefumi Mori, and Masataka Tomari. Professors Mori and Tomari kindly informed him a property of discrete fibers. Professor Fujiki pointed out a mistake in an earlier version at a seminar talk. Professor Fujimoto informed him the problem of Ueno. He had meaningful discussion with Professors Fujimoto and Miyaoka very often. He is also grateful for the encouragement of Professors Mori and Miyaoka.

The first version [N5] of this article appeared in the preprint series of Research Institute for Mathematical Sciences (RIMS) in April 1996. The author gave a series of talks on the article in a seminar at RIMS between November 1999 and June 2000. By the experience, he could modify some mistakes in the version and could enlarge the story to the present form. He thanks to the attendants: Professors Fujimoto, Osamu Fujino, Daisuke Matsushita and Hiromichi Takagi. He is also grateful to Professor Fujimoto for the careful reading of the manuscript. He thanks very much to Professor Mitsuru Kawazoe who pointed out a mistake in 7.6.7 of the last version.

\section{$\S 0.3 . \quad$ Notation}

Analytic space. A complex analytic space is always assumed to be paracompact and Hausdorff. A variety is an irreducible and reduced analytic space. A Zariski-open subset is the complement of an analytic subset.

Disc. The unit disc is denoted by $\boldsymbol{\Delta}:=\{t \in \mathbb{C}|| t \mid<1\}$ and the punctured disc $\boldsymbol{\Delta} \backslash\{0\}$ is denoted by $\boldsymbol{\Delta}^{\star}$. The upper-half plane and the universal covering map of $\boldsymbol{\Delta}^{\star}$ are written by

$$
\mathrm{e}: \mathbb{H}:=\{z \in \mathbb{C} \mid \operatorname{Im} z>0\} \ni z \longmapsto \mathrm{e}(z):=\exp (2 \pi \sqrt{-1} z) \in \boldsymbol{\Delta}^{\star} .
$$

The $n$-fold product $\boldsymbol{\Delta}^{n}=\boldsymbol{\Delta} \times \cdots \times \boldsymbol{\Delta}$ is called an $n$-dimensional unit polydisc. 
Exponential sequence. We write $\mathbb{C}^{\star}=\mathbb{C} \backslash\{0\}$ for the complex number field $\mathbb{C}$. For a complex analytic variety $X, \mathcal{O}_{X}$ denotes the sheaf of germs of holomorphic functions. $\mathcal{O}_{X}^{\star}$ is the sheaf of germs of unit (or invertible) holomorphic functions whose abelian group structure is given by the multiplication. Let $\mathcal{O}_{X} \rightarrow \mathcal{O}_{X}^{\star}$ be the homomorphism given by $f \mapsto \mathrm{e}(f)=\exp (2 \pi \sqrt{-1} f)$. The induced exact sequence

$$
0 \rightarrow \mathbb{Z}_{X} \rightarrow \mathcal{O}_{X} \rightarrow \mathcal{O}_{X}^{\star} \rightarrow\{1\}=0
$$

is called the exponential sequence of $X$.

Complex. Let $K^{\bullet}=\left[\cdots \rightarrow K^{p} \stackrel{\mathrm{d}^{p}}{\rightarrow} K^{p+1} \rightarrow \cdots\right]$ be a complex of objects of an abelian category $\mathcal{A}$. The shift $K^{\bullet}[k]$ by an integer $k$ is defined by

$$
\left(K^{\bullet}[k]\right)^{p}=K^{p+k}, \quad \mathrm{~d}_{K \bullet[k]}^{p}=(-1)^{k} \mathrm{~d}_{K^{\bullet}}^{p+k} .
$$

$K^{\bullet}$ is called bounded if $K^{p}=0$ for $p \ll 0$ and for $p \gg 0$. A morphism of complexes $K_{1}^{\bullet} \rightarrow K_{2}^{\bullet}$ is called a quasi-isomorphism if it induces isomorphisms on cohomologies. The derived category $\mathcal{D}(\mathcal{A})$ is the localization of the category of complexes of $\mathcal{A}$ by quasi-isomorphisms. We write $K_{1}^{\bullet} \sim_{\text {qis }} K_{2}^{\bullet}$ if $K_{1}^{\bullet}$ and $K_{2}^{\bullet}$ are quasi-isomorphic. There is a notion of distinguished triangle

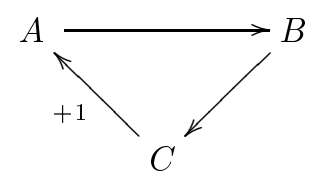

in $\mathcal{D}(\mathcal{A})$. We call it simply by a triangle and write it in the form

$$
\cdots \stackrel{+1}{\longrightarrow} A \rightarrow B \rightarrow C \stackrel{+1}{\longrightarrow} \cdots
$$

For an integer $k$, the truncations $\tau_{\leq k}$ and $\tau_{\geq k}$ are defined by

$$
\begin{aligned}
& \tau_{\leq k} K^{\bullet}:=\left[\cdots \rightarrow K^{p} \rightarrow K^{p+1} \rightarrow \cdots \rightarrow K^{k-1} \rightarrow \operatorname{Kerd}^{k} \rightarrow 0 \rightarrow \cdots\right], \\
& \tau_{\geq k} K^{\bullet}:=\left[\cdots \rightarrow 0 \rightarrow \operatorname{Imd}^{k-1} \rightarrow K^{k} \rightarrow \cdots \rightarrow K^{p} \rightarrow K^{p+1} \rightarrow \cdots\right] .
\end{aligned}
$$

These are well-defined in $\mathcal{D}(\mathcal{A})$ and there is a natural triangle

$$
\cdots \stackrel{+1}{\longrightarrow} \tau_{\leq k} K^{\bullet} \rightarrow K^{\bullet} \rightarrow \tau_{\geq k+1} K^{\bullet} \stackrel{+1}{\longrightarrow} \cdots
$$

Hypercohomology. Let $F^{\bullet}$ be a bounded complex of sheaves of abelian groups on an analytic space $X$. Let $R \Gamma$ be the derived functor of the global section functor $\Gamma$ of $X: \Gamma(F)=H^{0}(X, F)$ for a sheaf $F$. The hypercohomology group $H^{p}\left(R \Gamma\left(F^{\bullet}\right)\right)$ is denoted by $H^{p}\left(X, F^{\bullet}\right)$. 
Local cohomology. Let $Z \subset X$ be a closed subset and let $F^{\bullet}$ be a bounded complex of sheaves of abelian groups on $X$. For the complement $U=$ $X \backslash Z$ and for the embedding $j: U \hookrightarrow X$, there is a natural morphism $F^{\bullet} \rightarrow R j_{*}\left(\left.F^{\bullet}\right|_{U}\right)$ in the derived category of sheaves of abelian groups on $X$. An object $R \underline{\Gamma}_{Z}\left(F^{\bullet}\right)$ of the derived category is defined by the triangle

$$
\cdots \stackrel{+1}{\longrightarrow} R \underline{\Gamma}_{Z}\left(F^{\bullet}\right) \rightarrow F^{\bullet} \rightarrow R j_{*}\left(\left.F^{\bullet}\right|_{U}\right) \stackrel{+1}{\longrightarrow} \cdots
$$

The $p$-th cohomology sheaf $\mathcal{H}^{p}\left(R \underline{\Gamma}_{Z}\left(F^{\bullet}\right)\right)$ is denoted by $\mathcal{H}_{Z}^{p}\left(F^{\bullet}\right)$. If $F$ is a sheaf of abelian groups, then $\mathcal{H}_{Z}^{p}(F)$ is the $p$-th local cohomology sheaf supported in $Z$. Note that $R \underline{\Gamma}_{Z}$ is right adjoint to $R i_{*}$ for the closed immersion $i: Z \rightarrow X$. For the derived functor $R \Gamma$ above, the derived functor $R \Gamma_{Z}:=R \Gamma \circ R \underline{\Gamma}_{Z}$ calculates local cohomology groups supported in $Z$. $H^{p}\left(R \Gamma_{Z}\left(F^{\bullet}\right)\right)$ is denoted by $H_{Z}^{p}\left(X, F^{\bullet}\right)$.

Topological dualizing complex. Let $\phi: X \rightarrow$ Spec $\mathbb{C}$ be the natural morphism from a complex analytic space. Let $\boldsymbol{\omega}_{X}^{\text {top }}$ denote the twisted inverse $\phi ! \mathbb{Z}$ defined by Verdier [V2]. We call $\boldsymbol{\omega}_{X}^{\text {top }}$ by the topological dualizing complex of $X$. If $X$ is non-singular of dimension $d$, then $\boldsymbol{\omega}_{X}^{\text {top }} \sim_{\text {qis }} \mathbb{Z}_{X}[2 d]$. There is a natural morphism $R f_{!} \boldsymbol{\omega}_{X}^{\text {top }} \rightarrow \boldsymbol{\omega}_{Y}^{\text {top }}$ called trace map for a morphism $f: X \rightarrow Y$ of complex analytic spaces. Moreover, $R \underline{\Gamma}_{Z}\left(\boldsymbol{\omega}_{X}^{\text {top }}\right) \sim_{\text {qis }}$ $\boldsymbol{\omega}_{Z}^{\text {top }}$ for a closed subspace $Z \subset X$.

Residue field. For a point $x$ of an analytic space $X$, the residue filed $\mathcal{O}_{X, x} / \mathfrak{m}_{x}$ is denoted by $\mathbb{C}(x)$.

Torsion. Let $M$ be an abelian group and let $m$ be a positive integer. ${ }_{m} M$ denotes the subgroup $\{x \in M \mid m x=0\}$. The torsion-part $M_{\text {tor }}$ is the union $\bigcup_{m>0} M$. For the subgroup $\boldsymbol{\mu}_{m}:=m^{-1} \mathbb{Z} / \mathbb{Z} \subset \mathbb{Q} / \mathbb{Z},{ }_{m} M \simeq$ $\operatorname{Tor}_{1}\left(\boldsymbol{\mu}_{m}, M\right)$ and $M_{\text {tor }} \simeq \operatorname{Tor}_{1}(\mathbb{Q} / \mathbb{Z}, M)$ hold.

Meromorphic mappings. A holomorphic mapping (map) $f: X \rightarrow Y$ of complex analytic varieties is often called a morphism. A meromorphic mapping (map) $f: X \cdots \rightarrow Y$ is defined as a closed analytic subvariety $\Gamma_{f} \subset X \times Y$ in which the first projection $\Gamma_{f} \rightarrow X$ is proper and is an isomorphism over a dense Zariski-open subset of $X$. $f$ is called proper if $\Gamma_{f} \rightarrow Y$ is proper. $f$ is called a bimeromorphic mapping (map) if $\Gamma_{f} \rightarrow Y$ is proper and is an isomorphism over a dense Zariski-open subset of $Y$. In the scheme theory, 'meromorphic map' corresponds to 'strictly rational map' and 'bimeromorphic map' corresponds to 'proper birational map' in the sense of Iitaka [I, Section 2.12]. A meromorphic function on $X$ is regarded as a meromorphic mapping $X \cdots \rightarrow \mathbb{P}^{1}$ into the projective line. 
Section. Let $f: X \rightarrow Y$ be a holomorphic mapping of complex analytic varieties. A (holomorphic) section of $f$ is a holomorphic mapping $\sigma: Y \rightarrow X$ such that $f \circ \sigma=\mathrm{id}_{Y}$; the identity mapping. This is also called a global section over $Y$. A subvariety $\Sigma \subset X$ is also called a section if the composite $\Sigma \subset X \rightarrow Y$ is an isomorphism. A meromorphic section of $f$ is a meromorphic mapping $\sigma: Y \cdots \rightarrow X$ such that $f \circ \sigma=\operatorname{id}_{Y}$. A subvariety $\Sigma \subset X$ is also called a meromorphic section if $\Sigma \rightarrow Y$ is a bimeromorphic morphism. $f$ is called to have local holomorphic (resp. meromorphic) sections over $Y$ if there is an open covering $\left\{Y_{\lambda}\right\}$ of $Y$ such that each $f^{-1} Y_{\lambda} \rightarrow Y_{\lambda}$ have holomorphic (resp. meromorphic) sections.

Reflexive sheaf. Let $X$ be a normal variety and let $\mathcal{F}$ be a coherent $\mathcal{O}_{X^{-}}$ module. The dual $\mathcal{H o m}_{\mathcal{O}_{X}}\left(\mathcal{F}, \mathcal{O}_{X}\right)$ is denoted by $\mathcal{F}^{\vee}$. The double-dual of $\mathcal{F}$ is $\mathcal{F}^{\vee \vee} . \mathcal{F}$ is called a reflexive sheaf if $\mathcal{F} \leadsto \mathcal{F}^{\vee \vee}$. If $\mathcal{F}$ is reflexive, then $\mathcal{H}_{Z}^{p}(\mathcal{F})=0$ for $p \leq 1$, for any analytic subset $Z \subset X$ of codimension greater than one. For a reflexive sheaf $\mathcal{L}$ of rank one and for an integer $m$, the double dual of $\mathcal{L}^{\otimes m}$ is denoted by $\mathcal{L}^{[m]}$. A reflexive sheaf $\mathcal{L}$ of rank one is called $\mathbb{Q}$-invertible if, locally on $X$, there is an integer $m \neq 0$ such that $\mathcal{L}^{[m]}$ is invertible.

Cartier and Weil divisors. Let $X$ be a normal variety. A prime divisor is an irreducible subvariety of codimension one. A Weil divisor $B$ is an element

$$
\left(b_{\Gamma}\right) \in \prod_{\Gamma: \text { prime divisors }} \mathbb{Z}
$$

that is locally finite on $X$, i.e, the support

$$
\operatorname{Supp} B:=\bigcup_{b_{\Gamma} \neq 0} \Gamma
$$

is an analytic subset. We usually write $B$ as a formal combination $\sum b_{\Gamma} \Gamma$. The coefficient $b_{\Gamma}$ is written by mult ${ }_{\Gamma} B$. A reduced divisor is a Weil divisor $B$ with Supp $B \neq \emptyset$ and mult $_{\Gamma} B=0$ or 1 for any $\Gamma$. The reduced divisor is identified with $\operatorname{Supp} B$. We write $B \geq B^{\prime}$ or $B^{\prime} \leq B$ for two Weil divisors $B, B^{\prime}$, if $\operatorname{mult}_{\Gamma} B \geq \operatorname{mult}_{\Gamma} B^{\prime}$ for any $\Gamma$. If $B \geq 0$, then $B$ is called an effective divisor.

The group of Weil divisors of $X$ is denoted by $\operatorname{WDiv}(X)$. It forms a sheaf $w \mathcal{D}_{i v_{X}}$ of $X$ with $H^{0}\left(X, w \mathcal{D} i v_{X}\right) \simeq \operatorname{WDiv}(X)$. The sheaf of germs of meromorphic functions of $X$ is denoted by $\mathfrak{M}_{X}$. We define $\mathfrak{M}_{X}^{\star}$ to be its subsheaf (as sets) consisting of invertible meromorphic functions. The 
abelian group structure of $\mathfrak{M}_{X}^{\star}$ is derived from the multiplication. The sheaf $\mathcal{D} i v_{X}$ of germs of Cartier divisors is defined to be the quotient $\mathfrak{M}_{X}^{\star} / \mathcal{O}_{X}^{\star}$. A Cartier divisor of $X$ is an element of $\operatorname{Div}(X):=H^{0}\left(X, \mathcal{D}_{i} v_{X}\right)$. For a prime divisor $\Gamma$ and for a meromorphic function $\varphi \in H^{0}\left(X, \mathfrak{M}_{X}^{\star}\right)$, let $\operatorname{ord}_{\Gamma}(\varphi)$ be the order of zeros of $\varphi$ along $\Gamma$ (or the minus of order of poles). The Weil divisor

$$
\operatorname{div}(\varphi):=\sum \operatorname{ord}_{\Gamma}(\varphi) \Gamma
$$

is called a principal divisor. The map $\varphi \mapsto \operatorname{div}(\varphi)$ defines injective homomorphisms $\mathcal{D i v}_{X} \hookrightarrow w \mathcal{D i v}_{X}$ and $\operatorname{Div}(X) \hookrightarrow \operatorname{WDiv}(X) . \quad X$ is called locally factorial or locally $\mathbb{Q}$-factorial, if $\mathcal{D} i v_{X} \simeq w \mathcal{D}_{i v_{X}}$ or $\mathcal{D} i v_{X} \otimes \mathbb{Q} \simeq$ $w \mathcal{D} i v_{X} \otimes \mathbb{Q}$, respectively. $X$ is locally factorial if and only if every local rings $\mathcal{O}_{X, x}$ are UFD. Let $j^{\circ}: X^{\circ} \hookrightarrow X$ be the open immersion from a nonsingular Zariski-open subset with $\operatorname{codim}\left(X \backslash X^{\circ}\right) \geq 2$. Then $w \mathcal{D} i v_{X} \simeq$ $j_{*}^{\circ} \mathcal{D} i v_{X^{\circ}}$ and $\operatorname{WDiv}(X) \simeq \operatorname{WDiv}\left(X^{\circ}\right)=\operatorname{Div}\left(X^{\circ}\right)$. A Weil divisor $B$ defines a reflexive sheaf $\mathcal{O}_{X}(B)$ of rank one by

$$
H^{0}\left(U, \mathcal{O}_{X}(B)\right)=\left\{\varphi \in H^{0}\left(U, \mathfrak{M}_{X}^{\star}\right)|\operatorname{div}(\varphi) \geq B|_{U}\right\} \cup\{0\} .
$$

The sheaf $\mathcal{O}_{X}(B)$ is invertible if and only if $B$ is Cartier. If $B$ is Cartier, then $\mathcal{O}_{X}(B)$ is determined by the connecting homomorphism $\operatorname{Div}(X) \rightarrow$ $\operatorname{Pic}(X)=H^{1}\left(X, \mathcal{O}_{X}^{\star}\right)$ of the exact sequence

$$
0=\{1\} \rightarrow \mathcal{O}_{X}^{\star} \rightarrow \mathfrak{M}_{X}^{\star} \rightarrow \mathcal{D i v}_{X} \rightarrow 0 .
$$

Normal crossing divisor. Let $X$ be a non-singular variety of dimension $d$ and let $D$ be a reduced divisor. $D$ is called a normal crossing divisor, if $D$ is locally defined as $\operatorname{div}\left(z_{1} z_{2} \cdots z_{l}\right)$ for a coordinate system $\left(z_{1}, z_{2}, \ldots, z_{d}\right)$ of $X$ and for some $1 \leq l \leq d$. $D$ is called a simple normal crossing divisor if $D$ is normal crossing and if all the irreducible components of $D$ are nonsingular.

Round-up and round-down. The round-up $\ulcorner r\urcorner$ and the round-down $\left.{ }_{\llcorner}\right\lrcorner$of a real number $r$ are defined by

$$
\ulcorner r\urcorner:=\min \{n \in \mathbb{Z} \mid n \geq r\}, \quad \text { and } \quad\left\llcorner r_{\lrcorner}:=\max \{n \in \mathbb{Z} \mid n \leq r\} .\right.
$$

A $\mathbb{Q}$-divisor of $X$ is an element of $\operatorname{Wiv}(X, \mathbb{Q})=H^{0}\left(X, w \mathcal{D} i v_{X} \otimes \mathbb{Q}\right)$. The round-up and the round-down of a $\mathbb{Q}$-divisor $B=\sum b_{\Gamma} \Gamma$ are defined by

$$
\left.\ulcorner B\urcorner:=\sum\left\ulcorner b_{\Gamma}\right\urcorner \Gamma, \quad \text { and } \quad{ }_{\llcorner} B\right\lrcorner:=\sum{ }_{\llcorner} b_{\Gamma} \Gamma .
$$


Desingularization. For a variety $V$, Sing $V$ denotes the singular locus of $V$. A bimeromorphic morphism $\mu: Y \rightarrow V$ is called a desingularization or a resolution of singularities, if $Y$ is non-singular and $\mu$ is isomorphic over $V \backslash \operatorname{Sing} V$. The existence of desingularization is proved by Hironaka [H1], [H2].

VHS. A $\mathbb{Z}$-polarized variation of Hodge structure $H$ of rank two and of weight one defined over a complex variety $S$ consists of a locally constant system $H$ of a free abelian group of rank two, a skew-symmetric bilinear form $Q: H \times H \rightarrow \mathbb{Z}_{S}$ inducing an isomorphism $\bigwedge^{2} H \leadsto \mathbb{Z}_{S}$, and of a subbundle $\mathcal{F}^{1}(\mathcal{H})$ of $\mathcal{H}:=H \otimes \mathcal{O}_{S}$ such that $\left(H_{s}, Q_{s}, \mathcal{F}^{1}(\mathcal{H}) \otimes \mathbb{C}(s)\right)$ forms a polarized Hodge structure of weight one for every $s \in S$ (cf. [G3], [S3]). We call a $\mathbb{Z}$-polarized variation of Hodge structure of rank two and of weight one by $V H S$ for short.

\section{$\S 1 . \quad \partial$-space}

We introduce the category $\partial \mathrm{SP}$ of complex analytic $\partial$-spaces and the $\partial$ étale topology. In the category $\mathcal{A B}$ of complex analytic spaces with boundary, we define special morphisms: $\partial$-isomorphisms, $\partial$-open immersions, and $\partial$-étale morphisms. The $\partial \mathrm{sP}$ is defined as the localization of $\mathcal{A B}$ by $\partial$-isomorphisms and the $\partial$-étale topology is defined by $\partial$-étale morphisms. A $\partial$-space $\underline{X}$ is an object of $\partial \mathrm{sP}$. A reduced analytic $\operatorname{space} \operatorname{sp}(\underline{X})$ is associated and an element $x$ of $\operatorname{sp}(\underline{X})$ is called a point of $\underline{X}$. The local profinite fundamental group $\hat{\pi}_{1}^{\text {loc }}(\underline{X} ; x)$ is defined by $\partial$-étale morphisms over $x$.

\section{$\S 1.1$ Category of $\partial$-spaces}

Let $X$ be a complex analytic space and let $B$ be a closed analytic subset. $B$ is called nowhere-dense in $X$ if $X \backslash B$ is dense. If $B$ is nowhere-dense and if $\Gamma$ is an irreducible component of $X$, then $\Gamma \cap B$ is also nowhere-dense in $\Gamma$. In particular, $\Gamma \backslash B \neq \emptyset$.

Definition. The category $\mathcal{A B}$ of complex analytic spaces with boundary is defined as follows:

- An object is a pair $[X, B]$ consisting of a complex analytic space $X$ and a nowhere-dense closed analytic subset $B$;

- A morphism $f:[X, B] \rightarrow[Y, D]$ is defined to be a holomorphic mapping $X \rightarrow Y$ satisfying $f^{-1}(D) \subset B$. 
An object $[X, B]$ is called a complex analytic space with boundary and $B$ is called its boundary.

By considering objects $[X, B]$ with $B=\emptyset$, we have a natural fully faithful functor from the category $\mathcal{A} n$ of complex analytic spaces into $\mathcal{A B}$. Note that fiber products always exist in $\mathcal{A B}$. In fact, for two morphisms $[X, B] \rightarrow[Z, \Delta]$ and $[Y, D] \rightarrow[Z, \Delta]$ in $\mathcal{A B}$, we have the fiber product $X \times_{Z} Y$ in $\mathcal{A} n$ and a closed subset $p_{1}^{-1}(B) \cup p_{2}^{-1}(D)$, where $p_{1}: X \times_{Z} Y \rightarrow X$ and $p_{2}: X \times_{Z} Y \rightarrow Y$ are projections. Let $E$ be the union of all the irreducible components of $X{ }{ }_{Z} Y$ that are contained in $p_{1}^{-1}(B) \cup p_{2}^{-1}(D)$ and let $E^{\star}$ be its interior: this is the maximal open subset of $X \times_{Z} Y$ contained in $E$. Then the fiber product $[X, B] \times_{[Z, \Delta]}[Y, D]$ in $\mathcal{A B}$ should be

$$
\left[X \times_{Z} Y \backslash E^{\star}, p_{1}^{-1}(B) \cup p_{2}^{-1}(D) \backslash E^{\star}\right] .
$$

Definition 1.1.1. Let $f:[X, B] \rightarrow[Y, D]$ be a morphism in $\mathcal{A B}$ such that

(1) $f$ has only discrete fibers, and

(2) $f^{-1}(D)=B$.

It is called a $\partial$-étale morphism, a $\partial$-open immersion, and a $\partial$-isomorphism according to the following conditions:

d-étale morphism: $X \backslash B \rightarrow Y \backslash D$ is an étale morphism, i.e., a local isomorphism.

D-open immersion: $X \backslash B \rightarrow Y \backslash D$ is an open immersion.

$\partial$-isomorphism: $X \backslash B \rightarrow Y \backslash D$ is an isomorphism and $X \rightarrow Y$ is a finite (proper) morphism.

Let $P$ be one of the three conditions above. Then the following properties hold:

- An identity mapping satisfies $P$;

- The composite of morphisms satisfying $P$ also satisfies $P$;

- The condition $P$ is stable under base change.

Two pairs $[X, B]$ and $[Y, D]$ are called $\partial$-isomorphic, if there exist $\partial$ isomorphisms $[Z, \Delta] \rightarrow[X, B]$ and $[Z, \Delta] \rightarrow[Y, D]$ from another pair $[Z, \Delta]$. The relation being $\partial$-isomorphic is an equivalence relation on the objects of 
$\mathcal{A B}$ and an equivalence class is called a complex analytic $\partial$-space $(\partial$-space, for short). A $\partial$-space is written by an underlined capital letter, e.g., $\underline{X}$. The $\partial$ space corresponding to $[X, B]$ is denoted by $(X, B)$. If $\underline{X}=(X, B)$ for a pair $[X, B]$, then $[X, B]$ is called a realization of $\underline{X}$ and we write $[X, B] \in \underline{X}$. For any realizations $[X, B]$ of a fixed $\underline{X}$, the open subspaces $X \backslash B$ are canonically isomorphic. It is denoted by $\underline{X}^{\star}$ and is called by the interior or the open part of $\underline{X}$.

Definition. The category $\partial \mathrm{sP}$ of (complex analytic) $\partial$-spaces is defined as follows: An object is a $\partial$-space. For two $\partial$-spaces $\underline{X}:=(X, B)$ and $\underline{Y}:=$ $(Y, D)$, the set $\operatorname{Hom}_{\partial \mathrm{SP}}(\underline{X}, \underline{Y})$ of morphisms is well-defined to be

$$
\underset{\left[X^{\prime}, B^{\prime}\right] \rightarrow[X, B]}{\lim _{\longrightarrow}} \operatorname{Hom}_{\mathcal{A B}}\left(\left[X^{\prime}, B^{\prime}\right],[Y, D]\right),
$$

where the direct limit is taken over all the $\partial$-isomorphisms $\left[X^{\prime}, B^{\prime}\right] \rightarrow[X, B]$.

A $\partial$-space $(X, B)$ with $B=\emptyset$ is considered as an analytic space $X$. Then there is a fully faithful functor $\mathcal{A} n \hookrightarrow \partial \mathrm{sP}$. Fiber products also exist in $\partial \mathrm{sP}$, which are induced from those in $\mathcal{A B}$. However, these are different from usual fiber products.

Example. Let $\boldsymbol{\Delta}$ be a unit disc $\{t \in \mathbb{C}|| t \mid<1\}$. We shall consider two morphisms $C_{1}:=\boldsymbol{\Delta} \ni t \mapsto t^{m} \in \boldsymbol{\Delta}=: C_{3}$ and $C_{2}:=\boldsymbol{\Delta} \ni t \mapsto t^{n} \in$ $\boldsymbol{\Delta}=C_{3}$ for mutually coprime positive integers $m$ and $n$. Then the usual fiber product $C_{1} \times{ }_{C_{3}} C_{2}$ is an irreducible curve $\Gamma$ in $C_{1} \times C_{2} \simeq \Delta^{2}$ defined by $x^{m}=y^{n}$ for a coordinate system $(x, y)$ of $\boldsymbol{\Delta}^{2}$. But the fiber product $\left(C_{1},\{0\}\right) \times{ }_{\left(C_{3},\{0\}\right)}\left(C_{2},\{0\}\right)$ is isomorphic to $(\boldsymbol{\Delta},\{0\})$ and the projections to $\left(C_{1},\{0\}\right)$ and $\left(C_{2},\{0\}\right)$ are given by $t \mapsto t^{n}$ and $t \mapsto t^{m}$, respectively. Here $\boldsymbol{\Delta} \rightarrow \Gamma$ is the normalization.

Definition. An analytic space with boundary $[X, B]$ is called locally connected at a point $x \in X$ (with respect to the boundary $B$ ), if for any open neighborhood $\mathcal{U}$ of $x$, there is an open subneighborhood $\mathcal{U}^{\prime} \subset \mathcal{U}$ with $\mathcal{U}^{\prime} \backslash B$ being connected. $[X, B]$ is called locally connected, if it is locally connected at every points of $X$.

Remark. In the case $X$ is reduced and $B$ is the singular locus of $X$, $[X, B]$ is locally connected at $x$ if and only if $X$ is locally irreducible at $x$. In particular, the locally connectedness is not an open condition.

Lemma 1.1.2. Let $[X, B]$ be a complex analytic space with boundary, $f:[Y, D] \rightarrow[X, B]$ a D-étale morphism, and let $x \in f(Y) \subset X$ be a point at 
which $[X, B]$ is locally connected. Then $f(Y)$ is a neighborhood of $x$. Suppose further that $f$ is a $\partial$-open immersion. Then

(1) $f^{-1}(x)$ consists of one point, and

(2) for an open neighborhood $\mathcal{V}$ of $f^{-1}(x)$, there is an open neighborhood $\mathcal{U}$ of $x$ such that $f^{-1}(\mathcal{U}) \subset \mathcal{V}$ and that $f^{-1}(\mathcal{U}) \rightarrow \mathcal{U}$ is a finite morphism.

In particular, if $[X, B]$ is locally connected, then $f(Y)$ is open. If further $f$ is a $\partial$-open immersion, then $f$ is a homeomorphism onto $f(Y)$.

Proof. For a point $y \in f^{-1}(x)$, there exist open neighborhoods $\mathcal{V}$ and $\mathcal{U}$ of $y$ and $x$, respectively, such that $f$ induces a finite morphism $\mathcal{V} \rightarrow \mathcal{U}$ and that $\mathcal{U} \backslash B$ is connected. This property is derived from the conditions: $f^{-1}(x)$ is discrete and $[X, B]$ is locally connected at $x$. Thus $\mathcal{V} \backslash D \rightarrow \mathcal{U} \backslash B$ is a finite surjective étale morphism. Hence $\mathcal{U} \subset f(Y)$. If $f$ is a $\partial$-open immersion, then $Y \backslash D \rightarrow X \backslash B$ is an open immersion. Therefore $f^{-1}(x)=\{y\}$ and $f^{-1}(\mathcal{U}) \backslash D=\mathcal{V} \backslash D$. Thus $f^{-1}(\mathcal{U})=\mathcal{V}$, since $D$ is nowhere-dense. In particular, $f^{-1}(\mathcal{U}) \rightarrow \mathcal{U}$ is a finite morphism.

Let $j: X \backslash B \hookrightarrow X$ be the open immersion for an analytic space with boundary $[X, B]$. The image of $\mathcal{O}_{X} \rightarrow j_{*} \mathcal{O}_{X \backslash B}$ is the structure sheaf of a closed analytic subspace $X^{\prime}$. Since $B$ is nowhere-dense, $X$ and $X^{\prime}$ are homeomorphic and $\left[X^{\prime}, B\right] \rightarrow[X, B]$ is a $\partial$-isomorphism. The $[X, B]$ is called refined if $X \simeq$ $X^{\prime}$, i.e., the homomorphism $\mathcal{O}_{X} \rightarrow j_{*} \mathcal{O}_{X \backslash B}$ is injective.

Lemma 1.1.3. Let $[X, B]$ be a complex analytic space with boundary and let $x \in X$ be a point. Then there exist an open neighborhood $U$ of $x$ and a $\partial$-isomorphism $f:[Y, D] \rightarrow[U, B \cap U]$ such that $[Y, D]$ is refined and is locally connected at every point of $f^{-1}(x)$.

Proof. We may assume that $X$ is refined. By considering the irreducible decomposition of the germ $(X, x)$, we have an open neighborhood $U$ of $x$ and a finitely many closed analytic subspaces $U_{r} \subset U(1 \leq r \leq k)$ such that

(1) $U=\bigcup_{r=1}^{k} U_{r}$,

(2) restriction homomorphisms induce an injection $\mathcal{O}_{U} \hookrightarrow \bigoplus_{r=1}^{k} \mathcal{O}_{U_{r}}$,

(3) $U_{r}$ is locally irreducible at $x$ for any $r$. 
Since $B$ is nowhere-dense, $U_{r} \backslash B$ is non-empty. We may assume that $U_{r} \backslash B$ is connected and the immersion $\lambda_{r}: U_{r} \backslash B \hookrightarrow U_{r}$ induces an injection $\mathcal{O}_{U_{r}} \hookrightarrow$ $\lambda_{r *} \mathcal{O}_{U_{r} \backslash B}$. Let us consider the following commutative diagram:

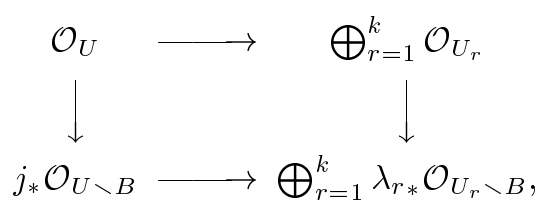

and an $\mathcal{O}_{U}$-algebra $\mathcal{A}$ defined by

$$
\mathcal{A}:=j_{*} \mathcal{O}_{U \backslash B} \cap \bigoplus_{r=1}^{k} \mathcal{O}_{U_{r}} .
$$

Then $\mathcal{A}$ is a coherent $\mathcal{O}_{U}$-module. Thus we have a finite morphism $f: Y \rightarrow U$ with a commutative diagram:

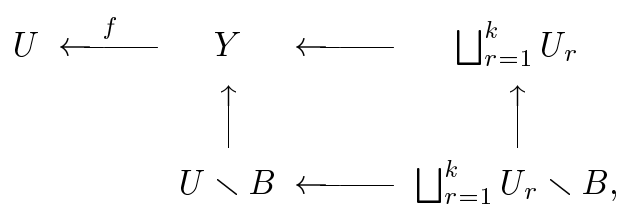

such that $\mathcal{O}_{U} \subset f_{*} \mathcal{O}_{Y}=\mathcal{A} \subset \bigoplus_{r=1}^{k} \mathcal{O}_{U_{r}}$. In particular, $f:\left[Y, f^{-1}(B)\right] \rightarrow$ $[U, B \cap U]$ is a $\partial$-isomorphism and $\left[Y, f^{-1}(B)\right]$ is refined. We shall show $\left[Y, f^{-1}(B)\right]$ is locally connected at every point $y \in f^{-1}(x)$. Suppose that $V \backslash f^{-1}(B)$ is not connected for a connected open neighborhood $V$ of $y$. By replacing $V$, we may assume that non-empty intersections $V \cap U_{r}=V \cap f^{-1}\left(U_{r}\right)$ are connected. Now we have two open subsets $W_{1}^{\star}, W_{2}^{\star}$ with $V \backslash f^{-1}(B)=$ $W_{1}^{\star} \sqcup W_{2}^{\star}$. Let us consider the following sets:

$$
R:=\left\{1 \leq r \leq k \mid V \cap U_{r} \neq \emptyset\right\}, \quad R_{i}:=\left\{r \in R \mid V \cap\left(U_{r} \backslash B\right) \subset W_{i}^{\star}\right\},
$$

for $i=1,2$. Let $W_{i}$ be the subspace of $V$ whose structure sheaf is the image of $\mathcal{O}_{V} \rightarrow \bigoplus_{r \in R_{i}} \mathcal{O}_{V \cap U_{r}}$. Then $W_{i} \backslash B \simeq W_{i}^{\star}$. Therefore $\mathcal{O}_{W_{1}} \oplus \mathcal{O}_{W_{2}} \subset$ $\left.\left.\left(j_{*} \mathcal{O}_{X \backslash B}\right)\right|_{V} \cap\left(\bigoplus_{r=1}^{k} \mathcal{O}_{U_{r}}\right)\right|_{V}$. Hence $\mathcal{O}_{V}=\mathcal{O}_{W_{1}} \oplus \mathcal{O}_{W_{2}}$ and $V=W_{1} \sqcup W_{2}$. This contradicts the property: $V$ is connected. Therefore $\left[Y, f^{-1}(B)\right]$ is locally connected at $y$.

Definition. An analytic space with boundary $[X, B]$ is called top, if for a $\partial$-isomorphism $f:[Y, D] \rightarrow[X, B]$, there is a $\partial$-isomorphism $g:[X, B] \rightarrow$ $[Y, D]$ such that $f \circ g$ is the identity mapping. 
A top realization $[X, B]$ of $\partial$-space $\underline{X}$ is unique up to isomorphisms in $\mathcal{A B}$. If it exists, then

$$
\operatorname{Hom}_{\partial \mathrm{SP}}(\underline{X},(Y, D)) \simeq \operatorname{Hom}_{\mathcal{A B}}([X, B],[Y, D])
$$

for any $[Y, D]$.

Lemma 1.1.4. If the interior of a $\partial$-space is a reduced analytic space, then there is a top realization.

Proof. Let $[X, B]$ be an analytic space with boundary with $X \backslash B$ being reduced. We may assume that $[X, B]$ is refined and hence $X$ is reduced. Let $q: W \rightarrow X$ be the normalization and let $j: X \backslash B \hookrightarrow X$ be the open immersion. Then we have a commutative diagram:

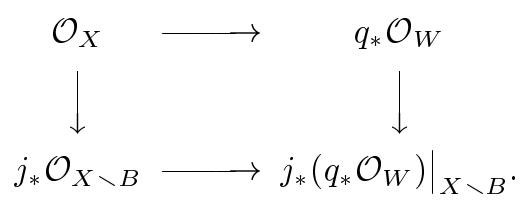

Here, every arrows are injective. The $\mathcal{O}_{X}$-algebra

$$
\mathcal{A}:=j_{*} \mathcal{O}_{X \backslash B} \cap q_{*} \mathcal{O}_{W}
$$

is a coherent $\mathcal{O}_{X}$-module. Thus we have a finite morphism $p: X^{\prime} \rightarrow X$ such that $\mathcal{A} \simeq p_{*} \mathcal{O}_{X^{\prime}}$. Here $\left[X^{\prime}, B^{\prime}\right] \rightarrow[X, B]$ is a $\partial$-isomorphism for $B^{\prime}=p^{-1}(B)$. For a $\partial$-isomorphism $[Y, D] \rightarrow\left[X^{\prime}, B^{\prime}\right], W$ is also a normalization of $Y$. Thus we have a unique factorization $\left[X^{\prime}, B^{\prime}\right] \rightarrow[Y, D]$. Hence $\left[X^{\prime}, B^{\prime}\right]$ is a top realization.

Definition. A $\partial$-space $\underline{X}$ is said to be reduced, if $X$ is reduced for a realization $[X, B]$ of $\underline{X}$. This condition is equivalent to that $\underline{X}^{\star}$ is reduced. For a $\partial$-space $\underline{X}$, the reduced $\partial$-space $\left(X_{\text {red }}, B\right)$ is independent of the choice of realizations $[X, B] \in \underline{X}$. This $\partial$-space is denoted by $\underline{X}_{\text {red }}$.

Example. If $\underline{X}$ is not reduced, then we have no top realization in general. For example, let $C$ be a non-singular curve, $x$ a point and let $\mathcal{F}$ be a non-zero locally free sheaf of finite rank on $C$. We can give an algebra structure on $\mathcal{O}_{C} \oplus \mathcal{F}$ as follows: For $(a, v),(b, w) \in \mathcal{O}_{C} \oplus \mathcal{F}$, the multiplication is given by $(a b, b v+a w)$. Then we have a non-reduced curve $C(\mathcal{F})$ such that $C \subset C(\mathcal{F})$ and its nil-radical is just $\mathcal{F}$. Let us consider the pair $[C(\mathcal{F}), x]$. For arbitrary injection $\mathcal{F} \hookrightarrow \mathcal{G}$ of locally free sheaves whose cokernel is supported only at $\{x\}$, we have a $\partial$-isomorphism $[C(\mathcal{G}), x] \rightarrow[C(\mathcal{F}), x]$. Thus it is impossible to obtain the top realization of $(C(\mathcal{F}), x)$. 
Lemma 1.1.5.

(1) The top realization of a $\partial$-space is locally connected.

(2) If $[X, B]$ is locally connected at a point $x$ and if $\pi:[Z, \Delta] \rightarrow\left[X_{\mathrm{red}}, B\right]$ is the $\partial$-isomorphism from the top realization of $\left(X_{\mathrm{red}}, B\right)$, then $\pi^{-1}(x)$ consists of one point. In particular, if $[X, B]$ is locally connected, then $\pi: Z \rightarrow X_{\text {red }}$ is a homeomorphism.

Proof. (1) is derived from 1.1.3. In (2), $\left[X_{\text {red }}, B\right]$ is also locally connected at $x$. Thus the assertion follows 1.1.2.

Lemma 1.1.6. Let $f:[Y, D] \rightarrow[X, B]$ be a $\partial$-open immersion.

(1) For a point $x \in f(Y) \subset X$, there is an open neighborhood $\mathcal{U}$ of $x$ such that $f^{-1}(\mathcal{U}) \rightarrow \mathcal{U}$ is a finite morphism.

(2) If $f(Y)=X$, then $f$ is a $\partial$-isomorphism.

(3) The second projection

$$
p_{2}:[Y, D] \times_{[X, B]}[Y, D] \rightarrow[Y, D]
$$

is a $\partial$-isomorphism.

Proof. (1) Let $\mu:\left[X^{\prime}, B^{\prime}\right] \rightarrow[\mathcal{U}, B \cap \mathcal{U}]$ be a $\partial$-isomorphism such that $\mathcal{U}$ is an open neighborhood of $x$ and that $\left[X^{\prime}, B^{\prime}\right]$ is locally connected at any point of $\mu^{-1}(x)$ (cf. 1.1.3). Then by the proof of 1.1.2, the second projection

$$
[Y, D] \times{ }_{[X, B]}\left[X^{\prime}, B^{\prime}\right] \rightarrow\left[X^{\prime}, B^{\prime}\right]
$$

is a finite morphism, if we replace $\mathcal{U}$ by another open neighborhood of $x$. Thus $f^{-1}(\mathcal{U}) \rightarrow \mathcal{U}$ is a finite morphism.

(2) is a consequence of (1), and (3) is a special case of (2).

\section{Definition.}

(1) A morphism $\underline{Y} \rightarrow \underline{X}$ of $\partial$-spaces is called a $\partial$-open immersion if it is induced from a $\partial$-open immersion of each realizations. The $\underline{Y}$ is called a $\partial$-open subspace of $\underline{X}$.

(2) A $\partial$-étale morphism $\underline{Y} \rightarrow \underline{X}$ is defined to be a morphism induced from a $\partial$-étale morphism of each realizations. The $\underline{Y}$ is called a $\partial$-étale space over $\underline{X}$. 
The second projection $\underline{U} \times \underline{X} \underline{U} \rightarrow \underline{U}$ is a $\partial$-isomorphism for a $\partial$-open subspace $\underline{U}$ of $\underline{X}$ by 1.1.6.

Let $[X, B]$ be a complex analytic space with boundary and let $([Y, D], y, f)$ be a triplet consisting of a complex analytic space with boundary $[Y, D]$, a point $y \in Y$, and a $\partial$-open immersion $f:[Y, D] \rightarrow[X, B]$ such that $[Y, D]$ is locally connected at $y$. A morphism $h:\left(\left[Y_{1}, D_{1}\right], y_{1}, f_{1}\right) \rightarrow\left(\left[Y_{2}, D_{2}\right], y_{2}, f_{2}\right)$ of such triplets is defined to be a $\partial$-open immersion $h:\left[Y_{1}, D_{1}\right] \rightarrow\left[Y_{2}, D_{2}\right]$ such that $f_{2} \circ h=f_{1}$ and $h\left(y_{1}\right)=y_{2}$. Two triplets are called equivalent if there exist morphisms from another triplet to each triplets.

Lemma 1.1.7. Let $\underline{X}=(X, B)$ be a $\partial$-space and let $\pi:[Z, \Delta] \rightarrow$ $\left[X_{\mathrm{red}}, B\right]$ be the top realization of $\left(X_{\mathrm{red}}, B\right)$. Then $Z$ is set-theoretically identified with the set of all the equivalence classes of the triplets $([Y, D], y, f)$.

Proof. For a triplet $([Y, D], y, f)$, we have a $\partial$-isomorphism

$$
q:[Z, \Delta] \times{ }_{\left[X_{\text {red }}, B\right]}\left[Y_{\text {red }}, D\right] \rightarrow\left[Y_{\text {red }}, D\right]
$$

and a $\partial$-open immersion

$$
p:[Z, \Delta] \times{ }_{\left[X_{\text {red }}, B\right]}\left[Y_{\text {red }}, D\right] \rightarrow[Z, \Delta] .
$$

Then $q^{-1}(y)$ consists of one point, since $[Y, D]$ is locally connected at $y$. Thus $([Y, D], y, f)$ defines a point $p\left(q^{-1}(y)\right)$ of $Z$. This is independent of the choice of equivalent triplets. A point $z \in Z$ defines an equivalence class of triplets as follows: Let $x=\pi(z) \in X$ be the image. By 1.1.3, there exist an open neighborhood $U$ of $x$ and a $\partial$-isomorphism $f:[Y, D] \rightarrow[U, B \cap U]$ such that $[Y, D]$ is locally connected at every point of $f^{-1}(x)$. There is a $\partial$-isomorphism $h:\left[\pi^{-1}\left(U_{\text {red }}\right), \Delta \cap \pi^{-1}\left(U_{\text {red }}\right)\right] \rightarrow\left[Y_{\text {red }}, D\right]$. By 1.1.2 or $1.1 .5, h^{-1} f^{-1}(x) \rightarrow$ $f^{-1}(x)$ is bijective. Thus $z \in Z$ is determined by the triplet $([Y, D], y, f)$ for a point $y \in Y$.

Definition. For a $\partial$-space $\underline{X}$, let $[Z, \Delta]$ be the top realization of $\underline{X}_{\text {red }}$. The reduced analytic space $Z$ is called the space of $\underline{X}$ and is denoted by $\operatorname{sp}(\underline{X})$. An element of $\operatorname{sp}(\underline{X})$ is called a point of $\underline{X}$. The $\underline{X}$ is called connected if $\operatorname{sp}(\underline{X})$ is connected, equivalently $\underline{X}^{\star}$ is connected.

Lemma 1.1.8. Let $\underline{X}$ be a complex analytic $\partial$-space.

(1) If $[X, B]$ is a locally connected realization of $\underline{X}$ and if $\underline{U} \rightarrow \underline{X}$ is a $\partial$ open immersion, then there is an open subset $U \subset X$ such that $\underline{U} \rightarrow \underline{X}$ is induced from the $\partial$-open immersion $[U, B \cap U] \rightarrow[X, B]$. 
(2) If $\underline{U} \rightarrow \underline{X}$ is a $\partial$-open immersion, then $\operatorname{sp}(\underline{U}) \rightarrow \operatorname{sp}(\underline{X})$ is an open immersion.

(3) If $\operatorname{sp}\left(\underline{U}_{1}\right)=\operatorname{sp}\left(\underline{U}_{2}\right)$ in $\operatorname{sp}(\underline{X})$ for $\partial$-open subspaces $\underline{U}_{1}$ and $\underline{U}_{2}$ of $\underline{X}$, then $\underline{U}_{1}$ and $\underline{U}_{2}$ are $\partial$-isomorphic over $\underline{X}$.

Proof. (1) There is a realization $f:\left[U^{\prime}, \Delta\right] \rightarrow[X, B]$ of $\underline{U} \rightarrow \underline{X}$. The image $U:=f\left(U^{\prime}\right)$ is an open subset by 1.1.2. Thus $\left[U^{\prime}, \Delta\right] \rightarrow[U, B \cap U]$ is a $\partial$-isomorphism by 1.1.6. In particular, $\operatorname{sp}(\underline{U}) \rightarrow \operatorname{sp}(\underline{X})$ is injective.

(2) We may assume that $\underline{X}$ and $\underline{U}$ are reduced. Hence the $\partial$-open immersion is induced from that of each top realizations. Thus (2) is a consequence of (1).

(3) Let $\underline{U}_{3}$ be the fiber product $\underline{U}_{1} \times_{\underline{X}} \underline{U}_{2}$ and let $p_{i}: \underline{U}_{3} \rightarrow \underline{U}_{i}$ be the $i$-th projection for $i=1,2$. Then $p_{1}$ and $p_{2}$ are $\partial$-isomorphisms by 1.1.6.

A $\partial$-open subspace $\underline{U}$ of $\underline{X}$ is called a $\partial$-open neighborhood of $x \in \operatorname{sp}(\underline{X})$ if $x \in \operatorname{sp}(\underline{U})$.

Lemma 1.1.9. $\quad$ Let $\underline{X}$ be a complex analytic $\partial$-space and let $x$ be a point of $\underline{X}$. Then for any open neighborhood $\mathcal{U}$ of $x$ in $\operatorname{sp}(\underline{X})$, there exist a $\partial$-open neighborhood $\underline{U}$ of $x$ such that $\operatorname{sp}(\underline{U}) \subset \mathcal{U}$.

Proof. Let $[X, B]$ be a realization of $\underline{X}$ that is locally connected at the image $\bar{x}=\mu(x)$ under the morphism $\mu: \operatorname{sp}(\underline{X}) \rightarrow X_{\text {red }}$. Then there is an open neighborhood $U$ of $\bar{x}$ such that $\mu^{-1}(U) \subset \mathcal{U}$ by 1.1.2. Thus $\operatorname{sp}(\underline{U}) \subset \mathcal{U}$ for $\underline{U}=(U, B \cap U)$.

Thus the topology of $\operatorname{sp}(\underline{X})$ is generated by $\operatorname{sp}(\underline{U})$ for $\partial$-open subspaces $\underline{U}$.

Problem. For any $\partial$-space $\underline{X}$, does there exist a locally connected realization $[X, B]$ such that $X_{\text {red }} \simeq \operatorname{sp}(\underline{X})$ as reduced analytic spaces?

\section{$\S 1.2$ Profinite fundamental group}

We introduce and study profinite fundamental groups of $\partial$-spaces by using $\partial$-étale morphisms.

Lemma 1.2.1. For a d-étale morphism $\underline{Y} \rightarrow \underline{X}$, the induced morphism $\operatorname{sp}(\underline{Y}) \rightarrow \operatorname{sp}(\underline{X})$ is an open map. 
Proof. Let $f:[X, B] \rightarrow[Y, D]$ be a realization of the morphism such that $f$ is $\partial$-étale. Then $\left[X_{\text {red }}, B\right] \rightarrow\left[Y_{\text {red }}, D\right]$ is also $\partial$-étale. Thus we may assume that $\underline{X}$ and $\underline{Y}$ are reduced and that $[X, B]$ and $[Y, D]$ are top realizations. Then the assertion follows 1.1.2.

A morphism $\underline{Y} \rightarrow \underline{X}$ of $\partial$-spaces is called surjective and finite, respectively, if the induced morphism $\operatorname{sp}(\underline{Y}) \rightarrow \operatorname{sp}(\underline{X})$ is so. If a $\partial$-étale morphism $\underline{Y} \rightarrow \underline{X}$ is finite and if $\underline{X}$ is connected, then it is surjective by 1.2.1.

Definition. Let $\underline{X}$ be a connected $\partial$-space. A $\partial$-étale morphism $\underline{Y} \rightarrow \underline{X}$ is called Galois, if there is a left action of a discrete group $G$ on $\underline{Y}$ in the category $\partial \mathrm{sP}$ such that the morphism

$$
G \times \underline{Y} \ni(g, y) \mapsto(g y, y) \in \underline{Y} \times \underline{X} \underline{Y}
$$

is an isomorphism in $\partial \mathrm{sP}$. In this case, $\underline{Y}^{\star}$ is étale and Galois over the image of $\underline{Y}^{\star} \rightarrow \underline{X}^{\star}$. The Galois group $G$ is denoted by $\operatorname{Gal}(\underline{Y} / \underline{X})$.

Lemma 1.2.2. Let $f: \underline{Y} \rightarrow \underline{X}$ be a $\partial$-étale morphism of connected $\partial$ spaces. Suppose that the second projection $\underline{Y} \times \underline{X} \underline{Y} \rightarrow \underline{Y}$ is a finite morphism. Then there exists a $\partial$-étale finite morphism $\underline{Z} \rightarrow \underline{Y}$ from a connected $\partial$-space $\underline{Z}$ such that the composite $\underline{Z} \rightarrow \underline{X}$ is Galois.

Proof. If $f$ itself is Galois, we have nothing to prove. Otherwise, $\underline{Y} \times \underline{X}$ $\underline{Y}$ has a connected component which is not isomorphic to $\underline{Y}$ by the second projection. Let $\underline{Y}^{(1)}$ be one of such component. Then the second projection $\underline{Y}^{(1)} \times \underline{X} \underline{Y}^{(1)} \rightarrow \underline{Y}^{(1)}$ is also finite. If $\underline{Y}^{(1)} \rightarrow \underline{X}$ is Galois, we take $\underline{Z}=\underline{Y}^{(1)}$. Otherwise, we can choose a connected component $\underline{Y}^{(2)}$ of $\underline{Y}^{(1)} \times_{X} \underline{Y}^{(1)}$ which is not isomorphic to $\underline{Y}^{(1)}$. Next we shall examine $\underline{Y}^{(2)} \rightarrow \underline{X}$ to be Galois or not. By continuing the process, we have a sequence of $\partial$-étale finite morphisms $\underline{Y}^{(k)} \rightarrow \underline{Y}^{(k-1)} \rightarrow \cdots \rightarrow \underline{Y}$. But this is not an infinite sequence, since the length of the restriction to $\underline{X}^{\star}$ is finite. Thus we can take $\underline{Z}=\underline{Y}^{(k)}$ for some $k$.

The $\underline{Z}$ obtained by the method of 1.2.2 is minimal in all such Galois morphisms. This is called the Galois closure of $f$.

Lemma 1.2.3. Let $f: \underline{Y} \rightarrow \underline{X}$ be a $\partial$-étale Galois morphism with a finite Galois group $\operatorname{Gal}(\underline{Y} / \underline{X})=G$. If $H \subset G$ is a subgroup, then there exists a $\partial$-étale morphism $\underline{Z} \rightarrow \underline{X}$ with a $\partial$-étale finite Galois morphism $\underline{Y} \rightarrow \underline{Z}$ such that $\operatorname{Gal}(\underline{Y} / \underline{Z})=H$ and the composite $\underline{Y} \rightarrow \underline{Z} \rightarrow \underline{X}$ is the original $f$. 
Proof. Since $G$ is a finite group, we can find a realization $[Y, D] \in \underline{Y}$ such that $G$ acts holomorphically on $Y$. Let $Z$ be the quotient of $Y$ by $H$ and let $\Delta \subset Z$ be the image of $D$ under the quotient morphism. Then $\underline{Z}:=(Z, \Delta)$ satisfies the condition.

Corollary 1.2.4. Let $f: \underline{Y} \rightarrow \underline{X}$ be a $\partial$-étale morphism. Assume that the second projection $\underline{Y} \times \underline{X} \underline{Y} \rightarrow \underline{Y}$ is a finite morphism. Then $f$ is the composite of a finite $\partial$-étale morphism $\underline{Y} \rightarrow \underline{U}$ and a $\partial$-open immersion $\underline{U} \rightarrow \underline{X}$.

Proof. We may assume that $\underline{X}$ and $\underline{Y}$ are connected. Let $\underline{Z} \rightarrow \underline{Y} \rightarrow \underline{X}$ be the Galois closure with $G=\operatorname{Gal}(\underline{Z} / \underline{X})$. Let $\underline{U}$ be the quotient of $\underline{Z}$ by $G$ by 1.2.3. Then $\underline{U} \rightarrow \underline{X}$ is a $\partial$-open immersion.

Problem. Let $\underline{X}$ be a connected $\partial$-space and let $U \rightarrow \underline{X}^{\star}$ be a finite étale morphism. Then does there exist a $\partial$-space $\underline{Y}$ finite and $\partial$-étale over $\underline{X}$ such that $\underline{Y}^{\star}$ is isomorphic to $U$ over $\underline{X}^{\star}$ ?

We have an affirmative answer in the case $\underline{X}^{\star}$ is a normal variety, by a theorem of Grauert-Remmert [G2].

A pointed $\partial$-space is a pair $(\underline{Y} ; y)$ consisting of a $\partial$-space $\underline{Y}$ and a point $y \in \operatorname{sp}(\underline{Y})$. A morphism of pointed $\partial$-spaces should be a morphism of $\partial$-spaces preserving the given points. Let $\underline{X}$ be a connected $\partial$-space and let $x$ be a point of the interior $\underline{X}^{\star}$. We shall define the profinite fundamental group of $(\underline{X} ; x)$ to be

$$
\hat{\pi}_{1}(\underline{X} ; x):=\lim _{\longleftarrow} \operatorname{Gal}(\underline{Y} / \underline{X}),
$$

where the limit is taken over all the pointed $\partial$-spaces $(\underline{Y} ; y)$ over $(\underline{X} ; x)$ such that $\underline{Y}$ is connected and $\underline{Y} \rightarrow \underline{X}$ is finite $\partial$-étale and Galois.

Let $\pi_{1}\left(\underline{X}^{\star}, x\right)$ be the usual fundamental group, $\widetilde{X} \rightarrow \underline{X}^{\star}$ the universal covering mapping and let $\widetilde{x} \in \widetilde{X}$ be a point over $x$. For a finite $\partial$-étale morphism $f: \underline{Y} \rightarrow \underline{X}$ and for a point $y \in f^{-1}(x)$, there exists uniquely a morphism $\pi_{y}: \widetilde{X} \rightarrow \underline{Y}^{\star}$ over $\underline{X}^{\star}$ that sends $\widetilde{x}$ to $y$. Thus the set $\operatorname{Hom}_{\underline{X}}(\widetilde{X}, \underline{Y})$ of morphisms $\widetilde{X} \rightarrow \underline{Y}$ over $\underline{X}$ is identified with the fiber $f^{-1}(x)$. Let $L_{\gamma}: \widetilde{X} \rightarrow \widetilde{X}$ be the left action of $\gamma \in \pi_{1}\left(\underline{X}^{\star}, x\right)$. For a morphism $\varphi \in \operatorname{Hom}_{\underline{X}}(\tilde{X}, \underline{Y})$, we define $\varphi^{\gamma}:=\varphi \circ L_{\gamma}$. Then $f^{-1}(x)$ admits a right action of $\pi_{1}\left(\underline{X}^{\star}, x\right)$. By 1.2.2, there is a finite $\partial$-étale Galois morphism $\underline{Z} \rightarrow \underline{X}$ satisfying the following conditions:

(1) $\underline{Z}$ is connected;

(2) For a point $z \in \underline{Z}^{\star}$ and for a point $y \in \underline{Y}^{\star}$ both lying over $x$, there exists uniquely a morphism $\underline{Z} \rightarrow \underline{Y}$ over $\underline{X}$ which sends $z$ to $y$. 
If we fix a point $z \in \underline{Z}^{\star}$ lying over $x$, then the fiber $f^{-1}(x)$ is also identified with the set $\operatorname{Hom}_{\underline{X}}(\underline{Z}, \underline{Y})$ of morphisms over $\underline{X}$. Thus there is a group homomorphism $\pi_{1}\left(\underline{X}^{\star}, x\right) \rightarrow \operatorname{Gal}(\underline{Z} / \underline{X})$ (which depends on the choice of $z$ ) such that the action of $\pi_{1}\left(\underline{X}^{\star}, x\right)$ on $f^{-1}(x)$ is derived from that of $\operatorname{Gal}(\underline{Z} / \underline{X})$. In particular, we have a natural homomorphism $\pi_{1}\left(\underline{X}^{\star}, x\right) \rightarrow \hat{\pi}_{1}(\underline{X} ; x)$.

Definition. For a topological group $\Pi$, the category Fin( $\Pi)$ of finite discrete sets with continuous right action of $\Pi$ is defined as follows: an object is a pair $(\mathcal{S}, \rho)$ consisting of a finite discrete set $\mathcal{S}$ and an anti-group homomorphism $\rho: \Pi \rightarrow \operatorname{Aut}(\mathcal{S})$ such that

$$
\mathcal{S} \times \Pi \ni(s, p) \rightarrow \rho(p)(s) \in \mathcal{S}
$$

is continuous. A morphism $\left(\mathcal{S}_{1}, \rho_{1}\right) \rightarrow\left(\mathcal{S}_{2}, \rho_{2}\right)$ is defined to be a map $f: \mathcal{S}_{1} \rightarrow$ $\mathcal{S}_{2}$ satisfying $f \circ \rho_{1}(p)=\rho_{2}(p) \circ f$ for any $p \in \Pi$. The subcategory consisting of all the objects $(\mathcal{S}, \rho)$ with transitive action of $\rho(\Pi)$ is denoted by $\operatorname{Fin}^{\text {trans }}(\Pi)$.

Lemma 1.2.5. The category $\operatorname{Fin}\left(\hat{\pi}_{1}(\underline{X} ; x)\right)$ is equivalent to the category of $\partial$-spaces finite and $\partial$-étale over $\underline{X}$. Here, the subcategory $\operatorname{Fin}^{\text {trans }}\left(\hat{\pi}_{1}(\underline{X} ; x)\right)$ is equivalent to the category of connected $\partial$-spaces finite and $\partial$-étale over $\underline{X}$.

Proof. For a finite $\partial$-étale morphism $f: \underline{Y} \rightarrow \underline{X}$, the fiber $f^{-1}(x)$ admits a continuous right action of $\hat{\pi}_{1}(\underline{X} ; x)$, by the argument above. Let $\mathcal{S}$ be a finite discrete set with a continuous right action of $\hat{\pi}_{1}(\underline{X} ; x)$. By considering the decomposition by orbits, we may assume that the action is transitive. Then there exist a finite $\partial$-étale Galois morphism $\underline{Z} \rightarrow \underline{X}$ from a connected $\partial$-space $\underline{Z}$ and a point $z \in \underline{Z}^{\star}$ lying over $x$ such that the action is derived from that of $\operatorname{Gal}(\underline{Z} / \underline{X})$ on $\mathcal{S}$. Let $U \subset \operatorname{Gal}(\underline{Z} / \underline{X})$ be the stabilizer of a point of $\mathcal{S}$. Then $\mathcal{S} \simeq U \backslash \operatorname{Gal}(\underline{Z} / \underline{X})$ as sets with right action of $\operatorname{Gal}(\underline{Z} / \underline{X})$. We associate $U$ with the quotient $\partial$-space $U \backslash \underline{Z}$, which is finite $\partial$-étale over $\underline{X}$. Thus we have the equivalence.

Let $x$ be any point of $\underline{X}$. We fix a continuous path $\Phi:[0,1] \rightarrow \operatorname{sp}(\underline{X})$ such that $\Phi(0)=x$ and $\Phi(t) \in \underline{X}^{*}$ for $t \neq 0$. For a $\partial$-open neighborhood $\underline{U}$ of $x$, there is a number $0<t_{0}<1$ such that $\Phi(t) \in \operatorname{sp}(\underline{U})$ for any $t \leq t_{0}$. The profinite fundamental groups $\hat{\pi}_{1}(\underline{U} ; \Phi(t))$ for $0<t<t_{0}$ are isomorphic to $\hat{\pi}_{1}\left(\underline{U} ; \Phi\left(t_{0}\right)\right)$ by the path $\Phi$. We consider couples $(\underline{U}, t)$ consisting of a $\partial$-open neighborhood $\underline{U}$ of $x$ and a number $t$ such that $\Phi\left(t^{\prime}\right) \in \operatorname{sp}(\underline{U})$ for any $t^{\prime} \leq t$. For two couples $\left(\underline{U}_{1}, t_{1}\right)$ and $\left(\underline{U}_{2}, t_{2}\right)$, we denote $\left(\underline{U}_{1}, t_{1}\right) \prec\left(\underline{U}_{2}, t_{2}\right)$ if $\underline{U}_{1} \subset \underline{U}_{2}$ and if $t_{1} \leq t_{2}$. By considering the projective system $\left\{\hat{\pi}_{1}(\underline{U} ; \Phi(t))\right\}$ induced from $\prec$, 
we define the local profinite fundamental group by

$$
\hat{\pi}_{1}^{\text {loc }}(\underline{X} ; x, \Phi):=\lim _{\leftarrow} \hat{\pi}_{1}(\underline{U} ; \Phi(t)) .
$$

Lemma 1.2.6. If $\Phi_{1}$ and $\Phi_{2}$ are two continuous paths $[0,1] \rightarrow \operatorname{sp}(\underline{X})$ such that $\Phi_{1}(0)=\Phi_{2}(0)=x$ and that $\Phi_{1}(t), \Phi_{2}(t) \in \underline{X}^{\star}$ for $t \neq 0$, then there is an isomorphism between $\hat{\pi}_{1}^{\text {loc }}\left(\underline{X} ; x, \Phi_{1}\right)$ and $\hat{\pi}_{1}^{\text {loc }}\left(\underline{X} ; x, \Phi_{2}\right)$.

Proof. We may assume that $x \notin \underline{X}^{\star}$. Let $\mathcal{U}$ be an open neighborhood of $x$ in $\operatorname{sp}(\underline{X})$ such that there is a closed embedding $\mathcal{U} \hookrightarrow \Delta^{n}$ into an $n$ dimensional unit polydisc $\boldsymbol{\Delta}^{n}$ sending $x$ to the origin. By a coordinate system $\left(z_{1}, z_{2}, \ldots, z_{n}\right)$ of $\boldsymbol{\Delta}^{n}$ at $x=0$, we define $\rho(z):=\sum\left|z_{i}\right|^{2}$. Let $B_{\varepsilon}$ be the ball $\left\{z \in \boldsymbol{\Delta}^{n} \mid \rho(z)<\varepsilon\right\}$ and let $S_{\varepsilon}$ be the sphere $\left\{z \in \boldsymbol{\Delta}^{n} \mid \rho(z)=\varepsilon\right\}$ for a positive number $\varepsilon<1$. Then by the existence of Whitney stratifications and by Thom's first isotropy lemma, there exist positive numbers $\varepsilon_{0}<\varepsilon_{1}$ and a homeomorphism

$$
\varphi: B_{\varepsilon_{0}} \cap \mathcal{U} \cap \underline{X}^{\star} \rightarrow\left(S_{\varepsilon_{1}} \cap \mathcal{U} \cap \underline{X}^{\star}\right) \times\left(0, \varepsilon_{0}\right)
$$

such that $p_{2} \circ \varphi=\rho$ for the second projection $p_{2}$. Then for some positive number $\delta<1, \Phi_{1}$ and $\Phi_{2}$ restricted to $[0, \delta]$ are considered to be paths starting from the vertex in the cone

$$
\mathcal{C}:=\left(S_{\varepsilon_{1}} \cap \mathcal{U} \cap \underline{X}^{\star}\right) \times\left[0, \varepsilon_{0}\right) /\left(S_{\varepsilon_{1}} \cap \mathcal{U} \cap \underline{X}^{\star}\right) \times\{0\}
$$

of $S_{\varepsilon_{1}} \cap \mathcal{U} \cap \underline{X}^{\star}$. Thus there is a homotopy $h:[0, \delta] \times[0,1] \rightarrow \mathcal{C}$ such that $h(t, 0)=\Phi_{1}(t), h(t, 1)=\Phi_{2}(t)$ for any $t \in[0, \delta]$ and $h(0, s)=0$ for any $s \in[0,1]$. Hence we have an isomorphism $\hat{\pi}_{1}^{\text {loc }}\left(\underline{X} ; x, \Phi_{1}\right) \simeq \hat{\pi}_{1}^{\text {loc }}\left(\underline{X} ; x, \Phi_{2}\right)$ by the homotopy $h$.

We denote $\hat{\pi}_{1}^{\text {loc }}(\underline{X} ; x)=\hat{\pi}_{1}^{\text {loc }}(\underline{X} ; x, \Phi)$ when we consider only the group structure.

Definition 1.2.7. A germ of pointed $\partial$-spaces is an equivalence class of pointed $\partial$-spaces with respect to the following relation: Two pointed $\partial$ spaces $\left(\underline{Y}_{1} ; y_{1}\right)$ and $\left(\underline{Y}_{2} ; y_{2}\right)$ are equivalent if there exist $\partial$-open immersions $\left(\underline{Y}_{3} ; y_{3}\right) \rightarrow\left(\underline{Y}_{i} ; y_{i}\right)$ for $i=1,2$, from another pointed $\partial$-space. A $\partial$-étale morphism $(\underline{Y} ; y) \rightarrow(\underline{X} ; x)$ is called a $\partial$-étale neighborhood of $x$. A germ of $\partial$-étale neighborhoods of $x$ is a germ of pointed $\partial$-spaces étale over $(\underline{X} ; x)$.

Lemma 1.2.8. The following two categories are equivalent: 
(1) The category of germs of $\partial$-étale neighborhoods of $x$;

(2) $\operatorname{Fin}^{\operatorname{trans}}\left(\hat{\pi}_{1}^{\text {loc }}(\underline{X} ; x)\right)$.

Proof. The functors between two categories below give the equivalence.

$(1) \Longrightarrow(2)$ Let $(\underline{Y} ; y) \rightarrow(\underline{X} ; x)$ be a $\partial$-étale neighborhood. Since we consider only germs, we may assume that $\underline{X}$ and $\underline{Y}$ are connected, $f^{-1}(x)=$ $\{y\}$ for the morphism $f: \operatorname{sp}(\underline{Y}) \rightarrow \operatorname{sp}(\underline{X})$, and that $f: \underline{Y} \rightarrow \underline{X}$ is a finite $\partial-$ étale morphism. There exist a path $\Psi:[0,1] \rightarrow \operatorname{sp}(\underline{Y})$ and a homeomorphism $\varphi:[0,1] \rightarrow[0, \varepsilon]$ for $0<\varepsilon<1$ such that $f \circ \Psi=\Phi \circ \varphi$. Thus by 1.2.5, we can attach a finite discrete set $\mathcal{S}$ with a transitive continuous right action of $\hat{\pi}_{1}(\underline{X} ; \Phi(\varepsilon))$ to $\underline{Y} / \underline{X}$. It also admits a transitive continuous right action of $\hat{\pi}_{1}^{\text {loc }}(\underline{X} ; x)$ by definition. Since $\mathcal{S}$ is essentially the fiber $f^{-1}(\Phi(\varepsilon))$, this is independent of the choice of $\Psi$ and $\varepsilon$.

$(2) \Longrightarrow(1)$ Let $\mathcal{S}$ be a finite discrete set with a transitive continuous right action of $\hat{\pi}_{1}^{\text {loc }}(\underline{X} ; x)$. Then the action is derived from $\hat{\pi}_{1}(\underline{U} ; \Phi(t))$ for a $\partial$-open neighborhood of $x$ and for some $0<t<1$. Thus by 1.2 .5 , it is associated with a finite $\partial$-étale morphism $(\underline{Y} ; y) \rightarrow(\underline{U} ; x)$, where $\underline{Y}$ is connected. The germ of this étale neighborhood does not depend on the choice of $\underline{U}$ and $t$.

\section{§2. $\partial$-étale Cohomology}

The $\partial$-étale topology of the category $\partial \mathrm{sP}$ is defined by $\partial$-étale morphisms. A $\partial$-étale covering family of a $\partial$-space $\underline{X}$ is a collection of $\partial$-étale morphisms $\left\{h_{i}: \underline{U}_{i} \rightarrow \underline{X}\right\}_{i \in I}$ such that

$$
\bigcup_{i \in I} h_{i}\left(\operatorname{sp}\left(\underline{U}_{i}\right)\right)=\operatorname{sp}(\underline{X}) .
$$

In Section 2, we shall study sheaves (of abelian groups) on $\underline{X}$ with respect to the $\partial$-étale topology and their cohomology groups. For a presheaf $F$, the stalk $F_{x}$ at a point $x \in \operatorname{sp}(\underline{X})$ is defined as a discrete $\hat{\pi}_{1}^{\text {loc }}(\underline{X} ; x)$-module. The enough-injectiveness of the category of sheaves on $\underline{X}$ is proved and the cohomology groups $H^{i}(\underline{X}, F)$ are defined. The Čech cohomology group $\check{H}^{p}(\underline{X}, F)$ is shown to be isomorphic to $H^{p}(\underline{X}, F)$. Leray's spectral sequence exists for a morphism of $\partial$-spaces. In particular, for the morphism $\varepsilon: \underline{X}=(X, B) \rightarrow X$ to a realization, $H^{i}(\underline{X}, F)$ are calculated by $H^{p}\left(X, R^{q} \varepsilon_{*} F\right)$. If $[X, B]$ is locally connected at $x$, then the stalk of $R^{q} \varepsilon_{*} F$ at $x$ is isomorphic to the continuous group cohomology $H_{\text {cont. }}^{q}\left(\hat{\pi}_{1}^{\text {loc }}(\underline{X} ; x), F_{x}\right)$. Coherent $\mathcal{O}_{\underline{X}}$-sheaves are also studied. 


\section{§2.1. Sheaf}

A presheaf $F$ of abelian groups of $\underline{X}$ is a contra-variant functor from the category $(\partial$-ét $) / \underline{X}$ of $\partial$-spaces $\partial$-étale over $\underline{X}$ to the category of abelian groups. This is called a sheaf if for a $\partial$-étale morphism $\underline{U} \rightarrow \underline{X}$ and for a $\partial$-étale covering family $\left\{\underline{U}_{i} \rightarrow \underline{U}\right\}_{i \in I}$, the sequence

$$
0 \rightarrow F(\underline{U}) \rightarrow \prod_{i \in I} F\left(\underline{U}_{i}\right) \rightrightarrows \prod_{i, j \in I} F\left(\underline{U}_{i} \times_{\underline{U}} \underline{U}_{j}\right)
$$

is exact.

Remark. Let $\pi: \underline{Y} \rightarrow \underline{X}$ be a $\partial$-étale Galois morphism with the Galois group $G$. If $F$ is a presheaf of $\underline{X}$, then $F(\underline{Y})$ is a right $G$-module, since $G \times \underline{Y} \simeq$ $\underline{Y} \times \underline{X} \underline{Y}$. If $F$ is a sheaf and if $\pi$ is surjective, then the $G$-invariant part of $F(\underline{Y})$ is $F(\underline{X})$.

Example. Let $\underline{X}$ be a $\partial$-space and let $M$ an abelian group. For a $\partial$-étale morphism $\underline{U} \rightarrow \underline{X}$, we attach the group

$$
\lim _{[U, \Delta] \in \underline{U}} H^{0}(U, M) .
$$

This forms a sheaf $M_{\underline{X}}$ called the constant sheaf. If $\underline{U}$ is connected, then $M_{\underline{X}}(\underline{U}) \simeq M$. For a $\partial$-étale morphism $\underline{U} \rightarrow \underline{X}$, we attach the group

$$
\lim _{[U, \Delta] \in \underline{U}} H^{0}\left(U, \mathcal{O}_{U}\right) .
$$

This forms a sheaf of rings in $\partial$-étale topology. This is called the structure sheaf and is denoted by $\mathcal{O}_{\underline{X}}$.

We introduce Čech cohomology groups. Let $F$ be a presheaf of $\underline{X}$ and let $\underline{\mathcal{U}}:=\left\{\underline{U}_{\alpha} \rightarrow \underline{X}\right\}_{\alpha \in A}$ be a $\partial$-étale covering family of $\underline{X}$. For $\boldsymbol{\alpha}=\left(\alpha_{0}, \alpha_{1}, \ldots\right.$, $\left.\alpha_{q}\right) \in A^{q+1}$, we define

$$
\underline{U}_{\alpha}:=\underline{U}_{\alpha_{0}} \times \underline{X} \underline{U}_{\alpha_{1}} \times_{\underline{X}} \cdots \times{ }_{\underline{X}} \underline{U}_{\alpha_{q}} .
$$

The group of $q$-th $\check{C}$ ech cochains is defined to be

$$
C^{q}(\underline{\mathcal{U}} / \underline{X}, F):=\prod_{\boldsymbol{\alpha} \in A^{q+1}} F\left(\underline{U}_{\boldsymbol{\alpha}}\right) .
$$

Then we have the Čech complex $C \bullet \underline{\mathcal{U}} / \underline{X}, F)$ and its cohomology groups $\check{H}^{q}$ $(\underline{\mathcal{U}} / \underline{X}, F)$ as usual. A refinement of $\underline{\mathcal{U}}$ is a $\partial$-étale covering family $\underline{\mathcal{V}}=\left\{\underline{V}_{\beta} \rightarrow\right.$ 
$\underline{X}\}_{\beta \in B}$ of $\underline{X}$ with a map $\alpha: B \rightarrow A$ and with $\partial$-étale morphisms $\varphi_{\beta}: \underline{V}_{\beta} \rightarrow$ $\underline{U}_{\alpha(\beta)}$ over $\underline{X}$. For the refinement, we have homomorphisms $\check{H}^{q}(\underline{\mathcal{U}} / \underline{X}, F) \rightarrow$ $\check{H}^{q}(\underline{\mathcal{V}} / \underline{X}, F)$ as usual, which do not depend on the choices of $\alpha$ and $\varphi_{\beta}$. For a given $\partial$-étale covering family, we have a refinement $\left\{\underline{V}_{\beta} \rightarrow \underline{X}\right\}$ such that each $\underline{V}_{\beta}$ is $\partial$-étale Galois over $\underline{X}$ with a finite Galois group. Thus we may assume that $\underline{V}_{\beta}$ is determined by a finite quotient group of $\hat{\pi}_{1}^{\text {loc }}\left(\underline{X} ; x_{\beta}\right)$ for some point $x_{\beta}$ of $\underline{X}$. Therefore we can define the inductive limit

$$
\check{H}^{q}(\underline{X}, F):=\lim _{\longrightarrow} \check{H}^{q}(\underline{\mathcal{U}} / \underline{X}, F)
$$

and call this the $q$-th $\check{C}$ ech cohomology group.

Lemma 2.1.1. Let $\underline{U} \rightarrow \underline{X}$ be a surjective $\partial$-étale Galois morphism with the Galois group $G$. Then for a sheaf $F$ of $\underline{X}$, we have

$$
\check{H}^{p}(\underline{U} / \underline{X}, F) \simeq H^{p}(G, F(\underline{U})),
$$

where we consider $\{\underline{U} \rightarrow \underline{X}\}$ as a $\partial$-étale covering family and the right hand side is the group cohomology of the right $G$-module $F(\underline{U})$.

Proof. For a non-negative integer $q$, we have an isomorphism

$$
G^{q} \times \underline{U} \simeq \underline{U} \times_{\underline{X}} \underline{U} \times_{\underline{X}} \cdots \times_{\underline{X}} \underline{U} \quad((q+1) \text {-fold fiber product }) .
$$

Thus $C^{q}(\underline{U} / \underline{X}, F) \simeq \operatorname{Map}\left(G^{q}, F(\underline{U})\right)$. The complex $\operatorname{Map}\left(G^{\bullet}, F(\underline{U})\right)$ is nothing but the complex defining $H^{p}(G, F(\underline{U}))$, which is derived from the nonhomogeneous free resolution of the trivial $G$-module $\mathbb{Z}$.

We define presheaves $\underline{\mathcal{H}}^{q}(F)$ of $\underline{X}$ for non-negative integers $q$ by

$$
\underline{\mathcal{H}}^{q}(F)(\underline{U}):=\check{H}^{q}(\underline{U}, F)
$$

for $\partial$-étale morphisms $\underline{U} \rightarrow \underline{X}$. In the case $q=0$, the following properties are well-known:

(1) $\check{\mathcal{H}}^{0}(F)$ is a separated presheaf, i.e.,

$$
0 \rightarrow \underline{\mathcal{H}}^{0}(F)(\underline{U}) \rightarrow \prod_{i \in I} \check{\mathcal{H}}^{0}(F)\left(\underline{U}_{i}\right)
$$

is exact for a $\partial$-étale covering family $\left\{\underline{U}_{i} \rightarrow \underline{U}\right\}_{i \in I}$;

(2) If $F$ itself is a separated presheaf, then $\underline{\mathcal{H}}^{0}(F)$ is a sheaf; 
(3) If $F$ is a sheaf, then $F \simeq \underline{\mathcal{H}}^{0}(F)$;

(4) For an exact sequence of presheaves $0 \rightarrow F \rightarrow G \rightarrow H$, the induced sequence $0 \rightarrow \underline{\mathcal{H}}^{0}(F) \rightarrow \underline{\mathcal{H}}^{0}(G) \rightarrow \underline{\mathcal{H}}^{0}(H)$ is also exact.

Thus $F^{a}:=\underline{\mathcal{H}}^{0}\left(\underline{\mathcal{H}}^{0}(F)\right)$ is the sheafification of $F$.

The stalk $F_{x / \underline{X}}$ in the weak sense of a presheaf $F$ at a point $x \in \operatorname{sp}(\underline{X})$ is defined to be the inductive limit

$$
F_{x / \underline{X}}:=\lim _{\longrightarrow} F(\underline{U})
$$

for $\partial$-open neighborhoods $\underline{U}$ of $x$. We attach the stalk $F_{v / \underline{V}}$ in the weak sense to a germ $(\underline{V} ; v)$ of a $\partial$-étale neighborhood of $x$. Then by considering 1.2 .8 , we can define an abelian group $F_{x}[\mathcal{S}]$ for a finite discrete set $\mathcal{S}$ with a transitive continuous action of $\hat{\pi}_{1}^{\text {loc }}(\underline{X} ; x)$. In particular, $F_{x}[\Gamma]$ is a right $\Gamma$-module for a finite quotient group $\Gamma$ of $\hat{\pi}_{1}^{\text {loc }}(\underline{X} ; x)$. If $F$ is a sheaf and if $\Gamma^{\prime}$ is a subgroup of $\Gamma$, then the $\Gamma^{\prime}$-invariant part of $F_{x}[\Gamma]$ is $F_{x}\left[\Gamma^{\prime} \backslash \Gamma\right]$. The stalk $F_{x}$ is defined to be the inductive limit

$$
F_{x}:=\lim _{\longrightarrow} F_{x}[\Gamma]
$$

for finite quotient groups $\hat{\pi}_{1}^{\text {loc }}(\underline{X} ; x) \rightarrow \Gamma$. This is a discrete $\hat{\pi}_{1}^{\text {loc }}(\underline{X} ; x)$-module. If $F$ is a sheaf, then the $F_{x}[\Gamma]$ is identified with the invariant part of $F_{x}$ by the action of the kernel of $\hat{\pi}_{1}^{\text {loc }}(\underline{X} ; x) \rightarrow \Gamma$. If $(\underline{V} ; v) \rightarrow(\underline{U} ; u)$ is $\partial$-étale, then we have an isomorphism $F_{u} \simeq F_{v}$ as abelian groups. The support of $F, \operatorname{Supp} F$, is defined to be $\left\{x \in \operatorname{sp}(\underline{X}) \mid F_{x} \neq 0\right\}$. For a section $s \in F(\underline{U})$, the germ $s_{u}$ at $u \in \operatorname{sp}(\underline{U})$ is defined to be the image of $s$ by $F(\underline{U}) \rightarrow F_{u}$. The following lemma is proved by a standard argument.

\section{Lemma.}

(1) Let $F \rightarrow G$ be a homomorphism of presheaves of $\underline{X}$ where $G$ is a sheaf. Then this is the sheafification of $F$ if and only if this induces isomorphisms $F_{x} \simeq G_{x}$ for any point $x$ of $\underline{X}$.

(2) Let $0 \rightarrow F \rightarrow G \rightarrow H \rightarrow 0$ be a sequence of sheaves on $\underline{X}$. Then it is exact if and only if $0 \rightarrow F_{x} \rightarrow G_{x} \rightarrow H_{x} \rightarrow 0$ is exact for any $x \in \operatorname{sp}(\underline{X})$.

(3) Let $F$ be a presheaf of a $\partial$-space $\underline{X}, F^{a}$ the sheafification of $F$ and let $x$ be a point of $\underline{X}$. Then the stalk $F_{x / \underline{X}}^{a}$ in the weak sense is isomorphic to the $\hat{\pi}_{1}^{\mathrm{loc}}(\underline{X} ; x)$-invariant part of $F_{x} . \underline{F}$ or a $\partial$-étale morphism $\underline{U} \rightarrow \underline{X}$, we have 
the following identification:

$$
\begin{aligned}
F^{a}(\underline{U})= & \left\{\left(s_{u}\right) \in \prod_{u \in \operatorname{sp}(\underline{U})} F_{u / \underline{U}}^{a} \mid \text { for any } u,\right. \\
& \text { there exist a } \partial \text {-étale neighborhood } \tau: \underline{V} \rightarrow \underline{U} \\
& \text { of } u \text { and an element } t \in F(\underline{V}) \\
& \text { such that } \left.t_{v}=s_{\tau(v)} \text { for any } v \in \operatorname{sp}(\underline{V})\right\} .
\end{aligned}
$$

The stalk $F_{x}$ is a discrete right $\hat{\pi}_{1}^{\text {loc }}(\underline{X} ; x)$-module for a sheaf $F$ of $\underline{X}$. Conversely, we can construct a sheaf $i_{x} m$ of $\underline{X}$ from a discrete right $\hat{\pi}_{1}^{\text {loc }}(\underline{X} ; x)$ module $m$ as follows: For a $\partial$-étale morphism $\underline{U} \rightarrow \underline{X}$, we set:

$$
i_{x} m(\underline{U}):=\prod_{\operatorname{sp}(\underline{U}) \ni u \mapsto x} m^{\hat{\pi}_{1}^{\text {loc }}(\underline{U} ; u)},
$$

where $m^{\Pi}$ is the $\Pi$-invariant part of $m$ for a subgroup $\Pi \subset \hat{\pi}_{1}^{\text {loc }}(\underline{X} ; x)$. Then $i_{x} m$ is a sheaf and $\operatorname{Supp} i_{x} m=\{x\}$. Therefore we have:

Lemma. The category of discrete right $\hat{\pi}_{1}^{\text {loc }}(\underline{X} ; x)$-modules and the category of sheaves of $\underline{X}$ supported in $\{x\}$, are equivalent.

For a sheaf $F$, we have the sheaf $\prod_{x \in \operatorname{sp}(\underline{X})} i_{x} F_{x}$ and an injection $F \hookrightarrow$ $\prod_{x \in \operatorname{sp}(\underline{X})} F_{x}$. Thus the category of sheaves on $\underline{X}$ has enough injectives. In fact, if $m$ is an injective discrete $\hat{\pi}_{1}^{\text {loc }}(\underline{X} ; x)$-module, then $i_{x} m$ is an injective sheaf on $\underline{X}$. Therefore we can consider right derived functors, especially cohomology groups of sheaves.

Definition. The right derived functors for the global section functor given by $F \mapsto F(\underline{X})$ are denoted by $H^{i}(\underline{X}, F)$. These are called $\partial$-étale cohomology groups of $F$ over $\underline{X}$.

Let $f: \underline{Y} \rightarrow \underline{X}$ be a morphism of $\partial$-spaces. Then for a sheaf $F$ of $\underline{Y}$, the direct image sheaf $f_{*} F$ is defined by:

$$
f_{*} F(\underline{U})=F(\underline{Y} \times \underline{X} \underline{U})
$$

for any $\partial$-étale morphism $\underline{U} \rightarrow \underline{X}$. We also define the direct image sheaf with proper support $f_{!} F$ as follows:

$$
f_{!} F(\underline{U})=\{s \in F(\underline{Y} \times \underline{X} \underline{U}) \mid \operatorname{Supp}(s) \rightarrow \operatorname{sp}(\underline{U}) \text { is proper }\} .
$$


Conversely, for a sheaf $G$ of $\underline{X}$, we have the pullback sheaf $f^{-1} G$. This is defined to be the sheafification of the presheaf

$$
\underline{V} \mapsto \underset{\underline{V} \rightarrow \underline{\underline{U} \rightarrow \underline{X}}}{\lim _{\underline{X}}} G(\underline{U}),
$$

where the limit is taken over $\partial$-étale morphisms $\underline{U} \rightarrow \underline{X}$ with factorizations $\underline{V} \rightarrow$ $\underline{U}$ of the composite $\underline{V} \rightarrow \underline{Y} \rightarrow \underline{X}$. Then there exist canonical homomorphisms $f^{-1} f_{*} F \rightarrow F$ and $G \rightarrow f_{*} f^{-1} G$, by which $f_{*}$ and $f^{-1}$ are adjoint to each other:

$$
\operatorname{Hom}_{\underline{X}}\left(F, f_{*} G\right) \simeq \operatorname{Hom}_{\underline{Y}}\left(f^{-1} F, G\right) .
$$

If $f$ is a $\partial$-étale morphism, we sometimes write $f^{-1} F$ by $\left.F\right|_{\underline{Y}}$, since this is the restriction to $(\partial$-ét) $/ \underline{Y}$.

Lemma 2.1.2. Let $f: \underline{Y} \rightarrow \underline{X}$ be a $\partial$-étale morphism and let $F$ and $G$ be sheaves of $\underline{Y}$ and $\underline{X}$, respectively. Then there exist canonical homomorphisms $F \rightarrow f^{-1}\left(f_{!} F\right)$ and $f_{!}\left(f^{-1} G\right) \rightarrow G$ by which $f_{!}$and $f^{-1}$ are adjoint, i.e.,

$$
\operatorname{Hom}_{\underline{X}}\left(f_{!} F, G\right) \simeq \operatorname{Hom}_{\underline{Y}}\left(F, f^{-1} G\right) .
$$

Proof. The left adjoint $f_{\sharp}^{a}$ of $f^{-1}$ is constructed by the following Kan's process (cf. [S5]): For a $\partial$-étale morphism $\underline{U} \rightarrow \underline{X}$, let $I_{\underline{U} / \underline{X}}^{f}$ be the category of pairs $\langle\underline{V} / \underline{Y}, \phi\rangle$ such that $\underline{V} \rightarrow \underline{Y}$ is a $\partial$-étale morphism and $\phi: \underline{U} \rightarrow \underline{V}$ is a $\partial$-étale morphism over $\underline{X}$. A morphism $\left\langle\underline{V}_{1} / \underline{Y}, \phi_{1}\right\rangle \rightarrow\left\langle\underline{V}_{2} / \underline{Y}, \phi_{2}\right\rangle$ is defined to be a $\partial$-étale morphism $h: \underline{V}_{1} \rightarrow \underline{V}_{2}$ such that $\phi_{2}=h \circ \phi_{1}$. For a sheaf $F$ of $\underline{Y}$, we can define a presheaf of $\underline{X}$ by

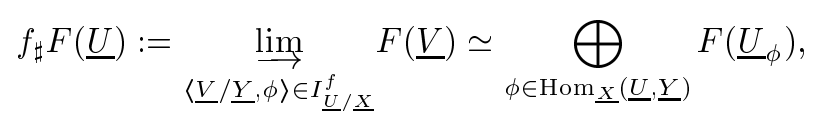

where $\underline{U}_{\phi}$ denotes the $\partial$-étale morphism $\phi: \underline{U} \rightarrow \underline{Y}$. Then the sheafification $\left(f_{\sharp} F\right)^{a}$ induces the left adjoint functor $f_{\sharp}^{a}$. We have a natural homomorphism $f_{\sharp}^{a} F \rightarrow f_{!} F$ for a sheaf $F$ of $\underline{Y}$. By comparing their stalks, we have an isomorphism $f_{\sharp}^{a} F \simeq f_{!} F$.

Remark. The enough-injectiveness of the category of sheaves of $\underline{X}$ is also derived from 2.1.2, since $f ! \mathbb{Z}_{\underline{U}}$ for all $\partial$-étale morphisms $f: \underline{U} \rightarrow \underline{X}$ form a generator of the category.

Corollary 2.1.3. Let $\underline{U} \rightarrow \underline{X}$ be a $\partial$-étale morphism and let $F$ be a sheaf of $\underline{X}$. 
(1) If $F$ is an injective sheaf, then so is $\left.F\right|_{\underline{U}}$.

(2) The functors $F \mapsto H^{i}\left(\underline{U},\left.F\right|_{\underline{U}}\right)(i \geq 0)$ are the right derived functors of $F \mapsto F(\underline{U})$.

For a morphism $f: \underline{X} \rightarrow \underline{Y}$ of $\partial$-spaces, the right derived functors for $f_{*}$ are called the higher direct images and denoted by $R^{i} f_{*} F$. There is a natural morphism $\varepsilon=\varepsilon_{X}: \underline{X}=(X, B) \rightarrow(X, \emptyset)$ for a realization $[X, B]$ of $\underline{X}$. The category of $\partial$-étale morphisms over $(X, \emptyset)$ is equivalent to that of étale morphisms over $X$. Thus the category of sheaves of $(X, \emptyset)$ is equivalent to that of $X$, since an étale morphism is a local isomorphism. Therefore the direct image $\varepsilon_{*} F$ is the functor $F$ restricted to the category of open subspaces of $X$.

\section{§2.2. Čech cohomology and right derived functor cohomology}

For a presheaf $F$ of $\underline{X}$, we define the presheaves $\underline{\mathcal{H}}^{q}(F)$ of $\underline{X}$ for $q \geq 0$ by:

$$
\underline{\mathcal{H}}^{q}(F)(\underline{U})=H^{q}(\underline{U}, F),
$$

for a $\partial$-étale morphism $\underline{U} \rightarrow \underline{X}$. If $F$ is a sheaf, then $F \simeq \underline{\mathcal{H}}^{0}(F)$. Let $\underline{\mathcal{V}}:=\left\{\underline{V}_{\beta} \rightarrow \underline{X}\right\}$ be a $\partial$-étale covering family of $\underline{X}$. Let $\underline{C^{q}}(\underline{\mathcal{V}} / \underline{X}, F)$ be the presheaf of $\underline{X}$ defined by

$$
\underline{C}^{q}(\underline{\mathcal{V}} / \underline{X}, F)(\underline{U}):=C^{q}(\underline{\mathcal{V}} \times \underline{X} \underline{U} / \underline{U}, F)
$$

for $\partial$-étale morphisms $\underline{U} \rightarrow \underline{X}$, where $\underline{\mathcal{V}} \times \underline{X} \underline{U}$ is the $\partial$-étale covering family $\left\{\underline{V}_{\beta} \times \underline{X} \underline{U} \rightarrow \underline{U}\right\}$ of $\underline{U}$. Then $\underline{C} \bullet(\underline{\mathcal{V}} / \underline{X}, F)$ is a complex of presheaves. The $q$-th cohomology presheaf is denoted by $\check{\mathcal{H}}^{q}(\underline{\mathcal{V}} / \underline{X}, F)$. In particular, $\check{\mathcal{H}}^{q}(\underline{\mathcal{V}} / \underline{X}, F)(\underline{X})$ $=\check{H}^{q}(\underline{\mathcal{V}} / \underline{X}, F)$.

Lemma 2.2.1. $\quad$ Let $I$ be an injective sheaf on $\underline{X}$ and let $\underline{\mathcal{U}}:=\left\{\underline{U}_{\alpha} \rightarrow\right.$ $\underline{X}\}_{\alpha \in A}$ be a $\partial$-étale covering family of $\underline{X}$. Then $\breve{H}^{i}(\underline{\mathcal{U}} / \underline{X}, I)=0$ for $i>0$.

Proof. For a non-negative integer $q$, let $C_{q}$ be the sheaf

$$
C_{q}:=\bigoplus_{\boldsymbol{\alpha} \in A^{q+1}} f_{\boldsymbol{\alpha}} ! \mathbb{Z}_{\underline{U}_{\alpha}}
$$

where $f_{\boldsymbol{\alpha}}: \underline{U}_{\boldsymbol{\alpha}} \rightarrow \underline{X}$ is the induced morphism. Then we have an exact sequence

$$
\cdots \rightarrow C_{q} \rightarrow C_{q-1} \rightarrow \cdots \rightarrow C_{0} \rightarrow \mathbb{Z}_{\underline{X}} \rightarrow 0
$$


such that, for a sheaf $F$, the Čech cohomology group $\check{H}^{q}(\underline{\mathcal{U}} / \underline{X}, F)$ is the $q$-th cohomology group of the induced complex

$$
0 \rightarrow \operatorname{Hom}\left(C_{0}, F\right) \rightarrow \operatorname{Hom}\left(C_{1}, F\right) \rightarrow \cdots \rightarrow \operatorname{Hom}\left(C_{q}, F\right) \rightarrow \cdots
$$

If $I$ is an injective sheaf, then $\operatorname{Hom}(\bullet, I)$ is an exact functor. Hence $\check{H}^{i}(\underline{\mathcal{U}} / \underline{X}, I)$ $=0$ for $i>0$.

Lemma 2.2.2. Let $[X, B]$ be a realization of $\underline{X}, \varepsilon: \underline{X} \rightarrow X$ the natural morphism, and let $\underline{\mathcal{U}}:=\left\{\underline{U}_{\alpha} \rightarrow \underline{X}\right\}_{\alpha \in A}$ be a $\partial$-étale covering family of $\underline{X}$. Then we have the following spectral sequences for a sheaf $F$ of $\underline{X}$ :

$$
\begin{aligned}
E_{2}^{p, q}(\underline{\mathcal{U}} / \underline{X})=\check{H}^{p}\left(\underline{\mathcal{U}} / \underline{X}, \underline{\mathcal{H}}^{q}(F)\right) & \Longrightarrow H^{p+q}(\underline{X}, F) ; \\
E_{2}^{p, q}(\underline{X})=\check{H}^{p}\left(\underline{X}, \underline{\mathcal{H}}^{q}(F)\right) & \Longrightarrow H^{p+q}(\underline{X}, F) ; \\
E_{2}^{p, q}(X)=\check{H}^{p}\left(X, \varepsilon_{*} \underline{\mathcal{H}}^{q}(F)\right) & \Longrightarrow H^{p+q}(\underline{X}, F) .
\end{aligned}
$$

Proof. Let

$$
0 \rightarrow F \rightarrow I^{0} \rightarrow I^{1} \rightarrow \cdots \rightarrow I^{p} \rightarrow \cdots
$$

be an injective resolution of $F$. The presheaf $\underline{\mathcal{H}}^{q}(F)$ is the $q$-th cohomology of the complex $I^{\bullet}$ of presheaves. We consider the double complex

$$
K^{p, q}:=\check{H}^{p}\left(\underline{\mathcal{U}} / \underline{X}, I^{q}\right) .
$$

Then $H^{p}\left(K^{\bullet}, q\right)=0$ for $p>0$ by 2.2.1. Since $H^{0}\left(K^{\bullet, q}\right)=H^{0}\left(\underline{X}, I^{q}\right)$, the $p$-th cohomology of the total complex of $K^{\bullet \bullet \bullet}$ is isomorphic to $H^{p}(\underline{X}, F)$. On the other hand, $H^{q}\left(K^{p, \bullet}\right)$ is isomorphic to $C^{p}\left(\underline{\mathcal{U}} / \underline{X}, \underline{\mathcal{H}}^{q}(F)\right)$. Thus we have the first spectral sequence $E_{2}^{p, q}(\underline{\mathcal{U}} / \underline{X})$. By taking the inductive limit for refinements of $\underline{\mathcal{V}}$, we have the second sequence $E_{2}^{p, q}(\underline{X})$. Let $\left\{U_{\alpha}\right\}$ be an open covering of $X$. Then $\varepsilon^{-1}\left(U_{\alpha}\right)$ form a $\partial$-étale covering family $\underline{\mathcal{U}}$ of $\underline{X}$. Then

$$
\check{H}^{p}\left(\underline{\mathcal{U}} / \underline{X}, \underline{\mathcal{H}}^{q}(F)\right) \simeq \check{H}^{p}\left(\left\{U_{\alpha}\right\}, \varepsilon_{*} \underline{\mathcal{H}}^{q}(F)\right)
$$

for any $p, q$. Thus we have the third sequence $E_{2}^{p, q}(X)$.

Corollary 2.2.3. Let $f: \underline{Y} \rightarrow \underline{X}$ be a morphism of $\partial$-étale spaces and let $F$ be a sheaf on $\underline{Y}$. Then there exists Leray's spectral sequence:

$$
E_{2}^{p, q}=H^{p}\left(\underline{X}, R^{q} f_{*} F\right) \Longrightarrow H^{p+q}(\underline{Y}, F) .
$$


Proof. It is enough to show that $H^{r}\left(\underline{X}, f_{*} I\right)=0$ for $r>0$ and for any injective sheaf $I$ of $\underline{Y}$. We know $\check{H}^{p}\left(\underline{X}, f_{*} I\right)=0$ for $p>0$ by 2.2.1. Let us consider the spectral sequence

$$
E_{2}^{p, q}(\underline{X})=\check{H}^{p}\left(\underline{X}, \underline{\mathcal{H}}^{q}\left(f_{*} I\right)\right) \Longrightarrow E^{p+q}=H^{p+q}\left(\underline{X}, f_{*} I\right) .
$$

Then $E_{2}^{p, 0}(\underline{X})=0$ for $p>0$. Moreover, $E_{2}^{0, q}(\underline{X})=\check{H}^{0}\left(\underline{X}, \underline{\mathcal{H}}^{q}\left(f_{*} I\right)\right)=0$ for $q>0$, since the sheafification of $\underline{\mathcal{H}}^{q}\left(f_{*} I\right)$ is zero. In particular, $E_{2}^{1,0}(\underline{X})=$ $E_{2}^{0,1}(\underline{X})=0$. Suppose that for a positive integer $r, E_{2}^{p, q}(\underline{X})=0$ for $0<$ $p+q<r$. Then $E^{i}=0$ for any $0<i<r$. Thus $\underline{\mathcal{H}}^{i}\left(f_{*} I\right)=0$ by 2.1.3. Therefore $E_{2}^{p, q}(\underline{X})=0$ also for $p+q=r$. Hence we have $E^{r}=0$ for any $r>0$.

Lemma 2.2.4. $\quad$ Let $\underline{U}$ be a $\partial$-étale neighborhood of a point $x$ of $\underline{X}$ and let

$$
\underline{W} \rightarrow \underline{U}^{q+1}:=\underline{U} \times \underline{X} \underline{U} \times_{\underline{X}} \cdots \times_{\underline{X}} \underline{U} \quad((q+1) \text {-fold fiber product })
$$

be a $\partial$-étale morphism for a non-negative integer $q \geq 0$ such that $\operatorname{sp}(\underline{W}) \rightarrow$ $\operatorname{sp}\left(\underline{U}^{q+1}\right)$ is surjective over $x$. Then there exist a $\partial$-étale neighborhood $\underline{V}$ of $x$ and a $\partial$-étale morphism $\underline{V} \rightarrow \underline{U}$ over $\underline{X}$ such that

(1) the induced morphism $\operatorname{sp}(\underline{V}) \rightarrow \operatorname{sp}(\underline{U})$ is surjective over $x$,

(2) there is a factorization $\underline{V}^{q+1} \rightarrow W \rightarrow \underline{U}^{q+1}$.

Proof. By taking base changes, we may assume that $\underline{U}$ is a $\partial$-étale Galois neighborhood determined by a finite quotient group $\Gamma$ of $\hat{\pi}_{1}^{\text {loc }}(\underline{X} ; x)$ and that $\underline{W} \rightarrow \underline{X}$ is a finite $\partial$-étale morphism. Since $\underline{U}^{q+1} \simeq \Gamma^{q} \times \underline{U}$ is a disjoint union of copies of $\underline{U}$,

$$
\underline{W}=\bigsqcup_{\gamma \in \Gamma^{q}} \underline{W}_{\gamma},
$$

for $\partial$-étale finite morphisms $\underline{W}_{\gamma} \rightarrow \underline{U}$. We may assume that each $\underline{W}_{\gamma}$ is also determined by a finite quotient group $\Gamma_{\gamma}$ of $\hat{\pi}_{1}^{\text {loc }}(\underline{X} ; x)$. There are surjections $\hat{\pi}_{1}^{\text {loc }}(\underline{X} ; x) \rightarrow \Gamma_{\gamma} \rightarrow \Gamma$. Hence we have a finite quotient group $\Gamma^{\prime}$ of $\hat{\pi}_{1}^{\text {loc }}(\underline{X} ; x)$ with $\hat{\pi}_{1}^{\text {loc }}(\underline{X} ; x) \rightarrow \Gamma^{\prime} \rightarrow \Gamma_{\gamma}$ for any $\gamma \in \Gamma^{q}$. Let $\underline{V} \rightarrow \underline{X}$ be a $\partial$-étale Galois neighborhood corresponding to $\Gamma^{\prime}$. Then it factors through each $\underline{W}_{\gamma} \rightarrow \underline{X}$. Let $\underline{V} \rightarrow \underline{U}$ be a $\partial$-étale Galois morphism corresponding to the $\Gamma^{\prime} \rightarrow \Gamma$. Then $\underline{V}^{q+1} \simeq \Gamma^{\prime q} \times \underline{V}$ and we can take a factor $\Gamma^{\prime q} \times \underline{V} \rightarrow \underline{W}$ of $\underline{V}^{q+1} \rightarrow \underline{U}^{q+1}$.

Corollary 2.2.5. $\quad$ Let $F$ be a presheaf of $\underline{X}$ and let $\varepsilon: \underline{X} \rightarrow X$ be the natural morphism to a realization $[X, B]$ of $\underline{X}$. 
(1) If $F_{x}=0$ for a point $x \in \operatorname{sp}(\underline{X})$, then for any $q$, we have

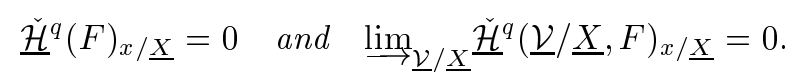

(2) Let $F^{a}$ be the sheafification of $F$. Then for any point $x \in \operatorname{sp}(\underline{X})$ and for any $q \geq 0$, we have isomorphisms

$$
\lim _{\underline{\underline{\mathcal{V}}} / \underline{\underline{X}}} \underline{\mathcal{H}}^{q}(\underline{\mathcal{V}} / \underline{X}, F)_{x / \underline{X}} \simeq \underline{\mathcal{H}}^{q}(F)_{x / \underline{X}} \simeq \underline{\mathcal{H}}^{q}\left(F^{a}\right)_{x / \underline{X}} .
$$

(3) The group of (2) is also isomorphic to the continuous group cohomology

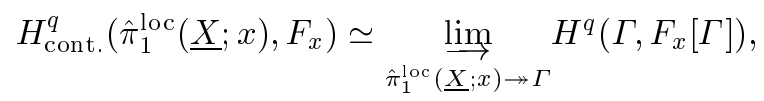

where the limit is taken over all the finite quotient groups of $\hat{\pi}_{1}^{\operatorname{loc}}(\underline{X} ; x)$.

(4) If $F$ is a sheaf, then $R^{p} \varepsilon_{*} F$ is the sheafification of $\varepsilon_{*} \check{\mathcal{H}}^{p}(F)$.

(5) If $F$ is a sheaf and if $[X, B]$ is locally connected at a point $\bar{x} \in X$, then

$$
\left(R^{p} \varepsilon_{*} F\right)_{\bar{x}} \simeq H_{\text {cont. }}^{p}\left(\hat{\pi}_{1}^{\text {loc }}(\underline{X} ; x), F_{x}\right),
$$

where $x \in \operatorname{sp}(\underline{X})$ is the unique point lying over $\bar{x}$.

Proof. (1) Let $\underline{U} \rightarrow \underline{X}$ be a $\partial$-étale neighborhood of $x$ and let $s \in$ $C^{q}(\underline{U} / \underline{X}, F)=F\left(\underline{U}^{q+1}\right)$. Then there is a $\partial$-étale neighborhood $\underline{V}$ over $\underline{U}$ of $x$ such that the restriction $s_{\underline{V}} \in C^{q}(\underline{V} / \underline{X}, F)$ is zero, by 2.2.4.

(2) By (1), we have

$$
\begin{aligned}
\lim _{\underline{\underline{\mathcal{V}}} / \underline{X}} \underline{\underline{\mathcal{H}}}^{q}(\underline{\mathcal{V}} / \underline{X}, F)_{x / \underline{X}} & \simeq \underline{\lim }_{\underline{\underline{\mathcal{V}}} / \underline{X}} \check{\check{\mathcal{H}}}^{q}\left(\underline{\mathcal{V}} / \underline{X}, F^{a}\right)_{x / \underline{X}}, \\
\underline{\check{\mathcal{H}}}^{q}(F)_{x / \underline{X}} & \simeq \underline{\check{\mathcal{H}}}^{q}\left(F^{a}\right)_{x / \underline{X}} .
\end{aligned}
$$

Thus we may assume that $F$ is a sheaf. By localizing spectral sequences in 2.2.2, we have the following two spectral sequences of presheaves of $\underline{X}$ :

$$
\lim _{\underline{\underline{\mathcal{V}}} / \underline{\underline{X}}} \underline{\check{\mathcal{H}}}^{p}\left(\underline{\mathcal{V}} / \underline{X}, \underline{\mathcal{H}}^{q}(F)\right) \Longrightarrow \underline{\mathcal{H}}^{p+q}(F), \quad \text { and } \quad \underline{\mathcal{H}}^{p}\left(\underline{\mathcal{H}}^{q}(F)\right) \Longrightarrow \underline{\mathcal{H}}^{p+q}(F) \text {. }
$$

Since $\underline{\mathcal{H}}^{q}(F)_{x}=0$ for $q>0$, we have the isomorphisms

$$
\lim _{\underline{\mathcal{V}} / \underline{X}} \underline{\check{\mathcal{H}}}^{q}(\underline{\mathcal{V}} / \underline{X}, F)_{x / \underline{X}} \simeq \underline{\check{\mathcal{H}}}^{q}(F)_{x / \underline{X}} \simeq \underline{\mathcal{H}}^{q}\left(F^{a}\right)_{x / \underline{X}} .
$$

(3) We may assume that $F$ is a sheaf. By definition,

$$
\check{\check{\mathcal{H}}}^{q}(F)_{x / \underline{X}}=\underline{\lim }_{\underline{U} / \underline{X}} \check{H}^{q}(\underline{U} / \underline{X}, F),
$$


where the limit is taken over all the $\partial$-étale neighborhoods of $x$. If $\underline{U} \rightarrow \underline{X}$ is a $\partial$-étale Galois neighborhood with the Galois group $\Gamma$, then $\check{H}^{q}(\underline{U} / \underline{X}, F) \simeq$ $H^{q}(\Gamma, F(\underline{U}))$ by 2.1.1. Thus we have $\underline{\mathcal{H}}^{q}(F)_{x / \underline{X}} \simeq H_{\text {cont. }}^{q}\left(\hat{\pi}_{1}^{\text {loc }}(\underline{X} ; x), F_{x}\right)$.

(4) For a point $y \in X$, let us choose an open neighborhood $\mathcal{U}$ and a $\partial$ isomorphism $f:[Y, D] \rightarrow[\mathcal{U}, B \cap \mathcal{U}]$ such that $[Y, D]$ is locally connected at any point of $f^{-1}(y)=\left\{y_{1}, y_{2}, \ldots, y_{l}\right\}$. Then by (2), we have

$$
\left(\varepsilon_{*} \underline{\mathcal{H}}^{q}(F)\right)_{y} \simeq \bigoplus_{i=1}^{r} \check{\mathcal{H}}^{q}(F)_{y_{i}} \simeq \bigoplus_{i=1}^{r} \underline{\mathcal{H}}^{q}(F)_{y_{i}} \simeq\left(\varepsilon_{*} \underline{\mathcal{H}}^{q}(F)\right)_{y} .
$$

Therefore the stalk of the natural homomorphism $\varepsilon_{*} \underline{\check{\mathcal{H}}}^{q}(F) \rightarrow \varepsilon_{*} \underline{\mathcal{H}}^{q}(F)$ is an isomorphism for any point $y \in X$. Since $R^{p} \varepsilon_{*} F$ is the sheafification of $\varepsilon_{*} \underline{\mathcal{H}}^{p}(F)$, it is also the sheafification of $\varepsilon_{*} \underline{\mathcal{H}}^{q}(F)$.

(5) is derived from (3) and (4).

Corollary 2.2.6. $\quad$ Let $F$ be a sheaf of abelian groups on a $\partial$-space $\underline{X}$ and let $[X, B]$ be a realization of $\underline{X}$. Then for the natural morphism $\varepsilon: \underline{X} \rightarrow X$, we have

$$
\left(R \varepsilon_{*} F\right) \stackrel{\mathrm{L}}{\otimes} \mathbb{Q} \sim_{\text {qis }} \varepsilon_{*}(F \otimes \mathbb{Q}) .
$$

In particular, if $F$ is a sheaf of $\mathbb{Q}$-vector spaces, then $H^{p}(\underline{X}, F) \simeq H^{p}\left(X, \varepsilon_{*} F\right)$.

Proof. Let $G$ be a finite group and let $M$ be a $G$-module. Then $H^{0}(G, M)$ $\otimes \mathbb{Q} \simeq H^{0}(G, M \otimes \mathbb{Q})$ and $H^{p}(G, M) \otimes \mathbb{Q}=H^{p}(G, M \otimes \mathbb{Q})=0$ for $p>$ 0 . Since an inductive limit and a tensor product are commutative, we have $\left(R^{p} \varepsilon_{*} F\right)_{x} \otimes \mathbb{Q}=R^{p} \varepsilon_{*}(F \otimes \mathbb{Q})_{x}$ for $p \geq 0$ and $\left(R^{p} \varepsilon_{*} F\right)_{x} \otimes \mathbb{Q}=0$ for $p>0$, for any point $x \in \operatorname{sp}(\underline{X})$.

Example. Let $\underline{X}$ be a $\partial$-space with a realization $[X, B]$ and let $\varepsilon: \underline{X} \rightarrow$ $X=[X, \emptyset]$ be the natural morphism. If $M$ is a $\mathbb{Q}$-vector space, then $H^{p}(\underline{X}$, $\left.M_{\underline{X}}\right) \simeq H^{p}(X, M)$ for any $p \geq 0$, by 2.2.6. Similarly for an $\mathcal{O}_{\underline{X}}$-module $F$, we have $H^{p}(\underline{X}, F) \simeq H^{p}\left(X, \varepsilon_{*} F\right)$.

Lemma 2.2.7. Let $\underline{\mathcal{U}}=\left\{\underline{U}_{\alpha} \rightarrow \underline{X}\right\}_{\alpha \in A}$ and $\underline{\mathcal{V}}=\left\{\underline{V}_{\beta} \rightarrow \underline{X}\right\}_{\beta \in B}$ be two $\partial$-étale covering families of $\underline{X}$ such that $\underline{\mathcal{V}}$ is a refinement of $\underline{\mathcal{U}}$. Then there is a spectral sequence

$$
E_{2}^{p, q}=\check{H}^{p}\left(\underline{\mathcal{U}} / \underline{X}, \underline{\mathcal{H}}^{q}(\underline{\mathcal{V}} / \underline{X}, F)\right) \Longrightarrow \check{H}^{p+q}(\underline{\mathcal{V}} / \underline{X}, F)
$$

for a presheaf $F$ of $\underline{X}$. 
Proof. Let us consider

$$
K^{p, q}:=C^{p}\left(\underline{\mathcal{U}} / \underline{X}, \underline{C}^{q}(\underline{\mathcal{V}} / \underline{X}, F)\right)=\prod_{\alpha \in A^{p+1}} \prod_{\beta \in B^{q+1}} F\left(\underline{U}_{\alpha} \times_{\underline{X}} \underline{V}_{\beta}\right),
$$

for $p, q \geq 0$. Then $K^{p, q}$ form a double complex. Let $\underline{\mathcal{U}} \cup \underline{\mathcal{V}}$ be the union of the two $\partial$-étale covering families. Since $\underline{\mathcal{U}}$ and $\underline{\mathcal{V}}$ are refinements, we have a natural surjective homomorphism

$$
C^{r}(\underline{\mathcal{U}} \cup \underline{\mathcal{V}} / \underline{X}, F) \rightarrow C^{r}(\underline{\mathcal{U}} / \underline{X}, F) \oplus C^{r}(\underline{\mathcal{V}} / \underline{X}, F)
$$

for $r \geq 0$. Let $L^{\bullet}=L^{\bullet}(\underline{\mathcal{U}}, \underline{\mathcal{V}}, F)$ be the kernel complex of

$$
C^{\bullet}(\underline{\mathcal{U}} \cup \underline{\mathcal{V}} / \underline{X}, F) \rightarrow C^{\bullet}(\underline{\mathcal{U}} / \underline{X}, F) \oplus C^{\bullet}(\underline{\mathcal{V}} / \underline{X}, F) .
$$

Then the shift $L^{\bullet}[1]$ is isomorphic to the total complex of $K^{\bullet}, \bullet$. Since $\underline{\mathcal{V}}$ is a refinement of $\underline{\mathcal{U}}$, the union $\underline{\mathcal{U}} \cup \underline{\mathcal{V}}$ is also a refinement of $\underline{\mathcal{U}}$. Hence the homomorphisms $\breve{H}^{r}(\underline{\mathcal{U}} \cup \underline{\mathcal{V}} / \underline{X}, F) \rightarrow \check{H}^{r}(\underline{\mathcal{U}} / \underline{X}, F)$ are isomorphic. Thus

$$
\check{H}^{r}(\underline{\mathcal{V}} / \underline{X}, F) \simeq H^{r+1}(L \bullet(\underline{\mathcal{U}}, \underline{\mathcal{V}}, F)) .
$$

We have the expected spectral sequence since $H^{q}\left(K^{p, \bullet}\right) \simeq C^{p}\left(\underline{\mathcal{H}} / \underline{X}, \underline{\mathcal{H}}^{q}\right.$ $(\underline{\mathcal{V}} / \underline{X}, F))$.

We recall the following well-known:

Lemma 2.2.8. Let $X$ be a para-compact and Hausdorff topological space. Assume that the sheafification of a presheaf $G$ of $X$ is zero. Then all the $\check{C}$ ech cohomology groups $\check{H}^{i}(X, G)$ are zero.

Now we are ready to prove the following:

Theorem 2.2.9. The Čech cohomology groups are isomorphic to the cohomology groups induced as right derived functors. More precisely, for any presheaf $F^{\prime}$ and its sheafification $F=F^{\prime a}$, we have canonical isomorphisms

$$
\check{H}^{p}\left(\underline{X}, F^{\prime}\right) \simeq \check{H}^{p}(\underline{X}, F) \simeq H^{p}(\underline{X}, F)
$$

for $p \geq 0$.

Proof. Let $F^{\prime}$ be a presheaf of $\underline{X}$ such that $F_{x}^{\prime}=0$ for any $x \in \operatorname{sp}(\underline{X})$. Let $\varepsilon: \underline{X} \rightarrow X$ be the natural morphism for a realization $[X, B]$ of $\underline{X}$. Then by 2.2 .5 ,

$$
\left(\varepsilon_{*} \lim _{\underline{\underline{\mathcal{V}}} / \underline{X}} \check{\mathcal{\mathcal { H }}}^{q}\left(\underline{\mathcal{V}} / \underline{X}, F^{\prime}\right)\right)_{x}=0
$$


for any point $x \in X$. Let $\mathcal{U}=\left\{U_{i}\right\}_{i \in I}$ be an open covering of $X$ and let $\varepsilon^{-1} \mathcal{U}$ be the induced $\partial$-étale covering family $\left\{\left(U_{i}, B \cap U_{i}\right) \rightarrow \underline{X}\right\}$. Then we have a spectral sequence

$$
E_{2}^{p, q}(\mathcal{U})=H^{p}\left(\varepsilon^{-1} \mathcal{U}, \underline{\lim }_{\underline{\mathcal{V}} / \underline{X}} \underline{\check{\mathcal{H}}}^{q}\left(\underline{\mathcal{V}} / \underline{X}, F^{\prime}\right)\right) \Longrightarrow \check{H}^{p+q}\left(\underline{X}, F^{\prime}\right)
$$

by 2.2.7. Since the inductive $\operatorname{limit} \lim _{\longrightarrow} E_{2}^{p, q}(\mathcal{U})$ for open coverings $\mathcal{U}$ is isomorphic to the usual Čech cohomology group

$$
H^{p}\left(X, \varepsilon_{*} \underline{\lim }_{\underline{\underline{\mathcal{V}}} / \underline{\underline{X}}} \check{\check{\mathcal{H}}}^{q}\left(\underline{\mathcal{V}} / \underline{X}, F^{\prime}\right)\right),
$$

we have $\lim _{\longrightarrow} E_{2}^{p, q}(\mathcal{U})=0$ for any $p, q$ by 2.2.8. Thus $\check{H}^{p}\left(\underline{X}, F^{\prime}\right)=0$.

Therefore, for any presheaf $F^{\prime}$ and its sheafification $F=F^{\prime a}$, we have $\check{H}^{p}\left(\underline{X}, F^{\prime}\right) \simeq \check{H}^{p}(\underline{X}, F)$. Let us consider any short exact sequence

$$
0 \rightarrow F \rightarrow G \rightarrow H \rightarrow 0
$$

of sheaves of $\underline{X}$. Then, for the cokernel $F^{\prime \prime}$ of $G \rightarrow H$ as the presheaf, we have $F_{x}^{\prime \prime}=0$ for any $x \in \operatorname{sp}(\underline{X})$. Therefore we have a long exact sequence:

$$
\cdots \rightarrow \check{H}^{q}(\underline{X}, F) \rightarrow \check{H}^{q}(\underline{X}, G) \rightarrow \check{H}^{q}(\underline{X}, H) \rightarrow \check{H}^{q+1}(\underline{X}, F) \rightarrow \cdots
$$

Since $\check{H}^{0}(\underline{X}, F)=F(\underline{X})$ for any sheaf $F$ and since $\check{H}^{q}(\underline{X}, G)=0$ for any injective sheaf $G$ by 2.2 .1 , we see that $\check{H}^{q}(\underline{X}, F) \simeq H^{q}(\underline{X}, F)$ for any $q$ and for any sheaf $F$ on $\underline{X}$.

\section{$\S 2.3$. Coherent sheaf}

Proposition 2.3.1. Let $\underline{X}$ be a complex analytic $\partial$-space. Let $\varepsilon_{X}$ denote the natural morphism $\underline{X}=(X, B) \rightarrow X=(X, \emptyset)$ for a realization $[X, B]$. Then the following conditions are equivalent:

(1) There is a top realization $[Z, \Delta]$ of $\underline{X}$ such that $[U, \Delta \cap U]$ is also a top realization for any open subset $U \subset Z$;

(2) For any realization $[X, B], \varepsilon_{X *} \mathcal{O}_{\underline{X}}$ is a coherent $\mathcal{O}_{X}$-module;

(3) There is a realization $[X, B]$ of $\underline{X}$ such that $\varepsilon_{X *} \mathcal{O}_{\underline{X}}$ is coherent.

Proof. (1) $\Longrightarrow(2)$ Let $[Z, \Delta]$ be the top realization. Then the natural homomorphism $\mathcal{O}_{Z} \rightarrow \varepsilon_{Z *} \mathcal{O}_{\underline{X}}$ is an isomorphism. Let $[X, B]$ be a realization of 
$\underline{X}$. Then there is a unique $\partial$-isomorphism $\mu:[Z, \Delta] \rightarrow[X, B]$. Hence $\varepsilon_{X *} \mathcal{O}_{\underline{X}} \simeq$ $\mu_{*} \varepsilon_{Z *} \mathcal{O}_{\underline{X}} \simeq \mu_{*} \mathcal{O}_{Z}$. This is coherent.

$(2) \Longrightarrow(3)$ is clear.

$(3) \Longrightarrow(1)$ There is a $\partial$-isomorphism $\mu:[Z, \Delta] \rightarrow[X, B]$ such that $\mu_{*} \mathcal{O}_{Z} \simeq$ $\varepsilon_{X *} \mathcal{O}_{\underline{X}}$ as $\mathcal{O}_{X}$-algebras. The direct image of the natural homomorphism $\mathcal{O}_{Z} \rightarrow$ $\varepsilon_{Z *} \mathcal{O}_{\underline{X}}$ under $\mu_{*}$ is an isomorphism. Since $\mu$ is a finite morphism, we have $\mathcal{O}_{Z} \simeq \varepsilon_{Z *} \mathcal{O}_{\underline{X}}$. If $f:[Y, D] \rightarrow[U, \Delta \cap U]$ is a $\partial$-isomorphism for an open subset $U \subset Z$, then there is a sequence of homomorphisms $\left.\mathcal{O}_{U} \rightarrow f_{*} \mathcal{O}_{Y} \rightarrow \varepsilon_{Z *} \mathcal{O}_{\underline{X}}\right|_{U}$. Hence $f$ admits a splitting $[U, \Delta \cap U] \rightarrow[Y, D]$. Thus $[U, \Delta \cap U]$ is a top realization.

A reduced $\partial$-space satisfies the condition above.

Definition. An $\mathcal{O}_{\underline{X}}$-module $\mathcal{F}$ is called coherent if the following two conditions are satisfied:

(1) $\mathcal{F}$ is $\partial$-étale locally finitely generated;

(2) Let $\underline{U} \rightarrow \underline{X}$ be a $\partial$-étale morphism and let $\left.\mathcal{O}_{\underline{U}}^{\oplus r} \rightarrow \mathcal{F}\right|_{\underline{U}}$ be an $\mathcal{O}_{\underline{U}}$-linear homomorphism. Then the kernel is also $\partial$-étale locally finitely generated.

Let $\underline{X}$ be a $\partial$-space such that for any $\partial$-étale morphism $\underline{U} \rightarrow \underline{X}, \underline{U}$ satisfies the condition of 2.3.1. Let $[X, B]$ be the top realization of $\underline{X}$. Then $\mathcal{O}_{X} \simeq \varepsilon_{*} \mathcal{O}_{\underline{X}}$ for the natural morphism $\varepsilon=\varepsilon_{X}: \underline{X} \rightarrow X$ (cf. 2.3.1). Let $\varepsilon^{*}$ denote the right exact functor $F \mapsto \varepsilon^{-1} F \otimes_{\varepsilon^{-1}} \mathcal{O}_{X} \mathcal{O}_{\underline{X}}$ from the category of $\mathcal{O}_{X}$-modules to that of $\mathcal{O}_{\underline{X}}$-modules.

Lemma 2.3.2. Let $\underline{X}=(X, B)$ and $\varepsilon: \underline{X} \rightarrow X$ be as above and let $\mathcal{F}$ be an $\mathcal{O}_{\underline{X}}$-module. Then $\mathcal{F}$ is coherent if and only if there exist a $\partial$-étale covering family $\left\{\underline{U}_{\lambda} \rightarrow \underline{X}\right\}$ and coherent sheaves $F_{\lambda}$ of $U_{\lambda}=\operatorname{sp}\left(\underline{U}_{\lambda}\right)$ such that

$$
\left.\mathcal{F}\right|_{\underline{U}_{\lambda}} \simeq \varepsilon_{U_{\lambda}}^{*} F_{\lambda}
$$

for the natural morphism $\varepsilon_{U_{\lambda}}: \underline{U}_{\lambda} \rightarrow U_{\lambda}$.

Proof. If $\mathcal{F}$ is coherent, then for any point $x \in \underline{X}$, there exist an open neighborhood $V$, a finite Galois covering $\tau: U \rightarrow V$ étale outside $B$, and an exact sequence

$$
\left.\mathcal{O}_{\underline{U}}^{\oplus s} \rightarrow \mathcal{O}_{\underline{U}}^{\oplus r} \rightarrow \mathcal{F}\right|_{\underline{U}} \rightarrow 0
$$

for $\underline{U}=\left(U, \tau^{-1} B\right)$. By taking the exact functor $\varepsilon_{U *}$ (cf. 2.2.6), we have

$$
\mathcal{O}_{U}^{\oplus s} \rightarrow \mathcal{O}_{U}^{\oplus r} \rightarrow \varepsilon_{U *}\left(\left.\mathcal{F}\right|_{\underline{U}}\right) \rightarrow 0 .
$$


Hence $F_{U}:=\varepsilon_{U *}\left(\left.\mathcal{F}\right|_{\underline{U}}\right)$ is a coherent $\mathcal{O}_{U}$-module. Next we apply $\varepsilon_{U}^{*}$. Then we have an isomorphism $\left.\mathcal{F}\right|_{\underline{U}} \simeq \varepsilon_{U}^{*} F_{U}$.

Conversely, suppose that $\left.\mathcal{F}\right|_{\underline{U}} \simeq \varepsilon_{U}^{*} F$ for the finite Galois covering $\tau: U \rightarrow$ $V$ étale outside $B$ and for a coherent sheaf $F$ of $U$ with an exact sequence

$$
\mathcal{O}_{U}^{\oplus s} \rightarrow \mathcal{O}_{U}^{\oplus r} \rightarrow F \rightarrow 0
$$

Then $\left.\mathcal{F}\right|_{\underline{U}}$ is finitely generated, $F_{U}:=\varepsilon_{U *}\left(\left.\mathcal{F}\right|_{\underline{U}}\right)$ is also a coherent sheaf of $U$, and $\left.\mathcal{F}\right|_{\underline{U}}=\varepsilon_{U}^{*} F_{U}$. If $\left.\mathcal{O}_{\underline{U}}^{\oplus t} \rightarrow \mathcal{F}\right|_{\underline{U}}$ is an $\mathcal{O}_{\underline{U}}$-linear homomorphism, then it is determined by an $\mathcal{O}_{U}$-linear homomorphism $\mathcal{O}_{U}^{\oplus t} \rightarrow F_{U}$. Therefore the kernel is also finitely generated.

In particular, $\mathcal{O}_{\underline{X}}$ is coherent. A lemma of Serre on the heredity of coherency on short exact sequence also holds.

Corollary. Under the situation above, $\varepsilon_{*} \mathcal{F}$ is coherent for a coherent $\mathcal{O}_{\underline{X}}$-module $\mathcal{F}$.

\section{$\S 3 . \quad$ Cohomology Groups of Toroidal Embeddings}

We shall calculate some $\partial$-étale cohomology groups on the $\partial$-space $\underline{X}=$ $(X, B)$ associated with a toroidal embedding $X^{\star}=X \backslash B \subset X$. The local cohomology sheaf $\mathcal{H}_{B}^{2}\left(\mathbb{Z}_{X}\right)$ is canonically isomorphic to the sheaf $\mathcal{H}_{B}^{0}\left(\mathcal{D} i v_{X}\right)$ of germs of Cartier divisors supported in $B$. In the $\partial$-étale version, $\mathcal{H}_{B}^{2}\left(\mathbb{Z}_{\underline{X}}\right) \simeq$ $\mathcal{H}_{B}^{0}\left(\mathcal{D} i v_{\underline{X}}\right)$ turns to be a sheaf of $\mathbb{Q}$-vector spaces. In particular, a $\mathbb{Q}$-Cartier divisor on $X$ whose fractional part is supported in $B$ is regarded as a Cartier divisor of $\underline{X}$. This correspondence of divisors is generalized in Section 3.5: we show that a reflexive sheaf of $\underline{X}$ corresponds to a parabolic sheaf of $X$.

\section{§3.1. Torus embedding}

Let $N$ be a free abelian group of $\operatorname{rank} l$ and let $M$ be the dual $\operatorname{Hom}_{\mathbb{Z}}(N, \mathbb{Z})$. A convex rational polyhedral cone $\sigma \subset \mathrm{N} \otimes \mathbb{R}$ is written by

$$
\sigma=\sum_{i=1}^{k} \mathbb{R}_{\geq 0} \nu_{i}=\left\{\sum_{i=1}^{k} r_{i} \nu_{i} \mid r_{i} \in \mathbb{R}_{\geq 0}\right\}
$$

for some $\nu_{i} \in \mathrm{N}$. We assume that $\left\{\mathbb{R}_{\geq 0} \nu_{1}, \ldots, \mathbb{R}_{\geq 0} \nu_{k}\right\}$ is the set of onedimensional faces in $\sigma, \nu_{i}$ are primitive elements of $\mathrm{N}$, and that $\sigma$ is strictly convex, i.e., $\sigma \cap(-\sigma)=\{0\}$. The $\nu_{i}$ are called vertices of $\sigma$. The dual cone 
$\sigma^{\vee} \subset \mathrm{M} \otimes \mathbb{R}$ consists of linear functions on $\mathbf{N} \otimes \mathbb{R}$ that are non-negative over $\sigma$. Then $\sigma^{\vee}$ is also a convex rational polyhedral cone in $\mathrm{M} \otimes \mathbb{R}$ and the semi-group $\sigma^{\vee} \cap \mathrm{M}$ is finitely generated. The semi-group ring $\mathbb{C}\left[\sigma^{\vee} \cap \mathrm{M}\right]$ and the group ring $\mathbb{C}[\mathrm{M}]$ define an open immersion Spec $\mathbb{C}[\mathrm{M}] \hookrightarrow \operatorname{Spec} \mathbb{C}\left[\sigma^{\vee} \cap \mathrm{M}\right]$ of affine schemes. We write the associated analytic spaces by

$$
\mathbb{T}_{\mathrm{N}}:=\operatorname{Spec} \mathbb{C}[\mathrm{M}]^{\text {an }} \quad \text { and } \quad \mathbb{T}_{\mathrm{N}}(\sigma):=\operatorname{Spec} \mathbb{C}\left[\sigma^{\vee} \cap \mathrm{M}\right]^{\text {an }} .
$$

The algebraic torus $\mathbb{T}_{\mathrm{N}} \simeq \mathrm{N} \otimes \mathbb{C}^{\star}$ acts on $\mathbb{T}_{\mathrm{N}}(\sigma)$ and the open immersion $\mathbb{T}_{\mathrm{N}} \hookrightarrow \mathbb{T}_{\mathrm{N}}(\sigma)$ is $\mathbb{T}_{\mathrm{N}}$-equivariant. The open immersion is called an affine torus embedding. Let $\mathrm{N}(\sigma)$ be the subgroup $\mathrm{N} \cap(\sigma+(-\sigma))$. Then $\mathbb{T}_{\mathrm{N}}(\sigma) \simeq \mathbb{T}_{\mathrm{N}(\sigma)}(\sigma) \times$ $\mathbb{T}_{\mathrm{N} / \mathrm{N}(\sigma)}$. A face $\tau \prec \sigma$ defines a $\mathbb{T}_{\mathrm{N}}$-equivariant open immersion $\mathbb{T}_{\mathrm{N}}(\tau) \subset \mathbb{T}_{\mathrm{N}}(\sigma)$. The complement $\mathbb{O}_{\sigma}$ of the union of all the $\mathbb{T}_{N}(\tau)$ with $\tau \prec \sigma, \tau \neq \sigma$, is the unique closed orbit of $\mathbb{T}_{N}$ in $\mathbb{T}_{N}(\sigma)$. Any orbit in $\mathbb{T}_{N}(\sigma)$ is of the form $\mathbb{O}_{\tau}$ for some $\tau \prec \sigma$. More explicitly, $\mathbb{O}_{\tau}=\operatorname{Spec} \mathbb{C}\left[\tau^{\perp} \cap \mathrm{M}\right]^{\text {an }} \simeq \mathbb{T}_{\mathrm{N} / \mathrm{N}(\tau)}$, where $\tau^{\perp}$ denotes the vector subspace of $\mathrm{M} \otimes \mathbb{R}$ consisting of functionals vanishing along $\tau$. Note that $\operatorname{Hom}\left(\tau^{\perp} \cap \mathrm{M}, \mathbb{Z}\right) \simeq \mathrm{N} / \mathrm{N}(\tau)$. The immersion $\mathbb{O}_{\tau} \subset \mathbb{T}_{\mathrm{N}}(\sigma)$ is induced from the ring homomorphism $f: \mathbb{C}\left[\sigma^{\vee} \cap \mathrm{M}\right] \rightarrow \mathbb{C}\left[\tau^{\perp} \cap \mathrm{M}\right]$ defined by

$$
\mathbb{C}\left[\tau^{\perp} \cap \mathrm{M}\right] \ni f(m)=\left\{\begin{array}{lll}
0, & \text { if } & m \notin \tau^{\perp} \cap \mathrm{M} ; \\
m, & \text { if } & m \in \tau^{\perp} \cap \mathrm{M},
\end{array}\right.
$$

for $m \in \sigma^{\vee} \cap \mathrm{M}$. The closure $\overline{\mathbb{O}}_{\tau}$ is of dimension $l-\operatorname{dim} \tau$ and is isomorphic to $\mathbb{T}_{\mathrm{N} / \mathrm{N}(\tau)}(\bar{\sigma})$ for the image $\bar{\sigma} \subset \mathrm{N} / \mathrm{N}(\tau) \otimes \mathbb{R}$ of $\sigma$. For a vertex $\nu_{i}, \mathbb{O}_{\nu_{i}}$ and $\mathbb{T}_{\mathrm{N}}\left(\nu_{i}\right)$ stand for $\mathbb{O}_{\tau}$ and $\mathbb{T}_{\mathrm{N}}(\tau)$, respectively, for $\tau=\mathbb{R}_{\geq 0} \nu_{i}$. Let $B_{i}$ be the prime divisor $\overline{\mathbb{O}}_{\nu_{i}}$. We define a filtration

$$
\mathbb{T}_{\mathrm{N}}(\sigma)_{\bullet}: \mathbb{T}_{\mathrm{N}}(\sigma)=\mathbb{T}_{\mathrm{N}}(\sigma)_{l} \supset \mathbb{T}_{\mathrm{N}}(\sigma)_{l-1} \supset \cdots \supset \mathbb{T}_{\mathrm{N}}(\sigma)_{i} \supset \cdots
$$

by setting

$$
\mathbb{T}_{\mathrm{N}}(\sigma)_{l-i}:=\bigcap_{1 \leq \lambda_{1}<\lambda_{2}<\cdots<\lambda_{i} \leq k} B_{\lambda_{1}} \cap B_{\lambda_{2}} \cap \cdots \cap B_{\lambda_{i}} .
$$

$\mathbb{T}_{\mathrm{N}}(\sigma)_{i}$ is the union of all the orbits of dimension $\leq i$. The stratum $\mathbb{T}_{\mathrm{N}}(\sigma)_{i} \backslash$ $\mathbb{T}_{\mathrm{N}}(\sigma)_{i-1}$ is the union of $i$-dimensional orbits. Thus $\mathbb{T}_{\mathrm{N}}(\sigma)$ • gives a Whitney stratification.

Remark. The variety $X=\mathbb{T}_{\mathrm{N}}(\sigma)$ has only rational singularities, i.e., $\mathcal{O}_{X} \sim_{\text {qis }} R \mu_{*} \mathcal{O}_{Y}$ for a desingularization $\mu: Y \rightarrow X$ (cf. [K6]).

In Section 3.1, except for 3.1.5, we assume that $\mathrm{N}(\sigma)=\mathrm{N}$, i.e., $\sigma$ generates $\mathrm{N} \otimes \mathbb{R}$. Then the closed orbit $\mathbb{O}_{\sigma}$ consists of one point which we denote by 0 . 
Let $\left\{m_{1}, m_{2}, \ldots, m_{r}\right\}$ be a generator of the semi-group $\sigma^{\vee} \cap \mathrm{M}$. We denote by $x^{m} \in \mathbb{C}[\mathrm{M}]$ the monomial corresponding to $m$. Then we have a closed embedding $\mathbb{T}_{\mathrm{N}}(\sigma) \hookrightarrow \mathbb{C}^{r}$ by $x^{m_{j}}$ which sends 0 to 0 . Let Int $\sigma$ denote the interior of $\sigma$. We fix an element $v \in \operatorname{Int} \sigma$ and set $v_{j}:=\left\langle m_{j}, v\right\rangle>0$, where $\langle$,$\rangle stands for the natural pairing \mathrm{M} \times \mathrm{N} \rightarrow \mathbb{Z}$. Let $\boldsymbol{\Delta}_{\alpha}$ for $\alpha>0$ be the following polydisc in $\mathbb{C}^{r}$ :

$$
\boldsymbol{\Delta}_{\alpha}:=\left\{\left(y_{1}, y_{2}, \ldots, y_{r}\right) \in \mathbb{C}^{r}|| y_{j} \mid<\alpha^{v_{j}} \text { for any } j\right\} .
$$

We denote the intersections with $\boldsymbol{\Delta}_{\alpha}$ as follows:

$$
\mathbb{T}_{\mathrm{N}}(\sigma)^{<\alpha}:=\boldsymbol{\Delta}_{\alpha} \cap \mathbb{T}_{\mathrm{N}}(\sigma), \quad \mathbb{T}_{\mathrm{N}}^{<\alpha}:=\boldsymbol{\Delta}_{\alpha} \cap \mathbb{T}_{\mathrm{N}}, \quad \mathbb{T}_{\mathrm{N}}(\sigma)_{i}^{<\alpha}:=\boldsymbol{\Delta}_{\alpha} \cap \mathbb{T}_{\mathrm{N}}(\sigma)_{i}
$$

There is an isomorphism $\mathbb{T}_{\mathrm{N}}^{<1} \simeq \mathbb{T}_{\mathrm{N}}^{<\alpha}$ given by

$$
\left(y_{1}, y_{2}, \ldots, y_{r}\right) \mapsto\left(\alpha^{v_{1}} y_{1}, \alpha^{v_{2}} y_{2}, \ldots, \alpha^{v_{r}} y_{r}\right)
$$

Example. Let $\mathrm{N}$ be a free abelian group generated by $\nu_{1}, \nu_{2}, \ldots, \nu_{l}$ and let $\sigma$ be the first quadrant $\sum_{i=1}^{l} \mathbb{R}_{\geq 0} \nu_{i}$. For the point $v=\nu_{1}+\nu_{2}+\cdots+\nu_{l} \in$ Int $\sigma$, the open subset $\mathbb{T}_{\mathrm{N}}(\sigma)^{<\alpha}$ is just the open polydisc

$$
\left\{\left(s_{1}, s_{2}, \ldots, s_{l}\right) \in \mathbb{C}^{l}|| s_{i} \mid<\alpha \text { for any } i\right\} .
$$

Definition. Let the abelian group $\mathrm{N}$ of rank $l$, the strictly convex rational polyhedral cone $\sigma$ with $\mathrm{N}(\sigma)=\mathrm{N}$, the vector $v \in \operatorname{Int} \sigma$, and the generator $m_{\bullet}=\left\{m_{1}, m_{2}, \ldots, m_{r}\right\}$ of $\sigma^{\vee} \cap \mathrm{M}$ be as above. An open immersion $X^{\star} \subset X$ into a normal analytic space together with a point $x \in B=X \backslash X^{\star}$ is called an $n$-dimensional toroidal embedding of type $\left(\mathrm{N}, l, \sigma, v, m_{\bullet}\right)$ or of type $(\mathrm{N}, l, \sigma)$ for short, if there is an isomorphism $X \simeq \mathbb{T}_{\mathbb{N}}(\sigma)^{<1} \times \Delta^{n-l}$ such that $X^{\star}$ corresponds to $\mathbb{T}_{\mathrm{N}} \times \boldsymbol{\Delta}^{n-l}$ and that $x$ corresponds to $(0,0)$ for the zero-dimensional orbit $0 \in \mathbb{T}_{\mathrm{N}}(\sigma)$ and for the origin $0 \in \boldsymbol{\Delta}^{n-l}$.

The $\mathbb{T}_{\mathrm{N}}(\sigma)$ is identified with the set $\operatorname{Hom}\left(\sigma^{\vee} \cap \mathrm{M}, \mathbb{C}\right)$ of semi-group homomorphisms into the multiplicative semi-group $\mathbb{C}=(\mathbb{C}, \times)$. By considering the multiplicative semi-group $\mathbb{R}_{\geq 0}=\left(\mathbb{R}_{\geq 0}, \times\right)$, we define

$$
\operatorname{Mc}_{N}(\sigma):=\operatorname{Hom}\left(\sigma^{\vee} \cap \mathrm{M}, \mathbb{R}_{\geq 0}\right)
$$

(cf. [O1], [O2]). This is realized as a subset of $\mathbb{T}_{\mathrm{N}}(\sigma)$ and as the quotient space of $\mathbb{T}_{\mathrm{N}}(\sigma)$ by the the action of compact torus

$$
\mathrm{N} \otimes \mathrm{S}^{1} \simeq \operatorname{Hom}\left(\mathrm{M}, \mathrm{S}^{1}\right) \subset \mathrm{N} \otimes \mathbb{C}^{\star} \simeq \mathbb{T}_{\mathrm{N}}
$$


where $\mathrm{S}^{1}$ stands for the circle as well as the unitary group $\mathrm{U}(1)$. The quotient map

$$
\nu: \mathbb{T}_{\mathrm{N}}(\sigma) \rightarrow \mathrm{Mc}_{\mathrm{N}}(\sigma)
$$

is induced from the norm map $\mathbb{C} \ni z \mapsto|z| \in \mathbb{R}_{\geq 0}$ and is proper and open. An orbit $\mathbb{O}_{\tau}$ is identified with $\operatorname{Hom}\left(\tau^{\perp} \cap \mathrm{M}, \mathbb{C}^{\star}\right) \simeq \mathrm{N} / \mathrm{N}(\tau) \otimes \mathbb{C}^{\star}$. The immersion $\mathbb{O}_{\tau}=\operatorname{Hom}\left(\tau^{\perp} \cap \mathrm{M}, \mathbb{C}^{\star}\right) \subset \mathbb{T}_{\mathrm{N}}(\sigma)=\operatorname{Hom}\left(\sigma^{\vee} \cap \mathrm{M}, \mathbb{C}\right)$ is described as the zero extension: For $w \in \tau^{\perp} \cap \mathrm{M} \rightarrow \mathbb{C}^{\star}$, the semi-group homomorphism $\tilde{w}: \sigma^{\vee} \cap \mathrm{M} \rightarrow$ $\mathbb{C}$ is defined by

$$
\tilde{w}(m)=\left\{\begin{array}{lll}
0, & \text { if } & m \notin \tau^{\perp} \cap \mathbf{M} ; \\
w(m), & \text { if } & m \in \tau^{\perp} \cap \mathbf{M} .
\end{array}\right.
$$

The image $\boldsymbol{\nu}\left(\mathbb{O}_{\tau}\right)$ is written by

$$
\boldsymbol{\nu}\left(\mathbb{O}_{\tau}\right)=\operatorname{Hom}\left(\tau^{\perp} \cap \mathrm{M}, \mathbb{R}_{>0}\right) \simeq \mathrm{N} / \mathrm{N}(\tau) \otimes \mathbb{R}_{>0},
$$

where we consider $\mathbb{R}_{>0}$ as a multiplicative group. We have $\mathbb{O}_{\tau}=\boldsymbol{\nu}^{-1}\left(\boldsymbol{\nu}\left(\mathbb{O}_{\tau}\right)\right)$ and $\mathbb{O}_{\tau} \simeq \boldsymbol{\nu}\left(\mathbb{O}_{\tau}\right) \times\left(\mathrm{N} / \mathrm{N}(\tau) \otimes \mathrm{S}^{1}\right)$. The images $\operatorname{Mc}_{\mathrm{N}}(\sigma)_{i}:=\boldsymbol{\nu}\left(\mathbb{T}_{\mathrm{N}}(\sigma)_{i}\right)$ define a filtration of $\operatorname{Mc}_{N}(\sigma)$. The stratum $\operatorname{Mc}_{N}(\sigma)_{i} \backslash \operatorname{Mc}_{N}(\sigma)_{i-1}$ is a disjoint union of $\boldsymbol{\nu}\left(\mathbb{O}_{\tau}\right)$ with $\operatorname{dim} \tau=l-i$. Thus $\mathbb{T}_{\mathrm{N}}(\sigma)_{i} \backslash \mathbb{T}_{\mathrm{N}}(\sigma)_{i-1}$ is topologically a trivial fiber bundle over $\operatorname{Mc}_{N}(\sigma)_{i} \backslash \mathrm{Mc}_{\mathrm{N}}(\sigma)_{i-1}$ with fiber $\left(\mathrm{S}^{1}\right)^{i}=\mathrm{S}^{1} \times \cdots \times \mathrm{S}^{1}$ (i-fold product). The image $\operatorname{Mc}_{\mathrm{N}}(\sigma)^{<\alpha}$ of $\mathbb{T}_{\mathrm{N}}(\sigma)^{<\alpha}$ is identified with

$$
\operatorname{Mc}_{\mathrm{N}}(\sigma)^{<\alpha}=\left\{w \in \operatorname{Hom}\left(\sigma^{\vee} \cap \mathrm{M}, \mathbb{R}_{\geq 0}\right) \mid w\left(m_{j}\right)<\alpha^{v_{j}} \text { for any } j\right\}
$$

and $\mathbb{T}_{\mathrm{N}}(\sigma)^{<\alpha}=\boldsymbol{\nu}^{-1}\left(\mathrm{Mc}_{\mathrm{N}}(\sigma)^{<\alpha}\right)$.

Claim 3.1.1. Let $\bar{v}$ and $\bar{\sigma}$ be the images of $v$ and $\sigma$, respectively, under the projection $\mathrm{N} \otimes \mathbb{R} \rightarrow \mathrm{N} / \mathrm{N}(\tau) \otimes \mathbb{R}$. Then $\operatorname{Mc}_{\mathrm{N}}(\sigma)^{<\alpha} \cap \boldsymbol{\nu}\left(\mathbb{O}_{\tau}\right)$ is identified with

$$
-(\log \alpha) \bar{v}+\operatorname{Int} \bar{\sigma} \subset \mathrm{N} / \mathrm{N}(\tau) \otimes \mathbb{R} .
$$

Proof. For a point $w \in \boldsymbol{\nu}\left(\mathbb{O}_{\tau}\right)=\operatorname{Hom}\left(\tau^{\perp} \cap \mathrm{M}, \mathbb{R}_{>0}\right), w$ is contained in $\operatorname{Mc}_{\mathrm{N}}(\sigma)^{<\alpha}$ if and only if $w\left(m_{j}\right)<\alpha^{v_{j}}$ for any $m_{j} \in \tau^{\perp}$. This condition is also written as $\log w\left(m_{j}\right)<\left\langle m_{j}, v\right\rangle \log \alpha$. Let $\boldsymbol{\nu}\left(\mathbb{O}_{\tau}\right) \rightarrow \mathrm{N} / \mathrm{N}(\tau) \otimes \mathbb{R}$ be the homeomorphism

$$
\operatorname{Hom}\left(\tau^{\perp} \cap \mathrm{M}, \mathbb{R}_{>0}\right) \ni w \longmapsto-\log w \in \operatorname{Hom}\left(\tau^{\perp} \cap \mathrm{M}, \mathbb{R}\right) .
$$

Then the condition is equivalent to that $(\log \alpha) \bar{v}-\log w \in \mathrm{N} / \mathrm{N}(\tau) \otimes \mathbb{R}$ is contained in Int $\bar{\sigma}$.

In particular, any connected component of $\operatorname{Mc}_{\mathrm{N}}(\sigma)_{i} \backslash \mathrm{Mc}_{\mathrm{N}}(\sigma)_{i-1}$ and its intersection with $\operatorname{Mc}_{\mathrm{N}}(\sigma)^{<\alpha}$ are contractible. 
Corollary 3.1.2. The open subsets $\mathbb{T}_{\mathrm{N}}(\sigma)^{<\alpha}$ form a base of open neighborhoods of 0 . For a connected component $U$ of $\mathbb{T}_{\mathrm{N}}(\sigma)_{i} \backslash \mathbb{T}_{\mathrm{N}}(\sigma)_{i-1}, U$ and $U \cap \mathbb{T}_{\mathrm{N}}(\sigma)_{i}^{<\alpha}$ are both homotopically equivalent to an $i$-dimensional compact torus $\mathrm{S}^{1} \times \cdots \times \mathrm{S}^{1}$.

Lemma 3.1.3. For a face $\tau \prec \sigma$, the universal covering space of $\mathbb{O}_{\tau} \cap$ $\mathbb{T}_{\mathrm{N}}(\sigma)^{<\alpha}$ is isomorphic to the domain

$$
\mathbb{H}_{\mathrm{N} / \mathrm{N}(\tau)}(\operatorname{Int} \bar{\sigma}):=\mathrm{N} / \mathrm{N}(\tau) \otimes \mathbb{R}+\sqrt{-1} \operatorname{Int} \bar{\sigma} \subset \mathrm{N} / \mathrm{N}(\tau) \otimes \mathbb{C}
$$

Proof. The space $\mathbb{O}_{\tau}^{<\alpha}:=\mathbb{O}_{\tau} \cap \mathbb{T}_{\mathrm{N}}(\sigma)^{<\alpha}$ is homotopically equivalent to $\mathbb{O}_{\tau} \simeq \mathrm{N} / \mathrm{N}(\tau) \otimes \mathbb{C}^{\star}$. Let $\mathrm{e}_{\mathrm{N} / \mathrm{N}(\tau)}: \mathrm{N} / \mathrm{N}(\tau) \otimes \mathbb{C} \rightarrow \mathrm{N} / \mathrm{N}(\tau) \otimes \mathbb{C}^{\star}$ be the morphism id $\otimes$ e for the exponential mapping e: $\mathbb{C} \ni z \mapsto \exp (2 \pi \sqrt{-1} z) \in \mathbb{C}^{\star}$. We have

$$
x^{m_{i}}\left(\mathrm{e}_{\mathrm{N} / \mathrm{N}(\tau)}(u)\right)=\left\{\begin{array}{lll}
0, & \text { if } & m_{i} \notin \tau^{\perp} \cap \mathrm{M} ; \\
\mathrm{e}\left(\left\langle m_{i}, u\right\rangle\right), & \text { if } & m_{i} \in \tau^{\perp} \cap \mathrm{M},
\end{array}\right.
$$

for $u \in \mathrm{N} / \mathrm{N}(\tau) \otimes \mathbb{C}$. Therefore,

$$
\begin{aligned}
\mathrm{e}_{\mathrm{N} / \mathrm{N}(\tau)}^{-1}\left(\mathbb{O}_{\tau}^{<\alpha}\right)=\left\{u \in \mathrm{N} / \mathrm{N}(\tau) \otimes \mathbb{C} \mid-2 \pi\left\langle m_{i}, \operatorname{Im} u\right\rangle\right. \\
\left.\quad<\left\langle m_{i}, v\right\rangle \log \alpha \text { for } m_{i} \in \tau^{\perp}\right\} \\
=\{u \in \mathrm{N} / \mathrm{N}(\tau) \otimes \mathbb{C} \mid(\log \alpha) \bar{v}+2 \pi \operatorname{Im} u \in \operatorname{Int} \bar{\sigma}\} \\
=\mathbb{H}_{\mathrm{N} / \mathrm{N}(\tau)}(\operatorname{Int} \bar{\sigma})-\frac{\sqrt{-1}}{2 \pi}(\log \alpha) \bar{v} .
\end{aligned}
$$

This is the universal covering space of $\mathbb{O}_{\tau}^{<\alpha}$.

A bounded complex $F^{\bullet}$ of sheaves of abelian groups on $\mathbb{T}_{N}(\sigma)$ is called constructible with respect to the filtration $\mathbb{T}_{\mathrm{N}}(\sigma)$. if the cohomology sheaves of the complex restricted to any strata $\mathbb{T}_{\mathrm{N}}(\sigma)_{i} \backslash \mathbb{T}_{\mathrm{N}}(\sigma)_{i-1}$ are locally constant systems of finitely generated abelian groups (cf. [G1]).

Lemma 3.1.4. Let $F^{\bullet}$ be a constructible bounded complex of sheaves on $\mathbb{T}_{\mathrm{N}}(\sigma)$. Then we have isomorphisms

$$
H^{p}\left(\mathbb{T}_{\mathrm{N}}(\sigma), F^{\bullet}\right) \simeq H^{p}\left(\mathbb{T}_{\mathrm{N}}(\sigma)^{<\alpha}, F^{\bullet}\right) \simeq \mathcal{H}^{p}\left(F^{\bullet}\right)_{0},
$$

for $\alpha>0$ and for any $p$. In particular,

$$
H^{p}\left(\mathbb{T}_{\mathrm{N}}(\sigma)^{<\alpha}, F^{\bullet}\right) \otimes \mathbb{Q} \simeq H^{p}\left(\mathbb{T}_{\mathrm{N}}(\sigma)^{<\alpha}, F^{\bullet} \stackrel{\mathrm{L}}{\otimes} \mathbb{Q}\right)
$$


Proof. Suppose first that $F^{\bullet} \sim_{\text {qis }} R j_{i *} F_{0}^{\bullet}$ for a bounded complex $F_{0}^{\bullet}$ on $\mathbb{T}_{\mathrm{N}}(\sigma)_{i} \backslash \mathbb{T}_{\mathrm{N}}(\sigma)_{i-1}$ with locally constant cohomology sheaves, where $j_{i}$ denotes the immersion $\mathbb{T}_{\mathrm{N}}(\sigma)_{i} \backslash \mathbb{T}_{\mathrm{N}}(\sigma)_{i-1} \hookrightarrow \mathbb{T}_{\mathrm{N}}(\sigma)$. Then we have

$$
\begin{aligned}
H^{p}\left(\mathbb{T}_{\mathrm{N}}(\sigma), F^{\bullet}\right) & \simeq H^{p}\left(\mathbb{T}_{\mathrm{N}}(\sigma)_{i} \backslash \mathbb{T}_{\mathrm{N}}(\sigma)_{i-1}, F_{0}^{\bullet}\right) \\
& \simeq H^{0}\left(\operatorname{Mc}_{\mathrm{N}}(\sigma)_{i} \backslash \operatorname{Mc}_{\mathrm{N}}(\sigma)_{i-1}, R^{p} \boldsymbol{\nu}_{*} F_{0}^{\bullet}\right), \\
H^{p}\left(\mathbb{T}_{\mathrm{N}}(\sigma)^{<\alpha}, F^{\bullet}\right) & \simeq H^{p}\left(\mathbb{T}_{\mathrm{N}}(\sigma)_{i}^{<\alpha} \backslash \mathbb{T}_{\mathrm{N}}(\sigma)_{i-1}, F_{0}^{\bullet}\right) \\
& \simeq H^{0}\left(\operatorname{Mc}_{\mathrm{N}}(\sigma)_{i}^{<\alpha} \backslash \operatorname{Mc}_{\mathrm{N}}(\sigma)_{i-1}, R^{p} \boldsymbol{\nu}_{*} F_{0}^{\bullet}\right) .
\end{aligned}
$$

Since $R^{p} \boldsymbol{\nu}_{*} F_{0}^{\bullet}$ are constant sheaves, we have the isomorphism for $F^{\bullet}$.

Next we consider general $F^{\bullet}$. We have a triangle

$$
\cdots \stackrel{+1}{\longrightarrow} R{\underline{\mathbb{T}_{\mathrm{N}}(\sigma)_{l-1}}}\left(F^{\bullet}\right) \rightarrow F^{\bullet} \rightarrow R j_{l-1}\left(\left.F^{\bullet}\right|_{\mathbb{T}_{\mathrm{N}}(\sigma) \backslash \mathbb{T}_{\mathrm{N}}(\sigma)_{l-1}} \stackrel{+1}{\longrightarrow} \cdots\right.
$$

Then every complex is constructible and the statement holds on the third complex. By the induction on the dimension of the support of cohomology sheaves, we have the required isomorphisms.

Corollary 3.1.5. $\quad$ Let $\sigma \subset \mathrm{N} \otimes \mathbb{R}$ be a strictly convex rational polyhedral cone. Then the homomorphism

$$
H^{p}\left(\mathbb{T}_{\mathrm{N}}(\sigma), \mathbb{Z}\right) \rightarrow H^{p}\left(\mathbb{T}_{\mathrm{N}}, \mathbb{Z}\right)
$$

is isomorphic to the natural injection $\bigwedge^{p}\left(\sigma^{\perp} \cap \mathrm{M}\right) \rightarrow \bigwedge^{p} \mathrm{M}$.

Proof. There is an isomorphism $\mathbb{T}_{\mathrm{N}}(\sigma) \simeq \mathbb{T}_{\mathrm{N}(\sigma)}(\sigma) \times \mathbb{T}_{\mathrm{N} / \mathrm{N}(\sigma)}$, where $\operatorname{Hom}(\mathrm{N} / \mathrm{N}(\sigma), \mathbb{Z}) \simeq \sigma^{\perp} \cap \mathrm{M}$. We have $H^{p}\left(\mathbb{T}_{\mathrm{N}(\sigma)}(\sigma), \mathbb{Z}\right)=0$ for $p>0$ by 3.1.4. Thus the homomorphism in question is derived from the projection $\mathbb{T}_{\mathrm{N}} \rightarrow \mathbb{T}_{\mathrm{N} / \mathrm{N}(\sigma)}$.

\section{$\S 3.2$. Normal varieties with boundary}

Let us consider a complex analytic space with boundary $[V, D]$ such that $V$ is normal and that the open part $V^{\star}:=V \backslash D$ is non-singular. This is a top realization of the $\partial$-space $\underline{V}=(V, D)$. Let $j: V^{\star} \hookrightarrow V$ denote the open immersion. The analytic subset $Z:=\operatorname{Sing} V \cup \operatorname{Sing} D$ satisfies $\operatorname{codim} Z \geq 2$. Let $V^{\circ}:=V \backslash Z, D^{\star}:=D \cap V^{\circ}$ and let $j^{\circ}: V^{\circ} \hookrightarrow V, j^{\star}: V^{\star} \hookrightarrow V^{\circ}$ be the associated open immersions.

Let $\Omega_{V^{\circ}}^{p}\left(\log D^{\star}\right)$ be the sheaf of germs of logarithmic $p$-forms along $D^{\star}$. We define $\hat{\Omega}_{V}^{p}(\log D)$ to be the direct image $j_{*}^{\circ} \Omega_{V^{\circ}}^{p}\left(\log D^{\star}\right)$. Then $\hat{\Omega}_{V}^{p}(\log D)$ are 
reflexive sheaves, since $\Omega_{V^{\circ}}^{p}\left(\log D^{\star}\right)$ extends to the coherent sheaf $\mu_{*} \Omega_{Y}^{p}\left(\log \mu^{-1}\right.$ $D$ ) for a desingularization $\mu: Y \rightarrow V$ with $\mu^{-1} D$ being normal crossing. Let $X$ be an affine torus embedding $\mathbb{T}_{\mathrm{N}}(\sigma)$ and let $B$ be the complement of $\mathbb{T}_{\mathrm{N}}$. Then we have a natural isomorphism

$$
\hat{\Omega}_{X}^{p}(\log B) \simeq \mathcal{O}_{X} \otimes_{\mathbb{Z}} \bigwedge^{p} \mathrm{M}
$$

Moreover, we have the so-called logarithmic de Rham complex

$$
\hat{\Omega}_{X}^{\bullet}(\log B)=\left[\cdots \rightarrow \hat{\Omega}_{X}^{p}(\log B) \stackrel{\mathrm{d}}{\rightarrow} \hat{\Omega}_{X}^{p+1}(\log B) \rightarrow \cdots\right] .
$$

There is a natural quasi-isomorphism

$$
R j_{*} \mathbb{C}_{X^{\star}} \sim_{\text {qis }} \hat{\Omega}_{X}^{\bullet}(\log B)
$$

by [D2], [D3], [D1, Section 15]. In particular, the natural morphism $R j_{*} \mathbb{C}_{X^{\star}} \rightarrow$ $R j_{*} \mathcal{O}_{X^{\star}}$ is decomposed as $R j_{*} \mathbb{C}_{X^{\star}} \rightarrow \mathcal{O}_{X} \rightarrow j_{*} \mathcal{O}_{X^{\star}} \simeq_{\text {qis }} R j_{*} \mathcal{O}_{X^{\star}}$. This is generalized to 3.2 .2 below.

Definition 3.2.1. $\quad$ Let $k$ be a positive integer. A normal variety $V$ is said to have only $k$-rational singularities if for a desingularization $\mu: Y \rightarrow V$, $R^{i} \mu_{*} \mathcal{O}_{Y}=0$ for $0<i \leq k$.

Lemma 3.2.2. If $V$ has only $k$-rational singularities, then the morphism $\tau_{\leq k} R j_{*} \mathbb{C}_{V^{\star}} \rightarrow R j_{*} \mathcal{O}_{V^{\star}}$ factors through $\mathcal{O}_{V} \rightarrow R j_{*} \mathcal{O}_{V^{\star}}$. In particular, $\tau_{\leq k+1} R \underline{\Gamma}_{D}\left(\mathbb{C}_{V}\right) \rightarrow R \underline{\Gamma}_{D}\left(\mathcal{O}_{V}\right)$ is zero in the derived category.

Proof. Let $\mu: M \rightarrow V$ be a bimeromorphic morphism from a non-singular variety such that $\mu^{-1} D$ is a normal crossing divisor and that $\mu$ is isomorphic over $V^{\star}$. Then for the open immersion $j_{M}: V^{\star} \simeq M \backslash \mu^{-1} D \hookrightarrow M$, we have the factorization $R j_{M *} \mathbb{C}_{V^{*}} \rightarrow \mathcal{O}_{M} \rightarrow R j_{M_{*}} \mathcal{O}_{M^{\star}}$ as above. By taking $R \mu_{*}$, we also have $R j_{*} \mathbb{C}_{V^{\star}} \rightarrow R \mu_{*} \mathcal{O}_{M} \rightarrow R j_{*} \mathcal{O}_{V^{\star}}$. The result follows the assumption $\mathcal{O}_{V} \sim_{\text {qis }} \tau_{\leq k} R \mu_{*} \mathcal{O}_{M}$. Since $R \underline{\Gamma}_{D}\left(R j_{*} \mathbb{C}_{V^{\star}}\right) \sim_{\text {qis }} 0$, we have

$$
\tau_{\leq k+1} R \underline{\Gamma}_{D}\left(\tau_{\leq k} R j_{*} \mathbb{C}_{V^{*}}\right) \sim_{\text {qis }} \tau_{\leq k+1}\left(R \underline{\Gamma}_{D}\left(\tau_{\geq k+1} R j_{*} \mathbb{C}_{V^{*}}\right)[-1]\right) \sim_{\text {qis }} 0 .
$$

Thus $\tau_{\leq k+1} R \underline{\Gamma}_{D}$ of the composite $\mathbb{C}_{V} \rightarrow \tau_{\leq k} R j_{*} \mathbb{C}_{V^{\star}} \rightarrow \mathcal{O}_{V}$ is zero.

Definition. For the sheaf $\mathfrak{M}_{V}^{\star}$ of germs of invertible meromorphic functions of $V$, we define

$$
\mathcal{O}_{V}(* D)^{\star}:=\mathfrak{M}_{V}^{\star} \cap j_{*} \mathcal{O}_{V^{\star}}^{\star} \subset j_{*} \mathfrak{M}_{V^{\star}}^{\star}
$$


The sheaf $\mathcal{D} i v_{V}$ of germs of Cartier divisors is defined to be $\mathfrak{M}_{V}^{\star} / \mathcal{O}_{V}^{\star}$. Thus the sheaf $\mathcal{O}_{V}(* D)^{\star}$ is the kernel of the composite

$$
\mathfrak{M}_{V}^{\star} \rightarrow \mathcal{D} i v_{V} \rightarrow{\mathcal{D} i v_{V}} / \mathcal{H}_{D}^{0}\left(\mathcal{D}^{*} v_{V}\right) .
$$

\section{Lemma 3.2.3.}

(1) Let $\mu: Y \rightarrow V$ be a bimeromorphic morphism from a normal variety that is isomorphic over $V^{\star}$. Then $\mathcal{O}_{V}(* D)^{\star} \simeq \mu_{*} \mathcal{O}_{Y}\left(* \mu^{-1} D\right)^{\star}$.

(2) Assume that $V$ has only 1-rational singularities. Then $\mathcal{O}_{V}(* D)^{\star}$ is isomorphic to the mapping cone of $\tau_{\leq 1} R j_{*} \mathbb{Z}_{V^{\star}} \rightarrow \mathcal{O}_{V}$, which is induced from $\tau_{\leq 1} R j_{*} \mathbb{C}_{V^{*}} \rightarrow \mathcal{O}_{V}$ in 3.2.2.

(3) Assume that $V$ has only 1-rational singularities. Let $\mathcal{H}_{D}^{0}\left(\mathcal{D}_{i v_{V}}\right) \rightarrow$ $\mathcal{H}_{D}^{1}\left(\mathcal{O}_{V}^{\star}\right)$ and $\mathcal{H}_{D}^{1}\left(\mathcal{O}_{V}^{\star}\right) \rightarrow \mathcal{H}_{D}^{2}\left(\mathbb{Z}_{V}\right)$, respectively, be the connecting homomorphisms for the exact sequences

$$
0 \rightarrow \mathcal{O}_{V}^{\star} \rightarrow \mathfrak{M}_{V}^{\star} \rightarrow \mathcal{D} i v_{V} \rightarrow 0, \quad \text { and } \quad 0 \rightarrow \mathbb{Z}_{V} \rightarrow \mathcal{O}_{V} \rightarrow \mathcal{O}_{V}^{\star} \rightarrow 0 .
$$

Then the composite $\mathcal{H}_{D}^{0}\left(\mathcal{D} i v_{V}\right) \rightarrow \mathcal{H}_{D}^{2}\left(\mathbb{Z}_{V}\right)$ is an isomorphism.

Proof. (1) For the open immersion $j_{Y}: V^{\star} \leadsto \mu^{-1} V^{\star} \hookrightarrow Y$, we have $\mathcal{O}_{Y}\left(* \mu^{-1} D\right)^{\star}=\mathfrak{M}_{Y}^{\star} \cap j_{Y *} \mathcal{O}_{V^{\star}}^{\star}$. Thus $\mu_{*} \mathcal{O}_{Y}\left(* \mu^{-1} D\right)^{\star}=\mathfrak{M}_{V}^{\star} \cap j_{*} \mathcal{O}_{V^{\star}}^{\star}=$ $\mathcal{O}_{V}(* D)^{\star}$, since $\mu_{*} \mathfrak{M}_{Y}^{\star} \simeq \mathfrak{M}_{V}^{\star}$.

(2) Assume that the assertion holds when $V$ is non-singular and $D$ is normal crossing. Let $\mu: Y \rightarrow V$ be a bimeromorphic morphism from a nonsingular variety such that $\mu^{-1} D$ is normal crossing and that $Y \backslash \mu^{-1} D \simeq V \backslash D$. For the open immersion $j_{Y}: V^{\star} \hookrightarrow Y$, we have the triangle

$$
\cdots \stackrel{+1}{\longrightarrow} \tau_{\leq 1} R j_{Y *} \mathbb{Z}_{V^{\star}} \rightarrow \mathcal{O}_{Y} \rightarrow \mathcal{O}_{Y}\left(* \mu^{-1} D\right)^{\star} \stackrel{+1}{\longrightarrow} \cdots
$$

by the assumption. Applying $R \mu_{*}$, we have a triangle

$$
\cdots \stackrel{+1}{\longrightarrow} R \mu_{*}\left(\tau_{\leq 1} R j_{Y_{*}} \mathbb{Z}_{V^{\star}}\right) \rightarrow R \mu_{*} \mathcal{O}_{Y} \rightarrow R \mu_{*} \mathcal{O}_{Y}\left(* \mu^{-1} D\right)^{\star} \stackrel{+1}{\longrightarrow} \cdots
$$

From quasi-isomorphisms $\mathcal{O}_{V} \sim_{\text {qis }} \tau_{\leq 1} R \mu_{*} \mathcal{O}_{Y}$ and

$$
\tau_{\leq 1}\left(R \mu_{*}\left(\tau_{\leq 1} R j_{Y_{*}} \mathbb{Z}_{V^{\star}}\right)\right) \sim_{\text {qis }} \tau_{\leq 1} R j_{*} \mathbb{Z}_{V^{*}}
$$

we infer that $\mu_{*} \mathcal{O}_{Y}\left(* \mu^{-1} D\right)^{\star}$ is quasi-isomorphic to the mapping cone of $\tau_{\leq 1} R j_{*} \mathbb{Z}_{V^{\star}} \rightarrow \mathcal{O}_{V}$. Therefore, we are reduced to the case $V$ is non-singular and $D$ is normal crossing. Let $\mathcal{Z}^{1}(\log )$ denote the kernel of d: $\Omega_{V}^{1}(\log D) \rightarrow$ 
$\Omega_{V}^{2}(\log D)$. Then the truncation $\tau_{\leq 1} R j_{*} \mathbb{C}_{V^{\star}}$ is represented by the complex $\left[0 \rightarrow \mathcal{O}_{V} \rightarrow \mathcal{Z}^{1}(\log ) \rightarrow 0\right]$. Thus the mapping cone of $\tau_{\leq 1} R j_{*} \mathbb{C}_{V^{\star}} \rightarrow \mathcal{O}_{V}$ is $\mathcal{Z}^{1}(\log )$. Similarly, let $\mathcal{Z}^{1}$ denote the kernel of $\mathrm{d}: \Omega_{V^{\star}}^{1} \rightarrow \Omega_{V^{\star}}^{2}$. Then the mapping cone of $\tau_{\leq 1} R j_{*} \mathbb{C}_{V^{*}} \rightarrow R j_{*} \mathcal{O}_{V^{\star}} \sim_{\text {qis }} j_{*} \mathcal{O}_{V^{\star}}$ is quasi-isomorphic to $j_{*} \mathcal{Z}^{1}$. There is a homomorphism dlog: $j_{*} \mathcal{O}_{V^{\star}}^{\star} \rightarrow j_{*} \mathcal{Z}^{1}$. It appears in the commutative diagram

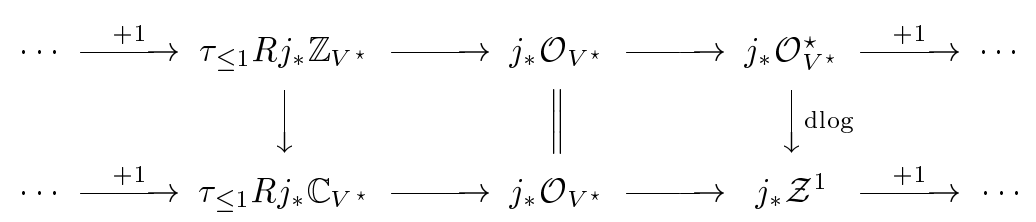

For a unit holomorphic function $u$ on $V^{\star}$, if $\operatorname{dlog} u=\mathrm{d} u / u$ is a logarithmic 1-form along $D$, then $u$ is meromorphic along $D$. Therefore, the kernel of $j_{*} \mathcal{O}_{V^{\star}}^{\star} \rightarrow j_{*} \mathcal{Z}^{1} / \mathcal{Z}^{1}(\log )$ is $\mathcal{O}_{V}(* D)^{\star}$. Therefore, we have a commutative diagram

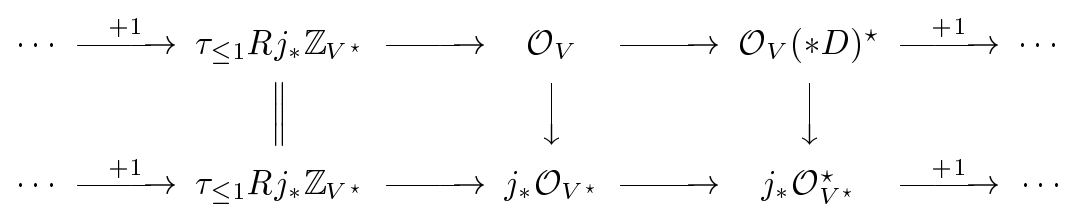

(3) The commutative diagram

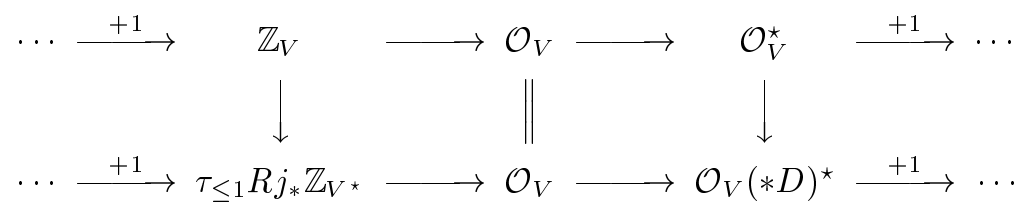

induces the isomorphism.

Remark. (2) is well-known in the study of Deligne-Beilinson cohomology groups ([B1], [E]). In fact, when $V$ is a projective variety, then $H^{0}\left(V, \mathcal{O}_{V}(* D)^{\star}\right)$ $=H_{\mathcal{D}}^{1}\left(V^{\star}, \mathbb{Z}(1)\right)$.

Remark. There is another proof of (3) for a toroidal embedding. Let $X^{\star} \subset X$ together with a point $x \in B$ be a toroidal embedding of type $(\mathrm{N}, l, \sigma)$. For the sheaf $\mathcal{O}_{X}(* B)^{\star} / \mathcal{O}_{X}^{\star} \simeq \mathcal{H}_{B}^{0}\left(\mathcal{D} i v_{X}\right)$, the stalk $\mathcal{H}_{B}^{0}\left(\mathcal{D} i v_{X}\right)_{x}$ is identified with $\mathrm{M}=\operatorname{Hom}(\mathrm{N}, \mathbb{Z})$. The connecting homomorphism $\mathcal{H}_{B}^{0}\left(\mathcal{D} i v_{X}\right) \rightarrow \mathcal{H}_{B}^{1}\left(\mathcal{O}_{X}^{\star}\right)$ is derived from an injection $\mathcal{O}_{X}(* B)^{\star} \subset j_{*} \mathcal{O}_{X}^{\star}$. Thus the image of $m \in \mathrm{M}$ in $\mathcal{H}_{B}^{2}\left(\mathbb{Z}_{X}\right) \simeq R^{1} j_{*} \mathbb{Z}_{X^{\star}}$ corresponds to the functional

$$
\mathrm{N} \simeq \pi_{1}\left(X^{\star}\right) \ni \gamma \longmapsto \frac{1}{2 \pi \sqrt{-1}} \int_{\gamma} \frac{\mathrm{d} x^{m}}{x^{m}},
$$


where $x^{m}$ stands for the meromorphic function corresponding to $m$. The functional is derived from the natural isomorphism $M \simeq \operatorname{Hom}(\mathrm{N}, \mathbb{Z})$. Therefore $\mathcal{H}_{B}^{0}\left(\mathcal{D} i v_{X}\right) \rightarrow \mathcal{H}_{B}^{2}\left(\mathbb{Z}_{X}\right)$ is an isomorphism.

The sheaf $w \mathcal{D} i v_{V}$ of germs of Weil divisors is isomorphic to $j_{*}^{\circ} \mathcal{D} i v_{V^{\circ}}$. Hence, we have an isomorphism $\mathcal{H}_{D}^{0}\left(w \mathcal{D} i v_{V}\right) \simeq j_{*}^{\circ} \mathcal{H}_{D^{\star}}^{0}\left(\mathcal{D} i v_{V^{\circ}}\right)$.

\section{Lemma 3.2.4.}

(1) $\tau_{\geq 1} R j_{*}^{\star} \mathbb{Z}_{V^{\star}} \quad \sim_{\text {qis }} \quad R \underline{\Gamma}_{D^{\star}}\left(\mathbb{Z}_{V^{\circ}}\right)[1] \quad \sim_{\text {qis }} \quad \mathbb{Z}_{D^{\star}}[-1] . \quad$ In particular, $R^{1} j_{*}^{\star} \mathbb{Z}_{V^{\star}} \simeq \mathbb{Z}_{D^{\star}}$.

(2) $\mathcal{H}_{D}^{0}\left(w \mathcal{D} i v_{V}\right) \simeq \nu_{*} \mathbb{Z}_{\tilde{D}} \simeq j_{*}^{\circ} \mathcal{H}_{D^{\star}}^{2}\left(\mathbb{Z}_{V^{\circ}}\right)$, for the normalization $\nu: \tilde{D} \rightarrow D$.

(3) Assume that $V$ has only $k$-rational singularities. Then

$$
\tau_{\leq k} R \underline{\Gamma}_{D}\left(\mathcal{O}_{V}^{\star}\right) \sim_{\text {qis }} \tau_{\leq k} R \underline{\Gamma}_{D}\left(\mathcal{O}_{V}\right) \oplus\left(\tau_{\leq k+1} R \underline{\Gamma}_{D}\left(\mathbb{Z}_{V}\right)\right)[1]
$$

(4) Assume that $V$ has only $k$-rational singularities for $k \geq 2$. Then $R^{p} j_{*}^{\circ} \mathcal{O}_{V^{\circ}}^{\star}$ $\simeq R^{p} j_{*}^{\circ} \mathcal{O}_{V^{\circ}} \oplus R^{p+1} j_{*}^{\circ} \mathbb{Z}_{V}$ for $p \leq k-1$.

(5) Assume that $V$ has only 1-rational singularities. Then $R^{1} j_{*}^{\circ} \mathbb{Z}_{V} \simeq \mathcal{H}_{Z}^{2}\left(\mathbb{Z}_{V}\right)$ $=0$ and $R^{1} \mu_{*} \mathbb{Z}_{Y}=0$ for a desingularization $\mu: Y \rightarrow V$.

Proof. (1) The first quasi-isomorphism is derived from the triangle

$$
\cdots \stackrel{+1}{\longrightarrow} R \underline{\Gamma}_{D^{\star}}\left(\mathbb{Z}_{V^{\circ}}\right) \rightarrow \mathbb{Z}_{V^{\circ}} \rightarrow R j_{*}^{\star} \mathbb{Z}_{V^{\star}} \stackrel{+1}{\longrightarrow} \cdots
$$

The second is induced from the natural quasi-isomorphism $R \underline{\Gamma}_{D^{\star}}\left(\boldsymbol{\omega}_{V^{\circ}}^{\text {top }}\right) \sim_{\text {qis }}$ $\boldsymbol{\omega}_{D^{\star}}^{\text {top }}$ (cf. Section 0.3).

(2) $B^{\star}$ itself is an element of $H^{0}\left(X^{\circ}, \mathcal{H}_{B}^{0}\left(\mathcal{D} i v_{X}\right)\right)$. Thus we have a homomorphism $\mathbb{Z}_{B^{\star}} \rightarrow \mathcal{H}_{B^{\star}}^{0}\left(\mathcal{D}_{i v_{X^{\circ}}}\right)$. This is an isomorphism by (1) and 3.2.3-(3). The application of $j_{*}^{\circ}$ to the isomorphism induces the expected isomorphisms.

(3) We have a triangle

$$
\cdots \stackrel{+1}{\longrightarrow} R \underline{\Gamma}_{D}\left(\mathbb{Z}_{V}\right) \rightarrow R \underline{\Gamma}_{D}\left(\mathcal{O}_{V}\right) \rightarrow R \underline{\Gamma}_{D}\left(\mathcal{O}_{V}^{\star}\right) \stackrel{+1}{\longrightarrow} \cdots
$$

from the exponential sequence of $V$. By 3.2.2, the morphism $\tau_{\leq k+1} R \underline{\Gamma}_{D}\left(\mathbb{Z}_{V}\right) \rightarrow$ $R \underline{\Gamma}_{D}\left(\mathcal{O}_{V}\right)$ is zero in the derived category.

(4) follows from the decomposition

$$
\tau_{\leq k} R \underline{\Gamma}_{Z}\left(\mathcal{O}_{V}^{\star}\right) \sim_{\text {qis }} \tau_{\leq k} R \underline{\Gamma}_{Z}\left(\mathcal{O}_{V}\right) \oplus\left(\tau_{\leq k+1} R \underline{\Gamma}_{Z}\left(\mathbb{Z}_{V}\right)\right)[1]
$$


that is induced from (3) and from $R \underline{\Gamma}_{Z} \sim_{\text {qis }} R \underline{\Gamma}_{Z} \circ R \underline{\Gamma}_{D}$.

(5) We may assume that $\mu^{-1} Z$ is a divisor by replacing $Y$. The exponential sequence of $Y$ induces an injection $R^{1} \mu_{*} \mathbb{Z}_{Y} \hookrightarrow R^{1} \mu_{*} \mathcal{O}_{Y}=0$. We have a triangle

$$
\cdots \stackrel{+1}{\longrightarrow} R \mu_{*} R \underline{\Gamma}_{\mu^{-1} Z}\left(\mathbb{Z}_{Y}\right) \rightarrow R \mu_{*} \mathbb{Z}_{Y} \rightarrow R j_{*}^{\circ} \mathbb{Z}_{V} \stackrel{+1}{\longrightarrow} \cdots
$$

and it induces an exact sequence

$$
0 \rightarrow R^{1} \mu_{*} \mathbb{Z}_{Y} \rightarrow R^{1} j_{*}^{\circ} \mathbb{Z}_{V^{\circ}} \rightarrow \mu_{*} \mathcal{H}_{\mu^{-1} Z}^{2}\left(\mathbb{Z}_{Y}\right) \rightarrow R^{2} \mu_{*} \mathbb{Z}_{Y}
$$

Here, the last homomorphism is decomposed as

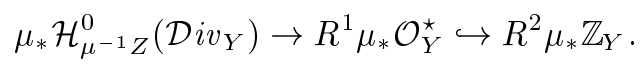

For a $\mu$-exceptional divisor $E=\sum a_{i} E_{i}$ of $Y$, if $\mathcal{O}_{Y}(E) \simeq \mathcal{O}_{Y}$, then $E=0$. Thus $0=R^{1} \mu_{*} \mathbb{Z}_{Y} \simeq R^{1} j_{*}^{\circ} \mathbb{Z}_{V^{\circ}}$.

Remark. Let $M$ be a non-singular complex analytic variety, $D$ a reduced divisor, and let $\nu: \tilde{D} \rightarrow D$ be the normalization. Let us consider the following commutative diagram:

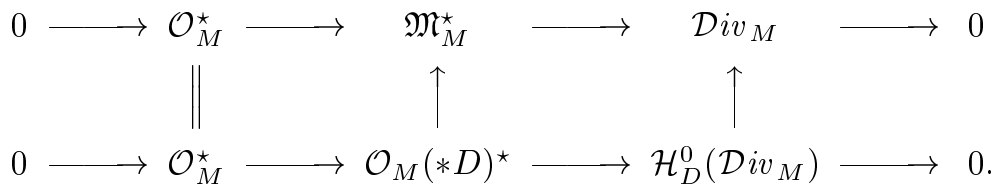

Then we have connecting homomorphisms $H^{p}(\tilde{D}, \mathbb{Z}) \rightarrow H^{p+1}\left(M, \mathcal{O}_{M}^{\star}\right)$. By combining with connecting homomorphisms $H^{p+1}\left(M, \mathcal{O}_{M}^{\star}\right) \rightarrow H^{p+2}(M, \mathbb{Z})$ of the exponential sequence of $M$, we have the so-called Gysin homomorphisms $H^{p}(\tilde{D}, \mathbb{Z}) \rightarrow H^{p+2}(M, \mathbb{Z})$.

We define $\operatorname{Div}_{D}(V):=H_{D}^{0}\left(V, \mathcal{D i v}_{V}\right)$ and $\operatorname{WDiv}_{D}(V):=H_{D}^{0}\left(V, w \mathcal{D} i v_{V}\right)$. These are the groups of Cartier and Weil divisors supported in $D$, respectively. In fact, $\operatorname{WDiv}_{D}(V) \simeq H^{0}(\tilde{D}, \mathbb{Z})$ by 3.2.4-(2). This is a free abelian group generated by irreducible components of $D$ provided that $D$ has only finitely many irreducible components. The sheaf $w \mathcal{D} i v_{V} / \mathcal{D} i v_{V}=\mathcal{H}_{Z}^{1}\left(\mathcal{D} i v_{V}\right)$ of local divisor class groups canonically contains the sheaf $\mathcal{H}_{D}^{0}\left(w \mathcal{D} i v_{V}\right) / \mathcal{H}_{D}^{0}\left(\mathcal{D} i v_{V}\right)=$ $\mathcal{H}_{Z}^{1}\left(\mathcal{H}_{D}^{0}\left(\mathcal{D} i v_{V}\right)\right)$. We have an exact sequence

$$
0 \rightarrow \mathcal{H}_{Z}^{1}\left(\mathcal{H}_{D}^{2}\left(\mathbb{Z}_{V}\right)\right) \rightarrow \mathcal{H}_{Z}^{3}\left(\mathbb{Z}_{V}\right) \rightarrow \mathcal{H}_{Z}^{0}\left(\mathcal{H}_{D}^{3}\left(\mathbb{Z}_{V}\right)\right)
$$

from the quasi-isomorphism $R \underline{\Gamma}_{Z}\left(\mathbb{Z}_{V}\right) \sim_{\text {qis }} R \underline{\Gamma}_{Z}\left(R \underline{\Gamma}_{D}\left(\mathbb{Z}_{V}\right)\right)$.

We introduce the following conditions for $[V, D]$ : 
Condition 3.2.5. $\quad \mathcal{H}_{Z}^{1}\left(\mathcal{H}_{D}^{0}\left(\mathcal{D} i v_{V}\right)\right) \rightarrow \mathcal{H}_{Z}^{3}\left(\mathbb{Z}_{V}\right)$ is an isomorphism.

Condition 3.2.6. The composite

$$
\operatorname{WDiv}_{D}(V) \rightarrow H^{0}\left(V, \mathcal{H}_{Z}^{1}\left(\mathcal{H}_{D}^{0}\left(\mathcal{D}_{i v_{V}}\right)\right)\right) \rightarrow H^{0}\left(V, \mathcal{H}_{Z}^{3}\left(\mathbb{Z}_{V}\right)\right)
$$

is surjective.

Remark. If $V$ has only 2-rational singularities, then $\mathcal{H}_{Z}^{1}\left(\mathcal{D}_{i v}\right) \simeq$ $\mathcal{H}_{Z}^{3}\left(\mathbb{Z}_{V}\right)$ by [F1, 6.1]. We will show in 3.3.1-(3) that if $V \backslash D \subset V$ is a toroidal embedding, then [V,D] satisfies 3.2.5. If $V$ is non-singular (cf. 3.2.7-(1)) or if $V \backslash D \subset V$ is a toroidal embedding without self-intersection in the sense of $[\mathrm{K} 6$, Chapter II], then $[V, D]$ satisfies both Conditions 3.2.5 and 3.2.6.

Example. Let $V$ be a normal surface with one $A_{1}$-singular point $P$ as the singular locus and let $D$ be an irreducible curve through $P$. Assume that, for the minimal desingularization $\mu: Y \rightarrow V$, the $(-2)$-curve $\mu^{-1} P$ intersects with the proper transform $D^{\prime}$ of $D$ at two points and the intersections are transversal. Then $D$ is a Cartier divisor and hence $\operatorname{WDiv}_{D}(V)=\operatorname{Div}_{D}(V)$, while the stalk of $\mathcal{H}_{Z}^{3}\left(\mathbb{Z}_{V}\right)$ at $P$ is isomorphic to $\mathbb{Z} / 2 \mathbb{Z}$. Thus $[V, D]$ does not satisfy 3.2.6.

Lemma 3.2.7. Let $\mu: Y \rightarrow V$ be a bimeromorphic morphism from a non-singular variety such that $Y \backslash \mu^{-1} D \rightarrow V^{\star}$ is an isomorphism. Let us consider the following pullback homomorphisms

$$
\begin{aligned}
\mu_{D}^{*}: H_{D}^{p}(V, \mathbb{Z}) \rightarrow H_{\mu^{-1} D}^{p}(Y, \mathbb{Z}) ; & \mu^{*}: H^{p}(V, \mathbb{Z}) \rightarrow H^{p}(Y, \mathbb{Z}) ; \\
\mu_{D, \star}^{*}: H_{D}^{p}\left(V, \mathcal{O}_{V}^{\star}\right) \rightarrow H_{\mu^{-1} D}^{p}\left(Y, \mathcal{O}_{Y}^{\star}\right) ; & \mu_{\star}^{*}: H^{p}\left(V, \mathcal{O}_{V}^{\star}\right) \rightarrow H^{p}\left(Y, \mathcal{O}_{Y}^{\star}\right) .
\end{aligned}
$$

(1) If $V$ is non-singular, then they are all injective.

(2) $\mu_{D}^{*}$ and $\mu^{*}$ are injective for $p \leq 1$. $\mu_{D, \star}^{*}$ and $\mu_{\star}^{*}$ are isomorphic for $p=0$.

(3) If $V$ has only 1-rational singularities, then $\mu_{D}^{*}$ and $\mu^{*}$ are injective for $p=2$, and $\mu_{D, \star}^{*}$ and $\mu_{\star}^{*}$ are injective for $p=1$.

(4) If $V$ has only 1-rational singularities and if $[V, D]$ satisfies both Conditions 3.2.5 and 3.2.6, then $\mu_{D}^{*}$ and $\mu^{*}$ are injective for $p=3$, and $\mu_{D, \star}^{*}$ and $\mu_{\star}^{*}$ are injective for $p=2$.

Proof. There is a commutative digram

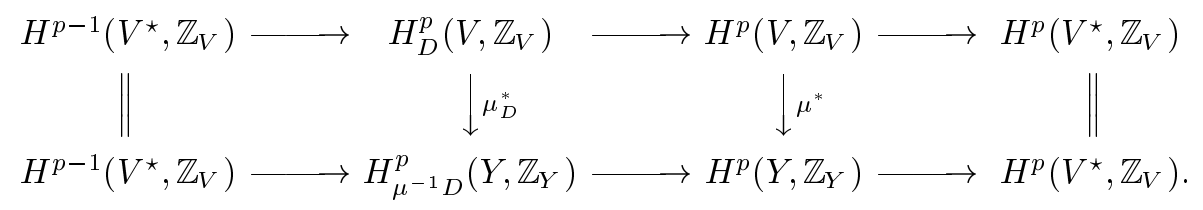


We infer that, if $\mu_{D}^{*}$ is injective for $p \leq k$ for some integer $k$, then $\mu^{*}$ is also injective for $p \leq k$. By considering a similar commutative diagram instead of $\mathbb{Z}$ by $\mathcal{O}^{\star}$, we see that if $\mu_{D, \star}^{*}$ is injective for $p \leq k$, then $\mu_{\star}^{*}$ is also injective for $p \leq k$.

(1) The trace map $R \mu_{*} \mathbb{Z}_{Y}[2 n] \sim_{\text {qis }} R \mu_{*} \omega_{Y}^{\text {top }} \rightarrow \boldsymbol{\omega}_{V}^{\text {top }} \sim_{\text {qis }} \mathbb{Z}_{V}[2 n]$ gives a splitting of $\mathbb{Z}_{V} \rightarrow R \mu_{*} \mathbb{Z}_{Y}$. Thus $H_{D}^{p}(V, \mathbb{Z})$ and $H^{p}(V, \mathbb{Z})$ are direct summands of $H_{\mu^{-1} D}^{p}(Y, \mathbb{Z})$ and $H^{p}(Y, \mathbb{Z})$, respectively. There is a splitting $R \underline{\Gamma}_{D}\left(\mathcal{O}_{V}^{\star}\right) \sim_{\text {qis }}$ $R \underline{\Gamma}_{D}\left(\mathcal{O}_{V}\right) \oplus R \underline{\Gamma}_{D}\left(\mathbb{Z}_{V}\right)[1]$ by 3.2.4-(3). Thus $\mu_{D, \star}^{*}$ is also injective for any $p$.

(2) The assertion follows $H_{D}^{0}(V, \mathbb{Z})=H_{D}^{1}(V, \mathbb{Z})=H_{D}^{0}\left(V, \mathcal{O}_{V}^{\star}\right)=0$ and $\mu_{*} \mathcal{O}_{Y}^{\star} \simeq \mathcal{O}_{V}^{\star}$.

(3) $\mu_{D}^{*}: H_{D}^{2}(V, \mathbb{Z}) \simeq H_{\mu^{-1} D}^{2}(Y, \mathbb{Z})$ is isomorphic to the injective pullback homomorphism $\operatorname{Div}_{D}(V) \rightarrow \operatorname{Div}_{\mu^{-1} D}(Y)$. The injectiveness of $\mu_{D, \star}^{*}$ follows from the decomposition $\tau_{\leq 1} R \underline{\Gamma}_{D}\left(\mathcal{O}_{V}^{\star}\right) \sim_{\text {qis }} \tau_{\leq 1} R \underline{\Gamma}_{D}\left(\mathcal{O}_{V}\right) \oplus\left(\tau_{\leq 2} R \underline{\Gamma}_{D}\left(\mathbb{Z}_{V}\right)\right)[1]$.

(4) By the argument above and by the decomposition $\tau_{\leq 2} R \underline{\Gamma}_{D}\left(\mathcal{O}_{V}^{\star}\right) \sim_{\text {qis }}$ $\tau_{\leq 2} R \underline{\Gamma}_{D}\left(\mathcal{O}_{V}\right) \oplus\left(\tau_{\leq 3} R \underline{\Gamma}_{D}\left(\mathbb{Z}_{V}\right)\right)[1]$, we have only to show $\mu_{D}^{*}$ is injective for $p=3$. Now there is an exact sequence

$$
0 \rightarrow \mathcal{H}_{D}^{2}\left(\mathbb{Z}_{V}\right) \rightarrow j_{*}^{\circ} \mathcal{H}_{D^{\star}}^{2}\left(\mathbb{Z}_{V^{\circ}}\right) \rightarrow \mathcal{H}_{Z}^{3}\left(\mathbb{Z}_{V}\right) \rightarrow 0
$$

by 3.2.5. Thus $\mathcal{H}_{D}^{3}\left(\mathbb{Z}_{V}\right) \rightarrow \mathcal{H}_{D}^{3}\left(R j_{*}^{\circ} \mathbb{Z}_{V}\right.$ ) is injective. By 3.2.6,

$$
H^{1}\left(V, \mathcal{H}_{D}^{2}\left(\mathbb{Z}_{V}\right)\right) \rightarrow H^{1}\left(V, j_{*}^{\circ} \mathcal{H}_{D^{\star}}^{2}\left(\mathbb{Z}_{V} \circ\right)\right)
$$

is also injective. There is a commutative diagram of exact sequences

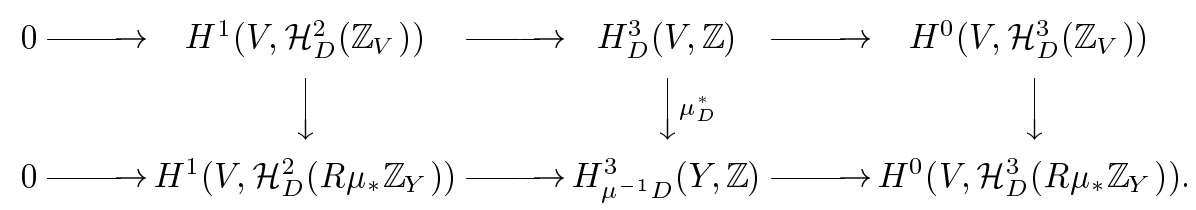

Since left and right vertical arrows are injective, the middle is also injective.

\section{§3.3. Toroidal embedding}

Let $X$ be an $n$-dimensional complex analytic normal variety and let $B$ a reduced divisor. We denote the complement $X \backslash B$ by $X^{\star}$ and denote the open immersion by $j: X^{\star} \hookrightarrow X$. We assume that the complement $X^{\star}$ defines a toroidal embedding $X^{\star} \subset X$ in the sense of [K6]. This is equivalent to that, for any point $x \in B$, there exists an open neighborhood $\mathcal{U}$ in $X$ such that $\mathcal{U} \cap X^{\star} \subset \mathcal{U}$ is an $n$-dimensional toroidal embedding of type (N, $\left.l, \sigma, v, m_{\bullet}\right)$ for a free abelian group $\mathrm{N}$ of rank $l \leq n$, a strictly convex rational polyhedral 
cone $\sigma \subset \mathrm{N} \otimes \mathbb{R}$ with $\mathbf{N}=\mathrm{N}(\sigma)$, a vector $v \in \operatorname{Int} \sigma$, and for a generator $m_{\bullet}$ of the semi-group $\sigma^{\vee} \cap \mathrm{N}$. By 3.1.2, there is a base of open neighborhoods $\mathcal{U}_{1} \supset \mathcal{U}_{2} \supset \cdots$ in $X$ such that $\mathcal{U}_{1} \cap X^{\star} \supset \mathcal{U}_{2} \cap X^{\star} \supset \cdots$ are all homotopically equivalent to $\mathrm{N} \otimes \mathrm{S}^{1}$. The rank $l=l(x)$ depends on $x$. Let $l(x):=0$ for $x \in X^{\star}$. For a non-negative integer $i \leq n$, let $X(B)_{i}$ be the subset of $X$ consisting of points $x$ with $l(x) \geq n-i$. Then the filtration

$$
X(B)_{\bullet}: X=X(B)_{n} \supset X(B)_{n-1} \supset \cdots \supset X(B)_{0}
$$

is a generalization of $\mathbb{T}_{\mathrm{N}}(\sigma)$ • of $\mathbb{T}_{\mathrm{N}}(\sigma)$. We infer that $B=X(B)_{n-1}$ and that $X(B)_{n-i}$ is locally the intersection of mutually distinct $i$-irreducible components of $B$ for $i>0$.

Notation. For the $n$-dimensional toroidal embedding $X^{\star}=X \backslash B \subset X$, we define $Z=Z(X, B)$ to be the analytic subset $X(B)_{n-2}=\operatorname{Sing} X \cup \operatorname{Sing} B$ $=\operatorname{Sing} B$. We set $X^{\circ}:=X \backslash Z$ and $B^{\star}:=B \backslash Z$. The related open immersions are denoted by $j^{\star}: X^{\star} \hookrightarrow X^{\circ}, j^{\circ}: X^{\circ} \hookrightarrow X$.

Lemma 3.3.1. Let $j: X^{\star}=X \backslash B \hookrightarrow X$ be a toroidal embedding as above and let $\mu: Y \rightarrow X$ be a bimeromorphic morphism from a non-singular variety that is an isomorphism over $X^{\star}$.

(1) The homomorphism $\bigwedge^{p} R^{1} j_{*} \mathbb{Z}_{X^{\star}} \rightarrow R^{p} j_{*} \mathbb{Z}_{X^{\star}}$ induced from cup product is an isomorphism.

(2) The homomorphism

$$
\mathcal{H}_{B}^{p}\left(\mathbb{Z}_{X}\right) \rightarrow \mathcal{H}_{B}^{p}\left(R j_{*}^{\circ} \mathbb{Z}_{X^{\circ}}\right) \simeq R^{p-2} j_{*}^{\circ} \mathbb{Z}_{B^{\star}}
$$

is injective for any $p \geq 2$. In other words,

$$
\mathcal{H}_{Z}^{p}\left(\mathbb{Z}_{X}\right) \rightarrow \mathcal{H}_{B}^{p}\left(\mathbb{Z}_{X}\right)
$$

is zero for any $p$.

(3) Let $\boldsymbol{\omega}_{X}^{\text {top }}$ be the topological dualizing complex of $X$. Then

$$
\mathcal{H}^{2-2 n}\left(\boldsymbol{\omega}_{X}^{\text {top }}\right) \simeq \mathcal{H}_{Z}^{3}\left(\mathbb{Z}_{X}\right) \simeq \mathcal{H}_{Z}^{1}\left(\mathcal{H}_{B}^{2}\left(\mathbb{Z}_{X}\right)\right) .
$$

In particular, $[X, B]$ satisfies the Condition 3.2.5.

(4) $\mu^{*}: \mathcal{H}_{B}^{p}\left(\mathbb{Z}_{X}\right) \rightarrow \mathcal{H}_{B}^{p}\left(R \mu_{*} \mathbb{Z}_{Y}\right)$ is injective for any $p$. If $[X, B]$ satisfies the Condition 3.2.6, then $H_{B}^{p}(X, \mathbb{Z}) \rightarrow H_{\mu^{-1} B}^{p}(Y, \mathbb{Z}), H^{p}(X, \mathbb{Z}) \rightarrow H^{p}(Y, \mathbb{Z})$ are injective for $p \leq 3$, and $H_{D}^{p}\left(X, \mathcal{O}_{X}^{\star}\right) \rightarrow H_{D}^{p}\left(Y, \mathcal{O}_{Y}^{\star}\right), H^{p}\left(X, \mathcal{O}_{X}^{\star}\right) \rightarrow$ $H^{p}\left(Y, \mathcal{O}_{Y}^{\star}\right)$ are injective for $p \leq 2$. 
(5) There is a canonical exact sequence:

$$
0 \rightarrow \mathcal{H}_{B}^{0}\left(\mathcal{D} i v_{X}\right) \rightarrow \mu_{*} \mathcal{H}_{\mu^{-1} B}^{0}\left(\mathcal{D}_{i v_{Y}}\right) \rightarrow R^{2} \mu_{*} \mathbb{Z}_{Y} \rightarrow 0
$$

(6) $H_{B}^{p}(X, \mathbb{Z}) \rightarrow H_{B}^{p}(X, \mathbb{Q})$ is injective for $p \leq 2$. If $[X, B]$ satisfies the Condition 3.2.6, then this is injective also for $p=3$.

Proof. (1) The stalk at a point is isomorphic to $\bigwedge^{p} H^{1}\left(\mathbb{T}_{\mathrm{N}}, \mathbb{Z}\right) \rightarrow H^{p}\left(\mathbb{T}_{\mathrm{N}}\right.$, $\mathbb{Z})$.

(2) The homomorphism (3.2) is isomorphic to $R^{p-1} j_{*} \mathbb{Z}_{X^{*}} \rightarrow$ $R^{p} j_{*}^{\circ}\left(R \underline{\Gamma}_{B^{\star}}\left(\mathbb{Z}_{X^{\circ}}\right)\right)$ derived from $R j_{*}^{\circ}$ of the triangle (3.1). We have only to consider the homomorphism of stalks at a point $x \in B$. Thus we may assume that the toroidal embedding is of type $(\mathrm{N}, l, \sigma)$. The homomorphism of stalks is isomorphic to

$$
H^{p-1}\left(X^{\star}, \mathbb{Z}\right) \rightarrow H_{B^{\star}}^{p}\left(X^{\circ}, \mathbb{Z}\right) \simeq \bigoplus_{i=1}^{k} H_{B_{i}^{\star}}^{p}\left(X^{\circ}, \mathbb{Z}\right),
$$

and hence to

$$
H^{p-1}\left(\mathbb{T}_{\mathrm{N}}, \mathbb{Z}\right) \rightarrow \bigoplus_{i=1}^{k} H_{\mathbb{O}_{\nu_{i}}}^{p}\left(\mathbb{T}_{\mathrm{N}}\left(\nu_{i}\right), \mathbb{Z}\right) .
$$

By 3.1.5, the kernel is

$$
\bigcap_{i=1}^{k} \bigwedge^{p-1}\left(\nu_{i}^{\perp} \cap \mathrm{M}\right)=0 .
$$

We have an exact sequence

$$
\cdots \rightarrow \mathcal{H}_{Z}^{p}\left(\mathbb{Z}_{X}\right) \rightarrow \mathcal{H}_{B}^{p}\left(\mathbb{Z}_{X}\right) \rightarrow R^{p} j_{*}^{\circ}\left(R \underline{\Gamma}_{B^{\star}}\left(\mathbb{Z}_{X^{\circ}}\right)\right) \simeq \mathcal{H}_{B}^{p}\left(R j_{*}^{\circ} \mathbb{Z}_{X^{\circ}}\right) \rightarrow \cdots
$$

by the quasi-isomorphism $R \underline{\Gamma}_{Z} \sim_{\text {qis }} R \underline{\Gamma}_{Z} \circ R \underline{\Gamma}_{B}$. Thus (3.3) is zero.

(3) There is a triangle

$$
\cdots \stackrel{+1}{\longrightarrow} \boldsymbol{\omega}_{Z}^{\mathrm{top}} \rightarrow \boldsymbol{\omega}_{X}^{\mathrm{top}} \rightarrow R j_{*}^{\circ} \mathbb{Z}_{X^{\circ}}[2 n] \stackrel{+1}{\longrightarrow} \cdots
$$

It induces isomorphisms $\mathcal{H}^{-2 n}\left(\boldsymbol{\omega}_{X}^{\text {top }}\right) \simeq \mathbb{Z}_{X}, \mathcal{H}^{1-2 n}\left(\boldsymbol{\omega}_{X}^{\text {top }}\right) \simeq R^{1} j_{*}^{\circ} \mathbb{Z}_{X^{\circ}}$, $\mathcal{H}^{2-2 n}\left(\boldsymbol{\omega}_{X}^{\text {top }}\right) \simeq R^{2} j_{*}^{\circ} \mathbb{Z}_{X^{\circ}}$, since $\mathcal{H}^{p}\left(\boldsymbol{\omega}_{Z}^{\text {top }}\right)=0$ for $p<-2 \operatorname{dim} Z=4-2 n$. There is a triangle

$$
\cdots \stackrel{+1}{\longrightarrow} R \underline{\Gamma}_{Z}\left(\mathbb{Z}_{X}\right) \rightarrow R \underline{\Gamma}_{B}\left(\mathbb{Z}_{X}\right) \rightarrow R \underline{\Gamma}_{B}\left(R j_{*}^{\circ} \mathbb{Z}_{X^{\circ}}\right) \stackrel{+1}{\longrightarrow} \cdots
$$

It induces an exact sequence

$$
0 \rightarrow \mathcal{H}_{B}^{2}\left(\mathbb{Z}_{X}\right) \rightarrow j_{*}^{\circ}\left(\left.\mathcal{H}_{B}^{2}\left(\mathbb{Z}_{X}\right)\right|_{X^{\circ}}\right) \rightarrow \mathcal{H}_{Z}^{3}\left(\mathbb{Z}_{X}\right) \rightarrow 0
$$


by $(2)$.

(4) In the case $X$ is non-singular, this is proved in 3.2.7-(1). Thus we may assume that $\mu$ is a desingularization. In particular, $\mu$ is isomorphic over $X^{\circ}$. Let us consider the composite $\mathbb{Z}_{X} \rightarrow R \mu_{*} \mathbb{Z}_{Y} \rightarrow R j_{*}^{\circ} \mathbb{Z}_{X^{\circ}}$. Thus the result follows from (2) and 3.2.7-(3).

(5) This is derived from the triangle

$$
\cdots \stackrel{+1}{\longrightarrow} R \underline{\Gamma}_{B}\left(\mathbb{Z}_{X}\right) \rightarrow R \underline{\Gamma}_{B}\left(R \mu_{*} \mathbb{Z}_{Y}\right) \rightarrow R \underline{\Gamma}_{B}\left(\tau_{\geq 2} R \mu_{*} \mathbb{Z}_{Y}\right) \stackrel{+1}{\longrightarrow} \cdots
$$

and from (4).

(6) Both cohomology groups are zero for $p \leq 1$. In the case $p=2$, $\mathcal{H}_{B}^{2}\left(\mathbb{Z}_{X}\right) \simeq R^{1} j_{*} \mathbb{Z}_{X^{\star}}$ is a sheaf of torsion-free abelian groups. Thus it is reduced to the injectiveness of $\mathcal{H}_{B}^{2}\left(\mathbb{Z}_{X}\right) \hookrightarrow \mathcal{H}_{B}^{2}\left(\mathbb{Q}_{X}\right) \simeq \mathcal{H}_{B}^{2}\left(\mathbb{Z}_{X}\right) \otimes \mathbb{Q}$. Finally, we consider the case $p=3$. We consider the commutative diagram

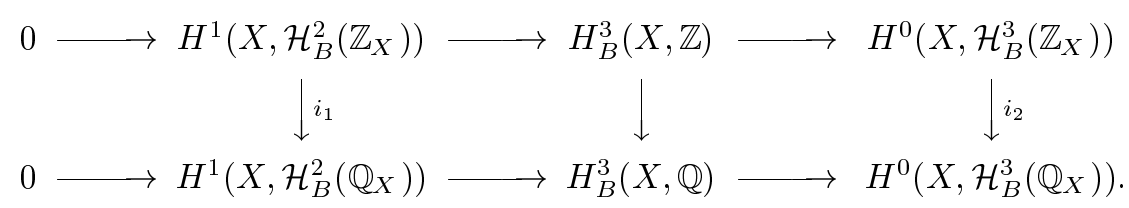

It is enough to show $i_{1}$ and $i_{2}$ are both injective. There is an injection

$$
H^{1}\left(X, \mathcal{H}_{B}^{2}\left(\mathbb{Z}_{X}\right)\right) \rightarrow H^{1}\left(X, j_{*}^{\circ} \mathcal{H}_{B^{\star}}^{2}\left(\mathbb{Z}_{X^{\star}}\right)\right) \simeq H^{1}(\tilde{B}, \mathbb{Z})
$$

for the normalization $\tilde{B} \rightarrow B$ by 3.2.6. Further $H^{1}(\tilde{B}, \mathbb{Z}) \rightarrow H^{1}(\tilde{B}, \mathbb{Q})$ is injective (cf. 3.3.2 below). Thus $i_{1}$ is injective. Since $\mathcal{H}_{B}^{3}\left(\mathbb{Z}_{X}\right)$ is a sheaf of torsion-free abelian group, $i_{2}$ is also injective.

Remark 3.3.2. $\quad$ Let $V$ be a complex analytic space and let $L_{1} \hookrightarrow L_{2}$ be an injection of abelian groups. Then the induced homomorphism $H^{1}\left(V, L_{1}\right) \rightarrow$ $H^{1}\left(V, L_{2}\right)$ is also injective. Because, $H^{0}\left(V, L_{2}\right) \simeq L_{2} \rightarrow H^{0}\left(V, L_{2} / L_{1}\right) \simeq L_{2} / L_{1}$ is surjective if $V$ is connected. In particular, $H^{1}(V, \mathbb{Z})$ is a torsion-free abelian group.

Corollary 3.3.3. $\quad$ Let $x$ be a point of $X$ for a toroidal embedding $X^{\star} \subset$ X.

(1) $\mathcal{H}_{Z}^{3}\left(\mathbb{Z}_{X}\right)_{x}=0$ if and only if the germ $(X, x)$ is non-singular.

(2) $\mathcal{H}_{Z}^{3}\left(\mathbb{Q}_{X}\right)_{x}=0$ if and only if $(X, x)$ is a quotient singularity.

Lemma 3.3.4. The vanishing $R^{p} j_{*}^{\circ} \mathcal{O}_{X^{\circ}}=0$ holds for $p \geq 2$. Assume that $X^{\star} \subset X$ is one of the following toroidal embeddings: 
(1) $X=\mathbb{T}_{\mathrm{N}}(\sigma), B=\mathbb{T}_{\mathrm{N}}(\sigma)_{l-1}$ for a free abelian group $\mathrm{N}$ of rank $l$ and for a strictly convex rational polyhedral cone $\sigma \subset \mathrm{N} \otimes \mathbb{R}$;

(2) $X^{\star} \subset X$ is a toroidal embedding of type $(\mathrm{N}, l, \sigma)$.

Then $H^{p}\left(X^{\circ}, \mathcal{O}_{X^{\circ}}\right)=0$ and $H^{q}\left(X, R^{i} j_{*}^{\circ} \mathcal{O}_{X^{\circ}}\right)=0$ hold for $p \geq 2, q \geq 1$, and for $i \geq 0$.

Proof. Suppose that $X=\mathbb{T}_{\mathrm{N}}(\sigma)$. We have

$$
X^{\circ}=\bigcup_{i=1}^{k} \mathbb{T}_{\mathrm{N}}\left(\nu_{i}\right), \quad X^{\star}=\mathbb{T}_{\mathrm{N}}, \quad \text { and } \quad B^{\star}=\bigsqcup_{i=1}^{k} \mathbb{O}_{\nu_{i}} \text {. }
$$

Hence

$$
H_{B^{\star}}^{p}\left(X^{\circ}, \mathcal{O}_{X^{\circ}}\right) \simeq \bigoplus_{i=1}^{k} H_{\mathbb{O}_{\nu_{i}}}^{p}\left(\mathbb{T}_{\mathrm{N}}\left(\nu_{i}\right), \mathcal{O}_{X}\right) .
$$

Since $\mathbb{T}_{N}\left(\nu_{i}\right)$ and $X^{\star}$ are Stein, the cohomology group vanishes for $p \geq 2$ by the long exact sequence:

$$
\cdots \rightarrow H^{p-1}\left(X^{\star}, \mathcal{O}_{X}\right) \rightarrow H_{\mathbb{O}_{\nu_{i}}}^{p}\left(\mathbb{T}_{\mathrm{N}}\left(\nu_{i}\right), \mathcal{O}_{X}\right) \rightarrow H^{p}\left(\mathbb{T}_{\mathrm{N}}\left(\nu_{i}\right), \mathcal{O}_{X}\right) \rightarrow \cdots
$$

The same argument works for the case (2). Thus we have $R^{p} j_{*}^{\circ} \mathcal{O}_{X^{\circ}}=0$ for $p \geq 2$ for general $X$. Again suppose that $X=\mathbb{T}_{\mathrm{N}}(\sigma)$. By considering Leray's spectral sequence

$$
E_{2}^{p, q}=H^{p}\left(X, R^{q} j_{*}^{\circ} \mathcal{O}_{X^{\circ}}\right) \Longrightarrow E^{p+q}=H^{p+q}\left(X^{\circ}, \mathcal{O}_{X^{\circ}}\right),
$$

we have $E_{2}^{p, 0}=0$ for $p>0, E_{2}^{p, q}=0$ for $q \geq 2$, and $E^{r}=0$ for $r \geq 2$. Thus $E_{2}^{p, 1} \simeq E_{\infty}^{p, 1}=0$ for $p>0$. This argument also works for the case (2).

The Picard group $\operatorname{Pic}(X)$ is defined to be $H^{1}\left(X, \mathcal{O}_{X}^{\star}\right)$. A reflexive sheaf $\mathcal{G}$ of $X$ is a coherent $\mathcal{O}_{X}$-module which is isomorphic to its double-dual. If $\mathcal{G}$ is of rank one, then $\left.\mathcal{G}\right|_{X^{\circ}}$ is an invertible sheaf and $\mathcal{G} \simeq j_{*}^{\circ}\left(\left.\mathcal{G}\right|_{X^{\circ}}\right)$. Let WPic $(X)$ denote the set of isomorphism classes of reflexive sheaves of rank one of $X$. It has a group structure such that the product is given by the double-dual of tensor product. Then $\operatorname{Pic}(X)$ is a subgroup. A Weil divisor $\Delta$ of $X$ naturally defines a reflexive sheaf $\mathcal{O}_{X}(\Delta)$ of rank one. Conversely, a section of a reflexive sheaf of rank one defines an effective Weil divisor. A natural injection $\mathbb{Z}_{X} \rightarrow \mathcal{O}_{X}$ is factored by $R j_{*} \mathbb{Z}_{X^{\star}} \rightarrow \mathcal{O}_{X}$ by 3.2.2. Let $\mathbb{Z}_{X}^{\bullet}=\tau_{\leq 2} R j_{*}^{\circ} \mathbb{Z}_{X^{\circ}}$ and let $\mathcal{O}_{X}^{\bullet}$ be the mapping cone of $\mathbb{Z}_{X}^{\bullet} \rightarrow \mathcal{O}_{X}$. Then we have

$$
\mathcal{H}^{p}\left(\mathbb{Z}_{X}^{\bullet}\right) \simeq\left\{\begin{array} { l l } 
{ \mathbb { Z } _ { X } , } & { \text { for } p = 0 , } \\
{ 0 , } & { \text { for } p = 1 , } \\
{ \mathcal { H } _ { Z } ^ { 3 } ( \mathbb { Z } _ { X } ) , } & { \text { for } p = 2 , }
\end{array} \text { and } \mathcal { H } ^ { p } ( \mathcal { O } _ { X } ^ { \bullet } ) \simeq \left\{\begin{array}{ll}
\mathcal{O}_{X}^{\star}, & \text { for } p=0, \\
\mathcal{H}_{Z}^{3}\left(\mathbb{Z}_{X}\right), & \text { for } p=1 .
\end{array}\right.\right.
$$


Lemma 3.3.5. The natural morphism $R \underline{\Gamma}_{B}\left(\mathbb{Z}_{X}^{\bullet}\right) \rightarrow R \underline{\Gamma}_{B}\left(\mathcal{O}_{X}\right)$ is zero in the derived category. In particular,

$$
R \underline{\Gamma}_{B}\left(\mathcal{O}_{X}^{\bullet}\right) \sim_{\text {qis }} R \underline{\Gamma}_{B}\left(\mathcal{O}_{X}\right) \oplus R \underline{\Gamma}_{B}\left(\mathbb{Z}_{X}^{\bullet}\right)[1]
$$

Proof. The morphism $\mathbb{Z}_{X}^{\bullet} \rightarrow \mathcal{O}_{X}$ is factored by $R j_{*}\left(\left.\mathbb{Z}_{X}^{\bullet}\right|_{X^{\star}}\right) \simeq R j_{*} \mathbb{Z}_{X^{\star}} \rightarrow$ $\mathcal{O}_{X}$. Hence $R \underline{\Gamma}_{B}\left(\mathbb{Z}_{X}^{\bullet}\right) \rightarrow \mathcal{O}_{X}$ is zero. Thus we have the property by taking $R \underline{\Gamma}_{B}$ to the zero map.

\section{Lemma 3.3.6.}

(1) We have an isomorphism $\mathcal{H}^{2}\left(R \underline{\Gamma}_{B}\left(\mathbb{Z}_{X}^{\bullet}\right)\right) \simeq j_{*}^{\circ} \mathcal{H}_{B^{\star}}^{2}\left(\mathbb{Z}_{X^{\circ}}\right)$. In particular, $H_{B}^{2}\left(X, \mathbb{Z}_{X}^{\bullet}\right) \simeq \operatorname{WDiv}_{B}(X) \simeq H^{0}(\tilde{B}, \mathbb{Z})$ and $\mathcal{H}^{1}\left(R \underline{\Gamma}_{B}\left(\mathcal{O}_{X}^{\bullet}\right)\right) \simeq \mathcal{H}_{B}^{1}\left(\mathcal{O}_{X}\right) \oplus$ $\mathcal{H}_{B}^{0}\left(w \mathcal{D} i v_{X}\right)$.

(2) There exists a commutative diagram of triangles:

$$
\begin{aligned}
& \cdots \stackrel{+1}{\longrightarrow} \mathcal{H}_{B}^{0}\left(\mathcal{D} i v_{X}\right)[-1] \longrightarrow \mathcal{H}_{B}^{0}\left(w \mathcal{D} i v_{X}\right)[-1] \oplus \mathcal{O}_{X}^{\star} \longrightarrow \mathcal{O}_{X}^{\bullet} \stackrel{+1}{\longrightarrow} \cdots \\
& \stackrel{+1}{\longrightarrow} \mathcal{D i v}_{X}[-1] \quad \longrightarrow \quad \operatorname{wDiv}_{X}[-1] \oplus \mathcal{O}_{X}^{\star} \quad \longrightarrow \mathcal{O}_{X}^{\bullet} \stackrel{+1}{\longrightarrow} \cdots
\end{aligned}
$$

(3) $\operatorname{WPic}(X) \simeq H^{1}\left(X, \mathcal{O}_{X}^{\bullet}\right)$.

(4) There is an injection $\operatorname{Pic}\left(X^{\circ}\right) / \operatorname{WPic}(X) \hookrightarrow H^{0}\left(X, R^{1} j_{*}^{\circ} \mathcal{O}_{X^{\circ}}\right)$.

Proof. (1) We have $\tau_{\leq 2} R \underline{\Gamma}_{B}\left(\mathbb{Z}_{X}^{\bullet}\right) \sim_{\text {qis }} \tau_{\leq 2} R \underline{\Gamma}_{B}\left(R j_{*}^{\circ} \mathbb{Z}_{X^{\circ}}\right)$. Thus

$$
\mathcal{H}^{2}\left(R \underline{\Gamma}_{B}\left(\mathbb{Z}_{X}^{\bullet}\right)\right) \simeq \mathcal{H}^{2}\left(R j_{*}^{\circ} R \underline{\Gamma}_{B^{\star}}\left(\mathbb{Z}_{X^{\circ}}\right)\right) \simeq j_{*}^{\circ} \mathcal{H}_{B^{\star}}^{2}\left(\mathbb{Z}_{X^{\circ}}\right) .
$$

(2) We have a morphism $\mathcal{H}_{B}^{0}\left(w \mathcal{D} i v_{X}\right)[-1] \rightarrow R \underline{\Gamma}_{B}\left(\mathcal{O}_{X}^{\bullet}\right)$ by (1), which induces the first triangle. We know $w \mathcal{D} i v_{X} / \mathcal{D}_{i v_{X}} \simeq \mathcal{H}_{B}^{0}\left(w \mathcal{D} i v_{X}\right) / \mathcal{H}_{B}^{0}\left(\mathcal{D}_{i} v_{X}\right)$ by 3.3.1-(3). This induces the second triangle.

(3) We have an injection WPic $(X) \hookrightarrow \operatorname{Pic}\left(X^{\circ}\right)$. An invertible sheaf of $X^{\circ}$ comes from WPic $(X)$ if and only if its image in $H^{0}\left(X, R^{1} j_{*}^{\circ} \mathcal{O}_{X^{\circ}}^{\star}\right)$ is contained in the subgroup $H^{0}\left(X, w \mathcal{D} i v_{X} / \mathcal{D}_{i v_{X}}\right)$. By the definition of $\mathcal{O}_{X}^{\bullet}$, we have a commutative diagram of triangles

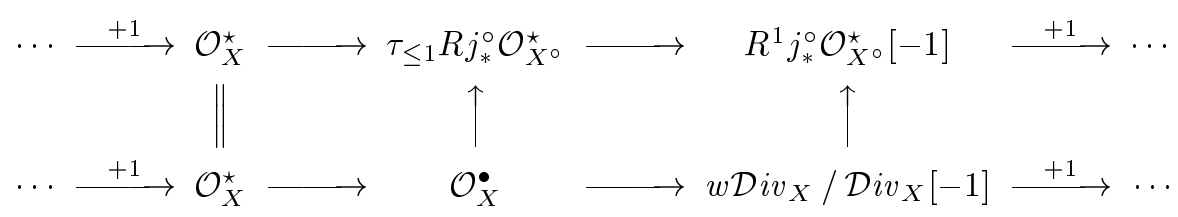


Thus $H^{1}\left(X, \mathcal{O}_{X}^{\bullet}\right) \simeq \operatorname{WPic}(X)$.

(4) The cokernel of $w \mathcal{D i v}_{X} / \mathcal{D i v}_{X} \rightarrow R^{1} j_{*}^{\circ} \mathcal{O}_{X^{\circ}}^{\star}$ is isomorphic to $R^{1} j_{*}^{\circ} \mathcal{O}_{X^{\circ}}$ by 3.2.4-(4).

\section{$\S 3.4 . \quad \partial$-spaces associated with toroidal embeddings}

Let $\underline{X}$ be the associated $\partial$-space $(X, B)$ and let $\underline{j}: X^{\star}=\left(X^{\star}, \emptyset\right) \hookrightarrow \underline{X}$ denote the $\partial$-open immersion. For the open subset $X^{\circ}$, let $\underline{j}^{\circ}: \underline{X}^{\circ}:=\left(X^{\circ}, B^{\star}\right) \hookrightarrow$ $\underline{X}$ be the induced $\partial$-open immersion. We always denote by $\varepsilon$ the morphism $\underline{X}=(X, B) \rightarrow X=(X, \emptyset)$.

Suppose that $X^{\star} \subset X$ together with $x \in X$ is an $n$-dimensional toroidal embedding of type $(\mathrm{N}, l, \sigma)$ as before. Then a $\partial$-étale finite morphism $\underline{Y} \rightarrow \underline{X}$ from a connected $\partial$-space is induced from its open part $Y^{\star} \rightarrow X^{\star}$, which is determined by a finite index subgroup $\mathrm{N}_{0}$ of $\mathrm{N} \simeq \pi_{1}\left(X^{\star}\right)$. Thus $\operatorname{sp}(\underline{Y})=Y$ for the toroidal embedding $Y^{\star} \subset Y$ associated with $\sigma \subset \mathrm{N}_{0} \otimes \mathbb{R}$ and $\underline{Y} \rightarrow \underline{X}$ is always Galois. The open immersion $Y^{\star} \subset Y$ together with the unique point $y$ lying over $x$ is a toroidal embedding of type $\left(\mathrm{N}_{0}, l, \sigma\right)$. The stalk $\mathcal{O}_{\underline{X}, x}$ is written as the inductive limit of $\mathcal{O}_{Y, y}$ for such $\partial$-étale neighborhood $(\underline{Y} ; y) \rightarrow(\underline{X} ; x)$. This is considered as the ring of Puiseux series with respect to monomials in $\sigma^{\vee} \cap \mathrm{M}_{\mathbb{Q}}$.

The sheaf $\hat{\Omega}_{\underline{X}}^{p}(\log B)$ of germs of logarithmic $p$-forms along $B$ is naturally defined as the sheafification of

$$
\underline{U} \longmapsto \lim _{[U, \Delta] \in \underline{U}} H^{0}\left(U, \hat{\Omega}_{U}^{p}(\log \Delta)\right)
$$

For a $\partial$-étale morphism $f:\left[U_{1}, \Delta_{1}\right] \rightarrow\left[U_{2}, \Delta_{2}\right]$ in which $U_{1} \backslash \Delta_{1} \subset U_{1}$ and $U_{2} \backslash$ $\Delta_{2} \subset U_{2}$ are toroidal embeddings, we have an isomorphism $f^{*} \hat{\Omega}_{U_{2}}^{p}\left(\log \Delta_{2}\right) \simeq$ $\hat{\Omega}_{U_{1}}^{p}\left(\log \Delta_{1}\right)$. Thus $\hat{\Omega}_{\underline{X}}^{p}(\log B) \simeq \varepsilon^{*} \hat{\Omega}_{X}^{p}(\log B)$ for the natural morphism $\varepsilon: \underline{X} \rightarrow$ $X$. In particular, this is a locally free $\mathcal{O}_{\underline{X}}$-module. We have a logarithmic de Rham complex

$$
\hat{\Omega}_{\underline{X}}^{\bullet}(\log B)=\left[\cdots \rightarrow \hat{\Omega}_{\underline{X}}^{p}(\log B) \stackrel{\mathrm{d}^{p}}{\rightarrow} \hat{\Omega}_{\underline{X}}^{p+1}(\log B) \rightarrow \cdots\right] .
$$

If $X^{\star} \subset X$ together with $x \in B$ be an $n$-dimensional toroidal embedding of type $(\mathrm{N}, l, \sigma)$, then $\hat{\Omega}_{\underline{X}}^{p}(\log B)_{x} \simeq \bigwedge^{p} \mathrm{M} \otimes \mathcal{O}_{\underline{X}, x}$. We infer that the natural morphism $\hat{\Omega}_{\underline{X}}^{\bullet}(\log B) \rightarrow R \underline{j}_{*} \Omega_{X^{\star}}^{\bullet} \sim_{\text {qis }} R \underline{j}_{*} \mathbb{C}_{X^{\star}}$ is quasi-isomorphic by considering similar quasi-isomorphisms over $Y=\operatorname{sp}(\underline{Y})$ for all $\partial$-étale neighborhoods $(\underline{Y} ; y) \rightarrow$ $(\underline{X} ; x)$. In particular, $R j_{*} \mathbb{C}_{\underline{X}} \rightarrow R \underline{j}_{*} \mathcal{O}_{X^{\star}}$ factors through $\mathcal{O}_{\underline{X}}$. 
We define the sheaf $\mathfrak{M}_{\underline{X}}$ of germs of meromorphic functions of $\underline{X}$ by

$$
\mathfrak{M}_{\underline{X}}(\underline{U})=\underset{[U, \Delta] \in \underline{U}}{\lim _{[}} H^{0}\left(U, \mathfrak{M}_{U}\right),
$$

for $\partial$-étale morphisms $\underline{U} \rightarrow \underline{X}$. Let $\mathfrak{M}_{\underline{X}}^{\star}$ be its subsheaf (as sets) consisting of invertible meromorphic functions, whose abelian group structure is derived from the multiplication. It contains the sheaf $\mathcal{O}_{\underline{X}}^{\star}$ of germs of invertible holomorphic functions as a subsheaf. We define the sheaf $\mathcal{D} i v_{\underline{X}}$ of germs of Cartier divisors of $\underline{X}$ to be $\mathfrak{M}_{\underline{X}}^{\star} / \mathcal{O}_{\underline{X}}^{\star}$ and define the sheaf $w \mathcal{D} i v_{\underline{X}}$ of germs of Weil divisors of $\underline{X}$ to be $\underline{j}_{*}^{\circ} \mathcal{D} i \underline{x}^{\circ}$. Let

$$
\mathcal{O}_{\underline{X}}(* B)^{\star}:=\mathfrak{M}_{\underline{X}}^{\star} \cap \underline{j}_{*} \mathcal{O}_{X^{\star}}^{\star} \subset \underline{j}_{*} \mathfrak{M}_{X^{\star}}
$$

be the sheaf of germs of meromorphic functions that are unit functions on $X^{\star}$. Then, by 3.2.3-(2), we have the triangle

$$
\cdots \stackrel{+1}{\longrightarrow} \tau_{\leq 1} R \underline{j}_{*} \mathbb{Z}_{X^{\star}} \rightarrow \mathcal{O}_{\underline{X}} \rightarrow \mathcal{O}_{\underline{X}}(* B)^{\star} \stackrel{+1}{\longrightarrow} \cdots
$$

Lemma 3.4.1. Let $\underline{X}=(X, B)$ for a toroidal embedding $j: X^{\star}=X \backslash$ $B \hookrightarrow X$.

(1) Let $x \in B$ be a point such that $\mathcal{U} \cap X^{\star} \subset \mathcal{U}$ is a toroidal embedding of type $(\mathrm{N}, l, \sigma)$ for an open neighborhood $\mathcal{U}$ of $x$. Then $\left(R^{p} \underline{j}_{*} \mathbb{Z}_{X^{\star}}\right)_{x} \simeq$ $\left(\varepsilon_{*} R^{p} \underline{j}_{*} \mathbb{Z}_{X^{\star}}\right)_{x} \simeq \bigwedge^{p} \mathrm{M} \otimes \mathbb{Q}$. In particular, $R \underline{\Gamma}_{B}\left(\mathbb{Z}_{\underline{X}}\right) \sim_{\text {qis }} R \underline{\Gamma}_{B}\left(\mathbb{Z}_{\underline{X}}\right) \stackrel{\mathrm{L}}{\otimes} \mathbb{Q}$ $\sim_{\text {qis }} R \underline{\Gamma}_{B}(\underline{\mathbb{Q}})$.

(2) $R^{p} \varepsilon_{*} \mathbb{Z}_{\underline{X}} \simeq \mathcal{H}_{B}^{p}\left(\mathbb{Z}_{X}\right) \otimes \mathbb{Q} / \mathbb{Z}$ for $p>0$. In particular, $R^{1} \varepsilon_{*} \mathbb{Z}_{\underline{X}}=0$.

(3) $R \underline{\Gamma}_{B}\left(\mathcal{O}_{\underline{X}}^{\star}\right) \sim_{\text {qis }} R \underline{\Gamma}_{B}\left(\mathcal{O}_{\underline{X}}\right) \oplus R \underline{\Gamma}_{B}\left(\mathbb{Z}_{\underline{X}}\right)[1]$.

(4) The composite $\mathcal{H}_{B}^{0}\left(\mathcal{D} i v_{\underline{X}}\right) \rightarrow \mathcal{H}_{B}^{1}\left(\mathcal{O}_{\underline{X}}^{\star}\right) \rightarrow \mathcal{H}_{B}^{2}\left(\mathbb{Z}_{\underline{X}}\right)$ is an isomorphism.

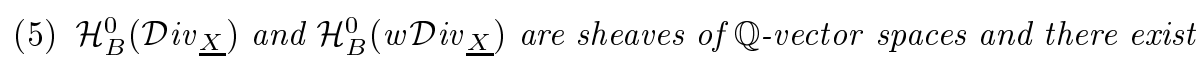
isomorphisms $\mathcal{H}_{B}^{0}\left(\mathcal{D} i v_{X}\right) \otimes \mathbb{Q} \simeq \varepsilon_{*} \mathcal{H}_{B}^{0}\left(\mathcal{D}_{i v_{X}}\right)$ and $\mathcal{H}_{B}^{0}\left(w \mathcal{D} i v_{X}\right) \otimes \mathbb{Q} \simeq$ $\varepsilon_{*} \mathcal{H}_{B}^{0}\left(w \mathcal{D} i v_{\underline{X}}\right)$.

(6) There exist injections $\varepsilon_{*} \mathcal{D}_{i v_{X}} \hookrightarrow \mathcal{D} i v_{X} \otimes \mathbb{Q}$ and $\varepsilon_{*} w \mathcal{D} i v_{\underline{X}} \hookrightarrow w \mathcal{D} i v_{X} \otimes$ $\mathbb{Q}$, and exact sequences

$$
\begin{aligned}
& 0 \rightarrow \mathcal{H}_{B}^{0}\left(\mathcal{D} i v_{\underline{X}}\right) \rightarrow\left(\mathcal{H}_{B}^{0}\left(\mathcal{D} i v_{X}\right) \otimes \mathbb{Q}\right) \oplus \mathcal{D} i v_{X} \rightarrow \varepsilon_{*} \mathcal{D} i v_{\underline{X}} \rightarrow 0 ; \\
& 0 \rightarrow \mathcal{H}_{B}^{0}\left(w \mathcal{D} i v_{\underline{X}}\right) \rightarrow\left(\mathcal{H}_{B}^{0}\left(w \mathcal{D} i v_{X}\right) \otimes \mathbb{Q}\right) \oplus w \mathcal{D} i v_{X} \rightarrow \varepsilon_{*} w \mathcal{D} i v_{\underline{X}} \rightarrow 0 \text {. }
\end{aligned}
$$


(7) $\mathcal{H}_{Z}^{2}\left(\mathbb{Z}_{\underline{X}}\right)=0$, the natural homomorphism $\mathcal{H}_{Z}^{p}\left(\mathbb{Z}_{\underline{X}}\right) \rightarrow \mathcal{H}_{B}^{p}\left(\mathbb{Z}_{\underline{X}}\right)$ is zero for any $p$, and there are isomorphisms

$$
\mathcal{H}_{B}^{0}\left(w \mathcal{D} i v_{\underline{X}}\right) / \mathcal{H}_{B}^{0}\left(\mathcal{D} i v_{\underline{X}}\right) \simeq w \mathcal{D} i v_{\underline{X}} / \mathcal{D} i v_{\underline{X}} \simeq \mathcal{H}_{Z}^{3}\left(\mathbb{Z}_{\underline{X}}\right) .
$$

In particular, $\varepsilon_{*}\left(w \mathcal{D} i v_{\underline{X}} / \mathcal{D}_{i v_{X}}\right) \simeq\left(w \mathcal{D}_{i v_{X}} / \mathcal{D} i v_{X}\right) \otimes \mathbb{Q}$.

(8) Let $\mu: Y \rightarrow X$ be a bimeromorphic morphism from a non-singular variety such that $\mu^{-1} B$ is a normal crossing divisor and that $\mu$ induces an isomorphism $Y \backslash \mu^{-1} B \rightarrow X \backslash B$. Then, for $\underline{\mu}: \underline{Y}=\left(Y, \mu^{-1} B\right) \rightarrow \underline{X}=(X, B)$, there is an exact sequence:

$$
0 \rightarrow \mathcal{H}_{B}^{0}\left(\mathcal{D} i v_{\underline{X}}\right) \rightarrow \underline{\mu}_{*} \mathcal{H}_{\mu^{-1} B}^{0}\left(\mathcal{D} i v_{\underline{Y}}\right) \rightarrow R^{2} \underline{\mu}_{*} \mathbb{Z}_{\underline{Y}} \rightarrow 0 .
$$

Proof. (1) By definition, the stalk $\left(R^{p} j_{*} \mathbb{Z}_{X^{\star}}\right)_{x}$ is the inductive limit of $\left(R^{p} j_{*}^{Y} \mathbb{Z}_{Y^{\star}}\right)_{y}$ for $\partial$-étale neighborhoods $(\underline{Y} ; y) \rightarrow(\underline{X} ; x)$, where $j^{Y}$ stands for the open immersion $Y^{\star} \hookrightarrow Y=\operatorname{sp}(\underline{Y})$. We may assume that $\underline{Y} \rightarrow \underline{X}$ is a finite $\partial$-étale covering corresponding to a subgroup $\mathrm{N}_{0} \subset \mathrm{N}$ of finite index. Thus the stalk is isomorphic to

$$
\underset{\mathrm{N}_{0} \subset \mathrm{N}}{\lim _{\longrightarrow}} \bigwedge^{p} \operatorname{Hom}\left(\mathrm{N}_{0}, \mathbb{Z}\right) \simeq \bigwedge^{p} \mathrm{M} \otimes \mathbb{Q} .
$$

We see that $\hat{\pi}_{1}^{\text {loc }}(\underline{X} ; x) \simeq \mathrm{N} \otimes \hat{\mathbb{Z}}$ for the profinite completion $\hat{\mathbb{Z}}$ of $\mathbb{Z}$. Since this is an abelian group, the action on the stalk is trivial. Therefore, $\left(R^{p} \underline{j}_{*} \mathbb{Z}_{X^{\star}}\right)_{x} \simeq$ $\left(\varepsilon_{*} R^{p} \underline{j}_{*} \mathbb{Z}_{X^{\star}}\right)_{x}$ by 2.2 .5 .

$(2)$ We have quasi-isomorphisms

$$
R \varepsilon_{*} R \underline{\Gamma}_{B}\left(\mathbb{Z}_{\underline{X}}\right) \sim_{\mathrm{qis}} R \varepsilon_{*} R \underline{\Gamma}_{B}\left(\underline{\mathbb{Q}_{X}}\right) \sim_{\mathrm{qis}} R \underline{\Gamma}_{B}\left(\mathbb{Q}_{X}\right)
$$

by (1) and by 2.2.6. The triangle

$$
\cdots \stackrel{+1}{\longrightarrow} R \underline{\Gamma}_{B}\left(\mathbb{Z}_{\underline{X}}\right) \rightarrow \mathbb{Z}_{\underline{X}} \rightarrow R \underline{j}_{*} \mathbb{Z}_{X^{\star}} \stackrel{+1}{\longrightarrow} \cdots
$$

induces an exact sequence

$$
\cdots \rightarrow R^{p-1} j_{*} \mathbb{Z}_{X^{\star}} \rightarrow \mathcal{H}_{B}^{p}\left(\mathbb{Q}_{X}\right) \rightarrow R^{p} \varepsilon_{*} \mathbb{Z}_{\underline{X}} \rightarrow R^{p} j_{*} \mathbb{Z}_{X^{\star}} \rightarrow \cdots
$$

By 3.3.1-(1), the stalk of $R^{q} j_{*} \mathbb{Z}_{X^{\star}} \simeq \mathcal{H}_{B}^{q+1}\left(\mathbb{Z}_{X}\right)$ for $q>0$ is of the form $\bigwedge^{q}$ M. Hence $R^{q} j_{*} \mathbb{Z}_{X^{*}} \rightarrow \mathcal{H}_{B}^{q+1}\left(\mathbb{Q}_{X}\right)$ are injective and we have $R^{p} \varepsilon_{*} \mathbb{Z}_{X} \simeq$ $\mathcal{H}_{B}^{p}\left(\mathbb{Z}_{X}\right) \otimes \mathbb{Q} / \mathbb{Z}$ for $p>0$.

(3) is derived from the factorization $R \underline{j}_{*} \mathbb{C}_{X^{\star}} \rightarrow \mathcal{O}_{\underline{X}} \rightarrow R \underline{j}_{*} \mathcal{O}_{X^{\star}}$ (cf. 3.2.2). 
(4) follows the same argument as 3.2.3-(3).

(5) is a consequence of (1) and (4).

(6) By applying $\varepsilon_{*}$ to the commutative diagram of exact sequence:

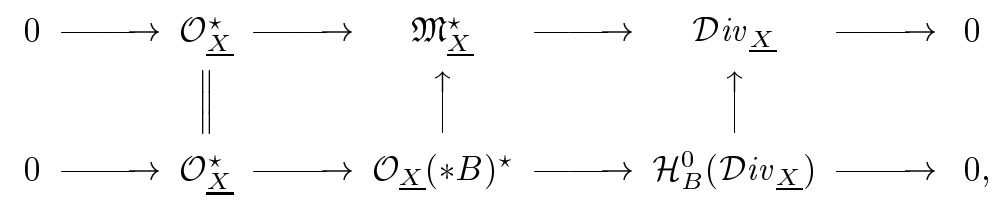

we have another commutative diagram of exact sequence:

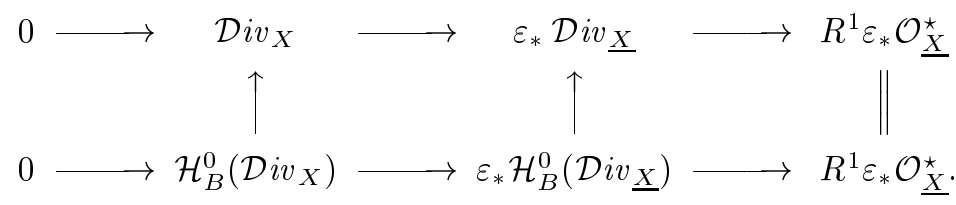

We have an isomorphism $R^{1} \varepsilon_{*} \mathcal{O}_{\underline{X}}^{\star} \simeq R^{2} \varepsilon_{*} \mathbb{Z}_{\underline{X}} \simeq \mathcal{H}_{B}^{2}\left(\mathbb{Z}_{X}\right) \otimes \mathbb{Q} / \mathbb{Z}$ by (2). Thus the right arrows of top and bottom sequences in (3.6) are both surjective. Hence we have the expected exact sequence for $\mathcal{D} i v_{\underline{X}}$ by (1) and (2). In order to show the sequence for $w \mathcal{D} i v_{\underline{X}}$ is derived from that on $X^{\circ}$, it is enough to prove that

$$
R^{1} j_{*}^{\circ} \mathcal{H}_{B^{\star}}^{2}\left(\mathbb{Z}_{X^{\circ}}\right) \rightarrow R^{1} j_{*}^{\circ} \mathcal{H}_{B^{\star}}^{2}\left(\mathbb{Q}_{X^{\circ}}\right)
$$

is injective. Since $\mathcal{H}_{B^{\star}}^{2}\left(\mathbb{Z}_{X^{\circ}}\right)$ is isomorphic to the constant sheaf $\mathbb{Z}_{B^{\star}}$, the homomorphism isomorphic to the injection $R^{1} j_{*}^{\circ} \mathbb{Z}_{B^{\star}} \rightarrow R^{1} j_{*}^{\circ} \mathbb{Q}_{B^{\star}}$ (cf. 3.3.2).

(7) $\mathcal{H}_{B}^{p}\left(\mathbb{Z}_{\underline{X}}\right) \rightarrow \mathcal{H}_{B}^{p}\left(R \underline{j}_{*}^{\circ} \mathbb{Z}_{\underline{X}^{\circ}}\right)$ is injective for any $p$ by 3.3.1-(2). Thus $\mathcal{H}_{Z}^{p}\left(\mathbb{Z}_{\underline{X}}\right) \rightarrow \mathcal{H}_{B}^{p}\left(\mathbb{Z}_{X}\right)$ is zero. The triangle

$$
\cdots \stackrel{+1}{\rightarrow} R \underline{\Gamma}_{Z}\left(\mathbb{Z}_{\underline{X}}\right) \rightarrow R \underline{\Gamma}_{B}\left(\mathbb{Z}_{\underline{X}}\right) \rightarrow R \underline{j}_{*}^{\circ} R \underline{\Gamma}_{B^{\star}}\left(\mathbb{Z}_{\underline{X}^{\circ}}\right) \stackrel{+1}{\longrightarrow} \cdots
$$

induces an exact sequence

$$
0 \rightarrow \mathcal{H}_{B}^{p}\left(\mathbb{Z}_{\underline{X}}\right) \rightarrow R^{p-2} \underline{j}_{*}^{\circ} \mathcal{H}_{B^{\star}}^{2}\left(\mathbb{Z}_{\underline{X}^{\circ}}\right) \rightarrow \mathcal{H}_{Z}^{p+1}\left(\mathbb{Z}_{\underline{X}}\right) \rightarrow 0 .
$$

Hence $\mathcal{H}_{Z}^{2}\left(\mathbb{Z}_{\underline{X}}\right)=0$ and $\mathcal{H}_{Z}^{1}\left(\mathcal{H}_{B}^{2}\left(\mathbb{Z}_{\underline{X}}\right)\right) \simeq \mathcal{H}_{Z}^{3}\left(\mathbb{Z}_{\underline{X}}\right)$.

(8) is derived by the same argument as 3.3.1-(5).

The group $\operatorname{Div}(X, \mathbb{Q})$ of $\mathbb{Q}$-Cartier divisors and the group $\operatorname{WDiv}(X, \mathbb{Q})$ of $\mathbb{Q}$-Weil divisors is defined to be $H^{0}\left(X, \mathcal{D} i v_{X} \otimes \mathbb{Q}\right)$ and $H^{0}\left(X, w \mathcal{D} i v_{X} \otimes \mathbb{Q}\right)$, respectively. Note that $\operatorname{Div}(X) \otimes \mathbb{Q} \nsucceq \operatorname{Div}(X, \mathbb{Q})$ for some non-compact analytic space $X$. We define similarly $\operatorname{Div}_{B}(X, \mathbb{Q}):=H_{B}^{0}\left(X, \mathcal{D} i v_{X} \otimes \mathbb{Q}\right)$ and $\operatorname{WDiv}_{B}(X, \mathbb{Q}):=H_{B}^{0}\left(X, w \mathcal{D} i v_{X} \otimes \mathbb{Q}\right)$. For the $\partial$-space, we also define the 
group $\operatorname{Div}(\underline{X})$ of Cartier divisors of $\underline{X}$ and the group $\operatorname{WDiv}(\underline{X})$ of Weil divisors of $\underline{X}$ by $\operatorname{Div}(\underline{X}):=H^{0}\left(\underline{X}, \mathcal{D}_{i v_{X}}\right)$ and $\operatorname{WDiv}(\underline{X}):=H^{0}\left(\underline{X}, w \mathcal{D} i v_{\underline{X}}\right)$. Similarly, we define $\operatorname{Div}_{B}(\underline{X}):=H_{B}^{0}\left(\underline{X}, \mathcal{D}_{i v_{\underline{X}}}\right)$ and $w \mathcal{D} i v_{B}(\underline{X}):=H_{B}^{0}\left(\underline{X}, w \mathcal{D} i v_{\underline{X}}\right)$. Then by 3.4.1, $H_{B}^{2}(\underline{X}, \mathbb{Z}) \simeq H^{0}\left(\underline{X}, \mathcal{H}_{B}^{2}\left(\mathbb{Z}_{\underline{X}}\right)\right) \simeq \operatorname{Div}_{B}(\underline{X}) \simeq \operatorname{Div}_{B}(X, \mathbb{Q})$ and $\operatorname{WDiv}_{B}(\underline{X}) \simeq \operatorname{WDiv}_{B}(X, \mathbb{Q})$. Moreover, 3.4.1-(6) implies that $\operatorname{WDiv}(\underline{X})$ can be identified with the group consisting of locally finite sums $\sum q_{i} \Gamma_{i}$ for prime divisors $\Gamma_{i}$ and for rational numbers $q_{i}$ such that $q_{i} \in \mathbb{Z}$ if $\Gamma_{i} \not \subset B$. The $\operatorname{sum} \sum q_{i} \Gamma_{i}$ in $\operatorname{WDiv}(\underline{X})$ is contained in $\operatorname{Div}(\underline{X})$ if and only if it is (locally) a $\mathbb{Q}$-Cartier divisor, i.e., $\operatorname{Div}(\underline{X})=\operatorname{Div}(X, \mathbb{Q}) \cap \operatorname{WDiv}(\underline{X}) \subset \operatorname{WDiv}(X, \mathbb{Q})$.

Theorem 3.4.2. $\quad$ Let $X^{\star}=X \backslash B \subset X$ be a toroidal embedding. Then the following two sequences are exact:

$$
\cdots \rightarrow H^{p-1}(\underline{X}, \mathbb{Z}) \rightarrow H_{B}^{p}(X, \mathbb{Z}) \rightarrow H_{B}^{p}(X, \mathbb{Q}) \oplus H^{p}(X, \mathbb{Z}) \rightarrow H^{p}(\underline{X}, \mathbb{Z}) \rightarrow \cdots
$$

$$
\cdots \rightarrow H^{p-1}\left(X^{\star}, \mathbb{Q}\right) \rightarrow H^{p}(\underline{X}, \mathbb{Z}) \rightarrow H^{p}(X, \mathbb{Q}) \oplus H^{p}\left(X^{\star}, \mathbb{Z}\right) \rightarrow H^{p}\left(X^{\star}, \mathbb{Q}\right) \rightarrow \cdots
$$

Moreover, we have an isomorphism $H^{1}(\underline{X}, \mathbb{Z}) \simeq H^{1}(X, \mathbb{Z})$ and an exact sequence:

$0 \rightarrow H_{B}^{2}(X, \mathbb{Z}) \rightarrow H^{2}(X, \mathbb{Z}) \oplus H_{B}^{2}(X, \mathbb{Q}) \rightarrow H^{2}(\underline{X}, \mathbb{Z}) \rightarrow H_{B}^{3}(X, \mathbb{Z}) \rightarrow H_{B}^{3}(X, \mathbb{Q})$.

If $[X, B]$ satisfies the Condition 3.2.6, then

$$
H^{2}(\underline{X}, \mathbb{Z}) \simeq\left(H^{2}(X, \mathbb{Z}) \oplus H_{B}^{2}(X, \mathbb{Q})\right) / H_{B}^{2}(X, \mathbb{Z}) .
$$

Proof. We have the quasi-isomorphism $R \varepsilon_{*} R \underline{\Gamma}_{B}\left(\mathbb{Z}_{\underline{X}}\right) \sim_{\text {qis }} R \underline{\Gamma}_{B}\left(\mathbb{Q}_{X}\right)$ by 3.4.1-(1). From the commutative diagram

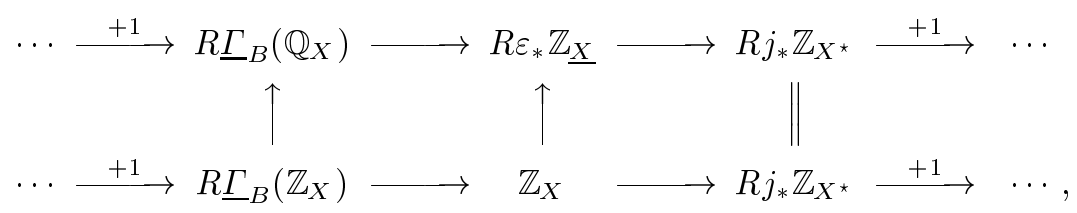

we have another triangle

$$
\cdots \stackrel{+1}{\rightarrow} R \underline{\Gamma}_{B}\left(\mathbb{Z}_{X}\right) \rightarrow R \underline{\Gamma}_{B}\left(\mathbb{Q}_{X}\right) \oplus \mathbb{Z}_{X} \rightarrow R \varepsilon_{*} \mathbb{Z}_{\underline{X}} \stackrel{+1}{\rightarrow} \cdots
$$

This induces the long exact sequence (3.7). Similarly, from the commutative diagram

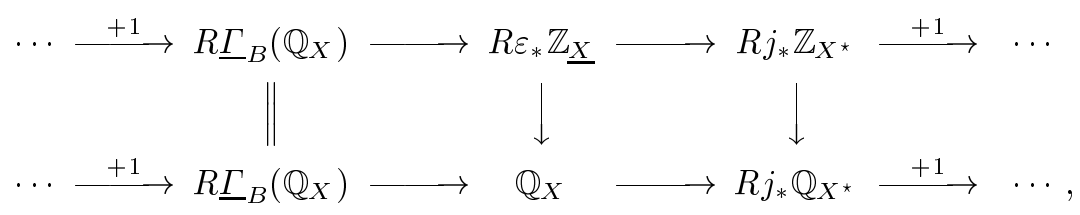


we have another triangle

$$
\cdots \stackrel{+1}{\longrightarrow} R \varepsilon_{*} \mathbb{Z}_{\underline{X}} \rightarrow R j_{*} \mathbb{Z}_{X^{\star}} \rightarrow R j_{*} \mathbb{Q}_{X^{\star}} \stackrel{+1}{\longrightarrow} \cdots
$$

and the long exact sequence (3.8).

The isomorphism for $H^{1}$ is derived from the vanishing $R^{1} \varepsilon_{*} \mathbb{Z}_{\underline{X}}=0$ by 3.4.1-(2) and from Leray's spectral sequence for $\varepsilon_{*}$.

For $H^{2}$, we look at the following exact sequence appearing in (3.7):

$$
H_{B}^{2}(X, \mathbb{Z}) \rightarrow H_{B}^{2}(X, \mathbb{Q}) \oplus H^{2}(X, \mathbb{Z}) \rightarrow H^{2}(\underline{X}, \mathbb{Z}) \rightarrow H_{B}^{3}(X, \mathbb{Z}) .
$$

The left homomorphism is injective by 3.3.1-(6). If $[X, B]$ satisfies the Condition 3.2.6, then the right homomorphism is surjective also by 3.3.1-(6).

Next, we shall study the Picard group $\operatorname{Pic}(\underline{X}):=H^{1}\left(\underline{X}, \mathcal{O}_{\underline{X}}^{\star}\right)$.

Proposition 3.4.3. Suppose that $[X, B]$ satisfies the Condition 3.2.6. Then we have the following two isomorphisms:

$$
\begin{aligned}
\operatorname{Pic}(\underline{X}) & \simeq\left(\operatorname{Div}_{B}(X, \mathbb{Q}) \oplus \operatorname{Pic}(X)\right) / \operatorname{Div}_{B}(X) ; \\
\operatorname{Div}(\underline{X}) & \simeq\left(\operatorname{Div}_{B}(X, \mathbb{Q}) \oplus \operatorname{Div}(X)\right) / \operatorname{Div}_{B}(X) .
\end{aligned}
$$

Proof. From the quasi-isomorphisms

$$
R \varepsilon_{*} R \underline{\Gamma}_{B}\left(\mathcal{O}_{\underline{X}}^{\star}\right) \sim_{\text {qis }} R \underline{\Gamma}_{B}\left(\mathcal{O}_{X}^{\star}\right) \stackrel{\mathrm{L}}{\otimes} \mathbb{Q} \sim_{\mathrm{qis}} R \underline{\Gamma}_{B}\left(\mathcal{O}_{X}^{\star} \otimes \mathbb{Q}\right)
$$

by 3.4.1, we have the following commutative diagram of triangles:

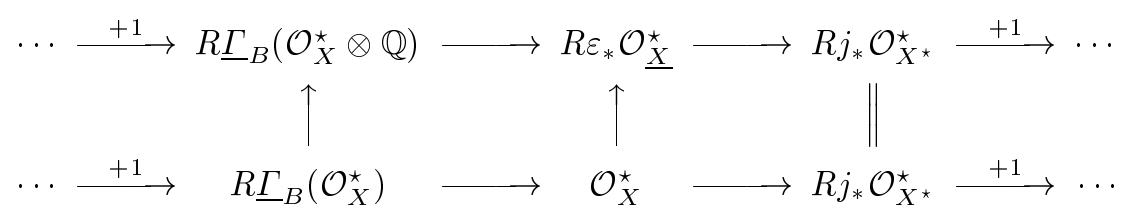

This induces another triangle

$$
\cdots \stackrel{+1}{\longrightarrow} R \underline{\Gamma}_{B}\left(\mathcal{O}_{X}^{\star}\right) \rightarrow R \underline{\Gamma}_{B}\left(\mathcal{O}_{X}^{\star} \otimes \mathbb{Q}\right) \oplus \mathcal{O}_{X}^{\star} \rightarrow R \varepsilon_{*} \mathcal{O}_{\underline{X}}^{\star} \stackrel{+1}{\longrightarrow} \cdots
$$

and the associated long exact sequence

$\cdots \rightarrow H_{B}^{1}\left(X, \mathcal{O}_{X}^{\star}\right) \rightarrow H_{B}^{1}\left(X, \mathcal{O}_{X}^{\star} \otimes \mathbb{Q}\right) \oplus \operatorname{Pic}(X) \rightarrow \operatorname{Pic}(\underline{X}) \rightarrow H_{B}^{2}\left(X, \mathcal{O}_{X}^{\star}\right) \rightarrow \cdots$

By 3.2.4, we have

$$
\begin{aligned}
H_{B}^{p}\left(X, \mathcal{O}_{X}^{\star}\right) & \simeq H_{B}^{p}\left(X, \mathcal{O}_{X}\right) \oplus H_{B}^{p+1}(X, \mathbb{Z}), \\
H_{B}^{p}\left(X, \mathcal{O}_{X}^{\star} \otimes \mathbb{Q}\right) & \simeq H_{B}^{p}\left(X, \mathcal{O}_{X}\right) \oplus H_{B}^{p+1}(X, \mathbb{Q}),
\end{aligned}
$$


for any $p$. The homomorphism $H_{B}^{p}(X, \mathbb{Z}) \rightarrow H_{B}^{p}(X, \mathbb{Q})$ is injective for $p \leq 3$ by 3.3.1-(6). Hence $H_{B}^{p}\left(X, \mathcal{O}_{X}^{\star}\right) \rightarrow H_{B}^{p}\left(X, \mathcal{O}_{X}^{\star} \otimes \mathbb{Q}\right)$ is injective for $p \leq 2$. Thus (3.9) follows.

The isomorphism (3.10) for $\operatorname{Div}(\underline{X})$ is derived from the exact sequence (3.4) in 3.4.1 and the injectiveness of $H^{1}\left(X, \mathcal{H}_{B}^{0}\left(\mathcal{D} i v_{X}\right)\right) \rightarrow H^{1}\left(X, \mathcal{H}_{B}^{0}\left(\mathcal{D} i v_{X}\right) \otimes \mathbb{Q}\right)$ proved in 3.3.1-(6).

Let $\mathbb{Z}_{\underline{X}}^{\bullet}=\tau_{\leq 2} R \underline{j}_{*}^{\circ} \mathbb{Z}_{\underline{X}}$ and let $\mathcal{O}_{\underline{X}}^{\bullet}$ be the mapping cone of the composite $\mathbb{Z}_{\underline{X}}^{\bullet} \rightarrow R \underline{j}_{*} \mathbb{C}_{X^{\star}} \rightarrow \mathcal{O}_{\underline{X}}$. Let WPic $(\underline{X})$ be the group of isomorphism classes of reflexive sheaves of rank one on $\underline{X}$. Then we have a triangle

$$
\cdots \stackrel{+1}{\longrightarrow} \mathcal{H}_{B}^{0}\left(\mathcal{D} i v_{\underline{X}}\right)[-1] \rightarrow \mathcal{H}_{B}^{0}\left(w \mathcal{D} i v_{\underline{X}}\right)[-1] \oplus \mathcal{O}_{\underline{X}}^{\star} \rightarrow \mathcal{O}_{\underline{X}}^{\bullet} \stackrel{+1}{\longrightarrow} \cdots
$$

and an isomorphism $\operatorname{WPic}(\underline{X}) \simeq H^{1}\left(\underline{X}, \mathcal{O}_{\underline{X}}^{\bullet}\right)$ as in 3.3.6. We can generalize 3.4.3 as follows:

\section{Theorem 3.4.4.}

$$
\begin{aligned}
& \operatorname{WDiv}(\underline{X}) \simeq\left(\operatorname{WDiv}_{B}(X, \mathbb{Q}) \oplus \operatorname{WDiv}(X)\right) / \operatorname{WDiv}_{B}(X) \\
& \operatorname{WPic}(\underline{X}) \simeq\left(\operatorname{WDiv}_{B}(X, \mathbb{Q}) \oplus \operatorname{WPic}(X)\right) / \operatorname{WDiv}_{B}(X) .
\end{aligned}
$$

Proof. (3.11) follows 3.4.3 for $X^{\circ}$. By comparing $\mathcal{O}_{X}^{\bullet}$ and $\mathcal{O}_{X}^{\bullet}$, we see that the mapping cone of $\mathcal{O}_{X}^{\bullet} \rightarrow \tau_{\leq 1} R \varepsilon_{*} \mathcal{O}_{\underline{X}}^{\bullet}$ is quasi-isomorphic to $\mathcal{H}_{B}^{0}\left(w \mathcal{D} i v_{X}\right) \otimes$ $\mathbb{Q} / \mathbb{Z}[-1]$. Thus we have a triangle

$$
\cdots \stackrel{+1}{\longrightarrow} \mathcal{H}_{B}^{0}\left(w \mathcal{D} i v_{X}\right)[-1] \rightarrow \mathcal{H}_{B}^{0}\left(w \mathcal{D} i v_{X}\right) \otimes \mathbb{Q}[-1] \oplus \mathcal{O}_{X}^{\bullet} \rightarrow \tau_{\leq 1} R \varepsilon_{*} \mathcal{O}_{\underline{X}}^{\bullet} \stackrel{+1}{\longrightarrow} \cdots
$$

This induces a commutative diagram of exact sequences:

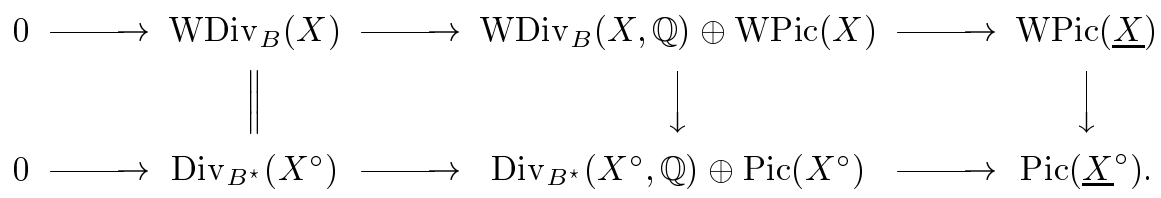

The bottom right arrow is surjective by 3.4.3. The cokernels of the middle and right vertical arrows are both contained in $H^{0}\left(X, R^{1} j_{*}^{\circ} \mathcal{O}_{X^{\circ}}\right)$, since the mapping cone of $\mathcal{O}_{\underline{X}}^{\circ} \rightarrow \tau_{\leq 1} R \underline{j}_{*}^{\circ} \mathcal{O}_{\underline{X}}^{\star}$ is quasi-isomorphic to $R^{1} \underline{j}_{*}^{\circ} \mathcal{O}_{\underline{X}}^{\circ}[-1]$. Hence $\operatorname{WDiv}_{B}(X, \mathbb{Q}) \oplus \underline{\mathrm{WPic}}(X) \rightarrow \mathrm{WPic}(\underline{X})$ is surjective.

Let $\mathrm{V}_{B}(X)$ and $\mathrm{V}_{B}(\underline{X})$, respectively, be the images of the natural homo-

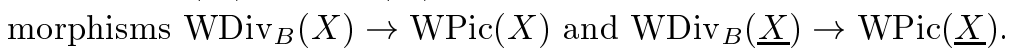


Corollary 3.4.5. Let $\mu: Y \rightarrow X$ be a bimeromorphic morphism from a non-singular variety such that $\mu$ is isomorphic outside $B$ and that $\mu^{-1} B$ is a normal crossing divisor. We denote $\underline{Y}=\left(Y, \mu^{-1} B\right)$. Then there exist canonical isomorphisms

$$
\operatorname{WPic}(X) / \mathrm{V}_{B}(X) \simeq \operatorname{WPic}(\underline{X}) / \mathrm{V}_{B}(\underline{X}) \simeq \operatorname{Pic}(\underline{Y}) / \mathrm{V}_{\mu^{-1} B}(\underline{Y}) .
$$

In particular, if $X$ has only quotient singularities, then

$$
\operatorname{Pic}(\underline{X}) / \mathrm{V}_{B}(\underline{X}) \simeq \operatorname{Pic}(\underline{Y}) / \mathrm{V}_{\mu^{-1} B}(\underline{Y}) .
$$

Proof. By 3.4.4, we have isomorphisms $\operatorname{WPic}(\underline{X}) / \mathrm{V}_{B}(\underline{X}) \simeq \operatorname{WPic}(X) /$ $\mathrm{V}_{B}(X)$ and $\operatorname{Pic}(\underline{Y}) / \mathrm{V}_{\mu^{-1} B}(\underline{Y}) \simeq \operatorname{Pic}(Y) / \mathrm{V}_{\mu^{-1} B}(Y)$. The surjective homomorphism $\mu_{*}: \operatorname{Pic}(Y) \rightarrow \operatorname{WPic}(X)$ induces an isomorphism $\operatorname{Pic}(Y) / \mathrm{V}_{\mu^{-1} B}(Y) \rightarrow$ $\mathrm{WPic}(X) / \mathrm{V}_{B}(X)$. If $X$ has only quotient singularities, then $\operatorname{Pic}(\underline{X}) \simeq$ $\operatorname{WPic}(\underline{X})$.

\section{$\S 3.5$. Reflexive sheaf and parabolic structure}

The notion of parabolic sheaf is introduced by Mehta and Seshadri [M2] on Riemann surfaces and is generalized to higher dimensions by Maruyama and Yokogawa [M1]. In [B2], Biswas shows that a lot of parabolic sheaves are considered as orbifold sheaves. Here, we consider this from the view point of $\partial$-étale topology.

Let $X$ be a normal variety and let $B$ be a reduced Weil divisor of $X$. We assume that, locally on $X, B$ is the support of an effective Cartier divisor. This condition is satisfied if $X \backslash B \hookrightarrow X$ is a toroidal embedding, for example. Let $\underline{X}$ denote the $\partial$-space $(X, B)$ and let $\varepsilon=\varepsilon_{X}$ be the natural morphism $\underline{X} \rightarrow X$.

Lemma. There is an inclusion $\operatorname{WDiv}_{B}(X, \mathbb{Q}) \subset \operatorname{WDiv}(\underline{X})$.

Proof. We can replace $X$ by an open neighborhood of any point. Then by the assumption, there is an effective divisor $D$ such that $\operatorname{Supp} D=B$ and that $\mathcal{O}_{X} \simeq \mathcal{O}_{X}(D)$. In this situation, for any positive integer $m$, we can construct a cyclic covering $\tau: Y \rightarrow X$ of degree $m$ branched only over $B$ such that $\tau^{*} D$ is divisible by $m$. This implies that $\mathcal{O}_{\underline{X}}(q D)$ is an invertible $\mathcal{O}_{\underline{X}}$-module for any $q \in \mathbb{Q}$. If $\Delta$ is a $\mathbb{Q}$-Weil divisor supported in $B$, then $\tau^{*} \Delta$ is a $\mathbb{Z}$-Weil divisor for a finite covering $\tau: Y \rightarrow X$ branched only over $B$. Thus $\Delta$ is a $\mathbb{Z}$-Weil divisor of $\underline{X}$.

A reflexive sheaf of $\underline{X}$ is a coherent reflexive $\mathcal{O}_{\underline{X}}$-module by definition. 
Lemma 3.5.1. Let $\mathcal{F}$ be a coherent $\mathcal{O}_{\underline{X}}$-module. Then $\mathcal{F}$ is reflexive if and only if, d-étale locally, $\mathcal{F} \simeq \varepsilon^{*} F$ for a reflexive $\mathcal{O}_{X}$-module $F$.

Proof. By 2.3.2, we may assume $\mathcal{F} \simeq \varepsilon^{*} F_{0}$ and $\mathcal{F}^{\vee}:=\mathcal{H o m}_{\mathcal{O}_{\underline{X}}}\left(\mathcal{F}, \mathcal{O}_{\underline{X}}\right) \simeq$ $\varepsilon^{*} F_{1}$ for coherent sheaves $F_{0}$ and $F_{1}$ of $X$. Moreover, we can assume that there is an exact sequence $\mathcal{O}_{X}^{\oplus s} \rightarrow \mathcal{O}_{X}^{\oplus r} \rightarrow F_{0} \rightarrow 0$. Thus the sequence $\mathcal{O}_{\underline{X}}^{\oplus s} \leftarrow$ $\mathcal{O}_{\underline{X}}^{\oplus r} \leftarrow \mathcal{F}^{\vee} \leftarrow 0$ is also exact. Hence $\varepsilon_{*} \mathcal{F}^{\vee} \simeq F_{0}^{\vee}$. Therefore, from the first, we may assume that $F_{1}=F_{0}^{\vee}$. By considering the same thing to $F_{1}$, we see that $\mathcal{F}$ is reflexive if and only if $F_{0}$ is so.

Corollary. The following properties hold for a reflexive sheaf $\mathcal{F}$ on $\underline{X}$ :

(1) $\varepsilon_{U *} \mathcal{F}$ is reflexive for a $\partial$-étale morphism $\underline{U} \rightarrow \underline{X}$ with $U=\operatorname{sp}(\underline{U})$.

(2) $\left.\mathcal{F} \simeq \underline{j}_{*}^{\circ} \mathcal{F}\right|_{\underline{X}^{\circ}}$.

The dual $\mathcal{G}^{\vee}=\mathcal{H}^{\circ} m_{\mathcal{O}_{\underline{X}}}\left(\mathcal{G}, \mathcal{O}_{\underline{X}}\right)$ of any coherent sheaf $\mathcal{G}$ is reflexive.

Let $\Delta$ be a $\mathbb{Q}$-Weil divisor contained in $\operatorname{WDiv}(\underline{X})=\operatorname{WDiv}(X)+\operatorname{WDiv}_{B}(X$, $\mathbb{Q})$. Then the sheaf $\mathcal{O}_{\underline{X}}(\Delta)$ is a reflexive sheaf of rank one. For a reflexive sheaf $\mathcal{F}$ of $\underline{X}$, we define $\mathcal{F}(\Delta)$ to be the double-dual of $\mathcal{F} \otimes \mathcal{O}_{\underline{X}}(\Delta)$. Similarly, for a reflexive sheaf $F$ and an effective divisor $D$ of $X$, we define $F(D)$ to be the double-dual of $F \otimes \mathcal{O}_{X}(D)$.

We consider parabolic sheaves in the following sense:

Definition 3.5.2. $\quad$ Let $F$ be a reflexive sheaf of $X$ and let $D$ be an effective Weil divisor supported in $B$. A parabolic structure of $F$ with respect to $D$ is a family $\left\{F_{t}\right\}$ of subsheaves of $j_{*}\left(\left.F\right|_{X^{\star}}\right)$ indexed by $t \in \mathbb{Q}$ satisfying the following conditions:

(1) $F_{t}$ are reflexive sheaves of $X$;

(2) $F_{t_{1}} \subset F_{t_{2}}$ for $t_{1}>t_{2}$;

(3) $F=F_{0}$;

(4) $F_{t+m}=F_{t}(-m D)$ for $m \in \mathbb{Z}$;

(5) Any point of $X$ has an open neighborhood $V$ such that, for any $t \in \mathbb{Q}$, there is a rational number $\delta>0$ satisfying $\left.F_{t-\delta}\right|_{V}=\left.F_{t}\right|_{V}$.

A reflexive sheaf endowed with a parabolic structure with respect to $B$ is simply called a parabolic sheaf. 
Remark. Let $F^{\prime} \subset F$ be an injection between reflexive sheaves on $X$ such that the support of $Q:=F / F^{\prime}$ is contained in $B$. Then, for a point $x \in \operatorname{Supp} Q$, an associated prime of the $\mathcal{O}_{X, x}$-module $Q_{x}$ corresponds to an irreducible component of the germ of $B$ at $x$. In particular, if $\operatorname{Supp} Q$ contains no irreducible components of $B$, then $F=F^{\prime}$.

Suppose that $B$ has only finitely many irreducible components and let $\left\{F_{t}\right\}$ be a parabolic structure with respect to an effective divisor $D$ supported in $B$. Then there exist a filtration of coherent subsheaves

$$
F_{0} / F_{1}=G_{0} \supset G_{1} \supset G_{2} \supset \cdots \supset G_{l} \supset G_{l+1}=0,
$$

and a set of rational numbers

$$
0 \leq \alpha_{0}<\alpha_{1}<\alpha_{2}<\cdots<\alpha_{l}<\alpha_{l+1}=1
$$

such that $F_{t} / F_{1}=G_{j}$ for $\alpha_{j-1}<t \leq \alpha_{j}$ and for $j \geq 1$ and that $F_{t} / F_{1}=G_{0}$ for $0 \leq t \leq \alpha_{0}$. We call $\left\{F_{t}\right\}$ is m-periodic if $\alpha_{i} \in(1 / m) \mathbb{Z}$. The condition is equivalent to: $F_{t}=F_{\ulcorner m t / m}$.

Lemma 3.5.3. Let $\mathcal{F}$ be a reflexive sheaf of $\underline{X}$ and let $D$ be an effective Weil divisor of $X$ supported in $B$. We set

$$
F_{t}:=\varepsilon_{*}(\mathcal{F}(-t D))
$$

for $t \in \mathbb{Q}$. Then $\left\{F_{t}\right\}$ is a parabolic structure with respect to $D$.

Proof. It suffices to show that, for any $t$, locally on $X$, there is rational number $\delta$ with $F_{t-\delta}=F_{t}$. For a point $x$ of $X$, there are an open neighborhood $V$, a finite Galois morphism $f: U \rightarrow V$ étale outside $B$, and a reflexive sheaf $F_{U}$ of $U$ such that $\left.\mathcal{F}\right|_{\underline{U}} \simeq \varepsilon_{\underline{U}}^{*} F_{U}$ for $\underline{U}=\left(U, B_{U}\right), B_{U}=f^{-1} B$, by 3.5.1. Let $F_{U, t}$ be the sheaf $\varepsilon_{U *} \mathcal{F}(t B)$, which admits a natural $G$-linearization for the Galois group $G$ of $f$. Then $F_{U, t}=F_{U}\left(\iota_{\llcorner}-t f^{*} D_{\lrcorner}\right)$, and $F_{t}$ is the $G$-invariant part of $f_{*} F_{U, t}$. Thus $F_{t} \simeq F_{t-\delta}$ for small $\delta>0$.

Lemma 3.5.4. Let $\left\{F_{t}\right\}$ be a parabolic structure of a reflexive sheaf $F_{0}$ of rank one with respect to $B$. Then there exists a reflexive sheaf $\mathcal{F} \in \operatorname{WPic}(\underline{X})$ of rank one uniquely up to isomorphisms such that $F_{t} \simeq \varepsilon_{*}(\mathcal{F}(-t B))$.

Proof. For an irreducible component $B_{i}$ of $B$, there is uniquely a rational number $0 \leq \beta_{i}<1$ such that $F_{\beta_{i}}=F_{0}$ and that the support of $F_{0} / F_{t}$ contains $B_{i}$ for $t>\beta_{i}$. Let $\Delta:=\sum_{i} \beta_{i} B_{i}$ and set $F_{t}^{\prime}:=F_{t}\left(-{ }_{\llcorner} \Delta-t B_{\lrcorner}\right)$. Then we have $F_{t}^{\prime}=F_{0}^{\prime}$ for any $t$. Hence $F_{t}=F_{0}^{\prime}\left({ }_{\llcorner} \Delta-t B_{\lrcorner}\right)$. The double-dual $\mathcal{F}$ of $\varepsilon^{*} F_{0}^{\prime} \otimes \mathcal{O}_{\underline{X}}(\Delta)$ satisfies the condition. 
Theorem 3.5.5. Let $\left\{F_{t}\right\}$ be a parabolic structure with respect to $B$. Then there exists a reflexive sheaf $\mathcal{F}$ of $\underline{X}$ uniquely up to isomorphisms such that $F_{t} \simeq \varepsilon_{*}(\mathcal{F}(-t B))$.

Proof. We consider the double-dual $\mathcal{F}_{t}^{\prime}$ of $\varepsilon^{*} F_{t}$ and

$$
\mathcal{F}_{t}^{\prime \prime}:=\sum_{0 \leq \lambda \leq 1} \mathcal{F}_{t-\lambda}^{\prime}(-\lambda B) \subset \mathcal{F}_{t}^{\prime}(B)
$$

Then we have $\mathcal{F}_{t+1}^{\prime \prime}=\mathcal{F}_{t}^{\prime \prime}(-B)$. For $0 \leq t \leq 1$,

$$
\begin{aligned}
\mathcal{F}_{t}^{\prime \prime} & =\sum_{-t \leq \mu \leq 0} \mathcal{F}_{-\mu}^{\prime}(-(t+\mu) B)+\sum_{0 \leq \mu \leq 1-t} \mathcal{F}_{-\mu}^{\prime}(-(t+\mu) B) \\
& =\sum_{0 \leq \mu \leq 1} \mathcal{F}_{-\mu}^{\prime}(-(t+\mu) B)=\mathcal{F}_{0}^{\prime \prime}(-t B) .
\end{aligned}
$$

If $\left\{F_{t}\right\}$ is $m$-periodic, then $\mathcal{F}_{t}^{\prime}=\mathcal{F}_{\ulcorner m t / m}^{\prime}$. Thus

$$
\mathcal{F}_{0}^{\prime \prime}=\sum_{s=0}^{m-1} \sum_{s / m \leq \lambda<(s+1) / m} \mathcal{F}_{\ulcorner-m \lambda\urcorner / m}^{\prime}(-\lambda B)=\sum_{s=0}^{m-1} \mathcal{F}_{-s / m}^{\prime}(-(s / m) B) .
$$

Hence $\mathcal{F}_{0}^{\prime \prime}$ is coherent. Let $\mathcal{F}$ be the double-dual of $\mathcal{F}_{0}^{\prime \prime}$. Then $\mathcal{F}(-t B)$ is the double-dual of $\mathcal{F}_{t}^{\prime \prime}$. The direct image $\varepsilon_{*} \mathcal{F}(-t B)$ is isomorphic to the doubledual of

$$
\sum_{0 \leq \lambda \leq 1} F_{t-\lambda}(\llcorner-\lambda\lrcorner B)
$$

If $0<\lambda \leq 1$, then $\left.F_{t-\lambda}\left({ }_{\llcorner}-\lambda\right\lrcorner B\right) \subset F_{t-1}(-B)=F_{t}$. Therefore, $\varepsilon_{*} \mathcal{F}(-t B)=$ $F_{t}$.

Suppose that $\mathcal{G}$ is another reflexive sheaf of $\underline{X}$ such that $F_{t} \simeq \varepsilon_{*} \mathcal{G}(-t B)$ as parabolic structures. Then we have injections $\overline{\mathcal{F}_{t}^{\prime}} \subset \mathcal{G}(-t B)$ for any $t$. Since

$$
\sum_{0 \leq \lambda \leq 1} \mathcal{G}(-(t-\lambda) B)(-\lambda B)=\mathcal{G}(-t B)
$$

we also have injections $\mathcal{F}(-t B) \subset \mathcal{G}(-t B)$. In order to show this is isomorphic, we may assume that $X$ is isomorphic to a polydisc and $B$ is a coordinate hyperplane. There is a finite cyclic covering $\tau: Y \rightarrow X$ étale outside $B$ satisfying the following condition: For the Galois group $\Gamma \simeq \mathbb{Z} / N \mathbb{Z}$ of $\tau$, there exist $\Gamma$-linearized reflexive sheaves $F_{Y}$ and $G_{Y}$ of $Y$ such that

$$
\left.\mathcal{F}\right|_{\underline{Y}} \simeq \varepsilon_{Y}^{*} F_{Y} \quad \text { and }\left.\quad \mathcal{G}\right|_{\underline{Y}} \simeq \varepsilon_{Y}^{*} G_{Y}
$$


Let $B_{Y}$ denote the pullback $\tau^{-1} B=\left(\tau^{*} B\right)_{\text {red }}$. Then $\varepsilon_{*} \mathcal{F}(-(i / N) B)$ is isomorphic to the $\Gamma$-invariant part of $\tau_{*} F_{Y}\left(-i B_{Y}\right)$. This is the eigenspace of $\tau_{*} F_{Y}$ with respect to the eigenvalue $\exp (2 \pi \sqrt{-1}(i / N))$. Since $\varepsilon_{*} \mathcal{F}(-t B) \simeq \varepsilon_{*} \mathcal{G}(-t B)$, we have $\tau_{*} F_{Y} \simeq \tau_{*} G_{Y}$. Therefore, $F_{Y} \simeq G_{Y}$ and $\mathcal{F} \simeq \mathcal{G}$.

Example 3.5.6. $\quad$ Let $X^{\star} \subset X$ be a toroidal embedding and let $\underline{X}=$ $(X, B)$ for $B=X \backslash X^{\star}$. Let $H$ be a locally constant system of a finitedimensional $\mathbb{C}$-vector space defined on $X^{\star}$. If the local monodromies are unipotent, then we have the canonical extension $\mathcal{H}_{X}^{\text {can }}$ of $\mathcal{H}=H \otimes \mathcal{O}_{X^{\star}}$ to $X$ in the sense of Deligne [D2] as a locally free $\mathcal{O}_{X}$-module. Even if the local monodromies of $H$ are only quasi-unipotent, we have the canonical extension $\mathcal{H}_{\underline{X}}^{\text {can }}$ as a $\partial$-étale locally free sheaf of $\underline{X}$. When $X$ is non-singular, the sheaf ${ }^{\ell} \mathcal{H}_{X}^{\text {can }}=\varepsilon_{*} \mathcal{H}_{\underline{X}}^{\text {can }}$ is locally free and is usually called the canonical extension in the sense of Deligne. We call this by the lower canonical extension (cf. [K9], [M9]).

Example 3.5.7. The sheaf $\Omega_{\underline{X}}^{1}$ of Kähler differentials is defined by the universal property for derivations to $\underline{\mathcal{O}_{X}}$-modules as usual. It is not necessarily coherent even if $X$ is non-singular. To see this, we consider the one-dimensional case: $X=\boldsymbol{\Delta}, B=\{0\}$. We can write $\Omega_{X}^{1}=\mathcal{O}_{X} \mathrm{~d} t$ for a coordinate function $t$ of $X$. Let $X^{\prime}=\Delta \rightarrow X$ be the cyclic covering $u \mapsto u^{m}=t$. Then

$$
\Omega_{X^{\prime}}^{1}=\mathcal{O}_{X^{\prime}} \mathrm{d} u=\mathcal{O}_{X^{\prime}} \frac{\mathrm{d} t}{t^{(1-1 / m)}}
$$

Hence the stalk $\Omega_{\underline{X}, 0}^{1}$ is isomorphic to

$$
\lim _{m \rightarrow \infty} \mathcal{O}_{\underline{X}}, 0 \frac{1}{t^{(1-1 / m)}} .
$$

Thus $\Omega_{\underline{X}}^{1}$ is not coherent. The double-dual of $\Omega_{\underline{X}}^{1}$ is isomorphic to $\hat{\Omega}_{\underline{X}}^{1}(\log B)$.

\section{$\S 4$. Local Nature of Variation of Hodge Structure}

Let $S^{\star} \subset S$ be a toroidal embedding. We consider a $\mathbb{Z}$-polarized variation of Hodge structure $H$ of rank two and of weight one defined over $S^{\star}$ (cf. [G3], [S3]): it consists of a locally constant system $H$ of a free abelian group of rank two, a skew-symmetric bilinear form $Q: H \times H \rightarrow \mathbb{Z}_{S^{\star}}$ inducing an isomorphism $\bigwedge^{2} H \leadsto \mathbb{Z}_{S^{\star}}$, and of a subbundle $\mathcal{F}^{1}(\mathcal{H})$ of $\mathcal{H}:=H \otimes \mathcal{O}_{S^{\star}}$ such that $\left(H_{s}, Q_{s}, \mathcal{F}^{1}(\mathcal{H}) \otimes \mathbb{C}(s)\right)$ forms a polarized Hodge structure of weight one for every $s \in S^{\star}$. We call by $V H S$ a $\mathbb{Z}$-polarized variation of Hodge structure of 
rank two and of weight one, for short. In Section 4, except 4.2, we shall study the local nature of $H$. Thus we mainly suppose that $S^{\star} \subset S$ together with a point $0 \in S$ is a $d$-dimensional toroidal embedding of type $(\mathrm{N}, l, \sigma)$. Thus we can write $S=\mathbb{T}_{\mathrm{N}}(\sigma)^{<1} \times \boldsymbol{\Delta}^{d-l}$. Note that the fundamental group $\pi_{1}\left(S^{\star}\right)$ of $S^{\star}$ is identified with N. Let $\left\{\nu_{1}, \nu_{2}, \ldots, \nu_{k}\right\}$ be the set of vertices of $\sigma$ and let

$$
D_{i}=D_{\nu_{i}}:=\left(\overline{\mathbb{O}}_{\nu_{i}} \cap \mathbb{T}_{\mathrm{N}}(\sigma)^{<1}\right) \times \boldsymbol{\Delta}^{d-l}
$$

denote the corresponding prime divisor to $\nu_{i}$. The complement $D:=S \backslash S^{\star}$ is written by $\sum_{i=1}^{k} D_{i}$. We set $S^{\circ}:=S \backslash \operatorname{Sing} D$. The open subset $S_{\nu_{i}}^{\circ}:=$ $\left(\mathbb{T}_{\mathrm{N}}\left(\nu_{i}\right) \cap \mathbb{T}_{\mathrm{N}}(\sigma)^{<1}\right) \times \boldsymbol{\Delta}^{d-l}$ is non-singular and its fundamental group is isomorphic to $\mathrm{N}\left(\nu_{i}\right)=\mathrm{N} \cap \mathbb{R} \nu_{i}=\mathbb{Z} \nu_{i}$. Note that $S_{\nu_{i}}^{\circ}=S^{\circ} \backslash\left(\cup_{j \neq i} D_{j}\right)$. According to 3.1 .3 , the universal covering mapping of $S^{\star}$ is given by

$$
\begin{aligned}
& \quad \tilde{\mathrm{e}}_{\mathrm{N}}: \mathbb{H}_{\mathrm{N}}(\operatorname{Int} \sigma) \times \Delta^{d-l} \ni z=\left(z^{\prime}, t^{\prime}\right) \longmapsto\left(\mathrm{e}_{\mathrm{N}}\left(z^{\prime}\right), t^{\prime}\right) \in \mathbb{T}_{\mathrm{N}}^{<1} \times \Delta^{d-l} \simeq S^{\star} \\
& \text { for } z^{\prime} \in \mathbb{H}_{\mathrm{N}}(\operatorname{Int} \sigma), t^{\prime} \in \boldsymbol{\Delta}^{d-l} .
\end{aligned}
$$

\section{§4.1. Monodromy and periods}

From the VHS $H$, we have a period mapping $\omega: \mathbb{H}_{\mathrm{N}}(\sigma) \times \Delta^{d-l} \rightarrow \mathbb{H}$ and a monodromy representation $\rho: \pi_{1}\left(S^{\star}\right)=\mathrm{N} \rightarrow \mathrm{SL}(2, \mathbb{Z})$ such that

$$
\omega(\gamma z)=\frac{a_{\gamma} \omega(z)+b_{\gamma}}{c_{\gamma} \omega(z)+d_{\gamma}}, \quad \text { for } \quad \rho(\gamma)=\left(\begin{array}{ll}
a_{\gamma} & b_{\gamma} \\
c_{\gamma} & d_{\gamma}
\end{array}\right) .
$$

Borel's lemma [S3, 4.5] asserts that $\rho\left(\nu_{i}\right)$ are all quasi-unipotent, since $\rho\left(\nu_{i}\right)$ is the local monodromy along $D_{i}$. Hence $\rho(\gamma)$ is quasi-unipotent for any $\gamma \in \mathbf{N}$, since $N$ is commutative. Any quasi-unipotent matrix in $\operatorname{SL}(2, \mathbb{Z})$ is conjugate to one of the matrices in Table 1 uniquely. Suppose that the image $\rho(\mathrm{N})$ of $\rho: \mathrm{N} \rightarrow \mathrm{SL}(2, \mathbb{Z})$ is a finite group. Then it is the cyclic group of order 1,2 , $3,4,6$, according as the image $\rho(\mathrm{N})$ is generated by the matrix $\mathrm{I}_{0}, \mathrm{I}_{0}^{*}, \mathrm{IV}^{*}$, III* $^{*}$ II $^{*}$ in Table 1 up to conjugates in $\operatorname{SL}(2, \mathbb{Z})$. If the order is $m$, then $\rho$ is essentially determined by a surjective group homomorphism $N \rightarrow \mathbb{Z} / m \mathbb{Z}$. Suppose next that $\rho(N)$ is infinite. Then there exist unique homomorphisms $\boldsymbol{a}: \mathrm{N} \rightarrow \mathbb{Z}, \boldsymbol{c}: \mathrm{N} \rightarrow \mathbb{Z} / 2 \mathbb{Z}$, and a matrix $P \in \mathrm{SL}(2, \mathbb{Z})$ such that

$$
P^{-1} \rho(\gamma) P=(-1)^{\boldsymbol{c}(\gamma)} \mathrm{I}_{\boldsymbol{a}(\gamma)}
$$

for $\gamma \in \mathrm{N}$. By a property of period mapping, we have $\boldsymbol{a}\left(\nu_{i}\right) \geq 0$, since $\rho\left(\nu_{i}\right)$ is the local monodromy in the right direction along $D_{i}$. Thus $\boldsymbol{a}$ is considered to be an element of $\sigma^{\vee} \cap \mathrm{M}$ such that $\boldsymbol{a}(\gamma)=\langle\boldsymbol{a}, \gamma\rangle$. 
Table 1. Monodromy matrices.

\begin{tabular}{|c|c|c|c|}
\hline $\mathrm{I}_{a} \quad(a \geq 0)$ & $\mathrm{II}$ & $\mathrm{III}$ & $\mathrm{IV}$ \\
\hline$\left(\begin{array}{ll}1 & a \\
0 & 1\end{array}\right)$ & $\left(\begin{array}{cc}1 & 1 \\
-1 & 0\end{array}\right)$ & $\left(\begin{array}{cc}0 & 1 \\
-1 & 0\end{array}\right)$ & $\left(\begin{array}{cc}0 & 1 \\
-1 & -1\end{array}\right)$ \\
\hline \hline $\mathrm{I}_{b}^{*}(b \geq 0)$ & $\mathrm{II}^{*}$ & $\mathrm{III}^{*}$ & $\mathrm{IV}^{*}$ \\
\hline$\left(\begin{array}{cc}-1 & -b \\
0 & -1\end{array}\right)$ & $\left(\begin{array}{cc}0 & -1 \\
1 & 1\end{array}\right)$ & $\left(\begin{array}{cc}0 & -1 \\
1 & 0\end{array}\right)$ & $\left(\begin{array}{cc}-1 & -1 \\
1 & 0\end{array}\right)$ \\
\hline
\end{tabular}

Definition 4.1.1 ([Type of monodromy representations]) •

(1) Suppose that $\rho(\mathrm{N})$ is finite. The monodromy representation $\rho: \mathrm{N} \rightarrow \operatorname{SL}(2$, $\mathbb{Z}$ ) is called of type $\mathrm{I}_{0}, \mathrm{I}_{0}^{(*)}, \mathrm{II}^{(*)}, \mathrm{III}^{(*)}, \mathrm{IV}^{(*)}$ according as: $\rho(\mathrm{N})$ is generated by a conjugate of the matrix $\mathrm{I}_{0}, \mathrm{I}_{0}^{*}, \mathrm{II}^{*}, \mathrm{III}^{*}, \mathrm{IV}^{*}$.

(2) Suppose that $\rho(N)$ is infinite and that $c: N \rightarrow \mathbb{Z} / 2 \mathbb{Z}$ is zero, i.e., any matrices in $\rho(N)$ is unipotent. Then $\rho$ is called of type $\mathrm{I}_{(+)}$. More precisely, it is called of type $\mathrm{I}_{\boldsymbol{a}}$ for the homomorphism $\boldsymbol{a}: \mathrm{N} \rightarrow \mathbb{Z}$. We define $\alpha$ to be the positive integer such that $\alpha^{-1} \boldsymbol{a}$ is primitive. In other words, $\alpha$ is the index of the image of $\boldsymbol{a}: \mathrm{N} \rightarrow \mathbb{Z}$ in $\mathbb{Z}$.

(3) Suppose that $\rho(N)$ is infinite and that $c: N \rightarrow \mathbb{Z} / 2 \mathbb{Z}$ is not zero. Then $\rho$ is called of type $I_{(+)}^{(*)}$. For $\boldsymbol{a}$ and $\boldsymbol{c}$, we define $\boldsymbol{a}^{*}: \mathrm{N} \rightarrow \mathbb{Z}$ by $\boldsymbol{a}^{*}(\gamma)=$ $(-1)^{\boldsymbol{c}(\gamma)} \boldsymbol{a}(\gamma)$. Then $\rho$ is called of type $\mathrm{I}_{(+)}^{(*)}(0), \mathrm{I}_{(+)}^{(*)}(1), \mathrm{I}_{(+)}^{(*)}(2)$ according as: $\boldsymbol{a}^{*} \equiv 0 \bmod 2, \boldsymbol{a}^{*} \equiv \boldsymbol{c} \bmod 2, \boldsymbol{a}^{*} \wedge \boldsymbol{c} \not \equiv 0 \bmod 2$. The case $\mathrm{I}_{(+)}^{(*)}(2)$ does not occur if $l=1$.

Remark. The definition of types is slightly different from that in [N4].

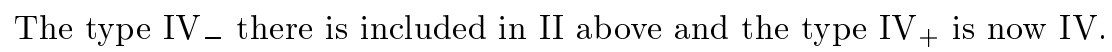

On the period mapping $\omega: \mathbb{H}_{\mathrm{N}}(\operatorname{Int} \sigma) \times \boldsymbol{\Delta}^{d-l} \rightarrow \mathbb{H}$, we have the following result by an argument of [N4]:

\section{Proposition 4.1.2.}

(1) If $H$ is of type $\mathrm{I}_{0}$ or of type $\mathrm{I}_{0}^{(*)}$, then $\omega$ descends to a holomorphic function on $S$.

(2) Suppose that $H$ is of type $\mathrm{I}_{(+)}$or of type $\mathrm{I}_{(+)}^{(*)}$. Let $\boldsymbol{a} \in \sigma^{\vee} \cap \mathrm{M}$ be the functional determined by the monodromy as before. Then there is a holomorphic 
function $h$ on $S$ such that $\operatorname{Im} h(t) \geq 0$ for $t \in S$ and that

$$
\omega(z)=\left\langle\boldsymbol{a}, z^{\prime}\right\rangle+h\left(\tilde{\mathrm{e}}_{\mathrm{N}}(z)\right) .
$$

(3) The $\boldsymbol{J}$-function is defined on $S^{\star}$ by $\boldsymbol{J}\left(\tilde{\mathrm{e}}_{\mathrm{N}}(z)\right)=\mathbf{j}(\omega(z))$ for the elliptic modular function $\mathbf{j}$. It extends to a holomorphic mapping $\boldsymbol{J}: S \rightarrow \mathbb{P}^{1}$.

\section{§4.2. Canonical extension}

The Hodge filtration $\mathcal{F}^{p}(\mathcal{H})$ of $\mathcal{H}$ is defined on $S^{\star}$. If $S$ is non-singular, we have canonical extensions of $\mathcal{H}$ and $\mathcal{F}^{p}(\mathcal{H})$ in the sense of Deligne [D2] by the nilpotent orbit theorem by Schmid [S3]. Even if $S$ has singularities, we have also locally free canonical extensions in the case $H$ has only unipotent monodromies. This is shown as follows: Suppose that $S$ is in the local situation: $S^{\star} \subset S$ is a toroidal embedding of type $(\mathrm{N}, l, \sigma)$. If $H$ is of type $\mathrm{I}_{0}$, then $H$ is originally defined on $S$ and thus the Hodge filter $\mathcal{F}^{1}(\mathcal{H})$ is defined naturally by the period function $\omega$. Suppose that $H$ is of type $\mathrm{I}_{(+)}$, more precisely of type $\mathrm{I}_{\boldsymbol{a}}$ for $0 \neq \boldsymbol{a} \in \sigma^{\vee} \cap \mathrm{M}$. We see that

$$
\log \rho(\gamma)=\boldsymbol{a}(\gamma) N \quad \text { for the matrix } \quad N:=\left(\begin{array}{ll}
0 & 1 \\
0 & 0
\end{array}\right)
$$

By 4.1.2, we have

$$
\exp \left(-\left\langle\boldsymbol{a}, z^{\prime}\right\rangle N\right) \cdot\left(\begin{array}{c}
\omega(z) \\
1
\end{array}\right)=\left(\begin{array}{c}
h(t) \\
1
\end{array}\right)
$$

for $t=\tilde{e}_{N}(z)$. This means that $\mathcal{H}=H \otimes \mathcal{O}_{S^{\star}}$ is a trivial module $\mathcal{O}_{S^{\star}}^{\oplus 2}$ in which $\mathcal{F}^{1}(\mathcal{H})$ is generated by the column vector ${ }^{\mathrm{t}}(h(t), 1)$. Hence we have naturally the canonical extension $\mathcal{H}_{S}^{\text {can }}=\mathcal{O}_{S}^{\oplus 2}$ and the extension $\mathcal{F}^{1}\left(\mathcal{H}_{S}^{\text {can }}\right)$ of $\mathcal{F}^{1}(\mathcal{H})$ as a subbundle of $\mathcal{H}_{S}^{\text {can }}$. This construction is compatible with the canonical extension over $S \backslash \operatorname{Sing} S$.

Even in the case the monodromy of $H$ is not unipotent, we can extend to $S$ the lower-canonical extensions defined over $S \backslash \operatorname{Sing} S$ by taking direct images for the open immersion. But they are not necessarily locally free. To see this, assume that $S^{\star} \subset S$ is a toroidal of type $(\mathrm{N}, l, \sigma)$. Let $\mathrm{N}_{\text {unip }} \subset \mathrm{N}$ be the submodule consisting of all $\gamma \in \mathrm{N}$ with $\rho(\gamma)$ being unipotent. The toric variety $\mathbb{T}_{\mathrm{N}_{\text {unip }}}(\sigma)$ induces a finite abelian covering $\tau: S^{\prime}=\mathbb{T}_{\mathrm{N}_{\text {unip }}}(\sigma)^{<1} \times \Delta^{d-l} \rightarrow S$ with the Galois group $\mathrm{N} / \mathrm{N}_{\text {unip }}$. The lower-canonical extensions ${ }^{\ell} \mathcal{H}_{S}^{\text {can }}$ and $\mathcal{F}^{p}\left({ }^{\ell} \mathcal{H}_{S}^{\text {can }}\right)$ are obtained as the $\mathrm{N} / \mathrm{N}_{\text {unip-invariant part of the direct images of }}$ 
canonical extensions defined on $S^{\prime}$. Hence these extensions are reflexive sheaves of $S$.

Next, we consider the $\partial$-space $\underline{S}:=(S, D)$ for a general toroidal embedding $S^{\star}=S \backslash D \subset S$, which is not necessarily of type $(\mathrm{N}, l, \sigma)$. Then we have the canonical extension $\mathcal{H}_{\underline{S}}^{\text {can }}$ as a locally free $\mathcal{O}_{\underline{S}}$-module (cf. 3.5.6). There is also the extended filter $\mathcal{F}^{1}\left(\mathcal{H}_{S}^{\text {can }}\right)$ as a subbundle. For the natural morphism $\varepsilon: \underline{S} \rightarrow S$, we have $\varepsilon_{*} \mathcal{H}_{\underline{S}}^{\text {can }} \simeq{ }^{{ }^{\ell}} \mathcal{H}_{S}^{\text {can }}$ and $\varepsilon_{*} \mathcal{F}^{1}\left(\mathcal{H}_{\underline{S}}^{\text {can }}\right) \simeq \mathcal{F}^{1}\left({ }^{\ell} \mathcal{H}_{S}^{\text {can }}\right)$.

Definition 4.2.1. We introduce the following sheaves:

$$
\begin{aligned}
\mathcal{L}_{H} & :=\mathcal{H} / \mathcal{F}^{1}(\mathcal{H}), \quad \mathcal{L}_{H / S}:={ }^{\ell} \mathcal{H}_{S}^{\text {can }} / \mathcal{F}^{1}\left({ }^{\ell} \mathcal{H}_{S}^{\text {can }}\right), \quad \text { and } \\
\mathcal{L}_{H / \underline{S}} & :=\mathcal{H}_{\underline{S}}^{\text {can }} / \mathcal{F}^{1}\left(\mathcal{H}_{\underline{S}}^{\text {can }}\right) .
\end{aligned}
$$

Note that $\varepsilon_{*} \mathcal{L}_{H / \underline{S}} \simeq \mathcal{L}_{H / S}$. The canonical extensions ${ }^{\ell} \mathcal{H}_{S}^{\text {can }}$ and $\mathcal{H}_{\underline{S}}^{\text {can }}$ have logarithmic connections

$$
\nabla_{S}:{ }^{\ell} \mathcal{H}_{S}^{\mathrm{can}} \rightarrow \hat{\Omega}_{S}^{1}(\log D) \otimes{ }^{\ell} \mathcal{H}_{S}^{\mathrm{can}}, \quad \text { and } \quad \nabla_{\underline{S}}: \mathcal{H}_{\underline{S}}^{\mathrm{can}} \rightarrow \hat{\Omega}_{\underline{S}}^{1}(\log D) \otimes \mathcal{H}_{\underline{S}}^{\mathrm{can}}
$$

such that $\nabla_{S}=\varepsilon_{*}\left(\nabla_{\underline{S}}\right)$. Then we have logarithmic de Rham complexes

$$
\hat{\Omega}_{S}^{\bullet}(\log D) \otimes{ }^{\ell} \mathcal{H}_{S}^{\text {can }}, \quad \text { and } \quad \hat{\Omega}_{\underline{S}}^{\bullet}(\log D) \otimes \mathcal{H}_{\underline{S}}^{\text {can }} .
$$

These are considered to be subcomplexes of $j_{*}\left(\Omega_{S^{\star}}^{\bullet} \otimes \mathcal{H}\right)$ and $\underline{j}_{*}\left(\Omega_{S^{\star}}^{\bullet} \otimes \mathcal{H}\right)$, respectively. We have natural quasi-isomorphisms

$$
j_{*}\left(\Omega_{S^{*}}^{\bullet} \otimes \mathcal{H}\right) \sim_{\text {qis }} R j_{*} H \stackrel{\mathrm{L}}{\otimes} \mathbb{C}, \quad \text { and } \quad \underline{j}_{*}\left(\Omega_{S^{*}}^{\bullet} \otimes \mathcal{H}\right) \sim_{\mathrm{qis}} R \underline{j}_{*} H \stackrel{\mathrm{L}}{\otimes} \mathbb{C} .
$$

As in the case of non-singular varieties with normal crossing divisors, we have:

Proposition 4.2.2. There exist quasi-isomorphisms

$$
\begin{aligned}
& R j_{*} H \stackrel{\mathrm{L}}{\mathbb{C}} \sim_{\text {qis }} \hat{\Omega}_{S}^{\bullet}(\log D) \otimes{ }^{\ell} \mathcal{H}_{S}^{\text {can }}, \quad \text { and } \\
& R \underline{j}_{*} H \stackrel{\mathrm{L}}{\otimes} \mathbb{C} \sim_{\text {qis }} \hat{\Omega}_{\underline{S}}^{\bullet}(\log D) \otimes \mathcal{H}_{\underline{S}}^{\text {can }} .
\end{aligned}
$$

Proof. It is enough to show the morphism

$$
\hat{\Omega}_{\underline{S}}^{\bullet}(\log D) \otimes \mathcal{H}_{\underline{S}}^{\text {can }} \rightarrow \underline{j}_{*}\left(\Omega_{S^{\star}}^{\bullet} \otimes \mathcal{H}\right)
$$

is quasi-isomorphic. Thus we may assume that $S^{\star} \subset S$ be a toroidal embedding of type $(\mathrm{N}, l, \sigma)$ and that $H$ is of type $\mathrm{I}_{\boldsymbol{a}}$ for some $\boldsymbol{a} \in \sigma^{\vee} \cap \mathrm{M}$. Let $\mu: Y \rightarrow S$ be a desingularization of $S$ corresponding to a subdivision of $\sigma \subset \mathrm{N} \otimes \mathbb{R}$ into 
a non-singular fan. Then $\mu^{-1} D$ is a normal crossing divisor, $\mathcal{H}_{Y}^{\text {can }} \simeq \mu^{*} \mathcal{H}_{S}^{\text {can }}$, and $\Omega_{Y}^{p}\left(\log \mu^{-1} D\right) \simeq \mu^{*} \hat{\Omega}_{S}^{p}(\log D)$ for any $p$. We have a quasi-isomorphism

$$
\Omega_{Y}^{\bullet}\left(\log \mu^{-1} D\right) \otimes \mathcal{H}_{Y}^{\text {can }} \simeq_{\text {qis }} j_{*}^{\prime}\left(\Omega_{S^{\star}}^{\bullet} \otimes \mathcal{H}\right)
$$

for the open immersion $j^{\prime}: S^{\star} \leadsto Y \backslash \mu^{-1} D \hookrightarrow Y$. Therefore,

$$
\hat{\Omega}_{S}^{\bullet}(\log D) \otimes \mathcal{H}_{S}^{\text {can }} \rightarrow j_{*}\left(\Omega_{S^{\star}}^{\bullet} \otimes \mathcal{H}\right)
$$

is quasi-isomorphic, since $S$ has only rational singularities. By considering $\partial-$ étale coverings over $[S, D]$, we have the similar quasi-isomorphism over $\underline{S}$.

Corollary 4.2.3. The natural morphism $R \underline{j}_{*} H \rightarrow R \underline{j}_{*} \mathcal{L}_{H} \sim_{\text {qis }} \underline{j}_{*} \mathcal{L}_{H}$ is decomposed into $R \underline{j}_{*} H \rightarrow \mathcal{L}_{H / \underline{S}} \rightarrow \underline{j}_{*} \mathcal{L}_{H}$. In particular, the morphisms $R \underline{\Gamma}_{D}\left(\underline{j}_{*} H\right) \rightarrow R \underline{\Gamma}_{D}\left(\underline{\mathcal{L}}_{H / \underline{S}}\right)$ and $R \underline{\Gamma}_{D}\left(j_{*} H\right) \rightarrow R \underline{\Gamma}_{D}\left(\mathcal{L}_{H / S}\right)$ are zero in the derived categories.

Lemma 4.2.4. Let $\mu: Y \rightarrow S$ be a bimeromorphic morphism from a non-singular variety such that $\mu^{-1} D$ is a normal crossing divisor. Let $H_{Y}$ denote the VHS $\mu^{-1} H$ defined on $Y \backslash \mu^{-1} D$. Then $\mathcal{L}_{H / S} \sim_{\text {qis }} R \mu_{*} \mathcal{L}_{H_{Y} / Y}$.

Proof. This means that $R^{p} \mu_{*} \mathcal{L}_{H_{Y} / Y}=0$ for $p>0$ and $\mu_{*} \mathcal{L}_{H_{Y} / Y} \simeq$ $\mathcal{L}_{H / S}$. The latter isomorphism holds if $\mu_{*} \mathcal{L}_{H^{\prime} / Y}$ is reflexive. Thus we may consider them locally on $S$. Then, there is a finite Galois covering $\tau: S^{\prime} \rightarrow$ $S$ étale outside $D$ such that $H^{\prime}:=\tau^{-1} H$ on $S^{\prime} \backslash \tau^{-1} D$ has only unipotent monodromies. Then $\mathcal{L}_{H^{\prime} / S^{\prime}}$ is an invertible sheaf and the invariant part of $\tau_{*} \mathcal{L}_{H^{\prime} / S^{\prime}}$ by the action of the Galois group $G$ is isomorphic to $\mathcal{L}_{H / S}$. Let $Y^{\prime}$ be the normalization of $Y \times{ }_{S} S^{\prime}$ and let $\mu^{\prime}: Y^{\prime} \rightarrow S^{\prime}$ be the induces morphism. Then $\mu^{\prime *} \mathcal{L}_{H^{\prime} / S^{\prime}} \simeq \mathcal{L}_{H_{Y^{\prime}}^{\prime} / Y^{\prime}}$ for the VHS $H_{Y^{\prime}}^{\prime}=\left(\mu^{\prime}\right)^{-1} H$ defined on $Y^{\prime} \times{ }_{S} S^{\star}$. Therefore $R^{p} \mu_{*}^{\prime} \mathcal{L}_{H_{Y^{\prime}}^{\prime} / Y^{\prime}}=0$ for $p>0$ and $\mu_{*}^{\prime} \mathcal{L}_{H_{Y^{\prime}}^{\prime} / Y^{\prime}} \simeq \mathcal{L}_{H^{\prime} / S^{\prime}}$, since $S^{\prime}$ has only rational singularities. By taking $G$-invariant parts, we have the vanishing $R^{p} \mu_{*} \mathcal{L}_{H_{Y} / Y}=0$ for $p>0$ and the local isomorphism $\mathcal{L}_{H / S} \simeq \mu_{*} \mathcal{L}_{H / Y}$.

Corollary 4.2.5. Suppose that $S$ is compact and connected.

(1) If the $\boldsymbol{J}$-function is non-trivial, then $H^{0}\left(S^{\star}, H\right)=0$.

(2) If $H$ is not trivial, then $H^{0}\left(S^{\star}, H\right)=0$.

(3) If $\operatorname{dim} S=1$ and if $H$ is not trivial, then $H^{2}\left(S, j_{*} H\right)$ is a finite group (cf. $[\mathrm{K} 7,11.7])$. 
Proof. (1) This is reduced to the vanishing $H^{0}\left(S, \mathcal{L}_{H / S}\right)=0$ by the injection $j_{*} H \rightarrow \mathcal{L}_{H / S}$. We may assume that $S$ is non-singular. Then

$$
\mathcal{L}_{H / S}^{\otimes(-12)} \simeq \boldsymbol{J}^{*} \mathcal{O}_{\mathbb{P}^{1}}(1) \otimes \mathcal{O}_{S}\left(\sum a_{i} D_{i}\right)
$$

for integers $0 \leq a_{i} \leq 10$ for irreducible components of $D_{i}$ (cf. [U1], [K2], [N4, Section 3]). If $H^{0}\left(S, \mathcal{L}_{H / S}\right) \neq 0$, then $H^{0}\left(\mathbb{P}^{1}, \mathcal{O}(1)\right)$ defines a non-constant holomorphic function on $S$. This is a contradiction.

(2) $H$ is trivial if and only if $H^{0}\left(S^{\star}, H\right)$ is of rank two. If $H^{0}\left(S^{\star}, H\right)$ contains a non-zero element, then it defines an extension

$$
0 \rightarrow \mathbb{Z}_{S^{\star}} \rightarrow H \rightarrow \mathbb{Z}_{S^{\star}} \rightarrow 0
$$

of local systems. Hence the monodromy representation $\pi_{1}\left(S^{\star}\right) \rightarrow \mathrm{SL}(2, \mathbb{Z})$ is equivalent to the induced representation

$$
\rho(\gamma)=\left(\begin{array}{cc}
1 & \phi(\gamma) \\
0 & 1
\end{array}\right)
$$

for the group homomorphism $\phi: \pi_{1}\left(S^{\star}\right) \rightarrow H_{1}\left(S^{\star}, \mathbb{Z}\right) \rightarrow \mathbb{Z}$ corresponding to the extension above. Since $\boldsymbol{J}$-function is constant by $(1)$, the period function $\omega(z)$ on the universal covering space of $S^{\star}$ is also constant. Hence $\phi=0$ and $H$ is trivial.

(3) We have $H^{2}\left(S, j_{*} H\right) \simeq H^{2}\left(S, j_{!} H\right) \simeq H_{c}^{2}\left(S^{\star}, H\right)$. By the Verdier duality

$$
R \Gamma_{c}\left(S^{\star}, H\right) \sim_{\text {qis }} R \operatorname{Hom}_{\mathbb{Z}}\left(R \Gamma\left(S^{\star}, H^{\vee}\right), \mathbb{Z}\right)[-2],
$$

or as a universal coefficient theorem, we have an exact sequence

$$
0 \rightarrow \operatorname{Ext}_{\mathbb{Z}}^{1}\left(H^{1}\left(S^{\star}, H^{\vee}\right), \mathbb{Z}\right) \rightarrow H_{c}^{2}\left(S^{\star}, H\right) \rightarrow \operatorname{Hom}_{\mathbb{Z}}\left(H^{0}\left(S^{\star}, H^{\vee}\right), \mathbb{Z}\right) \rightarrow 0 .
$$

Since $H^{1}\left(S^{\star}, H^{\vee}\right)$ is a finitely generated abelian group, $H_{c}^{2}\left(S^{\star}, H\right)$ is finite by (2).

\section{$\S 4.3$. Group cohomology}

According to [N4], we regard $\mathbb{Z}^{\oplus 2}$ as a right $\mathrm{SL}(2, \mathbb{Z})$-module. It turns to be an $\mathrm{N}$-module by the monodromy representation $\rho$. We infer that $p$-th cohomology group $H^{p}=H^{p}\left(S^{\star}, H\right)$ is isomorphic to $H^{p}\left(\pi_{1}\left(S^{\star}\right), \mathbb{Z}^{\oplus 2}\right)$ by a Hochschild-Serre spectral sequence.

Theorem 4.3.1. The group cohomologies $H^{p}=H^{p}\left(\pi_{1}\left(S^{\star}\right), \mathbb{Z}^{\oplus 2}\right)=$ $H^{p}\left(\mathrm{~N}, \mathbb{Z}^{\oplus 2}\right)$ are calculated as in Table 2. 
Table 2. List of cohomology groups.

\begin{tabular}{|c|c|c|c|}
\hline Type of monodromy & $H^{0}$ & $H^{1}$ & $H^{p}(p \geq 2)$ \\
\hline $\mathrm{I}_{0}$ & $\mathbb{Z}^{\oplus 2}$ & $\mathbb{Z}^{\oplus 2\left(\begin{array}{l}l \\
2\end{array}\right)}$ & $\mathbb{Z}^{\oplus 2\left(\begin{array}{l}l \\
p\end{array}\right)}$ \\
\hline $\mathrm{I}_{0}^{(*)}$ & 0 & $(\mathbb{Z} / 2 \mathbb{Z})^{\oplus 2}$ & $(\mathbb{Z} / 2 \mathbb{Z})^{\oplus 2\left(\begin{array}{c}l-1 \\
p-1\end{array}\right)}$ \\
\hline $\mathrm{II}^{(*)}$ & 0 & 0 & 0 \\
\hline $\mathrm{III}^{(*)}$ & 0 & $\mathbb{Z} / 2 \mathbb{Z}$ & $(\mathbb{Z} / 2 \mathbb{Z})^{\oplus\left(\begin{array}{l}l-1 \\
p-1\end{array}\right)}$ \\
\hline $\operatorname{IV}^{(*)}$ & 0 & $\mathbb{Z} / 3 \mathbb{Z}$ & $(\mathbb{Z} / 3 \mathbb{Z})^{\oplus\left(\begin{array}{l}l-1 \\
p-1\end{array}\right)}$ \\
\hline $\mathrm{I}_{(+)}$ & $\mathbb{Z}$ & $\mathbb{Z}^{\oplus l} \oplus \mathbb{Z} / \alpha \mathbb{Z}$ & $\mathbb{Z}^{\oplus\left(\begin{array}{l}l \\
p\end{array}\right)} \oplus(\mathbb{Z} / \alpha \mathbb{Z})^{\oplus\left(\begin{array}{l}l-1 \\
p-1\end{array}\right)}$ \\
\hline $\mathrm{I}_{(+)}^{(*)}(0)$ & 0 & $(\mathbb{Z} / 2 \mathbb{Z})^{\oplus 2}$ & $(\mathbb{Z} / 2 \mathbb{Z})^{\oplus 2\left(\begin{array}{l}l-1 \\
p-1\end{array}\right)}$ \\
\hline $\mathrm{I}_{(+)}^{(*)}(1)$ & 0 & $\mathbb{Z} / 4 \mathbb{Z}$ & $(\mathbb{Z} / 4 \mathbb{Z})^{\oplus\left(\begin{array}{l}l-1 \\
p-1\end{array}\right)}$ \\
\hline $\mathrm{I}_{(+)}^{(*)}(2)$ & 0 & $\mathbb{Z} / 2 \mathbb{Z}$ & $(\mathbb{Z} / 2 \mathbb{Z})^{\oplus\left(\begin{array}{l}l-1 \\
p-1\end{array}\right)}$ \\
\hline
\end{tabular}

In order to show 4.3.1, we consider Koszul complexes. Let $A$ be a commutative algebra with a unit and let $m$ be an $A$-module. We assume that $m$ is originally a right $A$-module. For a free $A$-module $\mathcal{E}$ of finite rank and for an element $\boldsymbol{b} \in \mathcal{E}$, the Koszul complex $\operatorname{Kos}_{\mathcal{E}}^{\bullet}(m, \boldsymbol{b})=\operatorname{Kos}_{A, \mathcal{E}}^{\bullet}(m, \boldsymbol{b})$ is defined as follows: the $p$-th module is

$$
\operatorname{Kos}_{\mathcal{E}}^{p}(m, \boldsymbol{b}):=\operatorname{Kos}_{A, \mathcal{E}}^{p}(m, \boldsymbol{b}):=m \otimes_{A} \bigwedge^{p} \mathcal{E}
$$

The differential d $\mathrm{d}^{p}$ is defined by $\mathbf{x} \mapsto \mathbf{x} \wedge \boldsymbol{b}$ for $\mathbf{x} \in \operatorname{Kos}_{\mathcal{E}}^{p}(m, \boldsymbol{b})$. If we choose a base of $\mathcal{E}$, then $\boldsymbol{b}$ corresponds to a row vector $\left(b_{1}, b_{2}, \ldots, b_{l}\right)$. If we denote by $\mathbf{x}_{i_{1}, i_{2}, \ldots, i_{p}} \in m$ the $\left(i_{1}, i_{2}, \ldots, i_{p}\right)$-th coefficient of $\mathbf{x}$ for $1 \leq i_{1}<i_{2}<\cdots<$ $i_{p} \leq l$, then the differential $\mathrm{d}^{p}$ is written by:

$$
\left(\mathrm{d}^{p}(\mathbf{x})\right)_{i_{0}, i_{1}, \ldots, i_{p}}=\sum_{j=0}^{p}(-1)^{p-j} \mathbf{x}_{i_{0}, i_{1}, \ldots, i_{j}, \ldots, i_{p}} b_{i_{j}} .
$$

We write the $p$-th cohomology group by $H^{p}(m, \boldsymbol{b})$. 
Lemma 4.3.2.

(1) If $A_{1}$ is an A-algebra such that $m$ is originally an $A_{1}$-module, then $\operatorname{Kos}_{\mathcal{E}}^{\bullet}(m$, $\boldsymbol{b})=\operatorname{Kos}_{A_{1} \otimes \mathcal{E}}\left(m, \boldsymbol{b}_{1}\right)$ for the image $\boldsymbol{b}_{1}$ of $\boldsymbol{b}$ under $\mathcal{E} \rightarrow A_{1} \otimes \mathcal{E}$.

(2) Suppose that $\mathcal{E}=\mathcal{E}_{1} \oplus \mathcal{E}_{2}$ for free $A$-modules $\mathcal{E}_{1}$ and $\mathcal{E}_{2}$ and that

$$
\boldsymbol{b}=\left(\boldsymbol{b}_{1}, \boldsymbol{b}_{2}\right) \in \mathcal{E}_{1} \oplus \mathcal{E}_{2}
$$

for $\boldsymbol{b}_{1} \in \mathcal{E}_{1}, \boldsymbol{b}_{2} \in \mathcal{E}_{2}$. Then $\operatorname{Kos}_{\mathcal{E}}^{\bullet}(m, \boldsymbol{b})$ is quasi-isomorphic to

$$
m \otimes \operatorname{Kos}_{\mathcal{E}_{1}}^{\bullet}\left(A, \boldsymbol{b}_{1}\right) \otimes \operatorname{Kos}_{\mathcal{E}_{2}}\left(A, \boldsymbol{b}_{2}\right) .
$$

In particular, if $\boldsymbol{b}_{2}=0$, then $H^{p}(m, \boldsymbol{b})$ is isomorphic to

$$
\bigoplus_{j \geq 0} H^{p-j}\left(m, \boldsymbol{b}_{1}\right) \otimes \bigwedge^{j} \mathcal{E}_{2}
$$

(3) If $\boldsymbol{b}^{\prime}=\boldsymbol{b} \cdot P$ for some right $A$-linear automorphism $P \in \operatorname{Aut}_{A}(\mathcal{E})$, then we have an isomorphism $\operatorname{Kos}_{\mathcal{E}}^{\bullet}\left(m, \boldsymbol{b}^{\prime}\right) \simeq \operatorname{Kos}_{\mathcal{E}}^{\bullet}(m, \boldsymbol{b})$.

We will find a resolution of the trivial $N$-module $\mathbb{Z}$ by free $\mathbb{Z}[\mathrm{N}]$-modules. Let us choose a generator $\left(\gamma_{1}, \gamma_{2}, \ldots, \gamma_{l}\right)$ of $\mathrm{N}$ as an abelian group of rank $l$. We set $\boldsymbol{\epsilon}: \mathbf{N} \rightarrow \mathbb{Z}[\mathbf{N}]$ to be the homomorphism defined by $\boldsymbol{\epsilon}\left(\gamma_{i}\right)=\gamma_{i}-1$ for $1 \leq i \leq l$. The $\epsilon$ does depend on the choice of generator. We define a (right) $\mathbb{Z}[\mathrm{N}]$-linear mapping

$$
\partial_{p+1}: \bigwedge^{p+1} \mathrm{~N} \otimes \mathbb{Z}[\mathrm{N}] \rightarrow \bigwedge^{p} \mathrm{~N} \otimes \mathbb{Z}[\mathrm{N}]
$$

from the composite of natural homomorphism $\bigwedge^{p+1} \mathrm{~N} \rightarrow \bigwedge^{p} \mathrm{~N} \otimes \mathrm{N}$ and id $\otimes$ $\boldsymbol{\epsilon}: \bigwedge^{p} \mathrm{~N} \otimes \mathrm{N} \rightarrow \bigwedge^{p} \mathrm{~N} \otimes \mathbb{Z}[\mathrm{N}]$. More explicitly, $\partial_{p+1}$ sends $e_{0} \wedge e_{1} \wedge \cdots \wedge e_{p}$ for $e_{i} \in \mathrm{N}$ to

$$
\sum_{j=0}^{p}(-1)^{p-j} e_{0} \wedge e_{1} \wedge \cdots \wedge \stackrel{\vee}{e_{j}} \wedge \cdots \wedge e_{p} \otimes \boldsymbol{\epsilon}\left(e_{j}\right)
$$

Then we have a resolution of $\mathbb{Z}$ :

$$
\cdots \rightarrow \bigwedge^{p+1} \mathrm{~N} \otimes \mathbb{Z}[\mathrm{N}] \stackrel{\partial_{p+1}}{\longrightarrow} \bigwedge^{p} \mathrm{~N} \otimes \mathbb{Z}[\mathrm{N}] \rightarrow \cdots \rightarrow \mathbb{Z}[\mathrm{N}] \rightarrow \mathbb{Z} \rightarrow 0 .
$$

Therefore for the right $\mathbb{Z}[\mathrm{N}]$-module $m:=\mathbb{Z}^{\oplus 2}, H^{p}(\mathrm{~N}, m)$ is the $p$-th cohomology group of the complex

$$
\left[\cdots \rightarrow \operatorname{Hom}_{\mathbb{Z}[\mathrm{N}]}\left(\bigwedge^{p} \mathrm{~N} \otimes \mathbb{Z}[\mathrm{N}], m\right) \stackrel{\mathrm{d}^{p}}{\rightarrow} \operatorname{Hom}_{\mathbb{Z}[\mathrm{N}]}\left(\bigwedge^{p+1} \mathrm{~N} \otimes \mathbb{Z}[\mathrm{N}], m\right) \rightarrow \cdots\right]
$$


Here $\mathrm{d}^{p}$ is described as follows: For $\mathbf{x} \in \operatorname{Hom}_{\mathbb{Z}[\mathrm{N}]}\left(\bigwedge^{p} \mathrm{~N} \otimes \mathbb{Z}[\mathrm{N}], m\right)$,

$$
\mathrm{d}^{p}(\mathbf{x})\left(e_{0} \wedge e_{1} \wedge \cdots \wedge e_{p}\right)=\sum_{j=0}^{l}(-1)^{p-j} \mathbf{x}\left(e_{0} \wedge e_{1} \wedge \cdots \wedge e_{j}^{\vee} \wedge \cdots \wedge e_{p}\right) \boldsymbol{\epsilon}\left(e_{j}\right) .
$$

Therefore, the complex is isomorphic to the Koszul complex

$$
\operatorname{Kos}_{\mathrm{M} \otimes \mathbb{Z}[\mathrm{N}]}^{\bullet}(m, \boldsymbol{\epsilon}) .
$$

We denote $\mathbf{i}:=\sqrt{-1} \in \mathbb{C}$ and $\boldsymbol{\omega}:=\exp (2 \pi \sqrt{-1} / 3) \in \mathbb{C}$. We introduce a commutative algebra $A$ depending on the type of monodromies as follows:

$$
A:= \begin{cases}\mathbb{Z}, & \text { in the cases } \mathrm{I}_{0}, \mathrm{I}_{0}^{(*)}, \\ \mathbb{Z}[\boldsymbol{\omega}], & \text { in the cases } \mathrm{II}^{(*)}, \mathrm{IV}^{(*)} \\ \mathbb{Z}[\mathbf{i}], & \text { in the case } \mathrm{III}^{(*)}, \\ \mathbb{Z}[\varepsilon] /\left(\varepsilon^{2}\right), & \text { in the cases } \mathrm{I}_{(+)}, \mathrm{I}_{(+)}^{(*)}\end{cases}
$$

Then we can consider $m=\mathbb{Z}^{\oplus 2}$ as an $A$-module by regarding $\mathbf{i}, \boldsymbol{\omega}$ and $\varepsilon$ as:

$$
\mathbf{i} \leftrightarrow\left(\begin{array}{cc}
0 & -1 \\
1 & 0
\end{array}\right), \boldsymbol{\omega} \leftrightarrow\left(\begin{array}{cc}
-1 & -1 \\
1 & 0
\end{array}\right), \varepsilon \leftrightarrow\left(\begin{array}{ll}
0 & 1 \\
0 & 0
\end{array}\right) .
$$

Thus there is an algebra homomorphism $\tilde{\rho}: \mathbb{Z}[\mathrm{N}] \rightarrow A$, from which the $\mathbb{Z}[\mathrm{N}]$ module structure of $m$ is derived. More precisely, the $\tilde{\rho}(\gamma)$ is determined by the type of the matrix $\rho(\gamma)$ as in Table 3 . If the type of the monodromy is neither $\mathrm{I}_{0}$

Table 3. Image of $\gamma$.

\begin{tabular}{|c|c|c|c|c|c|c|c|c|c|c|}
\hline Type of $\gamma$ & $\mathrm{I}_{0}$ & $\mathrm{I}_{0}^{*}$ & II & II $^{*}$ & III & III $^{*}$ & IV & IV $^{*}$ & $\mathrm{I}_{a}$ & $\mathrm{I}_{a}^{*}$ \\
\hline$\tilde{\rho}(\gamma)$ & 1 & -1 & $-\boldsymbol{\omega}$ & $-\boldsymbol{\omega}^{2}$ & $-\mathbf{i}$ & $\mathbf{i}$ & $\boldsymbol{\omega}^{2}$ & $\boldsymbol{\omega}$ & $1+a \varepsilon$ & $-(1+a \varepsilon)$ \\
\hline
\end{tabular}

nor $\mathrm{I}_{0}^{(*)}$, then $m$ is isomorphic to $A$ as an $A$ module. We set $\boldsymbol{b} \in \mathrm{M} \otimes A$ to be the image of $\boldsymbol{\epsilon} \in \mathrm{M} \otimes \mathbb{Z}[\mathrm{N}]$ under id $\otimes \tilde{\rho}: \mathrm{M} \otimes \mathbb{Z}[\mathrm{N}] \rightarrow \mathrm{M} \otimes A$. Then $\operatorname{Kos}_{\mathrm{M} \otimes \mathbb{Z}[\mathrm{M}]}(m, \boldsymbol{\epsilon})$ is isomorphic to $\operatorname{Kos}_{\mathbf{M} \otimes A}^{\bullet}(m, \boldsymbol{b})$ and hence $H^{p}=H^{p}\left(\pi_{1}\left(S^{\star}\right), \mathbb{Z}^{\oplus 2}\right) \simeq H^{p}(m, \boldsymbol{b})$.

Proof of 4.3.1.

The case $\mathrm{I}_{0}$. We have $\boldsymbol{b}=0$ and hence

$$
\operatorname{Kos}_{\mathrm{M} \otimes A}^{\bullet}(m, \boldsymbol{b}) \simeq \bigoplus_{j=0}^{l} m \otimes_{\mathbb{Z}} \bigwedge^{j} \mathrm{M}[-j] .
$$




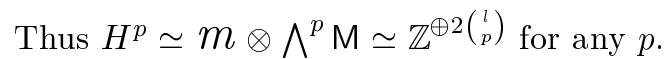

The cases $\mathrm{I}_{0}^{(*)}, \mathrm{II}^{(*)}$, $\mathrm{III}^{(*)}, \mathrm{IV}^{(*)}$. For the generator $\left(\gamma_{1}, \gamma_{2}, \ldots, \gamma_{l}\right)$ of $\mathrm{N}$, we have $\boldsymbol{b}\left(\gamma_{j}\right)=\tilde{\rho}\left(\gamma_{j}\right)-1$. Hence there is an a matrix $P \in \operatorname{GL}(l, A)$ such that

$$
\left(\boldsymbol{b}\left(\gamma_{1}\right), \boldsymbol{b}\left(\gamma_{2}\right), \ldots, \boldsymbol{b}\left(\gamma_{l}\right)\right)=(\beta, 0, \ldots, 0) \cdot P
$$

for an element $\beta \in A$. Here we can choose $\beta$ as follows:

$$
\beta= \begin{cases}2, & \text { in the case } \mathrm{I}_{0}^{(*)} \\ \boldsymbol{\omega}, & \text { in the case } \mathrm{II}^{(*)} \\ \mathbf{i}-1, & \text { in the case } \mathrm{III}^{(*)} \\ \boldsymbol{\omega}-1, & \text { in the case } \mathrm{IV}^{(*)}\end{cases}
$$

By 4.3.2, we have

$$
H^{p}(m, \boldsymbol{b}) \simeq \bigoplus_{j \geq 0} H^{p-j}(m, \beta) \otimes \bigwedge^{j} A^{\oplus(l-1)} .
$$

Here $H^{p}(m, \beta)=0$ for $p \neq 1$ and $H^{1}(m, \beta)=m \otimes A / \beta A$ is isomorphic to the following abelian groups:

$$
m \otimes A / \beta A \simeq \begin{cases}(\mathbb{Z} / 2 \mathbb{Z})^{\oplus 2}, & \text { in the case } \mathrm{I}_{0}^{(*)} ; \\ 0, & \text { in the cases } \mathrm{II}^{(*)} \\ \mathbb{Z} / 2 \mathbb{Z}, & \text { in the case } \mathrm{III}^{(*)} \\ \mathbb{Z} / 3 \mathbb{Z}, & \text { in the case } \mathrm{IV}^{(*)}\end{cases}
$$

The case $\mathrm{I}_{(+)}$. We infer that $m \simeq A$ as an $A$-module and that

$$
\left(\boldsymbol{b}\left(\gamma_{1}\right), \boldsymbol{b}\left(\gamma_{2}\right), \ldots, \boldsymbol{b}\left(\gamma_{l}\right)\right)=(\alpha \varepsilon, 0, \ldots, 0) \cdot P
$$

for $\alpha=\operatorname{gcd} \boldsymbol{a}$ and for some $P \in \mathrm{GL}(l, A)$. Thus by 4.3.2,

$$
H^{p} \simeq \bigoplus_{j \geq 0} H^{p-j}(A, \alpha \varepsilon) \otimes \bigwedge^{j} A^{\oplus(l-1)} .
$$

We have $H^{0}(A, \alpha \varepsilon) \simeq \varepsilon A=\mathbb{Z}, H^{1}(A, \alpha \varepsilon) \simeq A / \alpha \varepsilon \simeq \mathbb{Z} \oplus \mathbb{Z} / \alpha \mathbb{Z}$, and $H^{p}(A$, $\alpha \varepsilon)=0$ for $p \geq 2$. Thus $H^{0} \simeq \mathbb{Z}$ and for $p \geq 1$,

$$
H^{p} \simeq \mathbb{Z}^{\oplus\left(\begin{array}{c}
l-1 \\
p
\end{array}\right)} \oplus(\mathbb{Z} \oplus \mathbb{Z} / \alpha \mathbb{Z})^{\oplus\left(\begin{array}{c}
l-1 \\
p-1
\end{array}\right)} \simeq \mathbb{Z}^{\oplus\left(\begin{array}{l}
l \\
p
\end{array}\right)} \oplus(\mathbb{Z} / \alpha \mathbb{Z})^{\oplus\left(\begin{array}{c}
l-1 \\
p-1
\end{array}\right)} .
$$


The cases $\mathrm{I}_{(+)}^{(*)}(0)$ and $\mathrm{I}_{(+)}^{(*)}(1)$. Let $\hat{\boldsymbol{c}}: \mathrm{N} \rightarrow \mathbb{Z} \subset A$ be the homomorphism defined by

$$
\hat{\boldsymbol{c}}\left(\gamma_{j}\right)= \begin{cases}0, & \text { if } \boldsymbol{c}\left(\gamma_{j}\right)=0 \\ 1, & \text { if } \boldsymbol{c}\left(\gamma_{j}\right) \neq 0 .\end{cases}
$$

This also depends on the choice of generator $\left\{\gamma_{j}\right\}$. Then $\boldsymbol{b}=-2 \hat{\boldsymbol{c}}+\boldsymbol{\varepsilon} \boldsymbol{a}^{*}$ and

$$
\left(\boldsymbol{b}\left(\gamma_{1}\right), \boldsymbol{b}\left(\gamma_{2}\right), \ldots, \boldsymbol{b}\left(\gamma_{l}\right)\right)=(\beta, 0, \ldots, 0) \cdot P
$$

for some $\beta \in A$ and for some $P \in \operatorname{GL}(l, A)$. We can choose $\beta$ as follows:

$$
\beta= \begin{cases}2, & \text { in the case } \mathrm{I}_{(+)}^{(*)}(0) \\ 2+\varepsilon, & \text { in the case } \mathrm{I}_{(+)}^{(*)}(1) .\end{cases}
$$

Thus by 4.3 .2 , we have

$$
H^{p} \simeq \bigoplus_{j \geq 0} H^{p-j}(A, \beta) \otimes \bigwedge^{j} A^{\oplus(l-1)} .
$$

Here $H^{p}(A, \beta)=0$ for $p \neq 1$ and $H^{1}(A, \beta) \simeq A / \beta A$ is isomorphic to the following abelian groups:

$$
A / \beta A \simeq \begin{cases}(\mathbb{Z} / 2 \mathbb{Z})^{\oplus 2}, & \text { in the case } \mathrm{I}_{(+)}^{(*)}(0) \\ \mathbb{Z} / 4 \mathbb{Z}, & \text { in the case } \mathrm{I}_{(+)}^{(*)}(1) .\end{cases}
$$

The case $\mathrm{I}_{(+)}^{(*)}(2)$. The condition $\hat{\boldsymbol{c}} \wedge \boldsymbol{a}^{*} \not \equiv 0 \bmod 2$ implies that there exist a matrix $P \in \mathrm{GL}(l, A)$ such that

$$
\left(\boldsymbol{b}\left(\gamma_{1}\right), \boldsymbol{b}\left(\gamma_{2}\right), \ldots, \boldsymbol{b}\left(\gamma_{l}\right)\right)=(-2, \varepsilon, 0, \ldots, 0) \cdot P .
$$

By 4.3.2, we have

$$
H^{p} \simeq \bigoplus_{j \geq 0} H^{p-j}(A,(-2, \varepsilon)) \otimes \bigwedge^{j} A^{\oplus(l-2)} .
$$

Moreover we have $H^{p}(A,(-2, \varepsilon))=\mathbb{Z} / 2 \mathbb{Z}$ for $p=1,2$ and $H^{p}(A,(-2, \boldsymbol{\varepsilon}))=0$ for other $p$. Thus

$$
H^{p} \simeq \mathbb{Z} / 2 \mathbb{Z}^{\oplus\left(\begin{array}{l}
l-2 \\
p-1
\end{array}\right)} \oplus \mathbb{Z} / 2 \mathbb{Z}^{\oplus\left(\begin{array}{l}
l-2 \\
p-2
\end{array}\right)} \simeq \mathbb{Z} / 2 \mathbb{Z}^{\oplus\left(\begin{array}{l}
l-1 \\
p-1
\end{array}\right) .}
$$


Leray's spectral sequence

$$
E_{2}^{p, q}=H^{p}\left(S^{\circ}, R^{q} j_{*}^{\star} H\right) \Longrightarrow E^{p+q}=H^{p+q}\left(S^{\star}, H\right)
$$

for the immersion $j^{\star}: S^{\star} \hookrightarrow S^{\circ}$ induces a long exact sequence:

$$
\cdots \rightarrow H^{p}\left(S^{\circ}, j_{*}^{\star} H\right) \rightarrow H^{p}\left(S^{\star}, H\right) \rightarrow H^{p-1}\left(S^{\circ}, R^{1} j_{*}^{\star} H\right) \rightarrow H^{p+1}\left(S^{\circ}, j_{*}^{\star} H\right) \rightarrow \cdots,
$$

since $R^{q} j_{*}^{\star} H=0$ for $q \geq 2$. The support of $R^{1} j_{*}^{\star} H$ is contained in $D^{\star}=$ $\bigsqcup_{i=1}^{k} D_{i}^{\star}$. Thus for a vertex $\nu_{i}$, we have natural homomorphisms

$$
E^{p}=H^{p}\left(S^{\star}, H\right) \rightarrow E_{2}^{(i) p-1,1}:=H^{p-1}\left(D_{i}^{\star},\left.R^{1} j_{*}^{\star} H\right|_{D_{i}^{\star}}\right) .
$$

As in the proof of 4.3.1, we consider $\mathbb{Z}^{\oplus 2}$, a fiber of $H$, as an $A$-module $m$. The cohomology group $H^{p}\left(S^{\star}, H\right)$ is calculated by the Koszul complex $\operatorname{Kos}_{\mathrm{M} \otimes A}^{\bullet}(m, \boldsymbol{b})$. Suppose that $\boldsymbol{b}\left(\nu_{i}\right)=\tilde{\rho}\left(\nu_{i}\right)-1$. This is satisfied if $\nu_{i}=\gamma_{j}$ for some $j$ or if the monodromy of $H$ is unipotent. Let $\boldsymbol{b}^{(i)}$ be the homomorphism $\mathrm{N} / \mathrm{N}\left(\nu_{i}\right) \rightarrow A / \boldsymbol{b}\left(\nu_{i}\right)$ determined by the commutative diagram:

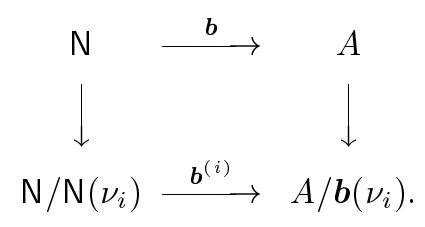

Then $\boldsymbol{b}^{(i)}$ is considered as an element of $\left(\nu_{i}^{\perp} \cap \mathrm{M}\right) \otimes A / \boldsymbol{b}(i)$. We define a morphism of complexes

$$
\operatorname{Kos}_{\mathrm{M} \otimes A}^{\bullet}(m, \boldsymbol{b}) \rightarrow \operatorname{Kos}_{\left(\nu_{i}^{\perp} \cap \mathrm{M}\right) \otimes A}^{\bullet}\left(m \otimes_{A} A / \boldsymbol{b}\left(\nu_{i}\right), \boldsymbol{b}^{(i)}\right)[-1]
$$

as follows: The homomorphism of $p$-th level

$$
m \otimes_{A}\left(\bigwedge^{p} \mathrm{M} \otimes A\right) \rightarrow m \otimes_{A}\left(\bigwedge^{p-1}\left(\nu_{i}^{\perp} \cap \mathrm{M}\right) \otimes A / \boldsymbol{b}\left(\nu_{i}\right)\right)
$$

is induced from the surjection $\bigwedge^{p} \mathrm{M} \rightarrow \mathrm{N}\left(\nu_{i}\right)^{\vee} \otimes \bigwedge^{p-1}\left(\nu_{i}^{\perp} \cap \mathrm{M}\right)$ and from the isomorphism $\mathrm{N}\left(\nu_{i}\right)^{\vee} \leadsto \mathbb{Z}$ which is the dual of $\mathbb{Z} \ni 1 \mapsto \nu_{i} \in \mathrm{N}\left(\nu_{i}\right)$.

Lemma 4.3.3. Suppose that $\boldsymbol{b}\left(\nu_{i}\right)=\tilde{\rho}\left(\nu_{i}\right)-1$. Then the homomorphism (4.2) is described as the $H^{p}$ of the morphism of complexes

$$
\operatorname{Kos}_{\mathrm{M} \otimes A}^{\boldsymbol{B}}(m, \boldsymbol{b}) \rightarrow \operatorname{Kos}_{\left(\nu_{i}^{\perp} \cap \mathrm{M}\right) \otimes A}^{\bullet}\left(m \otimes_{A} A / \boldsymbol{b}\left(\nu_{i}\right), \boldsymbol{b}^{(i)}\right)[-1] .
$$


Proof. We consider the open subset $S_{\nu_{i}}^{\circ}=S^{\circ} \backslash\left(\bigcup_{j \neq i} D_{j}\right)$. We denote the immersion $S^{\star} \hookrightarrow S_{\nu_{i}}^{\circ}$ by $j_{i}^{\circ}$. Then $\left.\left.R^{1} j_{*}^{\star} H\right|_{D_{i}^{\star}} \simeq R^{1} j_{i *}^{\circ} H\right|_{D_{i}^{\star}}$ and the homomorphism (4.2) is derived from Leray's spectral sequence for $j_{i}^{\circ}$. The immersion $j_{i}^{\circ}: S^{\star} \hookrightarrow S_{\nu_{i}}^{\circ}$ is homotopically equivalent to the projection $\mathrm{N} \otimes_{\mathbb{Z}} \mathrm{S}^{1} \rightarrow \mathrm{N} / \mathrm{N}\left(\nu_{i}\right) \otimes_{\mathbb{Z}} \mathrm{S}^{1}$. Thus Leray's spectral sequence for $j_{i}^{\circ}$ is isomorphic to that for the projection and for a local constant system of $N \otimes_{\mathbb{Z}} \mathrm{S}^{1}$ defined by the same monodromy representation $\pi_{1}\left(S^{\star}\right)=\mathrm{N} \rightarrow \mathrm{SL}(2, \mathbb{Z})$ as $H$. The spectral sequence is then expressed as the following Hochschild-Serre's spectral sequence:

$$
E_{2}^{p, q}=H^{p}\left(\mathrm{~N} / \mathrm{N}\left(\nu_{i}\right), H^{q}\left(\mathrm{~N}\left(\nu_{i}\right), m\right)\right) \Longrightarrow H^{p+q}(\mathrm{~N}, m) .
$$

We have $H^{p}\left(m, \boldsymbol{b}\left(\nu_{i}\right)\right) \simeq H^{p}\left(\mathrm{~N}\left(\nu_{i}\right), m\right)$, since $\boldsymbol{b}\left(\nu_{i}\right)=\tilde{\rho}\left(\nu_{i}\right)-1$. The Koszul complex $\operatorname{Kos}_{\mathrm{M} \otimes A}^{\bullet}(m, \boldsymbol{b})$ is isomorphic to the total complex of

$$
m \otimes \operatorname{Kos}_{A}^{\bullet}\left(A, \boldsymbol{b}\left(\nu_{i}\right)\right) \otimes \operatorname{Kos}_{\left(\nu_{i}^{\perp} \cap \mathrm{M}\right) \otimes A}^{\bullet}\left(A, \boldsymbol{b}^{\prime}\right)
$$

for some $\boldsymbol{b}^{\prime} \in\left(\nu_{i}^{\perp} \cap \mathrm{M}\right) \otimes A$ with $\boldsymbol{b}^{(i)}=\boldsymbol{b}^{\prime} \bmod \boldsymbol{b}\left(\nu_{i}\right)$. For the complex $\operatorname{Kos}_{A}^{\bullet}\left(m, \boldsymbol{b}\left(\nu_{i}\right)\right)$, we have a triangle

$$
\cdots \stackrel{+1}{\longrightarrow} m^{\boldsymbol{b}\left(\nu_{i}\right)} \rightarrow \operatorname{Kos}_{A}^{\bullet}\left(m, \boldsymbol{b}\left(\nu_{i}\right)\right) \rightarrow m / m \boldsymbol{b}\left(\nu_{i}\right)[-1] \stackrel{+1}{\longrightarrow} \cdots,
$$

where $M^{\boldsymbol{b}\left(\nu_{i}\right)}=\left\{x \in m \mid x \boldsymbol{b}\left(\nu_{i}\right)=0\right\}$. This induces the triangle

$$
\begin{aligned}
\cdots \stackrel{+1}{\rightarrow} \operatorname{Kos}_{\left(\nu_{i}^{\perp} \cap \mathrm{M}\right) \otimes A}^{\bullet}\left(m^{\boldsymbol{b}\left(\nu_{i}\right)}, \boldsymbol{b}^{\prime}\right) \rightarrow \operatorname{Kos}_{\mathrm{M} \otimes A}^{\bullet}(m, \boldsymbol{b}) \rightarrow \\
\rightarrow \operatorname{Kos}_{\left(\nu_{i}^{\perp} \cap \mathrm{M}\right) \otimes A}^{\bullet}\left(m \otimes A / \boldsymbol{b}\left(\nu_{i}\right), \boldsymbol{b}^{(i)}\right)[-1] \stackrel{+1}{\rightarrow} \ldots
\end{aligned}
$$

The associated long exact sequence is isomorphic to the long exact sequence derived from Hochschild-Serre's spectral sequence above. Because, the morphism of complexes is naturally derived from a double complex given by resolutions of $\mathbb{Z}$ by free $\mathbb{Z}[\mathrm{N}]$-modules and free $\mathbb{Z}\left[\mathrm{N} / \mathrm{N}\left(\nu_{i}\right)\right]$-modules.

Suppose that $H$ is of type $\mathrm{I}_{\boldsymbol{a}}$ for an element $0 \neq \boldsymbol{a} \in \sigma^{\vee} \cap \mathrm{M}$. Let $\boldsymbol{u}=\alpha^{-1} \boldsymbol{a}$ be the primitive element. The set $\boldsymbol{a}^{\perp} \cap \sigma=\{\nu \in \sigma \mid \boldsymbol{a}(\nu)=0\}$ is a face of $\sigma$. The abelian group $\mathrm{N}\left(\boldsymbol{a}^{\perp} \cap \sigma\right)$ was defined to be $\mathrm{N} \cap\left(\boldsymbol{a}^{\perp} \cap \sigma+\left(-\boldsymbol{a}^{\perp} \cap \sigma\right)\right)$. We define $l_{+}:=l-\operatorname{rank} \mathrm{N}\left(\boldsymbol{a}^{\perp} \cap \sigma\right)$ and define $k_{+}$to be the number of indices $1 \leq i \leq k$ with $a_{i}:=\boldsymbol{a}\left(\nu_{i}\right)>0$. Then $l_{+} \leq k_{+}$and $l-l_{+} \leq k-k_{+}$ hold. Note that $l=k$ if and only if $(S, 0)$ is a quotient singularity. Let $S^{\natural}$ be the complement of $\bigcup_{a_{i}>0} D_{i}$ in $S$ and let $j^{\natural}: S^{\natural} \hookrightarrow S$ denote the open immersion. Then the toroidal embedding $S^{\star} \subset S^{\natural}$ is associated with the cone $\boldsymbol{a}^{\perp} \cap \sigma \subset \mathrm{N} \otimes \mathbb{R}$. In particular, $S^{\natural}$ is homotopically equivalent to

$$
\mathbb{T}_{\mathrm{N}\left(\boldsymbol{a}^{\perp} \cap \sigma\right)}\left(\boldsymbol{a}^{\perp} \cap \sigma\right) \times \mathbb{T}_{\mathrm{N} / \mathrm{N}\left(\boldsymbol{a}^{\perp} \cap \sigma\right)} .
$$


We know that $R \Gamma\left(S^{\star}, H\right)$ is quasi-isomorphic to the Koszul complex $\operatorname{Kos}_{\mathrm{M} \otimes A}^{\bullet}(A, \boldsymbol{a} \varepsilon)$ for $A=\mathbb{Z}[\varepsilon]$. We have the following triangle

$$
\cdots \stackrel{+1}{\rightarrow} \operatorname{Kos}_{\mathrm{M}}^{\bullet}(\mathbb{Z}, 0) \rightarrow \operatorname{Kos}_{\mathrm{M} \otimes A}^{\bullet}(A, \boldsymbol{a} \varepsilon) \rightarrow \operatorname{Kos}_{\mathrm{M}}^{\bullet}(\mathbb{Z}, 0) \stackrel{\wedge \boldsymbol{a}}{\longrightarrow} \operatorname{Kos}_{\mathrm{M}}^{\bullet}(\mathbb{Z}, 0)[1] \rightarrow \cdots
$$

Hence $H^{p}\left(S^{\star}, H\right)$ is isomorphic to

$$
\operatorname{Ker}\left(\bigwedge^{p} \mathrm{M} \stackrel{\wedge a}{\longrightarrow} \bigwedge^{p+1} \mathrm{M}\right) \oplus \operatorname{Coker}\left(\bigwedge^{p-1} \mathrm{M} \stackrel{\wedge a}{\longrightarrow} \bigwedge^{p} \mathrm{M}\right)
$$

Next, we consider the complex $\operatorname{Kos}_{\left(\nu_{i}^{\perp} \cap M\right) \otimes A}^{\bullet}\left(A / \boldsymbol{b}\left(\nu_{i}\right), \boldsymbol{b}^{(i)}\right)$ for $\boldsymbol{b}=\boldsymbol{a} \varepsilon$. Here $\boldsymbol{b}\left(\nu_{i}\right)=\boldsymbol{a}\left(\nu_{i}\right) \boldsymbol{\varepsilon}$, and $\boldsymbol{b}^{(i)}=\boldsymbol{a}^{(i)} \boldsymbol{\varepsilon}$ is determined by the commutative diagram

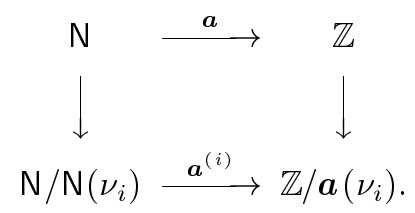

Then we have a triangle

$$
\begin{aligned}
\cdots \stackrel{+1}{\rightarrow} \operatorname{Kos}_{\nu_{i}^{\perp} \cap \mathrm{M}}^{\bullet}\left(\mathbb{Z} / a_{i} \mathbb{Z}, 0\right) \rightarrow \operatorname{Kos}_{\left(\nu_{i}^{\perp} \cap \mathrm{M}\right) \otimes A}^{\bullet}\left(A / a_{i} \varepsilon A, \boldsymbol{a}^{(i)} \varepsilon\right) \rightarrow \\
\quad \rightarrow \operatorname{Kos}_{\nu_{\frac{1}{i}}^{\bullet} \cap \mathrm{M}}^{\bullet}(\mathbb{Z}, 0) \stackrel{\wedge \boldsymbol{a}^{(i)}}{\longrightarrow} \operatorname{Kos}_{\nu^{\perp} \cap \mathrm{M}}^{\bullet}\left(\mathbb{Z} / a_{i} \mathbb{Z}, 0\right)[1] \rightarrow \cdots
\end{aligned}
$$

Lemma 4.3.4. Suppose that $H$ is of type $\mathrm{I}_{\boldsymbol{a}}$ for $0 \neq \boldsymbol{a} \in \sigma^{\vee} \cap \mathrm{M}$.

(1) $H^{1}\left(S^{\circ}, j_{*}^{\star} H\right)$ is torsion-free of rank $k_{+}-1$.

(2) $H^{1}\left(S^{\star}, H\right) \simeq \mathbb{Z} \oplus \mathrm{M} / \mathbb{Z} \boldsymbol{a}$.

(3) $H^{0}\left(S^{\circ}, R^{1} j_{*}^{\star} H\right) \simeq \mathbb{Z}^{\oplus k} \oplus \bigoplus_{a_{i}>0} \mathbb{Z} / a_{i} \mathbb{Z}$.

(4) The rank of $H^{2}\left(S^{\circ}, j_{*}^{\star} H\right)$ is $\left(\begin{array}{c}l_{+} \\ 2\end{array}\right)+k-l$. The torsion part of $H^{2}\left(S^{\circ}, j_{*}^{\star} H\right)$ is mapped to zero in $H^{2}\left(S^{\star}, H\right)$. If $S$ is non-singular, then $H^{2}\left(S^{\circ}, j_{*}^{\star} H\right)$ is torsion-free.

(5) $H^{2}\left(S^{\star}, H\right) \simeq \mathrm{M} / \mathbb{Z} \boldsymbol{u} \oplus \bigwedge^{2} \mathrm{M} /(\mathrm{M} \wedge \boldsymbol{a})$.

Proof. We have known (2) and (5) by the Koszul complex. The homomorphisms (4.2) for $p=1,2$ are described as follows:

Case $p=1$. We have isomorphisms $E^{1} \simeq \mathbb{Z} \oplus \mathrm{M} / \mathbb{Z} \boldsymbol{a}$ and

$$
E_{2}^{(i) 0,1} \simeq \begin{cases}\mathbb{Z}, & \text { for } a_{i}=0 \\ \mathbb{Z} \oplus \mathbb{Z} / a_{i} \mathbb{Z}, & \text { for } a_{i}>0\end{cases}
$$


by Koszul complexes. In particular, we have (3). If $a_{i}>0$, then $E^{1} \rightarrow E_{2}^{(i) 0,1}$ is given as the direct sum of the identity $\mathbb{Z} \rightarrow \mathbb{Z}$ and $\mathrm{M} / \mathbb{Z} \boldsymbol{a} \rightarrow \mathbb{Z} / a_{i} \mathbb{Z}$ induced from $\nu_{i}: \mathrm{M} \rightarrow \mathbb{Z}$. If $a_{i}=0$, then $E^{1} \rightarrow E_{2}^{(i) 0,1}$ is induced from $\nu_{i}: \mathrm{M} / \mathbb{Z} \boldsymbol{a} \rightarrow \mathbb{Z}$.

Case $p=2$. For the primitive element $\boldsymbol{u}=\alpha^{-1} \boldsymbol{a}$, we have isomorphisms $E^{2} \simeq \mathrm{M} / \mathbb{Z} \boldsymbol{u} \oplus \bigwedge^{2} \mathrm{M} /(\mathrm{M} \wedge \boldsymbol{a})$ and

$$
E_{2}^{(i) 1,1} \simeq \begin{cases}\mathbb{Z} \oplus\left(\nu_{i}^{\perp} \cap \mathrm{M}\right) / \mathbb{Z} \boldsymbol{a}, & \text { if } \quad a_{i}=0 \\ \mathrm{M} / \mathbb{Z} \boldsymbol{u} \oplus\left(\left(\nu_{i}^{\perp} \cap \mathrm{M}\right) \otimes \mathbb{Z} / a_{i} \mathbb{Z}\right) / \mathbb{Z} \boldsymbol{a}^{(i)}, & \text { if } \quad a_{i}>0 .\end{cases}
$$

In the case $a_{i}=0$, i.e., $\boldsymbol{a} \in \nu_{i}^{\perp}, E^{2} \rightarrow E_{2}^{(i) 1,1}$ is given as the direct sum of $\mathrm{M} / \mathbb{Z} \boldsymbol{u} \rightarrow \mathbb{Z}$ induced from $\nu_{i}: \mathrm{M} \rightarrow \mathbb{Z}$ and $\bigwedge^{2} \mathrm{M} /(\mathrm{M} \wedge \boldsymbol{a}) \rightarrow\left(\nu_{i}^{\perp} \cap \mathrm{M}\right) / \mathbb{Z} \boldsymbol{a}$ induced from $\bigwedge^{2} \mathrm{M} \rightarrow \nu_{i}^{\perp} \cap \mathrm{M}$. In the case $a_{i}>0$, then $E^{2} \rightarrow E_{2}^{(i) 1,1}$ is given as the direct sum of the identity $\mathrm{M} / \mathbb{Z} \boldsymbol{u} \rightarrow \mathrm{M} / \mathbb{Z} \boldsymbol{u}$ and $\bigwedge^{2} \mathrm{M} /(\mathrm{M} \wedge \boldsymbol{a}) \rightarrow$ $\left(\left(\nu_{i}^{\perp} \cap \mathrm{M}\right) \otimes \mathbb{Z} / a_{i} \mathbb{Z}\right) / \mathbb{Z} \boldsymbol{a}^{(i)}$ induced from $\bigwedge^{2} \mathrm{M} \rightarrow\left(\nu_{i}^{\perp} \cap \mathrm{M}\right) \otimes \mathbb{Z} / a_{i} \mathbb{Z}$.

Therefore, $E^{1} \rightarrow E_{2}^{0,1}$ is isomorphic to the direct sum of $\mathbb{Z} \rightarrow \bigoplus_{a_{i}>0} \mathbb{Z}$ and $\mathrm{M} / \mathbb{Z} \boldsymbol{a} \rightarrow \bigoplus_{i=1}^{k} \mathbb{Z} / a_{i} \mathbb{Z}$. We have a commutative diagram of exact sequences:

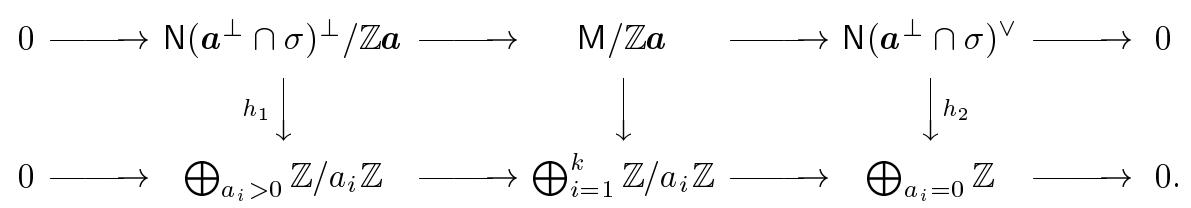

The right vertical arrow $h_{2}$ is injective and the torsion-part $\mathbb{Z} \boldsymbol{u} / \mathbb{Z} \boldsymbol{a}$ of $\mathrm{N}\left(\boldsymbol{a}^{\perp} \cap\right.$ $\sigma)^{\perp} / \mathbb{Z} \boldsymbol{a}$ is mapped injectively into $\bigoplus_{a_{i}>0} \mathbb{Z} / a_{i} \mathbb{Z}$ by the left arrow $h_{1}$. Hence the kernel of $h_{1}$ is isomorphic to $E_{2}^{1,0} \simeq H^{1}\left(S^{\circ}, j_{*}^{\star} H\right)$ and is a torsion-free abelian group of rank $l_{+}-1$. Thus (1) is proved. The cokernel of $\mathrm{M} / \mathbb{Z} \boldsymbol{a} \rightarrow \bigoplus_{i=1}^{k} \mathbb{Z} / a_{i} \mathbb{Z}$ is the direct sum $\mathcal{C}_{1} \oplus \mathcal{C}_{2}$ for $\mathcal{C}_{1}=$ Coker $h_{1}$ and $\mathcal{C}_{2}=$ Coker $h_{2}$. The $\mathcal{C}_{1}$ is a torsion group and the rank of $\mathcal{C}_{2}$ is $\left(k-k_{+}\right)-\left(l-l_{+}\right)$. If $S$ is non-singular, then $\mathcal{C}_{1}=0$. If $S^{\natural}$ is non-singular, then $\mathcal{C}_{2}=0$. Thus the cokernel of $E^{1} \rightarrow E_{2}^{0,1}$ is isomorphic to $\mathbb{Z}^{\oplus\left(k_{+}-1\right)} \oplus \mathcal{C}_{1} \oplus \mathcal{C}_{2}$ and is of rank $k-1-\left(l-l_{+}\right)$.

The homomorphism $E^{2} \rightarrow E_{2}^{1,1}$ is the direct sum of $\mathrm{M} / \mathbb{Z} \boldsymbol{u} \rightarrow \bigoplus_{a_{i}=0} \mathbb{Z} \oplus$ $\bigoplus \mathrm{M} / \mathbb{Z} \boldsymbol{u}$ and $\bigwedge^{2} \mathrm{M} / \mathrm{M} \wedge \boldsymbol{a} \rightarrow \bigoplus_{a_{i}=0}\left(\nu_{i}^{\perp} \cap \mathrm{M}\right) / \mathbb{Z} \boldsymbol{a} \oplus \bigoplus_{a_{i}>0}\left(\left(\nu_{i}^{\perp} \cap \mathrm{M}\right) \otimes \mathbb{Z} / a_{i} \mathbb{Z}\right) /$ $\mathbb{Z} \boldsymbol{a}^{(i)}$. Hence the kernel of the latter homomorphism is the kernel $E_{\infty}^{2,0}$ of $E^{2} \rightarrow E_{2}^{1,1}$. Thus (4) is reduced to the following claim.

Claim 4.3.5. We have an isomorphism

$$
E_{\infty}^{2,0} \simeq \bigcap_{i=1}^{k}\left(\bigwedge^{2}\left(\nu_{i}^{\perp} \cap \mathrm{M}\right)+\mathrm{M} \wedge \boldsymbol{a}\right) / \mathrm{M} \wedge \boldsymbol{a}
$$

Moreover, the abelian group is torsion-free of $\operatorname{rank}\left(\begin{array}{c}l_{+} \\ 2\end{array}\right)-\left(l_{+}-1\right)$. 
Proof. We have inclusions $a_{i} \bigwedge^{2} \mathrm{M} \subset \bigwedge^{2}\left(\nu_{i}^{\perp} \cap \mathrm{M}\right)+\mathrm{M} \wedge \boldsymbol{a}$. In fact, for $w \in \Lambda^{2} \mathrm{M}$, we define $\theta_{i} \in \mathrm{M}$ by the property $\theta_{i}(\nu)=w\left(\nu_{i}, \nu\right)$ for any $\nu \in \mathrm{N}$. Then $a_{i} w+\theta_{i} \wedge \boldsymbol{a} \in \bigwedge^{2}\left(\nu_{i} \cap \mathrm{M}\right)$. Hence we have the equality above. The torsion part of $E_{\infty}^{2,0}$ is contained in that of $\mathrm{M} \wedge \boldsymbol{u} / \mathrm{M} \wedge \boldsymbol{a}$. If $\theta \wedge \boldsymbol{u} \in \wedge^{2}\left(\nu_{i} \cap \mathrm{M}\right)$ for some $\theta \in \mathrm{M}$ and for $a_{i}>0$, then $\theta\left(\nu_{i}\right) \boldsymbol{u}(\nu)=\boldsymbol{u}\left(\nu_{i}\right) \theta(\nu)$ for any $\nu \in \mathrm{N}$. Thus $\theta \wedge \boldsymbol{u}=0$. Therefore, $E_{\infty}^{2,0}$ is torsion-free. In order to calculate the rank, we may replace $\boldsymbol{a}$ by $\boldsymbol{u}$. If $a_{i}>0$, then $\bigwedge^{2}\left(\nu_{i}^{\perp} \cap \mathrm{M}\right)+\mathrm{M} \wedge \boldsymbol{a}$ is a finite index subgroup of $\bigwedge^{2} \mathrm{M}$. We shall show

$$
\begin{aligned}
\bigcap_{a_{i}=0}\left(\bigwedge^{2}\left(\nu_{i}^{\perp} \cap \mathrm{M}\right)+\mathrm{M} \wedge \boldsymbol{u}\right) & =\bigcap_{a_{i}=0} \bigwedge^{2}\left(\nu_{i}^{\perp} \cap \mathrm{M}\right)+\mathrm{M} \wedge \boldsymbol{u} \\
& =\bigwedge^{2} \mathrm{~N}\left(\boldsymbol{a}^{\perp} \cap \sigma\right)^{\perp}+\mathrm{M} \wedge \boldsymbol{u} .
\end{aligned}
$$

Suppose that $w \in \bigwedge^{2} M$ is contained in the left hand side. Then $w-\theta_{i} \wedge \boldsymbol{u} \in$ $\bigwedge^{2}\left(\nu_{i}^{\perp} \cap \mathrm{M}\right)$ for some $\theta_{i} \in \mathrm{M}$. Thus $w\left(\nu_{i}, \nu\right)=\theta_{i}\left(\nu_{i}\right) \boldsymbol{u}(\nu)$. Let $\theta \in \mathrm{M}$ be determined by $w\left(\nu, x_{0}\right)=\theta(\nu)$ for a fixed $x_{0} \in \mathrm{M}$ with the property $\boldsymbol{u}\left(x_{0}\right)=1$. Then $w-\theta \wedge \boldsymbol{u} \in \bigwedge^{2}\left(\nu_{i}^{\perp} \cap \mathrm{M}\right)$ for any $i$ with $a_{i}=0$. Thus we have the equality above. Therefore, the rank of $E_{\infty}^{2,0}$ is equal to $\left(\begin{array}{c}l_{+} \\ 2\end{array}\right)-\left(l_{+}-1\right)$, since $\bigwedge^{2} \mathrm{~N}\left(\boldsymbol{a}^{\perp} \cap \sigma\right)^{\perp} \cap \mathrm{M} \wedge \boldsymbol{u}=\mathrm{N}\left(\boldsymbol{a}^{\perp} \cap \sigma\right)^{\perp} \wedge \boldsymbol{u}$.

Lemma 4.3.6. Suppose that $H$ is of type $\mathrm{I}_{\boldsymbol{a}}$ for $0 \neq \boldsymbol{a} \in \sigma^{\vee} \cap \mathrm{M}$. Let $\mathfrak{T}_{H / S^{\circ}}:=\left(R^{1} j_{*}^{\star} H\right)_{\text {tor }}$ be the torsion part of $R^{1} j_{*}^{\star} H$ as sheaf of abelian groups. Then we have the isomorphisms:

$$
\begin{aligned}
& H^{0}\left(S^{\circ}, \mathfrak{T}_{H / S^{\circ}}\right) \simeq \bigoplus_{a_{i}>0} \mathbb{Z} / a_{i} \mathbb{Z}, \quad \text { and } \\
& H^{1}\left(S^{\circ}, \mathfrak{T}_{H / S^{\circ}}\right)=\bigoplus_{a_{i}>0}\left(\nu_{i}^{\perp} \cap \mathrm{M}\right) \otimes \mathbb{Z} / a_{i} \mathbb{Z} .
\end{aligned}
$$

Proof. By the proof of $4.3 .3, R \Gamma\left(S^{\circ}, \mathfrak{T}_{H / S^{\circ}}\right)$ is quasi-isomorphic to

$$
\bigoplus_{a_{i}>0} \operatorname{Kos}_{\nu_{i}^{\perp} \cap M}^{\bullet}\left(\mathbb{Z} / a_{i} \mathbb{Z}, 0\right) .
$$

\section{$\S 4.4$. $\partial$-étale cohomology}

Let $\underline{S}=(S, D)$ be the associated $\partial$-space. We also denote $\underline{S}^{\circ}:=\left(S^{\circ}, D^{\star}\right)$. The related inclusions are written as follows:

$$
\begin{array}{lll}
j^{\star}: S^{\star} \hookrightarrow S^{\circ}, & j^{\circ}: S^{\circ} \hookrightarrow S, & j=j^{\circ} \circ j^{\star}: S^{\star} \hookrightarrow S, \\
\underline{j}^{\star}: \underline{S}^{\star} \hookrightarrow \underline{S}^{\circ}, & \underline{j}^{\circ}: \underline{S}^{\circ} \hookrightarrow \underline{S}, & \underline{j}:=\underline{j}^{\circ} \circ \underline{j}^{\star}: \underline{S}^{\star} \hookrightarrow \underline{S} .
\end{array}
$$


We shall calculate the stalks at 0 of cohomology groups such as $R^{p} \underline{j}_{*} H$.

Lemma 4.4.1. The stalk at the origin of the $R^{p} \underline{j}_{*} H$ is calculated as follows:

(1) In the case when every monodromy matrices have finite orders:

$$
\left(\underline{j}_{*} H\right)_{0} \simeq \mathbb{Z}^{\oplus 2}, \quad \text { and } \quad\left(R^{p} \underline{j}_{*} H\right)_{0} \simeq \mathbb{Q}^{\oplus 2\left(\begin{array}{l}
l \\
p
\end{array}\right)} \quad \text { for } \quad p \geq 1 .
$$

(2) In the other cases, i.e., $\mathrm{I}_{(+)}$and $\mathrm{I}_{(+)}^{(*)}$ :

$$
\begin{aligned}
& \left(\underline{j}_{*} H\right)_{0} \simeq \mathbb{Z}, \quad\left(R^{1} \underline{j}_{*} H\right)_{0} \simeq \mathbb{Q}^{\oplus l} \oplus \mathbb{Q} / \mathbb{Z}, \quad \text { and } \\
& \left(R^{p} \underline{j}_{*} H\right)_{0} \simeq \mathbb{Q}^{\oplus\left(\begin{array}{l}
l \\
p
\end{array}\right)} \quad \text { for } \quad p \geq 2 .
\end{aligned}
$$

In particular, for $p \geq 2, R^{p} \underline{j}_{*} H$ is a sheaf of $\mathbb{Q}$-vector spaces and there exist isomorphisms:

$$
\left(R^{p} j_{*} H\right) \otimes \mathbb{Q} \simeq R^{p} j_{*}(H \otimes \mathbb{Q}) \simeq \varepsilon_{*}\left(R^{p} \underline{j}_{*} H\right)
$$

Proof. A $\partial$-étale neighborhood of 0 in $\underline{S}$ is essentially given by a finite index subgroup $\mathrm{N}_{1}$ of N. Let $\underline{S}_{1} \rightarrow \underline{S}$ be the finite $\partial$-étale Galois covering corresponding to $\mathrm{N}_{1}$. Then $S_{1}=\operatorname{sp}\left(\underline{S}_{1}\right)$ is isomorphic to $\mathbb{T}_{\mathrm{N}_{1}}(\sigma)^{<1} \times \boldsymbol{\Delta}^{d-l}$. Let $S_{1}^{\star}$ be the open part of $\underline{S}_{1}, j_{1}: S_{1}^{\star} \hookrightarrow S_{1}$ the open immersion, and let $H_{1}$ be the pullback of $H$ by $S_{1}^{\star} \rightarrow S^{\star}$. The stalk $\left(R^{p} \underline{j}_{*} H\right)_{0}$ is the inductive limit of $\left(R^{p} \underline{j}_{1 *} H_{1}\right)_{0} \simeq H^{1}\left(S_{1}^{\star}, H_{1}\right)$ for finite index subgroups $\mathrm{N}_{1}$. Therefore, we may assume that $H$ has only unipotent monodromies. Thus we suppose that $H$ is of type $\mathrm{I}_{\boldsymbol{a}}$ for some $\boldsymbol{a} \in \sigma^{\vee} \cap \mathrm{M}$. Let $A$ be the algebra $\mathbb{Z}[\varepsilon] /\left(\varepsilon^{2}\right)$ and let us consider $m=\mathbb{Z}^{\oplus 2}$ as an $A$-module as follows: If $\boldsymbol{a}=0$, then the action of $\varepsilon$ on $m$ is zero. If $\boldsymbol{a} \neq 0$, then $m$ is the same $A$-module as in the proof of 4.3.1. Then the Koszul complex $\operatorname{Kos}_{\mathrm{M} \otimes A}^{\bullet}(m, \boldsymbol{a} \varepsilon)$ is quasi-isomorphic to $R \Gamma\left(S^{\star}, H\right)$. Let $M_{1}$ be the dual of $N_{1}$. Then there is a natural morphism of complexes

$$
\operatorname{Kos}_{\mathrm{M} \otimes A}^{\bullet}(m, \boldsymbol{a} \varepsilon) \rightarrow \operatorname{Kos}_{\mathrm{M}_{1} \otimes A}^{\bullet}(m, \boldsymbol{a} \varepsilon),
$$

whose $p$-th level is simply the inclusion $m \otimes \bigwedge^{p} \mathrm{M} \hookrightarrow m \otimes \bigwedge^{p} \mathrm{M}_{1}$. Therefore $\left(R \underline{j}_{*} H\right)_{0}$ is quasi-isomorphic to the inductive limit of the Koszul complexes. This is written as

$$
0 \rightarrow m \stackrel{\wedge a \varepsilon}{\longrightarrow} m \otimes \mathrm{M}_{\mathbb{Q}} \stackrel{\wedge a \varepsilon}{\longrightarrow} m \otimes \bigwedge^{2} \mathrm{M}_{\mathbb{Q}} \stackrel{\wedge a \varepsilon}{\longrightarrow} \cdots
$$


where $\mathrm{M}_{\mathbb{Q}}=\mathrm{M} \otimes \mathbb{Q}$. The complex may be expressed by $\operatorname{Kos}_{\mathrm{M}_{\mathbb{Q}} \otimes A}(m, \boldsymbol{a} \varepsilon)$. If $\boldsymbol{a}=0$, then $\left(R \underline{j}_{*} H\right)_{0}$ is quasi-isomorphic to

$$
\mathbb{Z}^{\oplus 2} \oplus \bigoplus_{p \geq 1} \bigwedge^{p} \mathrm{M} \otimes\left(\mathbb{Q}^{\oplus 2}\right)[-p] .
$$

Suppose that $\boldsymbol{a} \neq 0$. Then we have also a triangle

$\cdots \stackrel{+1}{\rightarrow} \operatorname{Kos}_{\mathrm{M}_{\mathbb{Q}}}(\mathbb{Z}, 0) \rightarrow \operatorname{Kos}_{\mathrm{M}_{\mathbb{Q}} \otimes A}(m, \boldsymbol{a} \varepsilon) \rightarrow \operatorname{Kos}_{\mathrm{M}_{\mathbb{Q}}}(\mathbb{Z}, 0) \stackrel{\wedge \boldsymbol{a}}{\longrightarrow} \operatorname{Kos}_{\mathrm{M}_{\mathbb{Q}}}(\mathbb{Z}, 0)[1] \rightarrow \cdots$

Hence $\left(\underline{j}_{*} H\right)_{0} \simeq \mathbb{Z},\left(R^{1} \underline{j}_{*} H\right)_{0} \simeq \mathbb{Q} \boldsymbol{a} \oplus \mathrm{M}_{\mathbb{Q}} / \mathbb{Z} \boldsymbol{a} \simeq \mathbb{Q}^{\oplus l} \oplus \mathbb{Q} / \mathbb{Z}$, and $\left(R^{p} \underline{j}_{*} H\right)_{0} \simeq$ $\left(R^{p} j_{*} H\right)_{0}^{*} \otimes \mathbb{Q}$ for $p \geq 2$.

Leray's spectral sequence:

$$
E_{2}^{p, q}=R^{p} \underline{j}_{*}^{\circ}\left(R^{q} \underline{j}_{*}^{\star} H\right) \Longrightarrow E^{p+q}=R^{p+q} \underline{j}_{*} H
$$

induces a long exact sequence:

$$
\cdots \rightarrow E_{2}^{p-2,1} \rightarrow E_{2}^{p, 0} \rightarrow E^{p} \rightarrow E_{2}^{p-1,1} \rightarrow E_{2}^{p+1,0} \rightarrow \cdots,
$$

since $R^{q} \underline{j}_{*}^{\star} H=0$ for $q \geq 2$.

Lemma 4.4.2. If $p+q \geq 2$, then $E_{2}^{p, q}=R^{p} \underline{j}_{*}^{\circ}\left(R^{q} \underline{j}_{*}^{\star} H\right)$ is a sheaf of $\mathbb{Q}$-vector spaces except for $(p, q)=(2,0)$. Moreover, the following properties hold:

(1) Suppose that $H$ has only monodromies of finite orders. Then $E_{2}^{p, q}$ is a sheaf of $\mathbb{Q}$-vector spaces for $p+q>0$.

(2) Suppose that $H$ is of type $\mathrm{I}_{\boldsymbol{a}}$ for $0 \neq \boldsymbol{a} \in \sigma^{\vee} \cap \mathrm{M}$.

(a) $\left(E_{2}^{1,0}\right)_{0}$ is a torsion-free abelian group with infinitely many generators and $\left(E_{2}^{1,0}\right)_{0} \otimes \mathbb{Q}$ isomorphic to $\left(\mathrm{N}\left(\boldsymbol{a}^{\perp} \cap \sigma\right)^{\perp} / \mathbb{Z} \boldsymbol{a}\right) \otimes \mathbb{Q}$.

(b) $\left(E_{2}^{0,1}\right)_{0}$ is isomorphic to $\bigoplus_{i=1}^{k} \mathbb{Q} \oplus \bigoplus_{a_{i}>0} \mathbb{Q} / a_{i} \mathbb{Z}$.

(c) $\left(E_{2}^{2,0}\right)_{0}$ is the sum of a $\mathbb{Q}$-vector space of dimension $\left(\begin{array}{c}l_{+} \\ 2\end{array}\right)+k-l$ and $a$ divisible group. If $S^{\natural}$ has only quotient singularities, then the divisible group is zero.

Remark. If $E_{2}^{p, q}$ is a sheaf of $\mathbb{Q}$-vector spaces, then, by 3.1.4, we have isomorphisms:

$$
\begin{aligned}
\left(R^{p} j_{*}^{\circ} R^{q} j_{*}^{\star} H\right) \otimes \mathbb{Q} & \simeq R^{p} j_{*}^{\circ}\left(\left(R^{q} j_{*}^{\star} H\right) \otimes \mathbb{Q}\right) \\
& \simeq R^{p} j_{*}^{\circ} R^{q} j_{*}^{\star}(H \otimes \mathbb{Q}) \\
& \simeq \varepsilon_{*}\left(R^{p} \underline{j}_{*}^{\circ} R^{q} \underline{j}_{*}^{\star} H\right) .
\end{aligned}
$$


Proof. We have only to check stalks at the origin $0 \in S$ and thus we replace the $E^{p}$ and $E_{2}^{p, q}$ by their stalks at 0 . Further, we may assume that $H$ is of type $\mathrm{I}_{\boldsymbol{a}}$. If $\boldsymbol{a}=0$, then $E^{p}$ and $E_{2}^{p, 1}$ are $\mathbb{Q}$-vector spaces by 4.4.1. Thus $E_{2}^{p, 0}$ also is a $\mathbb{Q}$-vector space for $p \geq 1$. Hence, we may assume that $\boldsymbol{a} \neq 0$. Let $\mathrm{N}_{1} \subset \mathrm{N}$ be a subgroup of finite index as in the proof of 3.1.4. Then there is a positive integer $n_{\mathrm{N}_{1}}(i)$ such that $\mathbb{R}_{\geq 0} \nu_{i} \cap \mathrm{N}_{1}=\mathbb{Z}_{\geq 0} n_{\mathrm{N}_{1}}(i) \nu_{i}$. Thus we have a commutative diagram

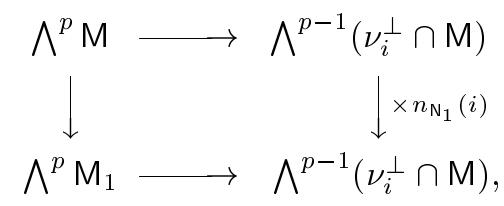

where the horizontal arrows are induced from $\nu_{i}: \mathrm{M} \rightarrow \mathbb{Z}$ and $n_{\mathrm{N}_{1}}(i) \nu_{i}: \mathrm{M}_{1} \rightarrow$ $\mathbb{Z}$. Then we have also a commutative diagram of complexes:

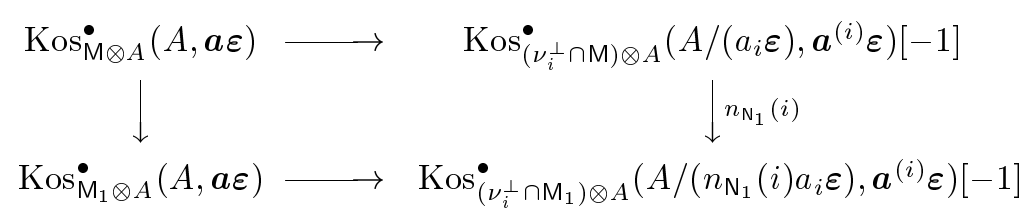

Let $L_{i}^{\bullet}$ denote the inductive limit of

$$
\operatorname{Kos}_{\left(\nu_{i}^{\perp} \cap \mathrm{M}\right) \otimes A}^{\bullet}\left(A /\left(a_{i} \varepsilon\right), \boldsymbol{a}^{(i)} \boldsymbol{\varepsilon}\right) \stackrel{n_{\mathrm{N}_{1}}(i)}{\longrightarrow} \operatorname{Kos}_{\left(\nu_{i}^{\perp} \cap \mathrm{M}_{1}\right) \otimes A}^{\bullet}\left(A /\left(n_{\mathrm{N}_{1}}(i) a_{i} \varepsilon\right), \boldsymbol{a}^{(i)} \boldsymbol{\varepsilon}\right)
$$

for all the finite index subgroups $\mathrm{N}_{1}$. Since the inductive limit $\lim _{\longrightarrow} A /\left(n_{\mathrm{N}_{1}}(i) a_{i} \varepsilon\right)$ is isomorphic to $A_{\mathbb{Q}} / a_{i} \varepsilon A$, where $A_{\mathbb{Q}}:=A \otimes \mathbb{Q}, L_{i}^{\bullet}$ is written as

$$
0 \rightarrow A_{\mathbb{Q}} / a_{i} \varepsilon A \stackrel{\wedge \boldsymbol{a}^{(i)}}{\longrightarrow} \mathrm{M}_{\mathbb{Q}} \otimes A /\left(a_{i} \varepsilon\right) \stackrel{\wedge \boldsymbol{a}^{(i)}}{\longrightarrow} \bigwedge^{2} \mathrm{M}_{\mathbb{Q}} \otimes A /\left(a_{i} \varepsilon\right) \stackrel{\wedge \boldsymbol{a}^{(i)}}{\longrightarrow} \cdots
$$

Then we may write

$$
L_{i}^{\bullet} \simeq \operatorname{Kos}_{\left(\nu_{i}^{\perp} \cap M_{\mathbb{Q}}\right) \otimes A}^{\bullet}\left(A_{\mathbb{Q}} / a_{i} \varepsilon A, \boldsymbol{a}^{(i)} \varepsilon\right) .
$$

The stalk $E_{2}^{p, 1}=\left(R^{p} \underline{j}_{*}^{\circ}\left(R^{1} \underline{j}_{*}^{\star} H\right)\right)_{0}$ is isomorphic to the direct sum of $p$-th cohomology groups $L_{i}^{\bullet}$ for $1 \leq i \leq k$. We have a triangle

$$
\begin{aligned}
\cdots \stackrel{+1}{\rightarrow} \operatorname{Kos}_{\nu_{i}^{\perp} \cap M_{\mathbb{Q}}}^{\bullet} & \left(\mathbb{Q} / a_{i} \mathbb{Z}, 0\right) \rightarrow L_{i}^{\bullet} \rightarrow \\
& \rightarrow \operatorname{Kos}_{\nu_{i}^{\perp} \cap M_{\mathbb{Q}}}^{\bullet}(\mathbb{Q}, 0) \stackrel{\wedge \boldsymbol{a}^{(i)}}{\longrightarrow} \operatorname{Kos}_{\nu_{i}^{\perp} \cap M_{\mathbb{Q}}}^{\bullet}\left(\mathbb{Q} / a_{i} \mathbb{Z}, 0\right)[1] \rightarrow \cdots
\end{aligned}
$$


If $a_{i}>0$, then $\operatorname{Kos}_{\nu_{i}^{\perp} \cap M_{\mathbb{Q}}}^{\bullet}\left(\mathbb{Q} / a_{i} \mathbb{Z}, 0\right)$ is isomorphic to the single complex $\mathbb{Q} / a_{i} \mathbb{Z}$. Thus, $H^{0}\left(L_{i}^{\bullet}\right) \simeq \mathbb{Q} \oplus \mathbb{Q} / a_{i} \mathbb{Z}$ and

$$
H^{p}\left(L_{i}^{\bullet}\right) \simeq \bigwedge^{p}\left(\nu_{i}^{\perp} \cap \mathrm{M}\right) \otimes \mathbb{Q} \simeq \mathbb{Q}^{\oplus\left(\begin{array}{c}
l-1 \\
p
\end{array}\right)}
$$

for $p>0$. If $a_{i}=0$, then $H^{0}\left(L_{i}^{\bullet}\right) \simeq \mathbb{Q}$ and

$$
\begin{aligned}
H^{p}\left(L_{i}^{\bullet}\right) \simeq & \operatorname{Ker}\left(\bigwedge^{p}\left(\nu_{i} \cap \mathrm{M}\right) \otimes \mathbb{Q} \stackrel{\wedge \boldsymbol{a}^{(i)}}{\longrightarrow} \bigwedge^{p+1}\left(\nu_{i} \cap \mathrm{M}\right) \otimes \mathbb{Q}\right) \\
& \oplus \operatorname{Coker}\left(\bigwedge_{p-1}^{p}\left(\nu_{i} \cap \mathrm{M}\right) \otimes \mathbb{Q} \stackrel{\wedge \boldsymbol{a}^{(i)}}{\longrightarrow} \bigwedge^{p}\left(\nu_{i} \cap \mathrm{M}\right) \otimes \mathbb{Q}\right) \\
\simeq & \bigwedge_{p}\left(\nu_{i} \cap \mathrm{M}\right) \otimes \mathbb{Q} \simeq \mathbb{Q}^{\oplus\left(\begin{array}{c}
l-1 \\
p
\end{array}\right)}
\end{aligned}
$$

for $p>0$. In particular, $E_{2}^{p, 1}$ is a $\mathbb{Q}$-vector space for $p \geq 1$. Thus $E_{2}^{p, 0}$ is also a $\mathbb{Q}$-vector space for $p \geq 3$. We also have isomorphisms $E^{1} \simeq \mathbb{Q} \boldsymbol{a} \oplus \mathrm{M}_{\mathbb{Q}} / \mathbb{Z} \boldsymbol{a}$ and $E_{2}^{0,1} \simeq \bigoplus_{a_{i}=0} \mathbb{Q} \oplus \bigoplus_{a_{i}>0}\left(\mathbb{Q} \oplus \mathbb{Q} / a_{i} \mathbb{Z}\right)$. The composite of $E^{1} \rightarrow E_{2}^{0,1}$ and the projection to the $i$-the direct summand is isomorphic to written by

$$
\mathbb{Q} \boldsymbol{a} \oplus \mathrm{M}_{\mathbb{Q}} / \mathbb{Z} \boldsymbol{a} \rightarrow \begin{cases}\mathbb{Q} & \text { for } a_{i}=0 \\ \mathbb{Q} \oplus \mathbb{Q} / a_{i} \mathbb{Z} & \text { for } a_{i}>0\end{cases}
$$

If $a_{i}=0$, then this homomorphism is induced from the evaluation map $\nu_{i}: \mathrm{M}_{\mathbb{Q}} /$ $\mathbb{Q} \boldsymbol{a} \rightarrow \mathbb{Q}$. If $a_{i}>0$, then this is the sum of the map $\mathbb{Q} \boldsymbol{a} \ni \boldsymbol{a} \mapsto 1 \in \mathbb{Q}$ and the map $\mathrm{M}_{\mathbb{Q}} / \mathbb{Z} \boldsymbol{a} \rightarrow \mathbb{Q} / a_{i} \mathbb{Z}$ induced from $\nu_{i}: \mathrm{M}_{\mathbb{Q}} \rightarrow \mathbb{Q}$. We have a commutative diagram

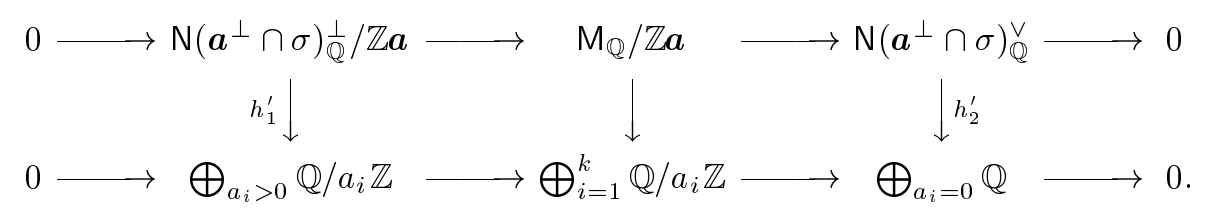

Here $h_{2}^{\prime}$ is injective and $h_{1}^{\prime}$ restricted to the torsion part $\mathbb{Q} \boldsymbol{a} / \mathbb{Z} \boldsymbol{a}$ is injective. Therefore, $E_{2}^{1,0}=H^{1}\left(\underline{S}^{\circ}, \underline{j}_{*}^{\star} H\right)$ is isomorphic to the kernel of $h_{1}$ and torsionfree. The cokernel of the middle arrow is isomorphic to $\mathcal{C}_{1}^{\prime} \oplus \mathcal{C}_{2}^{\prime}$, where $\mathcal{C}_{1}^{\prime}=$ Coker $h_{1}^{\prime}$ and $\mathcal{C}_{2}^{\prime}=$ Coker $h_{2}^{\prime}$. Here $\mathcal{C}_{2}^{\prime}$ is a $\mathbb{Q}$-vector space of dimension $k-k_{+}-$ $\left(l-l_{+}\right) . \mathcal{C}_{1}^{\prime}$ is a divisible group and is zero if $S^{\natural}$ has only quotient singularities. We have an exact sequence

$$
0 \rightarrow \mathbb{Q}^{\oplus\left(k_{+}-1\right)} \oplus \mathcal{C}_{1}^{\prime} \oplus \mathcal{C}_{2}^{\prime} \rightarrow E_{2}^{2,0} \rightarrow E_{\infty}^{2,0} \rightarrow 0 .
$$


Since $E^{2}$ and $E_{2}^{1,1}$ are $\mathbb{Q}$-vector spaces, $E_{\infty}^{2,0}$ is also a $\mathbb{Q}$-vector space and $\operatorname{dim} E_{\infty}^{2,0}=\left(\begin{array}{c}l_{+} \\ 2\end{array}\right)-\left(l_{+}-1\right)$ by 4.3.5. Thus $E_{2}^{2,0}$ is the direct sum of the divisible group $\mathcal{C}_{1}^{\prime}$ and a $\mathbb{Q}$-vector space of dimension $\left(\begin{array}{c}l_{+} \\ 2\end{array}\right)+k-l$.

Corollary 4.4.3. Let $\mathfrak{T}_{H / \underline{S}^{\circ}}$ be the torsion part of $R^{1} \underline{j}_{*}^{\star} H$ as a sheaf of abelian groups. Then we have the followings:

(1) The image of the composite $\underline{j}_{*}^{\circ} \mathfrak{T}_{H / \underline{S}^{\circ}} \hookrightarrow E_{2}^{0,1} \rightarrow E_{2}^{2,0}$ is the torsion part of $E_{2}^{2,0}$.

(2) $R^{p} \underline{j}_{*}^{\circ} \mathfrak{T}_{H / \underline{S}^{\circ}}=0$ for $p>0$.

Proof. We may assume the monodromy type is $\mathrm{I}_{\boldsymbol{a}}$ with $\boldsymbol{a} \neq 0$. As in the proof of 4.3 .3 and $4.4 .2,\left(R \underline{j}_{*}^{\circ} \mathfrak{T}_{H / \underline{S}^{\circ}}\right)_{0}$ is quasi-isomorphic to

$$
\bigoplus_{a_{i}>0} \operatorname{Kos}_{\nu_{i}^{\perp} \cap M_{\mathbb{Q}}}^{\bullet}\left(\mathbb{Q} / a_{i} \mathbb{Z}, 0\right)
$$

Hence $\left(\underline{j}_{*}^{\circ} \mathfrak{T}_{H / \underline{S}^{\circ}}\right)_{0} \simeq \bigoplus_{a_{i}>0} \mathbb{Q} / a_{i} \mathbb{Z}$. Thus (1) holds since the torsion part of $E_{2}^{2,0}$ is isomorphic to $\mathcal{C}_{1}^{\prime}$. Further, for $p>0$, we have

$$
\left(R^{p} \underline{j}_{*}^{\circ} \mathfrak{T}_{H / \underline{S}^{\circ}}\right)_{0} \simeq \bigoplus_{a_{i}>0} \bigwedge^{p}\left(\nu_{i}^{\perp} \cap \mathrm{M}\right) \otimes \mathbb{Q} \otimes \mathbb{Q} / a_{i} \mathbb{Z}=0 .
$$

\section{§5. Elliptic Fibration}

The notion of basic elliptic fibration plays an important role in the classification of elliptic fibration. A basic elliptic fibration is an elliptic fibration with a meromorphic section. This is bimeromorphically equivalent to a Weierstrass model. If we fix a VHS $H$ on the open part $S^{\star}$ for a toroidal embedding $S^{\star}=S \backslash D \subset S$, then there exists uniquely up to bimeromorphic equivalence over $S$, a basic elliptic fibration $\boldsymbol{p}: \boldsymbol{B}(H) \rightarrow S$. In Section 5.1, we recall Weierstrass models and properties of $\boldsymbol{B}(H)$. If $D$ is non-singular, then a non-singular relative minimal model of $\boldsymbol{B}(H)$ uniquely exists and it contains a so-called Néron model as a Zariski-open subset. As a classification of elliptic fibrations over $S$ with the same VHS as $H$ on $S^{\star}$, we introduce the set $\widetilde{\mathcal{E}}(S, D, H)$ of bimeromorphic equivalence classes of marked elliptic fibrations in Section 5.2 and some important subsets $\mathcal{E}(S, D, H), \mathcal{E}^{\operatorname{proj}}(S, D, H)$, and 
$\mathcal{E}_{0}(S, D, H)$. They are shown to have abelian group structures and are analogous to Weil-Châtelet groups or Tate-Shafarevich groups of elliptic curves defined in algebraic situation. The description of these groups corresponds to the classification. The sheaf $\mathfrak{S}_{H / S}$ of germs of meromorphic sections of the basic elliptic fibration has an abelian group structure and the cohomology group $H^{1}\left(S, \mathfrak{S}_{H / S}\right)$ is considered to be the set of meromorphic torsors of $\boldsymbol{B}(H)$. The subgroup $\mathcal{E}_{0}(S, D, H)$ consisting of elliptic fibrations having local meromorphic sections is realized as a subgroup of $H^{1}\left(S, \mathfrak{S}_{H / S}\right)$. If $D$ is non-singular, then $\mathcal{E}_{0}(S, D, H)=H^{1}\left(S, \mathfrak{S}_{H / S}\right)$.

\section{$\S 5.1 . \quad$ Weierstrass model}

Let $S$ be a normal complex analytic space. Let $\mathcal{L}$ be an invertible sheaf of $S$ and let $\boldsymbol{\alpha} \in H^{0}\left(S, \mathcal{L}^{\otimes(-4)}\right), \boldsymbol{\beta} \in H^{0}\left(S, \mathcal{L}^{\otimes(-6)}\right)$ be sections such that the zero locus of $4 \boldsymbol{\alpha}^{3}+27 \boldsymbol{\beta}^{2}$ is purely of codimension one. Let $D(\mathcal{L}, \boldsymbol{\alpha}, \boldsymbol{\beta})$ denote the $\operatorname{divisor} \operatorname{div}\left(4 \boldsymbol{\alpha}^{3}+27 \boldsymbol{\beta}^{2}\right)$. For such a triplet $(\mathcal{L}, \boldsymbol{\alpha}, \boldsymbol{\beta})$ above, the Weierstrass model $\mathbf{W}=\mathbf{W}_{S}(\mathcal{L}, \boldsymbol{\alpha}, \boldsymbol{\beta})$ is defined as follows [N3]: Let $\mathbf{p}: \mathbb{P} \rightarrow S$ be the projective bundle associated with the locally free sheaf $\mathcal{O}_{S} \oplus \mathcal{L}^{\otimes 2} \oplus \mathcal{L}^{\otimes 3}$. Here the tautological invertible sheaf $\mathcal{O}_{\mathbb{P}}(1)$ is determined by the isomorphism $\mathbf{p}_{*} \mathcal{O}_{\mathbb{P}}(1) \simeq \mathcal{O}_{S} \oplus \mathcal{L}^{\otimes 2} \oplus \mathcal{L}^{\otimes 3}$. According to the natural embeddings from $\mathcal{O}_{S}$, $\mathcal{L}^{\otimes 2}, \mathcal{L}^{\otimes 3}$ into $\mathcal{O}_{S} \oplus \mathcal{L}^{\otimes 2} \oplus \mathcal{L}^{\otimes 3}$, we have sections

$$
\begin{gathered}
\mathrm{Z} \in H^{0}\left(\mathbb{P}, \mathcal{O}_{\mathbb{P}}(1)\right), \quad \mathrm{X} \in H^{0}\left(\mathbb{P}, \mathcal{O}_{\mathbb{P}}(1) \otimes \mathbf{p}^{*} \mathcal{L}^{\otimes(-2)}\right), \\
\mathrm{Y} \in H^{0}\left(\mathbb{P}, \mathcal{O}_{\mathbb{P}}(1) \otimes \mathbf{p}^{*} \mathcal{L}^{\otimes(-3)}\right) .
\end{gathered}
$$

The Weierstrass model $\mathbf{W}$ is a divisor of $\mathbb{P}$ defined by the section

$$
\mathrm{Y}^{2} \mathrm{Z}-\left(\mathrm{X}^{3}+\boldsymbol{\alpha} \mathrm{XZ} \mathrm{Z}^{2}+\boldsymbol{\beta} \mathrm{Z}^{3}\right) \in H^{0}\left(\mathbb{P}, \mathcal{O}_{\mathbb{P}}(3) \otimes \mathbf{p}^{*} \mathcal{L}^{\otimes(-6)}\right) .
$$

The triplet $(\mathcal{L}, \boldsymbol{\alpha}, \boldsymbol{\beta})$ is called minimal if there is no prime divisor $\Gamma$ satisfying both $\operatorname{div}(\boldsymbol{\alpha}) \geq 4 \Gamma$ and $\operatorname{div}(\boldsymbol{\beta}) \geq 6 \Gamma$.

\section{Lemma 5.1.1.}

(1) $\mathbf{p}: \mathbf{W} \rightarrow S$ is a proper surjective flat Gorenstein morphism.

(2) $\omega_{\mathbf{W} / S} \simeq \mathbf{p}^{*} \mathcal{L}^{\otimes(-1)}$.

(3) $\mathbf{p}: \mathbf{W} \rightarrow S$ is smooth outside $D(\mathcal{L}, \boldsymbol{\alpha}, \boldsymbol{\beta})$.

(4) A fiber $\mathbf{W}_{s}=\mathbf{p}^{-1}(s)$ of $\mathbf{W} \rightarrow S$ is isomorphic to an irreducible plane cubic curve: an elliptic curve, a rational curve with one node, or a rational curve with one $(2,3)$-cusp. 
(5) The locus $\mathrm{X}=\mathrm{Z}=0$ defines a holomorphic section of $\mathbf{W} \rightarrow S$. This is called a canonical section. In each fiber $\mathbf{W}_{s}$, the intersection point is a point of inflection.

(6) Let $\mathbf{W}^{\sharp} \subset \mathbf{W}$ be the set of all points along which $\mathbf{W}$ is smooth over $S$. Then $\mathbf{W}^{\sharp} \rightarrow S$ has a structure of relative complex analytic Lie groups over $S$ (i.e., a group object in the category of complex analytic spaces over $S$ ) with the canonical section being zero.

(7) The (relative) left action of $\mathbf{W}^{\sharp}$ on $\mathbf{W}^{\sharp}$ over $S$ extends to that on $\mathbf{W}$.

(8) The relative tangent bundle of $\mathbf{W} \rightarrow S$ restricted to the canonical section is isomorphic to $\mathcal{L}$. Let $\mathbb{V}(\mathcal{L}) \rightarrow S$ be the (geometric) line bundle associated with $\mathcal{L}$. Then the relative exponential mapping $\mathbb{V}(\mathcal{L}) \rightarrow \mathbf{W}^{\sharp}$ is a surjective local isomorphism.

Proof. Locally $\mathbf{W} \rightarrow S$ is obtained as the pullback of a special Weierstrass model $\mathbf{W}(\mathcal{O}, x, y) \rightarrow \mathbb{C}^{2}$, where $(x, y)$ is a coordinate system of $\mathbb{C}^{2}$. Thus (1), (3), (4) hold. (2) is induced from the adjunction and the canonical bundle formula for a projective bundle. (5) is directly checked. (6) and (7) are essentially derived from the group structure of the non-singular part of a plane cubic curve with a point of inflection being zero. The first half of (8) is derived from (2). The latter half is also derived from a property of plane cubics.

We recall the following:

Theorem 5.1.2 ([N3, 2.1, 2.4]).

(1) Let $\pi: X \rightarrow S$ be an elliptic fibration between non-singular varieties. Suppose that $\pi$ is smooth outside an effective divisor $D$ of $S$ and that $\pi$ admits a holomorphic section $\boldsymbol{\sigma}: S \rightarrow X$. Then there exist a triplet $(\mathcal{L}, \boldsymbol{\alpha}, \boldsymbol{\beta})$ on $S$ and a bimeromorphic morphism $\mu: X \rightarrow \mathbf{W}_{S}(\mathcal{L}, \boldsymbol{\alpha}, \boldsymbol{\beta})$ over $S$ such that

(a) $\mathcal{L} \simeq \pi_{*}\left(\mathcal{O}_{X}(\boldsymbol{\sigma}(S)) \otimes \mathcal{O}_{\boldsymbol{\sigma}(S)}\right)$,

(b) $\mu$ is isomorphic over $S \backslash D$,

(c) $\mu \circ \sigma$ is the canonical section.

(2) Let $(\mathcal{L}, \boldsymbol{\alpha}, \boldsymbol{\beta})$ be a minimal triplet defined over a non-singular variety $S$ such that $D(\mathcal{L}, \boldsymbol{\alpha}, \boldsymbol{\beta})$ is a normal crossing divisor. Then the Weierstrass model $\mathbf{W}(\mathcal{L}, \boldsymbol{\alpha}, \boldsymbol{\beta})$ has only rational Gorenstein singularities. 
Next, we consider generalized Weierstrass models (cf. [N3, 3.1]). Let $\mathcal{L}$ be a reflexive sheaf of rank one. Suppose that it is $\mathbb{Q}$-invertible, i.e., the double-dual $\mathcal{L}^{[m]}$ of some multiple $\mathcal{L}^{\otimes m}$ is invertible but the index $m \neq 0$ exists locally on $S$. Let $\boldsymbol{\alpha} \in H^{0}\left(S, \mathcal{L}^{[-4]}\right)$ and $\boldsymbol{\beta} \in H^{0}\left(S, \mathcal{L}^{[-6]}\right)$ be sections such that $4 \boldsymbol{\alpha}^{3}+27 \boldsymbol{\beta}^{2}$ is not identically zero on $S$. We define $\mathbf{W}_{S}(\mathcal{L}, \boldsymbol{\alpha}, \boldsymbol{\beta})$ as follows: Suppose that $\mathcal{L}^{[m]}$ is invertible for a positive integer $m$. Then there is a finite Galois morphism $\tau: S^{\prime} \rightarrow S$ from a normal variety such that the double-dual $\mathcal{L}^{\prime}$ of $\tau^{*} \mathcal{L}$ is an invertible sheaf. Then we can define $\boldsymbol{\alpha}^{\prime}=\tau^{*} \boldsymbol{\alpha}$ and $\boldsymbol{\beta}^{\prime}=\tau^{*} \boldsymbol{\beta}$ as sections of $\mathcal{L}^{\prime \otimes(-4)}$ and $\mathcal{L}^{\prime \otimes(-6)}$, respectively. The Weierstrass model $\mathbf{W}^{\prime}=\mathbf{W}\left(\mathcal{L}^{\prime}, \boldsymbol{\alpha}^{\prime}, \boldsymbol{\beta}^{\prime}\right)$ admits a natural action of the Galois group of $\tau$ compatible with that on $S^{\prime}$. The quotient space $\mathbf{W}(\mathcal{L}, \boldsymbol{\alpha}, \boldsymbol{\beta})$ does not depend on the choice of the Galois covering $\tau: S^{\prime} \rightarrow S$. In general case, we can patch these local quotient spaces and obtain a global model $\mathbf{W}(\mathcal{L}, \boldsymbol{\alpha}, \boldsymbol{\beta}) \rightarrow S$. This is called a generalized Weierstrass model. The minimality of triplets $(\mathcal{L}, \boldsymbol{\alpha}, \boldsymbol{\beta})$ is similarly defined.

Now we restrict ourselves to the case that there is a reduced effective divisor $D$ such that $S^{\star}:=S \backslash D \subset S$ is a toroidal embedding. Let $H$ be a VHS defined over $S^{\star}$. There is a natural injection $H \hookrightarrow \mathcal{L}_{H}$. As a group object over $S^{\star}, H$ corresponds to a relative subgroup $\mathbb{V}(H)$ of the line bundle $\mathbb{V}\left(\mathcal{L}_{H}\right)$. The relative quotient group object $\boldsymbol{B}(H)^{\star}:=\mathbb{V}\left(\mathcal{L}_{H}\right) / \mathbb{V}(H)$ over $S^{\star}$ defines a smooth elliptic fibration $\boldsymbol{p}^{\star}: \boldsymbol{B}(H)^{\star} \rightarrow S^{\star}$ and its zero section. The sheaf $\mathfrak{S}_{H}$ of germs of sections of $\boldsymbol{B}(H)^{\star} \rightarrow S^{\star}$ is isomorphic to the cokernel of $H \hookrightarrow \mathcal{L}_{H}$. There is an isomorphism $R^{1} \boldsymbol{p}_{*}^{\star} \mathbb{Z}_{\boldsymbol{B}(H)^{\star}} \simeq H$ as VHS.

Definition. The $\boldsymbol{p}^{\star}: \boldsymbol{B}(H)^{\star} \rightarrow S^{\star}$ is called the smooth basic elliptic fibration associated with $H$. We sometimes write $\boldsymbol{B}^{\star}=\boldsymbol{B}(H)^{\star}$ if $H$ is fixed.

By 5.1.2, we have a triplet $\left(\mathcal{L}_{H}, \boldsymbol{\alpha}^{\star}, \boldsymbol{\beta}^{\star}\right)$ such that $\boldsymbol{B}(H)^{\star}$ is isomorphic to $\mathbf{W}(H)^{\star}:=\mathbf{W}\left(\mathcal{L}_{H}, \boldsymbol{\alpha}^{\star}, \boldsymbol{\beta}^{\star}\right)$ over $S^{\star}$, where the zero section is sent to the canonical section.

Let $\mathcal{L}_{H / S}$ be the reflexive sheaf defined in 4.2.1. By [N3, 2.5], there exist sections $\boldsymbol{\alpha} \in H^{0}\left(S, \mathcal{L}_{H / S}^{[-4]}\right)$ and $\boldsymbol{\beta} \in H^{0}\left(S, \mathcal{L}_{H / S}^{[-6]}\right)$ such that

(1) $\boldsymbol{\alpha}^{\star}=\left.\varepsilon^{4} \boldsymbol{\alpha}\right|_{S^{\star}}$ and $\boldsymbol{\beta}^{\star}=\left.\varepsilon^{6} \boldsymbol{\beta}\right|_{S^{\star}}$ for a nowhere-vanishing function $\boldsymbol{\varepsilon}$ on $S^{\star}$,

(2) $\left(\mathcal{L}_{H / S}, \boldsymbol{\alpha}, \boldsymbol{\beta}\right)$ is minimal.

In fact, $[\mathrm{N} 3,2.5]$ treated the case where $S$ is non-singular, and our case is reduced to the case, since $\mathcal{L}_{H / S}$ is reflexive. Moreover, $\mathcal{L}_{H / S}$ is $\mathbb{Q}$-invertible, since $\mathcal{L}_{H / \underline{S}}$ is invertible for $\underline{S}=(S, D)$. Therefore, the smooth elliptic fibration $\boldsymbol{p}^{\star}: \underline{\boldsymbol{B}}(H)^{\star} \rightarrow S^{\star}$ extends to the generalized Weierstrass model $\mathbf{W}(H):=$ 
$\mathbf{W}\left(\mathcal{L}_{H / S}, \boldsymbol{\alpha}, \boldsymbol{\beta}\right) \rightarrow S$. We call $\mathbf{p}: \mathbf{W}(H) \rightarrow S$ by the generalized Weierstrass model associated with $H$.

Remark. Suppose that the local monodromies of $H$ around $D$ are all unipotent. Let $\tau: S^{\prime} \rightarrow S$ be a holomorphic mapping such that $S^{\prime}$ is normal and that $\tau^{-1} S^{\star} \subset S^{\prime}$ is a toroidal embedding. Then for the pullback $H^{\prime}=\tau^{-1}(H)$ defined on $\tau^{-1} S^{\star}$, we have $\mathcal{L}_{H^{\prime} / S^{\prime}} \simeq \tau^{*} \mathcal{L}_{H / S}$. Thus $\mathbf{W}(H) \times_{S} S^{\prime}$ is isomorphic to $\mathbf{W}\left(H^{\prime}\right)$.

Theorem 5.1.3. For the generalized Weierstrass model $\mathbf{p}: \mathbf{W}(H)=$ $\mathbf{W}\left(\mathcal{L}_{H / S}, \boldsymbol{\alpha}, \boldsymbol{\beta}\right) \rightarrow S$ associated with $H$, let $\boldsymbol{\sigma}_{0}: S \rightarrow \mathbf{W}(H)$ be the canonical section and let $\phi_{0}$ be an isomorphism $R^{1} \mathbf{p}_{*}^{\star} \mathbb{Z}_{\mathbf{W}(H)^{\star}} \rightarrow H$ of VHS. Let $\boldsymbol{p}: \boldsymbol{B} \rightarrow S$ be an elliptic fibration having a meromorphic section $\boldsymbol{\sigma}: S \cdots \rightarrow \boldsymbol{B}$. Suppose that

(1) the restriction $p^{-1}\left(S^{\star}\right) \rightarrow S^{\star}$ is bimeromorphically equivalent to a smooth elliptic fibration $\boldsymbol{p}^{\prime \star}: \boldsymbol{B}^{\prime \star} \rightarrow S^{\star}$ over $S^{\star}$,

(2) there is an isomorphism $\phi: R^{1}{\boldsymbol{p}^{\prime \star}}^{\star} \mathbb{Z}_{\boldsymbol{B}^{\prime \star}} \leadsto H$ as VHS.

Then, there is a bimeromorphic mapping $\mu: \boldsymbol{B} \cdots \rightarrow \mathbf{W}(H)$ over $S$ such that $\mu \circ \boldsymbol{\sigma}$ is the canonical section and $\phi_{0}=\left.\phi \circ \mu^{*}\right|_{S^{\star}}$.

Proof. Let $\mu: \hat{S} \rightarrow S$ be a resolution of singularities such that $\hat{D}:=\mu^{-1} D$ is a normal crossing divisor and that $\hat{S} \backslash \hat{D} \simeq S^{\star}$ by $\mu$. The double-dual of $\mu_{*} \mathcal{L}_{H / \hat{S}}$ is $\mathcal{L}_{H / S}$. By [N3, 2.5], there are sections $\hat{\boldsymbol{\alpha}} \in H^{0}\left(\hat{S}, \mathcal{L}_{H / \hat{S}}^{\otimes(-4)}\right)$ and $\hat{\boldsymbol{\beta}} \in$ $H^{0}\left(\hat{S}, \mathcal{L}_{H / \hat{S}}^{\otimes(-6)}\right)$ corresponding to $\boldsymbol{\alpha}$ and $\boldsymbol{\beta}$, respectively. Then $\mathbf{W}\left(\mathcal{L}_{H / \hat{S}}, \hat{\boldsymbol{\alpha}}, \hat{\boldsymbol{\beta}}\right)$ is bimeromorphically equivalent to $\mathbf{W}\left(\mathcal{L}_{H / S}, \boldsymbol{\alpha}, \boldsymbol{\beta}\right)$ over $S$. Therefore, we may assume that $S$ is non-singular. By 5.1.2, we may assume that $\boldsymbol{B}=\mathbf{W}\left(\mathcal{L}^{\prime}, \boldsymbol{\alpha}^{\prime}, \boldsymbol{\beta}^{\prime}\right)$ for a minimal triplet $\left(\mathcal{L}^{\prime}, \boldsymbol{\alpha}^{\prime}, \boldsymbol{\beta}^{\prime}\right)$. Then $\mathcal{L}^{\prime} \simeq \mathcal{L}_{H / S}$. We have an isomorphism $\mathbf{W}\left(\mathcal{L}^{\prime}, \boldsymbol{\alpha}^{\prime}, \boldsymbol{\beta}^{\prime}\right) \simeq \mathbf{W}\left(\mathcal{L}_{H / S}, \boldsymbol{\alpha}, \boldsymbol{\beta}\right)$ preserving canonical sections by [N3, 2.5]. For an automorphism $\varphi: H \simeq H$ as VHS, we have an automorphism $f: \mathbf{W}\left(\mathcal{L}_{H / S}, \boldsymbol{\alpha}, \boldsymbol{\beta}\right) \leadsto \mathbf{W}\left(\mathcal{L}_{H / S}, \boldsymbol{\alpha}, \boldsymbol{\beta}\right)$ over $S$ such that $\left.f^{*}\right|_{S^{\star}}=\varphi$. Hence we have a required bimeromorphic mapping $\mu: B \cdots \rightarrow \mathbf{W}(H)$.

Definition. An elliptic fibration is called a basic elliptic fibration if it admits a meromorphic section. We call the elliptic fibration $\boldsymbol{p}: \boldsymbol{B} \rightarrow S$ satisfying the condition of 5.1 .3 by the basic elliptic fibration associated with $H$. We write $\boldsymbol{B}=\boldsymbol{B}(H)$ with respect to $H$.

If $\mathcal{L}_{H / S}$ is invertible, then $\mathbf{W}=\mathbf{W}\left(\mathcal{L}_{H / S}, \boldsymbol{\alpha}, \boldsymbol{\beta}\right)$ defined above is a usual Weierstrass model. The image of a holomorphic section $S \rightarrow \mathbf{W}$ is contained in 
the open subset $\mathbf{W}^{\sharp}$. Thus we can define the sheaf $\mathfrak{S}_{H / S}^{\mathbf{W}}$ of germs of holomorphic sections of $\mathbf{W} \rightarrow S$ from the group structure of $\mathbf{W}^{\sharp} \rightarrow S$. By the surjective exponential mapping $\mathbb{V}\left(\mathcal{L}_{H / S}\right) \rightarrow \mathbf{W}^{\sharp}$, we have a short exact sequence

$$
0 \rightarrow j_{*} H \rightarrow \mathcal{L}_{H / S} \rightarrow \mathfrak{S}_{H / S}^{\mathbf{W}} \rightarrow 0 .
$$

We thus define $\mathfrak{S}_{H / S}^{\mathbf{W}}$ by the exact sequence above also in the case $\mathcal{L}_{H / S}$ is not invertible.

Lemma 5.1.4. $\quad \mathfrak{S}_{H / S}^{\mathrm{W}}$ is isomorphic to the sheaf of germs of automorphisms $\varphi: \mathbf{W} \leadsto \mathbf{W}$ over $S$ such that $\left.\varphi^{*}\right|_{S^{\star}}$ is identical on $\left.R^{1} \mathbf{p}_{*} \mathbb{Z}_{\mathbf{W}}\right|_{S^{*}}$. This is also isomorphic to the sheaf of germs of holomorphic sections of $\mathbf{W} \rightarrow S$.

Proof. Let $\tau: \mathcal{U}^{\prime} \rightarrow \mathcal{U}$ be a finite Galois covering over an open neighborhood $\mathcal{U}$ of $s \in S$ such that it is étale outside $D$ and that the pullback $H^{\prime}=\tau^{-1} H$ defined on $\mathcal{U}^{\prime} \backslash \tau^{-1} D$ has only unipotent monodromies. Then $\mathcal{L}_{H^{\prime} / \mathcal{U}^{\prime}}$ is invertible and $\left.\mathcal{L}_{H / S}\right|_{\mathcal{U}}$ is the $G$-invariant part of $\tau_{*} \mathcal{L}_{H^{\prime} / S^{\prime}}$ for the Galois group $G$. Thus the $G$-invariant part of $\tau_{*} \mathfrak{S}_{H^{\prime} / \mathcal{U}^{\prime}}^{\mathbf{W}}$ is isomorphic to $\mathfrak{S}_{H / S}^{\mathbf{W}} \mid \mathcal{U}$. Let $\mathbf{W}^{\prime}:=\mathbf{W}_{\mathcal{U}^{\prime}}\left(\mathcal{L}_{H^{\prime} / \mathcal{U}^{\prime}}, \boldsymbol{\alpha}^{\prime}, \boldsymbol{\beta}^{\prime}\right)$ be the minimal Weierstrass model.

Let $\eta$ be a section of $\mathfrak{S}_{H / S}^{\mathbf{W}}$ defined over $\mathcal{U}$. As a section of $\tau_{*} \mathfrak{S}_{H^{\prime} / \mathcal{U}^{\prime}}^{\mathbf{W}}$, it defines a $G$-equivariant section $\sigma^{\prime}: \mathcal{U}^{\prime} \rightarrow \mathbf{W}^{\prime}$ of $\mathbf{W}^{\prime} \rightarrow \mathcal{U}^{\prime}$. Thus we have a holomorphic section of $\mathbf{W} \rightarrow S$ over $\mathcal{U}$. Further, $\eta$ acts on $\mathbf{W}^{\prime}$ as the translation mapping over $\mathcal{U}^{\prime}$ by $\boldsymbol{\sigma}^{\prime}$. This induces an automorphism of $\mathbf{W}$ over $\mathcal{U}$ that preserves the VHS $H$.

Let $\varphi$ be an automorphism $\mathbf{W} \rightarrow \mathbf{W}$ over $\mathcal{U}$ preserving $H$. Then, for the Galois covering $\mathcal{U}^{\prime} \rightarrow \mathcal{U}$, it defines an automorphism $\varphi^{\prime}: \mathbf{W}^{\prime} \simeq \mathbf{W}^{\prime}$, since $\mathbf{W}^{\prime} \rightarrow \mathbf{W}$ is finite over $\mathcal{U}$. For the canonical section $\sigma_{0}^{\prime}: \mathcal{U}^{\prime} \rightarrow \mathbf{W}^{\prime}$, let $\sigma^{\prime}:=$ $\varphi^{\prime} \circ \boldsymbol{\sigma}_{0}^{\prime}$. If $\boldsymbol{\sigma}^{\prime}=\boldsymbol{\sigma}_{0}^{\prime}$, then $\varphi^{\prime}$ is the identity of $\mathbf{W}^{\prime}$, since it preserves $H^{1}\left(\mathbf{W}_{s^{\prime}}^{\prime}, \mathbb{Z}\right)$ for any smooth fibers $\mathbf{W}_{s^{\prime}}^{\prime}$. Thus $\varphi^{\prime}$ is the translation mapping by the section $\boldsymbol{\sigma}^{\prime}$. By the construction, $\boldsymbol{\sigma}^{\prime}$ is a $G$-equivariant section of $\mathbf{W}^{\prime} \rightarrow \mathcal{U}^{\prime}$.

If $\boldsymbol{\sigma}$ is a holomorphic section of $\mathbf{W} \rightarrow S$ over $\mathcal{U}$, then it induces a holomorphic mapping $\sigma^{\prime}: \mathcal{U}^{\prime} \rightarrow \mathbf{W}^{\prime}$, since $\mathcal{U}^{\prime}$ is normal and $\mathbf{W}^{\prime} \rightarrow \mathbf{W}$ is finite over $\mathcal{U}$. Since $\boldsymbol{\sigma}^{\prime}$ is $G$-equivariant, it is considered to be a section of $\mathfrak{S}_{H / S}^{\mathbf{W}}$.

Let $\eta$ be an element of $H^{1}\left(S, \mathfrak{S}_{H / S}^{\mathbf{W}}\right)$. Then $\eta$ is represented by a cocycle $\left\{\eta_{\lambda, \mu}\right\}_{\lambda, \mu \in \Lambda}$ with respect to an open covering $\left\{S_{\lambda}\right\}_{\lambda \in \Lambda}$ of $S$, where $\eta_{\lambda, \mu}$ is a holomorphic section of $\mathbf{W} \rightarrow S$ over $S_{\lambda} \cap S_{\mu}$ satisfying $\eta_{\lambda, \mu}=-\eta_{\mu, \lambda}$ and $\eta_{\lambda, \mu}+\eta_{\mu, \nu}+\eta_{\nu, \lambda}=0$ on $S_{\lambda} \cap S_{\mu} \cap S_{\nu}$. By 5.1.4, we can glue $\mathbf{p}^{-1}\left(S_{\lambda}\right)=\mathbf{W}$ $\times_{S} S_{\lambda}$ by the cocycle. Then we have an elliptic fibration $\mathbf{p}^{\eta}: \mathbf{W}^{\eta} \rightarrow S$ that is smooth over $S^{\star}$ with $H\left(\mathbf{p}^{\eta}\right) \simeq H$. This depends only on the cohomology class $\eta$. There is a section of $\mathbf{p}^{\eta}$ if and only if $\eta=0$. 
Remark. The statement of $[\mathrm{N} 3,2.11]$ is not true. We must replace meromorphic sections by holomorphic sections.

\section{§5.2. Classification problem}

Let $S$ be a normal complex analytic variety and let $D$ be a reduced effective divisor such that $S^{\star}:=S \backslash D \subset S$ is a toroidal embedding. We denote the $\partial$-space $(S, D)$ by $\underline{S}$ as before. We want to classify elliptic fibrations $f: X \rightarrow S$ satisfying:

Condition 5.2.1. The restriction $f^{-1}\left(S^{\star}\right) \rightarrow S^{\star}$ is bimeromorphically equivalent to a smooth elliptic fibration over $S^{\star}$.

For the elliptic fibration $f: X \rightarrow S$ satisfying the condition 5.2.1, the smooth elliptic fibration $f^{\prime \star}: X^{\prime \star} \rightarrow S^{\star}$ bimeromorphically equivalent to $f^{-1}\left(S^{\star}\right)$ $\rightarrow S^{\star}$ over $S^{\star}$ is uniquely determined up to isomorphisms over $S^{\star}$. In particular, we can define a VHS

$$
H(f):=R^{1}{f^{\prime \star}}^{*} \mathbb{Z}_{X^{\prime \star}} .
$$

Note that if $X$ is non-singular, then $\left.H(f) \simeq\left(R^{1} f_{*} \mathbb{Z}_{X}\right)\right|_{S^{\star}}$ as local constant systems.

A VHS $H$ over $S^{\star}$ is determined by a period mapping and a monodromy representation. By a property of Weierstrass model, $H$ is also determined by a minimal triplet $(\mathcal{L}, \boldsymbol{\alpha}, \boldsymbol{\beta})$ consisting of a $\mathbb{Q}$-invertible reflexive sheaf $\mathcal{L}$ and sections $\boldsymbol{\alpha} \in H^{0}\left(S, \mathcal{L}^{[-4]}\right), \boldsymbol{\beta} \in H^{0}\left(S, \mathcal{L}^{[-6]}\right)$ such that $\operatorname{div}\left(4 \boldsymbol{\alpha}^{3}+27 \boldsymbol{\beta}^{2}\right) \subset D$. Thus the classification of such VHS is related to a kind of moduli problem. Hence, we fix such a VHS $H$ and consider the classification of marked elliptic fibrations (with respect to $(S, D, H)$ ) defined as follows: A marked elliptic fibration is a pair $(f: X \rightarrow S, \phi)$ consisting of an elliptic fibration $f: X \rightarrow S$ from a normal variety satisfying the condition 5.2.1 and of an isomorphism $\phi: H(f) \leadsto H$ as VHS. The $\phi$ is called a marking of $f$. Two marked elliptic fibrations $\left(f_{1}: X_{1} \rightarrow S, \phi_{1}\right)$ and $\left(f_{2}: X_{2} \rightarrow S, \phi_{2}\right)$ are called bimeromorphically equivalent over $S$, if there is a bimeromorphic mapping $\mu: X_{1} \cdots \rightarrow X_{2}$ over $S$ such that $\phi_{2}=\phi_{1} \circ \mu^{*}$. The marked elliptic fibration $(\boldsymbol{p}: \boldsymbol{B}(H) \rightarrow S, \phi)$ for a basic elliptic fibration $\boldsymbol{p}$ associated with $H$ is unique up to the bimeromorphic equivalence relation by 5.1.3.

Let $\widetilde{\mathcal{E}}(S, D, H)$ denote the set of bimeromorphic equivalence classes of all the marked elliptic fibrations with respect to $(S, D, H)$.

The set $\widetilde{\mathcal{E}}\left(S^{\star}, \emptyset, H\right)$ is identical to the set of torsors of the smooth basic elliptic fibration $\boldsymbol{p}^{\star}: \boldsymbol{B}(H)^{\star} \rightarrow S^{\star}$. Therefore, we have a one to one correspon- 
dence:

$$
\widetilde{\mathcal{E}}\left(S^{\star}, \emptyset, H\right) \longleftrightarrow H^{1}\left(S^{\star}, \mathfrak{S}_{H}\right)
$$

Remark 5.2.2. In purely algebraic context, Tate-Shafarevich group is similarly defined to $\widetilde{\mathcal{E}}(S, D, H)$. Let $\mathrm{S}$ be an irreducible normal separated algebraic scheme over Spec $\mathbb{C}$ and let $\mathrm{D}$ be a divisor such that $\mathrm{S}^{\star}:=\mathrm{S} \backslash \mathrm{D} \hookrightarrow \mathrm{S}$ is a toroidal embedding. Assume that $S=\mathrm{S}^{\mathrm{an}}, S^{\star}=\left(\mathrm{S}^{\star}\right)^{\text {an }}$, and $D=\mathrm{D}^{\text {an }}$. There is a basic elliptic fibration $\mathrm{p}: \mathrm{B} \rightarrow \mathrm{S}$ smooth over $\mathrm{S}^{\star}$ such that $\boldsymbol{p}=\mathrm{p}^{\text {an }}: \boldsymbol{B}(H)=$ $\mathrm{B}^{\text {an }} \rightarrow S$ is a basic elliptic fibration associated with $H$. Let $\eta$ denote the generic point of $S$ and let $B_{\eta}$ denote the generic fiber of $\mathrm{p}$. The curve $B_{\eta}$ is a smooth curve of genus one defined over the field $\mathbb{C}(\eta)=\mathbb{C}(S)$ that admits a rational point. Thus $\mathrm{B}_{\eta}$ is a group scheme over $\mathbb{C}(\eta)$. The Weil-Châtelet group $\mathrm{WC}\left(\mathrm{B}_{\eta}\right)$ is the group $H^{1}$ (Spec $\left.\mathbb{C}(\eta)_{\text {ét }}, \mathrm{B}_{\eta}\right)$ of isomorphism classes of torsors. The torsor is a pair $(C, \Phi)$ consisting of a smooth projective curve $C$ of genus one over $\mathbb{C}(\eta)$ and an isomorphism $\Phi: C \times_{\mathbb{C}(\eta)} C \leadsto \mathrm{B}_{\eta} \times_{\mathbb{C}(\eta)} C$ over $C$ via second projection that sends the diagonal to the zero section. Two pairs $\left(C_{1}, \Phi_{1}\right)$ and $\left(C_{2}, \Phi_{2}\right)$ are called isomorphic if there is an isomorphism $\mu: C_{1} \leadsto C_{2}$ such that $(\mathrm{id} \times \mu) \circ \Phi_{1}=\Phi_{2} \circ(\mu \times \mu)$. The Tate-Shafarevich group $\amalg_{\mathrm{S}}\left(\mathrm{B}_{\eta}\right)$ is the subgroup of $\mathrm{WC}\left(\mathrm{B}_{\eta}\right)$ consisting of étale locally trivial torsors. This is expressed as $H^{1}\left(\mathrm{~S}_{\text {ét }}, \iota_{*} \mathrm{~B}_{\eta}\right)$ for the morphism $\iota: \operatorname{Spec} \mathbb{C}(\eta) \rightarrow \mathrm{S}$ (cf. [D5]). In particular, $\amalg_{\mathrm{S}^{\star}}\left(\mathrm{B}_{\eta}\right)$ is similar to $\widetilde{\mathcal{E}}(S, D, H)$ or $\widetilde{\mathcal{E}}\left(S^{\star}, \emptyset, H\right)$ (cf. 7.2$)$.

Remark. In the complex analytic situation, the restriction map $\widetilde{\mathcal{E}}(S, D$, $H) \rightarrow \widetilde{\mathcal{E}}\left(S^{\star}, \emptyset, H\right)$ is not necessarily injective. For example, an elliptic surface with multiple fibers is constructed from an elliptic surface without multiple fibers by means of logarithmic transformations. Two surfaces are isomorphic outside the related fibers, but these are not bimeromorphically equivalent to each other.

Definition 5.2.3. Let $f: X \rightarrow S$ be a proper surjective morphism of normal complex analytic varieties.

(1) An invertible sheaf $\mathcal{A}$ of $X$ is called $f$-ample, if $\left.\mathcal{A}\right|_{f^{-1}(s)}$ is ample for any fiber $f^{-1}(s)$.

(2) $f$ is called a projective morphism, if there is an $f$-ample invertible sheaf on $X$.

(3) $f$ is called a locally projective morphism, if there is an open covering $\left\{S_{\lambda}\right\}$ of $S$ such that $f^{-1}\left(S_{\lambda}\right) \rightarrow S_{\lambda}$ is projective for any $\lambda$. 
(4) $f$ is called $B P$, if $f$ is bimeromorphically equivalent to a projective morphism over $S$.

(5) $f$ is called $L B P$, if there is an open covering $\left\{S_{\lambda}\right\}$ of $S$ such that $f^{-1}\left(S_{\lambda}\right) \rightarrow$ $S_{\lambda}$ is BP for any $\lambda$.

Remark $([\mathrm{N} 2,1.6]) . \quad$ If $\mathcal{A}$ is $f$-ample, then there exist an open covering $S=\bigcup S_{\lambda}$, positive integers $m_{\lambda}, n_{\lambda}$, and closed embeddings $f^{-1} S_{\lambda} \hookrightarrow \mathbb{P}^{n_{\lambda}} \times S_{\lambda}$ over $S_{\lambda}$ such that the pullback of the tautological invertible sheaf $\mathcal{O}(1)$ of $\mathbb{P}^{n_{\lambda}}$ to $f^{-1} S_{\lambda}$ is isomorphic to the restriction of $\mathcal{A}^{\otimes m_{\lambda}}$ to $f^{-1} S_{\lambda}$.

We introduce some important subsets of $\widetilde{\mathcal{E}}(S, D, H)$.

\section{Definition 5.2.4.}

$$
\begin{aligned}
& \mathcal{E}(S, D, H):=\{(f: X \rightarrow S, \phi) \in \widetilde{\mathcal{E}}(S, D, H) \mid f \text { is LBP }\} ; \\
& \mathcal{E}^{\operatorname{proj}}(S, D, H):=\{(f: X \rightarrow S, \phi) \in \widetilde{\mathcal{E}}(S, D, H) \mid f \text { is } \mathrm{BP}\} ; \\
& \widetilde{\mathcal{E}}_{0}\left(S, D, H ; S^{\nabla}\right):=\{(f: X \rightarrow S, \phi) \in \widetilde{\mathcal{E}}(S, D, H) \mid f \text { admits local } \\
&\text { meromorphic sections over any points of } \left.S^{\nabla}\right\} ; \\
& \mathcal{E}_{0}\left(S, D, H ; S^{\nabla}\right):= \mathcal{E}(S, D, H) \cap \widetilde{\mathcal{E}}_{0}\left(S, D, H ; S^{\nabla}\right) ; \\
& \mathcal{E}_{0}^{\text {proj }}\left(S, D, H ; S^{\nabla}\right):= \mathcal{E}^{\operatorname{proj}}(S, D, H) \cap \widetilde{\mathcal{E}}_{0}\left(S, D, H ; S^{\nabla}\right),
\end{aligned}
$$

for a Zariski-open subset $S^{\star} \subset S^{\nabla} \subset S$. We write $\mathcal{E}_{0}(S, D, H):=\widetilde{\mathcal{E}}_{0}(S, D, H ; S)$ $=\mathcal{E}_{0}(S, D, H ; S), \mathcal{E}_{0}^{\text {proj }}(S, D, H):=\mathcal{E}_{0}^{\text {proj }}(S, D, H ; S)$, for the sake of simplicity. Here, we denote by $(f: X \rightarrow S, \phi)$ the bimeromorphic equivalence class of $(f: X \rightarrow S, \phi)$ by abuse of notation.

Proposition 5.2.5. $\quad \mathcal{E}(S, D, H)$ has an abelian group structure with the class of basic elliptic fibration being zero. The restriction map $\mathcal{E}(S, D, H) \rightarrow$ $\mathcal{E}\left(S^{\star}, \emptyset, H\right) \leadsto H^{1}\left(S^{\star}, \mathfrak{S}_{H}\right)$ is a group homomorphism.

Proof. We shall define the addition + and the inverse $\eta \mapsto-\eta$ on $\mathcal{E}(S, D$, $H)$ in a natural way. Let $\eta=(f: X \rightarrow S, \phi)$ be a marked elliptic fibration. We set $-\eta:=(f: X \rightarrow S,-\phi)$ for the marking

$$
-\phi: H(f) \stackrel{\phi}{\rightarrow} H \stackrel{\times(-1)}{\longrightarrow} H .
$$

If $\eta$ is a basic elliptic fibration, then $\eta=-\eta$. Let $\eta_{1}=\left(f_{1}: X_{1} \rightarrow S, \phi_{1}\right)$ and $\eta_{2}=\left(f_{2}: X_{2} \rightarrow S, \phi_{2}\right)$ be two marked elliptic fibrations belonging to $\mathcal{E}(S, D, H)$. Let

$$
\eta_{i}^{\star}=\left(f_{i}^{\star}: X_{i}^{\star}=f_{i}^{-1} S^{\star} \rightarrow S^{\star}, \phi_{i}\right)
$$


be the restriction of $\eta_{i}$ to $\mathcal{E}\left(S^{\star}, \emptyset, H\right)$ for $i=1,2$. Assume that $f_{i}^{\star}$ are smooth. Then $\eta_{3}^{\star}:=\eta_{1}^{\star}+\eta_{2}^{\star}$ defines a marked smooth elliptic fibration $\eta_{3}^{\star}=\left(f_{3}^{\star}: X_{3}^{\star} \rightarrow\right.$ $\left.S^{\star}, \phi_{3}\right)$ and a morphism

$$
a^{\star}: X_{1}^{\star} \times_{S^{\star}} X_{2}^{\star} \rightarrow X_{3}^{\star}
$$

over $S^{\star}$ as a gluing of the addition map $\boldsymbol{B}(H)^{\star} \times_{S^{\star}} \boldsymbol{B}(H)^{\star} \rightarrow \boldsymbol{B}(H)^{\star}$. Let $Y$ denote the fiber product $X_{1} \times_{S} X_{2}, Y^{\star}:=X_{1}^{\star} \times_{S^{\star}} X_{2}^{\star}$ and let $\Gamma^{\star}$ denote $Y^{\star} \times_{X_{3}^{\star}} Y^{\star}$. Then $\Gamma^{\star} \subset Y^{\star} \times_{S^{\star}} Y^{\star}$ is proper and smooth over $Y^{\star}$ by the second projection and the fiber of the projection $\Gamma^{\star} \rightarrow Y^{\star}$ over $y \in Y^{\star}$ is isomorphic to the elliptic curve $E_{y}:=\left(a^{\star}\right)^{-1}\left(a^{\star}(y)\right)$. Therefore, it defines a morphism $h: Y^{\star} \rightarrow \mathcal{D}_{Y / S}$ into the relative Douady space $Y$ over $S$. Let $\pi: Y \rightarrow S$ be the structure morphism and let $Y_{y}$ denote the fiber $\pi^{-1}(\pi(y))$. Then the relative Zariski-tangent space of $\mathcal{D}_{Y / S}$ at $h(y)$ is isomorphic to $H^{0}\left(E_{y}, N_{E_{y} / Y_{y}}\right) \simeq H^{0}\left(E_{y}, \mathcal{O}_{E_{y}}\right) \simeq \mathbb{C}$. Thus $h$ is smooth and $h\left(Y^{\star}\right)$ is a connected component of $\mathcal{D}_{Y / S}$ near $h\left(Y^{\star}\right)$. By the construction, $h\left(Y^{\star}\right) \simeq X_{3}^{\star}$. By [F2], there is a subvariety $X_{3} \subset \mathcal{D}_{Y / S}$ proper over $S$ containing $h\left(Y^{\star}\right)$ as a Zariski-open subset. Let $\Gamma \subset Y \times_{S} X_{3}$ be the induced family of subspaces of $Y$. Then $\left.\Gamma\right|_{S^{\star}}$ is isomorphic to the graph of $a^{\star}: Y^{\star} \rightarrow X_{3}^{\star}$. In particular, the first projection $\Gamma \rightarrow Y$ is bimeromorphic. Therefore, $\Gamma$ defines a meromorphic mapping $a: Y \cdots \rightarrow X_{3}$ that is an extension of $a^{\star}$. We can define $\eta_{3}=\eta_{1}+\eta_{2}$ by the induced elliptic fibration $X_{3} \rightarrow S$. If $f_{i}^{\star}$ are not smooth over $S^{\star}$, then we replace $S^{\star}$ by an open dense subset over which $f_{i}^{\star}$ are both smooth and apply the same argument above. Then $\eta_{1}+\eta_{2}$ is similarly defined and is compatible with the addition of $H^{1}\left(S^{\star}, \mathfrak{S}_{H}\right)$.

Corollary 5.2.6. $\quad \widetilde{\mathcal{E}}(S, D, H)$ has a structure of an abelian group. The subsets $\mathcal{E}(S, D, H)$ and $\widetilde{\mathcal{E}}\left(S, D, H ; S^{\nabla}\right)$ are subgroups.

Proof. It is enough to show the addition + is naturally defined on $\widetilde{\mathcal{E}}(S, D$, $H)$. Let $(f: X \rightarrow S, \phi)$ be a marked elliptic fibration associated with $(S, D, H)$. By Hironaka's flattening [H3], there is a bimeromorphic morphism $\mu: \hat{S} \rightarrow S$ such that the main component $\hat{X}$ of $X \times_{S} \hat{S}$ induces a flat morphism $\hat{f}: \hat{X} \rightarrow \hat{S}$. We may assume that $\hat{S}$ is non-singular and that $\hat{D}:=\mu^{-1} D$ is a normal crossing divisor. We infer that $\hat{f}$ is a locally projective morphism, since $R^{1} \hat{f}_{*} \mathcal{O}_{\hat{X}}^{\star} \rightarrow$ $R^{2} \hat{f}_{*} \mathbb{Z}_{\hat{X}}$ is surjective. Thus $\left(\hat{X} \rightarrow \hat{S}, \mu^{*} \phi\right)$ defines an element of $\mathcal{E}(\hat{S}, \hat{D}, \hat{H})$ for the induced VHS $\hat{H}$ on $\mu^{-1} S^{\star}=\hat{S} \backslash \hat{D}$ and for the pullback $\mu^{*} \phi$ of the marking. The map $\mathcal{E}(S, D, H) \rightarrow \mathcal{E}(\hat{S}, \hat{D}, \hat{H})$ is a group homomorphism. Thus the addition + of $\widetilde{\mathcal{E}}(S, D, H)$ is induced from + of all $\mathcal{E}(\hat{S}, \hat{D}, \hat{H})$. The subset $\widetilde{\mathcal{E}}\left(S, D, H ; S^{\nabla}\right)$ is then the kernel of $\widetilde{\mathcal{E}}(S, D, H) \rightarrow \widetilde{\mathcal{E}}\left(S^{\nabla}, D \cap S^{\nabla}, H\right)$. 
Theorem 5.2.7 (cf. [K9]). Let $f: X \rightarrow S$ be an elliptic fibration satisfying 5.2.1. Suppose that $X$ is non-singular. Then $R^{p} f_{*} \mathcal{O}_{X}=0$ for $p \geq 2$ and $R^{1} f_{*} \mathcal{O}_{X} \simeq \mathcal{L}_{H / S}$.

Proof. There exist an elliptic fibration $\hat{f}: \hat{X} \rightarrow \hat{S}$ between non-singular varieties smooth outside a normal crossing divisor $\hat{D}$ of $\hat{S}$, a bimeromorphic morphism $\mu: \hat{S} \rightarrow S$, and a bimeromorphic morphism $\nu: \hat{X} \rightarrow X$ such that $\mu^{-1} D \subset \hat{D}, \mu$ is an isomorphism over $S^{\star}$, and that $f \circ \nu=\mu \circ \hat{f}$. Then we have $R^{p} \hat{f}_{*} \mathcal{O}_{\hat{X}}=0$ for $p \geq 2$ and $R^{1} \hat{f}_{*} \mathcal{O}_{\hat{X}} \simeq \mathcal{L}_{H / \hat{S}}$, by [N4, 3.2.3] (cf. [K9], [M9]). By the spectral sequence

$$
R^{p} \mu_{*} R^{q} \hat{f}_{*} \mathcal{O}_{\hat{X}} \Longrightarrow R^{p+q} f_{*} \mathcal{O}_{X}
$$

it suffices to show $R^{p} \mu_{*} \mathcal{L}_{H / \hat{S}}=0$ for $p>0$ and $\mu_{*} \mathcal{L}_{H / \hat{S}} \simeq \mathcal{L}_{H / S}$. These are done in 4.2.4.

Corollary 5.2.8. Let $f: X \rightarrow S$ be an elliptic fibration satisfying 5.2.1. Suppose that $X$ is non-singular and that there is a subvariety $T \subset X$ generically finite over $S$. Then $R f_{*} \mathcal{O}_{X} \sim_{\text {qis }} \mathcal{O}_{S} \oplus \mathcal{L}_{H / S}[-1]$ for $H=H(f)$.

Proof. Suppose that $S$ is non-singular. Let $Y \rightarrow T$ be a desingularization and let $h: Y \rightarrow S$ be the composite. Let $R$ be the ramification divisor of $h$ : $K_{Y} \sim h^{*} K_{X}+R$. Then we have an injection $\mathcal{O}_{Y} \hookrightarrow \mathcal{O}_{Y}(R)$ and a trace map $R h_{*} \mathcal{O}_{Y}(R) \rightarrow \mathcal{O}_{S}$. The composite

$$
\mathcal{O}_{S} \rightarrow R f_{*} \mathcal{O}_{X} \rightarrow R h_{*} \mathcal{O}_{Y} \rightarrow R h_{*} \mathcal{O}_{Y}(R) \rightarrow \mathcal{O}_{S}
$$

is the multiplication map by $\operatorname{deg} h>0$. Thus gives the splitting of $\mathcal{O}_{S} \rightarrow$ $R f_{*} \mathcal{O}_{S}$. Next, we consider general case. Let $\mu: \hat{S} \rightarrow S$ be a bimeromorphic morphism from a non-singular variety such that $\mu^{-1} D$ is a normal crossing divisor and let $\hat{f}: \hat{X} \rightarrow \hat{S}$ be an elliptic fibration from a non-singular variety bimeromorphically equivalent to the pullback of $f$ to $\hat{S}$. Then by 5.2 .7 ,

$$
R f_{*} \mathcal{O}_{X} \sim_{\text {qis }} R \mu_{*} R \hat{f}_{*} \mathcal{O}_{\hat{X}} \sim_{\text {qis }} R \mu_{*} \mathcal{O}_{\hat{S}} \oplus R \mu_{*} \mathcal{L}_{\hat{H} / \hat{S}}[-1] \sim_{\text {qis }} \mathcal{O}_{S} \oplus \mathcal{L}_{H / S}[-1]
$$

Remark 5.2.9. Let $f: X \rightarrow S$ be an elliptic fibration satisfying 5.2.1. Then the exponential sequence of $X$ induces a surjective homomorphism $R^{1} f_{*} \mathcal{O}_{X}^{\star} \rightarrow R^{2} f_{*} \mathbb{Z}_{X}$. Hence, we infer that if $X$ is a Kähler manifold, then $f$ is a locally projective morphism, by the argument of [N4, 3.3]. 
Notation 5.2.10.

(1) In what follows, we fix a normal complex analytic variety $S$ and a toroidal embedding $S^{\star} \subset S$. $D$ denotes the complement $S \backslash S^{\star}$. We set $S^{\circ}:=$ $S \backslash \operatorname{Sing} D$. The related open immersions are denoted by $j: S^{\star} \hookrightarrow S$, $j^{\star}: S^{\star} \hookrightarrow S^{\circ}$, and $j^{\circ}: S^{\circ} \hookrightarrow S$.

(2) For an open subset $U \subset S$, we denote by $\underline{U}$ the $\partial$-space $(U, D \cap U)$. For an open immersion $\lambda: U_{1} \hookrightarrow U_{2}$, the associated $\partial$-open immersion is denoted by $\underline{\lambda}$.

(3) We fix a $\mathbb{Z}$-polarized variation of Hodge structure $H$ of weight one and of rank two, defined on $S^{\star}$.

Note that if $S$ is non-singular, then $D$ is a normal crossing divisor. $S^{\circ}$ is always non-singular.

\section{$\S 5.3$. Minimal basic elliptic fibration over $S^{\circ}$}

Before studying elliptic fibrations over $S$, we discuss basic elliptic fibrations defined over $S^{\circ}$. This corresponds to the case $D$ is non-singular. The structure of these fibrations are well-known, but we present a brief explanation. The basic fibration $\boldsymbol{p}: \boldsymbol{B}(H) \rightarrow S$ is not uniquely determined up to biholomorphic equivalence relation over $S$, but the following 5.3.1 asserts that we can select its unique minimal model over $S^{\circ}$. A minimal model of an elliptic fibration $f: X \rightarrow S$ is defined to be an elliptic fibration $g: Y \rightarrow S$ satisfying the following conditions (cf. [K4], [N2]):

- $f$ and $g$ are bimeromorphically equivalent to each other over $S$;

- $Y$ has only terminal singularities and $K_{Y}$ is $g$-nef.

An elliptic fibration $g: Y \rightarrow S$ satisfying the latter condition above is called a minimal elliptic fibration.

Lemma 5.3.1. There exists a minimal basic elliptic fibration $\boldsymbol{p}^{\circ}: \boldsymbol{B}(H)^{\circ}$ $\rightarrow S^{\circ}$ of $\left.\boldsymbol{p}\right|_{S^{\circ}}$. This is a flat morphism and is determined uniquely up to isomorphisms over $S^{\circ}$. Further $\boldsymbol{B}(H)^{\circ}$ is non-singular.

Proof. Let $\mathbf{W}=\mathbf{W}(H)$ be the generalized Weierstrass model associated with $H$. By 5.1.1 and 5.1.2, $\mathbf{W}^{\circ}:=\mathbf{p}^{-1} S^{\circ}=\mathbf{W} \times{ }_{S} S^{\circ}$ has only rational Gorenstein singularities and the dualizing sheaf $\omega_{\mathbf{W}}$ 。 is relatively trivial over $S^{\circ}$. 
Since $D^{\star}$ is a smooth divisor, a singularity of $\mathbf{W}^{\circ}$ is isomorphic to the singularity $F \times \mathbb{C}^{d-1}$ for a rational double point singularity $F$ and $d=\operatorname{dim} S$. Thus we can resolve singularities of $\mathbf{W}^{\circ}$ over $S^{\circ}$ in a natural way to obtain the minimal model $\boldsymbol{B}(H)^{\circ}$ flat over $S^{\circ}$. The canonical bundle of $\boldsymbol{B}(H)^{\circ}$ is also relatively trivial over $S^{\circ}$. An irreducible curve in a singular fiber of $\boldsymbol{p}^{\circ}: \boldsymbol{B}(H)^{\circ} \rightarrow S^{\circ}$ is a fiber of the restriction of $p^{\circ}$ to a prime divisor that does not dominate $S^{\circ}$. Thus there is no small contraction and hence $\boldsymbol{B}(H)^{\circ}$ is the unique minimal model.

Remark.

(1) There is an isomorphism

$$
\omega_{\boldsymbol{B}(H)^{\circ}} \simeq\left(\boldsymbol{p}^{\circ}\right)^{*}\left(\omega_{S^{\circ}} \otimes \mathcal{L}_{H / S^{\circ}}^{\otimes(-1)}\right)
$$

(2) The canonical section of the Weierstrass model $\mathbf{W}^{\circ}$ lifts to a section of $\boldsymbol{B}(H)^{\circ}$ since $\mathbf{W}^{\circ}$ is non-singular along the section.

(3) A singular fiber of $\boldsymbol{p}^{\circ}: \boldsymbol{B}(H)^{\circ} \rightarrow S^{\circ}$ is isomorphic to one of the nonmultiple fibers of minimal elliptic surfaces (cf. [K7]).

(4) $p^{\circ-1} D^{\star}$ is not necessarily a normal crossing divisor.

We denote by $\left(\boldsymbol{B}^{\circ}\right)^{\sharp}$ the set of all the points of $\boldsymbol{B}^{\circ}:=\boldsymbol{B}(H)^{\circ}$ at which $\boldsymbol{p}^{\circ}$ is smooth.

Lemma 5.3.2. Let $\Gamma \subset \boldsymbol{B}^{\circ}$ be a meromorphic section of $\boldsymbol{p}^{\circ}: \boldsymbol{B}^{\circ} \rightarrow S^{\circ}$. Then $\Gamma$ is a holomorphic section, i.e., $\Gamma \rightarrow S^{\circ}$ is an isomorphism. In particular, $\Gamma \subset\left(\boldsymbol{B}^{\circ}\right)^{\sharp}$.

Proof. Assume the contrary. Then the canonical divisor $K_{\Gamma}$ is not relatively nef over $S^{\circ}$, thus there is a curve $\gamma$ contained in a fiber of $\boldsymbol{p}^{\circ}$ with the intersection number $K_{\Gamma} \cdot \gamma=\Gamma \cdot \gamma<0$. Over an open neighborhood of the point $\boldsymbol{p}^{\circ}(\gamma)$, there is a prime divisor $F$ of $\boldsymbol{B}^{\circ}$ such that $\gamma$ is a fiber of $F \rightarrow \boldsymbol{p}^{\circ}(F)$. Hence $F \subset \Gamma$. This is a contradiction.

Lemma 5.3.3. $\quad$ A bimeromorphic mapping $\boldsymbol{B}^{\circ} \cdots \rightarrow \boldsymbol{B}^{\circ}$ over $S^{\circ}$ is holomorphic.

Proof. Let $f$ be the bimeromorphic mapping. Then $f$ is an isomorphism in codimension one, since $\boldsymbol{B}^{\circ}$ is a relative minimal model. We may replace $S^{\circ}$ by 
an open subset and thus assume that $\boldsymbol{p}^{\circ}: \boldsymbol{B}^{\circ} \rightarrow S^{\circ}$ is the composite of a closed embedding $\boldsymbol{B}^{\circ} \hookrightarrow \mathbb{P}^{n} \times S^{\circ}$ for a projective space $\mathbb{P}^{n}$ and the second projection. If $f$ is not holomorphic, then the proper transform $A^{\prime}$ of a relatively ample divisor $A$ of $\boldsymbol{B}^{\circ}$ is not $\boldsymbol{p}^{\circ}$-nef. Thus $A^{\prime}$ contains a divisor $F$ with $\boldsymbol{p}^{\circ}(F) \neq S^{\circ}$ by the same argument as in 5.3.2. For a general relatively ample divisor $A, A^{\prime}$ is irreducible. This is a contradiction.

Proposition 5.3.4. The $S^{\star}$-group structure of $\boldsymbol{B}(H)^{\star}$ extends to an $S^{\circ}$-group structure of $\left(\boldsymbol{B}^{\circ}\right)^{\sharp}$. Moreover $\left(\boldsymbol{B}^{\circ}\right)^{\sharp}$ acts on $\boldsymbol{B}^{\circ}$ over $S^{\circ}$ compatibly with the open immersion $\left(\boldsymbol{B}^{\circ}\right)^{\sharp} \hookrightarrow \boldsymbol{B}^{\circ}$.

Proof. Let $\pi: T \rightarrow S^{\circ}$ be a morphism from a non-singular variety and let $T^{\star}:=\pi^{-1}\left(S^{\star}\right)$. We denote by $\boldsymbol{B}_{T}^{\circ}$ the normalization of the fiber product $\boldsymbol{B}^{\circ} \times S^{\circ} T$. The canonical section (zero section) $\boldsymbol{\Sigma} \subset \boldsymbol{B}^{\circ}$ of $\boldsymbol{p}^{\circ}: \boldsymbol{B}^{\circ} \rightarrow S^{\circ}$ induces a meromorphic section $\boldsymbol{\Sigma}_{T}$ of $\boldsymbol{p}_{T}^{\circ}: \boldsymbol{B}_{T}^{\circ} \rightarrow T$. For a meromorphic section $\boldsymbol{\sigma}: T \cdots \rightarrow \boldsymbol{B}_{T}^{\circ}$, we denote by $\Gamma_{\boldsymbol{\sigma}}$ the prime divisor of $\boldsymbol{B}_{T}^{\circ}$ defined as the image of $\boldsymbol{\sigma}$. For two meromorphic sections $\boldsymbol{\sigma}_{1}$ and $\boldsymbol{\sigma}_{2}$, let $\mathcal{N}$ be the reflexive sheaf

$$
\mathcal{N}:=\mathcal{O}_{B_{T}^{\circ}}\left(\Gamma_{\sigma_{1}}+\Gamma_{\sigma_{2}}-\boldsymbol{\Sigma}_{T}\right) .
$$

Since the restriction of $\mathcal{N}$ to a smooth fiber of $\boldsymbol{p}_{T}^{\circ}$ is an invertible sheaf of degree one, its the direct image $\left(\boldsymbol{p}_{T}^{\circ}\right)_{*} \mathcal{N}$ is a torsion-free sheaf of rank one. This is an invertible sheaf, since $\boldsymbol{p}_{T}^{\circ}$ is an equi-dimensional morphism. There is an effective divisor $\Gamma^{\prime}$ of $\boldsymbol{B}_{T}^{\circ}$ such that

$$
\mathcal{N} \simeq\left(\boldsymbol{p}_{T}^{\circ}\right)^{*}\left(\boldsymbol{p}_{T}^{\circ}\right)_{*} \mathcal{N} \otimes \mathcal{O}_{B_{T}^{\circ}}\left(\Gamma^{\prime}\right)
$$

The horizontal part of $\Gamma^{\prime}$ is a prime divisor dominating $T$ bimeromorphically. This is the meromorphic section corresponding to $\boldsymbol{\sigma}_{1}+\boldsymbol{\sigma}_{2}$ under the group structure $\boldsymbol{B}^{\star} \times_{S \star} T^{\star} \rightarrow T^{\star}$, where we write $\boldsymbol{B}^{\star}=\boldsymbol{B}(H)^{\star}$. Thus we can define the sum $\boldsymbol{\sigma}_{1}+\boldsymbol{\sigma}_{2}$ as a meromorphic section. For a meromorphic section $\boldsymbol{\sigma}$ of $\boldsymbol{p}_{T}^{\circ}$, let $\mathcal{M}$ be the reflexive sheaf

$$
\mathcal{M}:=\mathcal{O}_{B_{T}^{\circ}}\left(2 \Sigma_{T}-\Gamma_{\boldsymbol{\sigma}}\right) .
$$

Then its direct image $\left(\boldsymbol{p}_{T}^{\circ}\right)_{*} \mathcal{M}$ is an invertible sheaf. There is an effective divisor $\Gamma^{\prime \prime}$ of $\boldsymbol{B}_{T}^{\circ}$ such that

$$
\mathcal{M} \simeq\left(\boldsymbol{p}_{T}^{\circ}\right)^{*}\left(\boldsymbol{p}_{T}^{\circ}\right)_{*} \mathcal{M} \otimes \mathcal{O}_{B_{T}^{\circ}}\left(\Gamma^{\prime \prime}\right) .
$$

The horizontal part of $\Gamma^{\prime \prime}$ is a prime divisor dominating $T$ bimeromorphically. This is the meromorphic section corresponding to $-\boldsymbol{\sigma}$ under the group structure 
$\boldsymbol{B}^{\star} \times_{S^{\star}} T^{\star} \rightarrow T^{\star}$. Thus we can define $-\boldsymbol{\sigma}$ as a meromorphic section. If $\pi: T \rightarrow S^{\circ}$ is a smooth morphism, then $\boldsymbol{B}_{T}^{\circ} \simeq \boldsymbol{B}^{\circ} \times{ }_{S^{\circ}} T$ and $T \backslash T^{\star}$ is a non-singular divisor. Hence the meromorphic sections $\sigma_{1}, \sigma_{2}, \sigma_{1}+\sigma_{2}, \sigma$, and $-\boldsymbol{\sigma}$ are all holomorphic by 5.3.2.

Let us consider the case $T=\left(\boldsymbol{B}^{\circ}\right)^{\sharp} \times_{S^{\circ}} \boldsymbol{B}^{\circ}$. The projections $p_{1}: T \rightarrow$ $\left(\boldsymbol{B}^{\circ}\right)^{\sharp} \hookrightarrow \boldsymbol{B}^{\circ}$ and $p_{2}: T \rightarrow \boldsymbol{B}^{\circ}$ induce meromorphic sections $\boldsymbol{\sigma}_{1}$ and $\boldsymbol{\sigma}_{2}$ of $\boldsymbol{p}_{T}^{\circ}$, respectively. The meromorphic section $\boldsymbol{\sigma}_{1}+\boldsymbol{\sigma}_{2}$ induces a meromorphic mapping

$$
\mu:\left(\boldsymbol{B}^{\circ}\right)^{\sharp} \times_{S} \circ \boldsymbol{B}^{\circ} \cdots \rightarrow \boldsymbol{B}^{\circ}
$$

over $S^{\circ}$. This is an extension of the addition morphism $\boldsymbol{B}^{\star} \times_{S^{\star}} \boldsymbol{B}^{\star} \rightarrow \boldsymbol{B}^{\star}$ of the group structure $\boldsymbol{B}^{\star} \rightarrow S^{\star}$. The first projection $p_{1}$ and $\mu$ induce a meromorphic mapping

$$
\left(p_{1}, \mu\right):\left(\boldsymbol{B}^{\circ}\right)^{\sharp} \times_{S^{\circ}} \boldsymbol{B}^{\circ} \cdots \rightarrow\left(\boldsymbol{B}^{\circ}\right)^{\sharp} \times_{S^{\circ}} \boldsymbol{B}^{\circ}
$$

over $\left(\boldsymbol{B}^{\circ}\right)^{\sharp}$, which corresponds to the translation by $\boldsymbol{\sigma}_{1}+\boldsymbol{\sigma}_{2}$. This is holomorphic by 5.3.3. Thus $\mu$ is also holomorphic. Next, we consider the case $T=\boldsymbol{B}^{\circ}$ and a meromorphic section id of $\boldsymbol{p}_{T}^{\circ}$ corresponding to the identity mapping $T=\boldsymbol{B}^{\circ} \rightarrow \boldsymbol{B}^{\circ}$ (or the diagonal locus of $\boldsymbol{B}^{\circ} \times_{S^{\circ}} \boldsymbol{B}^{\circ}$ ). Then the "inverse" - id defines a meromorphic mapping $\boldsymbol{B}^{\circ} \cdots \rightarrow \boldsymbol{B}^{\circ}$ over $S^{\circ}$. This is holomorphic also by 5.3 .3 and is an extension of the inverse morphism $\boldsymbol{B}^{\star} \rightarrow \boldsymbol{B}^{\star}$ of the group structure $\boldsymbol{p}^{\star}: \boldsymbol{B}^{\star} \rightarrow S^{\star}$. In particular, $\left(\boldsymbol{B}^{\circ}\right)^{\sharp} \rightarrow S^{\circ}$ has the required group structure and $\left(\boldsymbol{B}^{\circ}\right)^{\sharp}$ acts on $\boldsymbol{B}^{\circ}$.

Remark. Let $\mathrm{S}$ be a normal integral scheme of finite type over $\mathbb{C}$ of dimension one and let $\mathrm{S}^{\star} \subset \mathrm{S}$ be a Zariski-open dense subset. Let $\mathrm{p}^{\star}: \mathrm{B}^{\star} \rightarrow \mathrm{S}^{\star}$ be an algebraic smooth basic elliptic fibration over $\mathbb{C}, S=\mathrm{S}^{\text {an }}, S^{\star}=\left(\mathrm{S}^{\star}\right)^{\text {an }}$, and let $H$ be the VHS defined by $\boldsymbol{p}^{\star}=\left(\mathrm{p}^{\star}\right)^{\text {an }}$. Then $\boldsymbol{B}(H)^{\star} \simeq\left(\mathrm{B}^{\star}\right)^{\text {an }}$ and $\left(\boldsymbol{B}^{\circ}\right)^{\sharp}$ corresponds to the Néron model of the generic fiber of $\mathrm{p}^{\star}$.

Corollary 5.3.5. Let $\boldsymbol{p}: \boldsymbol{B}(H) \rightarrow S$ be a basic elliptic fibration associated with $H$ and let $\boldsymbol{\sigma}: S \cdots \rightarrow \boldsymbol{B}(H)$ be a meromorphic section. Then there exist meromorphic mappings $\boldsymbol{B}(H) \times{ }_{S} \boldsymbol{B}(H) \cdots \rightarrow \boldsymbol{B}(H)$ and $\boldsymbol{B}(H) \cdots \rightarrow$ $\boldsymbol{B}(H)$ such that their restrictions to $S^{\star}$ are bimeromorphically equivalent to the multiplication mapping and the inverse mapping of the group structure of $\boldsymbol{B}(H)^{\star} \rightarrow S^{\star}$ with $\left.\boldsymbol{\sigma}\right|_{S^{\star}}$ being zero, respectively. In other words, $\boldsymbol{p}: \boldsymbol{B}(H) \rightarrow S$ has a meromorphic $S$-group structure.

Proof. $\boldsymbol{B}(H)$ is bimeromorphically equivalent to a generalized Weierstrass model $\mathbf{W}$ over $S$ and $\mathbf{W}^{\circ}=\mathbf{W} \times{ }_{S} S^{\circ}$ is bimeromorphically equivalent 
to $\boldsymbol{B}(H)^{\circ}$ over $S^{\circ}$. Since $\operatorname{codim}\left(\mathbf{W} \backslash \mathbf{W}^{\circ}\right) \geq 2$, the multiplication mapping and the inverse mapping extend to $\mathbf{W}$ as meromorphic mappings.

\section{Definition 5.3.6.}

(1) Let $\boldsymbol{p}^{\circ}: \boldsymbol{B}(H)^{\circ} \rightarrow S^{\circ}$ be the minimal basic elliptic fibration associated with $H$. For a holomorphic section of $\sigma^{\circ}$ of $\boldsymbol{p}^{\circ}$, the sheaf of germs of holomorphic sections of $\boldsymbol{p}^{\circ}$ has an abelian group structure with $\boldsymbol{\sigma}^{\circ}$ being zero. We denote the sheaf by $\mathfrak{S}_{H / S^{\circ}}$.

(2) Let $\boldsymbol{p}: \boldsymbol{B}(H) \rightarrow S$ be a basic elliptic fibration associated with $H$. For a meromorphic section $\boldsymbol{\sigma}: S \cdots \rightarrow \boldsymbol{B}(H)$ of $\boldsymbol{p}$, the sheaf of germs of meromorphic sections of $\boldsymbol{p}$ has an abelian group structure with $\boldsymbol{\sigma}$ being zero. We denote the sheaf by $\mathfrak{S}_{H / S}$.

There are natural isomorphisms:

$$
\left.\mathfrak{S}_{H / S}\right|_{S^{\circ}} \simeq \mathfrak{S}_{H / S^{\circ}}, \quad j_{*}^{\circ} \mathfrak{S}_{H / S^{\circ}} \simeq \mathfrak{S}_{H / S} .
$$

The second one follows the property $\operatorname{codim}\left(S \backslash S^{\circ}\right) \geq 2$.

Notation 5.3.7. In what follows, we fix a basic elliptic fibration $\boldsymbol{p}: \boldsymbol{B}$ $=\boldsymbol{B}(H) \rightarrow S$ associated with $H$ and a meromorphic section $\boldsymbol{\sigma}: S \cdots \rightarrow \boldsymbol{B}$. The image of $\boldsymbol{\sigma}$ is denoted by $\boldsymbol{\Sigma}$. $\boldsymbol{\sigma}$ and $\boldsymbol{\Sigma}$ are called the zero sections. We assume that $\boldsymbol{B}$ is non-singular and that $\boldsymbol{p}$ is smooth over $S^{\star}$. The minimal basic elliptic fibration over $S^{\circ}$ is always denoted by $\boldsymbol{p}^{\circ}: \boldsymbol{B}^{\circ} \rightarrow S^{\circ}$. Note that $p^{-1} S^{\circ}$ is not necessarily isomorphic to $\boldsymbol{B}^{\circ}$.

\section{$\S 5.4$. Fundamental diagram}

Let $f: X \rightarrow S$ be an LBP surjective morphism from a non-singular variety such that $f^{-1} S^{\star} \rightarrow S^{\star}$ is bimeromorphically equivalent to a smooth morphism. Then $H(f):=\left.R^{1} f_{*} \mathbb{Z}_{X}\right|_{S^{\star}}$ is a locally constant system. Let $i: X^{\star}:=f^{-1}\left(S^{\star}\right) \hookrightarrow X$ denote the open immersion. The quasi-isomorphism

$$
R f_{*} R \underline{\Gamma}_{f^{-1} D}\left(\mathbb{Z}_{X}\right) \sim_{\text {qis }} R \underline{\Gamma}_{D}\left(R f_{*} \mathbb{Z}_{X}\right)
$$

induces two spectral sequences

${ }_{\mathrm{I}} E_{2}^{p, q}=R^{p} f_{*} \mathcal{H}_{f^{-1} D}^{q}\left(\mathbb{Z}_{X}\right) \Longrightarrow E^{p+q}, \quad$ and $\quad{ }_{\mathrm{II}} E_{2}^{p, q}=\mathcal{H}_{D}^{p}\left(R^{q} f_{*} \mathbb{Z}_{X}\right) \Longrightarrow E^{p+q}$

Here, $E^{1}=0$ and $E^{2} \simeq{ }_{\mathrm{I}} E_{2}^{0,2}=f_{*} \mathcal{H}_{f^{-1} D}^{2}\left(\mathbb{Z}_{X}\right)$ hold. 
Lemma 5.4.1. ${ }_{\mathrm{II}} E_{2}^{p, 0} \rightarrow E^{p}$ is injective for any $p$. In particular, $\mathcal{H}_{D}^{0}\left(R^{1} f_{*} \mathbb{Z}_{X}\right)=0$.

Proof. We can localize $S$ and may assume that there exist a generically finite surjective morphism $\mu: Y \rightarrow S$ and a morphism $\sigma: Y \rightarrow X$ from a nonsingular variety $Y$ with $\mu=f \circ \sigma$. We have a trace map $R \mu_{*} \boldsymbol{\omega}_{Y}^{\text {top }} \rightarrow \boldsymbol{\omega}_{S}^{\text {top }}$ for the topological dualizing complexes by Verdier duality (cf. [V2]). Here $\boldsymbol{\omega}_{Y}^{\text {top }} \sim_{\text {qis }} \mathbb{Z}_{Y}[2 d]$ and there are natural morphisms $\mathbb{Z}_{S}[2 d] \rightarrow \boldsymbol{\omega}_{S}^{\text {top }}$ and $\boldsymbol{\omega}_{S}^{\text {top }} \rightarrow$ $R j_{*}^{\circ} \mathbb{Z}_{S^{\circ}}[2 d]$. The composite

$$
\mathbb{Z}_{S} \rightarrow R f_{*} \mathbb{Z}_{X} \rightarrow R \mu_{*} \mathbb{Z}_{Y} \rightarrow \boldsymbol{\omega}_{S}^{\mathrm{top}}[-2 d] \rightarrow R j_{*}^{\circ} \mathbb{Z}_{S^{\circ}}
$$

factors through the multiplication mapping $\mathbb{Z}_{S} \rightarrow \mathbb{Z}_{S}$ by the degree of $\mu$ and the natural morphism $\mathbb{Z}_{S} \rightarrow R j_{*}^{\circ} \mathbb{Z}_{S^{\circ}}$. We know that ${ }_{\mathrm{II}} E_{2}^{p, 0}=\mathcal{H}_{D}^{p}\left(\mathbb{Z}_{S}\right)$ are sheaves of torsion-free abelian groups and $\mathcal{H}_{D}^{p}\left(\mathbb{Z}_{S}\right) \rightarrow \mathcal{H}_{D}^{p}\left(R j_{*}^{\circ} \mathbb{Z}_{S^{\circ}}\right)$ are injective by 3.3.1. Thus we are done.

Remark 5.4.2. There is a trace map $R f_{*} \boldsymbol{\omega}_{X}^{\text {top }} \rightarrow \boldsymbol{\omega}_{S}^{\text {top }}$. The homomorphism $R^{\operatorname{dim} f} f_{*} \mathbb{Z}_{X} \rightarrow \mathbb{Z}_{S}$ obtained as $\mathcal{H}^{-2 d}$ is also called the trace map of $f$. If $f$ admits a meromorphic section $\Sigma \subset X$ and if $S$ is non-singular, then $\mathbb{Z}_{S} \rightarrow R f_{*} \mathbb{Z}_{X}$ has a splitting by the composite

$$
R f_{*} \mathbb{Z}_{\Sigma} \rightarrow R f_{*} \boldsymbol{\omega}_{\Sigma}^{\mathrm{top}}[-2 d] \rightarrow \boldsymbol{\omega}_{S}^{\mathrm{top}}[-2 d] \sim_{\mathrm{qis}} \mathbb{Z}_{S} .
$$

Lemma 5.4.3 (cf. [F5, (1.5)]). Let $h: Y \rightarrow V$ be a projective surjective morphism with connected fibers between normal varieties and let $D$ be a Cartier divisor of $Y$. Assume that

(1) V has only 1-rational singularities (cf. 3.2.1),

(2) $h(\operatorname{Supp} D) \neq V$,

(3) $D \cdot \gamma=0$ for any irreducible curves $\gamma$ contained in fibers of $h$.

Then, locally on $V$, there exists a positive integer $m$ such that $m D$ is the pullback of a Cartier divisor of $V$.

Proof. Let $\mu: \hat{V} \rightarrow V$ be a bimeromorphic morphism from a non-singular variety and let $\nu: \hat{Y} \rightarrow Y$ be a bimeromorphic morphism such that there is a morphism $\hat{h}: \hat{Y} \rightarrow \hat{V}$ with $\mu \circ \hat{h}=h \circ \nu$. Then the pullback $\nu^{*} D$ is a Cartier divisor not dominating $\hat{V}$ and $\nu^{*} D \cdot \hat{\gamma}=0$ for any curves $\hat{\gamma}$ contained fibers of $\hat{h}$. For a prime divisor $E$ contained in $\hat{h}\left(\operatorname{Supp} \nu^{*} D\right)$, let $a_{E}$ be the 
maximum of such rational numbers $r$ that $\nu^{*} D-r \hat{h}^{*} E$ is effective. Then we have $\nu^{*} D=\hat{h}^{*}\left(\sum a_{E} E\right)$ as $\mathbb{Q}$-divisors. In particular, there is a positive integer $m$ (locally on $S$ ) such that $m \nu^{*} D$ is the pullback of a Cartier divisor of $\hat{V}$. Thus we are reduced to the case $Y$ is non-singular and $h$ is bimeromorphic. Then the connecting homomorphism $R^{1} h_{*} \mathcal{O}_{Y}^{\star} \rightarrow R^{2} h_{*} \mathbb{Z}_{Y}$ of the exponential sequence is injective by the assumption (1). Since $D$ is $h$-numerically trivial, $D=0$ in any stalks $\left(R^{2} h_{*} \mathbb{Q}_{Y}\right)_{v}$ for $v \in V$. Hence, locally on $V, m D$ is the pullback of a Cartier divisor for some $m$.

Lemma 5.4.4. $\quad{ }_{\mathrm{II}} E_{2}^{1,1}=\mathcal{H}_{D}^{1}\left(R^{1} f_{*} \mathbb{Z}_{X}\right)$ is a sheaf of torsion abelian groups.

Proof. Let ${ }_{\mathrm{II}} F^{1}\left(E^{2}\right)$ be the kernel of $E^{2} \rightarrow{ }_{\mathrm{II}} E_{2}^{0,2}$. Then, by 5.4.1, we have an exact sequence

$$
0 \rightarrow{ }_{\mathrm{II}} E_{2}^{2,0} \rightarrow{ }_{\mathrm{II}} F^{1}\left(E^{2}\right) \rightarrow{ }_{\mathrm{II}} E_{2}^{1,1} \rightarrow 0 .
$$

Since $E^{2} \simeq f_{*} \mathcal{H}_{f^{-1} D}^{2}\left(\mathbb{Z}_{X}\right) \simeq f_{*} \mathcal{H}_{f^{-1} D}^{0}\left(\mathcal{D} i v_{X}\right)$, by 5.4.3, the kernel ${ }_{\mathrm{II}} F^{1}\left(E^{2}\right)$ is considered to be the sheaf of germs of $\mathbb{Q}$-Cartier divisors supported in $D$ whose pullback to $X$ are Cartier. Therefore, any stalk of ${ }_{\mathrm{II}} E_{2}^{1,1}$ is a torsion group.

Corollary 5.4.5. If one of the following conditions is satisfied, then $\mathcal{H}_{D}^{1}\left(R^{1} f_{*} \mathbb{Z}_{X}\right)=0$ and the sequence

$$
0 \rightarrow{ }_{\mathrm{II}} E_{2}^{2,0} \rightarrow E^{2} \rightarrow{ }_{\mathrm{II}} E_{2}^{0,2} \rightarrow{ }_{\mathrm{II}} E_{2}^{2,1}
$$

is exact:

(1) $S$ is non-singular and $f$ admits local meromorphic sections over the complement of an analytic subset of $S$ of codimension $\geq 2$;

(2) $f$ admits local meromorphic sections over $S$.

Proof. In the case (1), the assertion follows the argument of 5.4.4. For the case (2), we may localize $S$ and may assume that $f$ admits a meromorphic section. Let $Y \rightarrow X$ be a bimeromorphic morphism onto the meromorphic section from a non-singular variety and let $\mu: Y \rightarrow S$ be the composite. We may assume also that $\mu^{-1} D$ is a normal crossing divisor. There is a commutative diagram

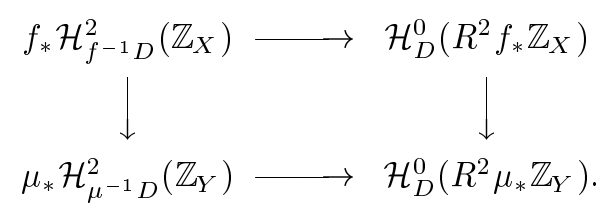


Here, the ${ }_{\text {II }} F^{1}\left(E^{2}\right)$ is the kernel of the top arrow. The kernel of the bottom arrow is $\mathcal{H}_{D}^{2}\left(\mathbb{Z}_{S}\right)={ }_{\mathrm{II}} E_{2}^{2,0}$ since $R^{1} \mu_{*} \mathbb{Z}_{Y}=0$. By the argument of 5.4.4, we infer that ${ }_{\mathrm{II}} E_{\infty}^{1,1}=0$ and hence ${ }_{\mathrm{II}} E_{2}^{1,1}=0$ by 5.4.1.

Let $f: X \rightarrow S$ be an LBP elliptic fibration satisfying 5.2.1 from a nonsingular variety. Suppose that $f$ is smooth over $S \backslash D^{\prime}$ for a divisor $D \subset D^{\prime} \subset$ $X$. We set $\mathcal{V}_{X}:=\mathcal{H}_{D^{\prime}}^{0}\left(R^{1} f_{*} \mathcal{O}_{X}^{\star}\right)$.

\section{Lemma 5.4.6.}

(1) Let $\mathcal{M}$ be an invertible sheaf of $X$. Its image under $H^{1}\left(X, \mathcal{O}_{X}^{\star}\right) \rightarrow H^{0}(S$, $\left.R^{1} \boldsymbol{p}_{*} \mathcal{O}_{X}^{\star}\right)$ is contained in $H^{0}\left(S, \mathcal{V}_{X}\right)$ if and only if, for any point $s \in S$, there exist an open neighborhood $\mathcal{U}$ and a Cartier divisor $E$ defined on $\boldsymbol{p}^{-1} \mathcal{U}$ such that $\operatorname{Supp} E \subset \boldsymbol{p}^{-1} D^{\prime}$ and that $\left.\mathcal{M}\right|_{f^{-1} \mathcal{U}} \simeq \mathcal{O}_{f^{-1} \mathcal{U}}(E)$.

(2) $\mathcal{V}_{X}$ does not depend on the choice of $D^{\prime}$.

(3) Let $f^{\prime}: X^{\prime} \rightarrow S$ be another elliptic fibration from a non-singular variety that is bimeromorphically equivalent to $f$ over $S$. Then $R^{1} f_{*} \mathcal{O}_{X}^{\star} / \mathcal{V}_{X} \simeq$ $R^{1} f_{*}^{\prime} \mathcal{O}_{X^{\prime}}^{\star} / \mathcal{V}_{X^{\prime}}$

Proof. (1) It is enough to show the 'only if' part. Let $\mathcal{N}$ be the doubledual of $f_{*} \mathcal{M}$. By 3.3.6, locally on $S, \mathcal{N} \simeq \mathcal{O}_{S}(\Delta)$ for a Weil divisor $\Delta$ supported in $D$. Thus we may assume that $\mathcal{N} \simeq \mathcal{O}_{S}$. Locally over $S$, there is an effective divisor $E^{\prime}$ satisfying codim $f\left(E^{\prime}\right) \geq 2, f\left(E^{\prime}\right) \subset D^{\prime}$, and $\mathcal{N} \simeq f_{*}\left(\mathcal{M} \otimes \mathcal{O}_{X}\left(E^{\prime}\right)\right)$. Since $f^{*} f_{*} \mathcal{M} \rightarrow \mathcal{M}$ is an isomorphism over $S \backslash D^{\prime}$, we have a local isomorphism $\mathcal{M} \simeq \mathcal{O}_{X}(E)$ for a divisor $E$ supported in $f^{-1} D^{\prime}$.

(2) If $f$ is a smooth morphism, then the trace map $R^{2} f_{*} \mathbb{Z}_{X} \rightarrow \mathbb{Z}_{S}$ is an isomorphism. In particular, $\mathcal{H}_{T}^{0}\left(R^{1} \boldsymbol{p}_{*} \mathcal{O}_{B}^{\star}\right)=0$ for any proper subset $T$. Therefore, for $D^{\prime} \subset D^{\prime \prime},\left.\mathcal{H}_{D^{\prime \prime}}^{0}\left(R^{1} f_{*} \mathcal{O}_{X}^{\star}\right)\right|_{S \backslash D^{\prime}}=0$. Thus $\mathcal{V}_{X} \simeq \mathcal{H}_{D^{\prime \prime}}^{0}\left(R^{1} f_{*} \mathcal{O}_{X}^{\star}\right)$.

(3) We may assume that there is a bimeromorphic morphism $\nu: X^{\prime} \rightarrow X$ over $S$. By (2), we may assume that $X^{\prime}$ is also smooth outside $D^{\prime}$. Then $\nu$ exceptional divisors define elements of $\mathcal{V}_{X^{\prime}}$. Thus we have the isomorphism.

We apply the argument above to the basic elliptic fibration $\boldsymbol{p}: \boldsymbol{B} \rightarrow S$, where $\boldsymbol{B}$ is non-singular and $\boldsymbol{p}$ is smooth over $S^{\star}$. Then we have the spectral sequences

$$
{ }_{\mathrm{I}} E_{2}^{p, q}=R^{p} \boldsymbol{p}_{*} \mathcal{H}_{\boldsymbol{p}^{-1} D}^{q}\left(\mathbb{Z}_{\boldsymbol{B}}\right) \Longrightarrow E^{p+q}, \quad \text { and } \quad{ }_{\mathrm{II}} E_{2}^{p, q}=\mathcal{H}_{D}^{p}\left(R^{q} \boldsymbol{p}_{*} \mathbb{Z}_{\boldsymbol{B}}\right) \Longrightarrow E^{p+q}
$$

from the quasi-isomorphism

$$
R \boldsymbol{p}_{*} R \underline{\Gamma}_{\boldsymbol{p}^{-1} D}\left(\mathbb{Z}_{\boldsymbol{B}}\right) \sim_{\mathrm{qis}} R \underline{\Gamma}_{D}\left(R \boldsymbol{p}_{*} \mathbb{Z}_{\boldsymbol{B}}\right) .
$$


The exponential sequence of $\boldsymbol{B}$ induces a long exact sequence

$$
0 \rightarrow R^{1} \boldsymbol{p}_{*} \mathbb{Z}_{\boldsymbol{B}} \rightarrow R^{1} \boldsymbol{p}_{*} \mathcal{O}_{\boldsymbol{B}} \rightarrow R^{1} \boldsymbol{p}_{*} \mathcal{O}_{\boldsymbol{B}}^{\star} \rightarrow R^{2} \boldsymbol{p}_{*} \mathbb{Z}_{\boldsymbol{B}} \rightarrow 0,
$$

by 5.2.7. The image of $R^{1} \boldsymbol{p}_{*} \mathcal{O}_{B} \rightarrow R^{1} \boldsymbol{p}_{*} \mathcal{O}_{B}^{\star}$ is isomorphic to $\mathfrak{S}_{H / S}^{\mathbf{W}}$ by 5.4.5. We set $\mathcal{V}_{\boldsymbol{B}}:=\mathcal{H}_{D}^{0}\left(R^{1} \boldsymbol{p}_{*} \mathcal{O}_{\boldsymbol{B}}^{\star}\right)$. Then, by 5.4.6, the quotient sheaf $R^{1} \boldsymbol{p}_{*} \mathcal{O}_{\boldsymbol{B}}^{\star} / \mathcal{V}_{\boldsymbol{B}}$ is uniquely determined by $H$. We have

$$
\mathcal{V}_{\boldsymbol{B}} \cap \mathfrak{S}_{H / S}^{\mathrm{W}}=\mathcal{H}_{D}^{0}\left(\mathfrak{S}_{H / S}^{\mathrm{W}}\right) \simeq \mathcal{H}_{D}^{1}\left(R^{1} \boldsymbol{p}_{*} \mathbb{Z}_{\boldsymbol{B}}\right)=0,
$$

by 5.4.5. In particular, the composite $\mathcal{V}_{\boldsymbol{B}} \rightarrow R^{1} \boldsymbol{p}_{*} \mathcal{O}_{\boldsymbol{B}}^{\star} \rightarrow R^{2} \boldsymbol{p}_{*} \mathbb{Z}_{\boldsymbol{B}}$ is injective and there is an exact sequence

$$
0 \rightarrow \mathfrak{S}_{H / S}^{\mathrm{W}} \rightarrow R^{1} \boldsymbol{p}_{*} \mathcal{O}_{B}^{\star} / \mathcal{V}_{B} \rightarrow R^{2} \boldsymbol{p}_{*} \mathbb{Z}_{\boldsymbol{B}} / \mathcal{V}_{\boldsymbol{B}} \rightarrow 0
$$

The quotient sheaf $R^{2} \boldsymbol{p}_{*} \mathbb{Z}_{\boldsymbol{B}} / \mathcal{V}_{\boldsymbol{B}}$ also does not depend on the choice of $\boldsymbol{B}$.

\section{Lemma 5.4.7.}

(1) $\mathcal{H}_{D}^{p}\left(\mathcal{O}_{S}^{\star}\right) \rightarrow \mathcal{H}^{p}\left(R \boldsymbol{p}_{*} R \underline{\Gamma}_{\boldsymbol{p}^{-1} D}\left(\mathcal{O}_{B}^{\star}\right)\right)$ is injective for any $p$. In particular, the sequence

$$
0 \rightarrow \mathcal{H}_{D}^{1}\left(\mathcal{O}_{S}^{\star}\right) \rightarrow \mathcal{H}^{1}\left(R \boldsymbol{p}_{*} R \underline{\Gamma}_{\boldsymbol{p}^{-1} D}\left(\mathcal{O}_{\boldsymbol{B}}^{\star}\right)\right) \rightarrow \mathcal{V}_{\boldsymbol{B}} \rightarrow 0
$$

is exact.

(2) For the spectral sequence ${ }_{\mathrm{II}} E_{2}^{p, q}=\mathcal{H}_{D}^{p}\left(R^{q} \boldsymbol{p}_{*} \mathbb{Z}_{\boldsymbol{B}}\right) \Longrightarrow E^{p+q}$ above, we have ${ }_{\mathrm{II}} E_{2}^{2,0}={ }_{\mathrm{II}} E_{\infty}^{2,0}, E^{2} /{ }_{\mathrm{II}} E_{\infty}^{2,0} \simeq{ }_{\mathrm{II}} E_{\infty}^{0,2} \simeq \mathcal{V}_{B}$.

Suppose that $[S, D]$ satisfies the condition 3.2.6. Then the following properties also hold:

(3) $H_{D}^{p}(S, \mathbb{Z}) \rightarrow H_{\boldsymbol{p}^{-1} D}^{p}(\boldsymbol{B}, \mathbb{Z})$ and $H^{p}(S, \mathbb{Z}) \rightarrow H^{p}(\boldsymbol{B}, \mathbb{Z})$ are injective for $p \leq 3$.

(4) $H_{D}^{p}\left(S, \mathcal{O}_{S}^{\star}\right) \rightarrow H_{\boldsymbol{p}^{-1} D}^{p}\left(\boldsymbol{B}, \mathcal{O}_{\boldsymbol{B}}^{\star}\right)$ and $H^{p}\left(S, \mathcal{O}_{S}^{\star}\right) \rightarrow H^{p}\left(\boldsymbol{B}, \mathcal{O}_{\boldsymbol{B}}^{\star}\right)$ are injective for $p \leq 2$. In particular, the sequence

$$
0 \rightarrow H_{D}^{1}\left(S, \mathcal{O}_{S}^{\star}\right) \rightarrow H_{\boldsymbol{p}^{-1} D}^{1}\left(\boldsymbol{B}, \mathcal{O}_{\boldsymbol{B}}^{\star}\right) \rightarrow H^{0}\left(S, \mathcal{V}_{\boldsymbol{B}}\right) \rightarrow 0
$$

is exact.

Proof. (3) and the injectiveness of (1), (4) follow from the existence of meromorphic section of $\boldsymbol{p}$ and 3.3.1. In particular, for the spectral sequences

$$
\begin{aligned}
\mathcal{H}_{D}^{p}\left(R^{q} \boldsymbol{p}_{*} \mathcal{O}_{\boldsymbol{B}}^{\star}\right) & \Longrightarrow \mathcal{H}^{p+q}\left(R \boldsymbol{p}_{*} R \underline{\Gamma}_{\boldsymbol{p}^{-1} D}\left(\mathcal{O}_{\boldsymbol{B}}^{\star}\right)\right), \\
H_{D}^{p}\left(S, R^{q} \boldsymbol{p}_{*} \mathcal{O}_{\boldsymbol{B}}^{\star}\right) & \Longrightarrow H_{\boldsymbol{p}^{-1} D}^{p+q}\left(\boldsymbol{B}, \mathcal{O}_{\boldsymbol{B}}^{\star}\right),
\end{aligned}
$$


we have exact sequences

$$
\begin{gathered}
0 \rightarrow \mathcal{H}_{D}^{1}\left(\mathcal{O}_{S}^{\star}\right) \rightarrow \mathcal{H}^{1}\left(R \boldsymbol{p}_{*} R \underline{\Gamma}_{\boldsymbol{p}^{-1} D}\left(\mathcal{O}_{\boldsymbol{B}}^{\star}\right)\right) \rightarrow \mathcal{H}_{D}^{0}\left(R^{1} \boldsymbol{p}_{*} \mathcal{O}_{\boldsymbol{B}}^{\star}\right)=\mathcal{V}_{\boldsymbol{B}} \rightarrow 0 \\
0 \rightarrow H_{D}^{1}\left(S, \mathcal{O}_{S}^{\star}\right) \rightarrow H_{\boldsymbol{p}^{-1} D}^{1}\left(\boldsymbol{B}, \mathcal{O}_{\boldsymbol{B}}^{\star}\right) \rightarrow H_{D}^{0}\left(S, R^{1} \boldsymbol{p}_{*} \mathcal{O}_{\boldsymbol{B}}^{\star}\right)=H^{0}\left(S, \mathcal{V}_{\boldsymbol{B}}\right) \rightarrow 0
\end{gathered}
$$

Thus (1) and (4) hold. The equality ${ }_{\mathrm{II}} E_{2}^{2,0}={ }_{\mathrm{II}} E_{\infty}^{2,0}$ of (2) follows from 3.3.1 or 5.4.1. We have a quasi-isomorphism

$$
R \underline{\Gamma}_{D}\left(R p_{*} \mathcal{O}_{B}^{\star}\right) \sim_{\mathrm{qis}} R \underline{\Gamma}_{D}\left(R p_{*} \mathcal{O}_{\boldsymbol{B}}\right) \oplus R \underline{\Gamma}_{D}\left(R p_{*} \mathbb{Z}_{\boldsymbol{B}}\right)[1]
$$

by 3.2.4. The second projection induces a commutative diagram of exact sequences

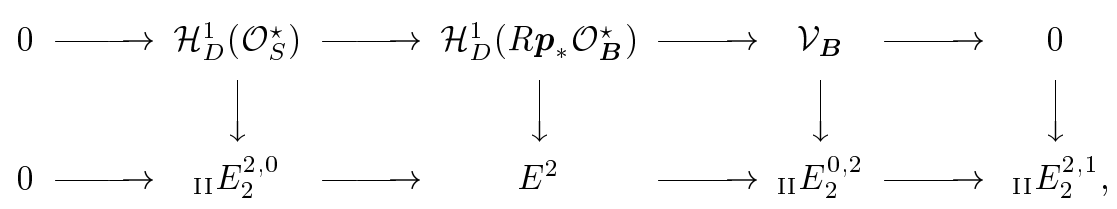

by 5.4.5. Here the first vertical arrow is isomorphic and the second vertical arrow is surjective. Thus $\mathcal{V}_{B} \simeq{ }_{\mathrm{II}} E_{\infty}^{0,2}$.

The trace map $R^{2} \boldsymbol{p}_{*} \mathbb{Z}_{\boldsymbol{B}} \rightarrow \mathbb{Z}_{S}$ derived from Verdier duality is an isomorphism over $S^{\star}$. The composite $R^{1} \boldsymbol{p}_{*} \mathcal{O}_{\boldsymbol{B}}^{\star} \rightarrow R^{2} \boldsymbol{p}_{*} \mathbb{Z}_{\boldsymbol{B}} \rightarrow \mathbb{Z}_{S}$ is the homomorphism measuring the degree of invertible sheaves restricted to smooth fibers. The trace map and the composite are surjective, since there is a meromorphic section of $\boldsymbol{p}$. In particular, ${ }_{\mathrm{II}} E_{2}^{1,2}=\mathcal{H}_{D}^{1}\left(R^{2} \boldsymbol{p}_{*} \mathbb{Z}_{\boldsymbol{B}}\right)=0$ and ${ }_{\mathrm{II}} E_{2}^{0,2}=$ $\mathcal{H}_{D}^{0}\left(R^{2} \boldsymbol{p}_{*} \mathbb{Z}_{\boldsymbol{B}}\right)$ is the kernel of the trace map.

Lemma 5.4.8. The kernel of $R^{1} \boldsymbol{p}_{*} \mathcal{O}_{B}^{\star} / \mathcal{V}_{B} \rightarrow \mathbb{Z}_{S}$ is isomorphic to $\mathfrak{S}_{H / S}$.

Proof. Let $\Psi: \mathfrak{S}_{H / S} \rightarrow R^{1} \boldsymbol{p}_{*} \mathcal{O}_{B}^{\star} / \mathcal{V}_{B}$ be a homomorphism defined as follows: Let $\Gamma \subset \boldsymbol{B}$ be a meromorphic section of $\boldsymbol{p}$. Then we attach an invertible sheaf $\mathcal{O}_{\boldsymbol{B}}(\Gamma-\boldsymbol{\Sigma})$, where $\boldsymbol{\Sigma}$ is the zero section. We define $\Psi(\Gamma)$ to be the invertible sheaf modulo $\mathcal{V}_{B}$. By localizing $S$, we have the sheaf homomorphism $\Psi$ that is injective. We shall show $\Psi\left(\mathfrak{S}_{H / S}\right)$ is the kernel. Let $\mathcal{M}$ be an invertible sheaf such that $\mathcal{M} \cdot \boldsymbol{p}^{-1}(s)=0$ for $s \in S^{\star}$. Then $\boldsymbol{p}_{*}\left(\mathcal{M} \otimes \mathcal{O}_{\boldsymbol{B}}(\boldsymbol{\Sigma})\right)$ is a torsion-free sheaf of rank one. Hence there is a meromorphic section $\Gamma \subset \boldsymbol{B}$ and a divisor $\Delta$ supported in $p^{-1} D$ such that $\mathcal{M} \otimes \mathcal{O}_{B}(\boldsymbol{\Sigma}) \simeq \mathcal{O}_{B}(\Gamma+\Delta)$. Hence $\Psi(\Gamma)=\mathcal{M}$ modulo $\mathcal{V}_{B}$. 
Let $\mathfrak{T}_{H / S}$ be the cokernel of $E^{2} \rightarrow{ }_{\mathrm{II}} E_{2}^{0,2}$. Then we have two exact sequences

$$
\begin{gathered}
0 \rightarrow \mathfrak{T}_{H / S} \rightarrow R^{2} \boldsymbol{p}_{*} \mathbb{Z}_{\boldsymbol{B}} / \mathcal{V}_{\boldsymbol{B}} \rightarrow \mathbb{Z}_{S} \rightarrow 0 \\
0 \rightarrow \mathfrak{S}_{H / S}^{\mathbf{W}} \rightarrow \mathfrak{S}_{H / S} \rightarrow \mathfrak{T}_{H / S} \rightarrow 0
\end{gathered}
$$

The first sequence is split by a meromorphic section of $\boldsymbol{p} . \mathfrak{T}_{H / S}$ also does not depend on the choice of $\boldsymbol{B}$. There is an injection

$$
\mathfrak{T}_{H / S} \hookrightarrow{ }_{\mathrm{II}} E_{2}^{2,1}=\mathcal{H}_{D}^{2}\left(R^{1} \boldsymbol{p}_{*} \mathbb{Z}_{\boldsymbol{B}}\right) \simeq R^{1} j_{*} H
$$

whose cokernel is ${ }_{\text {II }} E_{\infty}^{2,1}$. As a result, we have:

Theorem 5.4.9. Let $\boldsymbol{p}: \boldsymbol{B}=\boldsymbol{B}(H) \rightarrow S$ be a basic elliptic fibration associated with $H$ such that $\boldsymbol{B}$ is non-singular and that $\boldsymbol{p}$ is smooth over $S^{\star}$. Then we have a commutative diagram Figure 1 of exact sequences.

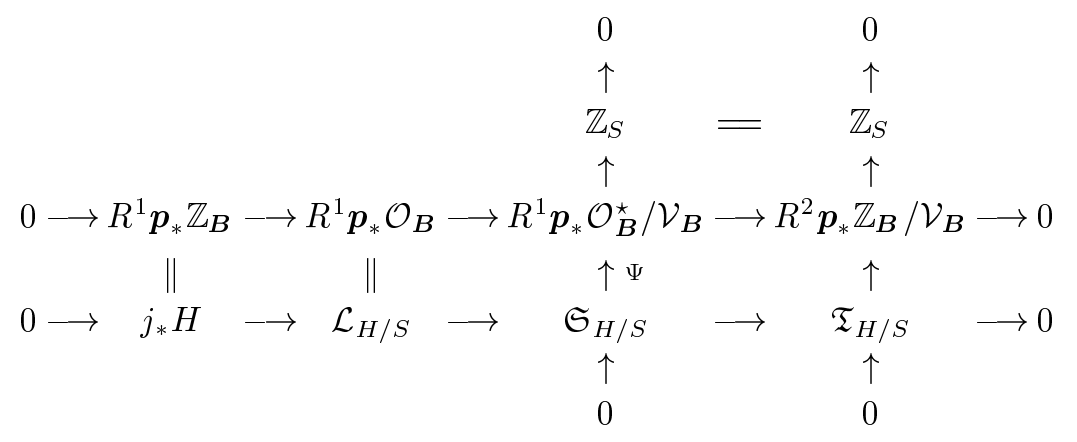

Figure 1.

Lemma 5.4.10. $\mathfrak{T}_{H / S^{\circ}}=\left.\mathfrak{T}_{H / S}\right|_{S^{\circ}}$ is isomorphic to the torsion part $\left(R^{1} j_{*}^{\star} H\right)_{\text {tor }}$.

Proof. We may assume $S=S^{\circ}$ and $\boldsymbol{p}: \boldsymbol{B} \rightarrow S$ is the minimal basic elliptic fibration. Thus ${ }_{\mathrm{II}} E_{2}^{2,0} \simeq \mathbb{Z}_{D}$. Let $\boldsymbol{p}^{-1} D=\sum_{i=1}^{h} C_{i}$ be the irreducible decomposition over a suitable open neighborhood of a point $s \in D$. Then $p^{-1}(s) \cap C_{i}$ are all irreducible curves. The stalk $\left(E^{2}\right)_{s}$ is a free abelian group of rank $h$ generated by $C_{i}$. The stalk $\left({ }_{\text {II }} E_{2}^{0,2}\right)_{s}$ is the kernel of the trace map $H^{2}\left(\boldsymbol{p}^{-1}(s), \mathbb{Z}\right) \rightarrow \mathbb{Z}$. Thus $\left({ }_{\mathrm{II}} E_{2}^{0,2}\right)_{s}$ is also a free abelian group of rank $h-1$. By 5.4.5, the cokernel $\left(\mathfrak{T}_{H / S}\right)_{s}$ of $\left(E^{2}\right)_{s} \rightarrow\left({ }_{\mathrm{II}} E_{2}^{0,2}\right)_{s}$ is a torsion group. On the other 
hand, $\left(E^{3}\right)_{s}$ is a torsion-free abelian group, since ${ }_{\mathrm{I}} E_{2}^{1,2}=R^{1} \boldsymbol{p}_{*} \mathcal{H}_{\boldsymbol{p}^{-1} D}^{2}\left(\mathbb{Z}_{\boldsymbol{B}}\right)$ and ${ }_{\mathrm{I}} E_{2}^{0,3}=\boldsymbol{p}_{*} \mathcal{H}_{\boldsymbol{p}^{-1} D}^{3}\left(\mathbb{Z}_{\boldsymbol{B}}\right)$ are sheaves of torsion-free abelian groups (cf. 3.3.2 and 3.3.1). The vanishing ${ }_{\mathrm{II}} E_{2}^{3,0}=\mathcal{H}_{D}^{3}\left(\mathbb{Z}_{S}\right)=0$ implies that there is an injection ${ }_{\mathrm{II}} E_{\infty}^{2,1} \rightarrow E^{3}$. Hence $\left({ }_{\mathrm{II}} E_{\infty}^{2,1}\right)_{s}$ is torsion-free. Therefore $\left(\mathfrak{T}_{H / S}\right)_{s}$ is the torsion part of $\left({ }_{\mathrm{II}} E_{2}^{2,1}\right)_{s} \simeq\left(R^{1} j_{*} H\right)_{s}$.

Corollary 5.4.11. $\quad \mathfrak{S}_{H / S^{\circ}}$ is characterized by the following condition as a subsheaf of $j_{*}^{\star} \mathfrak{S}_{H}$ containing $\mathfrak{S}_{H / S^{\circ}}^{\mathbf{W}}$ :

$$
\mathfrak{S}_{H / S^{\circ}} / \mathfrak{S}_{H / S^{\circ}}^{\mathbf{W}}=\left(j_{*}^{\star} \mathfrak{S}_{H} / \mathfrak{S}_{H / S^{\circ}}^{\mathbf{W}}\right)_{\text {tor }} .
$$

Proof. The commutative diagram of exact sequences

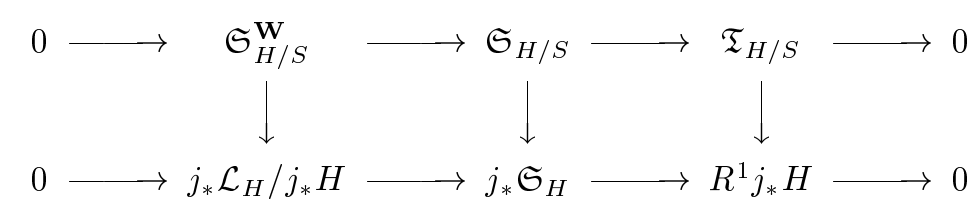

is derived from Figure 1. Then the assertion follows 5.4.10.

Let $S^{\Delta} \subset S$ be a Zariski-open subset such that $S^{\star} \subset S^{\Delta}$ and $\operatorname{codim}(S \backslash$ $\left.S^{\Delta}\right) \geq 2$. Let $j^{\Delta \star}: S^{\star} \hookrightarrow S^{\Delta}$ and $j^{\Delta}: S^{\Delta} \hookrightarrow S$ denote the open immersions.

Lemma 5.4.12. The natural homomorphism

$$
R^{q} j_{*}^{\Delta}\left(j_{*}^{\Delta \star} H\right) \rightarrow R^{q} j_{*}^{\Delta} \mathcal{L}_{H / S^{\Delta}}
$$

is zero for $q \geq 1$.

Proof. The morphism $R \underline{\Gamma}_{D}\left(j_{*} H\right) \rightarrow R \underline{\Gamma}_{D}\left(\mathcal{L}_{H / S}\right)$ is zero by 4.2.3. Let $Z^{\Delta}$ be the complement $S \backslash S^{\Delta}$. Then $R \underline{\Gamma}_{Z^{\Delta}}\left(j_{*} H\right) \rightarrow R \underline{\Gamma}_{Z^{\Delta}}\left(\mathcal{L}_{H / S}\right)$ is zero, since $R \underline{\Gamma}_{Z^{\Delta}} \sim_{\text {qis }} R \underline{\Gamma}_{Z^{\Delta}} \circ R \underline{\Gamma}_{D}$.

Proposition 5.4.13. Suppose that $S^{\Delta} \subset S^{\circ}$. Then there exists a commutative diagram of exact sequences:

$$
\begin{aligned}
& 0 \longrightarrow R^{1} j_{*}^{\Delta}\left(j_{*}^{\Delta \star} H\right) \longrightarrow \mathfrak{T}_{H / S} \longrightarrow j_{*}^{\Delta}\left(R^{1} j_{*}^{\Delta \star} H\right)_{\text {tor }} \longrightarrow R^{2} j_{*}^{\Delta}\left(j_{*}^{\Delta \star} H\right) \\
& 0 \longrightarrow R^{1} j_{*}^{\Delta}\left(j_{*}^{\Delta \star} H\right) \longrightarrow R^{1} j_{*} H \longrightarrow j_{*}^{\Delta}\left(R^{1} j_{*}^{\Delta \star} H\right) \longrightarrow R^{2} j_{*}^{\Delta}\left(j_{*}^{\Delta \star} H\right) \text {. }
\end{aligned}
$$

In particular, $\mathfrak{T}_{H / S}$ is isomorphic to the kernel of

$$
R^{1} j_{*} H \rightarrow j_{*}^{\circ}\left(R^{1} j_{*}^{\star} H /\left(R^{1} j_{*}^{\star} H\right)_{\text {tor }}\right) .
$$


Proof. Let $Z^{\Delta}$ be the complement of $S^{\Delta}$ in $S$. It is enough to show that $\mathcal{H}_{Z^{\Delta}}^{0}\left(\mathfrak{T}_{H / S}\right) \simeq \mathcal{H}_{Z^{\Delta}}^{0}\left(R^{1} j_{*} H\right)$ and that $\mathcal{H}_{Z^{\Delta}}^{1}\left(\mathfrak{T}_{H / S}\right) \rightarrow \mathcal{H}_{Z^{\Delta}}^{1}\left(R^{1} j_{*} H\right)$ is injective. We infer that the injection $\mathfrak{T}_{H / S} \hookrightarrow R^{1} j_{*} H$ is obtained as the composite

$$
\mathcal{H}_{D}^{0}\left(R^{2} \boldsymbol{p}_{*} \mathbb{Z}_{\boldsymbol{B}} / \mathcal{V}_{\boldsymbol{B}}\right) \rightarrow \mathcal{H}_{D}^{1}\left(\mathfrak{S}_{H / S}^{\mathbf{W}}\right) \rightarrow \mathcal{H}_{D}^{2}\left(j_{*} H\right)
$$

where the left homomorphism is derived from (5.1). Since $\mathfrak{S}_{H / S} \simeq j_{*}^{\Delta} \mathfrak{S}_{H / S^{\Delta}}$, we have an isomorphism $\mathcal{H}_{Z^{\Delta}}^{0}\left(\mathfrak{T}_{H / S}\right) \simeq \mathcal{H}_{Z^{\Delta}}^{1}\left(\mathfrak{S}_{H / S}^{\mathbf{W}}\right)$ and an injection $\mathcal{H}_{Z^{\Delta}}^{1}\left(\mathfrak{T}_{H / S}\right)$ $\hookrightarrow \mathcal{H}_{Z^{\Delta}}^{2}\left(\mathfrak{S}_{H / S}^{\mathbf{W}}\right)$ from (5.3). By 5.4.12, we have an isomorphism

$$
\mathcal{H}_{Z^{\Delta}}^{0}\left(\mathfrak{T}_{H / S}\right) \simeq \mathcal{H}_{Z^{\Delta}}^{1}\left(\mathfrak{S}_{H / S}^{\mathbf{W}}\right) \simeq \mathcal{H}_{Z^{\Delta}}^{2}\left(j_{*} H\right) \simeq \mathcal{H}_{Z^{\Delta}}^{0}\left(\mathcal{H}_{D}^{2}\left(j_{*} H\right)\right)
$$

Further we have an exact sequence

$$
0 \rightarrow \mathcal{H}_{Z^{\Delta}}^{2}\left(\mathcal{L}_{H / S}\right) \rightarrow \mathcal{H}_{Z^{\Delta}}^{2}\left(\mathfrak{S}_{H / S}^{\mathbf{W}}\right) \rightarrow \mathcal{H}_{Z^{\Delta}}^{3}\left(j_{*} H\right)
$$

Since $\mathcal{H}_{Z^{\Delta}}^{1}\left(\mathfrak{T}_{H / S}\right)$ is a sheaf of torsion groups by 5.4 .10 , the composite

$$
\mathcal{H}_{Z^{\Delta}}^{1}\left(\mathfrak{T}_{H / S}\right) \hookrightarrow \mathcal{H}_{Z^{\Delta}}^{2}\left(\mathfrak{S}_{H / S}^{\mathbf{W}}\right) \rightarrow \mathcal{H}_{Z^{\Delta}}^{3}\left(j_{*} H\right)
$$

is also injective. This is also obtained as the composite

$$
\mathcal{H}_{Z^{\Delta}}^{1}\left(\mathfrak{T}_{H / S}\right) \rightarrow \mathcal{H}_{Z^{\Delta}}^{1}\left(R^{1} j_{*} H\right) \simeq \mathcal{H}_{Z^{\Delta}}^{1}\left(\mathcal{H}_{D}^{2}\left(j_{*} H\right)\right) \rightarrow \mathcal{H}_{Z^{\Delta}}^{3}\left(j_{*} H\right),
$$

where the last homomorphism is derived from the spectral sequence associated with $R \underline{\Gamma}_{Z^{\Delta}}\left(R \underline{\Gamma}_{D}\left(j_{*} H\right)\right) \sim_{\text {qis }} R \underline{\Gamma}_{Z^{\Delta}}\left(j_{*} H\right)$. Thus we are done.

Corollary 5.4.14. Suppose that $S^{\star} \subset S$ together with $0 \in S$ is a ddimensional toroidal embedding of type $(\mathrm{N}, l, \sigma)$. Then

$$
\left(\mathfrak{T}_{H / S}\right)_{0} \simeq \begin{cases}0, & H \text { is of type } \mathrm{I}_{0} \\ N\left(\boldsymbol{a}^{\perp} \cap \sigma\right)^{\perp} / \mathbb{Z} \boldsymbol{a}, & H \text { is of type } \mathrm{I}_{\boldsymbol{a}} \text { for } \boldsymbol{a} \neq 0 \text { (cf. 4.3.4); } \\ \left(R^{1} j_{*} H\right)_{0}, & \text { otherwise. }\end{cases}
$$

Lemma 5.4.15. Suppose that $S=S^{\circ}$. Then we have the following isomorphisms:

$$
\begin{aligned}
& R^{1} j_{*} H \simeq j_{*}^{\Delta}\left(R^{1} j_{*}^{\Delta \star} H\right) ; \quad R^{2} j_{*}^{\Delta}\left(j_{*}^{\Delta \star} H\right)=0 ; \\
& R^{1} j_{*}^{\Delta} \mathcal{L}_{H / S^{\Delta}} \simeq R^{1} j_{*}^{\Delta} \mathfrak{S}_{H / S^{\Delta}}^{\mathbf{W}} \simeq R^{1} j_{*}^{\Delta} \mathfrak{S}_{H / S^{\Delta}} .
\end{aligned}
$$

In particular, $R^{1} j_{*}^{\Delta} \mathfrak{S}_{H / S^{\Delta}}$ is a sheaf of $\mathbb{C}$-vector spaces. 
Proof. We have $R^{1} j_{*} H \simeq j_{*}^{\triangleleft}\left(R^{1} j_{*}^{\Delta \star} H\right)$ since $R^{1} j_{*}^{\star} H$ is locally constant over $D$. Thus the edge sequence of Leray's spectral sequence for $R j_{*} H \sim_{\text {qis }}$ $R j_{*}^{\Delta}\left(R j_{*}^{\Delta \star} H\right)$ induces an injection $R^{2} j_{*}^{\Delta}\left(j_{*}^{\Delta \star} H\right) \rightarrow R^{2} j_{*} H$. We have $R^{2} j_{*} H=0$ since $S=S^{\circ}$. The sheaf $\mathfrak{T}_{H / S}=\left(R^{1} j_{*} H\right)_{\text {tor }}$ is also locally constant over $D$. Hence $\mathcal{H}_{Z^{\Delta}}^{1}\left(\mathfrak{T}_{H / S}\right)=0$ and

$$
j_{*}^{\Delta}\left(R^{1} j_{*}^{\Delta \star} H\right) \rightarrow j_{*}^{\Delta}\left(R^{1} j_{*}^{\Delta \star} H / \mathfrak{T}_{H / S^{\Delta}}\right)
$$

is surjective. Hence $\mathcal{H}_{Z^{\Delta}}^{2}\left(\mathfrak{T}_{H / S}\right) \rightarrow \mathcal{H}_{Z^{\triangleleft}}^{2}\left(R^{1} j_{*} H\right)$ is injective. We have the following commutative diagram

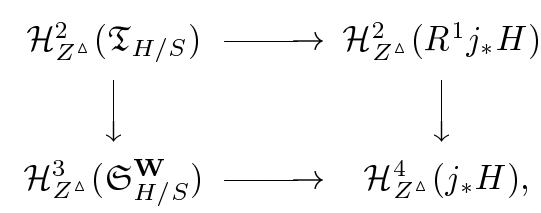

where the right arrow is " $E_{2}^{2,1} \rightarrow{ }^{\prime \prime} E_{2}^{4,0}$ for the spectral sequence

$$
{ }^{\prime \prime} E_{2}^{p, q}=\mathcal{H}_{Z^{\triangleleft}}^{p}\left(R^{q} j_{*} H\right) \Longrightarrow \mathcal{H}^{p+q}\left(R \underline{\Gamma}_{Z^{\triangleleft}}\left(R j_{*} H\right)\right)=0 .
$$

Since $R^{2} j_{*} H=0$, the right arrow is injective. Hence the left arrow is also injective. Therefore $\mathcal{H}_{Z^{\Delta}}^{2}\left(\mathfrak{S}_{H / S}^{\mathbf{W}}\right) \simeq \mathcal{H}_{Z^{\Delta}}^{2}\left(\mathfrak{S}_{H / S}\right)$. The rest isomorphism $\mathcal{H}_{Z^{\Delta}}^{2}\left(\mathcal{L}_{H / S}\right)$ $\simeq \mathcal{H}_{Z^{\Delta}}^{2}\left(\mathfrak{S}_{H / S}^{\mathbf{W}}\right)$ is derived from $\mathcal{H}_{Z^{\Delta}}^{3}\left(j_{*} H\right)=R^{2} j_{*}^{\Delta}\left(j_{*}^{\Delta \star} H\right)=0$.

\section{§5.5. Elliptic fibration having local meromorphic sections}

Proposition 5.5.1. There is an injective group homomorphism

$$
\mathcal{E}_{0}(S, D, H) \hookrightarrow H^{1}\left(S, \mathfrak{S}_{H / S}\right) .
$$

This is bijective if $S=S^{\circ}$.

Proof. We fix a basic elliptic fibration $\boldsymbol{p}: \boldsymbol{B} \rightarrow S$ associated with $H$. Let $(f: X \rightarrow S, \phi)$ be a marked elliptic fibration contained in $\mathcal{E}_{0}(S, D, H)$. Then there exist an open covering $\left\{U_{\alpha}\right\}$ of $S$ and meromorphic sections $\sigma_{\alpha}: U_{\alpha} \cdots \rightarrow$ $f^{-1}\left(U_{\alpha}\right)$. Thus there is a bimeromorphic mapping $h_{\alpha}: f^{-1}\left(U_{\alpha}\right) \cdots \rightarrow p^{-1}\left(U_{\alpha}\right)$ such that $h_{\alpha}^{*}$ induces $\phi$ over $U_{\alpha}$ and that $h_{\alpha} \circ \sigma_{\alpha}$ is the zero section. The transition mapping

$$
h_{\alpha} \circ h_{\beta}^{-1}: p^{-1}\left(U_{\alpha} \cap U_{\beta}\right) \cdots \rightarrow p^{-1}\left(U_{\alpha} \cap U_{\beta}\right)
$$

is the translation mapping $\operatorname{tr}\left(\eta_{\alpha, \beta}\right)$ of a meromorphic section $\eta_{\alpha, \beta}$ of $\boldsymbol{p}$ over $U_{\alpha} \cap U_{\beta}$. The cohomology class of $\left\{\eta_{\alpha, \beta}\right\}$ in $H^{1}\left(S, \mathfrak{S}_{H / S}\right)$ does not depend 
on the choice of $\left\{U_{\alpha}\right\}$ and $\left\{h_{\alpha}\right\}$. Thus we have an injection $\mathcal{E}_{0}(S, D, H) \hookrightarrow$ $H^{1}\left(S, \mathfrak{S}_{H / S}\right)$. By the construction and by 5.2 .5 , we infer that this is a group homomorphism. The converse construction has a problem on gluing $\boldsymbol{p}^{-1} U_{\alpha}$ by meromorphic translation mappings $\operatorname{tr}\left(\eta_{\alpha, \beta}\right)$. If $S=S^{\circ}$, then we can choose $\boldsymbol{p}: \boldsymbol{B} \rightarrow S$ to be the minimal basic elliptic fibration. Thus the meromorphic sections $\eta_{\alpha, \beta}$ are holomorphic by 5.3.2. Thus we can glue $\boldsymbol{p}^{-1}\left(U_{\alpha}\right)$.

Let $(f: X \rightarrow S, \phi)$ be a marked elliptic fibration belonging to $\mathcal{E}_{0}(S, D, H)$ such that $X$ is non-singular. Let $D^{\prime} \supset D$ be an analytic subset such that $f$ is smooth outside $D^{\prime}$. We define $\mathcal{V}_{X}:=\mathcal{H}_{D^{\prime}}^{0}\left(R^{1} f_{*} \mathcal{O}_{X}^{\star}\right)$. Then $R^{1} f_{*} \mathcal{O}_{X}^{\star} / \mathcal{V}_{X}$ does not depend on the choice of bimeromorphically equivalent non-singular models $X$ over $S$ by 5.4.6. The composite $\mathcal{V}_{X} \hookrightarrow R^{1} f_{*} \mathcal{O}_{X}^{\star} \rightarrow R^{2} f_{*} \mathbb{Z}_{X}$ is injective and $R^{1} f_{*} \mathbb{Z}_{X} \simeq j_{*} H$ by 5.4.5.

Lemma 5.5.2. Under the situation above, there exist a homomorphism $\Psi_{X}: \mathfrak{S}_{H / S} \rightarrow R^{1} f_{*} \mathcal{O}_{X}^{\star} / \mathcal{V}_{X}$ and a commutative diagram Figure 2 of exact sequences.

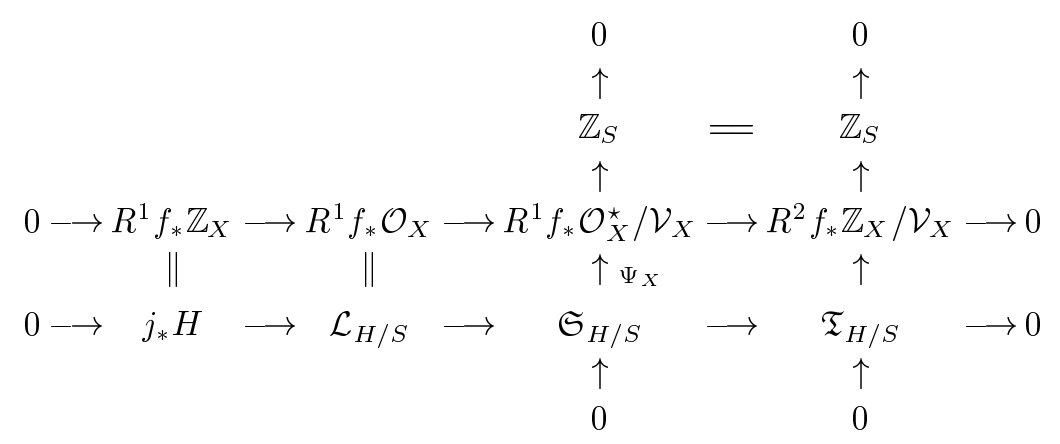

Figure 2.

Proof. Let $\left\{U_{\alpha}\right\}$ be an open covering of $S$ and let $\Sigma_{\alpha} \subset f^{-1}\left(U_{\alpha}\right)$ be a meromorphic section of $f^{-1}\left(U_{\alpha}\right) \rightarrow U_{\alpha}$. Let $h_{\alpha}: f^{-1}\left(U_{\alpha}\right) \cdots \rightarrow p^{-1}\left(U_{\alpha}\right)$ be the bimeromorphic mapping over $U_{\alpha}$ such that the proper transform of $\boldsymbol{\Sigma}_{\alpha}$ is the zero section $\boldsymbol{\Sigma} \cap \boldsymbol{p}^{-1}\left(U_{\alpha}\right)$ and that $h_{\alpha}$ preserves the marking $\phi$ of $H$. By 5.4.9, over $U_{\alpha}$, we have the homomorphism $\left(\Psi_{X}\right)_{\alpha}: \mathfrak{S}_{H / U_{\alpha}} \rightarrow$ $\left.\left(R^{1} f_{*} \mathcal{O}_{X}^{\star} / \mathcal{V}_{X}\right)\right|_{U_{\alpha}}$ with the desired property. Note that the $\left(\Psi_{X}\right)_{\alpha}$ is obtained as follows: A meromorphic section $\Gamma \subset \boldsymbol{p}^{-1}\left(U_{\alpha}\right)$ is mapped to the equivalence class of the invertible sheaf $\mathcal{O}\left(\Gamma_{\alpha}-\Sigma_{\alpha}\right)$, where $\Gamma_{\alpha}$ denotes the proper transform of $\Gamma$ by $h_{\alpha}^{-1}$. We have only to check $\left(\Psi_{X}\right)_{\alpha}=\left(\Psi_{X}\right)_{\beta}$ on $U_{\alpha} \cap U_{\beta}$ for any $\alpha, \beta$. 
We know that $h_{\beta} \circ h_{\alpha}^{-1}: \boldsymbol{p}^{-1}\left(U_{\alpha} \cap U_{\beta}\right) \cdots \rightarrow \boldsymbol{p}^{-1}\left(U_{\alpha} \cap U_{\beta}\right)$ is the translation mapping of a meromorphic section $\boldsymbol{\Sigma}_{\beta, \alpha} \subset \boldsymbol{p}^{-1}\left(U_{\alpha} \cap U_{\beta}\right)$. Since a translation mapping of an elliptic curve does not change invertible sheaves of degree zero, $\mathcal{O}\left(\Gamma_{\alpha}-\boldsymbol{\Sigma}_{\alpha}\right)$ and $\mathcal{O}\left(\Gamma_{\beta}-\boldsymbol{\Sigma}_{\beta}\right)$ are equivalent over $U_{\alpha} \cap U_{\beta}$ modulo $\mathcal{V}_{X}$. Hence we have a global homomorphism $\Psi_{X}$ with the desired property.

By the construction of $\Psi_{X}$, we have:

Corollary 5.5.3. The cohomology class in $H^{1}\left(S, \mathfrak{S}_{H / S}\right)$ corresponding to $(f: X \rightarrow S, \phi)$ by 5.5.1 is identical to the image of 1 under the connecting homomorphism $H^{0}(S, \mathbb{Z}) \rightarrow H^{1}\left(S, \mathfrak{S}_{H / S}\right)$ of the exact sequence:

$$
0 \rightarrow \mathfrak{S}_{H / S} \stackrel{\Psi_{X}}{\longrightarrow} R^{1} f_{*} \mathcal{O}_{X}^{\star} / \mathcal{V}_{X} \rightarrow \mathbb{Z}_{S} \rightarrow 0
$$

appearing in Figure 2.

Proposition 5.5.4. Let $(f: X \rightarrow S, \phi)$ be an element of $\mathcal{E}_{0}(S, D, H)$. Then $f$ is BP if and only if the corresponding cohomology class in $H^{1}\left(S, \mathfrak{S}_{H / S}\right)$ is a torsion element. Conversely, for a torsion element of $H^{1}\left(S, \mathfrak{S}_{H / S}\right)$, there is a marked elliptic fibration $(f: X \rightarrow S, \phi)$ belonging to $\mathcal{E}_{0}(S, D, H)$ such that $f$ is a BP elliptic fibration smooth outside $D$ and that $(f, \phi)$ represents the torsion element. In particular, we have an identification:

$$
\mathcal{E}_{0}^{\text {proj }}(S, D, H) \longleftrightarrow H^{1}\left(S, \mathfrak{S}_{H / S}\right)_{\text {tor }}
$$

Proof. If $f: X \rightarrow S$ is BP and if $X$ is non-singular, then there is an invertible sheaf $\mathcal{M}$ on $X$ whose restriction to a smooth fiber has positive degree. Since the degree is nothing but the image of the equivalence class of $\mathcal{M}$ in $R^{1} f_{*} \mathcal{O}_{X}^{\star} / \mathcal{V}_{X}$ under the surjection $R^{1} f_{*} \mathcal{O}_{X}^{\star} / \mathcal{V}_{X} \rightarrow \mathbb{Z}_{S}$. Therefore the corresponding cohomology class is a torsion element. Conversely, suppose that a cohomology class in $H^{1}\left(S, \mathfrak{S}_{H / S}\right)$ is a torsion element of order $m$. Let $\left\{U_{\alpha}\right\}$ be an open covering and let $\eta_{\alpha, \beta}$ be meromorphic sections of $\boldsymbol{p}$ over $U_{\alpha} \cap U_{\beta}$ representing the cohomology class. We want to patch $p^{-1}\left(U_{\alpha}\right)$ by the meromorphic translation mappings $\operatorname{tr}\left(\eta_{\alpha, \beta}\right)$. Here we consider the multiplication map $\boldsymbol{B} . \stackrel{m \times}{\longrightarrow} \boldsymbol{B}$ over $S$. The Stein factorization $\mu: \boldsymbol{B}^{\prime} \rightarrow \boldsymbol{B}$ is a finite morphism étale over $S^{\star}$. Let $\boldsymbol{p}^{\prime}: \boldsymbol{B}^{\prime} \rightarrow S$ denote the structure morphism. Then there is a commutative diagram

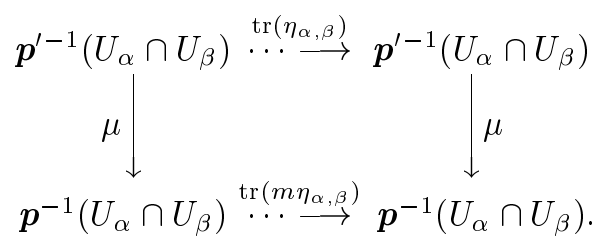


Since $\left\{m \eta_{\alpha, \beta}\right\}$ is cohomologous to zero, we can patch $\boldsymbol{p}^{-1}\left(U_{\alpha}\right)$. Hence we have an elliptic fibration $X^{\prime} \rightarrow S$ and a finite morphism $X^{\prime} \rightarrow \boldsymbol{B}$ over $S$. In particular, $X^{\prime} \rightarrow S$ is a BP morphism smooth outside $D$.

Proposition 5.5.5. Let $f^{\circ}: X^{\circ} \rightarrow S^{\circ}$ be an LBP elliptic fibration smooth outside $D^{\star}$. If a union of irreducible components of multiplicity 1 of $f^{*} D^{\star}$ dominates $D^{\star}$, then there exist local meromorphic sections of $f^{\circ}$ over any points of $S^{\circ}$.

Proof. We may assume that $S^{\circ}$ is a polydisc and $D^{\star}$ is a coordinate hyperplane and that $f^{\circ}$ is BP. Let $R \subset f^{*} D^{\star}$ be an irreducible component of multiplicity 1 dominating $D^{\star}$. For a desingularization $\widetilde{R} \rightarrow R$, the composite $\widetilde{R} \rightarrow D^{\star}$ is smooth outside a proper Zariski-closed subset $T \subset D^{\star} \subset S^{\circ}$. Thus $R \rightarrow D^{\star}$ admits local sections over $D^{\star} \backslash T . f^{\circ}$ is flat over a Zariski-open subset $S^{b} \subset S$ with $\operatorname{codim}\left(S \backslash S^{b}\right) \geq 2$. Let $S^{\Delta}$ denote the Zariski-open subset $S^{\mathrm{b}} \backslash T$ of $S^{\circ}$. Then the restriction of $f^{\circ}$ to $S^{\triangle}$ belongs to $\mathcal{E}_{0}\left(S^{\Delta}, D^{\star} \cap S^{\Delta}, H\right)$ and corresponds to a torsion element of $H^{1}\left(S^{b}, \mathfrak{S}_{H / S^{\circ}}\right)$. For the open immersion $j^{\circ \Delta}: S^{\Delta} \hookrightarrow S^{\circ}$, we have an isomorphism $\mathfrak{S}_{H / S^{\circ}} \simeq j_{*}^{\circ \Delta} \mathfrak{S}_{H / S^{\Delta}}$ and an exact sequence

$$
0 \rightarrow H^{1}\left(S^{\circ}, \mathfrak{S}_{H / S^{\circ}}\right) \rightarrow H^{1}\left(S^{\Delta}, \mathfrak{S}_{H / S^{\Delta}}\right) \rightarrow H^{0}\left(S^{\circ}, R^{1} j_{*}^{\circ \Delta} \mathfrak{S}_{H / S^{\Delta}}\right) .
$$

There is an isomorphism $H^{1}\left(S^{\circ}, \mathfrak{S}_{H / S^{\circ}}\right)_{\text {tor }} \simeq H^{1}\left(S^{\Delta}, \mathfrak{S}_{H / S^{\Delta}}\right)_{\text {tor }}$, since $R^{1} j_{*}^{\circ \Delta} \mathfrak{S}_{H / S^{\Delta}}$ is a sheaf of $\mathbb{Q}$-vector spaces by 5.4.15. Thus $f^{\circ}$ admits a meromorphic section over $S^{\Delta} \cap \mathcal{U}$ for an open neighborhood $\mathcal{U}$ of 0 . Since $f^{\circ}$ is $\mathrm{BP}$, we may assume that $\left(f^{\circ}\right)^{-1}(\mathcal{U})$ is bimeromorphically equivalent over $\mathcal{U}$ to a closed subvariety of $\mathbb{P}^{n} \times \mathcal{U}^{\circ}$ for some $n$. Since $\operatorname{codim}\left(S^{\circ} \backslash S^{\Delta}\right) \geq 2$, the meromorphic section extends to $\mathcal{U}$.

Corollary 5.5.6. Let $f^{\circ}: X^{\circ} \rightarrow S^{\circ}$ be an LBP elliptic fibration smooth outside $D^{\star}$ and let $U \subset S^{\circ}$ be the set of points over which $f^{\circ}$ admits local meromorphic sections. Then $U$ is a Zariski-open subset.

\section{$\S 6 . \quad$ Description of Global Structure}

In Section 5, we relate $\mathcal{E}_{0}(S, D, H)$ with $H^{1}\left(S, \mathfrak{S}_{H / S}\right)$. However, unfortunately, the method is not sufficient to classify other elliptic fibrations, for example, that have multiple fibers. In Section 6 , we realize $\mathcal{E}(S, D, H)$ as a subgroup of the $\partial$-étale cohomology group $H^{1}\left(\underline{S}, \mathfrak{S}_{H / \underline{S}}\right)$ for the sheaf $\mathfrak{S}_{H / \underline{S}}$ of germs of meromorphic sections in the $\partial$-étale topology on $\underline{S}=(S, D)$ defined below. Here, the group of BP-fibrations coincides with the torsion part of 
$H^{1}\left(\underline{S}, \mathfrak{S}_{H / \underline{S}}\right)$. The calculus in Section 6.2 is important for the proof and other applications.

For a basic elliptic fibration $\boldsymbol{p}: \boldsymbol{B}=\boldsymbol{B}(H) \rightarrow S$, let $\underline{\boldsymbol{B}}$ be the $\partial$-space $\left(\boldsymbol{B}, \boldsymbol{p}^{-1} D\right)$ and let $\underline{\boldsymbol{p}}$ be the induced morphism $\underline{\boldsymbol{B}} \rightarrow \underline{\boldsymbol{S}}$. The sheaf $\mathfrak{S}_{H / \underline{S}}$ of germs of meromorphic sections of $\underline{p}$ over $\underline{S}$ is defined as follows: For a $\partial$-étale morphism $\underline{U}=(U, \Delta) \rightarrow \underline{S}$ with $\underline{U}=\operatorname{sp}(\underline{U})$,

$$
\mathfrak{S}_{H / \underline{S}}(\underline{U}):=\{U \cdots \rightarrow \boldsymbol{B} \mid \text { meromorphic mapping over } S\} .
$$

\section{$\S 6.1$. Fundamental diagram in $\partial$-étale topology}

Let $\mathcal{E}_{1}(S, D, H)$ be the subset of $\mathcal{E}(S, D, H)$ consisting of marked elliptic fibrations $(f: X \rightarrow S, \phi)$ such that $f$ satisfies the following condition: For any point of $\underline{S}$, there is a $\partial$-étale neighborhood $(U, \Delta) \rightarrow \underline{S}$ such that $X \times_{S} U \rightarrow$ $U$ has a meromorphic section. $\mathcal{E}_{1}(S, D, H)$ is also a subgroup of $\mathcal{E}(S, D, H)$ (cf. 5.2.5). We set $\mathcal{E}_{1}^{\text {proj }}(S, D, H):=\mathcal{E}^{\operatorname{proj}}(S, D, H) \cap \mathcal{E}_{1}(S, D, H)$.

Let $f: X \rightarrow S$ be an elliptic fibration such that $X$ is non-singular, $f^{-1} D$ is a normal crossing divisor, and that $(f, \phi) \in \mathcal{E}_{1}(S, D, H)$ for some marking $\phi$. Let $\underline{X}:=\left(X, f^{-1} D\right)$ be the $\partial$-space and let $\underline{f}: \underline{X} \rightarrow \underline{S}$ be the induced morphism. Let $\underline{U}=\left(U, D_{U}\right) \rightarrow \underline{S}$ be a $\partial$-étale morphism, where $\left[U, D_{U}\right]$ is the top realization of $\underline{U}, X_{U}$ the normalization of the main component of the fiber product $X \times{ }_{S} U$, and let $f_{U}: X_{U} \rightarrow U$ denote the induced morphism by $f$. Then $X_{U}$ has only quotient singularities and $X_{U} \backslash f_{U}^{-1} D_{U} \hookrightarrow X_{U}$ is a toroidal embedding. The analytic space with boundary $\left[X_{U}, f_{U}^{-1} D_{U}\right]$ is the top realization of $\underline{X} \times \underline{S} \underline{U}$. We denote by $\underline{f}_{U}: \underline{X} \times \underline{S} \underline{U} \rightarrow \underline{U}$ the induced morphism.

Let $\mu: Y \rightarrow X_{U}$ be a bimeromorphic morphism from a non-singular variety such that $D_{Y}:=\mu^{-1} f_{U}^{-1} D_{U}$ is a normal crossing divisor and that $\mu$ is an isomorphism outside $D_{U}$. Let $\underline{Y}$ denote the $\partial$-space $\left(Y, D_{Y}\right)$ and let $\underline{\mu}: \underline{Y} \rightarrow$ $\underline{X} \times \underline{S} \underline{U}$ denote the induced morphism.

Lemma 6.1.1. We have the following isomorphisms, where $p \geq 0$ :

$$
\begin{array}{ll}
R^{1} f_{U *} \mathbb{Z}_{X_{U}} \simeq R^{1}\left(f_{U} \circ \mu\right)_{*} \mathbb{Z}_{Y}, & \left.\left(R^{1} \underline{f}_{*} \mathbb{Z}_{\underline{X}}\right)\right|_{\underline{U}} \simeq R^{1}\left(\underline{f}_{\underline{U}} \circ \underline{\mu}\right)_{*} \mathbb{Z}_{\underline{Y}}, \\
R^{p} f_{U *} \mathcal{O}_{X_{U}} \simeq R^{p}\left(f_{U} \circ \mu\right)_{*} \mathcal{O}_{Y}, & \left.\left(R^{p} \underline{f}_{*} \mathcal{O}_{\underline{X}}\right)\right|_{\underline{U}} \simeq R^{p}\left(\underline{f}_{\underline{U}} \circ \underline{\mu}\right)_{*} \mathcal{O}_{\underline{Y}} .
\end{array}
$$

Proof. We have $R^{1} \mu_{*} \mathbb{Z}_{Y}=0$ and $R^{p} \mu_{*} \mathcal{O}_{Y}=0$ for $p>0$, since $X_{U}$ has only rational singularities. By considering the toroidal embedding $X_{U} \backslash$ $f_{U}^{-1} D_{U} \subset X_{U}$, we also have $R^{1} \underline{\mu}_{*} \mathbb{Z}_{\underline{Y}}=0$ and $R^{p} \underline{\mu}_{*} \mathcal{O}_{\underline{Y}}=0$ for $p>0$ from 3.4.2 and 2.2.6. Thus we have the isomorphisms. 
As in Section 5.4, we consider the quasi-isomorphism

$$
R \underline{f}_{*} R \underline{\Gamma}_{f^{-1} D}\left(\mathbb{Z}_{\underline{X}}\right) \sim_{\text {qis }} R \underline{\Gamma}_{D}\left(R \underline{f}_{*} \mathbb{Z}_{\underline{X}}\right) .
$$

The cohomology sheaves have $\mathbb{Q}$-structures by 3.4.1. We have two spectral sequences

$\mathrm{I} \underline{E}_{2}^{p, q}=R^{p} \underline{f}_{*} \mathcal{H}_{f^{-1} D}^{q}\left(\mathbb{Z}_{\underline{X}}\right) \Longrightarrow \underline{E}^{p+q}, \quad$ and $\quad \mathrm{II}_{E_{2}^{p}}^{p, q}=\mathcal{H}_{D}^{p}\left(R^{q} \underline{f}_{*} \underline{\mathbb{Z}}_{\underline{X}}\right) \Longrightarrow \underline{E}^{p+q}$, where $\underline{E}^{1}=0$ and $\underline{E}^{2} \simeq{ }_{\mathrm{I}} \underline{E}_{2}^{0,2}=\underline{f}_{*} \mathcal{H}_{f^{-1} D}^{2}\left(\mathbb{Z}_{\underline{X}}\right)$. Let $\underline{U} \rightarrow \underline{X}$ and $\underline{Y} \rightarrow \underline{X} \times \underline{S} \underline{U}$ be as above. Then we have a similar quasi-isomorphism

$$
R\left(\underline{f}_{\underline{U}} \circ \underline{\mu}\right)_{*} R \underline{\Gamma}_{D_{Y}}\left(\mathbb{Z}_{\underline{Y}}\right) \sim_{\text {qis }} R \underline{\Gamma}_{D_{U}}\left(R\left(\underline{f}_{\underline{U}} \circ \underline{\mu}\right)_{*} \mathbb{Z}_{\underline{Y}}\right)
$$

and similar spectral sequences

$$
\begin{aligned}
& \mathrm{I}_{\underline{E}^{p, q}}=R^{p}\left(\underline{f}_{\underline{U}} \circ \underline{\mu}\right)_{*} \mathcal{H}_{D_{Y}}^{q}\left(\mathbb{Z}_{\underline{Y}}\right) \Longrightarrow \underline{\check{E}}^{p+q}, \\
& \mathrm{II} \underline{\check{E}}_{2}^{p, q}=\mathcal{H}_{D_{U}}^{p}\left(R^{q}\left(\underline{f}_{\underline{U}} \circ \underline{\mu}\right)_{*} \mathbb{Z}_{\underline{Y}}\right) \Longrightarrow \underline{\check{E}}^{p+q}
\end{aligned}
$$

for $\underline{f}_{\underline{U}} \circ \underline{\mu}: \underline{Y} \rightarrow \underline{U}$. There are natural homomorphisms

$$
\left.\mathrm{I} \underline{E}_{2}^{p, q}\right|_{\underline{U}} \rightarrow{ }_{\mathrm{I}} \underline{\check{E}}_{2}^{p, q},\left.\quad \mathrm{II} \underline{E}_{2}^{p, q}\right|_{\underline{U}} \rightarrow \mathrm{II}_{\check{E}_{2}^{p, q}}, \quad \text { and }\left.\quad \underline{E}^{p+q}\right|_{\underline{U}} \rightarrow \underline{\check{E}}^{p+q} .
$$

Claim 6.1.2. $\quad \mathrm{II} \underline{E}_{2}^{p, 0} \rightarrow \underline{E}^{p}$ is injective for any $p$. In particular, $\mathrm{II} \underline{E}_{2}^{0,1}=$ $\mathcal{H}_{D}^{0}\left(R^{1} \underline{f}_{*} \mathbb{Z}_{\underline{X}}\right)=0$.

Proof. Let $\varepsilon_{U}$ be the morphism $\underline{U} \rightarrow U=(U, \emptyset)$. The sheaves ${ }_{\mathrm{II}} \underline{E}_{2}^{p, 0}$ have $\mathbb{Q}$-structures by 3.4.1. Thus $\varepsilon_{U *}\left(\mathrm{II}_{\underline{E}^{p}}^{p, 0}\right) \rightarrow \varepsilon_{U *}\left(\underline{\check{E}}^{p}\right)$ is injective by 5.4.1. Since $\left.{ }_{\mathrm{II}} \underline{E}_{2}^{p, 0}\right|_{\underline{U}} \simeq{ }_{\mathrm{II}} \underline{\breve{E}}_{2}^{p, 0}, \mathrm{II}_{2} \underline{E}_{2}^{p, 0} \rightarrow \underline{E}^{p}$ is injective.

Lemma 6.1.3. The following sequence is exact:

$$
0 \rightarrow \mathrm{II}_{2}^{2,0} \underline{E}^{2} \rightarrow \underline{\mathrm{II}}_{2}^{E_{2}^{0,2}} \rightarrow{ }_{\mathrm{II}} \underline{E}_{2}^{2,1} .
$$

We have ${ }_{\mathrm{II}} \underline{E}_{\infty}^{1,1}=\mathcal{H}_{D}^{1}\left(R^{1} \underline{f}_{*} \underline{\mathbb{Z}}_{\underline{X}}\right)=0$. In particular, $R^{1} \underline{f}_{*} \mathbb{Z}_{\underline{X}} \rightarrow R^{1} \underline{f}_{*} \mathcal{O}_{\underline{X}}$ is isomorphic to $\underline{j}_{*} H \rightarrow \mathcal{L}_{H / \underline{S}}$ by the marking $\phi:\left.\left(R^{1} f_{*} \mathbb{Z}_{X}\right)\right|_{S^{*}} \widetilde{\sim} H$.

Proof. This follows the argument of 5.4.4 and 5.4.3. Because, if $\Delta \cdot \gamma=0$ for any irreducible curves $\gamma$ contained in fibers of $f_{U}$ for a Cartier divisor $\Delta$ with Supp $\Delta \subset D_{Y}$, then $\Delta=f^{*}\left(\Delta^{\prime}\right)$ for a $\mathbb{Q}$-Weil divisor $\Delta^{\prime}$ supported in $D_{U}$. Here $\Delta^{\prime}$ is $\mathbb{Q}$-Cartier and hence $\Delta^{\prime} \in \operatorname{Div}_{D_{U}}(\underline{U})$. Therefore, ${ }_{\mathrm{II}} \underline{E}_{\infty}^{1,1}={ }_{\mathrm{II}} \underline{E}_{2}^{1,1}=0$. 
The exponential sequence of $\underline{X}$ induces a long exact sequence:

$$
0 \rightarrow R^{1} \underline{f}_{*} \mathbb{Z}_{\underline{X}} \rightarrow R^{1} \underline{f}_{*} \mathcal{O}_{\underline{X}} \rightarrow R^{1} \underline{f}_{*} \mathcal{O}_{\underline{X}}^{\star} \rightarrow R^{2} \underline{f}_{*} \mathbb{Z}_{\underline{X}} \rightarrow 0,
$$

by 5.2.7. The first homomorphism is isomorphic to $\underline{j}_{*} H \rightarrow \mathcal{L}_{H / \underline{S}}$ by 6.1.3. Let $\mathfrak{S}_{H / \underline{S}}^{\mathbf{W}}$ be the cokernel of $\underline{j}_{*} H \rightarrow \mathcal{L}_{H / \underline{S}}$. Then we have the the following two short exact sequences:

$$
\begin{aligned}
0 \rightarrow R^{1} \underline{f}_{*} \mathbb{Z}_{\underline{X}} & \rightarrow R^{1} \underline{f}_{*} \mathcal{O}_{\underline{X}} \rightarrow \mathfrak{S}_{H / \underline{S}}^{\mathbf{W}} \rightarrow 0 ; \\
0 & \rightarrow \mathfrak{S}_{H / \underline{S}}^{\mathbf{W}} \rightarrow R^{1} \underline{f}_{*} \mathcal{O}_{\underline{X}}^{\star} \rightarrow R^{2} \underline{f}_{*} \mathbb{Z}_{\underline{X}} \rightarrow 0 .
\end{aligned}
$$

Let $D^{\prime} \supset D$ be a reduced divisor such that $f$ is smooth over $S \backslash D^{\prime}$. We define $\mathcal{V}_{\underline{X}}:=\mathcal{H}_{D^{\prime}}^{0}\left(R^{1} f_{*} \mathcal{O}_{\underline{X}}^{\star}\right)$. As in 5.4.6, it does not depend on the choice of $D^{\prime}$. We have $\mathcal{V}_{\underline{X}} \cap \mathfrak{S}_{H / \underline{S}}^{\mathbf{W}^{*}}=0$ since $\mathcal{H}_{D}^{0}\left(\mathfrak{S}_{H / \underline{S}}^{\mathbf{W}}\right) \simeq \mathcal{H}_{D}^{1}\left(R^{1} \underline{f}_{*} \mathbb{Z}_{\underline{X}}\right)=0$ by 6.1.3. Thus (6.3) induces an exact sequence

$$
0 \rightarrow \mathfrak{S}_{H / \underline{S}}^{\mathbf{W}} \rightarrow R^{1} \underline{f}_{*} \mathcal{O}_{\underline{X}}^{\star} / \mathcal{V}_{\underline{X}} \rightarrow R^{2} \underline{f}_{*} \mathbb{Z}_{\underline{X}} / \mathcal{V}_{\underline{X}} \rightarrow 0 \text {. }
$$

Lemma 6.1.4. Let $D_{U}^{\prime}$ be the pullback of $D^{\prime}$ by $U \rightarrow S$ and let

$$
\mathcal{V}_{\underline{Y}}:=\mathcal{H}_{D_{U}^{\prime}}^{0}\left(R^{1}\left(\underline{f}_{U} \circ \underline{\mu}\right)_{*} \mathcal{O}_{\underline{Y}}^{\star}\right) \text {. }
$$

Then the sheaf $R^{1} \underline{f}_{*} \mathcal{O}_{\underline{X}}^{\star} / \mathcal{V}_{\underline{X}}$ is associated with the presheaf

$$
\underline{U} \longmapsto \operatorname{WPic}\left(X_{U}\right) / \mathrm{V}_{f_{U}^{-1} D_{U}^{\prime}}\left(X_{U}\right) \simeq \operatorname{Pic}(Y) / \mathrm{V}_{\mu^{-1} f_{U}^{-1} D_{U}^{\prime}}(Y) .
$$

Moreover the following isomorphisms exist:

$$
\begin{aligned}
& \left.\left(R^{1} \underline{f}_{*} \mathcal{O}_{\underline{X}}^{\star} / \mathcal{V}_{\underline{X}}\right)\right|_{\underline{U}} \simeq R^{1}\left(\underline{f}_{\underline{U}} \circ \underline{\mu}\right)_{*} \mathcal{O}_{\underline{Y}}^{\star} / \mathcal{V}_{\underline{Y}}, \\
& \left.\left(R^{2} \underline{f}_{*} \mathbb{Z}_{\underline{X}} / \mathcal{V}_{\underline{X}}\right)\right|_{\underline{U}} \simeq R^{2}\left(\underline{f}_{\underline{U}} \circ \underline{\mu}\right)_{*} \mathbb{Z}_{\underline{Y}} / \mathcal{V}_{\underline{Y}} .
\end{aligned}
$$

Proof. The first assertion follows 3.4.5. The second isomorphisms are derived from the same argument as 5.4.6 and from 6.1.3.

We have a trace map $R^{2} \underline{f}_{*} \mathbb{Z}_{\underline{X}} \rightarrow \mathbb{Z}_{\underline{S}}$ which is just the restriction homomorphism

$$
R^{2} \underline{f}_{*} \mathbb{Z}_{\underline{X}} \rightarrow \underline{j}_{*}\left(\left.\left(R^{2} f_{*} \mathbb{Z}_{X}\right)\right|_{S^{*}}\right) \simeq \mathbb{Z}_{\underline{S}} .
$$

The composite $R^{1} \underline{f}_{*} \mathcal{O}_{\underline{X}}^{\star} \rightarrow R^{2} \underline{f}_{*} \mathbb{Z}_{\underline{X}} \rightarrow \mathbb{Z}_{\underline{S}}$ is the homomorphism measuring the degree of invertible sheaves restricted to smooth fibers. Since $(f: X \rightarrow$ $S, \phi) \in \mathcal{E}_{1}(S, D, H)$, the trace map is surjective. In particular, $\mathrm{II}_{E_{2}^{1,2}}=$ $\mathcal{H}_{D}^{1}\left(R^{2} \underline{f}_{*} \mathbb{Z}_{\underline{X}}\right)=0$ and ${ }_{\mathrm{II}} \underline{E}_{2}^{0,2}=\mathcal{H}_{D}^{0}\left(R^{2} \underline{f}_{*} \mathbb{Z}_{\underline{X}}\right)$ is the kernel of the trace map. 
Assume that $\boldsymbol{B}$ is non-singular and that $\boldsymbol{p}^{-1} D$ is a normal crossing divisor for the basic elliptic fibration $\boldsymbol{p}: \boldsymbol{B} \rightarrow S$. We follow the argument of 5.4.8. Then the kernel of $R^{1} \underline{\boldsymbol{p}}_{*} \mathcal{O}_{\underline{B}}^{\star} / \mathcal{V}_{\underline{B}} \rightarrow \mathbb{Z}_{\underline{S}}$ is isomorphic to $\mathfrak{S}_{H / \underline{S}}$ by a homomorphism

$$
\Psi_{\underline{B}}: \mathfrak{S}_{H / \underline{S}} \rightarrow R^{1} \underline{p}_{*} \mathcal{O}_{\underline{B}}^{\star} / \mathcal{V}_{\underline{B}}
$$

defined as follows: For a meromorphic section $\sigma: U \cdots \rightarrow \boldsymbol{B}$ of $\boldsymbol{p}: \boldsymbol{B} \rightarrow S$ over $U$, the image $\Gamma_{\sigma}=\sigma(U) \subset B_{U}$ is a prime divisor, where $B_{U}$ denotes the normalization of the main component of $B \times{ }_{S} U$. The pullback $\boldsymbol{\Sigma}_{U}$ of the canonical section $\boldsymbol{\Sigma} \subset \boldsymbol{B}$ of $\boldsymbol{p}$ is a meromorphic section of $\boldsymbol{p}_{U}: B_{U} \rightarrow U$. The homomorphism $\Psi_{\underline{B}}$ sends $\sigma$ to the equivalence class of the invertible sheaf

$$
\mathcal{O}_{\underline{B} \times \underline{S}}\left(\Gamma_{\sigma}-\Sigma_{U}\right) \text {. }
$$

Even if $f: X \rightarrow S$ does not admit a meromorphic section, we can define such a homomorphism $\Psi_{\underline{X}}$ that

$$
0 \rightarrow \mathfrak{S}_{H / \underline{S}} \stackrel{\Psi_{\underline{X}}}{\rightarrow} R^{1} \underline{f}_{*} \mathcal{O}_{\underline{X}}^{\star} / \mathcal{V}_{\underline{X}} \rightarrow \mathbb{Z}_{\underline{S}} \rightarrow 0
$$

is exact. This is because $(f: X \rightarrow S, \phi) \in \mathcal{E}_{1}(S, D, H)$ and we can apply the same argument as 5.5.2.

We have a quasi-isomorphism

$$
R \underline{\Gamma}_{D}\left(R \underline{f}_{*} \mathcal{O}_{\underline{X}}^{\star}\right) \sim_{\text {qis }} R \underline{\Gamma}_{D}\left(R \underline{f}_{*} \mathcal{O}_{\underline{X}}\right) \oplus R \underline{\Gamma}_{D}\left(R \underline{f}_{*} \underline{Z}_{\underline{X}}\right)[1]
$$

by 3.4.1. The second projection induces a commutative diagram of exact sequences

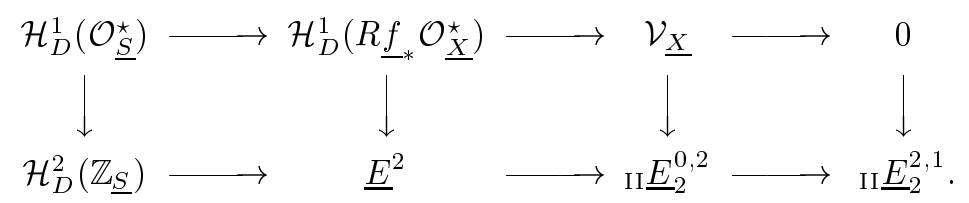

Hence $\mathcal{V}_{\underline{X}}$ is isomorphic to the image of $\underline{E}^{2} \rightarrow{ }_{\mathrm{II}} \underline{E}_{2}^{0,2}$. Let $\mathfrak{T}_{H / \underline{S}}$ be the cokernel of $\underline{E}^{2} \rightarrow \mathrm{II} \underline{E}_{2}^{0,2}$. Then we have two exact sequences

$$
\begin{gathered}
0 \rightarrow \mathfrak{T}_{H / \underline{S}} \rightarrow R^{2} \underline{f}_{*} \mathbb{Z}_{X} / \mathcal{V}_{\underline{X}} \rightarrow \mathbb{Z}_{\underline{S}} \rightarrow 0 ; \\
0 \rightarrow \mathfrak{S}_{H / \underline{S}}^{\mathbf{W}} \rightarrow \mathfrak{S}_{H / \underline{S}} \rightarrow \mathfrak{T}_{H / \underline{S}} \rightarrow 0 .
\end{gathered}
$$

Thus $\mathfrak{T}_{H / \underline{S}}$ also does not depend on the choice of $\underline{X}$. Further there is an injection

$$
\mathfrak{T}_{H / \underline{S}} \hookrightarrow \mathrm{II}_{2} \underline{E}_{2}^{0,2}=\mathcal{H}_{D}^{2}\left(R^{1} \underline{f}_{*} \mathbb{Z}_{\boldsymbol{B}}\right) \simeq R^{1} \underline{j}_{*} H
$$

whose cokernel is $\mathrm{II} \underline{E}_{\infty}^{2,1}$. Therefore, we have the following theorem from 5.4.9, 5.4.10, 5.4.11, 5.4.13, and 6.1.4. 
Theorem 6.1.5. Let $(f: X \rightarrow S, \phi)$ be a marked elliptic fibration in $\mathcal{E}_{1}(S, D, H)$ such that $X$ is non-singular and $f^{-1} D$ is a normal crossing divisor. Then we have a commutative diagram of exact sequences: Figure 3 . Further,

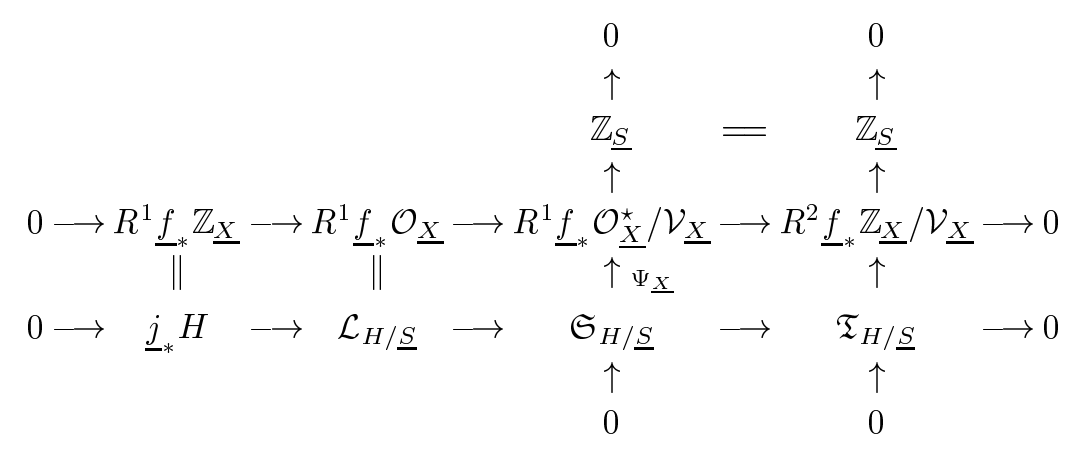

Figure 3.

the following properties are satisfied:

(1) $\mathfrak{T}_{H / \underline{S}^{\circ}}=\left.\left(\mathfrak{T}_{H / \underline{S}}\right)\right|_{\underline{S}^{\circ}}$ is isomorphic to the torsion part $\left(R^{1} \underline{j}_{*}^{\star} H\right)_{\mathrm{tor}}$.

(2) $\mathfrak{S}_{H / \underline{S}^{\circ}}$ is a subsheaf of $\underline{j}_{*}^{\star} \mathfrak{S}_{H}$ such that

$$
\mathfrak{S}_{H / \underline{S}^{\circ}} / \mathfrak{S}_{H / \underline{S}^{\circ}}^{\mathbf{W}}=\left(\underline{j}_{*}^{\star} \mathfrak{S}_{H} / \mathfrak{S}_{H / \underline{S}^{\circ}}^{\mathbf{W}}\right)_{\text {tor }} .
$$

(3) $\mathfrak{T}_{H / \underline{S}}$ is isomorphic to the kernel of

$$
R^{1} \underline{j}_{*} H \rightarrow \underline{j}_{*}^{\circ}\left(R^{1} \underline{j}_{*}^{\star} H /\left(R^{1} \underline{j}_{*}^{\star} H\right)_{\text {tor }}\right) .
$$

Corollary 6.1.6. $\quad$ Suppose that $S^{\star} \subset S$ together with a point $0 \in S$ is a $d$-dimensional toroidal embedding of type $(\mathrm{N}, l, \sigma)$. Then

$$
\left(\mathfrak{T}_{H / \underline{S}}\right)_{0} \simeq \begin{cases}0, & H \text { has only finite modomoromies; } \\ N\left(\boldsymbol{a}^{\perp} \cap \sigma\right)_{\mathbb{Q}}^{\perp} / \mathbb{Z} \boldsymbol{a}, & H \text { has infinite monodromies } \\ & \text { where } \boldsymbol{a} \text { is defined in } 4.3 .\end{cases}
$$

Let $S^{\star} \subset S^{\Delta} \subset S$ be the Zariski-open subset introduced in 5.4. Let $\underline{S}^{\Delta}$ denote the $\partial$-space $\left(S^{\Delta}, D \cap S^{\Delta}\right)$, and let $\underline{j}^{\Delta}: \underline{S}^{\Delta} \hookrightarrow \underline{S}$ and $\underline{j}^{\Delta \star}: S^{\star} \hookrightarrow \underline{S}^{\Delta}$ be the related $\partial$-open immersions. Then

$$
0 \rightarrow R^{p} \underline{j}_{*}^{\Delta} \mathcal{L}_{H / \underline{S}^{\Delta}} \rightarrow R^{p} \underline{j}_{*}^{\Delta} \mathfrak{S}_{H / \underline{S}^{\Delta}}^{\mathbf{W}} \rightarrow R^{p+1} \underline{j}_{*}^{\Delta}\left(\underline{j}_{*}^{\star} H\right) \rightarrow 0
$$

is a split exact sequence for $p>0$ by 4.2 .3 . 
Lemma 6.1.7. Suppose that $S^{\Delta} \subset S^{\circ}$. Then the following properties hold:

(1) The natural homomorphisms

$$
R^{p} \underline{j}_{*}^{\circ}\left(\underline{j}_{*}^{\star} H\right) \rightarrow R^{p} \underline{j}_{*}^{\Delta}\left(\underline{j}_{*}^{\Delta \star} H\right)
$$

are isomorphic for $p \leq 2$.

(2) The sequence

$$
0 \rightarrow R^{1} \underline{j}_{*}^{\Delta}\left(\underline{j}_{*}^{\Delta \star} H\right) \rightarrow \mathfrak{T}_{H / \underline{S}} \rightarrow \underline{j}_{*}^{\Delta} \mathfrak{T}_{H / \underline{S}^{\Delta}} \rightarrow R^{2} \underline{j}_{*}^{\Delta}\left(\underline{j}_{*}^{\Delta \star} H\right)
$$

is exact.

(3) The image of the composite

$$
\underline{j}_{*}^{\Delta} \mathfrak{T}_{H / \underline{S}^{\Delta}} \rightarrow R^{1} \underline{j}_{*}^{\Delta} \mathfrak{S}_{H / \underline{S}^{\Delta}}^{\mathbf{W}} \rightarrow R^{2} \underline{j}_{*}^{\Delta}\left(\underline{j}_{*}^{\Delta \star} H\right)
$$

is the torsion part of $R^{2} \underline{j}_{*}^{\Delta}\left(\underline{j}_{*}^{\Delta \star} H\right)$.

(4) The composite $R^{1} \underline{j}_{*}^{\Delta} \mathfrak{T}_{H / \underline{S}^{\Delta}} \rightarrow R^{2} \underline{j}_{*}^{\Delta} \mathfrak{S}_{H / \underline{S}^{\Delta}}^{\mathbf{W}} \rightarrow R^{3} \underline{j}_{*}^{\Delta}\left(\underline{j}_{*}^{\Delta \star} H\right)$ is injective. In particular,

$$
R_{\underline{j}_{*}^{1}}^{\Delta} \mathfrak{S}_{H / \underline{S}^{\Delta}}^{\mathbf{W}} \rightarrow R^{1} \underline{j}_{*}^{\Delta} \mathfrak{S}_{H / \underline{S}^{\Delta}}
$$

is surjective.

Proof. (1) Let $\underline{j}^{\circ \Delta}: \underline{S}^{\Delta} \rightarrow \underline{S}^{\circ}$ denote the $\partial$-open immersion. Then we have an isomorphism $R^{1} \underline{j}_{*}^{\star} H \simeq \underline{j}_{*}^{\circ \Delta}\left(R^{1} \underline{j}_{*}^{\Delta \star} H\right)$. In particular, $R^{1} \underline{j}_{*}^{\circ}\left(R^{1} \underline{j}_{*}^{\star} H\right) \rightarrow$ $R^{1} \underline{j}_{*}^{\Delta}\left(R^{1} \underline{j}_{*}^{\Delta \star} H\right)$ is injective. By considering two spectral sequences:

$$
R^{p} \underline{j}_{*}^{\Delta}\left(R^{q} \underline{j}_{*}^{\Delta \star} H\right) \Longrightarrow R^{p+q} \underline{j}_{*} H, \quad \text { and } \quad R^{p} \underline{j}_{*}^{\circ}\left(R^{q} \underline{j}_{*}^{\star} H\right) \Longrightarrow R^{p+q} \underline{j}_{*} H
$$

we have the expected isomorphisms.

(2) is derived from (1) and from the diagram of 5.4.13.

(3) By (1), we may assume $S^{\circ}=S^{\Delta}$. Then this follows 4.4.3.

(4) Let us consider the commutative diagram

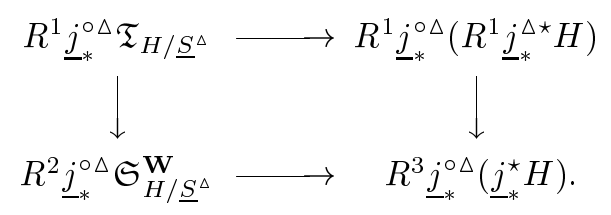


The top arrow is injective, since $R^{1} j_{*}^{\star} H$ is locally constant on $D^{\star}$. The right vertical arrow is an isomorphism since $R^{p} \underline{j}_{*}^{\star} H=0$ for $p \geq 2$. Next, we consider the commutative diagram

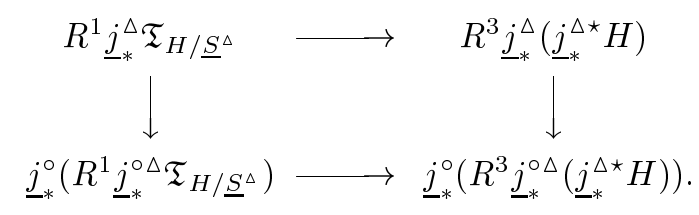

The bottom arrow is injective by the previous diagram. The left vertical arrow is an isomorphism since $R^{p} \underline{j}_{*}^{\circ} \mathfrak{T}_{H / \underline{S}^{\circ}}=0$ for $p>0$ by 4.4.3. Thus we are done.

Lemma 6.1.8. $\quad R^{1} \underline{j}_{*}^{\Delta} \mathfrak{S}_{H / \underline{S}^{\Delta}}$ is a sheaf of $\mathbb{Q}$-vector spaces.

Proof. Let $Z^{\Delta}$ be the complement $S \backslash S^{\Delta}$. Then

$$
R^{1} \underline{j}_{*}^{\Delta} \mathfrak{S}_{H / \underline{S}^{\Delta}} \simeq \mathcal{H}_{Z^{\Delta}}^{2}\left(\mathfrak{S}_{H / \underline{S}}\right) \simeq \mathcal{H}_{Z^{\Delta}}^{0}\left(\mathcal{H}_{Z^{\Delta} \cup \operatorname{Sing} D}^{2}\left(\mathfrak{S}_{H / \underline{S}}\right)\right) .
$$

Thus we are reduced to the case $S^{\Delta} \subset S^{\circ}$. Then it follows 6.1.7.

\section{$\S 6.2$. Calculation of cohomology groups}

\section{Definition 6.2.1.}

(1) Let $\mathbb{L}_{H / S}$ be the object of the derived category of sheaves of abelian groups of $S$ corresponding to the complex

$$
\left[\cdots \rightarrow 0 \rightarrow \mathcal{L}_{H / S} \rightarrow \mathfrak{S}_{H / S} \rightarrow 0 \rightarrow \cdots\right]
$$

where $\mathcal{L}_{H / S}$ lies in the degree zero.

(2) Let $\mathbb{L}_{H / S}^{\bullet}$ be the object of the derived category of sheaves of abelian groups of the $\partial$-space $\underline{S}$ corresponding to the complex

$$
\left[\cdots \rightarrow 0 \rightarrow \mathcal{L}_{H / \underline{S}} \rightarrow \mathfrak{S}_{H / \underline{S}} \rightarrow 0 \rightarrow \cdots\right]
$$

where $\mathcal{L}_{H / \underline{S}}$ lies in the degree zero.

(3) Let $\mathfrak{Q}_{H / S}$ be the cokernel of $\mathfrak{T}_{H / S} \rightarrow R^{1} j_{*} H$. This is ${ }_{\mathrm{II}} E_{\infty}^{2,1}$ of Section 5.4. Similarly, let $\mathfrak{Q}_{H / \underline{S}}$ be the cokernel of $\mathfrak{T}_{H / \underline{S}} \rightarrow R^{1} \underline{j}_{*} H$. 
Lemma 6.2.2. $\quad \mathfrak{Q}_{H / S}$ is a sheaf of torsion-free abelian groups supported in D. $\mathfrak{Q}_{H / \underline{S}}$ is a sheaf of $\mathbb{Q}$-vector spaces supported in $D$. There exist a canonical isomorphism

$$
\varepsilon_{*} \mathfrak{Q}_{H / \underline{S}} \simeq \mathfrak{Q}_{H / S} \otimes \mathbb{Q}
$$

for $\varepsilon: \underline{S} \rightarrow S$ and an exact sequence

$$
0 \rightarrow R^{1} j_{*}^{\circ}\left(j_{*}^{\star} H\right) \otimes \mathbb{Q} \rightarrow R^{1} j_{*} H \otimes \mathbb{Q} \rightarrow \mathfrak{Q}_{H / S} \otimes \mathbb{Q} \rightarrow 0
$$

Proof. The last exact sequence is derived from 5.4.13, since $\mathfrak{T}_{H / S^{\circ}}$ is a sheaf of torsion abelian groups. The natural homomorphism $R^{1} j_{*} H \rightarrow$ $\varepsilon_{*} R^{1} \underline{j}_{*} H$ induces $\mathfrak{T}_{H / S} \rightarrow \varepsilon_{*} \mathfrak{T}_{H / S}$ and $\mathfrak{Q}_{H / S} \rightarrow \varepsilon_{*} \mathfrak{Q}_{H / \underline{S}}$. Thus we can check only on the stalks at a point of $S$. Thus we may assume that $S$ and $D$ are as in Section 4 and we consider stalks at the origin $0 \in S$. Then the stalk $\left(\mathfrak{Q}_{H / \underline{S}}\right)_{0}$ is a $\mathbb{Q}$-vector space by 4.4.1. If $H$ is of type $\mathrm{I}_{0}$ or $\mathrm{I}_{(+)}$, then $\left(\mathfrak{Q}_{H / S}\right)_{0}$ is torsion-free and $\left(\mathfrak{Q}_{H / S}\right)_{0} \otimes \mathbb{Q} \simeq\left(\varepsilon_{*} \mathfrak{Q}_{H / \underline{S}}\right)_{0}$ by 4.3.1 and 4.4.1. Suppose that $H$ is not of type $\mathrm{I}_{0}$ nor $\mathrm{I}_{(+)}$. Then $\left(R^{1} j_{*} H\right)_{0}$ is a finite group. Thus $\left(\mathfrak{Q}_{H / S}\right)_{0}=0$ and

$$
\begin{aligned}
\left(R^{1} j_{*} H\right)_{0} \otimes \mathbb{Q} & \simeq\left(R^{1} j_{*}(H \otimes \mathbb{Q})\right)_{0} \simeq\left(\varepsilon_{*} R^{1} \underline{j}_{*}(H \otimes \mathbb{Q})\right)_{0} \\
& \simeq\left(\varepsilon_{*} R^{1} \underline{j}_{*} H\right)_{0} \otimes \mathbb{Q}=0
\end{aligned}
$$

by 2.2.6. We have a short exact sequence

$$
0 \rightarrow \mathfrak{T}_{H / \underline{S}} \otimes \mathbb{Q} \rightarrow\left(R^{1} \underline{j}_{*} H\right) \otimes \mathbb{Q} \rightarrow \mathfrak{Q}_{H / \underline{S}} \rightarrow 0 .
$$

Since $R^{1} \varepsilon_{*}\left(\mathfrak{T}_{H / \underline{S}} \otimes \mathbb{Q}\right)=0$, we have $\left(\varepsilon_{*} \mathfrak{Q}_{H / \underline{S}}\right)_{0}=0$.

Remark 6.2.3. Suppose that $S^{\star} \subset S$ together with a point $0 \in S$ is a $d$-dimensional toroidal embedding of type $(\mathrm{N}, l, \sigma)$. Then

$$
\left(\mathfrak{Q}_{H / S}\right)_{0} \simeq \begin{cases}\mathbb{Z}^{\oplus 2 l}, & H \text { is of type } \mathrm{I}_{0} ; \\ \mathbb{Z} \oplus N\left(\boldsymbol{a}^{\perp} \cap \sigma\right)^{\vee}, & H \text { is of type } \mathrm{I}_{\boldsymbol{a}} \text { for } \boldsymbol{a} \neq 0 \text { (cf. 4.3.4) } \\ 0, & \text { otherwise. }\end{cases}
$$

From the exact sequence: $0 \rightarrow H \rightarrow \mathcal{L}_{H} \rightarrow \mathfrak{S}_{H} \rightarrow 0$, we have quasiisomorphisms

$$
\begin{aligned}
& {\left[\cdots \rightarrow 0 \rightarrow j_{*} \mathcal{L}_{H} \rightarrow j_{*} \mathfrak{S}_{H} \rightarrow 0 \rightarrow \cdots\right] \sim_{\text {qis }} \tau_{\leq 1} R j_{*} H} \\
& {\left[\cdots \rightarrow 0 \rightarrow \underline{j}_{*} \mathcal{L}_{H} \rightarrow \underline{j}_{*} \mathfrak{S}_{H} \rightarrow 0 \rightarrow \cdots\right] \sim_{\text {qis }} \tau_{\leq 1} R \underline{j}_{*} H .}
\end{aligned}
$$

Thus there are natural morphisms $\mathbb{L}_{H / S}^{\bullet} \rightarrow \tau_{\leq 1} R j_{*} H$ and $\mathbb{L}_{H / \underline{S}}^{\bullet} \rightarrow \tau_{\leq 1} R \underline{j}_{*} H$. Their mapping cones are quasi-isomorphic to $\mathfrak{Q}_{H / S}[-1]$ and $\mathfrak{Q}_{H / \underline{S}}[-1]$, respectively. We have morphisms $R j_{*} H \rightarrow \mathcal{L}_{H / S}$ and $R \underline{j}_{*} H \rightarrow \mathcal{L}_{H / \underline{S}}$ by 4.2.3. 
Thus the morphisms $\mathbb{L}_{H / S}^{\bullet} \rightarrow \mathcal{L}_{H / S}$ and $\mathbb{L}_{H / \underline{S}}^{\bullet} \rightarrow \mathcal{L}_{H / \underline{S}}$ are decomposed into $\mathbb{L}_{H / S}^{\bullet} \rightarrow \tau_{\leq 1} R j_{*} H \rightarrow \mathcal{L}_{H / S}$ and $\mathbb{L}_{H / \underline{S}}^{\bullet} \rightarrow \tau_{\leq 1} R \underline{j}_{*} H \rightarrow \mathcal{L}_{H / \underline{S}}$, respectively. Hence we have the following two commutative diagrams of triangles:
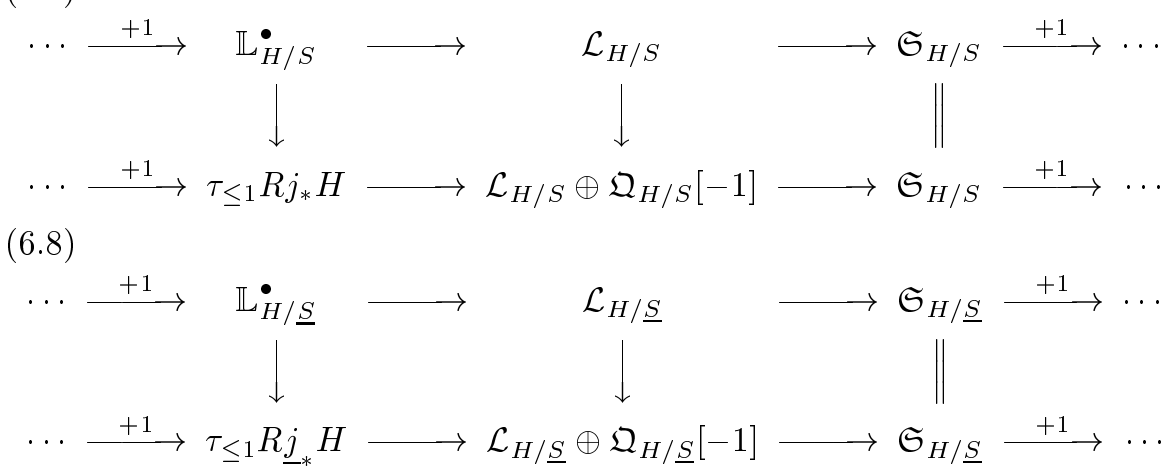

Definition 6.2.4. We denote the image of $H^{1}\left(S^{\star}, H\right) \rightarrow H^{1}\left(S, \mathcal{L}_{H / S}\right)$ $\oplus H^{0}\left(S, \mathfrak{Q}_{H / S}\right)$ by $C(H / S)$, and the image of $H^{1}\left(S^{\star}, H\right) \rightarrow H^{0}\left(S, \mathfrak{Q}_{H / S}\right)$ by $\bar{C}(H / S)$.

Theorem 6.2.5. We have the following long exact sequence:

$$
\begin{aligned}
H^{0}\left(S, \mathfrak{S}_{H / S}\right) \rightarrow H^{1}\left(S^{\star}, H\right) \rightarrow H^{1}\left(S, \mathcal{L}_{H / S}\right) \oplus H^{0}\left(S, \mathfrak{Q}_{H / S} \otimes \mathbb{Q}\right) \\
\rightarrow H^{1}\left(\underline{S}, \mathfrak{S}_{H / \underline{S}}\right) \rightarrow H^{2}\left(S^{\star}, H\right) \rightarrow H^{0}\left(S, R^{2} j_{*} H \otimes \mathbb{Q}\right) .
\end{aligned}
$$

In particular, the following two short exact sequences:

$$
\begin{aligned}
0 \rightarrow & C(H / S) \otimes \mathbb{Q} / \mathbb{Z} \rightarrow H^{1}\left(\underline{S}, \mathfrak{S}_{H / \underline{S}}\right)_{\text {tor }} \rightarrow H^{2}\left(S^{\star}, H\right)_{\text {tor }} \rightarrow 0 \\
& 0 \rightarrow \mathfrak{Q}_{H / S} \otimes \mathbb{Q} / \mathbb{Z} \rightarrow R^{1} \varepsilon_{*} \mathfrak{S}_{H / \underline{S}} \rightarrow\left(R^{2} j_{*} H\right)_{\text {tor }} \rightarrow 0 .
\end{aligned}
$$

Proof. We have isomorphisms $H^{1}\left(\underline{S}, \tau_{\leq 1} R \underline{j}_{*} H\right) \simeq H^{1}\left(\underline{S}, R \underline{j}_{*} H\right) \simeq$ $H^{1}\left(S^{\star}, H\right)$ and an exact sequence

$$
0 \rightarrow H^{2}\left(\underline{S}, \tau_{\leq 1} R \underline{j}_{*} H\right) \rightarrow H^{2}\left(\underline{S}, R \underline{j}_{*} H\right) \simeq H^{2}\left(S^{\star}, H\right) \rightarrow H^{0}\left(\underline{S}, R^{2} \underline{j}_{*} H\right) .
$$

Since $R^{2} \underline{j}_{*} H$ is a sheaf of $\mathbb{Q}$-vector spaces by 4.4 .1 , we have $\varepsilon_{*}\left(R^{2} \underline{j}_{*} H\right) \simeq$ $R^{2} j_{*} H \otimes \mathbb{Q}$. Hence the first exact sequence is derived. The torsion part $H^{2}\left(S^{\star}, H\right)_{\text {tor }}$ is contained in the kernel of $H^{2}\left(S^{\star}, H\right) \rightarrow H^{0}\left(S, R^{2} j_{*} H \otimes \mathbb{Q}\right)$. Since $H^{1}\left(S, \mathcal{L}_{H / S}\right)$ and $H^{1}\left(S, \mathfrak{Q}_{H / S} \otimes \mathbb{Q}\right)$ are $\mathbb{Q}$-vector space, the homomorphism $H^{1}\left(\underline{S}, \mathfrak{S}_{H / \underline{S}}\right)_{\text {tor }} \rightarrow H^{2}\left(S^{\star}, H\right)_{\text {tor }}$ is surjective. Further its kernel is coming from $C(H / S) \otimes \mathbb{Q}$. Thus we have the second sequence. The last sequence 
is also obtained by $R^{i} \varepsilon_{*}$. We note here that $R^{1} \varepsilon_{*} \mathcal{L}_{H / S}=0$, and the image of $\varepsilon_{*} \mathfrak{S}_{H / \underline{S}} \simeq \mathfrak{S}_{H / S} \rightarrow \mathcal{H}^{1}\left(R \varepsilon_{*} R \underline{j}_{*} H\right) \simeq R^{1} j_{*} H$ is $\mathfrak{T}_{H / S}$.

Let $\boldsymbol{p}: \boldsymbol{B}=\boldsymbol{B}(H) \rightarrow S$ be the basic elliptic fibration associated with $H$ (cf. 5.3.7). Let $i: \boldsymbol{B}^{\star} \hookrightarrow \boldsymbol{B}$ denote the open immersion. Then we have a natural morphism $R i_{*} \mathbb{Z}_{\boldsymbol{B}^{\star}} \rightarrow \mathcal{O}_{\boldsymbol{B}}$ as in 3.2.2.

Theorem 6.2.6. There are quasi-isomorphisms

$$
\begin{aligned}
& R \boldsymbol{p}_{*}^{\star} \mathbb{Z}_{\boldsymbol{B}^{\star}} \sim_{\text {qis }} \mathbb{Z}_{S^{\star}} \oplus H[-1] \oplus \mathbb{Z}_{S^{\star}}[-2] ; \\
& R \boldsymbol{p}_{*} \mathcal{O}_{\boldsymbol{B}} \sim_{\text {qis }} \mathcal{O}_{S} \oplus \mathcal{L}_{H / S}[-1] .
\end{aligned}
$$

The induced morphism $R j_{*} R \boldsymbol{p}_{*}^{\star} \mathbb{Z}_{\boldsymbol{B}^{\star}} \sim_{\text {qis }} R \boldsymbol{p}_{*} R i_{*} \mathbb{Z}_{\boldsymbol{B}^{\star}} \rightarrow R \boldsymbol{p}_{*} \mathcal{O}_{\boldsymbol{B}}$ (cf. 3.2.2) is decomposed into the natural morphisms $R j_{*} \mathbb{Z}_{S^{\star}} \rightarrow \mathcal{O}_{S}$ (cf. 3.2.2), $R j_{*} H[-1] \rightarrow$ $\mathcal{L}_{H / S}[-1]$ (cf. 4.2.3), and $R j_{*} \mathbb{Z}_{S^{\star}}[-2] \rightarrow 0$.

Proof. For the zero section $\boldsymbol{\Sigma} \subset \boldsymbol{B}, \boldsymbol{\Sigma} \rightarrow S$ is isomorphic over $S^{\star}$. Thus $\mathbb{Z}_{S^{\star}} \rightarrow R \boldsymbol{p}_{*}^{\star} \mathbb{Z}_{B^{\star}}$ admits a splitting. Similarly, the trace map $R \boldsymbol{p}^{\star} \boldsymbol{\omega}_{\boldsymbol{B}^{\star}}^{\text {top }} \rightarrow \boldsymbol{\omega}_{S^{\star}}^{\text {top }}$ admits a splitting. Hence we have (6.9). Let $\widehat{S} \rightarrow \boldsymbol{\Sigma}$ be a desingularization and let $\mu: \widehat{S} \rightarrow S$ denote the composite. Then $\mathcal{O}_{S} \rightarrow R p_{*} \mathcal{O}_{B} \rightarrow R \mu_{*} \mathcal{O}_{\widehat{S}} \sim_{\text {qis }} \mathcal{O}_{S}$ is identical. Thus we have (6.10) by 5.2.7. For the component $R j_{*} \mathbb{Z}_{S^{\star}}$ of $R j_{*} R \boldsymbol{p}_{*}^{\star} \mathbb{Z}_{\boldsymbol{B}^{\star}} \sim_{\text {qis }} R \boldsymbol{p}_{*} R i_{*} \mathbb{Z}_{\boldsymbol{B}^{\star}}$, the morphism into $R \boldsymbol{p}_{*} \mathcal{O}_{\boldsymbol{B}}$ factors through $\mathcal{O}_{S}$. For the component $R j_{*} \mathbb{Z}_{S^{*}}[-2]$, we consider the composite

$$
R i_{*} \mathbb{Z}_{\boldsymbol{\Sigma}^{\star}}[-2] \sim_{\mathrm{qis}} R i_{*} \boldsymbol{\omega}_{\boldsymbol{\Sigma}^{\star}}^{\mathrm{top}}[-2 d-2] \rightarrow R i_{*} \boldsymbol{\omega}_{\boldsymbol{B}^{\star}}^{\mathrm{top}}[-2 d-2] \sim_{\mathrm{qis}} R i_{*} \mathbb{Z}_{\boldsymbol{B}^{\star}} \rightarrow \mathcal{O}_{\boldsymbol{B}},
$$

for $\boldsymbol{\Sigma}^{\star}=\boldsymbol{\Sigma} \cap \boldsymbol{B}^{\star}$. This factors through $R \underline{\Gamma}_{\boldsymbol{\Sigma}}\left(\mathcal{O}_{\boldsymbol{B}}\right)$. The morphism $R \underline{\Gamma}_{\boldsymbol{\Sigma}}\left(R i_{*} \mathbb{Z}_{\boldsymbol{B}^{\star}}\right) \rightarrow R \underline{\Gamma}_{\boldsymbol{\Sigma}}\left(\mathcal{O}_{\boldsymbol{B}}\right)$ is zero as in 3.2 .2 and 4.2 .3 provided that $\boldsymbol{\Sigma} \cup$ $p^{-1} D$ is a normal crossing divisor. By replacing $\boldsymbol{B}$, we may assume this condition. Hence $R j_{*} \mathbb{Z}_{S^{*}}[-2] \rightarrow R \boldsymbol{p}_{*} \mathcal{O}_{B}$ is zero. There is a commutative diagram:

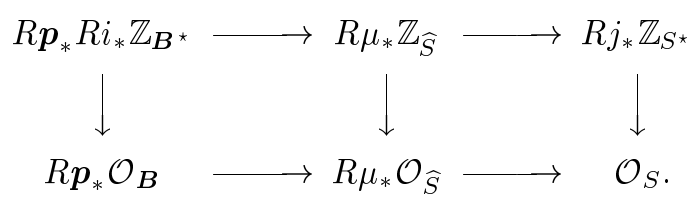

Since $H[-1] \rightarrow R \boldsymbol{p}_{*}^{\star} \mathbb{Z}_{\boldsymbol{B}^{\star}} \rightarrow \mathbb{Z}_{S^{\star}}$ is zero, the composite $R j_{*} H[-1] \rightarrow R \boldsymbol{p}_{*} \mathcal{O}_{\boldsymbol{B}} \rightarrow$ $\mathcal{O}_{S}$ is zero. The rest thing to show is that the induced morphism $R j_{*} H \rightarrow \mathcal{L}_{H / S}$ coincides with the morphism of 4.2.3. This is reduced to the case $S$ is nonsingular. Because, by a desingularization $\mu: \widehat{S} \rightarrow S$ that is isomorphic over $S^{\star}$, $R j_{*} H \rightarrow R p_{*} \mathcal{O}_{B}$ is written as $R \mu_{*}$ of the similar morphism over $\widehat{S}$. Moreover, 
we may assume $S$ is a local object: $S$ is a unit polydisc and $D$ is a union of coordinate hyperplanes. By considering the unipotent reduction of $H$, we may further assume that $H$ is of type $\mathrm{I}_{0}$ or $\mathrm{I}_{(+)}$. This is because $R j_{*} H \rightarrow R \boldsymbol{p}_{*} \mathcal{O}_{\boldsymbol{B}}$ is written as $R \varepsilon_{*}$ of $R \underline{j}_{*} H \rightarrow R \underline{p}_{*} \mathcal{O}_{\underline{B}}$ for $\underline{p}: \underline{\boldsymbol{B}}=\left(\boldsymbol{B}, \boldsymbol{p}^{-1} D\right) \rightarrow \underline{S}$. We can also replace $\boldsymbol{B}$ by a bimeromorphic model that is isomorphic over $\boldsymbol{B}^{\star}$. Thus we assume that $\boldsymbol{p}: \boldsymbol{B} \rightarrow S$ is a toric model or a smooth model (cf. [N4]). We follow an argument in $[\mathrm{Z}, 15.5]$. Let $\Omega_{\boldsymbol{B} / S}^{1}\left(\log \boldsymbol{p}^{-1} D\right)$ be the cokernel of

$$
p^{*} \Omega_{S}^{1}(\log D) \rightarrow \Omega_{B}^{1}\left(\log p^{-1} D\right) .
$$

This is locally free by the figure of $p^{-1} D$ and is isomorphic to the relative dualizing sheaf $\omega_{B / S} \simeq \boldsymbol{p}^{*} \mathcal{L}_{H / S}^{-1}$. For the relative logarithmic de Rham complex $\Omega_{B / S}^{\bullet}\left(\log \boldsymbol{p}^{-1} D\right)$, there is a triangle

$$
\cdots \stackrel{+1}{\longrightarrow} \Omega_{B / S}^{1}\left(\log p^{-1} D\right)[-1] \rightarrow \Omega_{B / S}^{\bullet}\left(\log p^{-1} D\right) \rightarrow \mathcal{O}_{B} \stackrel{+1}{\longrightarrow} \cdots
$$

Since $R^{i} \boldsymbol{p}_{*} \omega_{\boldsymbol{B} / S}$ are torsion-free, we have isomorphisms

$$
R^{0} \boldsymbol{p}_{*} \Omega_{\boldsymbol{B} / S}^{\bullet}\left(\log \boldsymbol{p}^{-1} D\right) \simeq R^{2} \boldsymbol{p}_{*} \Omega_{\boldsymbol{B} / S}^{\bullet}\left(\log \boldsymbol{p}^{-1} D\right) \simeq \mathcal{O}_{S},
$$

an exact sequence

$$
0 \rightarrow R^{1} \boldsymbol{p}_{*} \omega_{\boldsymbol{B} / S} \rightarrow R^{1} \boldsymbol{p}_{*} \Omega_{\boldsymbol{B} / S}^{\bullet}\left(\log \boldsymbol{p}^{-1} D\right) \rightarrow R^{1} \boldsymbol{p}_{*} \mathcal{O}_{\boldsymbol{B}} \rightarrow 0,
$$

and vanishings $R^{q} \boldsymbol{p}_{*} \Omega_{\boldsymbol{B} / S}^{\bullet}\left(\log \boldsymbol{p}^{-1} D\right)=0$ for $q \geq 2$. Hence the Hodge spectral sequence

$$
R^{q} \boldsymbol{p}_{*} \Omega_{\boldsymbol{B} / S}^{p}\left(\log \boldsymbol{p}^{-1} D\right) \Longrightarrow R^{p+q} \boldsymbol{p}_{*} \Omega_{\boldsymbol{B} / S}^{\bullet}\left(\log \boldsymbol{p}^{-1} D\right)
$$

degenerates at $E_{1}$ and the locally free sheaf $R^{q} \boldsymbol{p}_{*} \Omega_{B / S}^{\bullet}\left(\log \boldsymbol{p}^{-1} D\right)$ is isomorphic to the canonical extension $\mathcal{H}_{S}^{(q) \text { can }}$ of $R^{q} \boldsymbol{p}_{*}^{\star} \mathbb{Z}_{\boldsymbol{B}^{\star}} \otimes \mathcal{O}_{S^{\star}}$. Here

$$
\mathcal{H}_{S}^{(q) \mathrm{can}} \simeq \begin{cases}\mathcal{O}_{S}, & q=0,2 \\ \mathcal{H}_{S}^{\text {can }}, & q=1 ; \\ 0, & \text { otherwise }\end{cases}
$$

Let $L^{p}$ be the filtration of $\Omega_{\boldsymbol{B}}^{\bullet}\left(\log \boldsymbol{p}^{-1} D\right)$ defined by

$$
L^{p}\left(\Omega_{\boldsymbol{B}}^{\bullet}\left(\log \boldsymbol{p}^{-1} D\right)\right)=\boldsymbol{p}^{*} \Omega_{S}^{p}(\log D) \wedge \Omega_{\boldsymbol{B}}^{\bullet-p}\left(\log \boldsymbol{p}^{-1} D\right) .
$$

Then we have quasi-isomorphisms

$$
\operatorname{Gr}_{L}^{p}\left(\Omega_{\boldsymbol{B}}^{\bullet}\left(\log \boldsymbol{p}^{-1} D\right)\right) \sim_{\mathrm{qis}} \boldsymbol{p}^{*} \Omega_{S}^{p}(\log D) \stackrel{\mathrm{L}}{\otimes} \Omega_{\boldsymbol{B} / S}^{\bullet}\left(\log \boldsymbol{p}^{-1} D\right)[-p] .
$$


For the spectral sequence ${ }_{L} E_{1}^{p, q}$ associated with $L$, we have

$$
\begin{aligned}
{ }_{L} E_{1}^{p, q}=R^{p+q} \boldsymbol{p}_{*} \operatorname{Gr}_{L}^{p}\left(\Omega_{\boldsymbol{B}}^{\bullet}\left(\log \boldsymbol{p}^{-1} D\right)\right) & \simeq \Omega_{S}^{p}(\log D) \otimes R^{q} \boldsymbol{p}_{*} \Omega_{\boldsymbol{B} / S}^{\bullet}\left(\log \boldsymbol{p}^{-1} D\right) \\
& \simeq \Omega_{S}^{p}(\log D) \otimes \mathcal{H}_{S}^{(q) \operatorname{can}},
\end{aligned}
$$

where the complex ${ }_{L} E_{1}^{\bullet, q}$ is isomorphic to the logarithmic de Rham complex $\Omega_{S}^{\bullet}(\log D) \otimes \mathcal{H}_{S}^{(q) \text { can }}$ defined by the logarithmic connection $\nabla$. For the filtration $\operatorname{Dec}(L)$, we have quasi-isomorphisms

$$
\begin{aligned}
& \operatorname{Gr}_{\operatorname{Dec}(L)}^{p} R \boldsymbol{p}_{*} \Omega_{\boldsymbol{B}}^{\bullet}\left(\log \boldsymbol{p}^{-1} D\right) \\
& \quad \rightarrow \Omega_{S}^{\bullet}(\log D) \otimes \mathcal{H}_{S}^{(-p) \operatorname{can}}[p] \sim_{\text {qis }} R j_{*}\left(R^{-p} \boldsymbol{p}_{*}^{\star} \mathbb{C}_{\boldsymbol{B}^{\star}}\right)[p] .
\end{aligned}
$$

Since the induced filtration $L$ on $\mathcal{O}_{B}$ by the natural morphism $\Omega_{B}^{\bullet}\left(\log p^{-1} D\right) \rightarrow$ $\mathcal{O}_{B}$ is trivial, we have $\operatorname{Gr}_{\operatorname{Dec}(L)}^{p} R \boldsymbol{p}_{*} \mathcal{O}_{\boldsymbol{B}} \sim_{\text {qis }} R^{-p} \boldsymbol{p}_{*} \mathcal{O}_{\boldsymbol{B}}[p]$. Therefore, $R j_{*}(H \otimes$ $\mathbb{C}) \rightarrow R^{1} \boldsymbol{p}_{*} \mathcal{O}_{B} \simeq \mathcal{L}_{H / S}$ is the same morphism as 4.2.3.

Remark. The relation between logarithmic de Rham complexes both on $\boldsymbol{B}$ and on $S$ is mentioned in [Z, 15.5 and 2.16] for general fibration $\boldsymbol{B} \rightarrow S$ over a non-singular curve $S$. The theory of mixed Hodge modules [S1] by Saito treats more general object and shows the similar compatibility at least in the category of algebraic varieties.

Lemma 6.2.7. $\quad \mathcal{O}_{S}(* D)^{\star} \simeq \boldsymbol{p}_{*} \mathcal{O}_{\boldsymbol{B}}\left(* \boldsymbol{p}^{-1} D\right)^{\star}$.

Proof. We consider the basic elliptic fibration $\boldsymbol{p}: \boldsymbol{B} \rightarrow S$. We have a triangle

$$
\cdots \stackrel{+1}{\longrightarrow} \tau_{\leq 2} R j_{*} R \boldsymbol{p}_{*}^{\star} \mathbb{Z}_{\boldsymbol{B}^{\star}} \rightarrow R \boldsymbol{p}_{*} \mathcal{O}_{\boldsymbol{B}} \rightarrow \tau_{\leq 1} R \boldsymbol{p}_{*} \mathcal{O}_{\boldsymbol{B}}\left(* \boldsymbol{p}^{-1} D\right)^{\star} \stackrel{+1}{\longrightarrow} \cdots
$$

The left complex is quasi-isomorphic to $\tau_{\leq 2} R j_{*} \mathbb{Z}_{S^{\star}} \oplus \tau_{\leq 1} R j_{*} H[-1] \oplus \mathbb{Z}_{S}[-2]$ by 6.2.6. Since $j_{*} H \rightarrow R^{1} \boldsymbol{p}_{*} \mathcal{O}_{B} \simeq \mathcal{L}_{H / S}$ is injective, we have a triangle

$$
\cdots \stackrel{+1}{\longrightarrow} \tau_{\leq 1} R j_{*} \mathbb{Z}_{S^{\star}} \rightarrow \boldsymbol{p}_{*} \mathcal{O}_{\boldsymbol{B}} \rightarrow \boldsymbol{p}_{*} \mathcal{O}_{\boldsymbol{B}}\left(* \boldsymbol{p}^{-1} D\right)^{\star} \stackrel{+1}{\longrightarrow} \cdots
$$

and the isomorphism $\mathcal{O}_{S}(* D)^{\star} \simeq \boldsymbol{p}_{*} \mathcal{O}_{\boldsymbol{B}}\left(* \boldsymbol{p}^{-1} D\right)^{\star}$ by 3.2.3.

From the morphism $R i_{*} \mathbb{Z}_{\boldsymbol{B}^{\star}} \rightarrow \mathcal{O}_{\boldsymbol{B}}$, we have a triangle:

$$
\cdots \stackrel{+1}{\longrightarrow} R i_{*} \mathbb{Z}_{\boldsymbol{B}^{\star}} \rightarrow \mathcal{O}_{\boldsymbol{B}} \oplus R \underline{\Gamma}_{\boldsymbol{p}^{-1} D}\left(\mathbb{Z}_{\boldsymbol{B}}\right)[1] \rightarrow \mathcal{O}_{\boldsymbol{B}}^{\star} \stackrel{+1}{\longrightarrow} \cdots
$$


Combining with a similar triangle for $S$ and $D$, we have a commutative diagram of exact sequences

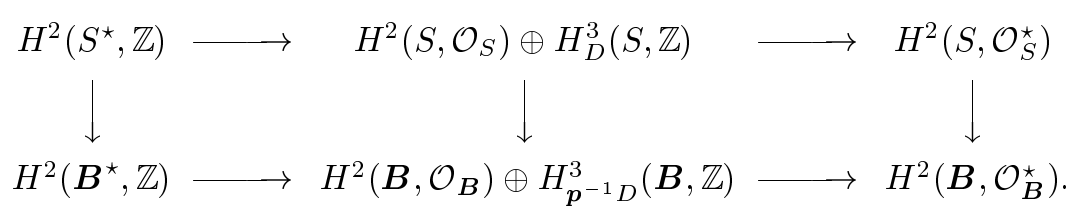

If $[S, D]$ satisfies the condition 3.2.6, then every vertical arrows are injective by 3.3.1 and 5.4.7.

\section{Lemma 6.2.8.}

(1) There is a triangle

$$
\begin{aligned}
\cdots \stackrel{+1}{\longrightarrow} \boldsymbol{p}_{*} \mathcal{H}_{\boldsymbol{p}^{-1} D}^{2}\left(\mathbb{Z}_{\boldsymbol{B}}\right)[-1] & \rightarrow \tau_{\leq 1} R \boldsymbol{p}_{*} \mathcal{O}_{\boldsymbol{B}}^{\star} \\
& \rightarrow \mathcal{O}_{S}(* D)^{\star} \oplus\left(\mathfrak{S}_{H / S} \oplus \mathbb{Z}_{S}\right)[-1] \stackrel{+1}{\longrightarrow} \cdots
\end{aligned}
$$

(2) There exist a triangle

$$
\begin{aligned}
\cdots \stackrel{+1}{\longrightarrow} \boldsymbol{p}_{*} \mathcal{H}_{\boldsymbol{p}^{-1} D}^{2}\left(\mathbb{Z}_{\boldsymbol{B}}\right)[-2] & \rightarrow \tau_{\leq 2} R \boldsymbol{p}_{*} \mathbb{Z}_{\boldsymbol{B}} \\
& \rightarrow \tau_{\leq 1} R j_{*} \mathbb{Z}_{S^{\star}} \oplus \mathbb{L}_{H / S}[-1] \oplus \mathbb{Z}_{S}[-2] \stackrel{+1}{\longrightarrow} \cdots
\end{aligned}
$$

and a commutative diagram of triangles

$$
\begin{aligned}
& \cdots \stackrel{+1}{\longrightarrow} \mathcal{V}_{\boldsymbol{B}}[-2] \longrightarrow R^{1} \boldsymbol{p}_{*} \mathcal{O}_{\boldsymbol{B}}^{\star}[-2] \longrightarrow \mathfrak{S}_{H / S}[-2] \oplus \mathbb{Z}_{S}[-2] \stackrel{+1}{\longrightarrow} \cdots \\
& \cdots \stackrel{+1}{\longrightarrow} \mathcal{V}_{\boldsymbol{B}}[-2] \longrightarrow \tau_{\geq 1} \tau_{\leq 2} R \boldsymbol{p}_{*} \mathbb{Z}_{\boldsymbol{B}} \longrightarrow \mathbb{L}_{H / S}^{\bullet}[-1] \oplus \mathbb{Z}_{S}[-2] \stackrel{+1}{\longrightarrow} \cdots
\end{aligned}
$$

(3) If $[S, D]$ satisfies the condition (3.2.6), then the following sequence is exact:

$$
0 \rightarrow \operatorname{Div}_{\boldsymbol{p}^{-1} D}(\boldsymbol{B}) / \operatorname{Div}_{D}(S) \rightarrow \operatorname{Pic}(\boldsymbol{B}) / \operatorname{Pic}(S) \rightarrow H^{0}\left(S, \mathfrak{S}_{H / S}\right) \oplus \mathbb{Z} \rightarrow 0 .
$$

(4) If $[S, D]$ satisfies the condition (3.2.6), then the torsion-free group $C(H / S)$ is isomorphic to the image of

$$
H^{2}\left(\boldsymbol{B}^{\star}, \mathbb{Z}\right) / H^{2}\left(S^{\star}, \mathbb{Z}\right) \rightarrow H^{2}\left(\boldsymbol{B}, \mathcal{O}_{\boldsymbol{B}}\right) / H^{2}\left(S, \mathcal{O}_{S}\right) \oplus H_{\boldsymbol{p}^{-1} D}^{3}(\boldsymbol{B}, \mathbb{Z}) / H_{D}^{3}(S, \mathbb{Z}) .
$$

Proof. (1) Let us consider the following morphism induced from (6.11):

$$
\boldsymbol{p}_{*} \mathcal{H}_{\boldsymbol{p}^{-1} D}^{2}\left(\mathbb{Z}_{\boldsymbol{B}}\right)[-1] \sim_{\text {qis }}\left(\tau_{\leq 2} R \boldsymbol{p}_{*} R \underline{\Gamma}_{\boldsymbol{p}^{-1} D}\left(\mathbb{Z}_{\boldsymbol{B}}\right)\right)[1] \rightarrow \tau_{\leq 1} R \boldsymbol{p}_{*} \mathcal{O}_{\boldsymbol{B}}^{\star}
$$


and let $\mathcal{C}^{\bullet}$ be its mapping cone. Note that the mapping cone of similar morphism $\mathcal{H}_{D}^{2}\left(\mathbb{Z}_{S}\right)[-1] \rightarrow \mathcal{O}_{S}^{\star}$ is quasi-isomorphic to $\mathcal{O}_{S}(* D)^{\star}$ by 3.2.3. Since the image of the induced morphism $\boldsymbol{p}_{*} \mathcal{H}_{\boldsymbol{p}^{-1} D}^{2}\left(\mathbb{Z}_{\boldsymbol{B}}\right) \rightarrow R^{1} \boldsymbol{p}_{*} \mathcal{O}_{B}^{\star}$ from (6.12) is $\mathcal{V}_{\boldsymbol{B}}$, we have $\mathcal{H}^{0}\left(\mathcal{C}^{\bullet}\right)=\mathcal{O}_{S}(* D)^{\star}, \mathcal{H}^{1}\left(\mathcal{C}^{\bullet}\right)=\mathfrak{S}_{H / S} \oplus \mathbb{Z}_{S}, \mathcal{H}^{p}\left(\mathcal{C}^{\bullet}\right)=0$ for $p \geq 2$. In particular, there is a commutative diagram of triangles

$$
\begin{aligned}
& \begin{array}{cccc}
\longrightarrow \stackrel{+1}{\longrightarrow} & \left(\tau_{\leq 2} R \underline{\Gamma}_{D}\left(\mathbb{Z}_{S}\right)\right)[1] \\
\downarrow & \longrightarrow & \mathcal{O}_{S}^{\star} & \longrightarrow \mathcal{O}_{S}(* D)^{\star} \stackrel{+1}{\longrightarrow} \cdots \\
& & \downarrow & \downarrow
\end{array} \\
& \cdots \stackrel{+1}{\longrightarrow}\left(\tau_{\leq 2} R \underline{\Gamma}_{\boldsymbol{p}^{-1} D}\left(\mathbb{Z}_{\boldsymbol{B}}\right)\right)[1] \longrightarrow \tau_{\leq 1} R \boldsymbol{p}_{*} \mathcal{O}_{\boldsymbol{B}}^{\star} \longrightarrow \quad \mathcal{C}^{\bullet} \quad \stackrel{+1}{\longrightarrow} \cdots
\end{aligned}
$$

The zero section $\boldsymbol{\Sigma} \subset \boldsymbol{B}$ is bimeromorphic to $S$ and $\boldsymbol{\Sigma}^{\star}=\boldsymbol{\Sigma} \cap \boldsymbol{B}^{\star}$ is isomorphic to $S^{\star}$. Let $Y \rightarrow \boldsymbol{\Sigma}$ be a bimeromorphic morphism from a non-singular variety and let $\mu: Y \rightarrow \Sigma \rightarrow S$ be the composite. We may assume that $\mu^{-1} D$ is a normal crossing divisor. Note that $Y \backslash \mu^{-1} D \simeq S \backslash D=S^{\star}$. Let $j_{Y}: S^{\star} \hookrightarrow Y$ be the open immersion. Then we have $\mathcal{O}_{S}(* D)^{\star} \simeq \mu_{*} \mathcal{O}_{Y}\left(* \mu^{-1} D\right)^{\star}$ by 3.2.3. By the restrictions $R \boldsymbol{p}_{*} R \underline{\Gamma}_{\boldsymbol{p}^{-1} D}\left(\mathbb{Z}_{\boldsymbol{B}}\right) \rightarrow R \mu_{*} R \underline{\Gamma}_{\mu^{-1} D}\left(\mathbb{Z}_{Y}\right)$ and $R \boldsymbol{p}_{*} \mathcal{O}_{\boldsymbol{B}} \rightarrow R \mu_{*} \mathcal{O}_{Y}$, we have a morphism $\mathcal{C}^{\bullet} \rightarrow R \mu_{*} \mathcal{O}_{Y}\left(* \mu^{-1} D\right)^{\star}$. Since $\mu_{*} \mathcal{H}_{\mu^{-1} D}^{2}\left(\mathbb{Z}_{Y}\right) \rightarrow R^{2} \mu_{*} \mathbb{Z}_{Y}$ is surjective by 3.3.1-(5), we have another commutative diagram

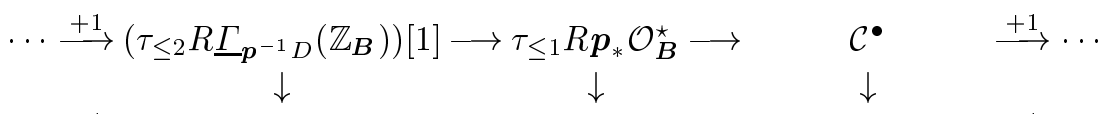

$$
\begin{aligned}
& \cdots \stackrel{+1}{\longrightarrow}\left(\tau_{\leq 2} R \underline{\Gamma}_{\mu^{-1} D}\left(\mathbb{Z}_{Y}\right)\right)[1] \longrightarrow \tau_{\leq 1} R \mu_{*} \mathcal{O}_{Y}^{\star} \longrightarrow \mu_{*} \mathcal{O}_{Y}\left(* \mu^{-1} D\right)^{\star} \stackrel{+1}{\longrightarrow} \cdots
\end{aligned}
$$

Thus $\mathcal{O}_{S}(* D)^{\star} \rightarrow \mathcal{C}^{\bullet}$ has a splitting and $\mathcal{C}^{\bullet} \sim_{\text {qis }} \mathcal{O}_{S}(* D)^{\star} \oplus\left(\mathfrak{S}_{H / S} \oplus \mathbb{Z}_{S}\right)[-1]$.

(2) The triangle

$$
\cdots \stackrel{+1}{\longrightarrow} R p_{*} R \underline{\Gamma}_{\boldsymbol{p}^{-1} D} \rightarrow R \boldsymbol{p}_{*} \mathbb{Z}_{\boldsymbol{B}} \rightarrow R \boldsymbol{p}_{*} R i_{*} \mathbb{Z}_{\boldsymbol{B}^{\star}} \stackrel{+1}{\longrightarrow} \cdots
$$

induces another triangle

$$
\begin{aligned}
\cdots \stackrel{+1}{\longrightarrow} \tau_{\leq 2} R \boldsymbol{p}_{*} R \underline{\Gamma}_{\boldsymbol{p}^{-1} D}\left(\mathbb{Z}_{\boldsymbol{B}}\right) & \rightarrow \tau_{\leq 2} R \boldsymbol{p}_{*} \mathbb{Z}_{\boldsymbol{B}} \\
& \rightarrow \tau_{\leq 1} R j_{*} \mathbb{Z}_{S^{\star}} \oplus \mathbb{L}_{H / S}^{\cdot}[-1] \oplus \mathbb{Z}_{S}[-2] \stackrel{+1}{\longrightarrow} \cdots
\end{aligned}
$$

This is because $\tau_{\leq 2} R \boldsymbol{p}_{*} R \underline{\Gamma}_{\boldsymbol{p}^{-1} D}\left(\mathbb{Z}_{\boldsymbol{B}}\right)$ is quasi-isomorphic to $\boldsymbol{p}_{*} \mathcal{H}_{\boldsymbol{p}^{-1} D}^{2}[-2]$ and the image of $\boldsymbol{p}_{*} \mathcal{H}_{\boldsymbol{p}^{-1} D}^{2} \rightarrow R^{2} \boldsymbol{p}_{*} \mathbb{Z}_{\boldsymbol{B}}$ is $\mathcal{V}_{\boldsymbol{B}}$. Combined with a triangle in the proof of $(1)$, we have the commutative diagram.

$(3) H_{D}^{3}(S, \mathbb{Z}) \rightarrow H_{\boldsymbol{p}^{-1} D}^{3}(\boldsymbol{B}, \mathbb{Z})$ and $H^{2}\left(S, \mathcal{O}_{S}^{\star}\right) \rightarrow H^{2}\left(\boldsymbol{B}, \mathcal{O}_{\boldsymbol{B}}^{\star}\right)$ are injective by 5.4 .7 . Thus we have an exact sequence

$$
\begin{aligned}
0 \rightarrow H_{\boldsymbol{p}^{-1} D}^{2}(\boldsymbol{B}, \mathbb{Z}) / H_{D}^{2}(S, \mathbb{Z}) \simeq H^{0} & \left(S, \mathcal{V}_{\boldsymbol{B}}\right) \\
& \rightarrow \operatorname{Pic}(\boldsymbol{B}) / \operatorname{Pic}(S) \rightarrow H^{0}\left(S, \mathfrak{S}_{H / S}\right) \oplus \mathbb{Z}
\end{aligned}
$$


from (6.13). Here, the right arrow is surjective, since an element of $H^{0}\left(S, \mathfrak{S}_{H / S}\right)$ defines a divisor of $\boldsymbol{B}$ by $\Psi$ (cf. 5.4.8) and since the invertible sheaf $\mathcal{O}_{\boldsymbol{B}}(\boldsymbol{\Sigma})$ attached to the zero section $\boldsymbol{\Sigma}$ goes to $1 \in \mathbb{Z}$.

(4) By (3), the cokernel of the homomorphism

$$
\operatorname{Pic}(\boldsymbol{B}) / \operatorname{Pic}(S) \rightarrow H^{2}\left(\boldsymbol{B}^{\star}, \mathbb{Z}\right) / H^{2}\left(S^{\star}, \mathbb{Z}\right) \simeq H^{1}\left(S^{\star}, H\right) \oplus H^{0}\left(S^{\star}, \mathbb{Z}\right)
$$

induced from (6.11) is isomorphic to $C(H / S)$.

Theorem 6.2.9. There is an exact sequence

$$
0 \rightarrow H^{0}\left(S, \mathfrak{S}_{H / S}\right) \otimes \mathbb{Q} / \mathbb{Z} \rightarrow \lim _{m} H^{1}\left(S^{\star}, H \otimes \boldsymbol{\mu}_{m}\right) \rightarrow H^{1}\left(\underline{S}, \mathfrak{S}_{H / \underline{S}}\right)_{\text {tor }} \rightarrow 0,
$$

where $\boldsymbol{\mu}_{m}:=m^{-1} \mathbb{Z} / \mathbb{Z} \subset \mathbb{Q} / \mathbb{Z}$. In particular, we also have an exact sequence

$$
0 \rightarrow \mathfrak{T}_{H / S} \otimes \mathbb{Q} / \mathbb{Z} \rightarrow R^{1} j_{*}(H \otimes \mathbb{Q} / \mathbb{Z}) \rightarrow R^{1} \varepsilon_{*} \mathfrak{S}_{H / \underline{S}} \rightarrow 0 .
$$

Suppose that $[S, D]$ satisfies the condition (3.2.6). Then for the basic elliptic fibration $\boldsymbol{p}: \boldsymbol{B} \rightarrow S$ associated with $H$, there is an exact sequence:

$$
\begin{aligned}
0 & \rightarrow \frac{\operatorname{Pic}(\boldsymbol{B})}{\mathrm{V}_{\boldsymbol{p}^{-1} D}(\boldsymbol{B})+\operatorname{Pic}(S)} \otimes \mathbb{Q} / \mathbb{Z} \rightarrow \lim _{m} \frac{H^{2}\left(\boldsymbol{B}^{\star}, \boldsymbol{\mu}_{m}\right)}{H^{2}\left(S^{\star}, \boldsymbol{\mu}_{m}\right)} \\
& \rightarrow H^{1}\left(\underline{S}, \mathfrak{S}_{H / \underline{S}}\right)_{\text {tor }} \rightarrow 0,
\end{aligned}
$$

where $\mathrm{V}_{\boldsymbol{p}^{-1} D}(\boldsymbol{B})$ is the image of $\operatorname{Div}_{\boldsymbol{p}^{-1} D}(\boldsymbol{B}) \rightarrow \operatorname{Pic}(\boldsymbol{B})$ (cf. 3.4.5).

Proof. Let us consider the multiplication mappings by a positive integer $m$ in the triangle

$$
\cdots \stackrel{+1}{\rightarrow} \tau_{\leq 1} R \underline{j}_{*} H \rightarrow \mathcal{L}_{H / \underline{S}} \oplus \mathfrak{Q}_{H / \underline{S}}[-1] \rightarrow \mathfrak{S}_{H / \underline{S}} \stackrel{+1}{\longrightarrow} \cdots .
$$

Then the map $\mathfrak{S}_{H / \underline{S}} \stackrel{\times m}{\longrightarrow} \mathfrak{S}_{H / \underline{S}}$ is surjective and its kernel ${ }_{m} \mathfrak{S}_{H / \underline{S}}$ is isomorphic to $\underline{j}_{*}\left(H \otimes \overline{\boldsymbol{\mu}}_{m}\right) \sim_{\text {qis }} R \underline{j}_{*}\left(H \otimes \boldsymbol{\mu}_{m}\right)$ by 4.4.1. Thus we have an exact sequence

$$
0 \rightarrow H^{0}\left(\underline{S}, \mathfrak{S}_{H / \underline{S}}\right) \otimes \boldsymbol{\mu}_{m} \rightarrow H^{1}\left(S^{\star}, H \otimes \boldsymbol{\mu}_{m}\right) \rightarrow{ }_{m} H^{1}\left(\underline{S}, \mathfrak{S}_{H / \underline{S}}\right) \rightarrow 0 .
$$

The inductive limit for $\boldsymbol{\mu}_{m} \subset \mathbb{Q} / \mathbb{Z}$ induces the first exact sequence (6.14). The second sequence (6.15) follows the isomorphism $\mathfrak{S}_{H / S} \otimes \mathbb{Q} / \mathbb{Z} \simeq \mathfrak{T}_{H / S} \otimes \mathbb{Q} / \mathbb{Z}$. The morphism $\mathcal{O}_{B}^{\star} \rightarrow R i_{*} \mathbb{Z}_{B^{\star}}[1]$ of (6.11) induces a homomorphism

$$
\operatorname{Pic}(\boldsymbol{B}) \otimes \boldsymbol{\mu}_{m} \rightarrow H^{2}\left(\boldsymbol{B}^{\star}, \boldsymbol{\mu}_{m}\right) \simeq H^{2}\left(S^{\star}, \boldsymbol{\mu}_{m}\right) \oplus H^{1}\left(S^{\star}, H \otimes \boldsymbol{\mu}_{m}\right) \oplus \boldsymbol{\mu}_{m}
$$


by (6.9). Then we have an exact sequence

$$
\frac{\operatorname{Div}_{\boldsymbol{p}^{-1} D}(\boldsymbol{B})}{\operatorname{Div}_{D}(S)} \otimes \boldsymbol{\mu}_{m} \rightarrow \frac{\operatorname{Pic}(\boldsymbol{B})}{\operatorname{Pic}(S)} \otimes \boldsymbol{\mu}_{m} \rightarrow \frac{H^{2}\left(\boldsymbol{B}^{\star}, \boldsymbol{\mu}_{m}\right)}{H^{2}\left(S^{\star}, \boldsymbol{\mu}_{m}\right)} \rightarrow{ }_{m} H^{1}\left(\underline{S}, \mathfrak{S}_{H / \underline{S}}\right) \rightarrow 0
$$

by 6.2 .8 . The third exact sequence $(6.16)$ is obtained by the inductive limit for $\boldsymbol{\mu}_{m} \subset \mathbb{Q} / \mathbb{Z}$.

Definition 6.2.10. Let $S^{\nabla} \subset S$ be a Zariski-open subset with $S^{\star} \subset$ $S^{\nabla}$. We define the group $\amalg\left(S^{\nabla} / S, H\right)$ to be the kernel of $H^{1}\left(\underline{S}, \mathfrak{S}_{H / \underline{S}}\right)_{\text {tor }} \rightarrow$ $H^{0}\left(S^{\nabla}, R^{1} \varepsilon_{*} \mathfrak{S}_{H / \underline{S}}\right)$. In the case $S^{\nabla}=S$, we simply write $\amalg(S, H):=\underline{\amalg}(S / S$, $H) \simeq H^{1}\left(S, \mathfrak{S}_{H / S}\right)_{\text {tor }}$.

Proposition 6.2.11. Let $S^{\nabla} \subset S$ be a Zariski-open subset with $S^{\star} \subset$ $S^{\nabla}$. Then, there is an exact sequence

$$
0 \rightarrow H^{0}\left(S, \mathfrak{S}_{H / S}\right) \otimes \mathbb{Q} / \mathbb{Z} \rightarrow \lim _{m} H^{1}\left(S^{\nabla}, \mathbb{L}_{H / S}^{\bullet} \stackrel{\mathrm{L}}{\otimes} \boldsymbol{\mu}_{m}\right) \rightarrow \amalg\left(S^{\nabla} / S, H\right) \rightarrow 0 .
$$

For the basic elliptic fibration $\boldsymbol{p}: \boldsymbol{B} \rightarrow S$ associated with $H$, suppose that $\boldsymbol{p}$ is flat over $S^{\nabla}$ and set $\boldsymbol{B}^{\nabla}:=p^{-1} S^{\nabla}$. Then we have an exact sequence

$$
(\operatorname{Pic}(\boldsymbol{B}) / \operatorname{Pic}(S)) \otimes \mathbb{Q} / \mathbb{Z} \rightarrow \varliminf_{\lim _{m}} H^{2}\left(\boldsymbol{B}^{\nabla}, \boldsymbol{\mu}_{m}\right) / H^{2}\left(S^{\nabla}, \boldsymbol{\mu}_{m}\right) \rightarrow \amalg\left(S^{\nabla} / S, H\right) .
$$

If $S$ is compact, then the cokernel of the right homomorphism is a finite group (cf. [D5, 2.24]).

Proof. For a positive integer $m$, we have quasi-isomorphisms

$$
\mathfrak{S}_{H / \underline{S}} \stackrel{\mathrm{L}}{\otimes} \boldsymbol{\mu}_{m}[-1] \sim_{\text {qis } m} \mathfrak{S}_{H / \underline{S}} \simeq \underline{j}_{*}\left(H \otimes \boldsymbol{\mu}_{m}\right) \sim_{\text {qis }} R \underline{j}_{*}\left(H \otimes \boldsymbol{\mu}_{m}\right) .
$$

They induce a triangle

$$
\cdots \stackrel{+1}{\longrightarrow} \mathfrak{S}_{H / S} \stackrel{\mathrm{L}}{\otimes} \boldsymbol{\mu}_{m} \rightarrow\left(\tau_{\leq 1} R j_{*}\left(H \otimes \boldsymbol{\mu}_{m}\right)\right)[1] \rightarrow_{m}\left(R^{1} \varepsilon_{*} \mathfrak{S}_{H / \underline{S}}\right) \stackrel{+1}{\longrightarrow} \cdots .
$$

By taking cohomology groups over $S^{\nabla}$, we have a commutative diagram of exact sequences

$$
\begin{aligned}
& 0 \longrightarrow H^{0}\left(S^{\nabla}, \mathfrak{S}_{H / S} \stackrel{\mathrm{L}}{\otimes} \boldsymbol{\mu}_{m}\right) \longrightarrow H^{1}\left(S^{\star}, H \otimes \boldsymbol{\mu}_{m}\right) \longrightarrow H^{0}\left(S^{\nabla},{ }_{m}\left(R^{1} \varepsilon_{*} \mathfrak{S}_{H / \underline{S}}\right)\right) \\
& \uparrow \quad \| \quad \uparrow \\
& 0 \longrightarrow H^{0}\left(S, \mathfrak{S}_{H / S}\right) \otimes \boldsymbol{\mu}_{m} \longrightarrow H^{1}\left(S^{\star}, H \otimes \boldsymbol{\mu}_{m}\right) \longrightarrow \quad{ }_{m} H^{1}\left(\underline{S}, \mathfrak{S}_{H / \underline{S}}\right),
\end{aligned}
$$


where the right bottom arrow is surjective. The first exact sequence (6.17) follows the quasi-isomorphism $\mathfrak{S}_{H / S} \stackrel{\mathrm{L}}{\otimes} \boldsymbol{\mu}_{m} \sim_{\text {qis }} \mathbb{L}_{H / S}^{\bullet} \stackrel{\mathrm{L}}{\otimes} \boldsymbol{\mu}_{m}[1]$. Let $\nu: \tilde{C} \rightarrow$ $p^{-1} D$ be the normalization. Then $\mathcal{H}_{\boldsymbol{p}^{-1} D}^{2}\left(\mathbb{Z}_{\boldsymbol{B}}\right) \simeq \nu_{*} \mathbb{Z}_{\tilde{C}}$ by 3.2 .4 , since $\boldsymbol{B}$ is non-singular. For simplicity, we write $\mathbb{Z}_{\tilde{C}}$ for $\nu_{*} \mathbb{Z}_{\tilde{C}}$. Since $R^{1}(\boldsymbol{p} \circ \nu)_{*} \mathbb{Z}_{\tilde{C}}$ is a sheaf of torsion-free abelian groups, we have

$$
\boldsymbol{p}_{*} \mathcal{H}_{\boldsymbol{p}^{-1} D}^{2}\left(\mathbb{Z}_{\boldsymbol{B}}\right) \stackrel{\mathrm{L}}{\otimes} \boldsymbol{\mu}_{m} \sim_{\text {qis }} \boldsymbol{p}_{*}\left(\mathbb{Z}_{\tilde{C}} \otimes \boldsymbol{\mu}_{m}\right) .
$$

For the normalization $\tilde{D} \rightarrow D$, we have only an injection $\mathcal{H}_{D}^{2}\left(\mathbb{Z}_{S}\right) \hookrightarrow \mathbb{Z}_{\tilde{D}}$. Now $S^{\nabla}$ is non-singular, since $\boldsymbol{p}$ is flat over it. Let $\tilde{D}^{\nabla} \subset \tilde{D}$ be the pullback of $D \cap S^{\nabla}$ and let $\tilde{C}^{\nabla} \subset \tilde{C}$ be the pullback of $C \cap \boldsymbol{B}^{\nabla}$. Then $\left.\mathcal{H}_{D}^{2}\left(\mathbb{Z}_{S}\right)\right|_{S^{\nabla}} \simeq \mathbb{Z}_{\tilde{D}^{\nabla}}$, $\left.\mathcal{H}_{D}^{2}\left(\mathbb{Z}_{S}\right)\right|_{S^{\nabla}} \otimes \stackrel{\mathrm{L}}{\otimes} \boldsymbol{\mu}_{m} \sim_{\text {qis }} \mathbb{Z}_{\tilde{D}^{\nabla}} \otimes \boldsymbol{\mu}_{m}$, and $\left.\mathcal{V}_{\boldsymbol{B}}\right|_{S^{\nabla}} \simeq \boldsymbol{p}_{*} \mathbb{Z}_{\tilde{C}^{\nabla}} / \mathbb{Z}_{\tilde{D}^{\nabla}}$ by 5.4.7. Since $\mathbb{Z}_{S^{\nabla}} \rightarrow R \boldsymbol{p}_{*} \mathbb{Z}_{\boldsymbol{B}^{\nabla}}$ has a splitting (cf. 5.4.2), we have an exact sequence

$$
\begin{aligned}
\frac{H^{0}\left(\tilde{C}^{\nabla}, \boldsymbol{\mu}_{m}\right)}{H^{0}\left(\tilde{D}^{\nabla}, \boldsymbol{\mu}_{m}\right)} & \rightarrow \frac{H^{2}\left(\boldsymbol{B}^{\nabla}, \boldsymbol{\mu}_{m}\right)}{H^{2}\left(S^{\nabla}, \boldsymbol{\mu}_{m}\right)} \\
& \rightarrow H^{1}\left(S^{\nabla}, \mathbb{L}_{H / S}^{\bullet} \otimes \boldsymbol{\mu}_{m}\right) \oplus \boldsymbol{\mu}_{m} \rightarrow H^{1}\left(S^{\nabla}, \mathcal{V}_{\boldsymbol{B}} \otimes \boldsymbol{\mu}_{m}\right)
\end{aligned}
$$

from 6.2.8-(2). Here, the last homomorphism is decomposed as

$$
H^{1}\left(S^{\nabla}, \mathbb{L}_{H / S}^{\bullet} \stackrel{\mathrm{L}}{\otimes} \boldsymbol{\mu}_{m}\right) \oplus \boldsymbol{\mu}_{m} \rightarrow H^{0}\left(S^{\nabla}, \mathfrak{T}_{H / S} \stackrel{\mathrm{L}}{\otimes} \boldsymbol{\mu}_{m}\right) \rightarrow H^{1}\left(S^{\nabla}, \mathcal{V}_{\boldsymbol{B}} \otimes \boldsymbol{\mu}_{m}\right) .
$$

Let $G\left(m, S^{\nabla}\right)$ be the image of the composite. Since $H^{1}\left(\tilde{C}^{\nabla}, \boldsymbol{\mu}_{m}\right) \rightarrow H^{1}\left(\tilde{C}^{\nabla} \cap\right.$ $\left.p^{-1} S^{\circ}, \boldsymbol{\mu}_{m}\right)$ is injective, we have injections

$$
\begin{aligned}
H^{1}\left(S^{\nabla}, \mathcal{V}_{\boldsymbol{B}} \otimes \boldsymbol{\mu}_{m}\right) & \hookrightarrow H^{1}\left(S^{\nabla} \cap S^{\circ}, \mathcal{V}_{\boldsymbol{B}} \otimes \boldsymbol{\mu}_{m}\right), \quad \text { and } \\
G\left(m, S^{\nabla}\right) & \hookrightarrow G\left(m, S^{\nabla} \cap S^{\circ}\right) .
\end{aligned}
$$

If $m$ is divisible by 12 , then $H^{0}\left(S^{\nabla} \cap S^{\circ}, \mathfrak{T}_{H / S} \stackrel{\mathrm{L}}{\otimes} \boldsymbol{\mu}_{m}\right) \simeq H^{1}\left(S^{\nabla} \cap S^{\circ}, \mathfrak{T}_{H / S^{\circ}}\right) \oplus$ $H^{0}\left(S^{\nabla} \cap S^{\circ}, \mathfrak{T}_{H / S^{\circ}}\right)$. Thus, $\lim _{m} G\left(m, S^{\nabla}\right)$ is a finite group provided that $S$ is compact. Now we have the following commutative diagram of exact sequences

$$
\begin{aligned}
& \frac{H^{0}(\tilde{C}, \mathbb{Z})}{H^{0}(\tilde{D}, \mathbb{Z})} \otimes \boldsymbol{\mu}_{m} \longrightarrow \frac{\operatorname{Pic}(\boldsymbol{B})}{\operatorname{Pic}(S)} \otimes \boldsymbol{\mu}_{m} \longrightarrow H^{0}\left(S, \mathfrak{S}_{H / S}\right) \otimes \boldsymbol{\mu}_{m} \oplus \boldsymbol{\mu}_{m}
\end{aligned}
$$

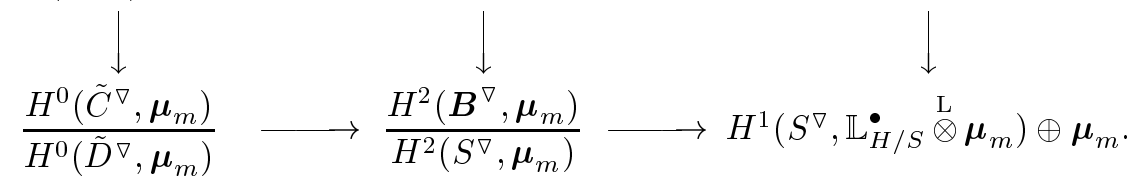

Here the left vertical arrow is surjective, since $\tilde{C}^{\nabla}$ is a Zariski-open subset of $\tilde{C}$. Hence the first exact sequence (6.17) induces the second sequence (6.18). 
The cokernel of

$$
\lim _{m} \frac{H^{2}\left(\boldsymbol{B}^{\nabla}, \boldsymbol{\mu}_{m}\right)}{H^{2}\left(S^{\nabla}, \boldsymbol{\mu}_{m}\right)} \rightarrow \amalg\left(S^{\nabla} / S, H\right)
$$

is isomorphic to $\lim _{m} G\left(m, S^{\nabla}\right)$.

Remark. Assume that there exist another open immersion $S^{\nabla} \hookrightarrow S^{\prime}$ and a bimeromorphic morphism $\mu: S \cdots \rightarrow S^{\prime}$ such that

(1) $S^{\nabla}$ is a Zariski-open subset of $S^{\prime}$,

(2) $S^{\star} \subset S^{\prime}$ is a toroidal embedding,

(3) $\left.\mu\right|_{S \nabla}$ is the identity of $S^{\nabla}$.

Then $\amalg\left(S^{\nabla} / S, H\right) \simeq \amalg\left(S^{\nabla} / S^{\prime}, H\right)$ by 6.2 .11 .

Theorem 6.2.12. Suppose that $S^{\star} \subset S$ together with a point $0 \in S$ is a d-dimensional toroidal embedding of type $(\mathrm{N}, l, \sigma)$. Then we have

$$
H^{1}\left(\underline{S}, \mathfrak{S}_{H / \underline{S}}\right) \simeq\left(R^{1} \varepsilon_{*} \mathfrak{S}_{H / \underline{S}}\right)_{0} \quad \text { and } \quad H^{1}\left(\underline{S}, \mathfrak{S}_{H / \underline{S}}\right) \simeq H^{1}\left(\underline{S}^{\circ}, \mathfrak{S}_{H / \underline{S}^{\circ}}\right)_{\text {tor }} .
$$

Let $\left\{\nu_{1}, \nu_{2}, \ldots, \nu_{k}\right\}$ be the set of all the vertices of $\sigma, \bigoplus_{i=1}^{k} \mathbb{Z} \nu_{i} \rightarrow \mathrm{N}$ the induced morphism, and let $\mathrm{M}=\operatorname{Hom}(\mathrm{N}, \mathbb{Z}) \rightarrow \mathrm{M}^{\prime}$ be its dual. Then the cohomology groups $H^{1}\left(\underline{S}, \mathfrak{S}_{H / \underline{S}}\right)$ and $H^{1}\left(\underline{S}^{\circ}, \mathfrak{S}_{H / \underline{S}^{\circ}}\right)$, and the restriction mapping $H^{1}\left(\underline{S}^{\circ}, \mathfrak{S}_{H / \underline{S}^{\circ}}\right) \rightarrow H^{1}\left(S^{\star}, \mathfrak{S}_{H}\right) \simeq H^{2}\left(S^{\star}, H\right)$ are described as follows:

- The case $H$ is of type $\mathrm{I}_{0}$. There are isomorphisms $H^{1}\left(\underline{S}, \mathfrak{S}_{H / \underline{S}}\right) \simeq \mathrm{M} \otimes$ $(\mathbb{Q} / \mathbb{Z})^{\oplus 2}, H^{1}\left(S^{\star}, \mathfrak{S}_{H}\right) \simeq \mathrm{M} \otimes \mathbb{Z}^{\oplus 2}$, and

$$
H^{1}\left(\underline{S}^{\circ}, \mathfrak{S}_{H / \underline{S}^{\circ}}\right) \simeq\left(\mathrm{M}_{\mathbb{Q}}^{\prime} / \mathrm{M}\right)^{\oplus 2} \oplus H^{1}\left(S^{\circ}, \mathcal{O}_{S^{\circ}}\right) .
$$

The restriction mapping $H^{1}\left(\underline{S}^{\circ}, \mathfrak{S}_{H / \underline{S}^{\circ}}\right) \rightarrow H^{1}\left(S^{\star}, \mathfrak{S}_{H}\right)$ is a zero map.

- The case $H$ is of type $\mathrm{I}_{(+)}$. Assume that $H$ is of type $\mathrm{I}_{\boldsymbol{a}}$ for $0 \neq \boldsymbol{a} \in \sigma^{\vee} \cap$ $\mathrm{M}$ and $\boldsymbol{a}=\alpha \boldsymbol{u}$ for a positive integer $\alpha>0$ and for a primitive element $\boldsymbol{u} \in \mathrm{M}$. For numbers $a_{i}=\left\langle\boldsymbol{a}, \nu_{i}\right\rangle$, let $\mathrm{M}^{\prime}=\mathrm{M}^{\natural} \oplus \mathrm{M}^{+}$be the direct sum, where $\mathrm{M}^{\natural}$ is dual to $\bigoplus_{a_{i}=0} \mathbb{Z} \nu_{i}$ and $\mathrm{M}^{+}$is dual to $\bigoplus_{a_{i}>0} \mathbb{Z} \nu_{i}$. Then

$$
\begin{aligned}
H^{1}\left(\underline{S}, \mathfrak{S}_{H / \underline{S}}\right) & \simeq \mathbb{Q} / \mathbb{Z} \oplus \operatorname{Hom}\left(\mathrm{N}\left(\boldsymbol{a}^{\perp} \cap \sigma\right), \mathbb{Q} / \mathbb{Z}\right) \oplus(\mathrm{M} \wedge \boldsymbol{u} / \mathrm{M} \wedge \boldsymbol{a}), \\
H^{1}\left(\underline{S}^{\circ}, \mathfrak{S}_{H / \underline{S}^{\circ}}\right) \simeq & H^{1}\left(S^{\circ}, \mathcal{O}_{S^{\circ}}\right) \oplus \mathrm{M}_{\mathbb{Q}}^{+} / \mathbb{Z} \boldsymbol{a} \oplus \mathrm{M}_{\mathbb{Q}}^{\natural} / \operatorname{Hom}\left(\mathrm{N}\left(\boldsymbol{a}^{\perp} \cap \sigma\right), \mathbb{Z}\right) \\
& \oplus \mathrm{M} / \mathbb{Z} \boldsymbol{u} \oplus \bigwedge^{2} \mathrm{M} / \mathrm{M} \wedge \boldsymbol{a} .
\end{aligned}
$$


The restriction map $H^{1}\left(\underline{S}^{\circ}, \mathfrak{S}_{H / \underline{S}^{\circ}}\right) \rightarrow H^{1}\left(S^{\star}, \mathfrak{S}_{H}\right)$ is the projection to the factor

$$
\mathrm{M} / \mathbb{Z} \boldsymbol{u} \oplus \bigwedge^{2} \mathrm{M} / \mathrm{M} \wedge \boldsymbol{a} \simeq H^{1}\left(S^{\star}, \mathfrak{S}_{H}\right) \simeq H^{2}\left(S^{\star}, H\right) .
$$

- Other cases. There are isomorphisms

$$
\begin{aligned}
H^{1}\left(\underline{S}, \mathfrak{S}_{H / \underline{S}}\right) & \simeq H^{2}\left(S^{\star}, H\right) \quad \text { and } \\
H^{1}\left(\underline{S}^{\circ}, \mathfrak{S}_{H / \underline{S}^{\circ}}\right) & \simeq H^{1}\left(S^{\circ}, \mathcal{L}_{H / S^{\circ}}\right) \oplus H^{2}\left(S^{\star}, H\right) .
\end{aligned}
$$

The restriction map $H^{1}\left(\underline{S}^{\circ}, \mathfrak{S}_{H / \underline{S}^{\circ}}\right) \rightarrow H^{1}\left(S^{\star}, \mathfrak{S}_{H}\right) \simeq H^{2}\left(S^{\star}, H\right)$ is the second projection.

Proof. We have $H^{1}\left(S, \mathcal{L}_{H / S}\right)=0$. Hence $H^{1}\left(S^{\star}, H\right) \rightarrow H^{1}\left(S^{\circ}, \mathcal{L}_{H / S^{\circ}}\right)$ is zero since it passes through $H^{1}\left(S, \mathcal{L}_{H / S}\right)$. Hence $H^{1}\left(\underline{S}^{\circ}, \mathfrak{S}_{H / S^{\circ}}\right)$ contains $H^{1}\left(S^{\circ}, \mathcal{L}_{H / S^{\circ}}\right)$ as a direct factor. The rest factor is an extension of $H^{2}\left(S^{\star}, H\right)$ by the cokernel of $H^{1}\left(S^{\star}, H\right) \rightarrow H^{0}\left(S^{\circ}, \mathfrak{Q}_{H / S^{\circ}} \otimes \mathbb{Q}\right)$, since $\left.R^{2} j_{*} H\right|_{S^{\circ}}=0$.

We have $H^{p}\left(S^{\star}, H\right) \simeq\left(R^{p} j_{*} H\right)_{0}$ for any $p$ and $H^{0}\left(S, \mathfrak{Q}_{H / S}\right) \simeq\left(\mathfrak{Q}_{H / S}\right)_{0}$ by 3.1.4. The isomorphism $H^{1}\left(\underline{S}, \mathfrak{S}_{H / \underline{S}}\right) \simeq\left(R^{1} \varepsilon_{*} \mathfrak{S}_{H / \underline{S}}\right)_{0}$ is derived from the commutative diagram

$$
\begin{aligned}
& H^{1}\left(S^{\star}, H\right) \longrightarrow H^{0}\left(S, \mathfrak{Q}_{H / S} \otimes \mathbb{Q}\right) \longrightarrow H^{1}\left(\underline{S}, \mathfrak{S}_{H / \underline{S}}\right) \longrightarrow H^{2}\left(S^{\star}, H\right)_{\text {tor }} \longrightarrow 0 \\
& \left(R^{1} j_{*} H\right)_{0} \longrightarrow\left(\mathfrak{Q}_{H / S}\right)_{0} \otimes \mathbb{Q} \quad \longrightarrow\left(R^{1} \varepsilon_{*} \mathfrak{S}_{H / \underline{S}}\right)_{0} \longrightarrow\left(R^{2} j_{*} H_{\text {tor }}\right)_{0} \longrightarrow 0
\end{aligned}
$$

obtained by 6.2.5. Thus $H^{1}\left(\underline{S}, \mathfrak{S}_{H / \underline{S}}\right)$ is a torsion group and hence $H^{1}\left(\underline{S}, \mathfrak{S}_{H / \underline{S}}\right)$ $\simeq H^{1}\left(\underline{S}^{\circ}, \mathfrak{S}_{H / \underline{S}^{\circ}}\right)_{\text {tor }}$ by 6.1.8. From 6.2.5, we infer that $H^{1}\left(\underline{S}, \mathfrak{S}_{H / \underline{S}}\right)$ is the extension of $H^{2}\left(S^{\star}, H\right)_{\text {tor }}$ by $C(H / S) \otimes \mathbb{Q} / \mathbb{Z}$. Since $C(H / S)$ is a free abelian group of finite rank, $H^{1}\left(\underline{S}, \mathfrak{S}_{H / \underline{S}}\right) \simeq C(H / S) \otimes \mathbb{Q} / \mathbb{Z} \oplus H^{2}\left(S^{\star}, H\right)_{\text {tor }}$.

Therefore, in order to calculate these cohomology groups, it is enough to describe the following two homomorphisms

$$
\begin{aligned}
& H^{1}\left(S^{\star}, H\right) \rightarrow H^{0}\left(S, \mathfrak{Q}_{H / S} \otimes \mathbb{Q}\right) ; \\
& H^{1}\left(S^{\star}, H\right) \rightarrow H^{0}\left(S^{\circ}, \mathfrak{Q}_{H / S^{\circ}} \otimes \mathbb{Q}\right) .
\end{aligned}
$$

If $H$ is neither of type $\mathrm{I}_{0}$ nor $\mathrm{I}_{(+)}$, then we have $\mathfrak{Q}_{H / S}=0$. If $H$ is of type $\mathrm{I}_{0}$, then $R^{1} j_{*} H \simeq \mathfrak{Q}_{H / S}$. Hence (6.19) is isomorphic to the natural injection $\mathrm{M}^{\oplus 2} \rightarrow \mathrm{M}_{\mathbb{Q}}^{\oplus 2}$. Since

$$
H^{0}\left(S^{\circ}, \mathfrak{Q}_{H / S^{\circ}}\right) \simeq \bigoplus_{i=0}^{k} H^{0}\left(D_{i}, \mathbb{Z}^{\oplus 2}\right) \simeq \mathrm{M}^{\prime \oplus 2}
$$


(6.20) is isomorphic to $\mathrm{M}^{\oplus 2} \rightarrow \mathrm{M}^{\prime \oplus 2}$. Suppose finally that $H$ is of type $\mathrm{I}_{\boldsymbol{a}}$ for $0 \neq \boldsymbol{a} \in \sigma^{\vee} \cap \mathrm{M}$. By 4.3.4 and 4.3.6 (or 4.4.2 and 4.4.3), we have isomorphisms

$$
\begin{aligned}
H^{0}\left(S, \mathfrak{Q}_{H / S} \otimes \mathbb{Q}\right) & \simeq \mathbb{Q} \oplus \operatorname{Hom}\left(\mathrm{N}\left(\boldsymbol{a}^{\perp} \cap \sigma\right), \mathbb{Q}\right), \quad \text { and } \\
H^{0}\left(S^{\circ}, \mathfrak{Q}_{H / S^{\circ}} \otimes \mathbb{Q}\right) & \simeq \mathrm{M}_{\mathbb{Q}}^{\prime},
\end{aligned}
$$

and we infer that $H^{0}\left(S, \mathfrak{Q}_{H / S} \otimes \mathbb{Q}\right) \rightarrow H^{0}\left(S^{\circ}, \mathfrak{Q}_{H / S^{\circ}} \otimes \mathbb{Q}\right)$ is isomorphic to the direct sum of $\mathbb{Q} \stackrel{\boldsymbol{a}}{\rightarrow} \mathrm{M}_{\mathbb{Q}}^{+}$and the natural inclusion $\operatorname{Hom}\left(\mathrm{N}\left(\boldsymbol{a}^{\perp} \cap \sigma\right), \mathbb{Q}\right) \rightarrow$ $\mathrm{M}_{\mathbb{Q}}^{\natural}$. The homomorphism (6.19) is written as $\mathbb{Z} \oplus \mathrm{M} / \mathbb{Z} \boldsymbol{a} \rightarrow \mathbb{Q} \oplus \operatorname{Hom}\left(\mathrm{N}\left(\boldsymbol{a}^{\perp} \cap\right.\right.$ $\sigma), \mathbb{Q})$ which is the sum of $\mathbb{Z} \rightarrow \mathbb{Q}$ and the natural homomorphism $\mathrm{M} / \mathbb{Z} \boldsymbol{a} \rightarrow$ $\operatorname{Hom}\left(\mathrm{N}\left(\boldsymbol{a}^{\perp} \cap \sigma\right), \mathbb{Q}\right)$. Hence the cokernel of $(6.19)$ is isomorphic to the direct $\operatorname{sum} \mathbb{Q} / \mathbb{Z} \oplus \operatorname{Hom}\left(\mathrm{N}\left(\boldsymbol{a}^{\perp} \cap \sigma\right), \mathbb{Q} / \mathbb{Z}\right)$ and the cokernel of $(6.20)$ is isomorphic to $\mathrm{M}_{\mathbb{Q}}^{+} / \mathbb{Z} \boldsymbol{a} \oplus \mathrm{M}_{\mathbb{Q}}^{\natural} / \operatorname{Hom}\left(\mathrm{N}\left(\boldsymbol{a}^{\perp} \cap \sigma\right), \mathbb{Z}\right)$.

Remark. There is another way to calculate $\left(R^{1} \varepsilon_{*} \mathfrak{S}_{H / \underline{S}}\right)_{0}$ by using $(6.15)$.

\section{$\S 6.3$. Description of $\mathcal{E}(S, D, H)$}

We assume that $p^{-1} D$ is a normal crossing divisor for our basic elliptic fibration $\boldsymbol{p}: \boldsymbol{B} \rightarrow S$. Recall that the fixed zero section $\boldsymbol{\sigma}$ of $\boldsymbol{p}$ defines a relative meromorphic group structure of $\boldsymbol{p}$.

Definition 6.3.1. A $\partial$-étale covering family $\left\{\varphi_{\alpha}: \underline{U}_{\alpha} \rightarrow \underline{S}\right\}_{\alpha \in \Lambda}$ is called good if the following two conditions are satisfied:

(1) Let $\left[U_{\alpha}, D_{\alpha}\right]$ be the top realization. Then the image $S_{\alpha}:=\varphi_{\alpha}\left(U_{\alpha}\right)$ is an open subset of $S$.

(2) The morphism $\varphi_{\alpha}: U_{\alpha} \rightarrow S_{\alpha}$ is a finite Galois covering.

For a good $\partial$-étale covering family $\left\{\underline{U}_{\alpha} \rightarrow \underline{S}\right\}_{\alpha \in \Lambda}$, let $H_{\alpha}$ be the pullback of $H$ to $U_{\alpha} \backslash D_{\alpha}$. Let $\boldsymbol{p}_{\alpha}: \boldsymbol{B}_{\alpha}=\boldsymbol{B}\left(H_{\alpha}\right) \rightarrow U_{\alpha}$ denote a basic elliptic fibration associated with $H_{\alpha}$. For two indices $\alpha, \beta \in \Lambda$, let $U_{\alpha, \beta}$ be the normalization of $U_{\alpha} \times_{S} U_{\beta}$. Then $\left[U_{\alpha, \beta}, D_{\alpha, \beta}\right]$ is the top realization of $\underline{U}_{\alpha} \times_{\underline{S}} \underline{U}_{\beta}$ for a naturally defined boundary $D_{\alpha, \beta}$. We have also the pullback $H_{\alpha, \beta}$ of $H$ to $U_{\alpha, \beta} \backslash D_{\alpha, \beta}$. Let $\boldsymbol{p}_{\alpha, \beta}: \boldsymbol{B}_{\alpha, \beta}=\boldsymbol{B}\left(H_{\alpha, \beta}\right) \rightarrow U_{\alpha, \beta}$ denote a basic elliptic fibration associated with $H_{\alpha, \beta}$. Note that $U_{\alpha, \alpha} \simeq G_{\alpha} \times U_{\alpha}$ for the Galois group $G_{\alpha}$ of $\varphi_{\alpha}: U_{\alpha} \rightarrow S_{\alpha}$. For a given $\partial$-étale covering family of $\underline{S}$, there is a finer and good $\partial$-étale covering family. Thus we need only good $\partial$-étale covering families in order to consider $\partial$-étale cohomology groups. 
Let $(f: X \rightarrow S, \phi)$ be a marked elliptic fibration belonging to $\mathcal{E}_{1}(S, D, H)$ such that $X$ is non-singular and that $f^{-1} D$ is a normal crossing divisor. Then the exact sequence

$$
0 \rightarrow \mathfrak{S}_{H / \underline{S}} \rightarrow R^{1} \underline{f}_{*} \mathcal{O}_{\underline{X}} / \mathcal{V}_{\underline{X}} \rightarrow \mathbb{Z}_{\underline{S}} \rightarrow 0
$$

defines a cohomology class $\eta(X / S, \phi)$ in $H^{1}\left(\underline{S}, \mathfrak{S}_{H / \underline{S}}\right)$ that depends on the bimeromorphic equivalence class of $(f, \phi)$. The $\eta(X / S, \phi)$ is also constructed as follows: By the assumption, there exist a good $\partial$-étale covering family $\left\{\underline{U}_{\alpha}=\left(U_{\alpha}, D_{\alpha}\right) \rightarrow \underline{S}\right\}$ as before and bimeromorphic mappings $h_{\alpha}: X_{\alpha} \cdots \rightarrow$ $\boldsymbol{B}_{\alpha}$ over $U_{\alpha}$ that preserves the marking $\phi$, where $X_{\alpha}$ denotes the normalization of the main component of $X \times_{S} U_{\alpha}$. The bimeromorphic mapping $h_{\beta} \circ h_{\alpha}^{-1}: \boldsymbol{B}_{\alpha, \beta} \cdots \rightarrow \boldsymbol{B}_{\beta, \alpha}$ is the translation mapping of a meromorphic section $\eta_{\alpha, \beta}$ of $\boldsymbol{B}_{\alpha, \beta} \rightarrow U_{\alpha, \beta}$, since this preserves the pullback $H_{\alpha, \beta}$ of $H$. By the construction of $\Psi_{\underline{X}}$, we infer that $\eta(X / S, \phi)$ above is determined by the cocycle $\left\{\eta_{\alpha, \beta}\right\}$ (cf. 5.5.2). Moreover, the map $\mathcal{E}_{1}(S, D, H) \rightarrow H^{1}\left(\underline{S}, \mathfrak{S}_{H / \underline{S}}\right)$ is a group homomorphism by 5.2 .5 .

Lemma 6.3.2. $\quad \mathcal{E}_{1}(S, D, H) \rightarrow H^{1}\left(\underline{S}, \mathfrak{S}_{H / \underline{S}}\right)$ is injective.

Proof. For two marked elliptic fibrations $\left(f_{1}: X_{1} \rightarrow S, \phi_{1}\right)$ and $\left(f_{2}: X_{2} \rightarrow\right.$ $\left.S, \phi_{2}\right)$ in $\mathcal{E}_{1}(S, D, H)$, suppose $\eta\left(X_{1} / S, \phi_{1}\right)=\eta\left(X_{2} / S, \phi_{2}\right)$. Then there exist a good $\partial$-étale covering family $\left\{\underline{U}_{\alpha} \rightarrow \underline{S}\right\}$ and bimeromorphic mappings

$$
h_{1, \alpha}: X_{1, \alpha} \cdots \rightarrow \boldsymbol{B}_{\alpha} \quad \text { and } \quad h_{2, \alpha}: X_{2, \alpha} \cdots \rightarrow \boldsymbol{B}_{\alpha}
$$

over $U_{\alpha}$ that preserve the marking $\phi$, where $X_{i, \alpha}$ denotes the normalization of the main component of $X \times{ }_{S} U_{\alpha}$, for $i=1,2$. The bimeromorphic mappings

$$
h_{i, \beta} \circ h_{i, \alpha}^{-1}: \boldsymbol{B}_{\alpha, \beta} \cdots \rightarrow \boldsymbol{B}_{\alpha, \beta}
$$

for $i=1,2$, respectively, are the translation mappings of meromorphic section $\eta_{\alpha, \beta}^{i}$ of $\boldsymbol{B}_{\alpha, \beta} \rightarrow U_{\alpha, \beta}$. By taking a finer $\partial$-étale covering family, we may assume that there is a collection of meromorphic sections $\boldsymbol{\sigma}_{\alpha}$ of $\boldsymbol{B}_{\alpha} \rightarrow U_{\alpha}$ such that

$$
\eta_{\alpha, \beta}^{1}-\eta_{\alpha, \beta}^{2}=p_{\alpha}^{*}\left(\boldsymbol{\sigma}_{\alpha}\right)-p_{\beta}^{*}\left(\boldsymbol{\sigma}_{\beta}\right)
$$

where $p_{\alpha}$ and $p_{\beta}$, respectively, stand for the projections $U_{\alpha, \beta} \rightarrow U_{\alpha}$ and $U_{\alpha, \beta} \rightarrow$ $U_{\beta}$. Thus we have a collection of bimeromorphic mappings $q_{\alpha}: X_{1, \alpha} \cdots \rightarrow X_{2, \alpha}$ such that the pullbacks of $q_{\alpha}$ and $q_{\beta}$ to $U_{\alpha, \beta}$ are same. Therefore we can glue these $q_{\alpha}$ to a global bimeromorphic mapping $X_{1} \cdots \rightarrow X_{2}$ over $S$. 
Lemma 6.3.3 (cf. 5.5.4).

$$
\mathcal{E}_{1}^{\text {proj }}(S, D, H)=H^{1}\left(\underline{S}, \mathfrak{S}_{H / \underline{S}}\right)_{\text {tor }} \cap \mathcal{E}_{1}(S, D, H) .
$$

If $(f: X \rightarrow S, \phi)$ is contained in the set above, then there is a finite surjective morphism $\tau: S^{\prime} \rightarrow S$ such that $\tau$ is étale outside $D$ and that $X \times{ }_{S} S^{\prime} \rightarrow S^{\prime}$ admits a meromorphic section.

Proof. Let $(f: X \rightarrow S, \phi)$ be a marked elliptic fibration contained in $\mathcal{E}(S, D, H)$. If $f$ is $\mathrm{BP}$ and if $X$ is non-singular, then there is an invertible sheaf of $X$ with positive degree on a smooth fiber. Thus if further $(f, \phi) \in$ $\mathcal{E}_{1}(S, D, H)$, then the cohomology class $\eta(X / S, \phi)$ is a torsion element. Therefore, we have $\mathcal{E}_{1}^{\text {proj }}(S, D, H) \subset H^{1}\left(\underline{S}, \mathfrak{S}_{H / \underline{S}}\right)_{\text {tor }}$.

Next, suppose that $(f, \phi) \in \mathcal{E}_{1}(S, D, H)$ and that the order $m$ of $\eta(X / S, \phi)$ is finite. There are a good $\partial$-étale covering family $\left\{\varphi_{\alpha}: \underline{U}_{\alpha} \rightarrow \underline{S}\right\}_{\alpha \in \Lambda}$ and a Čech cocycle $\left\{\eta_{\alpha, \beta}\right\}$ of $\mathfrak{S}_{H / \underline{S}}$ representing $\eta(X / S, \phi)$. Let $p_{\alpha}: \bar{U}_{\alpha, \beta} \rightarrow U_{\alpha}$ and $p_{\beta}: U_{\alpha, \beta} \rightarrow U_{\beta}$ stand for projections. We may assume that there are sections $\boldsymbol{\sigma}_{\alpha}$ of $\boldsymbol{B}_{\alpha} \rightarrow U_{\alpha}$ such that $m \eta_{\alpha, \beta}=p_{\beta}^{*}\left(\boldsymbol{\sigma}_{\beta}\right)-p_{\alpha}^{*}\left(\boldsymbol{\sigma}_{\alpha}\right)$ for any $\alpha$, $\beta \in \Lambda$. Let $\mu: \boldsymbol{B} \cdots \rightarrow \boldsymbol{B}$ be the multiplication mapping by $m$. Then we have $\mu \circ \operatorname{tr}\left(\eta_{\alpha, \beta}\right)=\operatorname{tr}\left(m \eta_{\alpha, \beta}\right) \circ \mu$ for the same multiplication mapping $\mu: \boldsymbol{B}_{\alpha, \beta} \cdots \rightarrow$ $\boldsymbol{B}_{\alpha, \beta}$. Therefore, the meromorphic mappings defined by

$$
X \times_{S} U_{\alpha} \stackrel{\operatorname{bim}}{\rightarrow} \boldsymbol{B}_{\alpha} \cdots \stackrel{\mu}{\rightarrow} \boldsymbol{B}_{\alpha} \stackrel{\operatorname{tr}\left(-\boldsymbol{\sigma}_{\alpha}\right)}{\longrightarrow} \boldsymbol{B}_{\alpha}
$$

can be patched to a generically finite meromorphic mapping $\mu_{X}: X \cdots \rightarrow$ $\boldsymbol{B}$ over $S$. Let $X^{\star} \rightarrow S^{\star}$ be a smooth elliptic fibration bimeromorphically equivalent to $f^{-1} S^{\star} \rightarrow S^{\star}$. Then $\mu_{X}$ induces a finite étale covering $X^{\star} \rightarrow \boldsymbol{B}^{\star}$. Let $S^{\prime \prime}$ be an irreducible component of the proper transform of the zero section $\boldsymbol{\Sigma}$ of $\boldsymbol{p}: \boldsymbol{B} \rightarrow S$ by $\mu_{X}$ and let $S^{\prime \prime} \rightarrow S^{\prime} \rightarrow S$ be the Stein factorization. Let $S^{\star \prime} \subset X^{\star}$ be the proper transform of $S^{\prime \prime} \cap f^{-1} S^{\star}$. Then $S^{\star \prime}$ is an irreducible component of the proper transform of $\boldsymbol{\Sigma} \cap \boldsymbol{B}^{\star}$. Since $\boldsymbol{\Sigma} \cap \boldsymbol{B}^{\star} \leadsto S^{\star}, S^{\star \prime} \rightarrow S^{\star}$ is a finite étale morphism. In particular, $S^{\prime} \times_{S} S^{\star} \simeq S^{\star \prime}$. Therefore the finite morphism $S^{\prime} \rightarrow S$ is étale outside $D$. tion 4].

The following theorem is proved in the case $S$ is non-singular in [N4, Sec-

Theorem 6.3.4.

$$
\mathcal{E}_{1}(S, D, H)=\mathcal{E}(S, D, H)
$$


This means that for any LBP elliptic fibration $f: X \rightarrow S$ satisfying the condition 5.2.1 and for any point $s \in S$, there exist a $\partial$-étale neighborhood $\left(U, D_{U}\right) \rightarrow(S, D)$ of $s$ and a meromorphic section of $X \times{ }_{S} U \rightarrow U$.

Proof. First we shall show $\mathcal{E}_{1}\left(S^{\circ}, D^{\star}, H\right)=\mathcal{E}\left(S^{\circ}, D^{\star}, H\right)$. Thus we may assume that $S$ and $D$ are non-singular, i.e., $S=S^{\circ}$. Since the property is local, we can localize $S$ if necessarily. Let $(X \rightarrow S, \phi)$ be a marked elliptic fibration in $\mathcal{E}(S, D, H)$. Then there exists locally over $S$ a finite branched covering $S^{\prime} \rightarrow S$ such that it is étale outside $D$ and that the general singular fiber of $X \times{ }_{S} S^{\prime} \rightarrow S^{\prime}$ is reduced. By 5.5.5, $X \times{ }_{S} S^{\prime} \rightarrow S^{\prime}$ admits a local meromorphic section. Hence $(X \rightarrow S, \phi) \in \mathcal{E}_{1}(S, D, H)$.

Next, we treat the general case. Let $(f: X \rightarrow S, \phi)$ be an element of $\mathcal{E}(S, D, H)$. Then its restriction to $S^{\circ}$ determines an element of $\mathcal{E}_{1}\left(S^{\circ}, D^{\star}, H\right) \simeq$ $H^{1}\left(\underline{S}^{\circ}, \mathfrak{S}_{H / \underline{S}^{\circ}}\right)$. There is an exact sequence:

$$
0 \rightarrow H^{1}\left(\underline{S}, \mathfrak{S}_{H / \underline{S}}\right) \rightarrow H^{1}\left(\underline{S}^{\circ}, \mathfrak{S}_{H / \underline{S}^{\circ}}\right) \rightarrow H^{0}\left(\underline{S}, R^{1} \underline{j}_{*}^{\circ} \mathfrak{S}_{H / \underline{S}^{\circ}}\right) .
$$

By 6.1.8, the image of the cohomology class $\eta\left(X^{\circ} / S^{\circ}, \phi\right)$ in any stalk of $R^{1} \underline{j}_{*}^{\circ} \mathfrak{S}_{H / \underline{S}^{\circ}}$ is zero. In particular, there is a $\partial$-étale covering family $\left\{\varphi_{\alpha}: \underline{U}_{\alpha}=\right.$ $\left.\left(U_{\alpha}, D_{\alpha}\right) \rightarrow \underline{S}\right\}$ such that $X \times_{S} U_{\alpha} \rightarrow U_{\alpha}$ admits a meromorphic section over $U_{\alpha}^{\circ}=U_{\alpha} \times{ }_{S} S^{\circ}$. Since $\operatorname{codim}\left(U_{\alpha} \backslash U_{\alpha}^{\circ}\right) \geq 2$, the meromorphic section extends to $U_{\alpha} \cdots \rightarrow X \times{ }_{S} U_{\alpha}$. Therefore $(f, \phi) \in \mathcal{E}_{1}(S, D, H)$.

We pose the following

Problem 6.3.5. Is the map $\mathcal{E}(S, D, H) \rightarrow H^{1}\left(\underline{S}, \mathfrak{S}_{H / \underline{S}}\right)$ bijective?

For an element $\eta \in H^{1}\left(\underline{S}, \mathfrak{S}_{H / \underline{S}}\right)$, we have a good $\partial$-étale covering family $\left\{\underline{U}_{\alpha} \rightarrow \underline{S}\right\}_{\alpha \in \Lambda}$ and a Čech cocycle $\left\{\eta_{\alpha, \beta}\right\}_{\alpha, \beta \in \Lambda}$ of $\mathfrak{S}_{H / \underline{S}}$ representing $\eta$. The $\eta_{\alpha, \alpha}$ is a meromorphic section of the pullback of $\boldsymbol{p}: \boldsymbol{B} \rightarrow S$ to $U_{\alpha, \alpha} \simeq G_{\alpha} \times U_{\alpha}$. Thus it defines a cocycle contained in $Z^{1}\left(G_{\alpha}, H^{0}\left(U_{\alpha}, \mathfrak{S}_{H_{\alpha} / U_{\alpha}}\right)\right)$. Therefore, we have a new meromorphic action of $G_{\alpha}$ on $\boldsymbol{B}_{\alpha}$ compatible with $U_{\alpha}$. Since $G_{\alpha}$ is a finite group, we can define the 'quotient' $G_{\alpha} \backslash \boldsymbol{B}_{\alpha}$ up to the bimeromorphic equivalence over $S_{\alpha} \simeq G_{\alpha} \backslash U_{\alpha}$. Let us choose a model $X_{\alpha}$ of $G_{\alpha} \backslash \boldsymbol{B}_{\alpha}$ such that $f_{\alpha}: X_{\alpha} \cdots \rightarrow S_{\alpha}$ is holomorphic. By the cocycle condition for $\eta_{\alpha, \alpha}$ and $\eta_{\alpha, \beta}$, there is a bimeromorphic mapping

$$
X_{\alpha} \times{ }_{S_{\alpha}}\left(S_{\alpha} \times{ }_{S} U_{\beta}\right) \cdots \rightarrow p^{-1}\left(S_{\alpha}\right) \times_{S} U_{\beta}
$$

over $S_{\alpha} \times{ }_{S} U_{\beta}$. Therefore we have meromorphic mappings

$$
h_{\alpha, \beta}: f_{\alpha}^{-1}\left(S_{\alpha} \cap S_{\beta}\right) \cdots \rightarrow f_{\beta}^{-1}\left(S_{\alpha} \cap S_{\beta}\right)
$$


such that $h_{\alpha, \beta} \circ h_{\beta, \gamma} \circ h_{\gamma, \alpha}=$ id over $S_{\alpha} \cap S_{\beta} \cap S_{\gamma}$. Hence if we choose models $X_{\alpha}$ so that $h_{\alpha, \beta}$ are all holomorphic, then we have a marked elliptic fibration $(f: X \rightarrow S, \phi)$ corresponding to $\eta$.

Theorem 6.3.6. The injection $\mathcal{E}\left(S^{\circ}, D^{\star}, H\right) \rightarrow H^{1}\left(\underline{S}^{\circ}, \mathfrak{S}_{H /} \underline{S}^{\circ}\right)$ is bijective. Further any cohomology class is attained by uniquely a relatively minimal locally projective elliptic fibration.

Proof. We may assume that $S=S^{\circ}$. Thus $D$ is non-singular. According to the argument above, it suffices to construct models $X_{\alpha}$ of $G_{\alpha} \backslash \boldsymbol{B}_{\alpha}$ such that $X_{\alpha} \rightarrow S_{\alpha}$ is minimal and that $h_{\alpha, \beta}$ are all holomorphic. The minimal models $X_{\alpha} \rightarrow S_{\alpha}$ are constructed in [N4, Section 5].

Proposition 6.3.7. Suppose that $m \eta \in \mathcal{E}(S, D, H)$ for an element $\eta \in$ $H^{1}\left(\underline{S}, \mathfrak{S}_{H / \underline{S}}\right)$ and for a positive integer $m$. Then $\eta \in \mathcal{E}(S, D, H)$. More precisely, if $\left(g: Y \rightarrow S, \phi_{g}\right)$ corresponds to $m \eta$, then there exist a marked elliptic fibration $\left(f: X \rightarrow S, \phi_{f}\right)$ corresponding to $\eta$ and a finite morphism $\mu: X \rightarrow Y$ over $S$ such that the homomorphism

$$
H \stackrel{\phi_{g}^{-1}}{\longrightarrow} H(g) \stackrel{\mu^{*}}{\longrightarrow} H(f) \stackrel{\phi_{f}}{\longrightarrow} H
$$

is the multiplication by $m$. In particular, if $g$ is smooth over $S^{\star}$, then $\left(f^{-1} S^{\star} \rightarrow\right.$ $\left.S^{\star}, \phi\right)$ is a marked smooth elliptic fibration corresponding to $\left.\eta\right|_{S^{\star}} \in \mathcal{E}\left(S^{\star}, \emptyset, H\right)$, and $\mu: X \rightarrow Y$ is the unique extension of a finite étale morphism $f^{-1} S^{\star} \rightarrow$ $g^{-1} S^{\star}$.

Proof. Let $\left\{\underline{U}_{\alpha} \rightarrow \underline{S}\right\}_{\alpha \in \Lambda}$ be a good $\partial$-étale covering family and let $\left\{\eta_{\alpha, \beta}\right\}_{\alpha, \beta \in \Lambda}$ be a Čech cocycle of $\mathfrak{S}_{H / \underline{S}}$ representing $\eta$. Let $X_{\alpha}$ and $h_{\alpha, \beta}$ be the varieties and the meromorphic mappings, respectively, defined as before. Let $(g: Y \rightarrow S, \phi)$ be a marked elliptic fibration corresponding to $m \eta$. The multiplication mapping $\boldsymbol{B} \cdots \rightarrow \boldsymbol{B}$ by $m$ induces meromorphic mappings $\mu_{\alpha}: X_{\alpha} \cdots \rightarrow g^{-1}\left(S_{\alpha}\right)$ such that $\mu_{\alpha} \circ h_{\alpha, \beta}=\mu_{\beta}$ over $S_{\alpha} \cap S_{\beta}$. By replacing $X_{\alpha}$, we may assume that $\mu_{\alpha}: X_{\alpha} \rightarrow g^{-1} S_{\alpha}$ are finite morphisms. Then $h_{\alpha, \beta}$ are holomorphic and thus we have a marked elliptic fibration $(f: X \rightarrow S, \phi)$ corresponding to $\eta$.

Remark. The multiplication mapping $\mathfrak{S}_{H / \underline{S}} \stackrel{\times m}{\longrightarrow} \mathfrak{S}_{H / \underline{S}}$ by a positive integer $m$ is surjective, the kernel ${ }_{m} \mathfrak{S}_{H / \underline{S}}$ is isomorphic to $\underline{j}_{*}\left(H \otimes \boldsymbol{\mu}_{m}\right)$, and 
$H^{1}\left(\underline{S},{ }_{m} \mathfrak{S}_{H / \underline{S}}\right) \simeq H^{1}\left(S^{\star}, H \otimes \boldsymbol{\mu}_{m}\right)$. Thus we have a commutative diagram

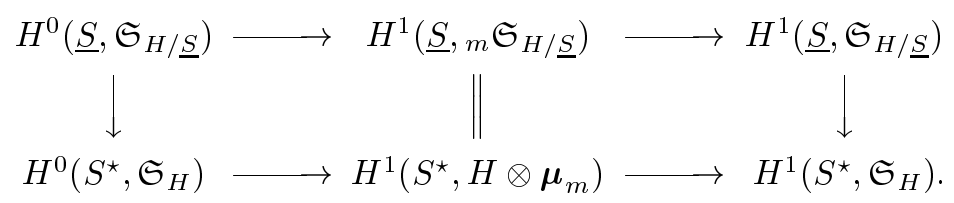

If $m \eta^{\prime}=m \eta$ and if $\left.\eta^{\prime}\right|_{S^{\star}}=\left.\eta\right|_{S^{\star}}$ for another $\eta^{\prime} \in H^{1}\left(\underline{S}, \mathfrak{S}_{H / \underline{S}}\right)$, then $\eta^{\prime}-\eta$ is coming from $\boldsymbol{\sigma}^{\star} \in H^{0}\left(S^{\star}, \mathfrak{S}_{H}\right)$. Let $(g: Y \rightarrow S, \phi)$ be a marked elliptic fibration representing $m \eta=m \eta^{\prime}$. Suppose that $g$ is smooth outside $S^{\star}$. By 6.3.7, there exist marked elliptic fibrations $(f: X \rightarrow S, \phi)$ and $\left(f^{\prime}: X^{\prime} \rightarrow S^{\prime}, \phi\right)$, and finite morphisms $\mu_{X}: X \rightarrow Y$ and $\mu_{X^{\prime}}: X^{\prime} \rightarrow Y$ such that $\eta=\eta(X / S, \phi)$, $\eta^{\prime}=\eta\left(X^{\prime} / S, \phi\right)$ and that $\mu_{X}$ and $\mu_{X^{\prime}}$ are derived from the multiplication mapping by $m$. Then we have an isomorphism $h^{\star}:\left.\left.X\right|_{S^{\star}} \leadsto X^{\prime}\right|_{S^{\star}}$ such that $\mu_{X^{\prime}} \circ h^{\star}=\operatorname{tr}\left(\boldsymbol{\sigma}^{\star}\right) \circ \mu_{X}$ for the translation mapping $\operatorname{tr}\left(\boldsymbol{\sigma}^{\star}\right): \boldsymbol{B}^{\star} \rightarrow \boldsymbol{B}^{\star}$.

Theorem 6.3.8. The subgroup $\mathcal{E}^{\operatorname{proj}}(S, D, H) \subset \mathcal{E}(S, D, H)$ is identified with the torsion part $H^{1}\left(\underline{S}, \mathfrak{S}_{H / \underline{S}}\right)_{\text {tor }}$. For an elliptic fibration $f: X \rightarrow S$ satisfying the condition 5.2.1, $f$ is $B P$ if and only if there is a prime divisor $\Gamma \subset X$ such that $f(\Gamma)=S$. If $f$ is smooth outside $S^{\star}$, we can choose $\Gamma$ to be étale over $S^{\star}$.

Proof. Let $m$ be the order of an element $\eta \in H^{1}\left(\underline{S}, \mathfrak{S}_{H / \underline{S}}\right)_{\text {tor }}$. Let $p: \boldsymbol{B} \rightarrow S$ be a basic elliptic fibration associated with $H$ such that $\boldsymbol{p}^{-1} S^{\star} \rightarrow S^{\star}$ is smooth. By 6.3.7, there exist a marked elliptic fibration $(f: X \rightarrow S, \phi)$ representing $\eta$ and a finite morphism $\mu: X \rightarrow \boldsymbol{B}$ of degree $m^{2}$ over $S$. Let $\boldsymbol{\Sigma} \subset \boldsymbol{B}$ be the zero section and let $\Gamma$ be an irreducible component of $\mu^{-1} \boldsymbol{\Sigma}$. Then $f(\Gamma)=S$ and $\Gamma$ is étale over $S^{\star}$.

By 6.2.12, we have

Corollary 6.3.9. Let $s \in S$ be a point. Then $\left(R^{1} \varepsilon_{*} \mathfrak{S}_{H / \underline{S}}\right)_{s}$ describes all the germs of marked projective elliptic fibrations defines near $s$ that is smooth over $S^{\star}$.

Theorem 6.3.10. $\quad$ Let $S^{\star} \subset S$ together with a point $0 \in S$ be a toroidal embedding of type $(\mathrm{N}, l, \sigma)$. Let $f: X \rightarrow S$ be a BP elliptic fibration satisfying 5.2.1. Suppose that $H(f)$ is not of type $\mathrm{I}_{(+)}^{(*)}$. If $f$ has no meromorphic section, then there exist a bimeromorphic mapping $M \rightarrow S$ and a prime divisor $\Gamma \subset M$ such that the singular fiber type along $\Gamma$ is ${ }_{m} \mathrm{I}_{a}$ for some $m>1, a \geq 0$. 
Proof.

Step 1. We follow the notation of 4.3. Let $\nu \in \sigma \cap \mathrm{N}$ be a primitive element. It corresponds to a prime divisor $\Gamma$ of a normal variety $V$ over $S$, where $\mu: V \rightarrow S$ is a bimeromorphic mapping obtained by a subdivision of $\sigma$ and hence $V^{\star}:=\mu^{-1} S^{\star} \leadsto S^{\star}$ and $V^{\star} \subset V$ is a toroidal embedding. Let $U \subset V$ be the complement of the reduced divisor $\left(\mu^{*} D\right)_{\mathrm{red}}-\Gamma$. Then $\Gamma^{\star}=\Gamma \cap U$ is non-singular and $j_{U}: V^{\star} \subset U$ is also a toroidal embedding. We have an exact sequence

$$
0 \rightarrow H^{0}\left(S, \mathfrak{T}_{H / S}\right) \otimes \mathbb{Q} / \mathbb{Z} \rightarrow H^{1}\left(S^{\star}, H \otimes \mathbb{Q} / \mathbb{Z}\right) \rightarrow H^{1}\left(\underline{S}, \mathfrak{S}_{H / \underline{S}}\right) \rightarrow 0
$$

by 6.2 .9 and an isomorphism $H^{0}\left(S, \mathfrak{S}_{H / S}\right) \otimes \mathbb{Q} / \mathbb{Z} \simeq H^{0}\left(S, \mathfrak{T}_{H / S}\right) \otimes \mathbb{Q} / \mathbb{Z}$. According to the argument of 4.3.3, we infer that the restriction homomorphism

$$
H^{1}\left(S^{\star}, H \otimes \mathbb{Q} / \mathbb{Z}\right) \rightarrow H^{0}\left(\Gamma^{\star}, R^{1} j_{U *}(H \otimes \mathbb{Q} / \mathbb{Z})\right)
$$

is written as $H^{1}$ of the following morphism of complexes:

$$
\operatorname{Kos}_{\mathrm{M} \otimes A}^{\bullet}(m \otimes \mathbb{Q} / \mathbb{Z}, \boldsymbol{b}) \rightarrow \operatorname{Kos}_{\left(\nu^{\perp} \cap \mathrm{M}\right) \otimes A}^{\bullet}\left(m \otimes_{A} A / \boldsymbol{b}(\nu) \otimes \mathbb{Q} / \mathbb{Z}, \boldsymbol{b}^{\prime}\right)[-1]
$$

where $\boldsymbol{b}^{\prime}$ is determined as the homomorphism $\mathrm{N} / \mathrm{N}(\nu) \rightarrow A / \boldsymbol{b}(\nu)$ inducing the commutative diagram

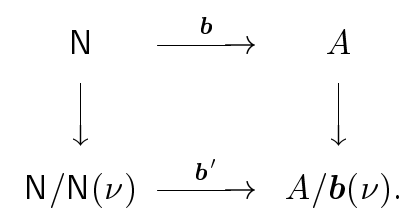

Step 2. The case $H$ has only unipotent monodromies. We have $\boldsymbol{b}=0$ in the case $\mathrm{I}_{0}$, and $\boldsymbol{b}=\boldsymbol{a} \boldsymbol{\varepsilon}$ in the case $\mathrm{I}_{\boldsymbol{a}}$. First we consider the case $\mathrm{I}_{0}$. Then (6.22) is isomorphic to

$$
\mathrm{M} \otimes(\mathbb{Q} / \mathbb{Z})^{\oplus 2} \rightarrow(\mathbb{Q} / \mathbb{Z})^{\oplus 2}
$$

induced by $\nu: \mathrm{M} \rightarrow \mathbb{Z}$. There is a generator $\left\{\gamma_{1}, \gamma_{2}, \ldots, \gamma_{l}\right\}$ of $\mathrm{N}$ such that $\gamma_{j} \in \sigma$ for any $j$. Hence if the elliptic fibration $f: X \rightarrow S$ is not basic, then the singular fiber type over $\Gamma$ is ${ }_{m} \mathrm{I}_{0}$ for some $m>0$ for some $\nu$. Next, we consider the case $\mathrm{I}_{\boldsymbol{a}}$ with $\boldsymbol{a} \neq 0$. Then (6.22) is isomorphic to

$$
\frac{\alpha^{-1} \mathrm{M}+\mathbb{Q} \boldsymbol{a}}{\mathrm{M}} \oplus \frac{\mathrm{M} \otimes \mathbb{Q}}{\mathrm{M}+\mathbb{Q} \boldsymbol{a}} \rightarrow \begin{cases}\alpha^{-1} \mathbb{Z} / \mathbb{Z} \oplus \mathbb{Q} / \mathbb{Z}, & \text { if } \boldsymbol{a}(\nu)=0 \\ \mathbb{Q} / \mathbb{Z}, & \text { if } \boldsymbol{a}(\nu)>0\end{cases}
$$


Here if $\boldsymbol{a}(\nu)=0$, then this is the sum of $\left(\alpha^{-1} \mathrm{M}+\mathbb{Q} \boldsymbol{a}\right) / \mathrm{M} \rightarrow \alpha^{-1} \mathbb{Z} / \mathbb{Z}$ and $\mathrm{M} \otimes \mathbb{Q} /(\mathrm{M}+\mathbb{Q} \boldsymbol{a}) \rightarrow \mathbb{Q} / \mathbb{Z}$ both of which are induced from $\nu: \mathrm{M} \rightarrow \mathbb{Z}$. If $\boldsymbol{a}(\nu)>$ 0 , then this is derived from the natural homomorphism $\left(\alpha^{-1} \mathrm{M}+\mathbb{Q} \boldsymbol{a}\right) / \mathrm{M} \hookrightarrow$ $\mathrm{M} \otimes \mathbb{Q} / \mathrm{M} \stackrel{\nu}{\rightarrow} \mathbb{Q} / \mathbb{Z}$. Let $\left(\alpha^{-1} \mathbf{x}+\lambda \boldsymbol{a}, \mathbf{y}\right)$ for $\lambda \in \mathbb{Q}, \mathbf{x} \in \mathrm{M}, \mathbf{y} \in \mathrm{M} \otimes \mathbb{Q}$ be a representative of $\left(\alpha^{-1} \mathrm{M}+\mathbb{Q} \boldsymbol{a}\right) / \mathrm{M} \oplus \mathrm{M} \otimes \mathbb{Q} /(\mathrm{M}+\mathbb{Q} \boldsymbol{a})$ whose image under the restriction mappings are zero for all primitive $\nu \in \sigma \cap \mathrm{M}$. Since $\alpha^{-1} \mathbf{x}(\nu)+$ $\lambda \boldsymbol{a}(\nu) \in \mathbb{Z}$ for all $\nu$, we infer that $\alpha^{-1} \mathbf{x}+\lambda \boldsymbol{a} \in \mathrm{M}$. Since $\mathbf{y}(\nu) \in \mathbb{Z}$ for $\nu$ with $\boldsymbol{a}(\nu)=0$, we infer that $\mathbf{y} \in \mathrm{M}+\mathrm{N}\left(\boldsymbol{a}^{\perp} \cap \sigma\right)^{\perp} \otimes \mathbb{Q}$. However, we have

$$
\frac{\mathrm{M}+\mathrm{N}\left(\boldsymbol{a}^{\perp} \cap \sigma\right)^{\perp} \otimes \mathbb{Q}}{\mathrm{M}+\mathbb{Q} \boldsymbol{a}} \simeq \mathrm{N}\left(\boldsymbol{a}^{\perp} \cap \sigma\right)^{\perp} / \mathbb{Z} \boldsymbol{a} \otimes \mathbb{Q} / \mathbb{Z} \simeq H^{0}\left(S, \mathfrak{T}_{H / S}\right) \otimes \mathbb{Q} / \mathbb{Z} .
$$

Therefore, the element of $H^{1}\left(S^{\star}, H \otimes \mathbb{Q} / \mathbb{Z}\right)$ corresponding to $\left(\alpha^{-1} \mathbf{x}+\lambda \boldsymbol{a}, \mathbf{y}\right)$ is coming from $H^{0}\left(S, \mathfrak{T}_{H / S}\right) \otimes \mathbb{Q} / \mathbb{Z}$. Consequently, the induced marked elliptic fibration is basic.

Step 3. Good choice of a generator of $\mathrm{N}$. We may assume the type of $H$ is one of $\mathrm{I}_{0}^{(*)}$, $\mathrm{III}^{(*)}, \mathrm{IV}^{(*)}$. Let $\mathrm{N}_{\text {unip }} \subset \mathrm{N}$ be the subgroup consisting of all $\gamma$ with $\rho(\gamma)$ being unipotent. Let $m$ be the order of $\mathrm{N} / \mathrm{N}_{\text {unip }}$. Then $m \in\{2,3,4\}$. There is a generator $\left\{\gamma_{1}, \gamma_{2}, \ldots, \gamma_{l}\right\}$ of $\mathrm{N}$ such that $\gamma_{1}$ generates $\mathrm{N} / \mathrm{N}_{\text {unip }} \simeq \mathbb{Z} / m \mathbb{Z}$ and that $\gamma_{j} \in \mathrm{N}_{\text {unip }}$ for $j \geq 2$. We want to change it to satisfy further condition: $\gamma_{j} \in \sigma$ for $j \geq 2$. For $i \geq 2$, we set

$$
\gamma_{i}^{\prime}=m c_{i} \gamma_{1}+\gamma_{i}
$$

for integers $c_{i}$. Then $\left\{\gamma_{1}, \gamma_{2}^{\prime}, \ldots, \gamma_{l}^{\prime}\right\}$ is also a generator of $\mathrm{N}$ and $\gamma_{j}^{\prime} \in \mathrm{N}_{\text {unip }}$ for $j \geq 2$. For some choice of $\left(c_{i}\right)$, the hyperplane cut $\sigma \cap \bigoplus_{i=2}^{l} \mathbb{R} \gamma_{i}^{\prime}$ is still a strictly convex rational polyhedral cone in $\bigoplus_{i=2}^{l} \mathbb{R} \gamma_{i}^{\prime}$. Hence there exist primitive vectors $\gamma_{2}^{\prime \prime}, \ldots, \gamma_{l}^{\prime \prime}$ in the hyperplane cut. Thus $\left\{\gamma_{1}, \gamma_{2}^{\prime \prime}, \ldots, \gamma_{l}^{\prime \prime}\right\}$ is a required generator.

Step 4. The cases $\mathrm{I}_{0}^{(*)}$, $\mathrm{III}^{(*)}, \mathrm{IV}^{(*)}$. As in the argument of Step 1, we consider the exact sequence (6.21), and the restriction homomorphism (6.22) for a primitive element $\nu \in \sigma \cap \mathrm{N}_{\text {unip }}$. By Step 3, we have a generator $\left\{\gamma_{1}, \gamma_{2}, \ldots\right.$, $\gamma_{l}$ \} of $\mathbf{N}$ such that $\gamma_{j} \in \sigma \cap \mathrm{N}_{\text {unip }}$ for $j \geq 2$. Then $\boldsymbol{b}\left(\gamma_{j}\right)=0$ for $j \geq 2$. The $\beta:=\boldsymbol{b}\left(\gamma_{1}\right)$ is -2 in the case $\mathrm{I}_{0}^{(*)}, \pm \mathbf{i}-1$ in the case $\mathrm{III}^{(*)}, \boldsymbol{\omega}^{ \pm 1}-1$ in the case $\mathrm{IV}^{(*)}$. We infer that $(6.22)$ is a factor of the homomorphism

$$
m \otimes \frac{\mathbb{Q} \boldsymbol{b}+\beta^{-1} \mathrm{M} \otimes A}{\mathbb{Q} \boldsymbol{b}+\mathrm{M} \otimes A} \rightarrow m \otimes A / \boldsymbol{b}\left(\gamma_{j}\right) A \otimes \mathbb{Q} / \mathbb{Z}
$$

derived from $\gamma_{j} \otimes \mathrm{id}: \mathrm{M} \otimes A \rightarrow A$. Let $\beta^{-1} \mathrm{x}$ for $\mathrm{x} \in \mathrm{M} \otimes A$ be a representative of the left hand side. Then the image is zero if and only if $\beta^{-1} \mathbf{x}\left(\gamma_{j}\right) \in A+\boldsymbol{b}\left(\gamma_{j}\right) A_{\mathbb{Q}}$. 
If the images are zero for any $j$, then $\beta^{-1} \underline{X} \in \mathbb{Q} \boldsymbol{b}+\mathrm{M} \otimes A$. Hence all the intersection of the kernels of $(6.22)$ is zero.

Example 6.3.11. Let us consider the following special case of $\mathrm{I}_{(+)}^{(*)}(0)$ : Let $S$ be a two-dimensional unit polydisc $\boldsymbol{\Delta}^{2}, D=D_{1}+D_{2}$ the union of coordinate hyperplanes, and assume that the monodromy type of $H$ is $\mathrm{I}_{0}^{*}$ along $D_{1}$ and $\mathrm{I}_{2}$ along $D_{2}$. Let $\gamma_{i} \in \mathrm{N} \simeq \mathbb{Z}^{\oplus 2}$ be an element corresponding to $D_{i}$ for $i=1,2$. Let $\left(e_{1}, e_{2}\right)$ be the base of $\mathrm{M}$ dual to $\left(\gamma_{1}, \gamma_{2}\right)$. Then $\boldsymbol{b}=-2 e_{1}+2 \varepsilon e_{2}$. Let $\nu \in \sigma \cap \mathrm{N}$ correspond to a prime divisor $\Gamma$ of a blown-up surface from $S$. Then the homomorphism (6.22) for $\Gamma$ is a factor of

$$
\frac{\mathbb{Q} \boldsymbol{b}+2^{-1} \mathrm{M} \otimes A}{\mathbb{Q} \boldsymbol{b}+\mathrm{M} \otimes A} \rightarrow A / \boldsymbol{b}(\nu) \otimes \mathbb{Q} / \mathbb{Z},
$$

for $A=\mathbb{Z}[\varepsilon]$. We have $\boldsymbol{b}(\nu) A_{\mathbb{Q}}=A_{\mathbb{Q}}$ if $e_{1}(\nu) \neq 0$ and $\boldsymbol{b}(\nu) A_{\mathbb{Q}}=\varepsilon A_{\mathbb{Q}}$ otherwise. The image of $\boldsymbol{v}:=2^{-1} \varepsilon e_{2} \in \mathbb{Q} \boldsymbol{b}+2^{-1} \mathrm{M} \otimes A$ is zero for any $\nu$, since $\boldsymbol{v}(\nu)=$ $2^{-1} \varepsilon e_{2}(\nu) \in \boldsymbol{b}(\nu) A_{\mathbb{Q}}$. However, $\boldsymbol{v} \notin \mathbb{Q} \boldsymbol{b}+\mathrm{M} \otimes A$. Hence, 6.3.10 does not hold in this case.

Concerning with Problem 6.3.5, we have

Theorem 6.3.12. Suppose that $\eta \in H^{1}\left(\underline{S}, \mathfrak{S}_{H / \underline{S}}\right)$ is mapped to a torsion element of $H^{1}\left(\underline{S}, \mathfrak{T}_{H / \underline{S}}\right)$ by $\mathfrak{S}_{H / \underline{S}} \rightarrow \mathfrak{T}_{H / \underline{S}}$. Then $\eta \in \mathcal{E}(S, D, H)$.

Proof. By 6.3.7, we may assume that $\eta$ is the image of $\theta \in H^{1}\left(\underline{S}, \mathfrak{S}_{H / S}^{\mathbf{W}}\right)$. A section of $\mathfrak{S}_{H / S}^{\mathbf{W}}$ over $S$ defines a holomorphic automorphism $\mathbf{W} \rightarrow \mathbf{W}$ as the translation for the generalized Weierstrass model $\mathbf{W}=\mathbf{W}(H)$ associated with $H$ by 5.1.4. In order to construct marked elliptic fibration representing $\eta$, let $\left\{\underline{U}_{\alpha} \rightarrow \underline{S}\right\}$ be a good $\partial$-étale covering family as before and let $\left\{\theta_{\alpha, \beta}\right\}$ be a Cech cocycle of $\mathfrak{S}_{H / \underline{S}}^{\mathbf{W}}$ representing $\theta$. We may assume that the local monodromies around $H_{\alpha}$ are all unipotent. Then we can replace a basic elliptic fibration $\boldsymbol{B}_{\alpha} \rightarrow U_{\alpha}$ by the minimal Weierstrass model $\mathbf{W}_{\alpha} \rightarrow U_{\alpha}$ associated with $H_{\alpha}$. Then $\mathbf{W}_{\alpha} \times_{U_{\alpha}} U_{\alpha, \beta} \simeq \mathbf{W}_{\beta} \times_{U_{\beta}} U_{\alpha, \beta}$ and it is isomorphic to the minimal Weierstrass model $\mathbf{W}_{\alpha, \beta}$ associated with $H_{\alpha, \beta}$. Since the translations by $\theta_{\alpha, \beta}$ are holomorphic, we have holomorphic quotients $X_{\alpha}:=G_{\alpha} \backslash \mathbf{W}_{\alpha}$ and holomorphic transition mappings $h_{\alpha, \beta}$. Thus we have the twist $\mathbf{W}^{\theta} \rightarrow S$ of $\mathbf{W}$ that represents $\theta$.

Corollary 6.3.13. Let $Z \subset D$ be the set of points $x$ around which $H$ is of type $\mathrm{I}_{(+)}$. If $\operatorname{dim} Z \leq 0$, then $\mathcal{E}(S, D, H)=H^{1}\left(\underline{S}, \mathfrak{S}_{H / \underline{S}}\right)$. 
Proof. If $x \notin Z$, then $\left(\mathfrak{T}_{H / \underline{S}}\right)_{x}$ is a torsion group and $2\left(\mathfrak{T}_{H / \underline{S}}\right)_{x}=0$. Let $2 \mathfrak{T}_{H / \underline{S}}$ be the image of the multiplication map $\mathfrak{T}_{H / \underline{S}} \rightarrow \mathfrak{T}_{H / \underline{S}}$ by 2 . Then $H^{1}\left(\underline{S}, 2 \mathfrak{T}_{H / \underline{S}}\right)=0$ by $\operatorname{dim} Z \leq 0$. Therefore, $2 H^{1}\left(\underline{S}, \mathfrak{T}_{H / \underline{S}}\right)=0$. Thus we are done by 6.3 .12 .

Example 6.3.14. Let $p: X_{\sigma} \rightarrow \boldsymbol{\Delta}^{2}=\left\{\left(t_{1}, t_{2}\right) \in \mathbb{C}|| t_{i} \mid<1(i=\right.$ $1,2)\}$ be the toric model (cf. [N4, Section 4]) associated with the sign function $\sigma: \mathbb{Z} \rightarrow\{1,2\}$ given by $\sigma(n)=1$ for $n$ odd, and $\sigma(n)=2$ for $n$ even. Then $\boldsymbol{p}$ has singular fibers of type $\mathrm{I}_{1}$ over both coordinate line $\left\{t_{i}=0\right\}(i=1,2)$. The $X_{\sigma}$ is defined as a quotient of $\mathcal{X}_{\sigma}$ which is a localization of a toric variety. We have an open covering $\left\{U_{n}\right\}$ of $\mathcal{X}_{\sigma}$ defined as follows:

$$
\begin{array}{ll}
U_{n}:=\left\{\left(t_{2}, \zeta_{n}, \eta_{n}\right) \in \mathbb{C}^{3}|| \zeta_{n} \eta_{n}|<1,| t_{2} \mid<1\right\} & \text { for } n \text { odd } \\
U_{n}:=\left\{\left(t_{1}, \zeta_{n}, \eta_{n}\right) \in \mathbb{C}^{3}|| \zeta_{n} \eta_{n}|<1,| t_{1} \mid<1\right\} & \text { for } n \text { even, }
\end{array}
$$

and the patching relation is given by: $\zeta_{n} \eta_{n}=t_{\sigma(n)}$ and $\zeta_{n+1} \eta_{n}=1$. There is an isomorphism $\mathcal{X}_{\sigma} \times \boldsymbol{\Delta}^{2}\left(\boldsymbol{\Delta}^{2}\right)^{\star} \simeq \mathbb{C}^{\star} \times\left(\boldsymbol{\Delta}^{2}\right)^{\star}$. Here, we can choose a coordinate of $\mathbb{C}^{\star}$ to be $s:=\zeta_{0}$, for example. Then $X_{\sigma}$ is obtained as the quotient of $\mathcal{X}_{\sigma}$ by the automorphism $s \mapsto s t_{1} t_{2}$. The meromorphic section $\left\{s=t_{1}\right\}$ of $\mathcal{X}_{\sigma} \rightarrow \boldsymbol{\Delta}^{2}$ induces a meromorphic section $\Gamma \subset X_{\sigma}$. Similarly, the section $\{s=1\}$ induces a holomorphic section $\boldsymbol{\Sigma} \subset X_{\sigma}$. We consider $\boldsymbol{\Sigma}$ as the canonical section. Let $g: X_{\sigma} \cdots \rightarrow X_{\sigma}$ be the translation by the meromorphic section $\Gamma$. For the induced VHS $H_{0}$, the group $\left(\mathfrak{T}_{H_{0} / \Delta^{2}}\right)_{0}$ is a free abelian group generated by $\Gamma$. Thus any compositions $g^{m}$ are not identical except for $m=0$. In fact, the $g^{m}$ corresponds to the meromorphic section $\left\{s=t_{1}^{m}\right\}$.

Let $M$ be a complex manifold with an element $c \in H^{1}(M, \mathbb{Z})$ of infinite order. For the base space $S=\Delta^{2} \times M$, we define a VHS $H$ to be the pullback of $H_{0}$ to $\left(\boldsymbol{\Delta}^{2}\right)^{\star} \times M$. We can find a cohomology class $\eta \in H^{1}\left(S, \mathfrak{S}_{H / S}\right)$ as the image of $\Gamma \otimes c \in H^{0}\left(\Delta^{2}, \mathfrak{S}_{H_{0} / S}\right) \otimes H^{1}(M, \mathbb{Z})$. There exist an open covering $\left\{V_{\alpha}\right\}_{\alpha \in \Lambda}$ of $M$ and integers $c_{\alpha, \beta}$ for $V_{\alpha} \cap V_{\beta} \neq \emptyset$ such that the collection $\left\{c_{\alpha, \beta}\right\}$ satisfies the cocycle condition:

$$
\begin{aligned}
c_{\alpha, \beta} & =-c_{\beta, \alpha}, \\
c_{\alpha, \beta}+c_{\beta, \gamma}+c_{\gamma, \alpha} & =0 \quad \text { for } \quad V_{\alpha} \cap V_{\beta} \cap V_{\gamma} \neq \emptyset,
\end{aligned}
$$

and that $\left\{c_{\alpha, \beta}\right\}$ represents $c$. If there is a marked elliptic fibration that induces $\eta$ under the map $\mathcal{E}(S, D, H) \rightarrow H^{1}\left(\underline{S}, \mathfrak{S}_{H / \underline{S}}\right)$, then it is bimeromorphic to $X_{\sigma} \times V_{\alpha}$ over $\Delta^{2} \times V_{\alpha}$ and the patchings are given by:

$$
X_{\sigma} \times\left(V_{\alpha} \cap V_{\beta}\right) \stackrel{g^{c} \alpha, \beta}{\cdots} \stackrel{\text { id }}{\longrightarrow} X_{\sigma} \times\left(V_{\alpha} \cap V_{\beta}\right)
$$


It seems to be impossible to patch them. We have another description of $\eta$. Let $\pi_{1}(M) \rightarrow \mathbb{Z}$ be the surjective homomorphism induced by $c$. This induces an étale covering $\lambda: \tilde{M} \rightarrow M$ with the Galois group isomorphic to $\mathbb{Z}$. Let $\theta$ be the generator 1 of the Galois group. Then we have a meromorphic automorphism

$$
X_{\sigma} \times \tilde{M} \stackrel{g \times \theta}{\longrightarrow} X_{\sigma} \times \tilde{M}
$$

which is not holomorphic. The $m$-times composite is not holomorphic except for $m=0$. If a marked elliptic fibration associated with $\eta$ exists, then it is bimeromorphically equivalent to the quotient of the meromorphic action $g \times \theta$ of $X_{\sigma} \times \tilde{M}$. Does the meromorphic quotient exist? In this case, the image of $\eta$ under $H^{1}\left(\underline{S}, \mathfrak{S}_{H / \underline{S}}\right) \rightarrow H^{2}\left(S^{\star}, H\right)$ is non-zero.

\section{$\S 7 . \quad$ Applications}

We consider the following applications: Ueno's extension problem (Section 7.1); a relation between Tate-Shafarevich group and our $\mathcal{E}(S, D, H)$ (Section 7.2); a generalization of some results of Gross [G5] on the study of minimal models with trivial canonical divisors and with elliptic fibration structures (Section 7.3); a characterization of Kähler morphisms (Section 7.4); a new description of logarithmic transformation by means of $\partial$-étale cohomology theory and its generalization to higher dimension (Section 7.5); and on the projectivity of logarithmic transform of elliptic surfaces (Section 7.6).

\section{$\S 7.1 . \quad$ Extension of elliptic fibrations}

Let $V$ be a normal complex analytic variety and let $D_{V}$ be a nowheredense analytic subset such that the complement $V^{\star}:=V \backslash D_{V}$ is non-singular. Suppose that a VHS $H$ is defined on $V^{\star}$. Let us consider the set $\mathcal{E}^{\operatorname{proj}}\left(V, D_{V}, H\right)$ of bimeromorphic equivalence classes of marked elliptic fibrations $(f: X \rightarrow$ $V, \phi)$ satisfying the following conditions:

(1) $f$ is $\mathrm{BP}$;

(2) The restriction $f^{-1} V^{\star} \rightarrow V^{\star}$ is bimeromorphically equivalent to a smooth elliptic fibration $f^{\prime \star}: X^{\prime \star} \rightarrow V^{\star}$

(3) $\phi$ is an isomorphism $H(f):=R^{1}{f^{\prime \star}}^{\star \star} \mathbb{Z}_{X^{\prime \star}} \leadsto H$ as VHS.

Let us fix a Zariski-open subset $V^{\circ} \subset V$ satisfying the following conditions:

(1) $V^{\star} \subset V^{\circ}$ and the complement $D_{V}^{\star}:=V^{\circ} \backslash V^{\star}$ is a normal crossing divisor. 
(2) $\operatorname{codim}\left(V \backslash V^{\circ}\right) \geq 2$.

We denote $\underline{V}^{\circ}:=\left(V^{\circ}, D_{V}^{\star}\right)$.

Theorem 7.1.1. There is an identification:

$$
\mathcal{E}^{\operatorname{proj}}\left(V, D_{V}, H\right) \longleftrightarrow H^{1}\left(\underline{V}^{\circ}, \mathfrak{S}_{H / \underline{V}^{\circ}}\right)_{\text {tor }}
$$

Proof. We have a bimeromorphic morphism $\mu: S \rightarrow V$ such that

(1) $S$ is non-singular,

(2) $\mu^{-1}\left(V^{\circ}\right) \rightarrow V^{\circ}$ is an isomorphism,

(3) $D:=\mu^{-1}\left(D_{V}\right)$ is a normal crossing divisor.

In particular, $S^{\star}=S \backslash D \simeq V^{\star}$ and $H$ is defined on $S^{\star}$. Then we can identify $\mathcal{E}^{\operatorname{proj}}\left(V, D_{V}, H\right)$ with $\mathcal{E}^{\operatorname{proj}}(S, D, H)$, by considering pullbacks and compositions. Therefore we have only to show that the restriction map $\mathcal{E}^{\text {proj }}(S, D, H)$ $\rightarrow \mathcal{E}^{\operatorname{proj}}\left(V^{\circ}, D_{V}^{\star}, H\right)$ is bijective. Note that the map is identified with

$$
H^{1}\left(\underline{S}, \mathfrak{S}_{H / \underline{S}}\right)_{\text {tor }} \rightarrow H^{1}\left(\underline{V}^{\circ}, \mathfrak{S}_{H / \underline{V}^{\circ}}\right)_{\text {tor }}
$$

by 6.3 .8 . We have a commutative diagram of exact sequences

$$
\begin{aligned}
& 0 \longrightarrow C(H / S) \otimes \mathbb{Q} / \mathbb{Z} \longrightarrow H^{1}\left(\underline{S}, \mathfrak{S}_{H / \underline{S}}\right)_{\text {tor }} \longrightarrow H^{2}\left(S^{\star}, H\right)_{\text {tor }} \longrightarrow 0 \\
& \downarrow \quad \downarrow \quad \| \\
& 0 \longrightarrow C\left(H / V^{\circ}\right) \otimes \mathbb{Q} / \mathbb{Z} \longrightarrow H^{1}\left(\underline{V}^{\circ}, \mathfrak{S}_{H / \underline{V}^{\circ}}\right)_{\text {tor }} \longrightarrow H^{2}\left(V^{\star}, H\right)_{\text {tor }} \longrightarrow 0
\end{aligned}
$$

by 6.2.5. Since $\operatorname{codim}\left(V \backslash V^{\circ}\right) \geq 2$, a meromorphic mapping $V^{\circ} \cdots \rightarrow \boldsymbol{B}$ over $S$ into the basic elliptic fibration $\boldsymbol{B} \rightarrow S$ associated with $H$ extends to $V \cdots \rightarrow \boldsymbol{B}$. Thus $H^{0}\left(S, \mathfrak{S}_{H / S}\right) \simeq H^{0}\left(V^{\circ}, \mathfrak{S}_{H / V^{\circ}}\right)$. Hence $C(H / S) \simeq C\left(H / V^{\circ}\right)$ and $(7.1)$ is bijective.

Corollary 7.1.2. $\quad$ Any smooth projective elliptic fibration over $V^{\star}$ extends to a BP elliptic fibration over $V$.

Proof. It suffices to show $H^{1}\left(\underline{V}^{\circ}, \mathfrak{S}_{H / \underline{V}^{\circ}}\right)$ tor $\rightarrow H^{1}\left(V^{\star}, \mathfrak{S}_{H}\right)_{\text {tor }}$ is surjective. By 6.2.5, this is surjective if $C\left(H / V^{\circ}\right) \rightarrow C\left(H / V^{\star}\right)$ is surjective. Since $C\left(H / V^{\circ}\right)$ and $C\left(H / V^{\star}\right)$ are both quotients of $H^{1}\left(V^{\star}, H\right)$, this is surjective.

Concerning with extension of non-BP elliptic fibrations, we have the following problem posed by Ueno (cf. [F4, II, 1.15]): 
Problem. Let $g^{\circ}: Y^{\circ} \rightarrow\left(\boldsymbol{\Delta}^{2} \backslash\{0\}\right)$ be a smooth elliptic fibration having no global sections. Then does it extend to an elliptic fibration over $\Delta^{2}$ ? The VHS $H\left(g^{\circ}\right)$ has only trivial monodromies, since $\boldsymbol{\Delta}^{2} \backslash\{0\}$ is simply connected. Let $H$ be the natural extension of $H\left(g^{\circ}\right)$ to $S$ as VHS. We can attach a cohomology class in $H^{1}\left(\left(\boldsymbol{\Delta}^{2} \backslash\{0\}\right), \mathfrak{S}_{H}\right)$ to $g^{\circ}$. We have an isomorphism $H^{1}\left(\left(\boldsymbol{\Delta}^{2} \backslash\{0\}\right), \mathfrak{S}_{H}\right) \simeq H^{1}\left(\left(\boldsymbol{\Delta}^{2} \backslash\{0\}\right), \mathcal{O}\right)$, which is an infinite-dimensional $\mathbb{C}$ vector space. By the assumption, the cohomology class is not a torsion element. Therefore, we can not extend $g^{\circ}$ as a projective morphism. Furthermore, by [N4, Section 3], it is also impossible to extend as a Kähler fibration. Therefore, if we have a positive answer to the problem, we will find an interesting nonKähler threefold. However, here we shall give a negative answer by using the $\partial$-étale cohomology theory. We can treat similar extension problem also for the case of other types of VHS and for higher dimensional case.

We assume that $S$ is a $d$-dimensional unit polydisc $\boldsymbol{\Delta}^{d}$ with a coordinate system $\left(t_{1}, t_{2}, \ldots, t_{d}\right)$ and that $D$ is the union of coordinate hyperplanes $D_{i}=$ $\left\{t_{i}=0\right\}$ for $1 \leq i \leq l$, where $1 \leq l \leq d$. Let us fix a Zariski-open subset $S^{\Delta} \subset S$ such that $S^{\star} \subset S^{\Delta}$ and $\operatorname{codim}\left(S \backslash S^{\Delta}\right) \geq 2$. The answer to the problem is negative by the following:

Theorem 7.1.3. Let $g: Y \rightarrow S^{\triangle}$ be an LBP elliptic fibration over $S^{\Delta}$ which is smooth over $S^{\star}$. Then the following two conditions are equivalent:

(1) There is an elliptic fibration $f: X \rightarrow S$ whose restriction $f^{-1} S^{\Delta} \rightarrow S^{\Delta}$ is bimeromorphically equivalent to $g$ over $S^{\triangle}$;

(2) $g$ is a BP elliptic fibration.

Proof. The implication $(2) \Longrightarrow(1)$ follows 7.1.1. We divide the proof of the other implication into the following 7 steps. Let $H=H(g)$ be the VHS defined on $S^{\star}$.

Step 1. We may assume that $S^{\triangle} \subset S^{\circ}$.

Suppose that the restriction of $f$ to the open subset $S^{\Delta \circ}:=S^{\triangle} \cap S^{\circ}$ is BP. Then the injection $H^{1}\left(\underline{S}^{\Delta}, \mathfrak{S}_{H / \underline{S}}\right) \rightarrow H^{1}\left(\underline{S}^{\Delta \circ}, \mathfrak{S}_{H / \underline{S}}\right)$ sends the cohomology class $[g]$ to a torsion element. Hence $g$ is BP by 6.3 .8 .

Step 2. We may assume that $H$ has only unipotent monodromies.

Let $\tau: S^{\prime} \simeq \boldsymbol{\Delta}^{d} \rightarrow S$ be the Kummer covering given by $\tau^{*} t_{i}=t_{i}^{m_{i}}$ for some positive integers $m_{i}$. We may assume that $\tau$ is étale outside $D$ and $\tau^{-1} H$ defined on $S^{\prime} \backslash \tau^{-1} D$ has only unipotent monodromies. If the pullback of $g$ to $\tau^{-1} S^{\Delta}$ is BP, then so is $g$. 


\section{Step 3. Flattening of $f$.}

We have a Zariski-open subset $S^{b} \subset S$ such that $\operatorname{codim}\left(S \backslash S^{b}\right) \geq 2$ and the restriction $f^{-1} S^{b} \rightarrow S^{b}$ is flat. By Hironaka's flattening [H3] of $f$, we have a bimeromorphic morphism $\mu: M \rightarrow S$ from a non-singular variety and an LBP elliptic fibration $h: X^{\prime} \rightarrow M$ satisfying the following conditions:

- $\mu$ induces an isomorphism $\mu^{-1} S^{b} \leadsto S^{b}$;

- $M \backslash \mu^{-1} S^{b}$ is a divisor $E=\sum_{j} E_{j}$;

- $D_{M}:=\mu^{-1}(D)$ is a simple normal crossing divisor on $M$;

- $\mu \circ h$ is bimeromorphically equivalent to $f$ over $S$.

Note that $D_{M}=\sum_{i=1}^{l} D_{i}^{\prime}+E$, where $D_{i}^{\prime}$ denotes the proper transform of $D_{i}$ in $M$. By 6.3 .2 and $6.3 .4, h$ defines a cohomology class $[h] \in H^{1}\left(\underline{M}, \mathfrak{S}_{H / \underline{M}}\right)$, where $H$ is the same VHS defined on $M \backslash D_{M} \simeq S^{\star}$ and $\underline{M}$ stands for the $\partial$-space $\left(M, D_{M}\right)$. The original fibration $g$ defines a cohomology class $[g] \in$ $H^{1}\left(\underline{S}^{\Delta}, \mathfrak{S}_{H / \underline{S}}\right)$. By the condition (1), the restrictions of $h$ and $g$ to the open subset $\mu^{-1}\left(S^{\Delta} \cap S^{b}\right) \simeq S^{\Delta} \cap S^{b}=: S^{\Delta b}$ are bimeromorphically equivalent. Thus they determine the same cohomology class in $H^{1}\left(\underline{S}^{\Delta b}, \mathfrak{S}_{H / S}\right)$. By $6.1 .8,[g]$ is a torsion element if and only if so is the image of $[h]$ under the restriction

$$
H^{1}\left(\underline{M}, \mathfrak{S}_{H / \underline{M}}\right) \rightarrow H^{1}\left(\underline{S}^{\Delta b}, \mathfrak{S}_{H / \underline{S}}\right) .
$$

Thus it is enough to show that the image of (7.2) is a torsion group.

Step 4.

Claim. Let $j_{M}: M \backslash D_{M} \hookrightarrow M$ be the open immersion.

(a) There is a point $x \in \mu^{-1}(0)$ such that for an open neighborhood $\mathcal{U}$ in $M$,

$$
\mu_{*}: \pi_{1}\left(\mathcal{U} \backslash D_{M}\right) \rightarrow \pi_{1}(S \backslash D)
$$

is an isomorphism.

(b) $H^{2}\left(S^{\star}, H \otimes \mathbb{Q}\right) \rightarrow H^{0}\left(M, R^{2} j_{M *} H \otimes \mathbb{Q}\right)$ is injective.

(c) The homomorphism $H^{1}\left(S^{\star}, H\right) \rightarrow H^{1}\left(S^{\Delta b}, \mathcal{L}_{H / S}\right)$ appearing in 6.2 .5 is a zero map.

Proof. (a) Let $W_{i}$ be the intersection $D_{1} \cap D_{2} \cap \cdots \cap D_{i}$ for $1 \leq i \leq l$ and let $W_{0}=S$. We can define inductively subvarieties $M=V_{0} \supset V_{1} \supset V_{2}$ $\supset \cdots \supset V_{l}$ satisfying the following condition: 
- $V_{i+1}$ is the proper transform of $W_{i+1}$ by the bimeromorphic morphism $\mu_{i}: V_{i} \rightarrow W_{i}$ induced from $\mu$, for $i<l$.

Then there exist uniquely irreducible components $\Gamma_{1}, \Gamma_{2}, \ldots, \Gamma_{l}$ of $D_{M}$ such that $V_{i}=\Gamma_{1} \cap \cdots \cap \Gamma_{i}$ for $i>0$. Let $x$ be a general point of $V_{l}$ and let $\mathcal{U}$ be an open neighborhood of $x$ in $M$ such that $D_{M} \cap \mathcal{U}=\left(\sum_{i=1}^{l} \Gamma_{i}\right) \cap \mathcal{U}$. We may assume that there is an isomorphism $\mathcal{U} \simeq \boldsymbol{\Delta}^{d}$ where $\Gamma_{i} \cap \mathcal{U}$ correspond to coordinate hyperplanes. It is enough to show $\mu^{*}: H^{1}(S \backslash D, \mathbb{Z}) \rightarrow H^{1}(\mathcal{U} \backslash$ $\left.D_{M}, \mathbb{Z}\right)$ is an isomorphism. By 3.1.4 and 3.2.4, this condition is equivalent to that the matrix $\left(a_{i, j}\right)_{1 \leq i, j \leq l}$ defined by

$$
\mu^{*} D_{i}=\left.\sum_{j=1}^{l} a_{i, j} \Gamma_{j}\right|_{\mathcal{U}}
$$

is non-singular. We have $a_{1,1}=1, a_{i, 1}=0$ for $i>1$. For $i>1$, we have

$$
\mu_{1}^{*}\left(\left.D_{i}\right|_{W_{1}}\right)=\left.\mu^{*} D_{i}\right|_{V_{1}}=\left.\sum_{j=2}^{l} a_{i, j} \Gamma_{j}\right|_{V_{1} \cap \mathcal{U}} .
$$

Hence, we have $a_{2,2}=1$ and $a_{i, 2}=0$ for $i>2$, since $\left.\Gamma_{2}\right|_{V_{1}}=V_{2}$ is the proper transform of $\left.D_{2}\right|_{W_{1}}=W_{2}$. Further, for $i>2$,

$$
\mu_{2}^{*}\left(\left.D_{i}\right|_{W_{2}}\right)=\left.\left(\mu_{1}^{*} D_{i}\right)\right|_{V_{2}}=\left.\sum_{j>2} a_{i, j} \Gamma_{j}\right|_{V_{2} \cap \mathcal{U}}
$$

In this way, we have $a_{i, i}=1$ and $a_{i, j}=0$ for $i>j$. Thus the matrix is non-singular.

(b) We have $H^{2}\left(S^{\star}, H\right) \simeq\left(R^{2} j_{*} H\right)_{0}$. By (a), we also have $\left(R^{2} j_{*} H\right)_{0} \simeq$ $\left(R^{2} j_{M *} H\right)_{x}$ for the point $x$.

(c) This is expressed as the composite

$$
H^{1}\left(S^{\star}, H\right) \rightarrow H^{1}\left(S, \mathcal{L}_{H / S}\right) \rightarrow H^{1}\left(S^{\Delta b}, \mathcal{L}_{H / S}\right),
$$

where $H^{1}\left(S, \mathcal{L}_{H / S}\right)=0$.

Step 5. More reduction. 
By 6.2.5 and Step 4-(b), we have a commutative diagram of exact sequences:

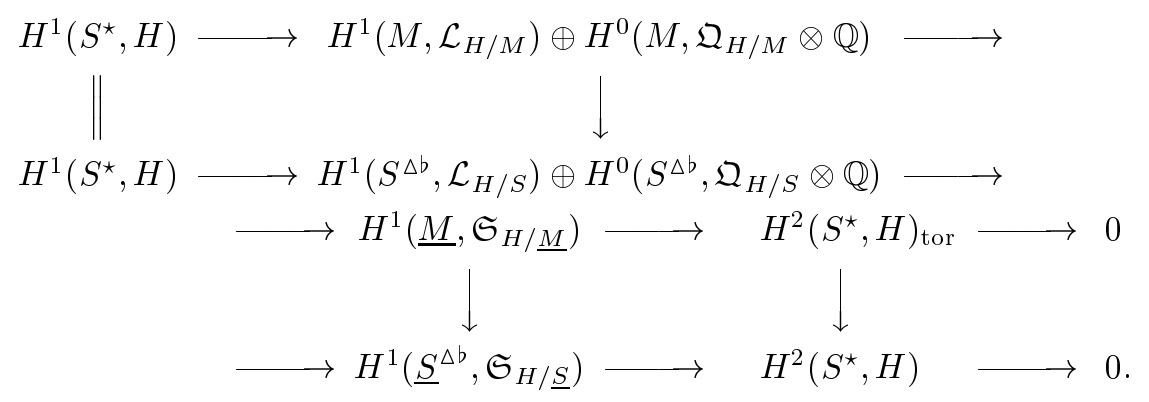

Thus, the image of (7.2) is a torsion group if and only if the image of

$$
H^{0}\left(M, \mathfrak{Q}_{H / M} \otimes \mathbb{Q}\right) \rightarrow H^{0}\left(S^{\Delta b}, \mathfrak{Q}_{H / S} \otimes \mathbb{Q}\right)
$$

is contained in the image of $H^{1}\left(S^{\star}, H \otimes \mathbb{Q}\right) \rightarrow H^{0}\left(S^{\Delta b}, \mathfrak{Q}_{H / S} \otimes \mathbb{Q}\right)$. Since $H^{1}\left(\underline{S}, \mathfrak{S}_{H / \underline{S}}\right)$ is a torsion group by 6.2 .12 , we have enough to show that the image of (7.3) coincides with the image of

$$
H^{0}\left(S, \mathfrak{Q}_{H / S} \otimes \mathbb{Q}\right) \rightarrow H^{0}\left(S^{\Delta b}, \mathfrak{Q}_{H / S} \otimes \mathbb{Q}\right) .
$$

Step 6 . The case $H$ is of type $\mathrm{I}_{0}$.

We have $\underline{j}_{*} H \simeq \mathbb{Z}_{\underline{S}}^{\oplus 2}$ and $\mathfrak{T}_{H / \underline{S}}=0$. Hence $R^{1} \underline{j}_{*} H \simeq \mathfrak{Q}_{H / \underline{S}}$ and $\varepsilon_{S *} \mathfrak{Q}_{H / \underline{S}} \simeq$ $\bigoplus_{i=1}^{l} \mathbb{Q}_{D_{i}}^{\oplus 2}$ for $\varepsilon_{S}: \underline{S} \rightarrow S$. Similarly, we have $\mathcal{L}_{H / \underline{M}} \simeq \mathcal{O}_{\underline{M}}, \underline{j}_{M *} H \simeq \mathbb{Z}_{\underline{M}}^{\oplus 2}$, $\mathfrak{T}_{H / \underline{M}}=0$, and an isomorphism

$$
\varepsilon_{M *} \mathfrak{Q}_{H / \underline{M}} \simeq \bigoplus \mathbb{Q}_{D_{i}^{\prime}}^{\oplus 2} \oplus \bigoplus \mathbb{Q}_{E_{j}}^{\oplus 2},
$$

where $\underline{j}_{M}: S^{\star} \simeq M \backslash D_{M} \rightarrow \underline{M}$ is the $\partial$-open immersion. Hence, (7.3) and (7.4) are both surjective.

Step 7 . The case $H$ is of type $\mathrm{I}_{(+)}$.

Suppose that $H$ is of type $\mathrm{I}_{\boldsymbol{a}}$ for $0 \neq \boldsymbol{a} \in \sigma^{\vee} \cap \mathrm{M}$ for the first quadrant $\sigma \subset \mathrm{N}$ $\otimes \mathbb{R}$ for the standard free group $\mathrm{N}=\bigoplus_{i=1}^{l} \mathbb{Z} \nu_{i}$ of rank $l$ and for its dual $\mathrm{M}$. Since $\mathrm{M} \simeq H^{1}\left(S^{\star}, \mathbb{Z}\right)$, the local system $H$ is determined by the extension

$$
0 \rightarrow \mathbb{Z}_{S^{\star}} \rightarrow H \rightarrow \mathbb{Z}_{S^{\star}} \rightarrow 0
$$

corresponding to $\boldsymbol{a}$. We also denote by $\boldsymbol{a}$ the connecting homomorphisms $R^{p} j_{*} \mathbb{Z}_{S^{\star}} \rightarrow R^{p+1} j_{*} \mathbb{Z}_{S^{\star}}$. The monodromy matrix around $D_{i}$ is of type $\mathrm{I}_{a_{i}}$ 
for $a_{i}=\left\langle\boldsymbol{a}, \nu_{i}\right\rangle$. Let $D^{+}$be the union of $D_{i}$ with $a_{i}>0, S^{\natural}:=S \backslash D^{+}$, and let $j^{\natural}: S^{\natural} \hookrightarrow S$ and $j^{\natural \star}: S^{\star} \hookrightarrow S^{\natural}$ be related immersions. Then we have the following exact sequences (cf. 4.3.4, 5.4.14 and 6.2.3):

$$
\begin{gathered}
0 \rightarrow \mathbb{Z}_{S} \rightarrow j_{*} H \rightarrow j_{!}^{\natural} \mathbb{Z}_{S^{\natural}} \rightarrow 0, \\
0 \rightarrow \mathbb{Z}_{D^{+}} \stackrel{a}{\rightarrow} R^{1} j_{*}^{\natural} \mathbb{Z}_{S^{\natural}} \rightarrow \mathfrak{T}_{H / S} \rightarrow 0, \\
0 \rightarrow \mathfrak{T}_{H / S} \oplus j_{*}^{\natural}\left(R^{1} j_{*}^{\natural \star} \mathbb{Z}_{S^{\star}}\right) \rightarrow R^{1} j_{*} H \rightarrow \mathbb{Z}_{D^{+}} \oplus j_{!}^{\natural}\left(R^{1} j_{*}^{\natural \star} \mathbb{Z}_{S^{\star}}\right) \rightarrow 0, \\
0 \rightarrow j_{*}^{\natural}\left(R^{1} j_{*}^{\natural \star} \mathbb{Z}_{S^{\star}}\right) \rightarrow \mathfrak{Q}_{H / S} \rightarrow \mathbb{Z}_{D^{+}} \oplus j_{!}^{\natural}\left(R^{1} j_{*}^{\natural \star} \mathbb{Z}_{S^{\star}}\right) \rightarrow 0 .
\end{gathered}
$$

We can consider similar sequences to $j_{M}: \mu^{-1} S^{\star} \hookrightarrow M$. The local monodromy around the exceptional divisor $E_{j}$ is of type $\mathrm{I}_{e_{j}}$ for some $e_{j} \geq 0$. Let $D_{M}^{+}:=$ $\mu^{-1} D^{+}$. This is the union of all $D_{i}^{\prime}$ with $a_{i}>0$ and all $E_{j}$ with $e_{j}>0$. We also define $M^{\natural}:=M \backslash D_{M}^{+}=\mu^{-1} S^{\natural}$ and denote the related injections by $j_{M}^{\natural}: M^{\natural} \hookrightarrow M$ and $j_{M}^{\natural \star}: S^{\star} \leadsto \mu^{-1} S^{\star} \hookrightarrow M^{\natural}$. Further, we write the restriction of $\mu$ to $M^{\natural}$ by $\mu^{\natural}$. Then we have an exact sequence

$$
0 \rightarrow j_{M *}^{\natural}\left(R^{1} j_{M *}^{\natural \star} \mathbb{Z}_{S^{\star}}\right) \rightarrow \mathfrak{Q}_{H / M} \rightarrow \mathbb{Z}_{D_{M}^{+}} \oplus j_{M !}^{\natural}\left(R^{1} j_{M *}^{\natural \star} \mathbb{Z}_{S^{*}}\right) \rightarrow 0 .
$$

Note that there is an isomorphism

$$
R^{1} j_{M *}^{\natural \star} \mathbb{Z}_{S^{\star}} \simeq \bigoplus_{a_{i}=0} \mathbb{Z}_{D_{i}^{\prime} \cap M^{\natural}} \oplus \bigoplus_{e_{j}=0} \mathbb{Z}_{E_{j} \cap M^{\natural}} .
$$

Since $H^{1}\left(D_{i}^{\prime}, \mathbb{Z}\right)=0$ and $H^{1}\left(E_{j}, \mathbb{Z}\right)=0$, we have $H^{1}\left(M, j_{M *}^{\natural}\left(R^{1} j_{M *}^{\natural \star} \mathbb{Q}_{S^{*}}\right)\right)=0$. We also have

$$
H^{0}\left(M, j_{M !}^{\natural}\left(R^{1} j_{M *}^{\natural \star} \mathbb{Q}_{S^{\star}}\right)\right) \simeq \bigoplus_{E_{j} \cap D_{M}^{+}=\emptyset} \mathbb{Q} .
$$

Thus we have a commutative diagram of exact sequences:

$$
\begin{aligned}
& 0 \longrightarrow \bigoplus_{a_{i}=0} \mathbb{Q} \quad \longrightarrow H^{0}\left(S, \mathfrak{Q}_{H / S} \otimes \mathbb{Q}\right) \longrightarrow \quad \mathbb{Q} \quad \longrightarrow 0 \\
& 0 \longrightarrow \bigoplus_{a_{i}=0} \stackrel{\downarrow}{\stackrel{Q}{\oplus}} \bigoplus_{e_{j}=0} \mathbb{Q} \longrightarrow H^{0}\left(M, \mathfrak{Q}_{H / M}^{\downarrow} \otimes \mathbb{Q}\right) \longrightarrow \mathbb{Q} \oplus \underset{E_{j} \cap D_{M}^{+}=\emptyset}{\stackrel{\downarrow}{\bigoplus}} \underset{\mathbb{Q}}{\bigoplus} 0 .
\end{aligned}
$$

Since $D_{i} \cap S^{\Delta b} \neq \emptyset$ and $E_{j} \cap S^{\Delta b}=\emptyset$, we have

$$
0 \rightarrow \bigoplus_{a_{i}=0} \mathbb{Q} \rightarrow H^{0}\left(S^{\Delta b}, \mathfrak{Q}_{H / S} \otimes \mathbb{Q}\right) \rightarrow \mathbb{Q} \oplus H^{0}\left(S^{\Delta b}, j_{!}^{\natural}\left(\bigoplus_{a_{i}=0} \mathbb{Q}_{D_{i} \cap S^{\natural}}\right)\right) \rightarrow 0 .
$$

Hence (7.3) and (7.4) have same images. 


\section{§7.2. Tate-Shafarevich group}

Suppose that $S$ is a projective variety. Let $S$ be a normal projective scheme over $\mathbb{C}$ with $\mathrm{S}^{\text {an }} \simeq S$. For a projective basic elliptic fibration $\boldsymbol{p}: \boldsymbol{B} \rightarrow S$ associated with $H$, we have a projective morphism $\mathrm{p}: \mathrm{B} \rightarrow \mathrm{S}$ of schemes over $\mathbb{C}$ such that $\mathrm{p}^{\text {an }} \simeq \boldsymbol{p}$. The generic fiber $\mathrm{B}_{\eta}$ of $\mathrm{p}$ is uniquely determined by $H$ and is an elliptic curve over the function field $\mathbb{C}(\mathrm{S})$. For Zariski-open subsets $S^{\star} \subset S^{\nabla}$ of $S$, let $\mathrm{S}^{\star} \subset \mathrm{S}^{\nabla}$ be corresponding Zariski-open subsets of S. We shall compare $\amalg\left(S^{\nabla} / S, H\right)$ defined in 6.2.10 with the Tate-Shafarevich group $\amalg_{S^{\nabla}}\left(\mathrm{B}_{\eta}\right)$ for the generic fiber $\mathrm{B}_{\eta}$. Previously, we defined a similar group $\mathcal{E}_{0}^{\text {proj }}\left(S, D, H ; S^{\nabla}\right) \subset \mathcal{E}^{\operatorname{proj}}(S, D, H)\left(\right.$ cf. 5.2.4). By 6.2.10, $\mathcal{E}_{0}^{\text {proj }}\left(S, D, H ; S^{\nabla}\right)$ is identified with $\amalg\left(S^{\nabla} / S, H\right)$.

Proposition 7.2.1. If $S^{\nabla}$ is non-singular, then

$$
\amalg\left(S^{\nabla} / S, H\right) \simeq \amalg_{S^{\nabla}}\left(\mathrm{B}_{\eta}\right) .
$$

Proof. We fix a bimeromorphic mapping $p^{-1} S^{\circ} \cdots \rightarrow \boldsymbol{B}^{\circ}$ over $S^{\circ}$ for basic elliptic fibrations $p: B \rightarrow S$ and $p^{\circ}: \boldsymbol{B}^{\circ} \rightarrow S^{\circ}$, where $p^{\circ}$ is minimal. Let $(f: X \rightarrow S, \phi)$ be a marked projective elliptic fibration contained in $\mathcal{E}_{0}^{\text {proj }}\left(S, D, H ; S^{\nabla}\right)$. Then the multiplication map by a positive integer $m$ induces a generically finite meromorphic mapping $\mu: X \cdots \rightarrow B$ over $S$. Let $\boldsymbol{\Sigma} \subset \boldsymbol{B}$ be the zero section of $\boldsymbol{p}$ and let $\boldsymbol{\Sigma}^{\circ}$ be the corresponding section of $\boldsymbol{p}^{\circ}$ over $S^{\circ}$. Let $\boldsymbol{\Sigma}^{\prime}$ be the proper transform of $\boldsymbol{\Sigma}$ in $X$. Let $\tau: T \rightarrow S$ be the Stein factorization of $\widetilde{\boldsymbol{\Sigma}}^{\prime} \rightarrow S$ from the normalization $\widetilde{\boldsymbol{\Sigma}}^{\prime}$ of $\boldsymbol{\Sigma}^{\prime}$. Let $T^{\sharp} \subset T$ be the maximal open subset of $T$ along which $\tau$ is étale. Then $X \times_{S} T^{\sharp} \rightarrow T^{\sharp}$ admits a meromorphic section. It suffices to show that $S^{\nabla} \subset \tau\left(T^{\sharp}\right)$. For a point $s \in S^{\nabla}$, we have an open neighborhood $\mathcal{U} \subset S^{\nabla}$ and a bimeromorphic mapping $\varphi: f^{-1} \mathcal{U} \cdots \rightarrow p^{-1} \mathcal{U}$. We may assume that $\mathcal{U}$ is isomorphic to a unit polydisc and that $D \cap \mathcal{U}$ is isomorphic to a union of coordinate hyperplanes. Then the generically finite meromorphic mapping $\rho=\mu \circ \varphi^{-1}: p^{-1} \mathcal{U} \cdots \rightarrow p^{-1} \mathcal{U}$ is composed of the multiplication mapping by $m$ and the translation by a section of $p$ over $\mathcal{U}$. Let $\rho^{\circ}: \boldsymbol{p}^{\circ-1} \mathcal{U}^{\circ} \cdots \rightarrow \boldsymbol{p}^{\circ-1} \mathcal{U}^{\circ}$ be the induced meromorphic mapping over $\mathcal{U}^{\circ}:=\mathcal{U} \cap S^{\circ}$ by $\rho$ and let $\boldsymbol{\Sigma}_{\mathcal{U}}^{\circ}$ be the proper transform of $\boldsymbol{\Sigma}^{\circ} \cap \boldsymbol{p}^{\circ-1} \mathcal{U}^{\circ}$ in $\boldsymbol{p}^{\circ-1} \mathcal{U}^{\circ}$ by $\rho^{\circ}$. The multiplication mapping $\boldsymbol{B}^{\circ} \cdots \rightarrow \boldsymbol{B}^{\circ}$ by $m$ is holomorphic over the Néron model $\left(\boldsymbol{B}^{\circ}\right)^{\sharp}$. Thus by 5.3.3, we have an irreducible component $V$ of $\boldsymbol{\Sigma}_{\mathcal{U}}^{\circ}$ such that $\boldsymbol{p}^{\circ}\left(V^{\sharp}\right)=\mathcal{U}^{\circ}$ for an open subset $V^{\sharp}$ along which $V \rightarrow \mathcal{U}^{\circ}$ is étale. The Stein factorization $V^{\prime} \rightarrow \mathcal{U}^{\circ}$ of $V \rightarrow \mathcal{U}^{\circ}$ is a finite Galois covering, since it is étale outside $D$ and since $\pi_{1}\left(\mathcal{U}^{\circ} \backslash D\right)$ is abelian. Thus $V^{\prime}$ is étale over $\mathcal{U}^{\circ}$ and hence $V^{\prime} \simeq \mathcal{U}^{\circ}$ since $\pi_{1}\left(\mathcal{U}^{\circ}\right)=\{1\}$. Therefore, $V$ is a holomorphic 
section of $\boldsymbol{p}^{\circ}$ over $\mathcal{U}^{\circ}$ by 5.3.2. Let $\Gamma_{\mathcal{U}}^{\circ}$ be the proper transform of $V$ in $f^{-1} \mathcal{U}^{\circ}$ by the bimeromorphic mapping $\varphi$ and let $\Theta_{\mathcal{U}^{\circ}}$ be the corresponding irreducible component of $\tau^{-1} \mathcal{U}^{\circ}$. Then $\Theta_{\mathcal{U}}$ oxtends to an irreducible component $\Theta_{\mathcal{U}}$ of $\tau^{-1} \mathcal{U}$ and $\Theta_{\mathcal{U}} \rightarrow \mathcal{U}$ is biholomorphic since it is finite and bimeromorphic. Hence $\Theta_{\mathcal{U}} \subset T^{\sharp}$ and $s \in \tau\left(T^{\sharp}\right)$.

The cohomological Brauer group $\mathrm{Br}^{\prime}(\mathrm{Y})$ of a non-singular algebraic variety (scheme) $\mathrm{Y}$ over $\mathbb{C}$ is defined to be $H^{2}\left(\mathrm{Y}_{\text {ét }}, \mathbb{G}_{\mathrm{m}}\right)$ for the group scheme $\mathbb{G}_{\mathrm{m}}:=$ Spec $\mathbb{C}\left[x, x^{-1}\right]$. This is a torsion group by [G6, II, 1.4].

Theorem 7.2.2 (cf. [D5, 1.17 and 2.24]). Let $\mathrm{S}^{\star} \subset \mathrm{S}$ be a toroidal embedding of algebraic varieties (schemes) over $\mathbb{C}$ such that $S=\mathrm{S}^{\mathrm{an}}, S^{\star}=\left(\mathrm{S}^{\star}\right)^{\text {an }}$. Let $\mathrm{p}: \mathrm{B} \rightarrow \mathrm{S}$ be an algebraic basic elliptic fibration such that $\mathrm{B}$ is non-singular and that $\boldsymbol{p}=\mathrm{p}^{\text {an }}$ is associated with $H$. We set $\boldsymbol{B}:=\mathrm{B}^{\mathrm{an}}, \mathrm{B}^{\star}:=\mathrm{p}^{-1} \mathrm{~S}^{\star}$, $B^{\star}:=\mathrm{B}^{\star a n}$.

(1) There is an isomorphism

$$
\mathrm{Br}^{\prime}\left(\mathrm{B}^{\star}\right) / \operatorname{Br}^{\prime}\left(\mathrm{S}^{\star}\right) \simeq H^{1}\left(\underline{S}, \mathfrak{S}_{H / \underline{S}}\right)_{\text {tor }} \simeq \amalg\left(S^{\star} / S, H\right) .
$$

(2) For a Zariski-open subset $\mathrm{S}^{\star} \subset \mathrm{S}^{\nabla} \subset \mathrm{S}$, assume that $\mathrm{p}$ is flat over $\mathrm{S}^{\nabla}$ and set $\mathrm{B}^{\nabla}:=\mathrm{p}^{-1} \mathrm{~S}^{\nabla}, S^{\nabla}=\left(\mathrm{S}^{\nabla}\right)^{\text {an }}$. Then $\amalg\left(S^{\nabla} / S, H\right)$ is an extension of a finite group by $\operatorname{Br}^{\prime}\left(\mathrm{B}^{\nabla}\right) / \mathrm{Br}^{\prime}\left(\mathrm{S}^{\nabla}\right)$.

Proof. By a comparison theorem, we have isomorphisms $H^{p}\left(\boldsymbol{B}^{\nabla}, \boldsymbol{\mu}_{m}\right) \simeq$ $H^{p}\left(\mathrm{~B}_{\text {ét }}^{\nabla}, \boldsymbol{\mu}_{m}\right)$ for $\boldsymbol{B}^{\nabla}:=\mathrm{B}^{\nabla}$ and $H^{p}\left(S^{\nabla}, \boldsymbol{\mu}_{m}\right) \simeq H^{p}\left(\mathrm{~S}_{\text {ét }}^{\nabla}, \boldsymbol{\mu}_{m}\right)$. The Kummer sequence $0 \rightarrow \boldsymbol{\mu}_{m} \rightarrow \mathbb{G}_{\mathrm{m}} \stackrel{\times m}{\longrightarrow} \mathbb{G}_{\mathrm{m}} \rightarrow 0$ of étale sheaves of $\mathrm{B}^{\nabla}$ induces an exact sequence

$$
0 \rightarrow \operatorname{Pic}\left(\mathrm{B}^{\nabla}\right) \otimes \mathbb{Q} / \mathbb{Z} \rightarrow \lim _{m} H^{2}\left(\mathrm{~B}_{\text {ét }}^{\nabla}, \boldsymbol{\mu}_{m}\right) \rightarrow \operatorname{Br}^{\prime}\left(\mathrm{B}^{\nabla}\right) \rightarrow 0 .
$$

There is an isomorphism $\operatorname{Pic}\left(\mathrm{B}^{\nabla}\right) \simeq \operatorname{Pic}(\boldsymbol{B}) / \mathrm{V}_{\boldsymbol{p}^{-1}\left(S \backslash S^{\nabla}\right)}(\boldsymbol{B})$. Thus (1) follows 6.2.9 and (2) follows 6.2.11.

We present some sufficient conditions for $\amalg\left(S^{\nabla} / S, H\right)$ to be a finite group for a Zariski-open subset $S^{\nabla}$ containing $S^{\star}$. Here $S$ is only an analytic space; not necessarily projective.

Lemma 7.2.3. $\amalg\left(S^{\nabla} / S, H\right)$ is a finite group provided that the following three conditions are satisfied:

(1) $H^{i}\left(S^{\star}, H\right)$ are finitely generated abelian groups for $i \leq 2$; 
(2) $H^{0}\left(S^{\nabla}, \mathfrak{Q}_{H / S}\right)$ is a finitely generated abelian group;

(3) $C(H / S) \rightarrow \bar{C}\left(H / S^{\nabla}\right)$ is injective.

Proof. Let us consider the commutative diagram

$$
\begin{aligned}
& 0 \longrightarrow C(H / S) \otimes \mathbb{Q} / \mathbb{Z} \quad \longrightarrow \quad H^{1}\left(\underline{S}, \mathfrak{S}_{H / \underline{S}}\right)_{\text {tor }} \quad \longrightarrow H^{2}\left(S^{\star}, H\right)_{\text {tor }} \\
& 0 \longrightarrow H^{0}\left(S^{\nabla}, \mathfrak{Q}_{H / S} \otimes \mathbb{Q} / \mathbb{Z}\right) \longrightarrow H^{0}\left(S^{\nabla}, R^{1} \varepsilon_{*} \mathfrak{S}_{H / \underline{S}}\right) .
\end{aligned}
$$

Under the conditions (1) and (2), the kernel of the second vertical arrow is a finite group if and only if $C(H / S) \rightarrow H^{0}\left(S^{\nabla}, \mathfrak{Q}_{H / S}\right)$ is injective.

Proposition 7.2.4. Suppose that $S$ is compact, $H^{1}\left(S, \mathcal{L}_{H / S}\right)=0$, and that the restriction map $H^{0}\left(S, \mathfrak{S}_{H / S}\right) \rightarrow H^{0}\left(S^{\nabla}, \mathfrak{S}_{H / S}\right)$ is an isomorphism. Then $\amalg\left(S^{\nabla} / S, H\right)$ is a finite group.

Proof. Since $H^{1}\left(S, \mathcal{L}_{H / S}\right)=0$, the homomorphism $H^{1}\left(S^{\star}, H\right) \rightarrow H^{1}\left(S^{\nabla}\right.$, $\left.\mathcal{L}_{H / S}\right)$ is also zero. Hence $C(H / S)=\bar{C}(H / S)$ and $C\left(H / S^{\nabla}\right)=\bar{C}\left(H / S^{\nabla}\right)$. Now $C(H / S) \simeq C\left(H / S^{\nabla}\right)$. Thus $C(H / S) \rightarrow \bar{C}\left(H / S^{\nabla}\right)$ is an isomorphism. Cohomology groups $H^{i}\left(S^{\star}, H\right)$ and $H^{0}\left(S^{\nabla}, \mathfrak{Q}_{H / S}\right)$ are all finitely generated since $S$ is compact. Thus the conditions of 7.2 .3 are all satisfied.

Let $V$ be a normal analytic variety, $D_{V}$ a reduced divisor, and let $H$ be a VHS defined on $V^{\star}=V \backslash D_{V}$ as in 7.1. For an open subset $U \subset V$, we define $\mathcal{E}_{0}^{\text {proj }}\left(V, D_{V}, H ; U\right)$ to be the subgroup of $\mathcal{E}^{\operatorname{proj}}\left(V, D_{V}, H\right)$ consisting of all marked elliptic fibrations $(f: X \rightarrow V, \phi)$ such that $f$ admits local meromorphic sections over any points of $U$. Let $V^{\circ} \subset V$ be a Zariski-open subset such that $V^{\star} \subset V^{\circ}, \operatorname{codim}\left(V \backslash V^{\circ}\right) \geq 2$, and that $D_{V} \cap V^{\circ}$ is non-singular.

Corollary 7.2.5. Let $\mu: S \rightarrow V$ be a bimeromorphic morphism from a non-singular variety such that $\mu$ is isomorphic over $V^{\star}$. If $V$ is compact and if $H^{1}\left(S, \mathcal{L}_{H / S}\right)=0$, then $\mathcal{E}_{0}^{\operatorname{proj}}\left(V, D_{V}, H ; V^{\circ}\right)$ is a finite group.

Proof. We may assume that $D:=\mu^{-1} D_{V}$ is a simple normal crossing divisor. Let $S^{\nabla}:=\mu^{-1} V^{\circ}$. Then $\mathcal{E}_{0}^{\text {proj }}\left(V, D_{V}, H ; V^{\circ}\right)$ is identified with $\mathcal{E}_{0}^{\text {proj }}\left(S, D, H ; S^{\nabla}\right)$. Since $\operatorname{codim}\left(V \backslash V^{\circ}\right) \geq 2, H^{0}\left(S, \mathfrak{S}_{H / S}\right) \rightarrow H^{0}\left(S^{\nabla}, \mathfrak{S}_{H / S}\right)$ is isomorphic. Hence, the assertion follows 7.2.4.

The following is a generalization of [G5, 3.2]. 
Theorem 7.2.6. $\quad$ Suppose that $S$ is compact and let $E$ be the complement $S \backslash S^{\nabla}$. Then the quotient group $\amalg\left(S^{\nabla} / S, H\right) / \amalg(S, H)$ is finite if the following two conditions are both satisfied:

(1) For the $\boldsymbol{J}$-function $S \rightarrow \mathbb{P}^{1}, E$ does not contain any connected component of $\boldsymbol{J}^{-1}(\infty)$;

(2) Let $\mathcal{U}(E)$ be the set of prime divisors $\Gamma \subset E$ such that there is an open neighborhood $\mathcal{U} \supset \Gamma, H$ extends to a VHS $\tilde{H}$ on $\mathcal{U}$, and that $\left.\tilde{H}\right|_{\Gamma}$ is a trivial constant system. For any prime divisor $\Gamma \in \mathcal{U}(E)$, there is an irreducible curve $C \subset \bigcup_{\Gamma^{\prime} \in \mathcal{U}(E)} \Gamma^{\prime}$ with $\Gamma \cdot C \neq 0$.

Proof. By 6.2.11, the quotient group is isomorphic to the cokernel of

$$
\lim _{m} H^{1}\left(S, \mathbb{L}_{H / S}^{\bullet} \stackrel{\mathrm{L}}{\otimes} \boldsymbol{\mu}_{m}\right) \rightarrow \underline{\lim }_{m} H^{1}\left(S^{\nabla}, \mathbb{L}_{H / S}^{\bullet} \stackrel{\mathrm{L}}{\otimes} \boldsymbol{\mu}_{m}\right),
$$

and hence is a subgroup of $\lim _{m} H_{E}^{2}\left(S, \mathbb{L}_{H / S}^{\bullet} \stackrel{\mathrm{L}}{\otimes} \boldsymbol{\mu}_{m}\right)$. The triangle

$$
\cdots \stackrel{+1}{\longrightarrow} \mathbb{L}_{H / S}^{\bullet} \rightarrow \tau_{\leq 1} R j_{*} H \rightarrow \mathfrak{Q}_{H / S}[-1] \stackrel{+1}{\longrightarrow} \cdots
$$

induces an exact sequence

$$
0 \rightarrow H_{E}^{0}\left(S, \mathfrak{Q}_{H / S} \otimes \boldsymbol{\mu}_{m}\right) \rightarrow H_{E}^{2}\left(S, \mathbb{L}_{H / S}^{\bullet} \stackrel{\mathrm{L}}{\otimes} \boldsymbol{\mu}_{m}\right) \rightarrow H_{E}^{0}\left(S,{ }_{m}\left(R^{2} j_{*} H\right)\right),
$$

because $R \underline{\Gamma}_{E}\left(\tau_{\geq 2} R j_{*} H\right)[-1] \simeq_{\text {qis }} R \underline{\Gamma}_{E}\left(\tau_{\leq 1} R j_{*} H\right)$. There is also a commutative diagram

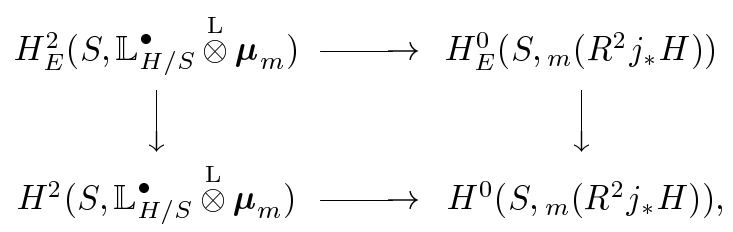

where the right vertical arrow is injective. Thus the quotient group is contained in the kernel of the composite

$$
\begin{aligned}
& \lim _{m} H_{E}^{0}\left(S, \mathfrak{Q}_{H / S} \otimes \boldsymbol{\mu}_{m}\right) \rightarrow \lim _{m} H_{E}^{2}\left(S, \mathbb{L}_{H / S}^{\bullet} \stackrel{\mathrm{L}}{\otimes} \boldsymbol{\mu}_{m}\right) \\
& \rightarrow \lim _{m} H^{2}\left(S, \mathbb{L}_{H / S}^{\bullet} \stackrel{\mathrm{L}}{\otimes} \boldsymbol{\mu}_{m}\right) .
\end{aligned}
$$

For a non-zero element $\theta \in \lim _{\rightarrow} H_{E}^{0}\left(S, \mathfrak{Q}_{H / S} \otimes \boldsymbol{\mu}_{m}\right)$, let $\mathcal{D}(E, \theta)$ be the set of prime divisors $\Gamma \subset E$ such that $\Gamma \subset \operatorname{Supp} \theta$ and let $\mathcal{D}(E)$ be the union of $\mathcal{D}(E, \theta)$ 
for all $\theta$. If $\Gamma \in \mathcal{D}(E, \theta)$, then $H$ is unipotent along $\Gamma$ : the local monodromies along $\Gamma^{\star}:=\Gamma \backslash \operatorname{Sing} D$ are unipotent. If $\Gamma^{\prime}$ is a prime divisor contained in $D$ with $\Gamma \cap \Gamma^{\prime} \neq \emptyset$, then by $6.2 .3, H$ is unipotent along $\Gamma^{\prime}$. Assume that $H$ is of type $\mathrm{I}_{(+)}$along $\Gamma$. If the monodromy along $\Gamma^{\prime}$ is of type $\mathrm{I}_{(+)}$, then $\Gamma^{\prime} \in \mathcal{D}(E, \theta)$ by 6.2.3. Thus any irreducible component of the connected component of $\boldsymbol{J}^{-1}(\infty)$ containing $\Gamma$ belongs to $\mathcal{D}(E, \theta)$. This contradicts the condition (1). Hence the monodromy along $\Gamma$ is trivial. Let $U_{\Gamma}$ denote the open subset $S^{\star} \cup$ $\Gamma^{\star}$. Then $H$ extends to a VHS $\tilde{H}$ on $U_{\Gamma}$ and $\left.\left.R^{1} j_{*} H\right|_{U_{\Gamma}} \simeq \mathfrak{Q}_{H / S}\right|_{U_{\Gamma}} \simeq \tilde{H} \otimes \mathbb{Z}_{\Gamma^{\star}}$. Hence $H^{0}\left(U_{\Gamma}, \mathfrak{Q}_{H / S} \otimes \boldsymbol{\mu}_{m}\right) \simeq H^{0}\left(\Gamma^{\star}, \tilde{H} \otimes \boldsymbol{\mu}_{m}\right)$. If the local constant system $\left.\tilde{H}\right|_{\Gamma^{\star}}$ is not trivial, then $H^{0}\left(\Gamma^{\star}, \tilde{H}\right)=0$ by 4.2 .5 . Thus $\lim _{m} H^{0}\left(\Gamma^{\star}, \tilde{H} \otimes \boldsymbol{\mu}_{m}\right) \simeq$ $H^{1}\left(\Gamma^{\star}, \tilde{H}\right)_{\text {tor }}$ is a finite group. Hence $\lim _{m} H_{E \backslash \Gamma^{\star}}^{0}\left(S, \mathfrak{Q}_{H / S} \otimes \boldsymbol{\mu}_{m}\right)$ is a subgroup of $\lim _{\rightarrow m} H_{E}^{0}\left(S, \mathfrak{Q}_{H / S} \otimes \boldsymbol{\mu}_{m}\right)$ of finite index. Thus, by replacing $E$ by $E \backslash \Gamma^{\star}$, we may assume that $\mathcal{D}(E) \subset \mathcal{U}(E)$. In other words, there is an open subset $U \subset S$ such that $\Gamma \subset U$ for any $\Gamma \in \mathcal{D}(E)$ and that $H$ extends to a VHS $\tilde{H}$ on $U$, where $\left.\tilde{H}\right|_{\Gamma}$ is a trivial local system for any $\Gamma \in \mathcal{D}(E)$. We have

$$
\left.\left.\left.\mathbb{L}_{H / S}^{\bullet}\right|_{U} \sim_{\text {qis }} j_{*} H\right|_{U} \sim_{\text {qis }} \tilde{H}\right|_{U} \quad \text { and }\left.\left.\left.\quad \mathfrak{Q}_{H / S}\right|_{U} \simeq R^{1} j_{*} H\right|_{U} \simeq\left(\mathbb{Z}_{D} \otimes \tilde{H}\right)\right|_{U} .
$$

In particular, $H_{E}^{0}\left(U, \mathfrak{Q}_{H / S} \otimes \boldsymbol{\mu}_{m}\right) \simeq H_{E}^{0}\left(U \cap D, \tilde{H} \otimes \boldsymbol{\mu}_{m}\right)$. There is a commutative diagram

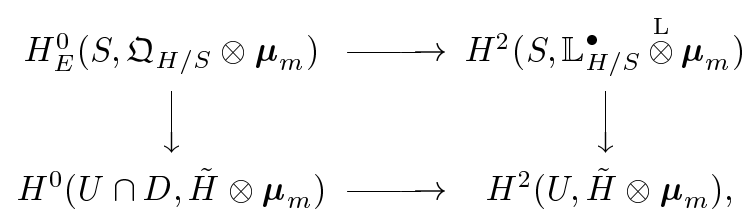

where the bottom arrow is derived from a Gysin map for $U \cap D \subset U$. Let $\nu: C \rightarrow U$ be the normalization of an irreducible curve such that $d:=\Gamma \cdot \nu_{*} C \neq$ 0 . The bottom arrow of the commutative diagram

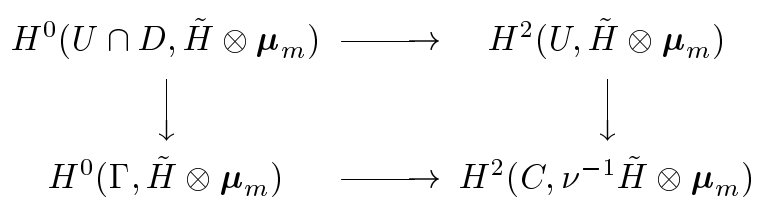

is isomorphic to the multiplication mapping $d \times: \boldsymbol{\mu}_{m}^{\oplus 2} \rightarrow \boldsymbol{\mu}_{m}^{\oplus 2}$. Hence the kernel of

$$
\lim _{m} H^{0}\left(\Gamma, \tilde{H} \otimes \boldsymbol{\mu}_{m}\right) \rightarrow \lim _{m} H^{2}\left(C, \nu^{-1} \tilde{H} \otimes \boldsymbol{\mu}_{m}\right)
$$

is isomorphic to $\boldsymbol{\mu}_{d}^{\oplus 2}$. The kernel of

$$
\lim _{m} H_{E}^{0}\left(S, \mathfrak{Q}_{H / S} \otimes \boldsymbol{\mu}_{m}\right) \rightarrow \bigoplus_{\Gamma \in \mathcal{D}(E)} \lim _{\rightarrow} H^{0}\left(\Gamma, \tilde{H} \otimes \boldsymbol{\mu}_{m}\right)
$$


is finite since $H_{E}^{0}\left(S, \mathfrak{Q}_{H / S}\right) \rightarrow \bigoplus_{\Gamma \in \mathcal{D}(E)} H^{0}(\Gamma, \tilde{H})$ is an injection to a finitely generated abelian group and since $H_{E}^{1}\left(S, \mathfrak{Q}_{H / S}\right)$ tor is a finite group. Therefore the kernel of (7.5) is finite.

\section{Remark.}

(1) The conditions (1) and (2) of 7.2.6 are satisfied if there is a bimeromorphic mapping $\mu: S \rightarrow V$ such that $E$ is $\mu$-exceptional.

(2) Under the assumption of 7.2.6, if $H^{1}\left(S, \mathcal{L}_{H / S}\right)=0$, then $\amalg\left(S^{\nabla} / S, H\right)$ is also a finite group. This is because $\amalg(S, H) \simeq H^{1}\left(S, \mathfrak{S}_{H / S}\right)_{\text {tor }}$ and there is an exact sequence $0 \rightarrow H^{0}\left(S, \mathfrak{Q}_{H / S}\right) \rightarrow H^{1}\left(S, \mathfrak{S}_{H / S}\right) \rightarrow H^{2}\left(S, \mathbb{L}_{H / S}^{\bullet}\right)$. In particular, we have another proof of 7.2.5.

\section{$\S 7.3 . \quad$ Minimal models with trivial canonical divisor}

A locally projective elliptic fibration over a normal surface has a standard elliptic fibration as a minimal model [N4, Appendix A]. A standard elliptic fibration $f: Y \rightarrow T$ has the following properties:

(1) $Y$ has only terminal singularities and is locally $\mathbb{Q}$-factorial;

(2) $f$ is equi-dimensional;

(3) $K_{Y} \sim_{\mathbb{Q}} f^{*}\left(K_{T}+\Delta\right)$ for an effective $\mathbb{Q}$-divisor $\Delta$ with $(T, \Delta)$ being logterminal.

We investigate similar elliptic fibrations over higher dimensional varieties but assuming stronger condition: $K_{Y}$ is relatively linearly equivalent to zero.

Let $\pi: Y \rightarrow V$ be a locally projective elliptic fibration between normal varieties such that $Y$ has only rational Gorenstein singularities and that the canonical sheaf $\omega_{Y}=\mathcal{O}_{Y}\left(K_{Y}\right)$ is $\pi$-trivial, i.e., $\mathcal{M}:=\pi_{*} \omega_{Y}$ is invertible and $\omega_{Y} \simeq \pi^{*} \mathcal{M}$. Then locally on $V$, there is an effective $\mathbb{Q}$-divisor $\Delta$ such that $K_{Y} \sim_{\mathbb{Q}} \pi^{*}\left(K_{V}+\Delta\right)$ and that $(V, \Delta)$ is log-terminal by [N3, 0.4]. In particular, $V$ has only rational singularities. Further, $R^{1} \pi_{*} \mathcal{O}_{Y} \simeq \omega_{V} \otimes \mathcal{M}^{-1}$ (cf. 5.2.7). Let $V^{\star} \subset V$ be a non-singular Zariski-open dense subset over which $\pi$ is smooth. Let $V^{\circ} \subset V$ be a non-singular Zariski-open subset containing $V^{\star}$ such that $\operatorname{codim}\left(V \backslash V^{\circ}\right) \geq 2$ and that $V^{\circ} \backslash V^{\star}$ is a non-singular divisor. Let $H$ denote the VHS over $V^{\star}$ induced from $\pi$. Then $\omega_{V} \otimes \mathcal{M}^{-1} \simeq j_{*}^{\circ} \mathcal{L}_{H / V^{\circ}}$ for $j^{\circ}: V^{\circ} \hookrightarrow V$ by 5.2 .7 . 
Proposition 7.3.1 (cf. [G5, 0.2]). Assume that $Y$ has only terminal singularities, $V$ is $\mathbb{Q}$-Gorenstein, and that $\pi$ is equi-dimensional. Then $V$ has only canonical singularities.

Proof. By the flattening of $\pi$ (cf. [H3]), there exist bimeromorphic morphisms $\mu: S \rightarrow V$ and $X \rightarrow Y \times_{V} S$ over $S$ from non-singular varieties $S$ and $X$, respectively, such that any exceptional divisor for $f: X \rightarrow S$ is exceptional for $\rho: X \rightarrow Y$. We may assume that $S^{\star}:=\mu^{-1}\left(V^{\star}\right)$ is isomorphic to $V^{\star}$ by $\mu$ and that $S \backslash S^{\star}$ is a normal crossing divisor. We write $S \backslash S^{\star}=\bigcup_{i} E_{i} \cup \bigcup_{\alpha} D_{\alpha}$ for $\mu$-exceptional prime divisors $E_{i}$ and for non-exceptional prime divisors $D_{\alpha}$. The singular fiber type of $f$ along $D_{\alpha}$ is not multiple, since $K_{Y}$ is relatively trivial. Thus $f_{*} \omega_{X} \simeq \omega_{S} \otimes \mathcal{L}_{H / S}^{-1}$ and

$$
\left(f_{*} \omega_{X}^{\otimes m}\right)^{\vee \vee} \simeq \omega_{S}^{\otimes m} \otimes \mathcal{L}_{H / S}^{\otimes(-m)} \otimes \mathcal{O}_{S}\left(\sum_{\llcorner} \sum m\left(1-1 / m_{i}\right) E_{i_{\lrcorner}}\right)
$$

for $m>1$, where $m_{i}$ is the multiplicity of singular fiber type along $E_{i}$. Let $L_{H / S}$ stand for formally a divisor with $\mathcal{O}_{S}\left(L_{H / S}\right) \simeq \mathcal{L}_{H / S}$. If it really exists, then it is determined up to the linear equivalence. Even if it does not exit, we consider $L_{H / S}$ formally as a divisor like a canonical divisor $K_{S}$. Similarly, let $M$ stand for a divisor of $V$ with $\mathcal{O}_{V}(M) \simeq \mathcal{M}$. By the choice of $\mu$, we have $\mathcal{M} \simeq \mu_{*}\left(\omega_{S} \otimes \mathcal{L}_{H / S}^{-1}\right)$ and

$$
\pi_{*} \omega_{Y}^{\otimes m} \simeq \mu_{*} \mathcal{O}_{S}\left(m K_{S}-m L_{H / S}+\sum m\left(1-1 / m_{i}\right) E_{i_{\lrcorner}}\right) .
$$

Let $E^{\text {b }}$ be the $\mu$-exceptional effective divisor determined by $K_{S}-L_{H / S} \sim \mu^{*} M+$ $E^{b}$.

Claim 7.3.2. For any $\mu$-exceptional prime divisor $E_{i}$,

$$
\operatorname{mult}_{E_{i}}\left(E^{b}+\sum\left(1-1 / m_{j}\right) E_{j}\right)>0 \text {. }
$$

Proof. We have $K_{X} \sim_{\mathbb{Q}} \rho^{*} K_{Y}+\sum b_{\beta} G_{\beta}$ for $\rho$-exceptional prime divisors $G_{\beta}$ and for positive rational numbers $b_{\beta}$. Since $\pi$ is equi-dimensional, for any $E_{i}$, there is a positive rational number $\delta_{i}$ such that $\sum b_{\beta} G_{\beta} \geq \delta_{i} f^{*} E_{i}$. Hence $K_{X}-\delta_{i} f^{*} E_{i} \geq \rho^{*} K_{Y} \sim f^{*} \mu^{*} M$ and

$$
f_{*} \mathcal{O}_{X}\left(m K_{X}\right) \otimes \mathcal{O}_{S}\left(-m \delta_{i} E_{i}\right) \supset \mu^{*} \mathcal{M}^{\otimes m}
$$

for $m>0$ with $m \delta_{i} \in \mathbb{Z}$. Thus

$$
\begin{gathered}
\mathcal{O}_{S}\left(m K_{S}-m L_{H / S}+\sum m\left(1-1 / m_{j}\right) E_{j}\right) \\
\simeq\left(f_{*} \omega_{X}^{\otimes m}\right)^{\vee \vee} \supset \mu^{*} \mathcal{M}^{\otimes m} \otimes \mathcal{O}_{S}\left(m \delta_{i} E_{i}\right) .
\end{gathered}
$$


Therefore, $E^{b}+\sum\left(1-1 / m_{j}\right) E_{j} \geq \delta_{i} E_{i}$.

Proof of 7.3.1 continued. Since $K_{V}$ is $\mathbb{Q}$-Cartier, $K_{S} \sim_{\mathbb{Q}} \mu^{*} K_{V}+\sum \varepsilon_{i} E_{i}$ for rational numbers $\varepsilon_{i}$. For a prime divisor $\Gamma$ of $S$, let $\sigma_{\Gamma}(L)$ stand for the relative $\sigma$-invariant [N6] of a $\mathbb{Q}$-divisor/line bundle $L$ of $S$ with respect to $\mu$. We have the formula

$$
\mathcal{L}_{H / S}^{\otimes(-12)} \simeq \boldsymbol{J}^{*} \mathcal{O}_{\mathbb{P}^{1}}(1) \otimes \mathcal{O}_{S}\left(\sum a_{i} E_{i}+\sum a_{\alpha} D_{\alpha}\right)
$$

for integers $0 \leq a_{i}, a_{\alpha} \leq 10$ (cf. [U1], [K2], [N4, Section 3]). Thus

$$
\begin{aligned}
\varepsilon_{i}+a_{i} / 12+1-1 / m_{i} & \geq \sigma_{E_{i}}\left(K_{S}-L_{H / S}+\sum\left(1-1 / m_{j}\right) E_{j}\right) \\
& =\operatorname{mult}_{E_{i}} E^{b}+1-1 / m_{i} .
\end{aligned}
$$

In particular, $\varepsilon_{i}+a_{i} / 12 \geq$ mult $_{E_{i}} E^{b} \geq 0$. Suppose that $m_{i}=1$. Then mult $_{E_{i}} E^{b} \geq 1$ by the claim above. Thus $\varepsilon_{i}+a_{i} / 12 \geq 1$ and hence $\varepsilon_{i}>0$, since $a_{i} \leq 10$. Next suppose that $m_{i} \geq 2$. Then $a_{i}=0$ and $\varepsilon_{i} \geq$ mult $_{E_{i}} E^{b} \geq 0$. Thus $V$ has only canonical singularities.

Corollary 7.3.3 (cf. [G5, 3.4]). Suppose that $\operatorname{dim} V=2$ and that $P \in$ $V$ is a singular point. Then $(V, P)$ is an $A_{m}$-singularity for some $m$, the $\boldsymbol{J}$ function is holomorphic at $P$, and $\boldsymbol{J}(P) \neq \infty$.

Proof. We use the same notation as in 7.3.1. We may replace $V$ by an open neighborhood of $P$. Thus we assume any exceptional divisors $E_{i}$ are contained in $E:=\mu^{-1}(P)$. We know that the negative part of the relative Zariski decomposition of $K_{S}-L_{H / S}+\sum\left(1-1 / m_{j}\right) E_{j}$ is $E^{b}+\sum\left(1-1 / m_{j}\right) E_{j}$. Thus by 7.3.2, $\left(K_{S}-L_{H / S}-E^{b}\right) \cdot E_{j}=0$ for any $j$. We write $\sum\left(a_{j} / 12+\right.$ $\left.\varepsilon_{j}\right) E_{j}-E^{b}=\sum \lambda_{j} E_{j}$. Then $\lambda_{i} \geq 0$ and

$$
(1 / 12) \boldsymbol{J}^{*} \mathcal{O}_{\mathbb{P}^{1}}(1) \cdot E_{i}+\sum\left(a_{\alpha} / 12\right) D_{\alpha} \cdot E_{i}+\sum \lambda_{j} E_{j} \cdot E_{i}=0
$$

for any $i$. Now $\varepsilon_{i}=0$ for some exceptional curve $E_{i}$ mapped to $P$. Thus $m_{i} \geq 2$ and $\lambda_{i}=0$. If $\lambda_{i}=0$ for some exceptional curve $E_{i}$, then $\boldsymbol{J}^{*} \mathcal{O}(1) \cdot E_{i}=0$, $D_{\alpha} \cdot E_{i}=0$ if $a_{\alpha} \neq 0$, and $E_{j} \cdot E_{i}=0$ if $\lambda_{j}>0$. Therefore, $\lambda_{i}=0$ for any exceptional divisor $E_{i} \subset \mu^{-1}(P)$ and $J\left(\mu^{-1}(P)\right)$ is a point. In particular, $\boldsymbol{J}: S \cdots \rightarrow \mathbb{P}^{1}$ is holomorphic at $P$. Moreover if $D_{\alpha} \cap \mu^{-1}(P) \neq \emptyset$, then $a_{\alpha}=0$. The fibration $f: X \rightarrow S$ defines a non-zero element of $\amalg\left(S^{\star} / S, H\right) / \amalg(S, H)$ since $m_{i} \geq 2$ for some $i$. By the argument of 7.2.6, $\amalg\left(S^{\star} / S, H\right) / \amalg(S, H)$ is contained in the kernel of

$$
\lim _{\rightarrow m} H_{E}^{0}\left(S, \mathfrak{Q}_{H / S} \otimes \boldsymbol{\mu}_{m}\right) \rightarrow \lim _{m} H^{2}\left(S, \mathbb{L}_{H / S}^{\bullet} \stackrel{\mathrm{L}}{\otimes} \boldsymbol{\mu}_{m}\right)
$$


for $E=\mu^{-1}(P)$. The argument also implies that $J(P) \neq \infty$ and that we can extend $H$ to a VHS $\tilde{H}$ on $S$, after replacing $V$ by an open neighborhood of $P$. Let $\sum q_{j} E_{j}$ for $q_{j} \in(\mathbb{Q} / \mathbb{Z})^{\oplus 2}$ correspond to the element of $\lim _{m} H_{E}^{0}\left(S, \mathfrak{Q}_{H / S} \otimes\right.$ $\left.\boldsymbol{\mu}_{m}\right)$ defined by $f$. Then $\sum q_{j} E_{j} \cdot E_{i} \equiv 0 \bmod \mathbb{Z}^{\oplus 2}$ for any $i$. Let $e$ be the number of irreducible components of $E$ and let $A$ be the $(e \times e)$-matrix whose $(i, j)$-coefficient is $E_{i} \cdot E_{j}$. Then $A: \mathbb{Z}^{\oplus e} \rightarrow \mathbb{Z}^{\oplus e}$ is injective since $A$ is negativedefinite. If for any element $\left(x_{j}\right) \in A^{-1} \mathbb{Z}^{\oplus e} \subset \mathbb{Q}^{\oplus e}$, the $i$-th coefficient $x_{i}$ is integral, then $m_{i}=1$. Thus $\varepsilon_{i}>0$ and $E_{i}$ is exceptional for the bimeromorphic morphism $S \rightarrow \hat{V}$ to the minimal desingularization $\hat{V}$ of $V$. If $(V, P)$ is not an $A_{m}$-type singularity, then there is a component $E_{i}$ such that $x_{i} \in \mathbb{Z}$ for any $\left(x_{j}\right) \in A^{-1} \mathbb{Z}^{\oplus e}$ and that $E_{i}$ is not exceptional for $S \rightarrow \hat{V}$.

We assume the following extra-conditions:

(1) $V$ is $\mathbb{Q}$-Gorenstein;

(2) $\pi$ is equi-dimensional.

Then, by (1), there is a generalized Weierstrass model $\mathbf{p}: \mathbf{W}=\mathbf{W}_{V}\left(\omega_{V} \otimes\right.$ $\left.\mathcal{M}^{-1}, \boldsymbol{\alpha}, \boldsymbol{\beta}\right) \rightarrow V$ associated with $H$.

Lemma 7.3.4. Under the situation above, $\mathbf{W}$ has only rational Gorenstein singularities and $\omega_{\mathbf{W}}=\mathcal{O}_{\mathbf{W}}\left(K_{\mathbf{W}}\right) \simeq \mathbf{p}^{*} \mathcal{M}$.

Proof. If $V$ is Gorenstein, then $\mathbf{W}$ is a usual Weierstrass model. Thus an open neighborhood of the canonical section $\boldsymbol{\Sigma}$ has only rational singularities. Let $\hat{V} \rightarrow V$ and $X \rightarrow \mathbf{W} \times_{V} \hat{V}$, respectively, be resolutions of singularities. Then for the composite $\mu: X \rightarrow \mathbf{W}, T:=\mu^{*} \boldsymbol{\Sigma}$ is non-singular and isomorphic to $\hat{S}$. Thus $R \mu_{*} \mathcal{O}_{T}(m T) \sim_{\text {qis }} \mathcal{O}_{\boldsymbol{\Sigma}}(m \boldsymbol{\Sigma})$ and $R f_{*} \mathcal{O}_{T}(m T) \sim_{\text {qis }} \mathbf{p}_{*} \mathcal{O}_{\boldsymbol{\Sigma}}(m \boldsymbol{\Sigma}) \simeq$ $\left(\omega_{V} \otimes \mathcal{M}^{-1}\right)^{\otimes m}$ for any integers $m$, where $f=\mathbf{p} \circ \mu$. Thus we infer that $\mathbf{W}$ has only rational singularities by the argument of $[\mathrm{N} 3,2.4]$. In non-Gorenstein case, there is a cyclic covering $\tau: V^{\prime} \rightarrow V$ locally on $V$ such that it is étale in codimension one and that $\omega_{V^{\prime}}$ is invertible. Let $Y^{\prime}$ denote the normalization of $Y \times_{V} V^{\prime}$. Then $Y^{\prime} \rightarrow Y$ is étale in codimension one, and hence $Y^{\prime}$ has only rational Gorenstein singularities. Thus the Weierstrass model $\mathbf{W}^{\prime}$ defined from the pullback of $H$ has only rational singularities. Therefore, $\mathbf{W}$ also has only rational singularities. In order to show $\omega_{\mathbf{W}} \simeq \mathbf{p}^{*} \mathcal{M}$, we may replace $V$ by $V^{\circ}$. Then this follows from 5.1.1.

If log-flip conjectures are true, then we have elliptic fibrations $Y \rightarrow V$ of this kind as minimal models. A projective variety $X$ has numerical Kodaira 
dimension $\kappa_{\sigma}(X)=0$ if and only if, for a non-singular projective model $Z$ of $X$, the function

$$
m \longmapsto \operatorname{dim} H^{0}\left(Z, \mathcal{O}_{Y}\left(m K_{Z}+A\right)\right)
$$

is bounded for any ample divisor $A$ and is non-trivial for some $A$ (cf. [N6]). If the existence and the termination of flips are proved for varieties birational to $X$, then the condition $\kappa_{\sigma}(X)=0$ is equivalent to that $X$ is birational to a normal projective variety $Y$ with only terminal singularities and with $K_{Y} \sim_{\mathbb{Q}} 0$.

Let $f: X \rightarrow S$ be an elliptic fibration between non-singular projective varieties such that $f$ is smooth outside a simple normal crossing divisor $D$ of $S$, the geometric genus $p_{g}(X)=1$, and that $\kappa_{\sigma}(X)=0$. Let $H=H(f)$ be the induced VHS defined on $S^{\star}=S \backslash D$ and let $\boldsymbol{p}: \boldsymbol{B} \rightarrow S$ be an associated basic elliptic fibration from a non-singular variety.

Over the open subset $S^{\circ}=S \backslash \operatorname{Sing} D$, we have a minimal elliptic fibration $f^{\circ}: X^{\circ} \rightarrow S^{\circ}$ which is bimeromorphically equivalent to $f^{-1} S^{\circ} \rightarrow S^{\circ}$ over $S^{\circ}$. Here the canonical bundle formula

$$
K_{X^{\circ}} \sim_{\mathbb{Q}}\left(f^{\circ}\right)^{*}\left(K_{S}-\mathcal{L}_{H / S}+\sum\left(1-1 / m_{i}\right) D_{i}^{\star}\right)
$$

holds for irreducible components $D_{i}^{\star}=D_{i} \cap S^{\circ}$. There is also an isomorphism

$$
\mathcal{L}_{H / S}^{\otimes(-12)} \simeq \boldsymbol{J}^{*} \mathcal{O}_{\mathbb{P}^{1}}(1) \otimes \mathcal{O}_{S}\left(\sum a_{i} D_{i}\right)
$$

for $0 \leq a_{i} \leq 10$ and for the $\boldsymbol{J}$-function $S \rightarrow \mathbb{P}^{1}$. There is a non-singular divisor $L$ such that $\mathcal{O}_{S}(L) \simeq \boldsymbol{J}^{*} \mathcal{O}_{\mathbb{P}}(1)$ and that $L+D$ is also a simple normal crossing divisor. We set

$$
\Delta_{H / S}:=(1 / 12)\left(L+\sum a_{i} D_{i}\right), \quad \text { and } \quad \Lambda_{X / S}:=\Delta_{H / S}+\sum\left(1-1 / m_{i}\right) D_{i} .
$$

Then $\left(S, \Delta_{H / S}\right)$ and $\left(S, \Lambda_{X / S}\right)$ are log-terminal pairs. We have

$$
K_{\mathbf{W}} \sim_{\mathbb{Q}} \mathbf{p}^{*}\left(K_{S}+\Delta_{H / S}\right) \quad \text { and } \quad K_{X} \sim_{\mathbb{Q}} f^{*}\left(K_{S}+\Lambda_{X / S}\right)+G
$$

for an $f$-exceptional divisor $\mathbb{Q}$-divisor $G$. In particular, the double-dual of $f_{*} \omega_{X}^{\otimes m}$ is isomorphic to $\mathcal{O}_{S}\left(m K_{S}+{ }_{\llcorner} m \Lambda_{X / S\lrcorner}\right)$ for $m \geq 0$.

Theorem 7.3.5. The equalities of Hodge numbers $h^{p, 0}(X)=h^{p, 0}(\boldsymbol{B})$ hold for any $p$, and $\kappa(\boldsymbol{B})=\kappa_{\sigma}(\boldsymbol{B})=0$. If the log-flip conjecture holds for varieties birational to $S$, then there exist an elliptic fibration $p: Y \rightarrow V$ between normal projective varieties and birational mappings $\nu: B \cdots \rightarrow Y, \mu: S \cdots \rightarrow$ $V$ satisfying the following conditions: 
(1) $\mu \circ \boldsymbol{p}=p \circ \nu$;

(2) $Y$ has only rational Gorenstein singularities with $K_{Y} \sim 0$;

(3) $V$ is $\mathbb{Q}$-factorial;

(4) $\mu^{-1}: V \cdots \rightarrow S$ contracts no prime divisors of $V$;

(5) Every prime divisor $D_{i}$ with $m_{i}>0$ is $\mu$-exceptional.

Proof. There are quasi-isomorphisms

$$
R f_{*} \mathcal{O}_{X} \sim_{\text {qis }} \mathcal{O}_{S} \oplus \mathcal{L}_{H / S}[-1] \sim_{\text {qis }} R \boldsymbol{p}_{*} \mathcal{O}_{B}
$$

by 5.2.8. Thus $h^{p, 0}(X)=h^{p, 0}(\boldsymbol{B})$. Further, $f_{*} \omega_{X / S} \simeq \boldsymbol{p}_{*} \omega_{\boldsymbol{B} / S} \simeq \mathcal{L}_{H / S}^{-1}$. Let p: $\mathbf{W}=\mathbf{W}(H) \rightarrow S$ be the minimal Weierstrass model associated with $H$. Since $\mathbf{W}$ has only canonical singularities, we have

$$
\boldsymbol{p}_{*}\left(\omega_{\boldsymbol{B} / S}^{\otimes m}\right) \simeq \mathbf{p}_{*}\left(\omega_{\mathbf{W} / S}^{\otimes m}\right) \simeq \mathcal{L}_{H / S}^{\otimes(-m)}
$$

for any $m \geq 1$. Since $\left(f_{*} \omega_{X}\right)^{\otimes m} \subset f_{*}\left(\omega_{X}^{\otimes m}\right)$, we have $\kappa(\boldsymbol{B})=0$.

By a flattening of $f$, there exist birational morphisms $\rho: \hat{S} \rightarrow S$ and $\lambda: \hat{X} \rightarrow X \times{ }_{S} \hat{S}$ such that

(1) $\hat{S}$ and $\hat{X}$ are non-singular projective,

(2) $\rho$ is isomorphic over $S^{\star}$,

(3) $\rho^{-1}(L+D)$ is a simple normal crossing divisor,

(4) the induced elliptic fibration $\hat{f}: \hat{X} \rightarrow \hat{S}$ is smooth outside $\rho^{-1} D$,

(5) $\hat{f}$-exceptional divisor is exceptional for $\hat{X} \rightarrow X$.

Let $\Delta_{H / \hat{S}}$ and $\Lambda_{\hat{X} / \hat{S}}$ be similarly defined $\mathbb{Q}$-divisors on $\hat{S}$. By considering the Weierstrass model over $\hat{S}$, we have

$$
\begin{gathered}
\kappa(\boldsymbol{B})=\kappa\left(K_{\hat{S}}+\Delta_{H / \hat{S}}\right)=\kappa\left(K_{S}+\Delta_{H / S}\right), \\
\kappa_{\sigma}(\boldsymbol{B})=\kappa_{\sigma}\left(K_{\hat{S}}+\Delta_{H / \hat{S}}\right)=\kappa_{\sigma}\left(K_{S}+\Delta_{H / S}\right) .
\end{gathered}
$$

For $K_{\hat{X}}$, we have

$$
K_{\hat{X}} \sim_{\mathbb{Q}} \hat{f}^{*}\left(K_{\hat{S}}+\Lambda_{\hat{X} / \hat{S}}\right)+\hat{G}
$$

for $\hat{f}$-exceptional $\mathbb{Q}$-divisor $\hat{G}$. Hence, for $m \geq 0$,

$$
f_{*} \omega_{X}^{\otimes m} \simeq \rho_{*} \mathcal{O}_{\hat{S}}\left(m K_{\hat{S}}+{ }_{\llcorner} m \Lambda_{\hat{X} / \hat{S}\lrcorner}\right) .
$$


Therefore, $\kappa\left(K_{\hat{S}}+\Lambda_{\hat{X} / \hat{S}}\right)=\kappa_{\sigma}\left(K_{\hat{S}}+\Lambda_{\hat{X} / \hat{S}}\right)=0$. Thus $\kappa_{\sigma}(\boldsymbol{B})=0$.

By [N6], there is a unique effective $\mathbb{Q}$-divisor $N$ such that $K_{S}+\Delta_{H / S} \sim_{\mathbb{Q}} N$. Here $N$ is the negative part of the Zariski-decomposition of $K_{S}+\Delta_{H / S}$. By replacing $S$ by $\hat{S}$, we may assume $\kappa\left(K_{S}+\Lambda_{X / S}\right)=\kappa_{\sigma}\left(K_{S}+\Lambda_{X / S}\right)=0$. Then $N+\sum\left(1-1 / m_{i}\right) D_{i}$ is the negative part of the Zariski-decomposition of $K_{S}+\Lambda_{X / S}$. Applying the log-minimal model program for $\left(S, \Lambda_{X / S}\right)$, we have a birational mapping $\mu: S \cdots \rightarrow V$ such that the pair $\left(V, \mu_{*} \Lambda_{X / S}\right)$ is log-terminal, $V$ is $\mathbb{Q}$-factorial, $\mu_{*}\left(N+\sum\left(1-1 / m_{i}\right) D_{i}\right)=0$, and that $\mu$ satisfies the conditions (4), (5) of the statement. In particular, $K_{V}+\mu_{*} \Delta_{H / S}=K_{V}+\mu_{*} \Lambda_{H / S} \sim_{\mathbb{Q}} 0$ and the double-dual of $\mu_{*} \mathcal{L}_{H / S}$ is isomorphic to $\omega_{V}$, since $p_{g}(\boldsymbol{B})=1$. Let $\mathbf{p}: \mathbf{W}=$ $\mathbf{W}_{S}\left(\mathcal{L}_{H / S}, \boldsymbol{\alpha}, \boldsymbol{\beta}\right) \rightarrow S$ be the minimal Weierstrass model associated with $H$ for $\boldsymbol{\alpha} \in H^{0}\left(S, \mathcal{L}_{H / S}^{\otimes(-4)}\right)$ and $\boldsymbol{\beta} \in H^{0}\left(S, \mathcal{L}_{H / S}^{\otimes(-6)}\right)$. We denote by same $\boldsymbol{\alpha}$ and $\boldsymbol{\beta}$ the corresponding sections in $H^{0}\left(V, \omega_{V}^{[-4]}\right)$ and $H^{0}\left(V, \omega_{V}^{[-6]}\right)$, respectively. Since $K_{V}$ is $\mathbb{Q}$-Cartier, we have a generalized Weierstrass model $Y:=\mathbf{W}_{V}\left(\omega_{V}, \boldsymbol{\alpha}, \boldsymbol{\beta}\right) \rightarrow$ $V$. Then $Y$ is birational to $\boldsymbol{B}$ and $K_{Y} \sim_{\mathbb{Q}} 0$. Hence $Y$ has only canonical singularities of index one with $K_{Y} \sim 0$.

Remark (cf. [N3]).

(1) Let $p: Y \rightarrow V$ be the basic elliptic fibration obtained in 7.3.5. Under the flip conjecture, there is a birational morphism $\beta: T \rightarrow V$ from a normal projective variety $T$ with only terminal singularities such that $T$ is $\mathbb{Q}$ factorial and $K_{T}$ is $\beta$-nef. The sections $\boldsymbol{\alpha}$ and $\boldsymbol{\beta}$ extend to sections of $\mathcal{O}_{T}\left(-4 K_{T}\right)$ and $\mathcal{O}_{T}\left(-6 K_{T}\right)$, since $\beta_{*} \mathcal{O}_{T}\left(-m K_{T}\right)$ is reflexive for $m \geq 0$. Thus for the generalized Weierstrass model $\mathbf{W}_{T}:=\mathbf{W}_{T}\left(\omega_{T}, \boldsymbol{\alpha}, \boldsymbol{\beta}\right), K_{\mathbf{W}_{T}}$ is linearly equivalent to 0 . Therefore, $\mathbf{W}_{T}$ has only rational Gorenstein singularities, since it is birational to $Y$. However, the divisor $D_{i}$ with multiplicity $m_{i}>0$ may not be exceptional for the birational mapping $S \cdots \rightarrow T$.

(2) Under the flip conjecture, we also have a relative minimal model of $T$ : there is a birational mapping $T \cdots \rightarrow R$ such that it is a composition of extremal divisorial contractions and flips with respect to canonical divisors and that any extremal ray of $R$ defines a contraction of fiber type. The sections $\boldsymbol{\alpha}$ and $\boldsymbol{\beta}$ also descend to sections of $\mathcal{O}_{R}\left(-4 K_{R}\right)$ and $\mathcal{O}_{R}\left(-6 K_{R}\right)$, respectively. Thus the generalized minimal model $\mathbf{W}_{R}:=\mathbf{W}_{R}\left(\omega_{R}, \boldsymbol{\alpha}, \boldsymbol{\beta}\right)$ has only rational Gorenstein singularities and $K_{\mathbf{W}_{R}}$ is linearly equivalent to zero.

By replacing $X$ by a birationally equivalent variety, we may assume that the composite $f_{V}=\mu \circ f: X \rightarrow V$ is holomorphic. We have a reduced effective 
divisor $D_{V}$ of $V$ such that $\operatorname{Supp} \mu_{*} \Delta_{H / S} \subset D_{V}$ and that $\mu^{-1}: V \cdots \rightarrow S$ is holomorphic over $V \backslash D_{V}$. Let $V^{\star}=V \backslash D_{V}$ and let $V^{\star} \subset V^{\circ} \subset V$ be a Zariskiopen subset such that $\operatorname{codim}\left(V \backslash V^{\circ}\right) \geq 2, B \cap V^{\circ}$ is non-singular, and that $\mu^{-1}: V \cdots \rightarrow S$ is holomorphic over $V^{\circ}$. Then, for a marking $\phi,\left(f_{V}: X \rightarrow\right.$ $V, \phi)$ belongs to $\mathcal{E}_{0}^{\text {proj }}\left(V, D_{V}, H_{V} ; V^{\circ}\right)$, where $H_{V}$ denotes the induced VHS defined on $V^{\star}$ from $H$. By 7.2 .5 or $7.2 .6, \mathcal{E}_{0}^{\text {proj }}\left(V, D_{V}, H_{V} ; V^{\circ}\right)$ is finite, if $h^{2,0}(X)=0$.

This observation in the case $\operatorname{dim} S=2$ is due to Gross [G5] and is the first step to show a kind of boundedness of Calabi-Yau threefolds with elliptic fibrations.

\section{§7.4. Kähler morphism}

Definition 7.4.1. Let $f: X \rightarrow S$ be a proper surjective morphism between normal complex analytic varieties.

(1) A real $C^{\infty}$-form $\omega$ on $X$ is called a Kähler form if there exist an open covering $\left\{X_{\lambda}\right\}$ of $X$ and strictly pluri-subharmonic functions $p_{\lambda}$ on $X_{\lambda}$ such that $\left.\omega\right|_{X_{\lambda}}=\sqrt{-1} \partial \bar{\partial} p_{\lambda}$, and that $\left.\left(p_{\lambda}-p_{\mu}\right)\right|_{X_{\lambda} \cap X_{\mu}}$ are pluri-harmonic.

(2) If there is an open covering $\left\{S_{\alpha}\right\}_{\alpha \in A}$ of $S$ such that $f^{-1}\left(S_{\alpha}\right)$ admit Kähler forms, then $f$ is called a locally Kähler morphism.

(3) A d-closed real $(1,1)$-form $\omega$ on $X$ is called a relative Kähler form if there exist an open covering $\left\{S_{\alpha}\right\}_{\alpha \in A}$ of $S$ and Kähler forms $\eta_{\alpha}$ on $S_{\alpha}$ such that $\left.\omega\right|_{X_{\alpha}}+f^{*} \eta_{\alpha}$ is a Kähler form for any $\alpha$, where $X_{\alpha}=f^{-1}\left(S_{\alpha}\right)$.

(4) If there is a relative Kähler form on $X$, then $f$ is called a Kähler morphism.

(5) An element $\xi$ in $H^{2}(X, \mathbb{R})$ is called a relative Kähler class if there is an open covering $\left\{S_{\alpha}\right\}_{\alpha \in A}$ of $S$ such that the restriction of $\xi$ in $H^{2}\left(X_{\alpha}, \mathbb{R}\right)$ is induced from a relative Kähler form for $X_{\alpha}=f^{-1}\left(S_{\alpha}\right) \rightarrow S_{\alpha}$.

(6) If there is a relative Kähler class, then $f$ is called a cohomologically Kähler morphism.

(7) $f$ is called a cohomologically projective morphism, if there exist a cohomology class $\xi \in H^{2}(X, \mathbb{Z})$ and an open covering $\left\{S_{\alpha}\right\}_{\alpha \in A}$ of $S$ such that the restriction of $\xi$ in $H^{0}\left(S_{\alpha}, R^{2} f_{*} \mathbb{Z}_{X}\right)$ is represented by the first Chern class of a relatively ample invertible sheaf on $f^{-1}\left(S_{\alpha}\right)$. 
(8) $f$ is called $B K$ if it is bimeromorphically equivalent over $S$ to a Kähler morphism. $f$ is called $L B K$, if $f$ is BK locally over $S$ : there is an open covering $\left\{S_{\lambda}\right\}$ of $S$ such that $f^{-1}\left(S_{\lambda}\right) \rightarrow S_{\lambda}$ is BK for any $\lambda$.

(9) $f$ is called $B C P$ and $B C K$, respectively, if $f$ is bimeromorphically equivalent over $S$ to a cohomologically projective morphism and a cohomologically Kähler morphism.

Remark. The composite of two Kähler morphisms is not necessarily a Kähler morphism but a locally Kähler morphism. In fact, this is Kähler over a relatively compact open subset. The same property holds for cohomologically Kähler morphisms.

Let $S^{\star}=S \backslash D \hookrightarrow S$ be a toroidal embedding as before and let $H$ be a VHS defined on $S^{\star}$. We use the same notation as before, e.g. $\underline{S}=(S, D)$, $j: S^{\star} \hookrightarrow S, \underline{j}: S^{\star} \hookrightarrow \underline{S}$, etc. We recall the complex $\mathbb{L}_{H / \underline{S}}^{\bullet}$ defined in 6.2.1. Let c: $H^{1}\left(\underline{S}, \mathfrak{S}_{H / \underline{S}}\right) \rightarrow H^{2}\left(\underline{S}, \mathbb{L}_{H / \underline{S}}^{\bullet}\right)$ denote the connecting homomorphism derived from

$$
\cdots \stackrel{+1}{\rightarrow} \mathbb{L}_{H / \underline{S}}^{\bullet} \rightarrow \mathcal{L}_{H / \underline{S}} \rightarrow \mathfrak{S}_{H / \underline{S}} \stackrel{+1}{\rightarrow} \cdots
$$

Let $(f: X \rightarrow S, \phi)$ be a marked elliptic fibration associated with $(S, D, H)$. If $f$ is a locally Kähler elliptic fibration, then it is locally projective by 5.2.9, [N4, Section 3]. Thus, if $f$ is LBK, then $(f: X \rightarrow S, \phi)$ belongs to $\mathcal{E}(S, D, H)$.

Proposition 7.4.2. For an element $\eta \in H^{1}\left(\underline{S}, \mathfrak{S}_{H / \underline{S}}\right)$, we consider the following five conditions:

(1) $\eta$ is represented by a BCP marked elliptic fibration;

(2) $\eta$ is represented by a BCK marked elliptic fibration;

(3) $\eta$ is represented by a marked elliptic fibration $(f: X \rightarrow S, \phi)$ such that $X$ is non-singular and that a cohomology class $\omega \in H^{2}(X, \mathbb{R})$ satisfies $\left.\operatorname{deg} \omega\right|_{X_{s}}>0$ for a general fiber $X_{s}=f^{-1}(s)$;

(4) c $(\eta)$ is sent to zero by $H^{2}\left(\underline{S}, \mathbb{L}_{H / \underline{S}}^{\bullet}\right) \rightarrow H^{2}\left(\underline{S}, \mathbb{L}_{H / \underline{S}}^{\bullet} \stackrel{\mathrm{L}}{\otimes} \mathbb{R}\right)$;

(5) c( $\eta)$ is a torsion element.

Then $(1) \Longrightarrow(2) \Longrightarrow(3) \Longrightarrow(4)$ hold. If $H^{p}\left(S^{\star}, H\right)$ are finitely generated abelian groups for $p \leq 2$, then $(4) \Longrightarrow(5)$ holds. If $[S, D]$ satisfies the condition 3.2.6, then $(5) \Longrightarrow(1)$ holds. 
Proof. $\quad(1) \Longrightarrow(2)$ and $(2) \Longrightarrow(3)$ are trivial.

$(3) \Longrightarrow(4)$ We may assume that $f^{-1} D$ is a normal crossing divisor. The $\eta$ is determined as the image of 1 under the connecting homomorphism $H^{0}(\underline{S}, \mathbb{Z}) \rightarrow H^{1}\left(\underline{S}, \mathfrak{S}_{H / \underline{S}}\right)$ of the exact sequence:

$$
0 \rightarrow \mathfrak{S}_{H / \underline{S}} \rightarrow R^{1} \underline{f}_{*} \mathcal{O}_{\underline{X}}^{\star} / \mathcal{V}_{\underline{X}} \rightarrow \mathbb{Z}_{\underline{S}} \rightarrow 0 .
$$

Let $\tilde{\mathbb{L}}_{\underline{X} / \underline{S}}^{\bullet}$ be the complex

$$
\left[\cdots \rightarrow 0 \rightarrow R^{1} \underline{f}_{*} \mathcal{O}_{\underline{X}} \rightarrow R^{1} \underline{f}_{*} \mathcal{O}_{\underline{X}}^{\star} / \mathcal{V}_{\underline{X}} \rightarrow 0 \rightarrow \cdots\right],
$$

where $R^{1} \underline{f}_{*} \mathcal{O}_{\underline{X}}$ lies in the degree zero. We have $\mathcal{H}^{0}\left(\tilde{\mathbb{L}}_{\underline{X} / \underline{S}}^{\bullet}\right) \simeq \underline{j}_{*} H$ and $\mathcal{H}^{1}\left(\tilde{\mathbb{L}}_{\underline{X} / \underline{S}}^{\bullet} \simeq R^{2} \underline{f}_{*} \mathbb{Z}_{\underline{X}} / \mathcal{V}_{\underline{X}}\right.$. Thus there exist a natural morphism

$$
\tau_{\geq 1} \tau_{\leq 2} R \underline{f}_{*} \mathbb{Z}_{\underline{X}} \rightarrow \tilde{\mathbb{L}}_{\underline{X} / \underline{S}}^{\bullet}[-1]
$$

and a triangle

$$
\cdots \stackrel{+1}{\rightarrow} \mathbb{L}_{H / \underline{S}}^{\bullet} \rightarrow \tilde{\mathbb{L}}_{\underline{X} / \underline{S}}^{\bullet} \rightarrow \mathbb{Z}_{\underline{S}}[-1] \stackrel{+1}{\rightarrow} \cdots
$$

Here, the composite $\tau_{\geq 1} \tau_{\leq 2} R \underline{f}_{*} \mathbb{Z}_{X} \rightarrow \mathbb{Z}_{\underline{S}}[-2]$ is derived from the trace map $R^{2} \underline{f}_{*} \mathbb{Z}_{\underline{X}} \rightarrow \mathbb{Z}_{\underline{S}}$. The $\omega \in \bar{H}^{2}(\bar{X}, \mathbb{R})$ goes to a positive number under the homomorphism

$$
H^{2}\left(\underline{S}, \tau_{\geq 1} \tau_{\leq 2} R \underline{f}_{*} \mathbb{R}_{\underline{X}}\right) \rightarrow H^{0}(\underline{S}, \mathbb{R}) \simeq \mathbb{R} .
$$

Hence $\mathrm{c}(\eta)$ goes to zero under $H^{2}\left(\underline{S}, \mathbb{L}_{H / \underline{S}}^{\bullet}\right) \rightarrow H^{2}\left(\underline{S}, \mathbb{L}_{H / \underline{S}}^{\bullet} \stackrel{\mathrm{L}}{\otimes} \mathbb{R}\right)$.

$(4) \Longrightarrow(5)$ under the assumption above. From the triangle

$$
\cdots \stackrel{+1}{\rightarrow} \mathbb{L}_{H / \underline{S}}^{\bullet} \rightarrow \tau_{\leq 1} R \underline{j}_{*} H \rightarrow \mathfrak{Q}_{H / \underline{S}} \stackrel{+1}{\longrightarrow} \cdots,
$$

we have a commutative diagram of exact sequences:

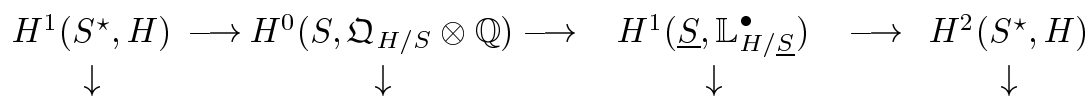

$$
\begin{aligned}
& H^{1}\left(S^{\star}, H_{\mathbb{R}}\right) \longrightarrow H^{0}\left(S, \mathfrak{Q}_{H / S} \otimes \mathbb{R}\right) \longrightarrow H^{1}\left(\underline{S}, \mathbb{L}_{H / \underline{S}}^{\bullet} \stackrel{\mathrm{L}}{\otimes} \mathbb{R}\right) \longrightarrow H^{2}\left(S^{\star}, H_{\mathbb{R}}\right),
\end{aligned}
$$

where $H_{\mathbb{R}}=H \otimes \mathbb{R}$. The universal coefficient theorem gives the isomorphism

$$
H^{p}\left(S^{\star}, H_{\mathbb{R}}\right) \simeq \operatorname{Hom}\left(H_{c}^{2 d-p}\left(S^{\star}, H^{\vee}\right), \mathbb{R}\right),
$$

for $H^{\vee}=\mathcal{H o m}\left(H, \mathbb{Z}_{S^{\star}}\right)$ and $d=\operatorname{dim} S$. Under the assumption, $H_{c}^{2 d-i}\left(S^{\star}, H^{\vee}\right)$ are finitely generated for $i \leq 1$. Hence $H^{p}\left(S^{\star}, H\right) \otimes \mathbb{R} \simeq H^{p}\left(S^{\star}, H_{\mathbb{R}}\right)$ for $p \leq 1$ 
and $H^{2}\left(S^{\star}, H\right) \otimes \mathbb{R} \rightarrow H^{2}\left(S^{\star}, H_{\mathbb{R}}\right)$ is injective. Further, $H^{0}\left(S, \mathfrak{Q}_{H / S} \otimes \mathbb{Q}\right) \rightarrow$ $H^{0}\left(S, \mathfrak{Q}_{H / S} \otimes \mathbb{R}\right)$ is also injective. Thus, $H^{2}\left(\underline{S}, \mathbb{L}_{H / \underline{S}}^{\bullet}\right) \otimes \mathbb{R} \rightarrow H^{2}\left(\underline{S}, \mathbb{L}_{H / \underline{S}}^{\bullet} \stackrel{\mathrm{L}}{\otimes} \mathbb{R}\right)$ is injective. Therefore, if $\mathrm{c}(\eta)$ goes to zero in $H^{2}\left(\underline{S}, \mathbb{L}_{H / \underline{S}}^{\bullet} \stackrel{\mathrm{L}}{\otimes} \mathbb{R}\right)$, then $\mathrm{c}(\eta)$ is a torsion element.

(5) $\Longrightarrow(1)$ under the assumption above. The image of $\eta$ under the homomorphism $H^{1}\left(\underline{S}, \mathfrak{S}_{H / \underline{S}}\right) \rightarrow H^{1}\left(\underline{S}, \mathfrak{T}_{H / \underline{S}}\right)$ is a torsion element, since so is c $(\eta)$. Thus by 6.3.12, $\eta$ is represented by a marked elliptic fibration $(f: X \rightarrow S, \phi) \in$ $\mathcal{E}(S, D, H)$. Suppose that $\mathrm{c}(m \eta)=0$ for a positive integer $m$. Then for a marked elliptic fibration $\left(X^{\prime} \rightarrow S, \phi\right)$ corresponding to $m \eta$, we have a generically finite meromorphic mapping $X \cdots \rightarrow X^{\prime}$ over $S$. Hence, we may assume that $\mathrm{c}(\eta)=0$. Then $\eta$ is the image of an element $\zeta \in H^{1}\left(S, \mathcal{L}_{H / S}\right)$ under $H^{1}\left(S, \mathcal{L}_{H / S}\right) \rightarrow H^{1}\left(\underline{S}, \mathfrak{S}_{H / \underline{S}}\right)$. Let $\mathbb{V}=\mathbb{V}\left(\mathcal{L}_{H / S^{\circ}}\right) \rightarrow S^{\circ}$ be the line bundle associated with the invertible sheaf $\mathcal{L}_{H / S^{\circ}}$ and let $\mathbb{V} \zeta \rightarrow S^{\circ}$ be its twist by $\zeta$. Then $\mathbb{V}^{\zeta}$ is isomorphic to the open subset $\mathbb{P}\left(\left.\mathcal{F}^{\zeta}\right|_{S^{\circ}}\right) \backslash \mathbb{P}\left(\mathcal{O}_{S^{\circ}}\right)$ for the extension

$$
0 \rightarrow \mathcal{L}_{H / S} \rightarrow \mathcal{F}^{\zeta} \rightarrow \mathcal{O}_{S} \rightarrow 0
$$

corresponding to $\zeta$. The image $\theta$ of $\zeta$ in $H^{1}\left(S, \mathfrak{S}_{H / S}^{\mathbf{W}}\right)$ defines the twist $\pi: \mathbf{W}^{\theta} \rightarrow$ $S$ of the minimal Weierstrass model $\mathbf{W} \rightarrow S$ associated with $H$. We may replace $X$ by $\mathbf{W}^{\theta}$. We have an exact sequence

$$
0 \rightarrow \mathfrak{S}_{H / S}^{\mathbf{W}} \rightarrow R^{1} \pi_{*} \mathcal{O}_{\mathbf{W}^{\theta}}^{\star} \rightarrow R^{2} \pi_{*} \mathbb{Z}_{\mathbf{W}^{\theta}} \simeq \mathbb{Z}_{S} \rightarrow 0 .
$$

The extension class is $\theta$. Let us consider Leray's spectral sequence

$$
E_{2}^{p, q}=H^{p}\left(S, R^{q} \pi_{*} \mathbb{Z}_{\mathbf{W}^{\theta}}\right) \Longrightarrow E^{p+q}=H^{p+q}\left(\mathbf{W}^{\theta}, \mathbb{Z}\right)
$$

Since $\theta$ is the image of $\zeta$, the generator $1 \in \mathbb{Z} \simeq E_{2}^{0,2}$ goes to zero in $E_{2}^{2,1}$. Hence $1 \in E_{3}^{0,2}$. We have a natural morphism $\mathbb{V}^{\zeta} \rightarrow \mathbf{W}^{\theta}$ whose image is the twist $\left(\left.\mathbf{W}^{\sharp}\right|_{S^{\circ}}\right)^{\theta}$. Since $\mathbb{V}^{\zeta} \rightarrow S^{\circ}$ is an affine bundle, $H^{p}\left(S^{\circ}, \mathbb{Z}\right) \rightarrow H^{p}\left(\mathbb{V}^{\zeta}, \mathbb{Z}\right)$ is an isomorphism for any $p$. By the condition 3.2.6, the restriction $H^{3}(S, \mathbb{Z}) \rightarrow$ $H^{3}\left(S^{\circ}, \mathbb{Z}\right)$ is injective. Therefore, the composite

$$
H^{3}(S, \mathbb{Z}) \rightarrow H^{3}\left(\mathbf{W}^{\theta}, \mathbb{Z}\right) \rightarrow H^{3}\left(\mathbb{V}^{\zeta}, \mathbb{Z}\right)
$$

is injective and hence $E_{2}^{p, 0}=E_{\infty}^{p, 0}$. Thus $1 \in E_{2}^{0,2}$ comes from $E^{2}=H^{2}\left(\mathbf{W}^{\theta}, \mathbb{Z}\right)$. Consequently, $\pi: \mathbf{W}^{\theta} \rightarrow S$ is cohomologically projective.

Lemma 7.4.3. Let $\eta=(f: X \rightarrow S, \phi)$ be a marked elliptic fibration such that $\mathrm{c}(\eta)$ is a torsion element. Then there exist a family of elliptic fibrations $\mathcal{X} \rightarrow S \times T$ and points $0, t \in T$ such that $\mathcal{X}_{0} \rightarrow S \times\{0\}$ is bimeromorphically equivalent to $f$ and that $\mathcal{X}_{t} \rightarrow S \times\{t\}$ is $B P$. 
Roughly speaking, a BCK elliptic fibration is deformed to a BP elliptic fibration up to bimeromorphic equivalence.

Proof. In viewing the exact sequence

$$
H^{1}\left(S, \mathcal{L}_{H / S}\right) \stackrel{\varphi}{\rightarrow} H^{1}\left(\underline{S}, \mathfrak{S}_{H / \underline{S}}\right) \stackrel{c}{\rightarrow} H^{2}\left(\underline{S}, \mathbb{L}_{H / \underline{S}}^{\bullet}\right),
$$

we find a positive integer $m$ and an element $\zeta \in H^{1}\left(S, \mathcal{L}_{H / S}\right)$ such that $\varphi(\zeta)=$ $m \eta$. Then $\eta-\varphi((1 / m) \zeta) \in H^{1}\left(\underline{S}, \mathfrak{S}_{H / \underline{S}}\right)_{\text {tor }}$. Thus it corresponds to a BP elliptic fibration. Let $p: S \times \mathbb{C} \rightarrow S$ be the first projection and let $p^{-1} H$ be the pullback of $H$ defined on $S^{\star} \times \mathbb{C}$. Let $\mathbb{C} \rightarrow H^{1}\left(S, \mathcal{L}_{H / S}\right)$ be the homomorphism sending 1 to $\zeta$. Then it defines a section

$$
\tilde{\zeta} \in H^{1}\left(S \times \mathbb{C}, \mathcal{L}_{p^{-1} H / S \times \mathbb{C}}\right), \quad \text { and } \quad \varphi(\tilde{\zeta}) \in H^{1}\left(\underline{S} \times \mathbb{C}, \mathfrak{S}_{p^{-1} H / \underline{S} \times \mathbb{C}}\right) .
$$

Let $(\mathcal{X} \rightarrow S \times \mathbb{C}, \phi)$ be the marked elliptic fibration corresponding to $\varphi(\tilde{\zeta})+$ $p^{*}(\eta)$. This exists by 6.3.12. We may assume that $\mathcal{X} \rightarrow \mathbb{C}$ is flat and $\mathcal{X}$ is smooth over $S^{\star} \times \mathbb{C}$. Then the fiber $\mathcal{X}_{t} \rightarrow S \times\{t\}$ corresponds to $\varphi(t \zeta)+\eta$. Thus $T=\mathbb{C}, 0 \in \mathbb{C}$, and $t=-1 / m \in \mathbb{C}$ satisfy the condition.

A compact complex variety is called to be in the class $\mathcal{C}$ if it is the image of a compact Kähler manifold under a meromorphic mapping [F2]. By [V1], a variety in the class $\mathcal{C}$ is bimeromorphically equivalent to a compact Kähler manifold.

Theorem 7.4.4. $\quad$ Let $S^{\star} \subset S$ be a d-dimensional toroidal embedding such that $S$ is compact and is in the class $\mathcal{C}$. Let $f: X \rightarrow S$ be an elliptic fibration that is smooth over $S^{\star}$. Then the following three conditions are equivalent:

(1) $X$ is in the class $\mathcal{C}$;

(2) $f$ is a BCK morphism;

(3) The homomorphism $H^{2 d}(S, \mathbb{C}) \rightarrow H^{2 d}(X, \mathbb{C})$ is injective.

Proof. (1) $\Longrightarrow(2)$ is trivial.

$(2) \Longrightarrow(3)$ We know $\mathcal{H}^{-2 d}\left(\boldsymbol{\omega}_{S}^{\text {top }}\right) \simeq \mathbb{Z}_{S}$ and $\mathcal{H}^{1-2 d}\left(\boldsymbol{\omega}_{S}^{\text {top }}\right)=0$. Hence, the Verdier duality $R \Gamma(S, \mathbb{Z}) \sim_{\text {qis }} R \operatorname{Hom}\left(R \Gamma\left(S, \boldsymbol{\omega}_{S}^{\text {top }}\right), \mathbb{Z}\right)$ and $H^{1}(S, \mathbb{Z})_{\text {tor }}=0$ induce an isomorphism

$$
H^{2 d}(S, \mathbb{Z}) \simeq \operatorname{Hom}\left(H^{0}(S, \mathbb{Z}), \mathbb{Z}\right) \simeq \mathbb{Z}
$$

The dual of the pullback $H^{2 d}(S, \mathbb{R}) \rightarrow H^{2 d}(X, \mathbb{R})$ is the homomorphism $H^{2}(X, \mathbb{R}) \rightarrow H^{0}(S, \mathbb{R})$ induced from the trace map $R f_{!} \boldsymbol{\omega}_{X}^{\text {top }} \rightarrow \boldsymbol{\omega}_{S}^{\text {top }}$. For 
a cohomology class $\omega \in H^{2}(X, \mathbb{R})$, the image in $H^{0}(S, \mathbb{R})$ is considered as $\left.\operatorname{deg} \omega\right|_{X_{s}}$ for a general fiber $X_{s}$. Thus $H^{2 d}(S, \mathbb{R}) \rightarrow H^{2 d}(X, \mathbb{R})$ is injective if $f$ is $\mathrm{BCK}$.

$(3) \Longrightarrow(1)$ We may assume that $S$ is a compact Kähler manifold. Let $H=H(f)$ and let $\eta \in H^{1}\left(\underline{S}, \mathfrak{S}_{H / \underline{S}}\right)$ be the cohomology class corresponding to $(f: X \rightarrow S, \phi)$ for a suitable marking $\phi$. Then $\mathrm{c}(m \eta)=0$ for a positive integer $m$ by 7.4 .2 and by the proof of $(2) \Longrightarrow(3)$ above. Let $\psi$ and $\varphi$ denote the homomorphisms $H^{1}\left(S, \mathcal{L}_{H / S}\right) \rightarrow H^{1}\left(\underline{S}, \mathfrak{S}_{H / \underline{S}}^{\mathbf{W}}\right)$ and $H^{1}\left(S, \mathcal{L}_{H / S}\right) \rightarrow H^{1}\left(\underline{S}, \mathfrak{S}_{H / \underline{S}}\right)$, respectively. Then $m \eta=\varphi(\zeta)$ for an element $\zeta \in H^{1}\left(S, \mathcal{L}_{H / S}\right)$. We set $\theta:=\psi(\zeta) \in H^{1}\left(S, \mathfrak{S}_{H / S}^{\mathbf{W}}\right)$ and let $\mathbf{W}^{\theta} \rightarrow S$ be the twist of the minimal Weierstrass model $\mathbf{W}=\mathbf{W}(H) \rightarrow S$ associated with $H$. Since there is a generically finite meromorphic mapping $X \cdots \rightarrow \mathbf{W}^{\theta}$ over $S$, we have only to prove that $\mathbf{W}^{\theta}$ is in the class $\mathcal{C}$. Let $\mathbb{C} \rightarrow H^{1}\left(S, \mathcal{L}_{H / S}\right)$ be the homomorphism sending 1 to $\zeta$. It defines a cohomology class $\tilde{\zeta} \in H^{1}\left(S \times \mathbb{C}, \mathcal{L}_{p^{-1} H / S \times \mathbb{C}}\right)$, where $p^{-1} H$ denotes the pullback of $H$ by the first projection $p: S^{\star} \times \mathbb{C} \rightarrow S^{\star}$. The restriction of $\tilde{\zeta}$ to $H^{1}\left(S \times\{t\}, \mathcal{L}_{H / S}\right)$ is $t \zeta$ for $t \in \mathbb{C}$. Let $\tilde{\theta}$ be the image $\psi(\tilde{\zeta})$ in $H^{1}\left(S \times \mathbb{C}, \mathfrak{S}_{p^{-1} H / S \times \mathbb{C}}^{\mathbf{W}}\right)$. Then we have the twist

$$
\widehat{\mathbf{W}}:=(\mathbf{W} \times \mathbb{C})^{\tilde{\theta}} \rightarrow S \times \mathbb{C}
$$

of the Weierstrass model $\mathbf{W} \times \mathbb{C} \rightarrow S \times \mathbb{C}$. The fiber of $\widehat{\mathbf{W}} \rightarrow S \times \mathbb{C}$ over a point $t \in \mathbb{C}$ is isomorphic to the twist $\mathbf{W}^{t \theta} \rightarrow S$, where $t \theta:=\psi(t \zeta)$. The composite $\pi: \widehat{\mathbf{W}} \rightarrow S \times \mathbb{C} \rightarrow \mathbb{C}$ is a locally trivial deformation of $\mathbf{W}$. By Hironaka's resolution of singularities, we have an open neighborhood $\mathcal{U}$ of the origin of $\mathbb{C}$ and a bimeromorphic morphism $\widehat{Y} \rightarrow \pi^{-1}(\mathcal{U})$ such that the composite $h: \widehat{Y} \rightarrow$ $\mathcal{U}$ is a smooth morphism. Here we may assume that the central fiber $Y_{0}=$ $h^{-1}(0) \rightarrow S \times\{0\}$ of $\widehat{Y} \rightarrow S \times \mathcal{U}$ is a projective morphism. In particular, $Y_{0}$ is a compact Kähler manifold. Hence, $Y_{1 / n}$ is a compact Kähler manifold for a positive integer $n$. Therefore $\mathbf{W}^{n^{-1} \theta}$ is in the class $\mathcal{C}$. Since there is a generically finite meromorphic mapping $\mathbf{W}^{n^{-1} \theta} \cdots \rightarrow \mathbf{W}^{\theta}, \mathbf{W}^{\theta}$ is also in the class $\mathcal{C}$.

Corollary 7.4.5 (Miyaoka [M6]). A compact elliptic surface is Kähler if and only if the first Betti number is even.

Proof. Let $f: X \rightarrow S$ be the elliptic surface and let $H=H(f)$ be the associated VHS. Then $f$ is isomorphic to the twist $\boldsymbol{B}^{\eta} \rightarrow S$ of the minimal basic elliptic fibration $\boldsymbol{B} \rightarrow S$ associated with $H$ for a cohomology class $\eta \in$ $H^{1}\left(\underline{S}, \mathfrak{S}_{H / \underline{S}}\right)$. We shall consider the following edge sequence:

$$
0 \rightarrow H^{1}(S, \mathbb{R}) \rightarrow H^{1}(X, \mathbb{R}) \rightarrow H^{0}\left(S, R^{1} f_{*} \mathbb{R}_{X}\right) \rightarrow H^{2}(S, \mathbb{R}) \rightarrow H^{2}(X, \mathbb{R}) .
$$


Suppose that $\eta=0$, i.e., $f$ is a basic fibration. Then $X$ is a projective surface. Therefore $\operatorname{dim} H^{0}\left(S, R^{1} f_{*} \mathbb{R}_{X}\right)=\operatorname{dim} H^{0}\left(S, j_{*} H \otimes \mathbb{R}\right)$ is even for any $\eta$. Since $H^{2}(S, \mathbb{R}) \simeq \mathbb{R}$, the first Betti number is even if and only if $H^{2}(S, \mathbb{R}) \rightarrow H^{2}(X, \mathbb{R})$ is injective. Thus by 7.4 .4 , it is also equivalent to: $X$ is in the class $\mathcal{C}$. Since a compact complex surface is in the class $\mathcal{C}$ if and only if it is Kähler (cf. [M5], [F3]), we are done.

Corollary 7.4.6. Let $\boldsymbol{p}: \boldsymbol{B} \rightarrow S$ be a minimal elliptic surface over a non-singular projective curve. Suppose that the VHS $H$ is not trivial. Then any element of $\mathcal{E}(S, D, H)$ represents a Kähler surface.

Proof. We have $H^{2}\left(S, j_{*} H\right)_{\mathbb{Q}}=0$ by 4.2.5. Hence $H^{2}\left(S, \mathbb{L}_{H / S}^{\bullet} \otimes \mathbb{Q}=0\right.$. Thus by 7.4.2 and 7.4.4, any element of $\mathcal{E}(S, D, H)$ represents a compact Kähler surface.

Example 7.4.7. Without the assumption of the compactness of $S$, a BCK elliptic fibration is not necessarily a BK morphism. Let $S:=\boldsymbol{\Delta}^{2} \backslash\{0\}$ and $D=\emptyset$. We fix a VHS $H$ on $S$, which is determined by a homomorphic function $S \rightarrow \mathbb{H}$. Then we have the smooth basic elliptic fibration $\boldsymbol{B} \rightarrow S$ as well as an exact sequence:

$$
0 \rightarrow H \simeq \mathbb{Z}_{S}^{\oplus 2} \rightarrow \mathcal{L}_{H} \simeq \mathcal{O}_{S} \rightarrow \mathfrak{S}_{H} \rightarrow 0 .
$$

Then $H^{1}\left(S, \mathcal{O}_{S}\right) \simeq H^{1}\left(S, \mathfrak{S}_{H}\right)$. Therefore, for any $\eta \in H^{1}\left(S, \mathfrak{S}_{H}\right)$, the corresponding twist $\boldsymbol{B}^{\eta} \rightarrow S$ is a BCK morphism. However if $\eta \neq 0$, then there is no $d$-closed $(1,1)$-form $\omega$ on $X:=\boldsymbol{B}^{\eta}$ such that $\left.\int \omega\right|_{F}>0$ for any fiber $F$ of $f: X \rightarrow S$. In fact, if there is such $\omega$, then the composite

$$
H^{1}\left(X, \mathcal{O}_{X} / \mathbb{R}_{X}\right) \rightarrow H^{2}(X, \mathbb{R}) \rightarrow H^{0}\left(S, R^{2} f_{*} \mathbb{R}_{X}\right) \simeq \mathbb{R}
$$

is surjective. Since $H^{i}(S, \mathbb{R})=0$ for $i=1,2$, we see that $H^{2}(X, \mathbb{R}) \simeq \mathbb{R}$. Hence $H^{2}(X, \mathbb{R}) \rightarrow H^{2}\left(X, \mathcal{O}_{X}\right)$ is a zero map. On the other hand, the $\eta$ is the image of 1 under the connecting homomorphism $H^{0}(S, \mathbb{Z}) \rightarrow H^{1}\left(S, \mathfrak{S}_{H}\right)$ of the exact sequence:

$$
0 \rightarrow \mathfrak{S}_{H} \rightarrow R^{1} f_{*} \mathcal{O}_{X}^{\star} \rightarrow R^{2} f_{*} \mathbb{Z}_{X} \simeq \mathbb{Z} \rightarrow 0 .
$$

There is a commutative diagram:

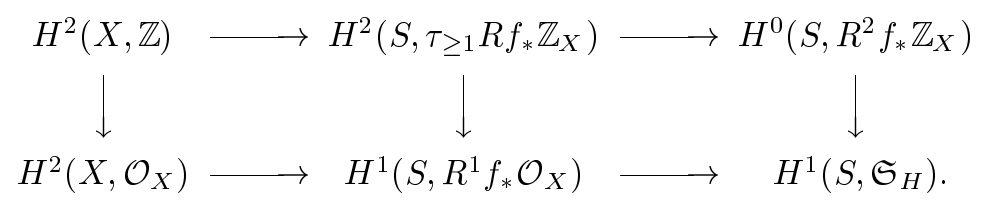

This contradicts $\eta \neq 0$. Hence $\boldsymbol{B}^{\eta}$ is not Kähler. 


\section{§7.5. New description of logarithmic transformations}

Let $j: S^{\star} \hookrightarrow S$ be a toroidal embedding, $D:=S \backslash S^{\star}$, and let $H$ be a VHS defined over $S^{\star}$. From the triangle (6.8), we have a homomorphism

$$
H^{0}\left(\underline{S}, \mathfrak{Q}_{H / \underline{S}}\right) \rightarrow H^{1}\left(\underline{S}, \mathfrak{S}_{H / \underline{S}}\right) .
$$

We shall relate the homomorphism above with logarithmic transformations [K8] along $D$. Let us consider the morphism $R j_{*} H \rightarrow \mathcal{L}_{H / S}$ appearing in 4.2.3. We define the sheaf $\mathfrak{S}_{H / S}^{\log }$ by the triangle

$$
\cdots \stackrel{+1}{\longrightarrow} \tau_{\leq 1} R j_{*} H \rightarrow \mathcal{L}_{H / S} \rightarrow \mathfrak{S}_{H / S}^{\log } \stackrel{+1}{\longrightarrow} \cdots
$$

By comparing with a similar triangle

$$
\cdots \stackrel{+1}{\longrightarrow} \tau_{\leq 1} R j_{*} H \rightarrow j_{*} \mathcal{L}_{H} \rightarrow j_{*} \mathfrak{S}_{H} \stackrel{+1}{\longrightarrow} \cdots,
$$

we can consider $\mathfrak{S}_{H / S}^{\log }$ as a subsheaf of $j_{*} \mathfrak{S}_{H}$. Then we have

$$
\mathfrak{S}_{H / S} \subset \mathfrak{S}_{H / S}^{\log }, \quad \mathfrak{S}_{H / S}^{\log } / \mathfrak{S}_{H / S}^{\mathbf{W}} \simeq R^{1} j_{*} H, \quad \text { and } \quad \mathfrak{S}_{H / S}^{\log } / \mathfrak{S}_{H / S} \simeq \mathfrak{Q}_{H / S} .
$$

We shall describe the stalk $\left(\mathfrak{S}_{H / S}^{\log }\right)_{P}$ at a point $P \in D$. If $\left(\mathfrak{Q}_{H / S}\right)_{P}=0$, then $\left(\mathfrak{S}_{H / S}^{\log }\right)_{P}=\left(\mathfrak{S}_{H / S}\right)_{P}$. Suppose that $\left(\mathfrak{Q}_{H / S}\right)_{P} \neq 0$. Then $H$ is of type $\mathrm{I}_{0}$ or $\mathrm{I}_{(+)}$ around $P$. Let $\mathcal{U} \ni P$ be an open neighborhood such that $\mathcal{U}^{\star}=\mathcal{U} \cap S^{\star} \subset \mathcal{U}$ is a toroidal embedding of type $(\mathrm{N}, l, \sigma): \mathcal{U}$ is isomorphic to $\mathbb{T}_{\mathrm{N}}(\sigma)^{<1} \times \boldsymbol{\Delta}^{d-l}$ and $\mathcal{U}^{\star}$ is isomorphic to $\mathbb{T}_{\mathrm{N}}^{<1} \times \boldsymbol{\Delta}^{d-l}$. Let $\tilde{\mathrm{e}}_{\mathrm{N}}: \widetilde{\mathcal{U}^{\star}} \rightarrow \mathcal{U}^{\star}$ be the universal covering mapping described by

$$
\begin{gathered}
\tilde{\mathrm{e}}_{\mathrm{N}}: \mathbb{H}_{\mathrm{N}}(\operatorname{Int} \sigma) \times \boldsymbol{\Delta}^{n-l} \ni z=\left(z^{\prime}, t^{\prime}\right) \longmapsto t=\left(\mathrm{e}_{N}\left(z^{\prime}\right), t^{\prime}\right) \in \mathbb{T}_{\mathrm{N}}^{<1} \times \boldsymbol{\Delta}^{d-l}, \\
\text { where } \quad \mathbb{H}_{\mathrm{N}}(\operatorname{Int} \sigma)=\mathrm{N} \otimes \mathbb{R}+\sqrt{-1} \operatorname{Int} \sigma \subset \mathrm{N} \otimes \mathbb{C} .
\end{gathered}
$$

Now $H$ is of type $\mathrm{I}_{\boldsymbol{a}}$ for some $\boldsymbol{a} \in \sigma^{\vee} \cap \mathrm{M}$. The period function $\omega: \widetilde{\mathcal{U}^{\star}} \rightarrow \mathbb{H}$ is written as

$$
\omega(z)=\left\langle\boldsymbol{a}, z^{\prime}\right\rangle+h\left(\tilde{\mathrm{e}}_{\mathrm{N}}(z)\right)
$$

for a holomorphic function $h(t)$ defined over $\mathcal{U}$. An element of $H^{0}\left(\mathcal{U}^{\star}, \mathfrak{S}_{H}\right)$ is represented by a holomorphic function $f(z)$ defined over $\widetilde{\mathcal{U}^{\star}}$ modulo $\mathbb{Z} \omega(z)+\mathbb{Z}$ which satisfies the condition

$$
f\left(z^{\prime}+\gamma, t^{\prime}\right)-f(z) \in \mathbb{Z} \omega(z)+\mathbb{Z} \quad \text { for any } \gamma \in \mathrm{N} .
$$


We denote the represented element of $H^{0}\left(\mathcal{U}^{\star}, \mathfrak{S}_{H}\right)$ by $[f(z)]$. An element $\boldsymbol{\theta} \in$ $\mathrm{M} \otimes \mathbb{C}$ defines a holomorphic function on $\widetilde{\mathcal{U}^{\star}}$ by $\hat{\boldsymbol{\theta}}(z):=\left\langle\boldsymbol{\theta}, z^{\prime}\right\rangle$. In the case $\boldsymbol{a}=0$, the holomorphic functions

$$
f\left(z ; \boldsymbol{\theta}_{1}, \boldsymbol{\theta}_{2}\right):=\hat{\boldsymbol{\theta}}_{1}(z) \omega(z)+\hat{\boldsymbol{\theta}}_{2}(z)
$$

for $\boldsymbol{\theta}_{1}, \boldsymbol{\theta}_{2} \in \mathrm{M}$ satisfy the condition (7.6). In fact,

$$
f\left(\left(z^{\prime}+\gamma, t^{\prime}\right) ; \boldsymbol{\theta}_{1}, \boldsymbol{\theta}_{2}\right)-f\left(z ; \boldsymbol{\theta}_{1}, \boldsymbol{\theta}_{2}\right)=\left\langle\boldsymbol{\theta}_{1}, \gamma\right\rangle h(t)+\left\langle\boldsymbol{\theta}_{2}, \gamma\right\rangle .
$$

In particular, the images of $\left[f\left(z ; \boldsymbol{\theta}_{1}, \boldsymbol{\theta}_{2}\right)\right]$ under $H^{0}\left(\mathcal{U}^{\star}, \mathfrak{S}_{H}\right) \rightarrow H^{1}\left(\mathcal{U}^{\star}, H\right) \simeq$ $\mathrm{M}^{\oplus 2}$ form a generator. In the case $\boldsymbol{a} \neq 0$, let $\alpha$ be the maximal positive integer such that $\boldsymbol{u}:=\alpha^{-1} \boldsymbol{a} \in \mathrm{M}$. The holomorphic functions

$$
f(z ; n, \boldsymbol{\theta}):=\frac{n}{2 \alpha} \omega(z)^{2}-\frac{n}{2} \hat{\boldsymbol{a}}(z)+\hat{\boldsymbol{\theta}}(z)
$$

for $n \in \mathbb{Z}$ and $\boldsymbol{\theta} \in \mathrm{M}$ satisfy (7.6). In fact,

$$
f\left(\left(z^{\prime}+\gamma, t^{\prime}\right) ; n, \boldsymbol{\theta}\right)-f(z ; n, \boldsymbol{\theta})=n\langle\boldsymbol{u}, \gamma\rangle \omega(z)+\alpha n \frac{\langle\boldsymbol{u}, \gamma\rangle(\langle\boldsymbol{u}, \gamma\rangle-1)}{2}+\langle\boldsymbol{\theta}, \gamma\rangle
$$

In particular, they form a generator of $H^{1}\left(\mathcal{U}^{\star}, H\right) \simeq \mathbb{Z} \oplus \mathrm{M} / \mathbb{Z} \boldsymbol{a}$.

Proposition 7.5.1. Suppose that the local monodromy type of $H$ around $P$ is $\mathrm{I}_{\boldsymbol{a}}$ for $\boldsymbol{a} \in \sigma^{\vee} \cap \mathrm{M}$. Then subgroups $\left(\mathfrak{S}_{H / S}\right)_{P},\left(\mathfrak{S}_{H / S}^{\log }\right)_{P} \subset\left(j_{*} \mathfrak{S}_{H}\right)_{P}$ are described as follows:

(1) In the case $\boldsymbol{a}=0$,

$$
\left(\mathfrak{S}_{H / S}\right)_{P}=\left(\mathfrak{S}_{H / S}^{\mathbf{W}}\right)_{P}, \quad\left(\mathfrak{S}_{H / S}^{\log }\right)_{P}=\left(\mathfrak{S}_{H / S}^{\mathbf{W}}\right)_{P}+\sum_{\boldsymbol{\theta}_{1}, \boldsymbol{\theta}_{2} \in \mathbf{M}} \mathbb{Z}\left[f\left(z ; \boldsymbol{\theta}_{1}, \boldsymbol{\theta}_{2}\right)\right] .
$$

(2) In the case $\boldsymbol{a} \neq 0$,

$$
\begin{aligned}
& \left(\mathfrak{S}_{H / S}\right)_{P}=\left(\mathfrak{S}_{H / S}^{\mathbf{W}}\right)_{P}+\sum_{\boldsymbol{\theta} \in \mathrm{N}\left(\boldsymbol{a}^{\perp} \cap \sigma\right)^{\perp}} \mathbb{Z}[f(z ; 0, \boldsymbol{\theta})] ; \\
& \left(\mathfrak{S}_{H / S}^{\log }\right)_{P}=\left(\mathfrak{S}_{H / S}^{\mathbf{W}}\right)_{P}+\sum_{n \in \mathbb{Z}, \boldsymbol{\theta} \in \mathbf{M}} \mathbb{Z}[f(z ; n, \boldsymbol{\theta})] .
\end{aligned}
$$

Proof. Let $F^{1 \bullet}:=F^{1}\left(\hat{\Omega}_{S}^{\bullet}(\log D) \otimes \mathcal{H}_{S}^{\text {can }}\right)$ be the subcomplex

$$
\left[\cdots \rightarrow 0 \rightarrow \mathcal{F}^{1}\left(\mathcal{H}_{S}^{\mathrm{can}}\right) \stackrel{\nabla}{\rightarrow} \hat{\Omega}_{S}^{1}(\log D) \otimes \mathcal{H}_{S}^{\mathrm{can}} \stackrel{\nabla}{\rightarrow} \hat{\Omega}_{S}^{2}(\log D) \otimes \mathcal{H}_{S}^{\mathrm{can}} \rightarrow \cdots\right]
$$

of the logarithmic de Rham complex $\hat{\Omega}_{S}^{\bullet}(\log D) \otimes \mathcal{H}_{S}^{\text {can }}$ defined by the GaussManin connection $\nabla$ with respect to $H$. Then the mapping cone of $R j_{*} H_{\mathbb{C}} \rightarrow$ 
$\mathcal{L}_{H / S}$ is quasi-isomorphic to $F^{1} \bullet[1]$. Therefore, the mapping cone of $\tau_{\leq 1} R j_{*} H_{\mathbb{C}}$ $\rightarrow \mathcal{L}_{H / S}$ is quasi-isomorphic to the first cohomology sheaf $\mathcal{H}^{1}\left(F^{1 \bullet}\right)$. Let $F_{\star}^{1 \bullet}$ be the subcomplex

$$
\left[\cdots \rightarrow 0 \rightarrow \mathcal{F}^{1}(\mathcal{H}) \stackrel{\nabla}{\rightarrow} \Omega_{S^{\star}}^{1} \otimes \mathcal{H} \stackrel{\nabla}{\rightarrow} \Omega_{S^{\star}}^{2} \otimes \mathcal{H} \rightarrow \cdots\right]
$$

of $\Omega_{S^{\star}}^{\bullet} \otimes \mathcal{H}$. The mapping cone of $\tau_{\leq 1} R j_{*} H_{\mathbb{C}} \rightarrow j_{*} \mathcal{L}_{H}$ is quasi-isomorphic to $\mathcal{H}^{1}\left(j_{*} F_{\star}^{1 \bullet}\right)$. There is a natural homomorphism $j_{*} \mathfrak{S}_{H} \rightarrow \mathcal{H}^{1}\left(j_{*} F_{\star}^{1 \bullet}\right)$ and an exact sequence

$$
0 \rightarrow \mathcal{H}^{1}\left(F^{1 \bullet}\right) \rightarrow \mathcal{H}^{1}\left(j_{*} F_{\star}^{1 \bullet}\right) \rightarrow j_{*} \mathcal{L}_{H} / \mathcal{L}_{H / S} \rightarrow 0 .
$$

The subsheaf $\mathfrak{S}_{H / S}^{\log }$ is characterized as the kernel of $j_{*} \mathfrak{S}_{H} \rightarrow \mathcal{H}^{1}\left(j_{*} F_{\star}^{1 \bullet}\right) /$ $\mathcal{H}^{1}\left(F^{1}\right)$. Let $\tilde{F}^{1 \bullet}$ be the pullback of $F_{\star}^{1 \bullet}$ to $\widetilde{\mathcal{U}^{\star}}$. The abelian group $A:=H^{0}\left(\widetilde{\mathcal{U}^{\star}}\right.$, $\left.\tilde{\mathrm{e}}_{\mathrm{N}}^{-1} H\right) \simeq \mathbb{Z}^{\oplus 2}$ admits a natural N-module structure and $R \Gamma\left(\mathcal{U}^{\star}, R j_{*} H\right)$ is quasiisomorphic to $R \Gamma(\mathrm{N}, A)=R \operatorname{Hom}_{\mathbb{Z}[\mathrm{N}]}(\mathbb{Z}, A) \simeq \operatorname{Kos}_{\mathrm{M} \otimes A}^{\bullet}(A, \boldsymbol{a} \varepsilon)$ under the identification $A=\mathbb{Z}[\varepsilon]$ (cf. 4.3). We have a commutative diagram of triangles

$$
\begin{array}{ccc}
\cdots \stackrel{+1}{\longrightarrow} \tau_{\leq 1} R \Gamma(\mathrm{N}, A \otimes \mathbb{C}) & \longrightarrow H^{0}\left(\mathcal{U}^{\star}, \mathcal{L}_{H}\right) & \longrightarrow H^{1}\left(\mathcal{U}^{\star}, F_{\star}^{1 \bullet}\right) \stackrel{+1}{\longrightarrow} \cdots \\
\downarrow & \stackrel{\downarrow}{\longrightarrow} \quad A \otimes \mathbb{C} & \longrightarrow H^{0}\left(\widetilde{\mathcal{U}^{\star}}, \tilde{\mathrm{e}}_{\mathrm{N}}^{-1} \mathcal{L}_{H}\right) \longrightarrow H^{1}\left(\widetilde{\mathcal{U}^{\star}}, \tilde{F}^{1 \bullet}\right) \stackrel{+1}{\longrightarrow} \cdots
\end{array}
$$

in the derived category of abelian groups. Let $\mathcal{C}$ be the cokernel of

$$
H^{0}\left(\widetilde{\mathcal{U}^{\star}}, \tilde{\mathrm{e}}_{\mathrm{N}}^{-1} \mathcal{F}^{1}(\mathcal{H})\right) \stackrel{\nabla}{\rightarrow} H^{0}\left(\widetilde{\mathcal{U}^{\star}}, \Omega_{\widetilde{\mathcal{U}}^{\star}}^{1} \otimes \tilde{\mathrm{e}}_{\mathrm{N}}^{-1} \mathcal{H}\right)
$$

and let us consider the composite

$$
\Phi: H^{0}\left(\widetilde{\mathcal{U}^{\star}}, \tilde{\mathrm{e}}_{\mathrm{N}}^{-1} \mathfrak{S}_{H}\right) \rightarrow H^{1}\left(\widetilde{\mathcal{U}^{\star}}, \tilde{F}^{1 \bullet}\right) \rightarrow H^{0}\left(\widetilde{\mathcal{U}^{\star}}, \Omega_{\widetilde{\mathcal{U}^{\star}}}^{1} \otimes \tilde{\mathrm{e}}_{\mathrm{N}}^{-1} \mathcal{H}\right) \rightarrow \mathcal{C}
$$

Here, $H^{1}\left(\widetilde{\mathcal{U}^{\star}}, \tilde{F}^{1 \bullet}\right) \rightarrow \mathcal{C}$ is injective. We have the following diagram

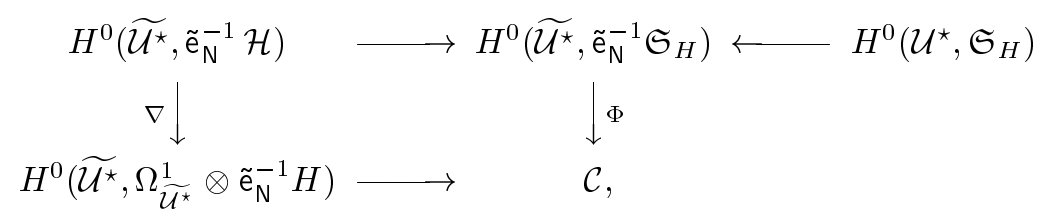

where the top left arrow is induced from the composite $\mathcal{H} \rightarrow \mathcal{L}_{H} \rightarrow \mathfrak{S}_{H}$ that is described as

$$
\left(\begin{array}{c}
\alpha(z) \\
\beta(z)
\end{array}\right) \longmapsto \beta(z) \omega(z)-\alpha(z) \bmod \mathbb{Z} \omega(z)+\mathbb{Z}
$$


under the isomorphisms

$$
\tilde{\mathrm{e}}_{\mathrm{N}}^{-1} \mathcal{H} \simeq \mathcal{O}_{\widetilde{\mathcal{U}^{\star}}}^{\oplus 2} \quad \text { and } \quad \tilde{\mathrm{e}}_{\mathrm{N}}^{-1} \mathfrak{S}_{H} \simeq \mathcal{O}_{\widetilde{\mathcal{U}^{\star}}} / \mathbb{Z} \omega(z)+\mathbb{Z} .
$$

Note that $\tilde{e}_{N}^{-1} \mathcal{F}^{1}(\mathcal{H}) \simeq \mathcal{O}_{\widetilde{\mathcal{U}^{*}}}$ is generated by the column vector ${ }^{\mathrm{t}}(\omega(z), 1)$.

Let $f(z)$ be a holomorphic function representing an element $[f]$ of $H^{0}\left(\mathcal{U}^{\star}, \mathfrak{S}_{H}\right)$, i.e., $f$ satisfies the condition (7.6). If $[f] \in H^{0}\left(\mathcal{U}, \mathfrak{S}_{H / S}^{\log }\right)$, then its image $\Phi([f])$ in $\mathcal{C}$ is coming from $H^{0}\left(\mathcal{U}, \hat{\Omega}_{S}^{1}(\log D) \otimes \mathcal{H}\right)$. Let $\mathfrak{S}_{P}^{\prime}$ denote the subgroup of the right hand side of the description of $\left(\mathfrak{S}_{H / S}^{\log }\right)_{P}$ in 7.5.1. We shall show that if $\Phi([f]) \in \mathcal{C}$ is coming from $H^{0}\left(\mathcal{U}, \hat{\Omega}_{S}^{1}(\log D) \otimes \mathcal{H}\right)$, then $[f] \in \mathfrak{S}_{P}^{\prime}$. Under the property, we have $\left(\mathfrak{S}_{H / S}^{\log }\right)_{P} \subset \mathfrak{S}_{P}^{\prime}$. This is enough, because the isomorphism $\mathfrak{S}_{P}^{\prime} /\left(\mathfrak{S}_{H / S}^{\mathbf{W}}\right)_{P} \simeq\left(R^{1} j_{*} H\right)_{P}$ implies $\left(\mathfrak{S}_{H / S}^{\log }\right)_{P}=\mathfrak{S}_{P}^{\prime}$.

We note that there is an isomorphism $\mathrm{M} \otimes \mathcal{O}_{\mathcal{U}}^{\oplus 2} \simeq \hat{\Omega}_{S}^{1}(\log D) \otimes \mathcal{H}_{S}^{\text {can }} \mid \mathcal{U}$ in which $\boldsymbol{\theta} \otimes{ }^{t}(u(t), v(t))$ for $\boldsymbol{\theta} \in \mathbf{M}, u(t), v(t) \in H^{0}\left(\mathcal{U}, \mathcal{O}_{\mathcal{U}}\right)$ corresponds to

$$
\left(\begin{array}{cc}
1 & \left\langle\boldsymbol{a}, z^{\prime}\right\rangle \\
0 & 1
\end{array}\right)\left(\begin{array}{l}
u(t) \\
v(t)
\end{array}\right)=\left(\begin{array}{c}
u(t)+\hat{\boldsymbol{a}}(z) v(t) \\
v(t)
\end{array}\right)
$$

as an element of $H^{0}\left(\widetilde{\mathcal{U}^{\star}}, \tilde{\mathrm{e}}_{\mathrm{N}}^{-1} \mathcal{H}\right) \simeq H^{0}\left(\widetilde{\mathcal{U}^{\star}}, \mathcal{O}\right)^{\oplus 2}$.

Suppose that $\boldsymbol{a}=0$. Then $\omega(z)=h(t)$. Since the functions $f\left(z ; \boldsymbol{\theta}_{1}, \boldsymbol{\theta}_{2}\right)$ form a generator of $H^{1}\left(\mathcal{U}^{\star}, H\right)$, we have $f(z)=f\left(z ; \boldsymbol{\theta}_{1}, \boldsymbol{\theta}_{2}\right)+\psi(t)$ for some $\boldsymbol{\theta}_{1}$, $\boldsymbol{\theta}_{2} \in \mathrm{M}$, and for a holomorphic function $\psi(t)$ defined over $\mathcal{U}^{\star}$. The element $[f]$ of $H^{0}\left(\mathcal{U}^{\star}, \mathfrak{S}_{H}\right)$ is coming from ${ }^{\mathrm{t}}(-f(z), 0)$ of $H^{0}\left(\widetilde{\mathcal{U}^{\star}}, \mathcal{O}^{\oplus 2}\right)$. We have

$$
\mathrm{d} f=h \mathrm{~d} \hat{\boldsymbol{\theta}}_{1}+\hat{\boldsymbol{\theta}}_{1} \mathrm{~d} h+\mathrm{d} \hat{\boldsymbol{\theta}}_{2}+\mathrm{d} \psi,
$$

in which $\mathrm{d} \hat{\boldsymbol{\theta}}_{i}$ for $i=1,2$ are logarithmic 1-forms on $\mathcal{U}$. The differential of $\xi(z)^{\mathrm{t}}(\omega(z), 1)$ for a holomorphic function $\xi(z)$ is written by ${ }^{\mathrm{t}}(\omega, 1) \mathrm{d} \xi+\xi^{\mathrm{t}}(\mathrm{d} \omega, 0)$. Since $\Phi([f])$ is contained in the image of $H^{0}\left(\mathcal{U}, \hat{\Omega}_{S}^{1}(\log D) \otimes \mathcal{H}\right)$, we have

$$
-\left(\begin{array}{c}
\mathrm{d} f \\
0
\end{array}\right)-\mathrm{d} \xi \otimes\left(\begin{array}{c}
\omega \\
1
\end{array}\right)-\xi\left(\begin{array}{c}
\mathrm{d} \omega \\
0
\end{array}\right) \in H^{0}\left(\mathcal{U}, \hat{\Omega}_{S}^{1}(\log D) \otimes \mathcal{H}_{S}^{\mathrm{can}}\right),
$$

for some holomorphic function $\xi(z)$ defined over $\widetilde{\mathcal{U}^{\star}}$. We infer that $\mathrm{d} f+h \mathrm{~d} \xi+$ $\xi \mathrm{d} h$ and $\mathrm{d} \xi$ are both logarithmic 1-forms on $\mathcal{U}$. In particular, $\xi(z)=\hat{\boldsymbol{v}}(z)+\varphi(t)$ for some $\boldsymbol{v} \in \mathrm{M} \otimes \mathbb{C}$ and for a holomorphic function $\varphi(t)$ defined over $\mathcal{U}$. Thus

$$
\mathrm{d} f+\xi \mathrm{d} h=h \mathrm{~d} \hat{\boldsymbol{\theta}}_{1}+\hat{\boldsymbol{\theta}}_{1} \mathrm{~d} h+\mathrm{d} \hat{\boldsymbol{\theta}}_{2}+\mathrm{d} \psi+\hat{\boldsymbol{v}} \mathrm{d} h+\varphi \mathrm{d} h
$$

and hence

$$
\left(\hat{\boldsymbol{\theta}}_{1}+\hat{\boldsymbol{v}}\right) \mathrm{d} h+\mathrm{d} \psi
$$


are logarithmic 1-forms. Since $\psi$ is defined over $\mathcal{U}^{\star}$, we infer that $\boldsymbol{\theta}_{1}+\boldsymbol{v}=0$ and that $\psi(t)$ is a holomorphic function $\mathcal{U}$. This implies that the element $[f(z)]$ of $\left(j_{*} \mathfrak{S}_{H}\right)_{P}$ is contained in $\mathfrak{S}_{P}^{\prime}$. Therefore, $\left(\mathfrak{S}_{H / S}^{\log }\right)_{P}=\mathfrak{S}_{P}^{\prime}$ as mentioned before. In this case $\boldsymbol{a}=0$, we also have $\left(\mathfrak{S}_{H / S}^{\mathbf{W}}\right)_{P}=\left(\mathfrak{S}_{H / S}\right)_{P}$ since $\left(\mathfrak{T}_{H / S}\right)_{P}=0$.

Next, suppose that $\boldsymbol{a} \neq 0$. Then $\omega(z)=\hat{\boldsymbol{a}}(z)+h(t)$ and $f(z)=f(z ; n, \boldsymbol{\theta})+$ $\psi(t)$ for $n \in \mathbb{Z}, \boldsymbol{\theta} \in \mathrm{M}$, and for a holomorphic function $\psi(t)$ defined on $\mathcal{U}^{\star}$. The element $[f]$ of $H^{0}\left(\mathcal{U}^{\star}, \mathfrak{S}_{H}\right)$ is coming from ${ }^{t}(-f(z), 0)$ of $H^{0}\left(\widetilde{\mathcal{U}^{\star}}, \mathcal{O}^{\oplus 2}\right)$. We have

$$
\mathrm{d} f=\frac{n}{\alpha} \omega \mathrm{d} \omega-\frac{n}{2} \mathrm{~d} \hat{\boldsymbol{a}}+\mathrm{d} \hat{\boldsymbol{\theta}}+\mathrm{d} \psi,
$$

in which $\mathrm{d} \hat{\boldsymbol{a}}, \mathrm{d} \hat{\boldsymbol{\theta}}$, and $\mathrm{d} \omega=\mathrm{d} \hat{\boldsymbol{a}}+\mathrm{d} h$ are logarithmic 1-forms on $\mathcal{U}$. If

$$
-\left(\begin{array}{c}
\mathrm{d} f \\
0
\end{array}\right)-\mathrm{d} \xi \otimes\left(\begin{array}{c}
\omega \\
1
\end{array}\right)-\xi\left(\begin{array}{c}
\mathrm{d} \omega \\
0
\end{array}\right) \in H^{0}\left(\mathcal{U}, \hat{\Omega}_{S}^{1}(\log D) \otimes \mathcal{H}_{S}^{\mathrm{can}}\right),
$$

for some holomorphic function $\xi(z)$ on $\widetilde{\mathcal{U}^{\star}}$, then $\mathrm{d} f+\omega \mathrm{d} \xi+\xi \mathrm{d} \omega$ and $\mathrm{d} \xi$ are both logarithmic 1-forms on $\mathcal{U}$. In particular, $\xi(z)=\hat{\boldsymbol{v}}(z)+\varphi(t)$ for some $\boldsymbol{v} \in \mathrm{M} \otimes \mathbb{C}$ and for a holomorphic function $\varphi(t)$ defined on $\mathcal{U}$. Thus

$$
\begin{aligned}
(n / \alpha) \hat{\boldsymbol{a}} \mathrm{d} \omega+\mathrm{d} \psi+\hat{\boldsymbol{a}} \mathrm{d} \xi+\hat{\boldsymbol{v}} \mathrm{d} \omega \\
=((n / \alpha) \hat{\boldsymbol{a}}+\hat{\boldsymbol{v}}) \mathrm{d} \hat{\boldsymbol{a}}+\hat{\boldsymbol{a}} \mathrm{d} \hat{\boldsymbol{v}}+((n / \alpha) \hat{\boldsymbol{a}}+\hat{\boldsymbol{v}}) \mathrm{d} h+\hat{\boldsymbol{a}} \mathrm{d} \varphi+\mathrm{d} \psi
\end{aligned}
$$

is a single valued logarithmic 1 -form on $\mathcal{U}$. Since it is invariant under the action of $\mathrm{N}$, we have

$$
\begin{aligned}
((n / \alpha)\langle\boldsymbol{a}, \gamma\rangle+\langle\boldsymbol{v}, \gamma\rangle) \mathrm{d} \hat{\boldsymbol{a}} & +\langle\boldsymbol{a}, \gamma\rangle \mathrm{d} \hat{\boldsymbol{v}} \\
+ & ((n / \alpha)\langle\boldsymbol{a}, \gamma\rangle+\langle\boldsymbol{v}, \gamma\rangle) \mathrm{d} h+\langle\boldsymbol{a}, \gamma\rangle \mathrm{d} \varphi=0
\end{aligned}
$$

for any $\gamma \in \mathrm{N}$. Hence $\boldsymbol{v}=-(n / 2 \alpha) \boldsymbol{a}$ and $\mathrm{d}((n / 2 \alpha) h+\varphi)=0$. Thus $\mathrm{d} \psi$ is a logarithmic 1-form and $\psi(t)$ is a holomorphic function on $\mathcal{U}$. This implies that the element $[f(z)]$ of $\left(j_{*} \mathfrak{S}_{H}\right)_{P}$ is contained in $\mathfrak{S}_{P}^{\prime}$. Therefore, $\left(\mathfrak{S}_{H / S}^{\log }\right)_{P}=\mathfrak{S}_{P}^{\prime}$ as mentioned before. The isomorphism $\mathfrak{S}_{P}^{\prime} /\left(\mathfrak{S}_{H / S}^{\mathbf{W}}\right)_{P} \simeq\left(R^{1} j_{*} H\right)_{P}$ sends $f(z ; n, \boldsymbol{\theta})$ to $(n, \boldsymbol{\theta} \bmod \boldsymbol{a})$ in $\mathbb{Z} \oplus \mathrm{M} / \mathbb{Z} \boldsymbol{a} \simeq\left(R^{1} j_{*} H\right)_{P}$. Hence $\left(\mathfrak{S}_{H / S}\right)_{P} /\left(\mathfrak{S}_{H / S}^{\mathbf{W}}\right)_{P}$ is generated by $[f(z ; 0, \boldsymbol{\theta})]$ for $\boldsymbol{\theta} \in \mathbf{N}\left(\boldsymbol{a}^{\perp} \cap \sigma\right)^{\perp}$.

Definition. A section of $\boldsymbol{p}^{\star}: \boldsymbol{B}^{\star} \rightarrow S^{\star}$ is called a logarithmic section if it contained in $H^{0}\left(S, \mathfrak{S}_{H / S}^{\log }\right) \subset H^{0}\left(S^{\star}, \mathfrak{S}_{H}\right)$. We call $\mathfrak{S}_{H / S}^{\log }$ the sheaf of germs of logarithmic sections.

The homomorphism

$$
H^{0}\left(S, \mathfrak{Q}_{H / S}\right)=0 \oplus H^{0}\left(S, \mathfrak{Q}_{H / S}\right) \rightarrow H^{1}\left(S, \mathfrak{S}_{H / S}\right)
$$


induced from the triangle (6.7) is a connecting homomorphism of the exact sequence

$$
0 \rightarrow \mathfrak{S}_{H / S} \rightarrow \mathfrak{S}_{H / S}^{\log } \rightarrow \mathfrak{Q}_{H / S} \rightarrow 0
$$

Next, we shall generalize the definition of $\mathfrak{S}_{H / S}^{\log }$ to the $\partial$-space $\underline{S}=$ $(S, D)$. Let $R \underline{j}_{*} H \rightarrow \mathcal{L}_{H / \underline{S}}$ be the morphism appearing in 4.2.3. Let the sheaf $\mathfrak{S}_{H / \underline{S}}^{\log }$ be defined by the triangle

$$
\cdots \stackrel{+1}{\longrightarrow} \tau_{\leq 1} R \underline{j}_{*} H \rightarrow \mathcal{L}_{H / \underline{S}} \rightarrow \mathfrak{S}_{H / \underline{S}}^{\log } \stackrel{+1}{\longrightarrow} \cdots
$$

Then $\mathfrak{S}_{H / \underline{S}}^{\log }$ is considered as a subsheaf of $\underline{j}_{*} \mathfrak{S}_{H}$. We have

$$
\mathfrak{S}_{H / \underline{S}} \subset \mathfrak{S}_{H / \underline{S}}^{\log }, \quad \mathfrak{S}_{H / \underline{S}}^{\log } / \mathfrak{S}_{H / \underline{S}}^{\mathbf{W}} \simeq R \underline{j}_{*} H, \quad \text { and } \quad \mathfrak{S}_{H / \underline{S}}^{\log } / \mathfrak{S}_{H / \underline{S}} \simeq \mathfrak{Q}_{H / \underline{S}} .
$$

For a point $P \in D$, let $\mathcal{U} \ni P$ be an open neighborhood such that $\mathcal{U}^{\star}=\mathcal{U} \cap S^{\star} \subset$ $\mathcal{U}$ is a toroidal embedding of type $(\mathrm{N}, l, \sigma)$. Let $\mathrm{N}_{\text {unip }} \subset \mathrm{N}$ be the maximal subgroup such that $\rho(\gamma)$ is unipotent for any $\gamma \in \mathbb{N}_{\text {unip }}$ for the monodromy representation $\rho: \mathrm{N} \rightarrow \mathrm{SL}(2, \mathbb{Z})$. As before, an element $\boldsymbol{\theta} \in \mathrm{N}_{\text {unip }} \otimes \mathbb{C}=\mathrm{N} \otimes \mathbb{C}$ induces a holomorphic function $\hat{\boldsymbol{\theta}}(z)=\left\langle\boldsymbol{\theta}, z^{\prime}\right\rangle$ on the universal covering space $\widetilde{\mathcal{U}^{\star}} \simeq \mathbb{H}_{\mathrm{N}}(\operatorname{Int} \sigma) \times \boldsymbol{\Delta}^{d-l}$. In the case the local monodromy of $H$ around $D$ near $P$ is finite, let $f\left(z ; \boldsymbol{\theta}_{1}, \boldsymbol{\theta}_{2}\right):=\hat{\boldsymbol{\theta}_{\mathbf{1}}}(z) \omega(z)+\hat{\boldsymbol{\theta}_{\mathbf{2}}}(z)$ for $\boldsymbol{\theta}_{1}, \boldsymbol{\theta}_{2} \in \mathrm{N} \otimes \mathbb{Q}$. Then the holomorphic function $f\left(z ; \boldsymbol{\theta}_{1}, \boldsymbol{\theta}_{2}\right)$ defines an element of $H^{0}\left(\mathcal{V}, \mathfrak{S}_{\lambda^{-1} H / \mathcal{V}}^{\log }\right)$ for a $\partial$-étale morphism $\lambda:\left[\mathcal{V}, \lambda^{-1} D\right] \rightarrow[\mathcal{U}, \mathcal{U} \cap D]$ associated with a finite index subgroup of N. Therefore,

$$
\left(\mathfrak{S}_{H / \underline{S}}^{\log }\right)_{P}=\left(\mathfrak{S}_{H / \underline{S}}^{\mathbf{W}}\right)_{P}+\sum_{\boldsymbol{\theta}_{1}, \boldsymbol{\theta}_{2} \in M \otimes \mathbb{Q}} \mathbb{Q}\left[f\left(z ; \boldsymbol{\theta}_{1}, \boldsymbol{\theta}_{2}\right)\right]
$$

as a subgroup of $\left(\underline{j}_{*} \mathfrak{S}_{H}\right)_{P}$. In the case the local monodromy of $H$ around $D$ is infinite near $P, \omega(z)=\hat{\boldsymbol{a}}(z)+h\left(\tilde{\mathrm{e}}_{\mathrm{N}}(z)\right)$ for some $0 \neq \boldsymbol{a} \in \sigma^{\vee} \cap \mathrm{M}$ and for some holomorphic function $h$ defined on $\mathcal{U}$. Let $\alpha$ be the maximal positive integer such that $\alpha^{-1} \boldsymbol{a}=\boldsymbol{u} \in \mathrm{M}$. We set $f(z ; n, \boldsymbol{\theta}):=(n / 2 \alpha) \omega(z)^{2}-(n / 2) \hat{\boldsymbol{a}}(z)+\hat{\boldsymbol{\theta}}(z)$ for $n \in \mathbb{Q}, \boldsymbol{\theta} \in \mathbf{N} \otimes \mathbb{Q}$. Then the holomorphic function $f(z ; n, \boldsymbol{\theta})$ on $\widetilde{\mathcal{U}^{\star}}$ defines an element of $\left(\mathfrak{S}_{H / \underline{S}}^{\log }\right)_{P}$ and moreover, we have

$$
\begin{aligned}
& \left(\mathfrak{S}_{H / \underline{S}}^{\log }\right)_{P}=\left(\mathfrak{S}_{H / \underline{S}}^{\mathbf{W}}\right)_{P}+\sum_{n \in \mathbb{Q}, \boldsymbol{\theta} \in \mathrm{M} \otimes \mathbb{Q}} \mathbb{Q}[f(z ; n, \boldsymbol{\theta})], \\
& \left(\mathfrak{S}_{H / \underline{S}}\right)_{P}=\left(\mathfrak{S}_{H / \underline{S}}^{\mathbf{W}}\right)_{P}+\sum_{\boldsymbol{\theta} \in \mathrm{N}\left(\boldsymbol{a}^{\perp \cap \sigma}\right)^{\perp} \otimes \mathbb{Q}} \mathbb{Q}[f(z ; 0, \boldsymbol{\theta})] .
\end{aligned}
$$

The homomorphism

$$
H^{0}\left(\underline{S}, \mathfrak{Q}_{H / \underline{S}}\right)=0 \oplus H^{0}\left(\underline{S}, \mathfrak{Q}_{H / \underline{S}}\right) \rightarrow H^{1}\left(\underline{S}, \mathfrak{S}_{H / \underline{S}}\right)
$$


induced from the triangle (6.8) is a connecting homomorphism of the exact sequence

$$
0 \rightarrow \mathfrak{S}_{H / \underline{S}} \rightarrow \mathfrak{S}_{H / \underline{S}}^{\log } \rightarrow \mathfrak{Q}_{H / \underline{S}} \rightarrow 0 .
$$

Two sheaves $\mathfrak{S}_{H / S}^{\log }$ and $\mathfrak{S}_{H / \underline{S}}^{\log }$ are related by

$$
\mathfrak{S}_{H / S}^{\log } \simeq \varepsilon_{*} \mathfrak{S}_{H / \underline{S}}^{\log }
$$

for the natural morphism $\varepsilon: \underline{S} \rightarrow S=(S, \emptyset)$.

Suppose that $S$ is a curve. Then the connecting homomorphism $\eta: H^{0}(S$, $\left.\mathfrak{Q}_{H / S} \otimes \mathbb{Q}\right) \rightarrow H^{1}\left(\underline{S}, \mathfrak{S}_{H / \underline{S}}\right)$ is considered to express logarithmic transformations [K8] as follows: Let $q \in H^{0}\left(\underline{S}, \mathfrak{Q}_{H / \underline{S}}\right) \simeq H^{0}\left(S, \mathfrak{Q}_{H / S} \otimes \mathbb{Q}\right)$ be an element supported only at a point $P \in D$. Let $\mathcal{U} \ni P$ be an open neighborhood $\mathcal{U} \simeq \boldsymbol{\Delta}$ such that $\mathcal{U}^{\star}=\mathcal{U} \cap S^{\star} \leadsto \boldsymbol{\Delta}^{\star}$. This is a toroidal embedding of type $(\mathrm{N}, l, \sigma)$ where $\mathbf{N}=\mathbb{Z}$ and $\sigma=\mathbb{R}_{\geq 0}$. The monodromy type of $H$ around $P$ is $\mathrm{I}_{a}$ for some integer $a \geq 0$.

In the case $a=0, q$ is represented by a holomorphic function $f(z)=$ $f\left(z ; \boldsymbol{\theta}_{1}, \boldsymbol{\theta}_{2}\right)$ for some rational numbers $\boldsymbol{\theta}_{1}, \boldsymbol{\theta}_{2} \in \mathrm{M} \otimes \mathbb{Q} \simeq \mathbb{Q}$. Let $m$ be a positive integer such that $m \boldsymbol{\theta}_{1}, m \boldsymbol{\theta}_{2} \in \mathrm{M}$. Let $\mathcal{V} \simeq \boldsymbol{\Delta} \ni u \mapsto u^{m} \in \boldsymbol{\Delta} \simeq \mathcal{U}$ be the cyclic covering of degree $m$ and let $\mathcal{V}^{\star}=\mathcal{V} \backslash\{0\}$. We have a morphism $\widetilde{\mathcal{U}^{\star}} \simeq$ $\mathbb{H} \ni z \mapsto \mathrm{e}(z / m) \in \mathcal{V}^{\star}$ as a universal covering map. Denoting $\underline{\mathcal{U}}:=(\mathcal{U},\{0\})$, $\underline{\mathcal{V}}:=(\mathcal{V},\{0\})$, we consider the single $\partial$-étale covering family $\{\underline{\mathcal{V}} \rightarrow \underline{\mathcal{U}}\}$. Then the image of $\eta(q)$ in $H^{1}\left(\underline{\mathcal{U}}, \mathfrak{S}_{H / \underline{S}}\right)$ is derived from a section of $H^{0}\left(\underline{\mathcal{V}} \times \underline{S} \underline{\mathcal{V}}, \mathfrak{S}_{H / \underline{S}}\right)$ corresponding to

$$
\begin{gathered}
\operatorname{sp}(\underline{\mathcal{V}} \times \underline{S} \underline{\mathcal{V}}) \simeq \mathbb{Z} / m \mathbb{Z} \times \mathcal{V} \ni(i, u) \mapsto f\left(z+i ; \boldsymbol{\theta}_{1}, \boldsymbol{\theta}_{2}\right)-f\left(z ; \boldsymbol{\theta}_{1}, \boldsymbol{\theta}_{2}\right) \\
=i \boldsymbol{\theta}_{1} \omega\left(u^{m}\right)+i \boldsymbol{\theta}_{2}
\end{gathered}
$$

This defines an action of $\mathbb{Z} / m \mathbb{Z}$ on $\boldsymbol{B} \times{ }_{S} \mathcal{V}$ by

$$
\boldsymbol{B} \times_{S} \mathcal{V} \ni(b, u) \longmapsto\left(\operatorname{tr}\left(\boldsymbol{\theta}_{1} \omega\left(u^{m}\right)+\boldsymbol{\theta}_{2}\right) b, \mathrm{e}(1 / m) u\right),
$$

where tr denotes the translation $\boldsymbol{B} \rightarrow \boldsymbol{B}$ by a section of $\boldsymbol{p}: \boldsymbol{B} \rightarrow S$. Let $X_{\mathcal{U}} \rightarrow \mathcal{U}$ be the quotient of $\boldsymbol{B} \times{ }_{S} \mathcal{V} \rightarrow \mathcal{V}$ by the action of $\mathbb{Z} / m \mathbb{Z}$. Then there is an isomorphism $\left.X_{\mathcal{U}}\right|_{\mathcal{U}^{\star}} \simeq \boldsymbol{B} \times{ }_{S} \mathcal{U}^{\star}$ given by

$$
(b, \mathrm{e}(z / m)) \longmapsto\left(\operatorname{tr}\left(-f\left(z ; \boldsymbol{\theta}_{1}, \boldsymbol{\theta}_{2}\right)\right) b, \mathrm{e}(z)\right) .
$$

Here, note that $f\left(z ; \boldsymbol{\theta}_{1}, \boldsymbol{\theta}_{2}\right)$ defines a section of $\boldsymbol{B} \times_{S} \mathcal{V}^{\star} \rightarrow \mathcal{V}^{\star}$. The elliptic fibration $\boldsymbol{B}^{\eta(q)} \rightarrow S$ corresponding to $\eta(q) \in H^{1}\left(\underline{S}, \mathfrak{S}_{H / \underline{S}}\right)$ is obtained by the gluing of $X_{\mathcal{U}}$ and $\boldsymbol{B} \times{ }_{S}(S \backslash\{P\})$ under the isomorphism above over $\mathcal{U}^{\star}$. 
Hence $\boldsymbol{B}^{\eta(q)} \rightarrow S$ is obtained by the logarithmic transformation associated with $f\left(z ; \boldsymbol{\theta}_{1}, \boldsymbol{\theta}_{2}\right)$.

In the case $a>0, q$ is represented by $f(z ; n, 0)$ for some $n \in \mathbb{Q}$. Let $m$ be the denominator of $n$. Let $\mathcal{V} \simeq \boldsymbol{\Delta} \ni u \mapsto u^{m} \in \boldsymbol{\Delta} \simeq \mathcal{U}$ be the cyclic covering of degree $m$ and let $\mathcal{V}^{\star}=\mathcal{V} \backslash\{0\}$. The $q$ is also represented by $f\left(z ; n, \theta_{n}\right)$ for $\theta_{n}:=-(1 / 2) n a(m-1)$. For the single $\partial$-étale covering family $\{\underline{\mathcal{V}}=(\mathcal{V},\{0\}) \rightarrow \underline{\mathcal{U}}=(\mathcal{U},\{0\})\}$, the image of $\eta(q)$ in $H^{1}\left(\underline{\mathcal{U}}, \mathfrak{S}_{H / \underline{S}}\right)$ is derived from a section of $H^{0}\left(\underline{\mathcal{V}} \times \underline{S} \underline{\mathcal{V}}, \mathfrak{S}_{H / \underline{S}}\right)$ corresponding to

$$
\begin{aligned}
\operatorname{sp}(\underline{\mathcal{V}} \times \underline{S} \underline{\mathcal{V}}) \simeq \mathbb{Z} / m \mathbb{Z} \times \mathcal{V} \ni(i, u) \mapsto f & \left(z+i ; n, \theta_{n}\right)-f\left(z ; n, \theta_{n}\right) \\
& =i n \omega(z)+n a \frac{i(i-1)}{2}+i \theta_{n}
\end{aligned}
$$

Let $\boldsymbol{B} \rightarrow \boldsymbol{\Delta}$ be the toric model [N4] associated with the period function $\omega(z)=$ $a z+h(t)$ and let $\mathcal{X} \rightarrow \boldsymbol{B}$ be the universal covering. Then $\left.\mathcal{X}\right|_{\boldsymbol{\Delta}^{\star}} \simeq \mathbb{C}^{\star} \times \boldsymbol{\Delta}^{\star}$ and the central fiber of $\mathcal{X} \rightarrow \Delta$ is a chain of infinitely many smooth rational curves. Let $s$ denote a coordinate of $\mathbb{C}^{\star}$. Then the quotient space of $\mathcal{X}$ by the action $s \mapsto s e(\omega(z))=s t^{a} \mathrm{e}(h(t))$ is the toric model $\boldsymbol{B}$. The section of $H^{0}\left(\underline{\mathcal{V}} \times_{\underline{S}} \underline{\mathcal{V}}, \mathfrak{S}_{H / \underline{S}}\right)$ above defines an action of $\mathbb{Z} / m \mathbb{Z}$ on $\boldsymbol{B} \times_{S} \mathcal{V}$ by

$$
\boldsymbol{B} \times_{S} \mathcal{V} \ni([s], u) \longmapsto\left(\left[s \mathrm{e}\left(n \omega(z)+\theta_{n}\right)\right], \mathrm{e}(1 / m) u\right),
$$

where $[s]$ denotes the image of $s \in \mathcal{X}$ in $\boldsymbol{B}$. Let $X_{\mathcal{U}} \rightarrow \mathcal{U}$ be the quotient of $\boldsymbol{B} \times{ }_{S} \mathcal{V} \rightarrow \mathcal{V}$ by the action of $\mathbb{Z} / m \mathbb{Z}$. Then there is an isomorphism $\left.X_{\mathcal{U}}\right|_{\mathcal{U}^{\star}} \simeq$ $\boldsymbol{B} \times{ }_{S} \mathcal{U}^{\star}$ given by

$$
(s, \mathrm{e}(z / m)) \longmapsto\left(\left[s \mathrm{e}\left(-f\left(z ; n, \theta_{n}\right)\right)\right], \mathrm{e}(z)\right) .
$$

The elliptic fibration $\boldsymbol{B}^{\eta(q)} \rightarrow S$ corresponding to $\eta(q) \in H^{1}\left(\underline{S}, \mathfrak{S}_{H / \underline{S}}\right)$ is obtained by the gluing of $X_{\mathcal{U}}$ and $\boldsymbol{B} \times{ }_{S}(S \backslash\{P\})$ under the isomorphism over $\mathcal{U}^{\star}$. Hence $\boldsymbol{B}^{\eta(q)} \rightarrow S$ is obtained by the logarithmic transformation associated with $f\left(z ; n, \theta_{n}\right)$.

Definition. For $q \in H^{0}\left(S, \mathfrak{Q}_{H / S} \otimes \mathbb{Q}\right)$, we define $L_{q}: H^{1}\left(\underline{S}, \mathfrak{S}_{H / \underline{S}}\right) \rightarrow$ $H^{1}\left(\underline{S}, \mathfrak{S}_{H / \underline{S}}\right)$ by $L_{q}(y)=y+\eta(q)$ for $\eta: H^{0}\left(S, \mathfrak{Q}_{H / S} \otimes \mathbb{Q}\right) \rightarrow H^{1}\left(\underline{S}, \mathfrak{S}_{H / \underline{S}}\right)$. We call $L_{q}$ the logarithmic transformation associated with $q$.

We still have the following problem related to 6.3.5.

Problem 7.5.2. $\quad$ For a marked elliptic fibration $(X \rightarrow S, \phi) \in \mathcal{E}(S, D, H)$ with the cohomology class $y \in H^{1}\left(\underline{S}, \mathfrak{S}_{H / \underline{S}}\right)$, is the logarithmic transform $L_{q}(y)=y+\eta(q)$ expressed by a marked elliptic fibration? 
Let $E \subset D$ be an analytic subset. We set $S^{\nabla}:=S \backslash E, \underline{S}^{\nabla}=\left(S^{\nabla}, D \cap S^{\nabla}\right)$. Suppose that $E$ contains the support of an element $q \in H^{0}\left(S, \mathfrak{Q}_{H / S} \otimes \mathbb{Q}\right)$. Then for any $y \in H^{1}\left(\underline{S}, \mathfrak{S}_{H / \underline{S}}\right)$, the difference $L_{q}(y)-y$ goes to zero by the restriction $H^{1}\left(\underline{S}, \mathfrak{S}_{H / \underline{S}}\right) \rightarrow H^{1}\left(\underline{S}^{\nabla}, \mathfrak{S}_{H / \underline{S}}\right)$. Therefore if both $y$ and $L_{q}(y)$ are contained in $\mathcal{E}(S, D, H)$, then for the representing elliptic fibrations $y=$ $(X \rightarrow S, \phi)$ and $L_{q}(y)=\left(X^{\prime} \rightarrow S, \phi^{\prime}\right)$, their restrictions are bimeromorphically equivalent over $S^{\nabla}$.

Let us consider the exact sequence

$H^{1}\left(S^{\star}, H\right) \rightarrow H^{1}\left(S, \mathcal{L}_{H / S}\right) \oplus H^{0}\left(S, \mathfrak{Q}_{H / S} \otimes \mathbb{Q}\right) \rightarrow H^{1}\left(\underline{S}, \mathfrak{S}_{H / \underline{S}}\right) \rightarrow H^{2}\left(S^{\star}, H\right)$

of 6.2.5. For $\mathfrak{K}=\mathbb{Z}, \mathbb{Q}, \mathbb{R}$, or $\mathbb{C}$, let $g_{\mathfrak{K}}$ denote the homomorphism $H^{1}\left(S^{\star}, H \otimes\right.$ $\mathfrak{K}) \rightarrow H^{1}\left(S, \mathcal{L}_{H / S}\right)$. Similarly, let $r_{\mathfrak{K}}$ denote the homomorphism $H^{1}\left(S^{\star}, H \otimes\right.$ $\mathfrak{K}) \rightarrow H^{0}\left(S, \mathfrak{Q}_{H / S} \otimes \mathfrak{K}\right)$. Recall that $C(H / S)$ is the image of $\left(g_{\mathbb{Z}}, r_{\mathbb{Z}}\right): H^{1}\left(S^{\star}, H\right)$ $\rightarrow H^{1}\left(S, \mathcal{L}_{H / S}\right) \oplus H^{0}\left(S, \mathfrak{Q}_{H / S}\right)$ and that $\bar{C}(H / S)$ is the image of $r_{\mathbb{Z}}$.

Proposition 7.5.3. Let $(f: X \rightarrow S, \phi)$ be a marked elliptic fibration with respect to $(S, D, H)$ with the cohomology class $y \in H^{1}\left(\underline{S}, \mathfrak{S}_{H / \underline{S}}\right)$. Let $q$ be an element of $H^{0}\left(S, \mathfrak{Q}_{H / S} \otimes \mathbb{Q}\right)$.

(1) Suppose that $X$ and $S$ are compact Kähler manifolds. Then $L_{q}(y)$ is represented by a marked elliptic fibration from a compact Kähler manifold if and only if $q$ is contained in $\bar{C}(H / S) \otimes \mathbb{Q}$.

(2) Suppose that $X$ and $S$ are non-singular projective varieties. Then $L_{q}(y)$ is represented by a marked elliptic fibration from a non-singular projective variety if and only if $q \in r_{\mathbb{Q}}\left(\operatorname{Ker} g_{\mathbb{Q}}\right)$.

Proof. (1) $L_{q}(y)$ is represented by a BCK morphism if and only if its image in $H^{2}\left(\underline{S}, \mathbb{L}_{H / \underline{S}}^{\bullet}\right)$ is torsion by 7.4.2. Since

$$
H^{1}\left(S^{\star}, H\right) \rightarrow H^{0}\left(S, \mathfrak{Q}_{H / S} \otimes \mathbb{Q}\right) \simeq H^{0}\left(\underline{S}, \mathfrak{Q}_{H / \underline{S}}\right) \rightarrow H^{2}\left(\underline{S}, \mathbb{L}_{H / \underline{S}}^{\bullet}\right)
$$

is exact, this is equivalent to $q$ is contained in $\bar{C}(H / S) \otimes \mathbb{Q}$.

(2) $L_{q}(y)$ is represented by a BP morphism if and only if $\eta(q)$ is a torsion element. The condition is equivalent to that $(0, q) \in H^{1}\left(S, \mathcal{L}_{H / S}\right) \oplus H^{0}\left(S, \mathfrak{Q}_{H / S}\right.$ $\otimes \mathbb{Q})$ is contained in $C(H / S) \otimes \mathbb{Q}$. In other words, $q$ is contained in the image of Ker $g_{\mathbb{Q}}$.

Let $\boldsymbol{p}: \boldsymbol{B} \rightarrow S$ be a basic elliptic fibration associated with $(S, D, H)$ such that $\boldsymbol{B}$ is non-singular and that $\boldsymbol{p}^{-1} D$ is a normal crossing divisor. Let $i: \boldsymbol{B}^{\star}=$ 
$\boldsymbol{B} \backslash \boldsymbol{p}^{-1} D \hookrightarrow \boldsymbol{B}$ be the open immersion and let us consider the mapping cone $\mathcal{O}_{B}^{\sharp}$ of the morphism $\tau_{\leq 2} R i_{*} \mathbb{Z}_{B^{\star}} \rightarrow \mathcal{O}_{B}$ (cf. 3.2.2 and 6.2.7). Similarly, we define $\mathcal{O}_{S}^{\sharp}$ to be the mapping cone of $\tau_{\leq 2} R j_{*} \mathbb{Z}_{S^{\star}} \rightarrow \mathcal{O}_{S}$.

Lemma 7.5.4. There is a natural quasi-isomorphism

$$
\tau_{\leq 1} R \boldsymbol{p}_{*} \mathcal{O}_{B}^{\sharp} \sim_{\text {qis }} \mathcal{O}_{S}^{\sharp} \oplus \mathfrak{S}_{H / S}^{\log }[-1] \oplus \mathbb{Z}_{S}[-1] .
$$

In particular,

$$
H^{1}\left(\boldsymbol{B}, \mathcal{O}_{\boldsymbol{B}}^{\sharp}\right) \simeq H^{1}\left(S, \mathcal{O}_{S}^{\sharp}\right) \oplus H^{0}\left(S, \mathfrak{S}_{H / S}^{\log }\right) \oplus \mathbb{Z} .
$$

Proof. We have a triangle

$$
\cdots \stackrel{+1}{\longrightarrow} \tau_{\leq 2}\left(R p_{*} R i_{*} \mathbb{Z}_{\boldsymbol{B}^{\star}}\right) \rightarrow R \boldsymbol{p}_{*} \mathcal{O}_{\boldsymbol{B}} \rightarrow \tau_{\leq 1} R \boldsymbol{p}_{*} \mathcal{O}_{\boldsymbol{B}}^{\sharp} \stackrel{+1}{\longrightarrow} \cdots
$$

The quasi-isomorphism is obtained by 6.2.6.

Remark. The cohomology group $H^{0}\left(\boldsymbol{B}, \mathcal{O}_{\boldsymbol{B}}^{\sharp}\right)$ is isomorphic to the group of meromorphic functions defined over $\boldsymbol{B}$ that are invertible on $\boldsymbol{p}^{-1} D(6.2 .7)$. When $\boldsymbol{B}$ is a projective variety, the cohomology group $H^{1}\left(\boldsymbol{B}, \mathcal{O}_{\boldsymbol{B}}^{\sharp}\right)$ is a DeligneBeilinson cohomology group $H_{\mathcal{D}}^{2}\left(\boldsymbol{B}^{\star}, \mathbb{Z}(1)\right)([\mathrm{B} 1],[\mathrm{E}])$.

\section{Lemma 7.5.5.}

(1) The triangle

$$
\cdots \stackrel{+1}{\longrightarrow} \tau_{\leq 2} R i_{*} \mathbb{Z}_{B^{\star}} \rightarrow \mathcal{O}_{B} \rightarrow \mathcal{O}_{B}^{\sharp} \stackrel{+1}{\longrightarrow} \cdots
$$

induces a triangle

$$
\cdots \stackrel{+1}{\longrightarrow} \tau_{\leq 2} R \boldsymbol{p}_{*} R i_{*} \mathbb{Z}_{\boldsymbol{B}^{\star}} \rightarrow R \boldsymbol{p}_{*} \mathcal{O}_{\boldsymbol{B}} \rightarrow \tau_{\leq 1} R \boldsymbol{p}_{*} \mathcal{O}_{B}^{\sharp} \stackrel{+1}{\longrightarrow} \cdots
$$

(2) The triangle

$$
\cdots \stackrel{+1}{\longrightarrow} \tau_{\leq 2} R i_{*} \mathbb{Z}_{\boldsymbol{B}^{\star}} \rightarrow \mathcal{O}_{\boldsymbol{B}} \oplus\left(\tau_{\leq 3} R \underline{\Gamma}_{\boldsymbol{p}^{-1} D}\left(\mathbb{Z}_{\boldsymbol{B}}\right)\right)[1] \rightarrow \mathcal{O}_{\boldsymbol{B}}^{\star} \stackrel{+1}{\longrightarrow} \cdots
$$

induces a triangle

$$
\begin{aligned}
\cdots \stackrel{+1}{\longrightarrow} \tau_{\leq 2} R \boldsymbol{p}_{*} R i_{*} \mathbb{Z}_{\boldsymbol{B}^{\star}} & \rightarrow R \boldsymbol{p}_{*} \mathcal{O}_{\boldsymbol{B}} \oplus\left(\tau_{\leq 3} R \underline{\Gamma}_{D}\left(\tau_{\leq 2} R \boldsymbol{p}_{*} \mathbb{Z}_{\boldsymbol{B}}\right)\right)[1] \\
& \rightarrow \tau_{\leq 1} R \boldsymbol{p}_{*} \mathcal{O}_{\boldsymbol{B}}^{\star} \stackrel{+1}{\longrightarrow} \cdots
\end{aligned}
$$


Proof. We have a triangle

$$
\cdots \stackrel{+1}{\longrightarrow}\left(\tau_{\leq 3} R \underline{\Gamma}_{\boldsymbol{p}^{-1} D}\left(\mathbb{Z}_{\boldsymbol{B}}\right)\right)[1] \rightarrow \mathcal{O}_{B}^{\star} \rightarrow \mathcal{O}_{\boldsymbol{B}}^{\sharp} \stackrel{+1}{\longrightarrow} \cdots,
$$

since $\tau_{\geq 1} \tau_{\leq 2} R i_{*} \mathbb{Z}_{\boldsymbol{B}^{\star}} \sim_{\text {qis }}\left(\tau_{\leq 3} R \underline{\Gamma}_{\boldsymbol{p}^{-1} D}\left(\mathbb{Z}_{\boldsymbol{B}}\right)\right)[1]$. By applying $R \boldsymbol{p}_{*}$ to $(7.9)$, we also have a triangle

$$
\cdots \stackrel{+1}{\longrightarrow} R p_{*}\left(\tau_{\leq 3} R \underline{\Gamma}_{p^{-1} D}\left(\mathbb{Z}_{\boldsymbol{B}}\right)\right)[1] \rightarrow R \boldsymbol{p}_{*} \mathcal{O}_{\boldsymbol{B}}^{\star} \rightarrow R \boldsymbol{p}_{*} \mathcal{O}_{\boldsymbol{B}}^{\sharp} \stackrel{+1}{\longrightarrow} \cdots
$$

Here the image of the homomorphism

$$
R^{1} \boldsymbol{p}_{*} \mathcal{O}_{\boldsymbol{B}}^{\star} \rightarrow R^{1} \boldsymbol{p}_{*} \mathcal{O}_{\boldsymbol{B}}^{\sharp} \simeq \mathcal{H}_{D}^{3}\left(\mathbb{Z}_{S}\right) \oplus \mathfrak{S}_{H / S}^{\log } \oplus \mathbb{Z}_{S}
$$

is $\mathfrak{S}_{H / S} \oplus \mathbb{Z}_{S}$ by 6.2.8. Thus its cokernel is isomorphic to

$$
\mathcal{H}_{D}^{3}\left(\mathbb{Z}_{S}\right) \oplus \mathfrak{Q}_{H / S} \subset \mathcal{H}^{3}\left(R \underline{\Gamma}_{D}\left(R p_{*} \mathbb{Z}_{\boldsymbol{B}}\right)\right),
$$

where $\mathcal{H}_{D}^{3}\left(\mathbb{Z}_{S}\right)={ }_{\text {II }} E_{\infty}^{3,0}, \mathfrak{Q}_{H / S}={ }_{\text {II }} E_{\infty}^{2,1}$ for the spectral sequence ${ }_{\text {II }} E_{2}^{p, q}$ in Section 5.4. Since ${ }_{\mathrm{II}} E_{2}^{1,2}=0$, the cokernel is the kernel of $\mathcal{H}^{3}\left(R \underline{\Gamma}_{D}\left(R \boldsymbol{p}_{*} \mathbb{Z}_{\boldsymbol{B}}\right)\right) \rightarrow$ $R^{3} \boldsymbol{p}_{*} \mathbb{Z}_{\boldsymbol{B}}$. Hence, we have another triangle

$$
\cdots \stackrel{+1}{\longrightarrow}\left(\tau_{\leq 3} R \underline{\Gamma}_{D}\left(\tau_{\leq 2} R \boldsymbol{p}_{*} \mathbb{Z}_{\boldsymbol{B}}\right)\right)[1] \rightarrow \tau_{\leq 1} R \boldsymbol{p}_{*} \mathcal{O}_{\boldsymbol{B}}^{\star} \rightarrow \tau_{\leq 1} R \boldsymbol{p}_{*} \mathcal{O}_{\boldsymbol{B}}^{\sharp} \stackrel{+1}{\longrightarrow} \cdots
$$

The triangle

$$
\cdots \stackrel{+1}{\longrightarrow} R \underline{\Gamma}_{D}\left(R \boldsymbol{p}_{*} \mathbb{Z}_{\boldsymbol{B}}\right) \rightarrow R \boldsymbol{p}_{*} \mathbb{Z}_{\boldsymbol{B}} \rightarrow R j_{*} R \boldsymbol{p}_{*}^{\star} \mathbb{Z}_{\boldsymbol{B}^{\star}} \stackrel{+1}{\longrightarrow} \cdots
$$

induces another triangle

$$
\cdots \stackrel{+1}{\longrightarrow} \tau_{\leq 3} R \underline{\Gamma}_{D}\left(\tau_{\leq 2} R \boldsymbol{p}_{*} \mathbb{Z}_{\boldsymbol{B}}\right) \rightarrow \tau_{\leq 2} R \boldsymbol{p}_{*} \mathbb{Z}_{\boldsymbol{B}} \rightarrow \tau_{\leq 2} R j_{*} R \boldsymbol{p}_{*}^{\star} \mathbb{Z}_{\boldsymbol{B}^{\star}} \stackrel{+1}{\longrightarrow} \cdots,
$$

since the image of

$$
\mathcal{H}^{2}\left(R j_{*} R \boldsymbol{p}_{*}^{\star} \mathbb{Z}_{\boldsymbol{B}^{\star}}\right) \simeq R^{2} j_{*} \mathbb{Z}_{S^{\star}} \oplus R^{1} j_{*} H \oplus \mathbb{Z}_{S} \rightarrow \mathcal{H}_{D}^{3}\left(R \boldsymbol{p}_{*} \mathbb{Z}_{\boldsymbol{B}}\right)
$$

is $\mathcal{H}_{D}^{3}\left(\mathbb{Z}_{S}\right) \oplus \mathfrak{Q}_{H / S}$. We have then triangles (7.7) and (7.8) by (7.10) and (7.11).

The cohomology group $H_{D}^{3}\left(S, \tau_{\leq 2} R p_{*} \mathbb{Z}_{\boldsymbol{B}}\right)$ is isomorphic to the kernel of $H_{\boldsymbol{p}^{-1} D}^{3}(\boldsymbol{B}, \mathbb{Z}) \rightarrow H^{3}(\boldsymbol{B}, \mathbb{Z})$. Here, we have an injection $H^{1}\left(S, \boldsymbol{p}_{*} \mathcal{H}_{\boldsymbol{p}^{-1} D}^{2}\left(\mathbb{Z}_{\boldsymbol{B}}\right)\right)$ $\rightarrow H_{D}^{3}\left(S, \tau_{\leq 2} R \boldsymbol{p}_{*} \mathbb{Z}_{\boldsymbol{B}}\right)$ from $E_{2}^{1,2} \rightarrow E^{3}$ for the spectral sequence

$$
E_{2}^{p, q}=H^{p}\left(S, \mathcal{H}^{q}\left(R \boldsymbol{p}_{*} R \underline{\Gamma}_{\boldsymbol{p}^{-1} D}\left(\mathbb{Z}_{\boldsymbol{B}}\right)\right)\right) \Longrightarrow E^{p+q}=H_{\boldsymbol{p}^{-1} D}^{p+q}(\boldsymbol{B}, \mathbb{Z}) .
$$

If $[S, D]$ satisfies the condition 3.2.6, by 5.4.7, we have another injection $H_{D}^{3}(S, \mathbb{Z})$ $\rightarrow H_{D}^{3}\left(S, \tau_{\leq 2} R \boldsymbol{p}_{*} \mathbb{Z}_{\boldsymbol{B}}\right) \subset H_{\boldsymbol{p}^{-1} D}^{3}(\boldsymbol{B}, \mathbb{Z})$. 
Definition. Suppose that $S$ is non-singular. We define an abelian group by

$$
\tilde{C}(\boldsymbol{B} / S):=\frac{H_{D}^{3}\left(S, \tau_{\leq 2} R \boldsymbol{p}_{*} \mathbb{Z}_{\boldsymbol{B}}\right)}{H^{1}\left(S, \boldsymbol{p}_{*} \mathcal{H}_{\boldsymbol{p}^{-1} D}^{2}\left(\mathbb{Z}_{\boldsymbol{B}}\right)\right)+H_{D}^{3}(S, \mathbb{Z})} .
$$

The triangles $(7.10)$ and $(7.11)$ induce homomorphisms $H^{1}\left(\boldsymbol{B}, \mathcal{O}_{\boldsymbol{B}}^{\sharp}\right) \rightarrow$ $H^{2}\left(\boldsymbol{B}^{\star}, \mathbb{Z}\right)$ and $H^{2}\left(\boldsymbol{B}^{\star}, \mathbb{Z}\right) \rightarrow \tilde{C}(\boldsymbol{B} / S)$, respectively.

Proposition 7.5.6. Suppose that $S$ is non-singular. Then there exists a natural injection $\tilde{C}(\boldsymbol{B} / S) \hookrightarrow H^{0}\left(S, \mathfrak{Q}_{H / S}\right)$. The image of $H^{2}\left(\boldsymbol{B}^{\star}, \mathbb{Z}\right) \rightarrow$ $\tilde{C}(\boldsymbol{B} / S) \subset H^{0}\left(S, \mathfrak{Q}_{H / S}\right)$ coincides with $\bar{C}(H / S)$. The image of $H^{1}\left(\boldsymbol{B}, \mathcal{O}_{\boldsymbol{B}}^{\sharp}\right) \rightarrow$ $\tilde{C}(\boldsymbol{B} / S) \subset H^{0}\left(S, \mathfrak{Q}_{H / S}\right)$ coincides with $r_{\mathbb{Z}}\left(\operatorname{Ker} g_{\mathbb{Z}}\right)$. In particular, the following conditions are equivalent:

(1) The image of $H^{1}\left(\boldsymbol{B}, \mathcal{O}_{\boldsymbol{B}}^{\sharp}\right) \rightarrow \tilde{C}(\boldsymbol{B} / S) \subset H^{0}\left(S, \mathfrak{Q}_{H / S}\right)$ is a finite index subgroup of $\bar{C}(H / S)$;

(2) The image of $H^{0}\left(S, \mathfrak{Q}_{H / S} \otimes \mathbb{Q}\right) \rightarrow H^{1}\left(\underline{S}, \mathfrak{S}_{H / \underline{S}}\right)$ is a torsion group;

(3) Any logarithmic transformation along D produces only a projective elliptic fibration.

Proof. We have triangle

$$
\begin{aligned}
\cdots \stackrel{+1}{\longrightarrow} \boldsymbol{p}_{*} \mathcal{H}_{\boldsymbol{p}^{-1} D}^{2}\left(\mathbb{Z}_{\boldsymbol{B}}\right)[-2] & \rightarrow \tau_{\leq 3} R \underline{\Gamma}_{D}\left(\tau_{\leq 2} R \boldsymbol{p}_{*} \mathbb{Z}_{\boldsymbol{B}}\right) \\
& \rightarrow\left(\mathcal{H}_{D}^{3}\left(\mathbb{Z}_{S}\right) \oplus \mathfrak{Q}_{H / S}\right)[-3], \stackrel{+1}{\longrightarrow} \cdots
\end{aligned}
$$

from the quasi-isomorphism $\tau_{\leq 2} R \underline{\Gamma}_{D}\left(\tau_{\leq 2} R \boldsymbol{p}_{*} \mathbb{Z}_{\boldsymbol{B}}\right) \sim_{\text {qis }} \tau_{\leq 2} R \boldsymbol{p}_{*} R \underline{\Gamma}_{\boldsymbol{p}^{-1} D}\left(\mathbb{Z}_{\boldsymbol{B}}\right)$ and an isomorphism $\mathcal{H}_{D}^{3}\left(\tau_{\leq 2} R \boldsymbol{p}_{*} \mathbb{Z}_{\boldsymbol{B}}\right) \simeq \mathcal{H}_{D}^{3}\left(\mathbb{Z}_{S}\right) \oplus \mathfrak{Q}_{H / S}$. Since $\mathbb{Z}_{S} \rightarrow R \boldsymbol{p}_{*} \mathbb{Z}_{\boldsymbol{B}}$ has a splitting, homomorphisms $H_{D}^{4}(S, \mathbb{Z}) \rightarrow H_{\boldsymbol{p}^{-1} D}^{4}(\boldsymbol{B}, \mathbb{Z})$ and $H^{2}\left(S, \mathcal{H}_{D}^{2}\left(\mathbb{Z}_{S}\right)\right)$ $\rightarrow H^{2}\left(S, \boldsymbol{p}_{*} \mathcal{H}_{\boldsymbol{p}^{-1} D}^{2}\left(\mathbb{Z}_{\boldsymbol{B}}\right)\right)$ are both injective. Thus we have an injection $\tilde{C}(\boldsymbol{B} / S)$ $\rightarrow H^{0}\left(S, \mathfrak{Q}_{H / S}\right)$. We have the decomposition $H^{2}\left(\boldsymbol{B}^{\star}, \mathbb{Z}\right) \simeq H^{2}\left(S^{\star}, \mathbb{Z}\right) \oplus H^{1}\left(S^{\star}\right.$, $H) \oplus H^{0}\left(S^{\star}, \mathbb{Z}\right)$. The composite $H^{2}\left(S^{\star}, \mathbb{Z}\right) \rightarrow \tilde{C}(\boldsymbol{B} / S)$ is zero since it factors $H_{D}^{3}(S, \mathbb{Z})$. A generator of $H^{0}\left(S^{\star}, \mathbb{Z}\right)$ comes from $c_{1}(\boldsymbol{\Sigma}) \in H^{2}(\boldsymbol{B}, \mathbb{Z})$. Thus the image of $H^{2}\left(\boldsymbol{B}^{\star}, \mathbb{Z}\right) \rightarrow \tilde{C}(\boldsymbol{B} / S)$ coincides with the image from $H^{1}\left(S^{\star}, H\right)$. This is $\bar{C}(H / S)$. The homomorphism $H^{1}\left(\boldsymbol{B}, \mathcal{O}_{\boldsymbol{B}}^{\sharp}\right) \rightarrow H^{2}\left(\boldsymbol{B}^{\star}, \mathbb{Z}\right)$ is written as the direct sum of $H^{1}\left(S, \mathcal{O}_{S}^{\sharp}\right) \rightarrow H^{2}\left(S^{\star}, \mathbb{Z}\right), H^{0}\left(S, \mathfrak{S}_{H / S}^{\log }\right) \rightarrow H^{1}\left(S^{\star}, H\right)$, and the identity $H^{0}(S, \mathbb{Z}) \rightarrow H^{0}\left(S^{\star}, \mathbb{Z}\right)$. Since

$$
H^{0}\left(S, \mathfrak{S}_{H / S}^{\log }\right) \rightarrow H^{1}\left(S^{\star}, H\right) \rightarrow H^{1}\left(S, \mathcal{L}_{H / S}\right)
$$

is exact, the image of $H^{2}\left(\boldsymbol{B}, \mathcal{O}_{\boldsymbol{B}}^{\sharp}\right) \rightarrow H^{0}\left(S, \mathfrak{Q}_{H / S}\right)$ is $r_{\mathbb{Z}}\left(\operatorname{Ker} g_{\mathbb{Z}}\right)$. 
Suppose that $S$ is a compact Kähler manifold. Then $H^{2}\left(\boldsymbol{B}^{\star}, \mathbb{Z}\right)$ has a mixed Hodge structure and $H^{2}\left(\boldsymbol{B}, \mathcal{O}_{\boldsymbol{B}}\right)$ is isomorphic to $\operatorname{Gr}_{F}^{0} H^{2}\left(\boldsymbol{B}^{\star}, \mathbb{C}\right)$ for the Hodge filtration $F$. Hence the image of $H^{1}\left(\boldsymbol{B}, \mathcal{O}_{\boldsymbol{B}}^{\sharp}\right) \rightarrow H^{2}\left(\boldsymbol{B}^{\star}, \mathbb{Z}\right)$ is $H^{2}\left(\boldsymbol{B}^{\star}, \mathbb{Z}\right) \cap F^{1} H^{2}\left(\boldsymbol{B}^{\star}, \mathbb{C}\right)$ and the kernel of $H^{1}\left(\boldsymbol{B}, \mathcal{O}_{\boldsymbol{B}}^{\sharp}\right) \rightarrow H^{2}\left(\boldsymbol{B}^{\star}, \mathbb{Z}\right)$ is generated by $\operatorname{Pic}^{0}(\boldsymbol{B})$.

Lemma 7.5.7. If $S$ is a compact Kähler manifold, then $r_{\mathbb{C}}\left(\operatorname{Ker} g_{\mathbb{C}}\right)=$ $\operatorname{Im} r_{\mathbb{C}}$

Proof. There is a splitting $H^{2}\left(\boldsymbol{B}^{\star}, \mathbb{C}\right)=\bigoplus I^{p, q}$ for vector spaces $I^{p, q}$ indexed by integers $p, q \geq 0$ with $p+q \geq 2$ such that

$$
\begin{aligned}
F^{k} H^{2}\left(\boldsymbol{B}^{\star}, \mathbb{C}\right) & =\bigoplus_{p \geq k} I^{p, q}, \quad W_{r} H^{2}\left(\boldsymbol{B}^{\star}, \mathbb{C}\right)=\bigoplus_{p+q \leq r} I^{p, q} \\
I^{p, q} & \equiv \overline{I^{q, p}} \quad \bmod \bigoplus_{p^{\prime}<p, q^{\prime}<q} I^{p^{\prime}, q^{\prime}}
\end{aligned}
$$

where $W$ stands for the weight filtration and $\bar{I}$ stands for the complex conjugate of $I$. We have $F^{3} H^{2}\left(\boldsymbol{B}^{\star}, \mathbb{C}\right)=H^{2}\left(B, F^{3} \Omega_{\boldsymbol{B}}^{\bullet}\left(\log \boldsymbol{p}^{-1} D\right)\right)=0$. The Leray spectral sequence

$$
E_{2}^{p, q}=H^{p}\left(\boldsymbol{B}, R^{q} i_{*} \mathbb{C}_{\boldsymbol{B}^{\star}}\right) \Longrightarrow H^{p+q}\left(\boldsymbol{B}^{\star}, \mathbb{C}\right)
$$

degenerates at $E_{3}$ and $\mathrm{Gr}_{q}^{W} H^{p+q}\left(\boldsymbol{B}^{\star}, \mathbb{C}\right) \simeq E_{3}^{2 p+q,-p}$ is a pure Hodge structure of weight $q$. Hence, $I^{p, q} \neq 0$ except for $(p, q)=(2,0),(1,1),(0,2),(2,1),(1,2)$, $(2,2)$. Thus $\operatorname{Gr}_{F}^{0} H^{2}\left(\boldsymbol{B}^{\star}, \mathbb{C}\right) \simeq I^{0,2}$ and Ker $g_{\mathbb{C}}=F^{1} H^{2}\left(\boldsymbol{B}^{\star}, \mathbb{C}\right)$. Further

$$
\operatorname{Ker} g_{\mathbb{C}} \rightarrow H^{2}\left(\boldsymbol{B}^{\star}, \mathbb{C}\right) / W_{2} H^{2}\left(\boldsymbol{B}^{\star} \mathbb{C}\right) \simeq I^{2,1} \oplus I^{1,2} \oplus I^{2,2}
$$

is surjective.

\section{§7.6. Logarithmic transformation on elliptic surfaces}

Proposition 7.6.1 (cf. [K5, 3.1 and 3.2]). Assume that $S$ is a nonsingular projective curve. Let $E$ be an elliptic curve and let $H$ be the trivial VHS $H=H^{1}(E, \mathbb{Z}) \otimes \mathbb{Z}_{S^{\star}}$. For a suitable isomorphism $H^{1}\left(E, \mathcal{O}_{E}\right) \simeq \mathbb{C}$, let $\Lambda$ be the image of $H^{1}(E, \mathbb{Z}) \rightarrow H^{1}\left(E, \mathcal{O}_{E}\right) \simeq \mathbb{C}$. Let us consider an element

$$
q \in H^{0}\left(S, \mathfrak{Q}_{H / S} \otimes \mathbb{Q}\right) \simeq H_{D}^{2}(S, \mathbb{Q}) \otimes \Lambda \simeq \operatorname{Div}_{D}(S, \mathbb{Q}) \otimes \Lambda .
$$

(1) The following conditions are equivalent: 
(1a) $\eta(q) \in H^{1}\left(\underline{S}, \mathfrak{S}_{H / \underline{S}}\right)=\mathcal{E}(S, D, H)$ represents a Kähler elliptic surface;

(1b) For the expression $q=\sum_{P \in D} q_{P} P$ for $q_{P} \in \Lambda \otimes \mathbb{Q}, \sum_{P} q_{P}=0$.

(2) The following conditions are equivalent:

(2a) $\eta(q) \in H^{1}\left(\underline{S}, \mathfrak{S}_{H / \underline{S}}\right)=\mathcal{E}(S, D, H)$ represents a projective elliptic surface;

(2b) There is a logarithmic 1 -form $\xi \in H^{0}\left(S, \Omega_{S}^{1}(\log D)\right)$ such that it is contained in $H^{1}\left(S^{\star}, \Lambda \otimes \mathbb{Q}\right)$ and that $q$ is its image under $H^{1}\left(S^{\star}, \Lambda \otimes\right.$ $\mathbb{Q}) \rightarrow H^{0}\left(S, R^{1} j_{*} \mathbb{Q}_{S^{\star}} \otimes \Lambda\right)$.

Proof. (1) (1a) is equivalent to that $q \in \bar{C}(H / S) \otimes \mathbb{Q}$. The assertion follows from the exact sequence

$$
H^{1}\left(S^{\star}, \mathbb{Z}\right) \rightarrow H^{0}\left(S, R^{1} j_{*} \mathbb{Z}_{S^{\star}}\right) \rightarrow H^{2}(S, \mathbb{Z}) \simeq \mathbb{Z} .
$$

(2) (2a) is equivalent to that $q \in r_{\mathbb{Q}}\left(\operatorname{Ker} g_{\mathbb{Q}}\right)$. Now $g$ is induced from $H^{1}\left(S^{\star}, \mathbb{Z}\right) \rightarrow H^{1}\left(S, \mathcal{O}_{S}\right)$ and $\Lambda \subset \mathbb{C}$ by the tensor product $H^{1}\left(S^{\star}, \mathbb{Z}\right) \otimes \Lambda \rightarrow$ $H^{1}\left(S, \mathcal{O}_{S}\right) \otimes_{\mathbb{C}} \mathbb{C}$. An element of Ker $g_{\mathbb{Q}}$ is represented by a logarithmic 1 -form $\xi \in H^{0}\left(S, \Omega_{S}^{1}(\log D)\right)$ such that $\xi \in H^{1}\left(S^{\star}, \mathbb{C}\right)$ is contained in $H^{1}\left(S^{\star}, \Lambda\right)$. Thus we are done.

Corollary 7.6.2 (cf. [K5, 4.2]). Under the same situation as 7.6.1, suppose that $S$ is isomorphic to the elliptic curve $E$ and $D=P_{1}+P_{2}$ for distinct two points. Suppose further $E$ has a complex multiplication. Then some $0 \neq q \in H^{0}\left(S, \mathfrak{Q}_{H / S} \otimes \mathbb{Q}\right)$ defines a projective elliptic surface if and only if $P_{1} \sim_{\mathbb{Q}} P_{2}$.

Proof. If $P_{1} \chi_{\mathbb{Q}} P_{2}$, then we have $H^{0}\left(S, \mathcal{O}_{S}(* D)^{\star}\right)=\mathbb{C}^{\star}$. Hence $H^{1}\left(S^{\star}\right.$, $\mathbb{Z}) \rightarrow H^{1}\left(S, \mathcal{O}_{S}\right)$ is injective. Now the image of $H^{1}\left(S^{\star}, \mathbb{Z}\right) \rightarrow H_{D}^{2}(S, \mathbb{Z})$ is of rank one. Let $\theta \in H^{1}\left(S^{\star}, \mathbb{Z}\right)$ be an element generating the image in $H_{D}^{2}(S, \mathbb{Z})$. Then the image of $H^{1}\left(S^{\star}, \mathbb{Z}\right) \rightarrow H^{1}\left(S, \mathcal{O}_{S}\right) \simeq \mathbb{C}$ is an abelian group of rank three generated by $\Lambda$ and $\theta$. Now $\Lambda_{\mathbb{Q}}=\Lambda \otimes \mathbb{Q}$ is a quadratic field. Hence $\emptyset=\theta \Lambda_{\mathbb{Q}} \cap \Lambda_{\mathbb{Q}} \subset \mathbb{C}$. Thus the kernel of $H^{1}\left(S^{\star}, \mathbb{Q}\right) \otimes \Lambda \rightarrow H^{1}\left(S, \mathcal{O}_{S}\right) \simeq \mathbb{C}$ is contained in $H^{1}(S, \mathbb{Q}) \otimes \Lambda$. Thus $r_{\mathbb{Q}}\left(\operatorname{Ker} g_{\mathbb{Q}}\right)=0$.

If $P_{1} \sim_{\mathbb{Q}} P_{2}$, then $H^{0}\left(S, \mathcal{O}_{S}(* D)^{\star}\right)$ contains a non-constant meromorphic function. Its image to $H_{D}^{2}(S, \mathbb{Z})$ is not zero. Thus we can find a non-zero element of $r_{\mathbb{Z}}\left(\operatorname{Ker} g_{\mathbb{Z}}\right)$.

Proposition 7.6.3. Let $\boldsymbol{p}: \boldsymbol{B} \rightarrow S$ be a modular elliptic surface (cf. $[\mathrm{S} 8])$ associated with a subgroup $\Gamma \subset \mathrm{SL}(2, \mathbb{Z})$ of finite index such that $-\mathrm{id} \notin \Gamma$, 
$\Gamma$ acts on $\mathbb{H}$ without fixed point, and the stabilizer of any cusp consists of unipotent matrices. Let $D \subset S$ be the set of cusps and let $H$ be the induced $V H S$ on $S^{\star}=S \backslash D$. Let $\mathfrak{G}_{k}(\Gamma)$ and $\mathfrak{S}_{k}(\Gamma)$, respectively, denote the spaces of (holomorphic) modular forms and cusp forms of weight $k$ with respect to $\Gamma$.

(1) $\mathfrak{G}_{k}(\Gamma) \simeq H^{0}\left(S, \mathcal{L}_{H / S}^{\otimes(-k)}\right)$ and $\mathfrak{S}_{k}(\Gamma) \simeq H^{0}\left(S, \mathcal{L}_{H / S}^{\otimes(-k)} \otimes \mathcal{O}_{S}(-D)\right)$;

(2) $\Omega_{S}^{1}(\log D) \simeq \omega_{S} \otimes \mathcal{O}_{S}(D) \simeq \mathcal{L}_{H / S}^{\otimes(-2)} . \quad$ In particular, $\mathfrak{G}_{2}(\Gamma) \simeq H^{0}(S$, $\left.\Omega_{S}^{1}(\log D)\right)$ and $\mathfrak{S}_{2}(\Gamma) \simeq H^{0}\left(S, \omega_{S}\right)$;

(3) For the mixed Hodge structure $H^{1}\left(S^{\star}, H\right)$, we have $F^{1}\left(H^{1}\left(S^{\star}, H\right)_{\mathbb{C}}\right) \simeq$ $\mathfrak{G}_{3}(\Gamma) ;$

(4) For the pure Hodge structure $H^{1}\left(S, j_{*} H\right)$ of weight two, its $(1,1)$-component is zero, (2,0)-component is isomorphic to $H^{0}\left(S, \omega_{S} \otimes \mathcal{L}_{H / S}^{-1}\right) \simeq \mathfrak{S}_{3}(\Gamma)$, and $(0,2)$-component is isomorphic to $H^{1}\left(S, \mathcal{L}_{H / S}\right)$.

Proof. (1) $\boldsymbol{p}$ is a natural compactification of $\Gamma \backslash(\mathbb{C} \times \mathbb{H}) \rightarrow \Gamma \backslash \mathbb{H}=S^{\star}$. For the universal covering mapping $\tau: \mathbb{H} \rightarrow S^{\star}, \mathcal{O}_{\mathbb{H}} \simeq \tau^{-1} \mathcal{L}_{H}$ is a $\Gamma$-linearized sheaf as follows:

$$
f^{\gamma}(z)=\left(c_{\gamma} z+d_{\gamma}\right) f(\gamma z), \quad \text { for } \gamma=\left(\begin{array}{ll}
a_{\gamma} & b_{\gamma} \\
c_{\gamma} & d_{\gamma}
\end{array}\right),
$$

where $f(z) \in H^{0}\left(\mathbb{H}, \mathcal{O}_{\mathbb{H}}\right)$ is a holomorphic function on $z \in \mathbb{H}$. Hence $H^{0}\left(S^{\star}\right.$, $\left.\mathcal{L}_{H}^{\otimes(-k)}\right)$ is the space of holomorphic functions $f(z)$ on $\mathbb{H}$ satisfying

$$
f(\gamma z)=\left(c_{\gamma} z+d_{\gamma}\right)^{k} f(z) .
$$

By the definition of $\mathcal{L}_{H / S}$ and by the unipotent property of $\Gamma$, we have the expected isomorphism.

(2) This is derived from the isomorphism $\Omega_{\mathbb{H}}^{1} \simeq \tau^{-1} \mathcal{L}_{H}^{\otimes(-2)}$ as $\Gamma$-linearized sheaves.

(3) The logarithmic de Rham complex $\Omega_{S}^{\bullet}(\log D) \otimes \mathcal{H}_{S}^{\text {can }}$ has a filtration

$$
\begin{aligned}
& F^{1}\left(\Omega_{S}^{\bullet}(\log D) \otimes \mathcal{H}_{S}^{\text {can }}\right)=\left[\cdots \rightarrow F^{1}\left(\mathcal{H}_{S}^{\text {can }}\right) \stackrel{\nabla_{S}}{\longrightarrow} \Omega_{S}^{1}(\log D) \otimes \mathcal{H}_{S}^{\text {can }} \rightarrow \cdots\right], \\
& F^{2}\left(\Omega_{S}^{\bullet}(\log D) \otimes \mathcal{H}_{S}^{\text {can }}\right)=\left[\cdots \rightarrow 0 \rightarrow \Omega_{S}^{1}(\log D) \otimes F^{1}\left(\mathcal{H}_{S}^{\text {can }}\right) \rightarrow \cdots\right] .
\end{aligned}
$$

Here $F^{1}\left(\mathcal{H}_{S}^{\text {can }}\right) \simeq \mathcal{L}_{H / S}^{-1}$. Now the composite $F^{1}\left(\mathcal{H}_{S}^{\text {can }}\right) \rightarrow \Omega_{S}^{1}(\log D) \otimes \mathcal{L}_{H / S}$ is injective since its restriction to $S^{\star}$ is a kind of Kodaira-Spencer mapping. By (2), it is an isomorphism. Thus $\operatorname{Gr}_{F}^{1}\left(\Omega_{S}^{\bullet}(\log D) \otimes \mathcal{H}_{S}^{\text {can }}\right) \sim_{\text {qis }} 0$. Hence

$$
\begin{aligned}
F^{1} H^{1}\left(S^{\star}, H_{\mathbb{C}}\right) & \simeq H^{1}\left(S, F^{1}\left(\Omega_{S}^{\bullet}(\log D) \otimes \mathcal{H}_{S}^{\text {can }}\right)\right) \\
& \simeq H^{0}\left(S, \Omega_{S}^{1}(\log D) \otimes \mathcal{L}_{H / S}^{-1}\right) \simeq \mathfrak{G}_{3}(\Gamma) .
\end{aligned}
$$


(4) The Leray spectral sequence

$$
E_{2}^{p, q}=H^{p}\left(S, R^{q} j_{*} H_{\mathbb{Q}}\right) \Longrightarrow H^{p+q}\left(S^{\star}, H_{\mathbb{Q}}\right)
$$

degenerates at $E_{3}$ and $E_{\infty}^{2 p+q,-p} \simeq \mathrm{Gr}_{1+q}^{W} H^{p+q}\left(S^{\star}, H_{\mathbb{Q}}\right)$ for the weight filtration $W$ of the mixed Hodge structure. Since the period of $H$ is non-constant, $H^{2}\left(S, j_{*} H\right) \simeq H^{2}\left(S, \mathbb{L}_{H / S}^{\bullet}\right)$ is a finite group by 4.2.5. Thus $\operatorname{Gr}_{2}^{W} H^{1}\left(S^{\star}, H_{\mathbb{Q}}\right) \simeq$ $H^{1}\left(S, j_{*} H_{\mathbb{Q}}\right)$ and $\operatorname{Gr}_{3}^{W} H^{1}\left(S^{\star}, H_{\mathbb{Q}}\right) \simeq H^{0}\left(S, R^{1} j_{*} H_{\mathbb{Q}}\right)$ and

$$
0 \rightarrow H^{1}\left(S, j_{*} H_{\mathbb{Q}}\right) \rightarrow H^{1}\left(S^{\star}, H_{\mathbb{Q}}\right) \rightarrow H^{0}\left(S, R^{1} j_{*} H_{\mathbb{Q}}\right) \rightarrow 0
$$

is an extension of mixed Hodge structures. Note that $\mathfrak{Q}_{H / S} \otimes \mathbb{Q} \simeq R^{1} j_{*} H_{\mathbb{Q}}$ in this case. We have $F^{1} H^{1}\left(S, j_{*} H_{\mathbb{C}}\right) \simeq F^{1} H^{1}\left(S^{\star}, H_{\mathbb{C}}\right) \cap H^{1}\left(S, j_{*} H_{\mathbb{C}}\right)$. From the residue isomorphism $\Omega_{S}^{1}(\log D) / \Omega_{S}^{1} \simeq \mathcal{O}_{D}$, we have a commutative diagram

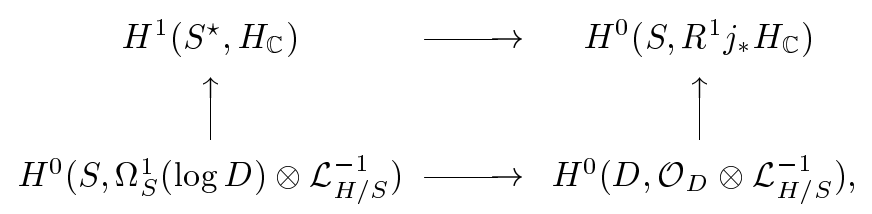

where the right vertical arrow is an isomorphism. Therefore,

$$
F^{1} H^{1}\left(S, j_{*} H_{\mathbb{C}}\right) \simeq H^{0}\left(S, \Omega_{S}^{1} \otimes \mathcal{L}_{H / S}^{-1}\right) \simeq \mathfrak{S}_{3}(\Gamma)
$$

and $\operatorname{Gr}_{F}^{0} H^{1}\left(S, j_{*} H_{\mathbb{C}}\right) \simeq \operatorname{Gr}_{F}^{0} H^{1}\left(S^{\star}, H_{\mathbb{C}}\right) \simeq H^{1}\left(S, \mathcal{L}_{H / S}\right)$. By the proof of $(3)$, we have $F^{1} H^{1}\left(S^{\star}, H_{\mathbb{C}}\right)=F^{2} H^{1}\left(S^{\star}, H_{\mathbb{C}}\right)$. Hence $F^{1} H^{1}\left(S, j_{*} H_{\mathbb{C}}\right)=F^{2} H^{1}(S$, $\left.j_{*} H_{\mathbb{C}}\right)$.

We recall some properties of the space of Eisenstein series. Let $N, k$ be integers greater than 2 and let $\Gamma(N)$ stand for the principal congruence modular group of level $N$. For $c, d \in \mathbb{Z}$, we consider the Eisenstein series

$$
G_{k}(z ; c, d ; N):=\sum_{\substack{(m, n) \equiv(c, d) \bmod N \\(m, n) \neq(0,0)}}(m z+n)^{-k},
$$

where $z \in \mathbb{H}$. Then $G_{k}(z ; c, d ; N)$ belongs to $\mathfrak{G}_{k}(\Gamma(N))$. Let $\mathcal{E}_{k}(N) \subset \mathfrak{G}_{k}(\Gamma(N))$ be the $\mathbb{C}$-subspace generated by the Eisenstein series. Then $\mathfrak{G}_{k}(\Gamma(N))=$ $\mathfrak{S}_{k}(\Gamma(N)) \oplus \mathcal{E}_{k}(N)$ and $\mathcal{E}_{k}(N)$ is the orthogonal complement of $\mathfrak{S}_{k}(\Gamma(N))$ with respect to the Petersson product. Hecke operators $T(n)$ with $(n, N)=1$ stabilize $\mathfrak{S}_{k}(\Gamma(N))$ and $\mathcal{E}_{k}(N)$ and are diagonalized in each spaces.

Lemma 7.6.4. $\quad A \mathbb{Q}$-vector subspace $\mathcal{E}_{k}(N)_{\mathbb{Q}} \subset \mathcal{E}_{k}(N)$ generates $\mathcal{E}_{k}(N)$ and the action of $T(n)$ with $(n, N)=1$ descends to $\mathcal{E}_{k}(N)_{\mathbb{Q}}$. 
Proof. The space of Dirichlet series associated with $\mathcal{E}_{k}(N)$ is generated by the series of the form $\left(t_{1} t_{2}\right)^{-s} L\left(\chi_{1}, s\right) L\left(\chi_{2}, s\right)$, where $t_{1}, t_{2} \mid N, \chi_{i}$ is the Dirichlet character modulo $N / t_{i}$ for $i=1,2, \chi_{1} \chi_{2}(-1)=(-1)^{k}, L(\chi, s)=$ $\sum_{n=1}^{\infty} \chi(n) n^{-s}$ (cf. [O4, Theorem 15]). Here, we have

$$
\begin{aligned}
\left(t_{1} t_{2}\right)^{-s} L\left(\chi_{1}, s\right) L\left(\chi_{2}, s\right) & =c \sum_{n=1}^{\infty} c(n) n^{-s} \\
c(n) & =\sum_{b_{i} \bmod N / t_{i}} \chi_{1}\left(b_{1}\right) \chi_{2}\left(b_{2}\right) a_{n}^{b_{1} t_{1}, b_{2} t_{2}}
\end{aligned}
$$

for $c \in \mathbb{Q}, a_{n}^{c, d} \in \mathbb{Z}$. Let

$$
f^{\chi_{1}, \chi_{2}}(z)=\sum_{n \geq 0} c(n) \mathrm{e}(n z / N)
$$

be the associated Eisenstein series. Then it is a common eigenfunction for $T(n)$ with $(n, N)=1$ and its eigenvalue $\lambda(n)=\lambda^{\chi_{1}, \chi_{2}}(n)$ satisfies $c(n)=c(1) \lambda(n)$. Let $U_{N} \subset \mathcal{E}_{k}(N)$ be the $\mathfrak{K}$-vector subspace of $\mathcal{E}_{k}(N)$ generated by $\left\{f^{\chi_{1}, \chi_{2}}\right\}$ for $\mathfrak{K}:=\mathbb{Q}(\mathrm{e}(1 / N))$. Then $\mathcal{E}_{k}(N) \simeq U_{N} \otimes_{\mathfrak{K}} \mathbb{C}$ and the action of $T(n)$ with $(n, N)=1$ descends to $U_{N}$. An element $\sigma \in \operatorname{Gal}(\mathfrak{K} / \mathbb{Q})$ acts on $U_{N}$ by

$$
\left(f^{\chi_{1}, \chi_{2}}\right)^{\sigma}(z)=\sum_{n \geq 0} c(n)^{\sigma} \mathrm{e}(n z / N)
$$

Then $\left(f^{\chi_{1}, \chi_{2}}\right)^{\sigma}=f^{\chi_{1}^{\sigma}, \chi_{2}^{\sigma}}$ for $\chi_{i}^{\sigma}(b)=\left(\chi_{i}(b)\right)^{\sigma}$. Hence $\left(\lambda^{\chi_{1}, \chi_{2}}(n)\right)^{\sigma}=\lambda^{\chi_{1}^{\sigma}, \chi_{2}^{\sigma}}(n)$. Thus $T(n)$ is commutative with the action of $\operatorname{Gal}(\mathfrak{K} / \mathbb{Q})$. Hence it descends to a rational structure.

Corollary 7.6.5. Let $\boldsymbol{p}: \boldsymbol{B} \rightarrow S$ be the modular elliptic surface associated with $\Gamma(N)$ for $N \geq 3$. Let $D \subset S$ be the set of cusps and let $H$ be the induced VHS on $S^{\star}=S \backslash D$. Then $\mathcal{E}_{3}(N) \subset \mathfrak{G}_{3}(\Gamma(N)) \subset H^{1}\left(S^{\star}, H_{\mathbb{C}}\right)$ is generated by a $\mathbb{Q}$-subspace of $H^{1}\left(S^{\star}, H_{\mathbb{Q}}\right)$. In particular, the exact sequence

$$
0 \rightarrow H^{1}\left(S, j_{*} H_{\mathbb{Q}}\right) \rightarrow H^{1}\left(S^{\star}, H_{\mathbb{Q}}\right) \rightarrow H^{0}\left(S, R^{1} j_{*} H_{\mathbb{Q}}\right) \rightarrow 0
$$

of mixed Hodge structures is split.

Proof. Hecke operators $T(n)$ acts on $H^{1}\left(S^{\star}, H\right) \simeq H^{1}\left(\Gamma(N), \mathbb{Z}^{\oplus 2}\right)$ compatibly with the inclusion $\mathfrak{G}_{3}(\Gamma(N)) \subset H^{1}\left(S^{\star}, H\right)_{\mathbb{C}}\left(\mathrm{cf}\right.$. [S7]). Thus $\mathcal{E}_{3}(N)_{\mathbb{Q}}$ satisfies the condition.

Theorem 7.6.6. Let $\boldsymbol{B} \rightarrow S$ be a modular elliptic surface associated with a subgroup $\Gamma \subset \mathrm{SL}(2, \mathbb{Z})$ of finite index such that $-\mathrm{id} \notin \Gamma$. Then any logarithmic transform only along singular fibers is a projective surface. 
Proof. Let $D \subset S$ be the set of points the fibers over which are singular, $j: S^{\star}=S \backslash D \subset S$ denote the immersion, and let $H$ be the induced VHS on $S^{\star}$. Then $\mathfrak{Q}_{H / S}$ is supported in points the singular fiber over which is of type $\mathrm{I}_{(+)}$.

There is an integer $N \geq 3$ with $\Gamma(N) \subset \Gamma$. Let $\boldsymbol{B}(N) \rightarrow S(N)$ be the elliptic modular surface associated with $\Gamma(N)$. Then $\boldsymbol{B}(N) \rightarrow S(N)$ is birational to the pullback of $\boldsymbol{B} \rightarrow S$ by the natural finite morphism $S(N) \rightarrow S$. An element $q \in H^{0}\left(S, \mathfrak{Q}_{H / S}\right)$ produces logarithmic transform $\boldsymbol{B}^{\eta(q)} \rightarrow S$ and $\boldsymbol{B}(N)^{\eta(q)} \rightarrow S(N)$. If $\boldsymbol{B}(N)^{\eta(q)}$ is projective, then so is $\boldsymbol{B}^{\eta(q)}$. Hence, we are reduced to the case $\Gamma=\Gamma(N)$. Then the assertion follows 7.5.3, 7.6.3 and 7.6.5.

Proposition 7.6.7. Let $\boldsymbol{p}: \boldsymbol{B} \rightarrow S$ be a minimal basic elliptic surface over a non-singular projective curve $S, D$ the set of points whose fibers are singular, and let $H$ be the induced VHS on $S^{\star}=S \backslash D$. Suppose that $H$ is non-trivial and $H^{1}\left(S, \mathcal{L}_{H / S}\right) \neq 0$. For a point $P \in S^{\star}$, we set $D(P):=D+P$, $S^{\star}(P):=S \backslash D(P)$, and $H(P):=\left.H\right|_{S^{\star}(P)}$. Then there exist a point $P \in S^{\star}$ and an element $q \in H_{P}^{0}\left(S, \mathfrak{Q}_{H(P) / S} \otimes \mathbb{Q}\right)$ such that $\eta(q) \in \mathcal{E}(S, D(P), H(P))$ represents an elliptic fibration not bimeromorphic to any projective surfaces.

Proof. There is a commutative diagram

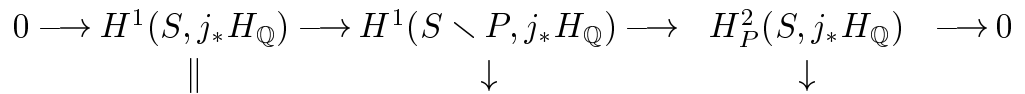

$$
\begin{aligned}
& 0 \longrightarrow H^{1}\left(S, j_{*} H_{\mathbb{Q}}\right) \longrightarrow H^{1}\left(S^{\star}(P), H_{\mathbb{Q}}\right) \longrightarrow H_{D(P)}^{2}\left(S, j_{*} H_{\mathbb{Q}}\right) \longrightarrow 0
\end{aligned}
$$

of mixed Hodge structures, where horizontal sequences are exact by $H^{2}(S$, $\left.j_{*} H_{\mathbb{Q}}\right)=0$ (cf. 4.2.5). We have $H_{P}^{0}\left(S, \mathfrak{Q}_{H(P) / S} \otimes \mathbb{Q}\right) \simeq H_{P}^{2}\left(S, j_{*} H_{\mathbb{Q}}\right)$. Note that $H^{1}\left(S, j_{*} H_{\mathbb{Q}}\right)$ and $H_{P}^{2}\left(S, j_{*} H_{\mathbb{Q}}\right)$ are pure Hodge structures of weight two and three, respectively. Let $I^{1,1}$ be the $(1,1)$-part of the pure Hodge structure $H^{1}\left(S, j_{*} H_{\mathbb{R}}\right)$. The intersection $I_{\mathbb{Q}}^{1,1}=I^{1,1} \cap H^{1}\left(S, j_{*} H_{\mathbb{Q}}\right)$ is a proper sub-Hodge structure of $H^{1}\left(S, j_{*} H_{\mathbb{Q}}\right)$. The $\eta(q)$ represents a projective surface if and only if the following sequence is split as mixed Hodge structures:

$$
0 \rightarrow H^{1}\left(S, j_{*} H_{\mathbb{Q}}\right) / I_{\mathbb{Q}}^{1,1} \rightarrow H^{1}\left(S \backslash P, j_{*} H_{\mathbb{Q}}\right) / I_{\mathbb{Q}}^{1,1} \rightarrow H_{P}^{2}\left(S, j_{*} H_{\mathbb{Q}}\right) \rightarrow 0 .
$$

Varying points $P$ in $S^{\star}$, we have exact sequences

$$
\begin{gathered}
0 \rightarrow H^{1}\left(S, j_{*} H\right) \otimes \mathbb{Q}_{S^{\star}} \rightarrow \tilde{H}_{\mathbb{Q}} \rightarrow H_{\mathbb{Q}} \rightarrow 0, \\
0 \rightarrow \frac{H^{1}\left(S, j_{*} H_{\mathbb{Q}}\right)}{I_{\mathbb{Q}}^{1,1}} \otimes \mathbb{Q}_{S^{\star}} \rightarrow \frac{\tilde{H}_{\mathbb{Q}}}{I_{\mathbb{Q}}^{1,1} \otimes \mathbb{Q}_{S^{\star}}} \rightarrow H_{\mathbb{Q}} \rightarrow 0,
\end{gathered}
$$


of admissible variations of mixed Hodge structures, where $\tilde{H}$ is defined as follows: Let $\pi_{1}: S \times S^{\star} \rightarrow S, \pi_{2}: S \times S^{\star} \rightarrow S^{\star}$ be projections and let $\Delta \subset S \times S^{\star}$ be the diagonal locus. We define

$$
\tilde{H}:=R^{1}\left(\pi_{2} \circ i\right)_{*}\left(\left.\pi_{1}^{-1}\left(j_{*} H\right)\right|_{S \times S^{\star} \backslash \Delta}\right),
$$

where $i: S \times S^{\star} \backslash \Delta \hookrightarrow S \times S^{\star}$ is the open immersion. The dual of the sequence (7.13) is an extension of the trivial variation $\left(H^{1}\left(S, j_{*} H_{\mathbb{Q}}\right) / I_{\mathbb{Q}}^{1,1}\right)^{\vee} \otimes \mathbb{Q}_{S^{\star}}$ of pure weight -2 by the variation $H_{\mathbb{Q}}^{\vee}$ of pure weight -3 . The extension is trivial if the stalks at all the points $P \in S^{\star}$ are trivial by [S2, 4.5]. Hence, it suffices to show that $(7.13)$ is not split on $S^{\star}$. We have a morphism

$$
H_{\mathbb{Q}} \rightarrow \frac{H^{1}\left(S, j_{*} H_{\mathbb{Q}}\right)}{I_{\mathbb{Q}}^{1,1}} \otimes \mathbb{Q}_{S^{\star}}[1] \rightarrow \frac{H^{1}\left(S^{\star}, H_{\mathbb{Q}}\right)}{I_{\mathbb{Q}}^{1,1}} \otimes \mathbb{Q}_{S^{\star}}[1]
$$

in the derived category from (7.13). It is enough to show (7.14) is not zero. The morphism is also obtained as follows: Let $R \Gamma_{c}\left(S^{\star}, H^{\vee}\right) \rightarrow R \Gamma\left(S^{\star}, H^{\vee}\right)$ be the natural morphism, where $\Gamma\left(S^{\star},-\right)$ denotes the functor taking global sections and $\Gamma_{c}\left(S^{\star},-\right)$ the functor taking global sections with compact support. It induces $R \Gamma_{c}\left(S^{\star}, H_{\mathbb{Q}}\right) \stackrel{\mathrm{L}}{\otimes} \mathbb{Q}_{S^{\star}} \rightarrow H_{\mathbb{Q}}^{\vee}$ as an evaluation map. We consider its dual $R \mathcal{H o m}{ }_{S}\left(-, \mathbb{Q}_{S^{*}}\right)$. By Verdier duality, we have

$$
\begin{gathered}
R \mathcal{H} \text { om }_{S^{\star}}\left(R \Gamma_{c}\left(S^{\star}, H_{\mathbb{Q}}^{\vee}\right) \stackrel{\mathrm{L}}{\otimes} \mathbb{Q}_{S^{\star}}, \mathbb{Q}_{S^{\star}}\right) \sim_{\text {qis }} R \operatorname{Hom}\left(R \Gamma_{c}\left(S^{\star}, H_{\mathbb{Q}}^{\vee}\right), \mathbb{Q}\right) \stackrel{\mathrm{L}}{\otimes} \mathbb{Q}_{S^{\star}} \\
\sim_{\text {qis }} R \Gamma\left(S^{\star}, R \mathcal{H} \text { om }\left(H_{\mathbb{Q}}^{\vee}, \omega_{S^{\star}}^{\text {top }} \stackrel{\mathrm{L}}{\otimes}\right)\right) \stackrel{\mathrm{L}}{\otimes} \mathbb{Q}_{S^{\star}} \sim_{\text {qis }} R \Gamma\left(S^{\star}, H_{\mathbb{Q}}\right) \otimes \mathbb{Q}_{S^{\star}}[2] .
\end{gathered}
$$

By 4.2.5, we have $R \Gamma\left(S^{\star}, H_{\mathbb{Q}}\right) \sim_{\text {qis }} H^{1}\left(S^{\star}, H_{\mathbb{Q}}\right)[-1]$. Hence the dual is the morphism $H_{\mathbb{Q}} \rightarrow H^{1}\left(S^{\star}, H_{\mathbb{Q}}\right) \otimes \mathbb{Q}_{S^{\star}}[1]$ related to 7.14 . The dual $R \operatorname{Hom}(-, \mathbb{Q})$ of $R \Gamma_{c}\left(S^{\star}, H^{\vee}\right) \rightarrow R \Gamma\left(S^{\star}, H^{\vee}\right)$ is nothing but the natural morphism

$$
R \Gamma_{c}\left(S^{\star}, H_{\mathbb{Q}}\right)[2] \sim_{\mathrm{qis}} H_{c}^{1}\left(S^{\star}, H_{\mathbb{Q}}\right)[1] \operatorname{to} R \Gamma\left(S^{\star}, H_{\mathbb{Q}}\right)[2] \sim_{\mathrm{qis}} H^{1}\left(S^{\star}, H_{\mathbb{Q}}\right)[1] .
$$

Since $H_{c}^{1}\left(S^{\star}, H_{\mathbb{Q}}\right) \rightarrow H^{1}\left(S^{\star}, H_{\mathbb{Q}}\right)$ is surjective and since $H^{1}\left(S, \mathcal{L}_{H / S}\right) \neq 0$, (7.14) is not zero. Thus we are done.

\section{References}

[A] Ash, A., Mumford, D., Rapoport, M. and Tai, Y., Smooth compactification of locally symmetric varieties, Lie groups: History, Frontiers and Applications vol. IV, Math. Sci. Press, 1975. 
[B1] Beilinson, A., Higher regulators and values of L-functions, J. Soviet Math., 30 (1985), 2036-2070.

[B2] Biswas, I., Parabolic bundles as orbifold bundles, Duke Math. J., 88 (1997), 305-325.

[C] Cox, D. A. and Zucker, S., Intersection numbers of sections of elliptic surfaces, Invent. Math., 53 (1979), 1-44.

[D1] Danilov, V., The geometry of toric varieties, Russian Math. Surveys, 33 (1978), 97-154.

[D2] Deligne, P., Équations Différentielles à Points Singuliers Réguliers, Lecture Notes in Math., 163, Springer-Verlag, 1970.

[D3] — Théorie de Hodge II, Publ. Math. I.H.E.S., 40 (1972), 5-57.

[D4] Doi, K. and Miyake, T., Automorphic forms and number theory (Japanese), Kinokuniya, 1976; English translation: T. Miyake, Modular Forms, Springer-Verlag, 1989.

[D5] Dolgachev, I. and Gross, M., Elliptic threefolds I: Ogg-Shafarevich theory, J. Alg. Geom., 3 (1994), 39-80.

[E] El Zein, F. and Zucker, S., Extendability of normal functions associated to algebraic cycles, in Topics in Transcendental Algebraic Geometry, Ann. of Math. Stud., 106, Princeton Univ. Press, 1984, pp. 269-288.

[F1] Flenner, H., Divisorenklassengruppen quasihomogener Singularitäten, J. Reine Angew. Math., 328 (1981), 128-160.

[F2] Fujiki, A., Closedness of the Douady spaces of compact Kähler spaces, Publ. RIMS. Kyoto Univ., 14 (1978), 1-52.

[F3] - Kählerian normal complex surfaces, Tôhoku Math. J., 35 (1983), 101-117.

[F4] Fujimoto, Y., Logarithmic transformations on elliptic fiber spaces; and II, J. Math. Kyoto Univ., 28 (1988), 91-110; Math. Ann., 288 (1990), 559-570.

[F5] Fujita, T., Zariski decomposition and canonical rings of elliptic threefolds, J. Math. Soc. Japan, 38 (1986), 19-37.

[G1] Goresky, M. and Macpherson, R., Intersection homology II, Invent. Math., 71 (1983), 77-129.

[G2] Grauert, H. and Remmert, R., Komplexe Räume, Math. Ann., 136 (1958), 245-318.

[G3] Griffiths, P., Periods of integrals on algebraic manifolds I, II and III, Amer. J. Math., 90 (1968), 568-626; ibid., 90 (1968), 805-865; Publ. Math. IHES., 38 (1970), 125-180.

[G4] Gross, M., Elliptic three-folds II: Multiple fibers, Trans. Amer. Math. Soc., 349 (1997), 3409-3468.

[G5] — A Aniteness theorem for elliptic Calabi-Yau threefolds, Duke Math. J., 74 (1994), 271-299.

[G6] Grothendieck, A., Le groupe de Brauer, I, II, III, in Dix Exposés sur la Cohomologie des Schémas, North-Holland, 1968, pp. 46-188.

[H1] Hironaka, H., Resolution of singularities of an algebraic variety over a field of characteristic zero, I, II, Ann. of Math., 79 (1964), 109-326.

[H2] — - Desingularization of complex-analytic varieties, Actes du Congrès International des Mathématiciens (Nice, 1970), 2, Gauthier-Villars, 1971, pp. 627-631.

[H3] - Flattening theorem in complex-analytic geometry, Amer. J. Math., 97 (1975), $503-547$.

[I] Iitaka, S., Algebraic Geometry, An Introduction to Birational Geometry of Algebraic Varieties, Grad. Text in Math., 76, Springer-Verlag, 1982.

[K1] Kawai, S., Elliptic fibre spaces over compact surfaces, Comment. Math. Univ. St. Paul., 15 (1967), 119-138.

[K2] Kawamata, Y., Kodaira dimension of certain algebraic fiber spaces, J. Fac. Sci. Univ. Tokyo, IA 30 (1983), 1-24.

[K3] —, Abundance theorem for minimal threefolds, Invent. Math., 108 (1992), 229246.

[K4] Kawamata, Y., Matsuda, K. and Matsuki, K., Introduction to the minimal model problem, in Algebraic Geometry Sendai 1985, Adv. Stud. Pure Math., 10, Kinokuniya and North-Holland, 1987, pp. 283-360.

[K5] Kawazoe, M., A criterion for algebraicity of certain analytic elliptic surfaces, Math. 
Ann., 303 (1995), 771-779.

[K6] Kempf, G., Knudsen, F., Mumford, D. and Saint-Donat, B., Toroidal Embeddings, Lecture Notes in Math., 339, Springer-Verlag, 1973.

[K7] Kodaira, K., On complex analytic surfaces II, III, Ann. of Math., 77 (1963), 563-626, ibid., 78 (1963), 1-40.

[K8] —— On the structure of compact complex analytic surfaces, I, Amer. J. Math., 86 (1964), 751-798.

[K9] Kollár, J., Higher direct images of dualizing sheaves II, Ann. of Math., 124 (1986), $171-202$.

[M1] Maruyama, M. and Yokogawa, K., Moduli of parabolic stable sheaves, Math. Ann., 293 (1992), 77-99.

[M2] Mehta, V. and Seshadri, C., Moduli of vector bundles on curves with parabolic structures, Math. Ann., 248 (1980), 205-239.

[M3] Milne, J., Étale cohomology, Princeton Math. Ser., 33, Princeton Univ. Press, 1980.

[M4] Miranda, R., Smooth models for elliptic threefolds, in The Birational Geometry of Degenerations, Progr. Math., 29, Birkhäuser, 1983, pp. 85-113.

[M5] Miyaoka, Y., Extension theorem for Kähler metrics, Proc. Japan Acad., 50 (1974), 407-410.

[M6] - Kähler metrics on elliptic surfaces, Proc. Japan Acad., 50 (1974), 533-536.

[M7] Mori, S., Threefolds whose canonical bundles are not numerically effective, Ann. of Math., 116 (1982), 133-176.

[M8] —_, Flip theorem and the existence of minimal models for 3-folds, J. AMS., 1 (1988), 117-253.

[M9] Moriwaki, A., Torsion freeness of higher direct images of canonical bundle, Math. Ann., 276 (1987), 385-398.

[N1] Nakamura, I., Relative compactification of the Néron model and its application, in Complex Analysis and Algebraic Geometry, Iwanami Shoten and Cambridge Univ. Press, 1977, pp. 207-225.

[N2] Nakayama, N., The lower semi-continuity of the plurigenera of complex varieties, in Algebraic Geometry Sendai 1985, Adv. Stud. Pure Math., 10, Kinokuniya and NorthHolland, 1987, pp. 551-590.

[N3] —_ On Weierstrass models, in Algebraic Geometry and Commutative Algebra in Honor of M. Nagata, Kinokuniya, 1987, pp. 405-431.

[N4] - Local structure of an elliptic fibration, Preprint RIMS Kyoto Univ. 1999, to appear in Adv. Stud. Pure Math.

[N5] ——, Global structure of an elliptic fibration, Preprint RIMS-1072 Kyoto Univ. 1996.

[N6] _ Z Zariski-decomposition and abundance, Preprint RIMS-1142 Kyoto Univ. 1997.

[N7] Néron, A., Modèles minimaux des variétés abéliennes sur les corps locaux et globaux, Publ. Math. IHES., 21 (1964).

[O1] Oda, T., Torus embeddings and applications (Based on joint work with Katsuya Miyake), Tata Inst. Fund. Res. 58, Springer-Verlag, 1978.

[O2] —_, Convex Bodies and Algebraic Geometry (Japanese), Kinokuniya 1985; English version: Convex Bodies and Algebraic Geometry - An Introduction to the Theory of Toric Varieties, Ergeb. Math. Grenzgeb. (3) 15, Springer-Verlag, 1988.

[O3] Ogg, A., Cohomology of abelian varieties over function fields, Ann. of Math., 76 (1962), 185-212.

[O4] ——, Modular Forms and Dirichlet Series, Benjamin, 1969.

[S1] Saito, M., Mixed Hodge modules, Publ. RIMS, Kyoto Univ., 26 (1990), 221-333.

[S2] - Hodge conjecture and mixed motives. I, in Complex geometry and Lie theory (Sundance, UT, 1989), Proc. Sympos. Pure Math., 53, Amer. Math. Soc. 1991, pp. 283303.

[S3] Schmid, W., Variation of Hodge structure: The singularities of the Period Mapping, Invent. Math., 22 (1973), 211-319.

[S4] Shafarevich, I., Principal homogeneous spaces over function fields, AMS Transl., 37 
(1964), 85-113.

[S5] Shatz, S., Profinite groups, Arithmetic, and Geometry, Ann. of Math. Stud. 67, Princeton Univ. Press, 1972.

[S6] Shimizu, H., Automorphic functions, I, II, III, (Japanese) Second edition, Iwanami Lectures on Fundamental Math. 8, Algebra, vii. Iwanami Shoten, 1984.

[S7] Shimura, G., Introduction to the arithmetic theory of automorphic functions, Publ. Math. Soc. Japan, No. 11, Iwanami Shoten and Princeton Univ. Press, 1971.

[S8] Shioda, T., On elliptic modular surfaces, J. Math. Soc. Japan, 24 (1972), 20-59

[T] Tate, J., On the conjecture of Birch and Swinnerton-Dyer and a geometric analog, Sem. Bourbaki 1965/66 n³06; also in Dix exposés sur cohomologie des schémas, NorthHolland, 1968, pp. 189-214.

[U1] Ueno, K., Classification of algebraic varieties, I, Compo. Math., 27 (1973), 277-342.

[U2] - Degeneration of elliptic surfaces and certain non-Kähler manifolds, in Classification of algebraic and analytic manifolds, Progr. Math., 39, Birkhäuser, 1983, pp. 545-556.

[V1] Varouchas, J., Stabilité de la classe des variétés kähleriennes pour les certains morphisms propres, Invent. Math., 77 (1984), 117-128.

[V2] Verdier, J.-L., Dualité dans la cohomologie des espaces localement compacts, Séminaire Bourbaki, 18e année 65/66, no. 300.

[Z] Zucker, S., Hodge theory with degenerating coefficients: $L_{2}$ cohomology in the Poincaré metric, Ann. of Math., 109 (1979), 415-476. 